\title{
Genetic influences on structural and functional brain maturation
}

\author{
Jalmar Teeuw
}





\section{GENETIC INFLUENCES ON STRUCTURAL AND FUNCTIONAL BRAIN MATURATION}

Jalmar Teeuw 
Genetic influences on structural and functional brain maturation

Research in this thesis was supported by the Netherlands Organization for Scientific Research Grants NWO 433-09-220 (to H.E.H.P.), NWO 51.02.060 (to H.E.H.P.), 668.772 (to D.I.B.); NWOMagW 480-04-004 (to D.I.B.); and NWO/SPI 56-464-14192 (to D.I.B.); the European Research Council Grant ERC-230374 (to D.I.B.).

Print: Gildeprint · www.gildeprint.nl

Cover: Photo by Grigory Bruev (iStock Photo)

Design by Jalmar Teeuw

ISBN: $\quad 978-90-393-7347-7$

Copyright (c) Jalmar Teeuw, 2020

All rights reserved. No part of this thesis may be reproduced or transmitted in any form or by any means, without prior written permission from the author. The copyrights of articles that have been published have been transferred to the respective journal. 


\title{
Genetic influences on structural and functional brain maturation
}

\author{
Genetische invloeden op structurele en \\ functionele ontwikkeling van de hersenen \\ (met een samenvatting in het Nederlands)
}

\section{Proefschrift}

ter verkrijging van de graad van doctor aan de Universiteit Utrecht op gezag van de rector magnificus, prof.dr. H.R.B.M. Kummeling, ingevolge het besluit van het college voor promoties in het openbaar te verdedigen op

maandag 30 november 2020 des middags te 2.30 uur

door

\section{Jalmar Teeuw}

geboren op 21 december 1987

te SCHIEDAM 


\section{Promotoren:}

Prof. dr. H.E. Hulshoff Pol

Prof. dr. D.I. Boomsma

\section{Copromotor:}

Dr. R.M. Brouwer 


\section{TABLE OF CONTENTS}

Chapter $1 \quad$ General introduction 7

Chapter $2 \quad$ Longitudinal development of cerebral cortical thickness 27

Chapter $3 \quad$ Reliability modelling of resting-state functional connectivity 55

Chapter $4 \quad$ Longitudinal development of cortical resting-state networks $\quad 75$

$\begin{array}{lll}\text { Chapter } 5 & \text { Progressive aging in schizophrenia } & 107\end{array}$

$\begin{array}{lll}\text { Chapter } 6 & \text { Highlights and general discussion } & 123\end{array}$

$\begin{array}{lll}\text { Appendices } & \text { Supplementary information to chapter 2 } & 139\end{array}$

Supplementary information to chapter $3 \quad 155$

Supplementary information to chapter 4

Supplementary information to chapter 5

$\begin{array}{ll}\text { References } & 273\end{array}$

$\begin{array}{ll}\text { Nederlandse samenvatting } & 319\end{array}$

$\begin{array}{ll}\text { Dankwoord } & 327\end{array}$

List of publications $\quad 333$

$\begin{array}{ll}\text { Curriculum vitae } & 339\end{array}$ 



\section{CHAPTER 1}

GENERAL INTRODUCTION 


\subsection{GENERAL INTRODUCTION}

Adolescent development is marked by significant transitions in behavioral, physical and physiological characteristics in mammals, including humans (Sawyer et al., 2018; Spear, 2011). Often associated by hormonal and physical changes with the onset of puberty typically around the age of 11 years in humans (Paula Abreu and Kaiser, 2016; Fredriks et al., 2000; Koenis et al., 2013), adolescence is characterized by changes in social, affective, and cognitive skills (Spear, 2000; Steinberg and Morris, 2001; Steinberg, 2005). Adolescence is associated with advances in critical thinking, the development of self-identity and morality, and attachment to peers in a transition to become an independent person, but can be accompanied with behavioral problems such as increased risk-taking, impulsivity, delinquency, and substance use (Spear, 2000; Steinberg and Morris, 2001; van Duijvenvoorde et al., 2016). These behavioral and physical changes during adolescence coincide with vast changes in the brain that continues to mature well into early adulthood (Durston et al., 2001; Peper et al., 2011; Peper et al., 2009; Brouwer et al., 2015; Lenroot and Giedd, 2006; Blakemore, 2012). Adolescence is also a period during which psychiatric disorders, such as schizophrenia, which are characterized by aberrant brain development, typically find their onset (Zahn-Waxler et al., 2008; Paus et al., 2008; de Wit et al., 2016; Smieskova et al., 2010; Rapoport et al., 2012). This heterogeneity in the many aspects of childhood and adolescent development results in considerable interindividual variation in developmental trajectories and outcome, that it has raised the question "why some children thrive and others don't"'.

An understanding of what motivates adolescent behavior or what causes aberrant development that eventually culminates in the onset of psychiatric disorders remains elusive. The field of imaging genetics combines methods from neuroimaging, such as magnetic resonance imaging, and genetics, such as twin studies and genome-wide association studies, to try to understand the role of the brain in the relation between genes, environment, and complex traits (Baaré et al., 2001; Hulshoff Pol et al., 2006; Glahn et al., 2007). Many structural and functional properties of the human brain are heritable (Peper et al., 2007; Jansen et al., 2015). Genetic influences may vary with age and also drive changes in the brain (i.e. brain plasticity; Pfefferbaum et al., 2004; Brans et al., 2008; van Soelen et al., 2012; Lessov-Schlaggar et al., 2012; van Soelen et al., 2013; Brouwer et al., 2014; Bootsman et al., 2015; Hedman et al., 2016; Brouwer et al., 2017; Brouwer et al., 2020). The expression of genes is highly regulated, especially during the early developmental stages (Kang et al., 2011; Jaffe et al., 2015). Environmental influences play a role in regulating gene expression, for example through epigenetic modifications (Jaenisch and Bird, 2003). This

\footnotetext{
${ }^{1}$ Consortium on Individual Development; https://individualdevelopment.nl/
} 
dynamic interplay between genetic and environmental influences on the brain is believed to explain why monozygotic twins with the same genetic background can be discordant for highly heritability disorders such as schizophrenia (Smith, 1970; van Os et al., 2008; van Nierop et al., 2013); and might also explain why some children thrive during adolescence and others don't.

In this dissertation, I continue the investigation into the developmental patterns of the brain in a longitudinal study of adolescent twins and look into the extent to which genes and the environment influence these patterns. Specifically, I investigate the development of grey matter thickness of the cerebral cortex and the development of functional connectivity within and between canonical cortical networks. In addition, I investigate the genetic and epigenetic contributions to accelerated brain maturation in patients with schizophrenia. This chapter provides an introduction to the topics of this study and concludes with an overview of the research questions that will be addressed in the subsequent chapters.

\subsection{MAGNETIC RESONANCE IMAGING OF THE BRAIN}

Magnetic resonance imaging (MRI) is a non-invasive imaging technology and is one of several techniques that can be used to study the brain (Kennedy et al., 2002; Ernst and Mueller, 2008; Dale et al., 2015). The brain can be imaged with MRI using several imaging contrasts that each capture different structural or functional properties of the brain (Figure 1.1).

\subsubsection{Brain structural MRI}

The morphology of the brain can be studied with high resolution $(<1 \mathrm{~mm})$ structural $\mathrm{T}_{1^{-}}$ weighted MRI (Figure 1.1A). Typically, image contrast is optimized to provide the best possible separation between the three major tissue types in the brain: gray matter, white matter, and cerebrospinal fluid (Symms et al., 2004; Heidemann et al., 2003). Advanced software tools are then applied to the images to produce tissue maps with estimates on the proportion of the tissue types within each voxel (Despotovic et al., 2015; e.g. Brouwer et al., 2010). After annotating the voxels with their corresponding brain structure, either manually, through projection to an atlas (Cabezas et al., 2011), or by automated delineation (e.g. Fischl et al., 2002; Patenaude et al., 2011), gray and white matter density and volumetric measures of the brain structures can be estimated. The tissue maps can be processed further to obtain estimates on the thickness, volume, and surface area of the cortex (e.g. Fischl et al., 2000; Fischl et al., 2004; Kim et al., 2005).

\subsubsection{Brain diffusion MRI}

The white matter structure of the brain can be studied more in-depth with diffusion-weighted MRI (Figure 1.1B). Diffusion imaging captures the Brownian motion of the protons in the brain (Beaulieu, 2002; Johansen-Berg and Behrens, Academic Press 2009). A diffusion tensor 


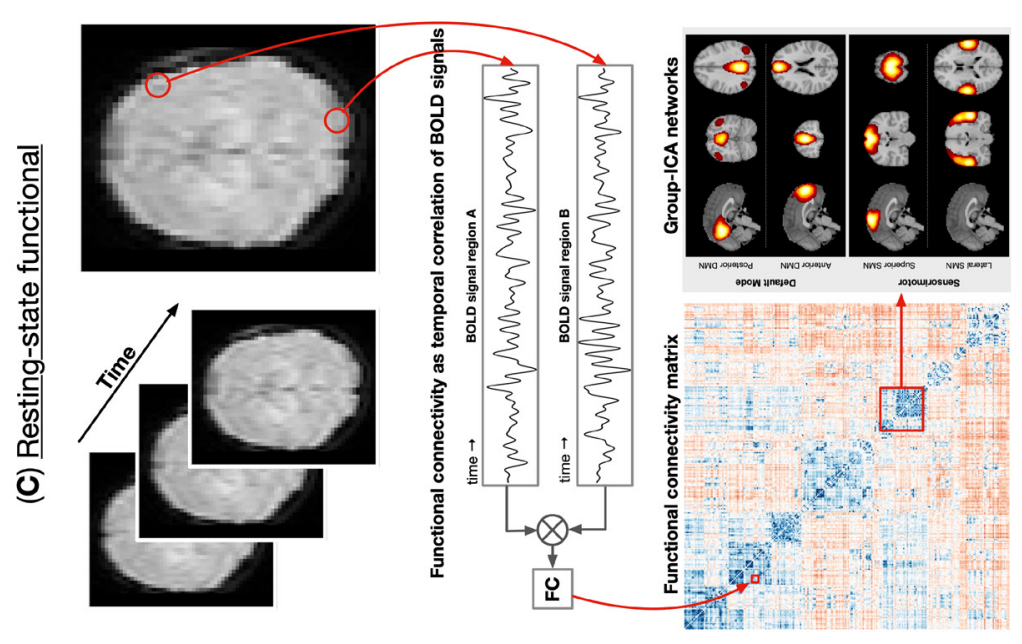

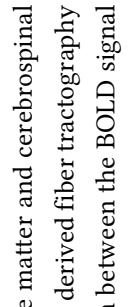

节志莺

矛范

घ वี

के क्षे छ

.ำ 코

흉 శ్రే:
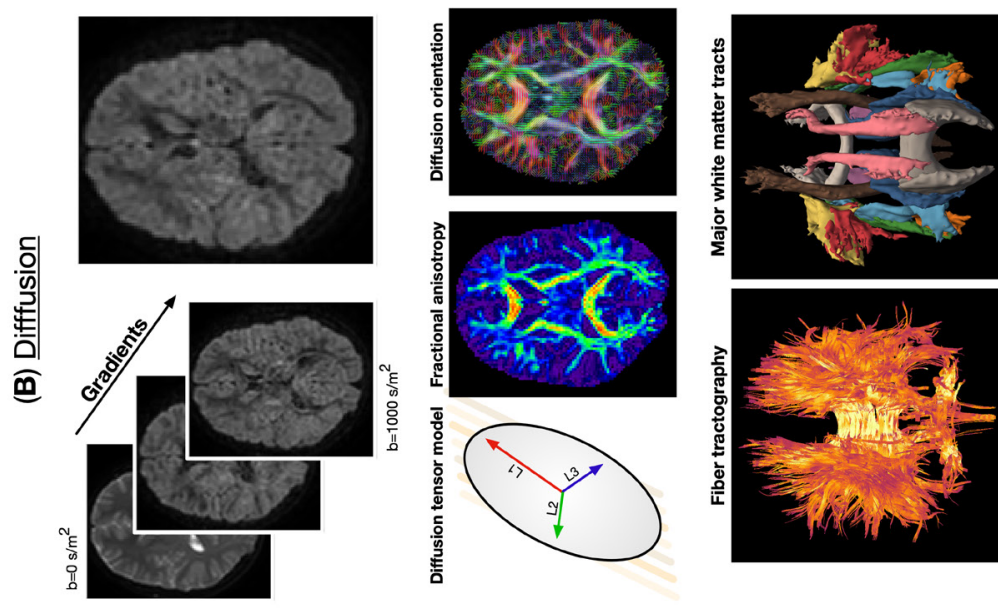

สิ

झ

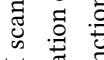

密完总

प्ञ त त

चี तु

承
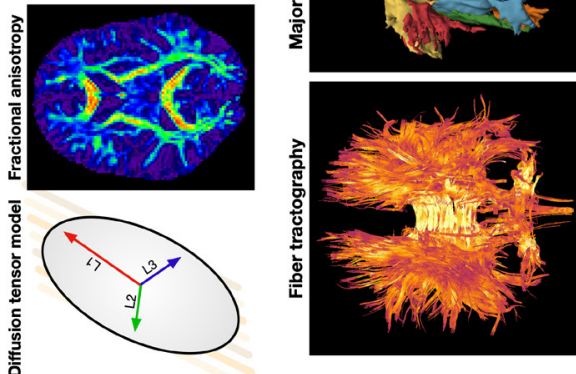

줄

苍

के

苾

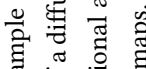

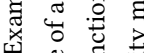

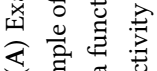

घ 㸚

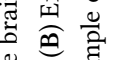
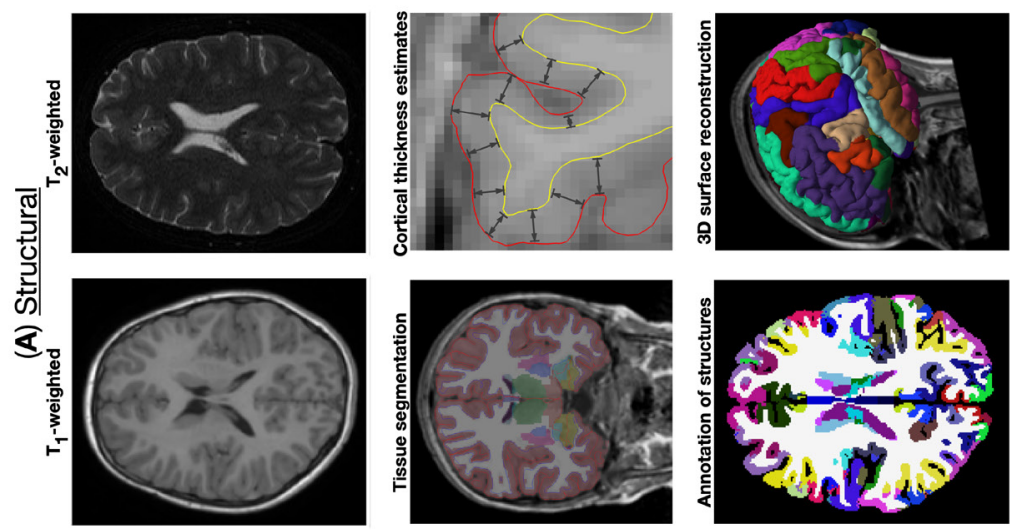

㟧芯

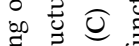

की

寻.

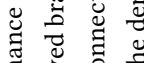

ฮี
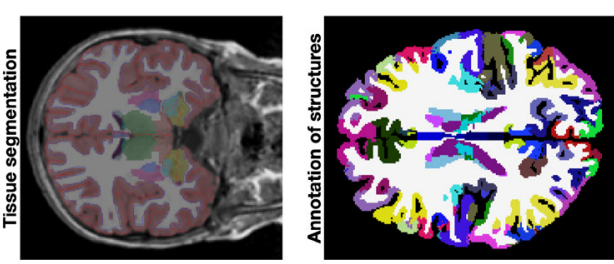

ठ

氙

矛

$\sum \sum^{\infty}$

-

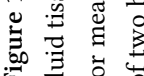


model can be applied to the data to describe the overall diffusion profile (Le Bihan et al., 2001; Mori and Zhang, 2006). Several local white matter structural integrity measures, such as fractional anisotropy (FA), mean diffusivity (MD) and radial diffusivity (RD), can be obtained from the diffusion tensor model at each voxel (Basser and Pierpaoli, 1996). Fractional anisotropy measures the directionality of diffusion and is associated with the degree of myelination and axon density in the brain (Friedrich et al., 2020; Chang et al., 2017). Parameters from the diffusion tensor model can also be used to trace the white matter pathways of the brain using fiber tractography (Basser et al., 2000), and provides a measure for structural connectivity between brain regions (Bullmore and Sporns, 2009).

\subsubsection{Brain functional MRI}

The activity of the brain can be measured with functional MRI (fMRI; Figure 1.1C). Active neurons consume glucose and oxygen that is transported by the vascular system (Watts et al., 2018). This process can be measured through changes in the blood-oxygenation level of veins surrounding brain tissue over time, and represents an indirect measure of neuronal activity both spatially and temporally (Logothetis, 2001; Logothetis and Wandell, 2004). In general, there are two types of functional MRI paradigms that are commonly used to study brain activity: task-based and resting-state. Task-based fMRI is recorded when the subjects are involved in a task; e.g. a motor task or gambling task (Soares et al., 2016; Bandettini, 2020). The correspondence between the measured BOLD response and the expected response to the task is used as an indication for the involvement of a brain region in the specific task (Worsley et al., 2002). However, the brain is alway active, even when "at rest" (Biswal et al., 1995; Tomasi et al., 2013; Kuzawa et al., 2014). In contrast to task-based fMRI, resting-state $\mathrm{fMRI}$ is the recording of the BOLD signal in the absence of any active task (Biswal et al., 1995; Fox and Riachle, 2007; Bijsterbosch et al., 2017). The absence of any task makes resting-state fMRI suitable across the entire age range and different patient populations. Function connectivity (FC) between two regions of the brain can be obtained by temporal correlation of the activity of those regions measured by the BOLD signal (Biswal et al., 1995; van den Heuvel and Hulshoff Pol, 2010). Head motion is more pronounced in children and may lead to biased estimation of results of long-distance connectivity when not properly controlled for (Power et al., 2012; Satterthwaite et al., 2012; van Dijk et al., 2012). Head motion can be countered by a simulation training session in a mock scanner or by (passive) viewing of a naturalistic video (Durston et al., 2009; Vanderwal et al., 2015). Nevertheless, data-driven methods have revealed functional networks of regions that are more strongly connected with each other than with regions of other networks and that show overlap with task-evoked activity (Beckmann et al., 2005; Damoiseaux et al., 2006; Yeo et al., 2011; Smith et al., 2009; Cole et al., 2014). These brain networks are already present in the second trimester of pregnancy and appear "adult-like" 
by the age of 2 years (Turk et al., 2019; Gao et al., 2015). Most focus has been on the defaultmode network of the brain (Raichle et al., 2001). This network consists of anterior and posterior regions of the brain that become more active during "rest", and has been associated with self-reflection and has been implicated in several disorders (AndrewsHanna et al., 2011; Whitfield-Gabrieli and Ford, 2012). Although there is no clear consensus on the number of networks in the brain, other established networks include the visual network, sensorimotor network, language network, frontoparietal networks, dorsal- and ventral attention networks, limbic network, and cerebellar network that have been associated with cognition and psychiatric- and neurological disorders (Damoiseaux et al., 2006; Rosazza and Minati, 2011; van den Heuvel and Hulshoff Pol, 2010; Menon, 2011).

\subsubsection{Reliable measurements}

To be able to determine the neural correlates of human behavior, brain and behavioral, variables need to be measured accurately and reliably. The relatively low sample sizes of most neuroimaging studies have impacted the fields of neuroimaging with poor reproducibility and possible false positive reports (Poldrack et al., 2017; Schnack, 2019; Button et al., 2013; Vul et al., 2009). The reliability of a measure is often determined by the intraclass correlation on repeated assessment of the measures (Chen et al., 2018). Although structural brain measures are often quite reliable (ICC $>0.8$; Nugent et al., 2012; Liem et al., 2015; Madan et al., 2017), their association with behavior can still fail replication due to sampling bias, variations in methodology, or incorrect statistics (Button et al., 2013; Boekel et al., 2015). In contrast, resting-state functional brain measures tend to have poor testretest reliability (ICC 0.3; Noble et al., 2019) and generally low sample sizes (Poldrack et al., 2017), that it is not surprising that larger studies fail to replicate previous findings (Button et al., 2013; Kruschwitz et al., 2018). Poor test-retest reliability can have many causes. For resting-state functional MRI in particular, the presence of confounding signals from non-neural origin, such as head motion, cardiovascular and respiratory system, account for at least half of the variation in the BOLD signal (Power et al., 2012; Bianciardi et al., 2009). Although many methods have been proposed to get rid of the unwanted signal, none of these methods address the problem in its entirety (Murphy et al., 2013; Ciric et al., 2017). Any residual noise that is left in the BOLD signal can be detrimental to the strength of the association that we can observe (Spearman, 1904). In Classical Test Theory, the "true" score of a measure can be obtained by controlling for random measurement error with repeated measures (Streiner, 2003). For associations, the "true" association can be obtained through disattenuation or the use of a measurement model (Leigh Wang, 2010; Cooper et al., 2019). These methods can thus be used to obtain estimates of the association between brain measures and behavior that are closer to the true value if the measures had been reliably quantified (van Baal et al., 1998; van Beijsterveldt et al., 2001). 


\subsection{TWIN STUDIES AND GENETICS}

To understand what motivates behavior or the manifestation of disorders, it helps to identify whether it is caused by intrinsic (nature; e.g. of biological origin such as genetics) or extrinsic (nurture; e.g. reaction to environment) factors. This understanding can also help to guide the design of diagnostics, interventions and therapies for behavioral problems and psychiatric disorders (Musci and Schlomer, 2018; Majewski et al., 2011). Twin and family studies are informative to the extent to which genetics and environment contribute to the variation of a trait in the population (Neale and Cardon, 1992). Twin studies can be considered as a precursor to gene discovery studies by identifying heritable traits that are most likely to produce positive results (Zondervan and Cardon, 2007). Genome-wide association studies (GWAS) can identify causal genetic variants of a trait (Hirschhorn and Daly, 2005). Based on the summary results of a GWAS, an individual's genetic susceptibility for a trait (e.g. polygenic risk score; PRS) can be estimated (Wray et al., 2007). Although human DNA is stable with few mutations throughout the lifespan of an individual (Kumar and Subramanian, 2002), its expression is highly regulated across the lifespan, regions of the brain, and specific to cell-type (Kang et al., 2011; Jaffe et al., 2015; Hawrylycz et al., 2015; Darmanis et al., 2015). Environmental factors can influence the expression of DNA through regulatory mechanisms, such as epigenetic modifications (Jaenisch and Bird, 2003). The interplay of genetics and environmental risk factors are believed to be responsible for why a psychiatric disorder manifests in one person but not another despite the identical genetic makeup of monozygotic twins (van Os et al., 2008; Stilo and Murray, 2019). This section describes the methodological principles behind heritability analysis in twin studies and the use of (epi)genome-wide association studies to determine individual (epi)genetic susceptibility scores of traits.

\subsubsection{Twin studies and heritability}

Genetic modelling of data from twins and siblings can provide information on the extent to what proportion of variation of a trait in the population is explained by genetic and environmental factors without knowing the genetic makeup of the subjects (Boomsma et al., 2002; Posthuma et al., 2000). Based on the fact that monozygotic (MZ) twins share $100 \%$ of their genetic material and that dizygotic (DZ) twins and full-blood siblings share on average $50 \%$ of their segregating genes, the phenotypic variance $(V)$ of a trait is typically decomposed into three variance components: additive genetic $(A)$, common environmental $(C)$, and unique environmental $(E)$ components. Influences from genetic factors are suggested when monozygotic twins are more alike than dizygotic twins. Common environmental influences represent sources of variance that are shared between members of the same family and cause the twins to be more alike than children growing up in 
different families. Unique environmental influences are what makes monozygotic- and dizygotic twins resemble each other less. Note that unique environmental influences are confounded by random measurement errors. The extended twin design, by including siblings of the twins, can aid in the detection of common environmental influences (Boomsma et al., 2002).

Genetic modelling of data from twins can be performed with structural equation modelling software, such as OpenMx (Neale et al., 2016). Several estimates can be obtained from a genetic model; e.g. heritability of a trait or genetic correlation between two traits. Heritability determines the degree to which a trait is determined by genetic factors. Heritability in its narrow sense $\left(a^{2}\right)$ is the standardized additive genetic component, or $a^{2}=\frac{A}{V}=\frac{A}{A+C+E}$. The common and unique environmental components can be standardized in similar fashion to obtain $c^{2}$ and $e^{2}$. The genetic correlation determines the extent to which genetic variation is shared between two traits; i.e. have a common causal genetic influence, either directly or indirectly (Figure 1.2). The genetic correlation between the two traits $x$ and $y$ can then be estimated as the standardized the standardized genetic covariation between the traits, or $r_{a}(x, y)=\frac{\operatorname{cov}\left(A_{x}, A_{y}\right)}{\sqrt{\operatorname{var}\left(A_{x}\right) * \operatorname{var}\left(A_{y}\right)}}$. The genetic variation between two traits can either be fully shared, partially shared, or completely independent. Sharing can represent pleiotropy, a causal effect where a gene affects more than one trait, and can be an indication of a common underlying biological process (Neale and Kendler, 1995; Mackay et al., 2009). This standardization can also be applied to the common and unique environmental components to obtain estimates for environmental correlations $r_{c}$ and $r_{e}$.

\subsubsection{Temporal dynamics of genetic and environmental influences of the brain}

Twin studies can also be informative regarding the temporal dynamics of the genetic and environmental influences or their interaction; e.g., the contribution of the genetic factor to general intelligence is known to increase with age, whereas the contribution of the common environmental factor decreases (Plomin et al., 1997; Bartels et al., 2002). Heritability of the cerebral cortical thickness of the brain can be compared at different ages either in a cross-sectional or longitudinal study design (Fjell et al., 2015; Fjell et al., 2020; Chouinard-Decorte et al., 2014; Lenroot et al., 2009; Panizzon et al., 2012). In a longitudinal twin study design, the role of genetic and environmental contributions that drive changes in the volumes of brain structures and the thickness of the cerebral cortex (i.e. brain plasticity; Pfefferbaum et al., 2004; Brans et al., 2008; van Soelen et al., 2012; LessovSchlaggar et al. 2012; van Soelen et al., 2013; Brouwer et al., 2014; Bootsman et al., 2015; Hedman et al., 2016; Brouwer et al., 2017). This is different from heritability at a single age because it is informative to what extent genes determine the rate of change rather than to 


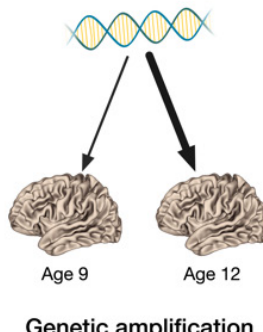

Genetic amplification

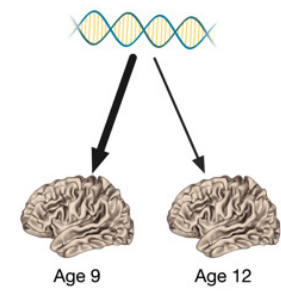

Genetic deamplification

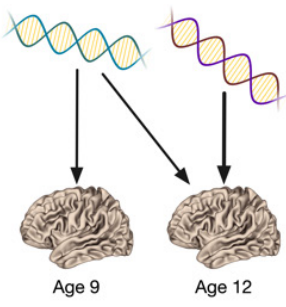

Genetic innovation

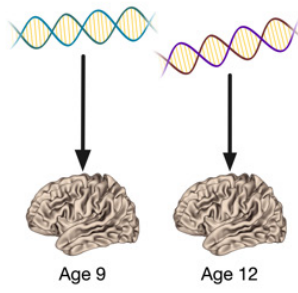

Genetic independence

Figure 1.2. Pleiotropy, partially shared and independent gene sets on two different traits or the same trait at different ages. In the case of amplification and deamplification, the same core set of genes influence the trait at both ages. However, in amplification, the genetic influences increase with age, whereas in deamplification the genetic influences decrease with age. In the case of genetic innovation, a novel set of genes influence the trait at different ages. Either a core set of genes influence both traits with additional contributions from a unique set of genes at one of the ages, or two fully distinct sets of genes influence the trait at each of the ages.

what extent genes determine the outcome at a specific moment; e.g. brain volume might be limited by intracranial volume and therefore share a genetic factor between the two traits, whereas genes that cause changes in brain volume during development or aging can be independent from the genes that influences intracranial volume (Brouwer et al., 2017). Although the outcome measure at any given age is the cumulative effect of the change rates preceding that age, these two measures can still vary in their genetic cause because the cumulative effect can be the result of different processes at different intervals during the lifespan. In a longitudinal twin design, the genetic correlation between repeated measures of the same trait can determine if the genes influencing the traits are independent of age or that different genes influence the trait at different ages (Figure 1.2). In addition, the extent to which the age-independent genes influence the trait at different ages can determine if the genes exert stable influence across the ages, or have waxing or waning influence on the trait (i.e. genetic amplification or deamplification of a core set of genes;

Figure 1.2; van Soelen et al., 2012).

\subsubsection{Genetic and epigenetic studies}

Where twin studies can inform if a trait is influenced by genetic factors, a gene discovery study, such as a genome-wide association study, is required to identify the potential individual genes (Hirschhorn and Daly, 2005). Microarray chips can detect single nucleotide polymorphisms (SNPs; i.e. variation in a single base pair) in the genome. However, most common SNPs have relatively low effect size (Park et al., 2011). Combined with performing millions of statistical tests across the genome that need to be accounted for by multiple comparison correction (typically a threshold of $\mathrm{p}<5 \times 10^{-8}$ is used), very large sample sizes are needed for discovery studies to find significant hits (Spencer et al., 2009). With the advent of large-scale genotyping, several world-wide collaborative efforts 
have been initiated to elucidate the genetic makeup of the brain and behavior (Thompson et al., 2020; Sullivan et al., 2018). These collaborative efforts have identified genetic variants that have been implicated in various traits, including schizophrenia, brain morphology and brain plasticity (Ripke et al., 2014; Pardiñas et al., 2018; Grasby et al., 2020; Satizabal et al., 2020; Brouwer et al., 2017). Subsequent identification of the causal gene is complicated by the majority of significant hits occurring in non-coding regions or spread across a wide range on the genome due to linkage disequilibrium (Edwards et al., 2013; Watanabe et al., 2017). An individual's genetic susceptibility (or polygenic risk score; PRS) for a trait can be obtained from the associations of the SNPs with the trait (Wray et al., 2007). In addition, a SNP-based heritability estimate for a trait can be computed (Speed et al., 2012). However, this heritability estimate is usually underestimated compared to twin studies (Manolio et al., 2009).

The expression of the human genome is highly regulated across the lifespan, regions of the brain, and is specific to each cell-type (Kang et al., 2011; Jaffe et al., 2015; Hawrylycz et al., 2015; Darmanis et al., 2015). The expression of genes can be influenced by environmental factors through regulatory mechanisms, such as epigenetic modifications, that facilitate genotype-by-environment interaction (Jaenisch and Bird, 2003; Ottman et al., 1996; Dick et al., 2011; Molenaar et al., 2016). This dynamic interplay of genetic and environmental influences may play an important role in differentiating outcomes in monozygotic twins, and can even vary between the sexes and across the lifespan (Fraga et al., 2005; Christensen et al., 2009; van Dongen et al., 2016). The methylation profiles in humans are partially determined by genetics (Shah et al., 2014; van Dongen et al., 2016). Similar to the genome, methylation of the epigenome can be measured with microarray chips that measure the binding of a methyl group to a cytosine-guanine dinucleotide (CpG) locus (Beck and Rakyan, 2008). It should be noted that there is an ongoing discussion whether peripheral tissues that can be easily obtained from a living specimen, such as blood or saliva, are representative for methylation for regions of the brain (Smith et al., 2015; Hannon et al., 2015). A "poly-epigenetic risk score" for a trait can be computed based on the associations of epigenetic loci with the trait from a discovery sample, similar to the polygenic risk score in genomic studies.

\subsection{ADOLESCENT BRAIN DEVELOPMENT}

Studying normative development of the brain in healthy children and adolescents is essential to understand when and how development is stunted in atypically developing children. Longitudinal studies are essential when studying development because the brain and behavior is dynamic across the ages (Haller et al., 2018). Cross-sectional studies on development (i.e. comparing two groups of subjects at different ages) are limited by the high interindividual variation and may produce false positive results due to sampling bias 
(i.e. the cohort effect; Mills and Tamnes, 2014; Crone and Elzinga, 2014; Telzer et al., 2018). In addition, the development trajectory from longitudinal studies is often more informative than individual measurements in aberrant development such as those linked to the development of psychiatric disorders (Paus et al., 2008; Shaw et al., 2010; Schnack et al., 2016). Determining the genetic and environmental contributions to brain development and behavior can help to identify their causal origin and aid in the design of diagnostic tools, interventions, and therapies (Musci and Schlomer, 2018; Majewski et al., 2011). This section provides a brief outline of the global developmental patterns observed in the brain during childhood and adolescence, and twin studies reporting on the heritability estimates of brain structure and function.

\subsubsection{Structural brain development}

Although the human brain has reached its adult-size by late childhood (Dekaban and Sadowsky, 1978; Hedman et al., 2012), the brain has a protracted development with vast developmental changes from the fetal period through childhood and adolescence and extending well into early adulthood (Stiles and Jernigan, 2010; Giedd and Rapoport, 2010; Brown and Jernigan, 2012; Mills et al., 2016). Overall, brain morphology is highly heritable with several genetic loci identified for typical and atypical development and across the lifespan (Peper et al., 2007; Blokland et al., 2012; Braber et al., 2013; Douet et al., 2014; Jansen et al., 2015; Strike et al., 2015; Alzheimer's Disease Neuroimaging Initiative et al., 2015; Kochunov et al., 2014; Brouwer et al., 2017; Thompson et al., 2020).

The global pattern of structural brain development is described by an increase of gray matter and white matter volume during childhood (Giedd et al., 1999; Mills et al., 2016). Around the end of childhood when puberty begins, total cortical gray matter starts to decrease while total white matter volume increases as myelination of white matter bundle continues throughout adolescence (Brouwer et al., 2012; van Soelen et al., 2013; Swagerman et al., 2014; Brouwer et al., 2015; Brouwer et al., 2017). This reduction in total grey matter volume and increasing myelination of white matter connections in the brain is accompanied by an unusual but characteristic decrease in grey matter cortical thickness during adolescence (van Soelen et al., 2012; Koenis et al., 2018). The decrease in gray matter cortical thickness is believed to be the result of pruning and white matter encroachment (Paus, 2010; Petanjek et al., 2011; Miller et al., 2012). Although no clear consensus has been reached whether cortical gray matter peaks during late childhood or shows a continuous decline from infancy (Walhovd et al., 2017). Recent studies covering wide age range from infancy to early adulthood are in favor of a continuous decline (Brown et al., 2012; Fjell et al., 2015; Wieringa et al., 2014). Similarly, although sex effects have been reported for cortical thickness (Lenroot and Giedd, 2010), this effect is not always observed (van Soelen 
et al., 2012). Sex effects of volumetric measures are largely attributed to intracranial volume that is positively associated with stature and where both measures share a common genetic background (Caspi et al., 2020; van Soelen et al., 2013). Sex effects are also determined by pubertal hormones that influence brain structure (Koolschijn et al., 2014); a review of the effect of pubertal hormones on brain structure suggesting that changes in sex hormones might trigger a reorganization of the structural brain (Peper et al., 2011). Gray matter volume of the hormone secreting pituitary glands in the brain is positively associated with levels of pubertal hormones estradiol, testosterone, luteinizing hormone, and follicle stimulating hormone in girls (Peper et al., 2010). Changes in pubertal hormone levels is positively associated with changes in gray matter density in both cortical and subcortical brain structures for follicle stimulating hormone levels in girls, and a negative association between gray matter density in frontal and parietal cortical region and estradiol levels in girls; these associations were mostly explained by unique environmental influences (Brouwer et al., 2015). White matter density is positively associated with luteinizing hormone with both measures sharing a common genetic background (Peper et al., 2008). Global and regional gray matter volumes are heritable in late childhood (Peper et al., 2009). Subcortical gray matter volume increases for the thalamus, hippocampus, amygdala and pallidum, and decreases in the caudate and nucleus accumbens, and are highly heritable at similar levels as during adulthood (Swagerman et al., 2014; Brouwer et al., 2020). The change rates of subcortical volume are also heritable with several genetic loci recently identified (Brouwer et al., 2017; Brouwer et al., 2020). White matter structural integrity and local and global efficiency measures of structural white matter brain network increase during early adolescence and are highly heritable (Brouwer et al., 2010; Brouwer et al., 2012; Koenis et al., 2015; Koenis et al., 2018). The gray matter thickness of the cerebral cortex shows a strong decline during adolescence (van Soelen et al., 2012). Cortical thickness and surface area are both highly heritable and influenced by distinct genetic factors (Hulshoff Pol et al., 2006; van Soelen et al., 2012; Panizzon et al., 2009). The heritability of cortical thickness is suggested to increase with age during development (Lenroot and Giedd, 2008; Lenroot et al., 2009; Schmitt et al., 2014). The rate of change in cortical thickness between the ages 9 and 12 years is also heritable, with a common genetic factor of decreasing influence over time and a unique genetic factor that is specific for cortical thickness at age 12 years indicating the influence of novel genes during adolescence brain development (van Soelen et al., 2012).

Many behavioral traits have been linked to structural properties of the brain, including cognition, affective, and social behavior (Paus, 2005; Mills et al., 2014). General cognitive abilities (including intelligence) are highly heritable (van Leeuwen et al., 2007; van Leeuwen et al., 2008; van Soelen et al., 2009; van Soelen, 2011). Intelligence is positively 
associated with global gray matter and white matter volume of the brain in children (van Leeuwen et al., 2009). With a negative association between intelligence and cortical gray matter thickness emerging around late childhood and early adolescence (Brouwer et al., 2014). The association persists during most of adolescence when cortical thickness shows a steep decline (van Soelen et al., 2012; Schnack et al., 2015), during which intelligence is positively associated with the rate of change in cortical thickness and surface area (Schnack et al., 2015). However, there is a cross-over effect around the end of adolescence and beginning of early adulthood where the association between cortical thickness and intelligence is reversed (Schnack et al., 2015). Heritability of change in cortical thickness also shares a common genetic background with intelligence (Brans et al. 2010). Local efficiency of structural white matter is positively associated with intelligence (Koenis et al., 2015; Koenis et al., 2018). Gestational age is negatively associated with cerebral volume, independent of intelligence (van Soelen et al., 2010). Aberrant development of the structural brain has been linked to the onset of psychiatric disorders such as schizophrenia, attentiondeficit hyper-activity (ADHD), and autism (Greenstein et al., 2006; Shaw et al., 2007; Zielinski et al., 2014; Paus et al., 2008). Psychiatric disorders share a common genetic architecture amongst each other and with the brain (The Brainstorm Consortium, 2018; de Zwarte et al., 2019; Hulshoff Pol et al, 2012). In addition, heritability of changes in cortical thickness has also been reported in monozygotic twins discordant for schizophrenia (Hedman et al., 2016).

\subsubsection{Functional brain development}

Although the functioning of the brain in children and adolescents has been studied extensively in cross-sectional studies (Johnson, 2001; Luna and Sweeney, 2006; Johnson et al., 2009; Crone and Dahl, 2012), not many longitudinal studies available or otherwise limited in sample size (<30 participants) to examine individual development (Crone and Elzinga, 2014). The longitudinal studies suggest that task activation in the cortical regions remain relatively stable, but that activation in subcortical nuclei show adaptive response with age in cognitive control and social-cognitive tasks, and social-affective and reward stimuli (Crone and Elzinga, 2014). The heritability of functional brain activity is generally lower than brain structure, with an indication that activity is influenced more strongly by common familial environment than additive genetics for certain tasks (Blokland et al., 2012; Douet et al., 2014; Jansen et al., 2015; Thompson et al., 2013). The development of restingstate functional connectivity is described by a large quantity of cross-sectional studies that report inconsistent results regarding the direction of change and affected connections (Ernst et al., 2015; Grayson and Fair, 2017; Stevens, 2016; Cao et al., 2016), with few longitudinal studies available to date to examine individual development (see Table 4.1 from Chapter 4 for overview; Teeuw et al., 2019). Based mostly on cross-sectional studies, the 
development of functional connectivity in children and adolescents is generally described by shifts from a local to a more distributed organization characterized by the segregation of functionally distinct regions and the integration of functionally related regions (Fair et al., 2007; Supekar et al., 2009; Kelly et al., 2009; Fair et al., 2009; Dosenbach et al., 2010). The heritability of resting-state functional connectivity is generally low with strong indication for common familial environment for certain network connections that is sometimes omitted from analysis (see Table 4.2 from Chapter 4 for overview; Teeuw et al., 2019).

The functional networks of the brain are already present in the second trimester of pregnancy and their spatial organization appear "adult-like" by the age of 2 years (Turk et al., 2019; Gao et al., 2011; Keunen et al., 2017; Thomason et al., 2015). Similarly, the spatial organization of functional networks is highly consistent throughout childhood and adolescence (Thomason et al., 2011). The development of these networks, with a protracted development of the executive control network during childhood, is partially determined by genetics (Gilmore et al., 2018; Gao et al., 2014). There are reports of longitudinal age-related increases in functional connectivity (or integration) within one part of the frontoparietal network, and default mode network, and decrease (or segregation) within the other of the frontoparietal network and the ventral attention network and between the frontoparietal and default mode network (Sherman et al., 2014; Wendelken et al., 2016; Long et al., 2017; Sylvester et al., 2017; Wendelken et al., 2017). Mixed results have been reported for corticalsubcortical connectivity (Strikwerda-Brown et al., 2015; Jalbrzikowski et al., 2017; Peters et al., 2017; van Duivenvoorde et al., 2019).

Heritability of functional connectivity range from $10 \%$ to $80 \%$ in adults, depending on the population, network connection, and methodology used (Adhikari et al., 2018; Fu et al., 2015; Ge et al., 2017; Colclough et al., 2017; Glahn et al., 2010; Korgaonkar et al., 2014; Meda et al., 2014; Sudre et al., 2017; Yang et al., 2016). Both genetic and common environmental factors influence functional connectivity within and between networks during early adulthood (Yang et al., 2016). Reports on the heritability of functional connectivity during childhood and adolescence are sparse. Genes explain up to $40 \%$ of individual difference in global efficiency at the age of 12 years (van den Heuvel et al., 2013). Similar magnitudes were reported for cortical-subcortical connections in younger children, aged 7 to 9 years, with heritability ranging from $32 \%$ to $67 \%$ (Achterberg et al., 2018). Another study found overall low heritability estimates $(<10 \%)$, with local peaks ranging between $55 \%$ to $83 \%$ (Fu et al., 2015). Age-dependent genetic effects on functional connectivity within cortical networks have been reported during infancy (Gao et al., 2014). However, it is unknown whether there are age-dependent dynamic influences of genes and environment on 
functional connectivity during childhood and adolescence due to the absence of any longitudinal twin studies during this developmental period.

Functional connectivity has been associated with a number of cognitive and behavioral traits in adults (Vaidya and Gordon, 2013; Basten et al., 2015; Shen et al., 2018; Smith et al., 2015; Toschi et al., 2018), and shyness, social and affective behavior, goal-directed cognition, motor development and neurocognition in children and adolescents (Sylvester et al., 2018; Kelly et al., 2009; Spreng et al., 2010; Marrus et al., 2018; Sripada et al., 2019). Alterations in functional connectivity been implicated in several neurodevelopmental psychiatric disorders such as autism, attention-deficit hyperactivity, schizophrenia, and major depressive disorder (Plitt et al., 2015; Mattfeld et al., 2014; Anticevic et al., 2015; Cullen et al., 2014), and behavioral problems in children and adolescents (Lu et al., 2015; Cohn et al., 2015; Whitfield-Gabrieli et al., 2020).

\subsection{SCHIZOPHRENIA}

Schizophrenia is a debilitating psychiatric disorder with a lifetime prevalence of about $1 \%$ that has a tremendous impact on the quality of life of the patients, their families, and society (Whiteford et al., 2013; Goldner et al., 2002). The disorder is mainly characterized by psychotic episodes (i.e. delusions and hallucinations), depression, and neurocognitive decline (Green, 1996; Heinrichs et al., 1998; van Os and Kapur, 2009). It is a highly hereditary disorder with a polygenic nature (Sullivan et al., 2003; Hilker et al., 2018; Ripke et al., 2014; Pardiñas et al., 2018) and shows genetic overlap with other psychiatric disorders, such as bipolar and depression, and cognitive functioning (Barkhuizen et al., 2020; Smeland et al., 2019; de Zwarte et al., 2019). Epigenetic regulation of genes associated with schizophrenia may explain the discordance in schizophrenia diagnosis for monozygotic twins despite their common genetic background (Hannon et al., 2016; van Os et al., 2008; van Nierop et al., 2013).

\subsubsection{Schizophrenia as a neuropsychiatric disorder of the brain}

Although schizophrenia has been extensively studied in the past, its aethiology remains largely unknown. The onset of psychiatric disorders such as schizophrenia typically occur around adolescence when a clinical diagnosis is made (Zahn-Waxler et al., 2008; Paus et al., 2008). Studies in populations at high risk for developing schizophrenia have reported aberrant changes of the brain compared to normative controls (de Wit et al., 2016; Smieskova et al., 2010; Rapoport et al., 2012). However, there are several indications of aberrant brain development as early as the fetal period (Debnath et al., 2015; Kim et al., 2015; Faa et al., 2016). Schizophrenia is therefore regarded as a neurodevelopmental disorder by some (Murray and Lewis, 1987; Weinberger, 1987). At the same time, the 
progressive changes of the brain persist for several years after the onset of psychosis (van Haren et al., 2008; van Haren et al., 2008; Hulshoff Pol and Kahn, 2008; Chiapponi et al., 2013). Patients with schizophrenia have an increased mortality risk, with their expected lifespan decreased on average by 15 to 20 years compared to the general population (Laursen et al., 2014; Hjorthøj et al., 2017). Combined with the neurocognitive decline that is reflective of the aging population has resulted in schizophrenia being regarded as a progressive aging disorder by others instead (Kirkpatrick et al., 2008; Olabi et al., 2011). Either way, schizophrenia is linked to wide-spread alteration in the brain (Haijma et al., 2013; van Haren et al., 2016; Mandl et al., 2008; Boos et al., 2013; van Erp et al., 2015; van Erp et al., 2018; Kelly et al., 2017). These alterations in the brain are heritability and share a common denominator with genes for schizophrenia (Hulshoff Pol et al., 2004; van Haren et al., 2012; Bohlken et al., 2016). In addition, environment factors influence the brain in schizophrenia through epigenetic modifications (Rijsdijk et al., 2005; van Haren et al., 2012; Jia et al., 2019).

\subsubsection{Progressive aging in schizophrenia}

The biological age of a subject can be predicted with reasonable accuracy from tissue samples by machine learning algorithms (Jylhävä et al., 2017; Cole et al., 2019). A classification algorithm can be trained on selected features from the brain, such as grey matter density, to predict a person's "biological” brain age (Cole and Franke, 2017; Franke and Gaser, 2019). Patients with schizophrenia show advanced brain age that is accelerated around the time of onset (Koutsouleris et al., 2014; Schnack et al., 2016; Nenadić et al., 2017; Kaufmann et al., 2019). Accelerated brain age predicts all-cause mortality (Cole et al., 2018), is highly heritable and has a genetic overlap with common brain disorders, including schizophrenia (Cole et al., 2017; Kaufmann et al., 2019). Similar to a person's brain age, the biological age of a person can be predicted from DNA methylation (Horvath, 2013; Hannum et al., 2013; Levine et al., 2018). Although previous studies have reported no significant accelerated epigenetic aging for schizophrenia patients (Viana et al., 2017; Voisey et al., 2017; McKinney et al., 2017; McKinney et al., 2018), a recent large-scale DNA methylation study has now demonstrated accelerated epigenetic age in schizophrenia (Ori et al., 2019). A prior study has reported a lack of significant association between brain age and epigenetic age in the healthy aging population (Cole et al., 2018). However, little is known about the association between brain age and epigenetic age in schizophrenia patients specifically.

\subsection{COHORT DESCRIPTIONS}

Three cohorts were studied in this thesis. First, the longitudinal adolescent twin BrainSCALE cohort that was used for studying the development of the brain during adolescence and its temporal dynamics of genetics and environmental influencing the brain (van Soelen et al., 2012). Secondly, a longitudinal cohort of schizophrenia and healthy 
controls that was used to study accelerated aging of the brain in relation to accelerated epigenetic aging and polygenic risk for schizophrenia (Schnack et al., 2016; Ori et al., 2019). Finally, the Human Connectome Project Young Adult cohort that was used to evaluate the effectiveness of reliability modeling of functional connectivity of the brain in relation to various traits (van Essen et al., 2013).

\subsubsection{The BrainSCALE cohort}

The BrainSCALE cohort is a longitudinal study of adolescent twins born between 19951996 whose parents registered them with the Netherlands Twin Register (NTR; Ligthart et al., 2019). Twin pairs who had an older sibling who was also willing to take part in the study were recruited through the NTR. The cohort was designed to investigate cognitive, behavioral, physical and physiological transitions during childhood and adolescence in relation to brain development and genetics. It is a collaborative effort between the University Medical Center Utrecht (UMCU) and Vrije Universiteit Amsterdam (VU Amsterdam). The subjects were selected to represent a cross-section of the Dutch population with an equal proportion of the sexes (52\% female; including 20 dizygotic twin pairs of opposite sex), a mixture of ethnical background and culture, normal cognitive functioning (intelligence quotient $101.9 \pm 14.75$ at baseline), and no prior history of physical or mental health problems. They were assessed when the twins were 9,12 , and 17 years of age; average ages were $9.3 \pm 0.28,12.2 \pm 0.35$ and $17.2 \pm 0.37$ years, with their older siblings on average $2.7 \pm 1.1$ years older. Extensive phenotypic information was collected about the children, including cognitive, behavioral, and neuropsychological tests, physical assessment, hormone levels, and magnetic resonance imaging scans of the brain (van Soelen et al., 2012; Koenis et al., 2013). The BrainSCALE study was approved by the Central Committee on Research Involving Human Subjects of The Netherlands (CCMO).

Over the years, the BrainSCALE cohort has been the foundation for previous dissertations by Marieke van Leeuwen (2008), Jiska Peper (2008), Inge van Soelen (2011), Suzanne Swagerman (2016), and Marinka Koenis (2017). In this dissertation, the longitudinal development and the temporal dynamics of genetic influences on cortical thickness was investigated for the twins at the ages 9, 12 and 17 years. The sex-specific longitudinal development of functional connectivity within and between cortical functional network from the resting-state functional MRI scans and the genetic and environmental influences on the stable and reliable component of functional connectivity from a measurement model were investigated for the twins at the ages 12 and 17 and their older sibling.

\subsubsection{The schizophrenia cohort}

The schizophrenia cohort used in this dissertation consists of a combination of two longitudinal schizophrenia cohorts acquired at UMCU (van Haren et al., 2007; Boos et al., 
2012). A total of 411 unrelated subjects (193 cases, 218 controls, $36 \%$ female) that had imaging data $(\mathrm{N}=411)$ and either epigenetic $(\mathrm{N}=172)$ or genetic $(\mathrm{N}=394)$ data available. The subjects were of European descent spanning a wide range of the adult lifespan (mean = 32.7 years, range $=[16.7-67.5]$ at baseline). For the majority of subjects (57\%) longitudinal imaging data was available (up to five scans), with a mean scanning interval of 3.4 years (range [0.9 - 7.0]). All patients met DSM-IV criteria for a nonaffective psychotic disorder (including schizophrenia, schizophreniform disorder or schizoaffective disorder). Written informed consent was obtained from all subjects, and both studies were approved by the Medical Ethics Committee for Research in Humans (METC) of the University Medical Center Utrecht. Data from this schizophrenia cohort has previously been used to investigate accelerated brain aging (Schnack et al., 2016), and accelerated epigenetic aging (Ori et al., 2019). In this dissertation, the association between accelerated aging of the brain, accelerated epigenetic aging, and polygenic risk for schizophrenia were investigated.

\subsubsection{The Human Connectome Project Young Adult cohort}

The Human Connectome Project is a large-scale endeavor to elucidate the structural and functional connections of the human brain that underlie behavior (van Essen et al., 2013). The Young Adult cohort is a publicly available high-quality multimodal neuroimaging dataset with rich and deep phenotyping of 1200 healthy adults within the age range of 2235 years. Subjects were recruited to reflect the ethnic diversity of America. Numerous studies have been published on this cohort. In this dissertation, the focus has been on the carefully prepared data package that provides data for 1003 related individuals (age $28.7 \pm$ 3.7 years; $53 \%$ female) from 429 families (siblings, including monozygotic- and dizygotic twins) with four complete runs ( 1 hour) of resting-state fMRI scans. The rich phenotyping of the subjects provided hundreds of measures, of which 110 measures from various domains, such as cognition, emotion, personality, alertness, language, motor skills, sensory, and physiology, were included in the analysis. The dataset was used to empirically evaluate the efficiency of reliability modelling of functional connectivity with a measurement model through its impact on behavioral utility and to replicate previously reported increased heritability estimates of the stable and reliable component of functional connectivity.

\subsection{OUTLINE OF THIS THESIS}

In chapter 2, I investigate the genetic influences on the grey matter thickness of the cerebral cortex throughout childhood and adolescence. A prior investigation of the development of the cerebral cortex in the BrainSCALE cohort had revealed genetic influences on the change rates of cortical thickness and indication of novel genetic influences between the ages 9 and 12 years (van Soelen et al., 2012). This research was 
extended by including the third assessment of the BrainSCALE cohort at age 17 years that covers a period of rapid decline in cortical thickness typically observed during adolescence.

In chapter 3 and chapter 4, I investigate resting-state functional connectivity in the brain. In chapter 3, I investigate the utility of a measurement model to obtain more reliable estimates of functional connectivity. These reliable estimates are used to reveal the "true" strength in associations with behavior and heritability of functional connectivity in the absence of measurement error. An empirical evaluation of the measurement model for various scan durations and sample sizes is performed on resting-state functional connectivity from the publicly available Human Connectome Project Young Adult cohort. In chapter 4, I investigate the development of resting-state functional connectivity within and between canonical cortical networks during adolescence in the BrainSCALE cohort. In addition, I applied a measurement model to the functional connectivity estimates, as described in chapter 3 , to investigate the genetic and environmental influences on the reliable and stable component of functional connectivity.

In chapter 5, I investigate the association between accelerated maturation of the brain and accelerated epigenetic aging in the blood of schizophrenia patients in relation to polygenic risk for schizophrenia.

Finally, in chapter 6, I summarize the results from chapter 2 through chapter 5 and discuss the significance of these results in a broader context of genetic and environmental influences on brain maturation. 



\section{CHAPTER 2}

\section{LONGITUDINAL DEVELOPMENT OF}

\section{CEREBRAL CORTICAL THICKNESS}

Original title: Genetic influences on the development of cerebral cortical thickness during childhood and adolescence in a Dutch longitudinal twin sample: the BrainSCALE study

Authors: Jalmar Teeuw ${ }^{1}$, Rachel M. Brouwer ${ }^{1}$, Marinka K.G. Koenis ${ }^{1}$, Suzanne C. Swagerman ${ }^{2}$, Dorret I. Boomsma르 and Hilleke E. Hulshoff $\mathrm{Pol}^{1}$

Affiliations: ${ }^{1}$ Department of Psychiatry, Brain Center Rudolf Magnus, University Medical Center Utrecht, Heidelberglaan 100, 5384 CX Utrecht, the Netherlands; ${ }^{2}$ Department of Biological Psychology, Vrije Universiteit Amsterdam, van der Boechorststraat 1, 1081 BT Amsterdam, the Netherlands

Published in 2018 in the journal Cerebral Cortex 29(3), 978-993. doi:10.1093/cercor/bhy005

Full reference: Teeuw, J., Brouwer, R., Koenis, M., Swagerman, S., Boomsma, D., Hulshoff Pol, H. (2018). Genetic Influences on the Development of Cerebral Cortical Thickness During Childhood and Adolescence in a Dutch Longitudinal Twin Sample: The Brainscale Study Cerebral Cortex 29(3), 978-993. doi:10.1093/cercor/bhy005 


\begin{abstract}
Previous studies have demonstrated that cortical thickness (CT) is under strong genetic control across the life span. However, little is known about genetic influences that cause changes in cortical thickness $(\triangle \mathrm{CT})$ during brain development. We obtained 482 longitudinal MRI scans at ages 9, 12, and 17 years from 215 twins and applied structural equation modelling to estimate genetic influences on (1) cortical thickness between regions and across time, and (2) changes in cortical thickness between ages. Although cortical thickness is largely mediated by the same genetic factor throughout late childhood and adolescence, we found evidence for influences of distinct genetic factors on regions across space and time. In addition, we found genetic influences for cortical thinning during adolescence that is mostly due to fluctuating influences from the same genetic factor, with evidence of local influences from a second emerging genetic factor. This fluctuating core genetic factor and emerging novel genetic factor might be implicated in the rapid cognitive and behavioral development during childhood and adolescence, and could potentially be targets for investigation into the manifestation of psychiatric disorders that have their origin in childhood and adolescence.
\end{abstract}

Keywords: cortex; development; twins; heritability; plasticity 


\subsection{INTRODUCTION}

The human brain changes substantially during development from fetus to newborn to adult. Non-invasive magnetic resonance imaging (MRI) has enabled the study of brain structure and function in healthy children and adolescents. Brain imaging studies from the past two decades have documented the changes that occur to the brain during development from childhood into early adulthood (Giedd et al. 2010). The size of the brain of a 9-yearold child is already at approximately $96 \%$ of its maximum size (Dekaban and Sadowsky 1978; Hedman et al. 2011), but continues to develop as the child transitions through adolescence and matures into adulthood. For example, total grey matter volume starts to decrease around the start of puberty, while total white matter volume continues to increase well into adulthood (Giedd et al. 1999; Mills et al. 2016). This reduction in total grey matter volume and increasing myelination of white matter connections in the brain is accompanied by an apparent decrease in grey matter cortical thickness during adolescence. Characterizing brain development in healthy children and adolescents is essential to understand when and how development is stunted in atypically developing children. Divergence from typical developmental trajectories has been associated with increased liability for psychiatric disorders (Greenstein et al. 2006; Shaw et al. 2007; Paus et al. 2008; Rapoport and Gogtay 2008; Zielinski et al. 2014; Giedd et al. 2015). Indeed, developmental trajectories and their underlying processes may be more informative about vulnerability for disease and clinical outcomes than absolute measures (Paus et al. 2008; Shaw et al. 2010; Schnack et al. 2015; 2016), highlighting the importance of longitudinal cohorts in developmental studies (Mills and Tamnes 2014).

The inclusion of twins in brain imaging studies has provided valuable information about the influences of genes and environment on brain development (Peper et al. 2007; Blokland et al. 2012; Braber et al. 2013; Douet et al. 2014; Jansen et al. 2015; Strike et al. 2015). Twin studies allow us to unravel genetic influences on the architecture of the brain and explain to what extent variation in brain measures are heritable, i.e. the extent to which individual differences can be attributed to genetic factors, or to common and unique environmental factors. The classical twin model allows for the study of genetic influences on the human brain by measuring similarities between monozygotic and dizygotic twins (Posthuma et al. 2000; Boomsma et al. 2002). Heritability estimates have revealed that most of the brain's structure is under genetic control (Peper et al. 2007; Blokland et al. 2012; Douet et al. 2014; Strike et al. 2015). Cortical thickness is found to have moderate to strong heritability (Thompson et al. 2001; Hulshoff Pol et al. 2006; van Soelen, Brouwer, van Baal, et al. 2012) and heritability of cortical thickness is suggested to increase with age (Lenroot and Giedd 2008; Schmitt et al. 2014). To date, there are few longitudinal studies which allow 
estimation of genetic influences on changes in cortical thickness (Brans et al. 2010; van Soelen, Brouwer, van Baal, et al. 2012; Hedman et al. 2016). We previously found evidence for heritability of cortical thinning in children between the ages 9 and 12 years (van Soelen, Brouwer, van Baal, et al. 2012). Of interest, at age 12 years, we found genetic innovation for cortical thickness in a prefrontal region, indicating that novel genetic factors become involved in the development of cortical thickness around the start of puberty. However, little is known about the dynamic landscape of genetic and environmental influences on cortical development during adolescence, a period with large cognitive and behavioral changes and a critical period for the manifestation of psychiatric disorders. In the current study, we report on the development of cortical thickness in the BrainSCALE twin cohort for which we measured the twins again at age 17 years, bringing the number of repeated assessments to three.

We investigated the spatiotemporal dynamics of genetic and environmental influences on cortical thickness. Specifically, we address the question of whether different genetic factors influence cortical thickness at different stages of childhood and adolescent brain development. Using twin modelling, we estimated genetic correlations between cortical regions to assess spatial genetic differentiation between regions within the same age and temporal genetic differentiation between regions at different ages. In addition, we extend on our previous findings on heritability of changes in cortical thickness between age 9 and 12 years (van Soelen, Brouwer, van Baal, et al. 2012) with new finding on heritability of changes in cortical thickness between age 12 and 17 years. We have included new estimates of our previous finding between age 9 and 12 years given the increase in power to detect smaller effect sizes with greater accuracy by including the third measurement.

\subsection{MATERIALS AND METHODS}

\subsubsection{Participants}

A total of 112 families consisting of twin pairs born in 1995-1996 and their older siblings, were invited to participate in the longitudinal BrainSCALE study on brain and cognitive development during childhood and adolescence (van Soelen, Brouwer, Peper, et al. 2012), a collaborative project between Netherland Twin Register (Boomsma et al. 2006; van Beijsterveldt et al. 2013) at the Vrije Universiteit (VU) Amsterdam and University Medical Center Utrecht (UMCU). The BrainSCALE cohort is a representative sample of mostly Caucasian typically-developing children from the Dutch population. The twins were around 9 years of age when they were assessed with a battery of cognitive tests and extensive MRI protocol at baseline measurement (Peper et al. 2009). Two follow-up measurements were conducted when the twins were around 12 and 17 years of age. Here 
we report results on a subsample of the BrainSCALE cohort that includes all twins. A total of 482 MRI scans from 215 participants (111 females and 104 males; approximately 16\% non-right-handed) between age 9 and 18 years were available for analysis (see also Supplementary Table A.1). Structural MRI scans were acquired for most of the subjects participating in the BrainSCALE study (94\%, 78\%, and 98\% respectively for each measurement; Supplementary Figure A.1). The decline in scan acquisition percentage at age 12 years was mostly due to exclusion of participants with dental braces incompatible with the magnetic field of the MR scanner. Other reasons for not acquiring scans include reluctance to participate and incomplete scans. In addition, a fraction of the acquired scans could not be processed due to scanning artefacts primarily related to head motion (9\%, $9 \%$, and $4 \%$ respectively for each measurement).

The study was approved by the Central Committee on Research Involving Human Subjects of The Netherlands (CCMO), and studies were performed in accordance with the Declaration of Helsinki. Parents signed informed consent forms for the children and for themselves. Children signed their own informed consent forms at the third measurement. Parents were financially compensated for travel expenses, and children received a present or gift voucher at the end of the testing days. In addition, a summary of cognition scores and a printed image of their T1 brain MRI scan, when available, were provided afterwards.

\subsubsection{MRI acquisition}

Participants underwent medical resonance imaging (MRI) on a 1.5 Tesla Philips Achieva scanner (Philips, Best, Netherlands) at the University Medical Center Utrecht (UMCU). For brain anatomy, a three-dimensional T1-weighted scan (Spoiled Gradient Echo; TE = $4.6 \mathrm{~ms}$; $\mathrm{TR}=30 \mathrm{~ms}$; flip angle $=30^{\circ} ; 160$ to 180 contiguous coronal slices of $1.2 \mathrm{~mm}$; in-plane resolution of $1.0 \times 1.0 \mathrm{~mm}^{2}$; acquisition matrix of $256 \times 256$ voxels; field-of-view of $256 \mathrm{~mm}$ with $70 \%$ scan percentage) of the whole head was acquired for each participant. The same scanners and scan sequence parameters were used at baseline and follow-up measurements to minimize the effect of differences in scan acquisition between measurements.

\subsubsection{Image processing}

Images were re-oriented to Talairach space without scaling, and corrected for inhomogeneities in the magnetic field (Sled et al. 1998). Quantitative assessment of intracranial volume (ICV) was performed as previously described for baseline (Peper et al. 2008) and follow-ups (van Soelen et al. 2013). Brain tissue was segmented into cerebrospinal fluid, grey matter and white matter using a partial volume segmentation algorithm that incorporates a non-uniform partial volume distribution (Brouwer et al. 2010). Cortical thickness was determined using a customized version of the CLASP algorithm designed at the McConnell Brain Imaging Centre, Montreal (Kim et al. 2005; Lerch et al. 2008). The grey 
and white matter segments obtained from our own partial volume segmentation algorithm were used to initialize the CLASP algorithm. A 3D surface was fitted to the white matter/grey matter interface to obtain the inner surface of the cortex. The outer cortical surface was obtained by expanding the inner surface outward until it fitted the grey matter/cerebrospinal fluid interface (Kim et al. 2005). Cortical thickness was defined at each vertex (40962 vertices per hemisphere) as the distance between the two surfaces. Changes in cortical thickness between ages were computed by taking the difference in estimates and converted to change in millimeter per year by dividing the difference by the scan interval between the ages in years. Cortical thickness measures were smoothed across the surface using a 20-mm full-width-at-half-maximum (FWHM) surface-based blurring kernel. This method of blurring simultaneously improves the chances of detecting population differences and follows the curvature of the surface to preserve any anatomical boundaries within the surface. The surfaces of the individuals were registered to an average surface (ICBM average surface template; Lyttelton et al. 2007) to allow for comparison between and within subjects across age. Non-smoothed cortical thickness mapped to ICBM average surface was used to compute mean cortical thickness for the major cortical lobes (frontal, parietal, temporal, and occipital lobes) and adjacent regions (insula and cingulate) obtained from the atlas provided by the CIVET software. The version of CIVET used in the analysis does not incorporate a dedicated longitudinal pipeline for simultaneous registration and classification of brain tissue. Nevertheless, cortical thickness estimates obtained using CIVET show high test-rest reliability (Jeon et al. 2017; Lewis et al. 2017), and a direct comparison of CIVET to FreeSurfer's longitudinal pipeline revealed no significant differences in effect sizes (Redolfi et al. 2015).

\subsubsection{Linear regression modelling}

Longitudinal mixed-effects models with cubic, quadratic, and linear age effects were fitted to the cortical thickness estimates while allowing for random intercept for individuals using the nlme package (Pinheiro et al. 2017) in R. The most parsimonious model was selected based on the log-likelihood ratio test.

\subsubsection{Genetic twin modelling}

Twin modelling can provide information on the variation of a trait in the population that can be explained by genetic factors (Posthuma et al. 2000; Boomsma et al. 2002). Based on the assumption that monozygotic twins share $100 \%$ of their genetic material and dizygotic twins share on average $50 \%$ of their segregating genes, the phenotypic variance $(V)$ of a trait is usually decomposed into three independent variance components: additive genetic $(A)$, common environmental $(C)$, and unique environmental $(E)$ components of variance. Additive genetic influences represent effects of multiple alleles at different loci across the 
genome that act in conjunction on the phenotypic trait. Common environmental influences represent sources of variance that are shared between twins of the same family and cause the twins to be more alike than children growing up in different families. Unique environmental influences are not shared by family members and may include measurement error (Falconer and Mackay 1996; Boomsma et al. 2002). If monozygotic twins resemble each other more than dizygotic twins for a given trait, then this difference is usually attributed to genetic influences. If both monozygotic and dizygotic twins are more alike in resemblance than expected based on genetics, common environmental influences are thought to play a role. Residual variation between twin pairs is attributed to unique environmental influences.

\subsubsection{Structural equation modelling}

Within structural equation modelling (SEM), the variance in univariate or multivariate phenotypes can be modelled as the combined effect of additive genetic factors, and common and unique environmental factors. These factors are modelled as latent variables with unit variance where path coefficients or factor loadings $a, c, e$ and quantify their respective influence on the phenotypic trait(s). The model is made identifiable by putting constraints on the correlation $\rho_{\widehat{A}}$ between the latent variable $\hat{A}$ of twin pairs; $\rho_{\hat{A}}=1.0$ for monozygotic twins, and $\rho_{\widehat{A}}=0.5$ for dizygotic twins. The correlation $\rho_{\hat{C}}$ between latent variable $\hat{C}$ of members of a twin pair is constrained to $\rho_{\hat{C}}=1.0$ for both monozygotic and dizygotic twins. The latent variable $\hat{E}$ is uncorrelated between individuals within and across twin pairs. The sum of the squared path coefficients $a^{2}, c^{2}$, and $e^{2}$, representing the variance components $A, C$, and $E$, is equal to the phenotypic variance $(V)$; i.e. $V=A+C+E=a^{2}+c^{2}+e^{2}$. Heritability $\left(h^{2}\right)$ of the trait is estimated as the proportion of phenotypic variance $(V)$ that is due to additive genetic variance $(A)$; i.e. $h^{2}=\frac{A}{V}=\frac{a^{2}}{a^{2}+c^{2}+e^{2}}$.

Nested models can be obtained by constraining parameters of interest in the model. Testing significance of nested models is performed using the log-likelihood ratio test. Statistical significance was determined by comparing the likelihood of the model fits from the model with and without a constraint on the parameter of interest. The difference in -2 times the $\log$ likelihood $(-2 L L)$ follows a $\chi^{2}$ distribution. For variance components (e.g. heritability estimates, but not correlations), $-2 L L$ asymptotically follows a 50:50 mixture of $\chi^{2}$ distributions with zero and one degree of freedom; effectively allowing $p$-values to be cut in half (Dominicus et al. 2006).

Structural equation models were defined using OpenMx version 2.2.6 (Boker et al. 2015), a package for structural equation modelling in $\mathrm{R}$ ( $\mathrm{R}$ Core Team 2015). Model fitting was performed using full-information maximum likelihood (FIML) to take advantage of all 
available information in case of missing data. E.g., when no data is available for one of the twins in a pair at any age, thereby creating a singleton "twin" at any or all three ages, FIML can still use the information from the available twin to improve the estimates of means and variances, thereby improving the overall fitting of the model parameters.

Based on our previous work (van Soelen, Brouwer, van Baal, et al. 2012) and the fact that evidence for common environmental influences on cortical thickness is limited in the literature, we assume that common environment latent variable $(\hat{C})$ could be dropped from our model. Indeed, based on the log-likelihood and Akaike Information Criterion, the longitudinal $A E$ model fitted the cortical thickness measurements better than $A C E, C E$, or $E$ on the global and lobar level (see Supplementary Table A.2). We therefore adopted the $A E$ model in all analyses.

\subsubsection{Longitudinal twin model to investigate heritability of changes in cortical thickness}

The phenotypic measurements of cortical thickness at the three ages were used to define a longitudinal twin model at every vertex of the brain surface model (Figure 2.1). From this longitudinal model, we obtained estimates for heritability of changes in cortical thickness; i.e. genetic factors influencing the rate of cortical thinning during childhood and adolescence. Subsequently, we attempt to identify if heritability of changes in cortical thickness are due to increasing (i.e. amplification) or decreasing (i.e. deamplification) influences of the same genetic factor influencing cortical thickness at both ages, or if heritability of changes in cortical thickness is due to the emergence of a novel genetic factor unique for the second age (i.e. genetic innovation; Figure 2.2). Genetic innovation can also represent the disappearance of a genetic factor unique for the first age, which unfortunately is indistinguishable in the current model due to symmetry. Derivation for the estimation of heritability of changes in cortical thickness are as follows. For simplification of the mathematical equations, we will refer to the measurement at age 9 years as wave 1 , refer to the measurement at age 12 years as wave 2, and refer to the measurement at age 17 years as wave 3 . Phenotypic variance of change $V_{\Delta i j}$ in cortical thickness between wave $i$ and wave $j$ can be derived from the phenotypic variance matrix $V$ of the cortical thickness measures as the sum of the phenotypic variance at the individual waves reduced by twice the phenotypic covariance between the two waves. The same rule applies to genetic $A_{\Delta_{i j}}$ and environmental $E_{\Delta_{i j}}$ variance of changes in cortical thickness. For example, the genetic variance of changes $A_{\Delta_{i j}}$ in cortical thickness between wave $i=1$ and wave $j=2$ is defined as

$$
A_{\Delta_{12}}=A_{11}+A_{22}-2 \cdot A_{12}
$$




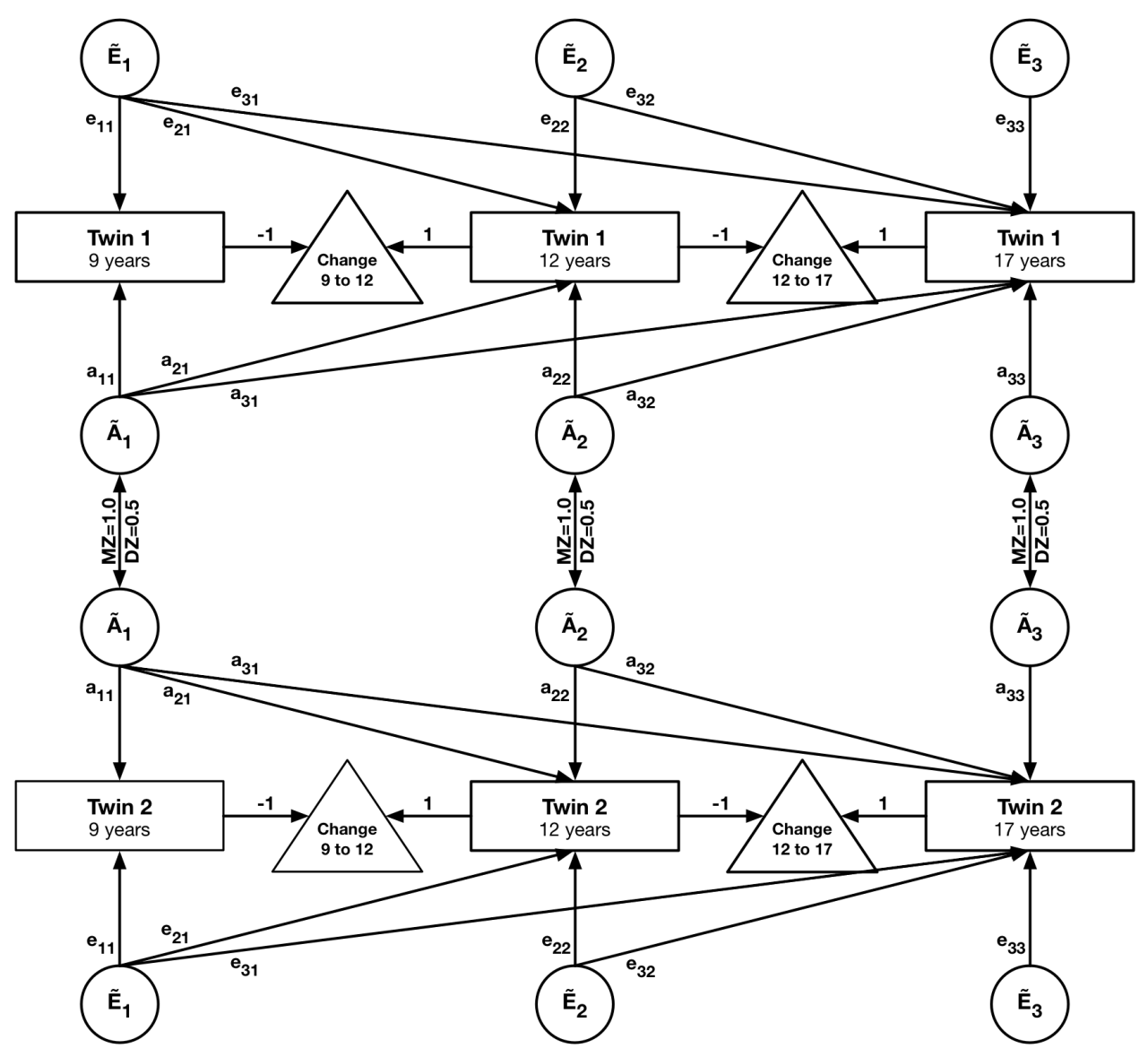

Figure 2.1. Path diagram of longitudinal AE model used to determine the heritability of changes in cortical thickness. The longitudinal cortical thickness measurements for a region of interest at age 9,12 , and 17 years are used as observed variables (rectangular boxes) for the first (upper half of path diagram) and the second twin (lower half of the path diagram) of each twin pair. Change rates in cortical thickness (triangles) are computed by the model as the difference between the observed variables Independent genetic factors $\hat{A}_{1}, \hat{A}_{2}$, and $\hat{A}_{3}$ (circles) load onto the longitudinal cortical thickness measurements through path coefficients. The genetic factor $\hat{A}_{1}$ represents genetic influences shared across all three ages through path coefficients $a_{11}, a_{21}$, and $a_{31}$. The genetic factor $\hat{A}_{2}$ represents genetic influences shared only between age 12 and 17 years through path coefficients $a_{22}$ and $a_{23}$. The genetic factor $\hat{A}_{3}$ represents genetic influences specific for age 17 years through path coefficient $a_{33}$. The same motif applies for the unique environmental factors $\hat{E}_{1}, \hat{E}_{2}$ and $\hat{E}_{3}$, and path coefficients $e_{11}, e_{21}, e_{31}, e_{22}, e_{23}$, and $e_{33}$. The model is made identifiable by constraining the correlation between genetic factors of both twins to 1.0 in case of monozygotic twins and 0.5 in case of dizygotic twins. 


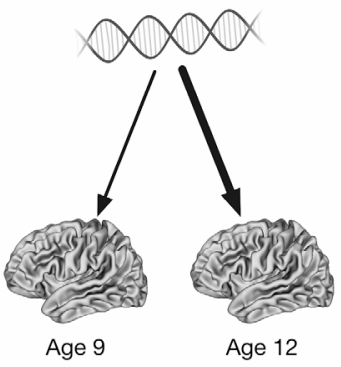

Amplifcation

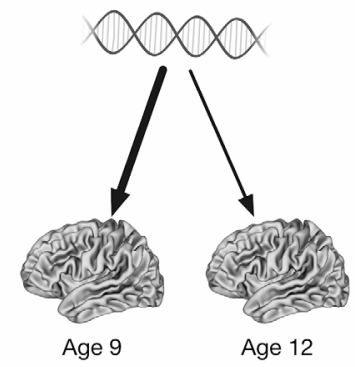

Deamplification

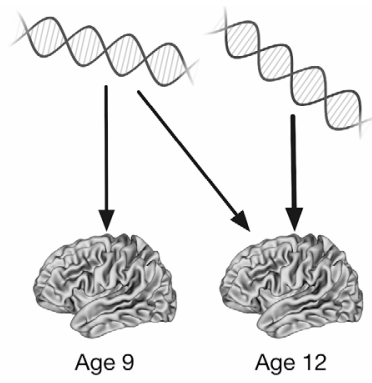

Genetic innovation

Figure 2.2. Schematic depiction of genetic amplification, deamplification, and innovation in an example with two ages. For genetic (de)amplification, the same genetic factor influences the phenotype during both ages, but to a greater extent during the second age compared to the first age for genetic amplification, and to a lesser extent during the second age compared to the first age for genetic deamplification. For genetic innovation, the same genetic factor may influence the phenotype during both ages, and an additional genetic factor that is distinct from the first genetic factor and unique to the second age influences the phenotype during the second age.

and the genetic variance of changes $A_{\Delta_{i j}}$ in cortical thickness between wave $i=2$ and wave $j=3$ is defined as

$$
A_{\Delta_{23}}=A_{22}+A_{33}-2 \cdot A_{23}
$$

where $A_{i j}$ is the genetic (co)variance in cortical thickness between wave $i$ and wave $j$. Heritability of changes $\left(h_{\Delta_{12}}^{2}=\frac{A_{\Delta_{12}}}{V_{\Delta_{12}}}\right)$ in cortical thickness between wave $i$ and wave $j$ is then the proportion of phenotypic variance of changes $V_{\Delta_{i j}}$ in cortical thickness between wave $i$ and wave $j$ due to additive genetic variance of changes $A_{\Delta_{i j}}$ in cortical thickness between wave $i$ and wave $j$ :

$$
h_{\Delta_{12}}^{2}=\frac{A_{\Delta_{12}}}{V_{\Delta_{12}}} \quad \text { and } \quad h_{\Delta_{23}}^{2}=\frac{A_{\Delta_{23}}}{V_{\Delta_{23}}}
$$

Using a similar rationale as in (van Soelen, Brouwer, van Baal, et al. 2012), genetic variance of changes in cortical thickness between wave 1 and 2 (i.e. $A_{\Delta_{12}}$ ) can be calculated as $a_{11}^{2}+$ $a_{22}^{2}+a_{21}^{2}-2 \cdot\left(a_{21} \cdot a_{11}\right)$ using path tracing rules; simplified, this gives $A_{\Delta_{12}}=a_{22}^{2}+\left(a_{11}-\right.$ $\left.a_{21}\right)^{2}$ (see Figure 2.1 for definition of the path coefficients $a_{i j}$ ). The first part of the equation represents the contribution of genetic factor $\widehat{A_{2}}$ specific for wave 2 , while the second part represents gradual changes in influences of genetic factor $\hat{A}_{1}$ on cortical thickness from wave 1 to wave 2 . When $a_{22}>0$ we speak of genetic innovation at wave 2 . When $a_{21}>a_{11}$ we speak of genetic amplification of factor $\hat{A}_{1}$ between wave 1 and wave 2 , and when $a_{21}<$ $a_{11}$ we speak of genetic deamplification of factor $\hat{A}_{1}$ between wave 1 and wave 2 . Using the same path tracing approach, genetic variance of change between wave 2 and 3 (i.e. $A_{\Delta_{23}}$ ) is 
calculated as $a_{22}^{2}+a_{21}^{2}+a_{33}^{2}+a_{32}^{2}+a_{31}^{2}-2 \cdot\left(a_{31} \cdot a_{21}+a_{32} \cdot a_{22}\right)$; simplified, this gives $A_{\Delta_{23}}=a_{33}^{2}+\left(a_{22}-a_{32}\right)^{2}+\left(a_{21}-a_{31}\right)^{2}$ (see Figure 2.1 for definition of the path coefficients $\left.a_{i j}\right)$. The first part of the equation represents the contribution of genetic factor $\hat{A}_{3}$ specific for wave 3 , the second part represents gradual changes in influences of genetic factor $\hat{A}_{2}$ on cortical thickness from wave 2 to wave 3 , and the third and last part represents gradual changes in influence of genetic factor $\hat{A}_{1}$ on cortical thickness from wave 2 to wave 3 . When $a_{33}>0$ we speak of genetic innovation at wave 3 . When $a_{31}>a_{21}$ we speak of genetic amplification of factor $\hat{A}_{1}$ between wave 2 and wave 3 , and when $a_{31}<a_{21}$ we speak of genetic deamplification of factor $\hat{A}_{1}$ between wave 2 and wave 3 . When $a_{32}>a_{22}$ we speak of genetic amplification of factor $\hat{A}_{2}$ between wave 2 and wave 3 , and when $a_{32}<a_{22}$ we speak of genetic deamplification of factor $\hat{A}_{2}$ between wave 2 and wave 3 .

\subsubsection{Determining the source of heritability of changes in cortical thickness}

Heritability of changes in cortical thickness can be the result of innovation of novel genetic factors or (de)amplification of existing genetic factors. We employed a step-wise nested model testing approach to determine the most likely origin of the heritability of changes in cortical thickness. First, we determined all vertices that show significant heritability of changes in cortical thickness (FDR adjusted $p<0.05$; Genovese et al. 2002) between wave 1 and 2 and similarly between wave 2 and 3. For those vertices, we first tested whether the heritability of changes in cortical thickness originated from innovation of genetic factors. Testing for innovation of genetic factor $\hat{A}_{2}$ at wave 2 was performed by comparing the model with path coefficient $a_{22}$ and $a_{32}$ constrained to zero to the base model without any constraints. Testing for innovation of genetic factor $\hat{A}_{3}$ at wave 3 was performed by comparing the model with path coefficient $a_{33}$ constrained to zero to the base model without any constraints.

If no evidence for innovation was found, we continued with testing for (de)amplification of existing genetic factors. All models testing for (de)amplification of existing genetic factors were compared to a reference model where no innovation of genetic factors was possible by constraining path coefficients $a_{22}, a_{32}$ and $a_{33}$ to zero. Testing for (de)amplification of genetic factor $\hat{A}_{1}$ between wave 1 and 2 was performed by additionally constraining path coefficient $a_{11}$ to be equal to $a_{21}$ in the model and comparing it to the reference model. Testing for (de)amplification of genetic factor $\hat{A}_{1}$ between wave 2 and wave 3 was performed in the same way by additionally constraining path coefficient $a_{21}$ to be equal to $a_{31}$ instead. 


\subsubsection{Statistical significance of parameters}

Statistical significance of heritability of cortical thickness (and heritability of changes in cortical thickness) was tested comparing the -2 log-likelihood of the unconstrained model to the -2 log-likelihood of the nested model with the heritability estimate at a given age (or interval) constrained to a fixed value of 0 . This statistic asymptotically follows a 50:50 mixture of $\chi^{2}$ distributions with zero and one degree of freedom; allowing $p$-values to be cut in half (Dominicus et al. 2006). Likewise, statistical significance of differences in heritability of cortical thickness between ages was determined by comparing the unconstrained model and a nested model in which the heritability estimates at the different ages were set to be equal.

2.2.5.5 Bivariate twin model to investigate overlap in genetic and environmental factors between brain regions

To investigate the presence of interrelationship in genetic and environmental factors between two brain regions across space and age, we estimated the genetic and environmental correlation for all possible pairs of regions of interest across the three waves. The genetic correlation $\left(r_{g}\right)$ between region $x$ at wave $i$ and region $y$ at wave $j$ is defined as

$$
r_{g}\left(x_{i}, y_{j}\right)=\frac{A_{x_{i} y_{j}}}{\sqrt{A_{x_{i}} \cdot A_{x_{i}}}}
$$

where $A_{x_{i} y_{j}}$ is the genetic covariance between the two regions, and $A_{x_{i}}$ and $A_{y_{j}}$ represent the genetic variances of the individual regions. The same definition applies to environmental correlation $\left(r_{e}\right)$ using environmental (co)variances $E_{x_{i} y_{j}}, E_{x_{i}}$ and $E_{y_{j}}$. Sequential bivariate analysis of all 630 unique pairwise bivariate models (6 lobar regions per hemisphere for each wave) was employed to populate a unitriangular matrix with dimensions $36 \times 36$ cells for the phenotypic $\left(r_{p h}\right)$, genetic $\left(r_{g}\right)$ and environmental $\left(r_{e}\right)$ correlation between regions of interest; a path diagram of the bivariate $A E$ model used in the analysis is presented in Supplementary Figure A.2. Statistical significance of a correlation was tested using a $\chi^{2}$ distribution with one degree of freedom on the difference in log-likelihood of the unconstrained model and a nested model with the correlation constrained to a fixed value of $-1,0$, or +1 . Correlation matrices were visualized using the corrplot package in R (Wei and Simko 2016). Next, we applied cluster analysis to the separate phenotypic and genetic correlation matrices to extract groups of regions with high phenotypic, genetic, or environmental similarity. 
2.2.6 Cluster analysis based on phenotypic, genetic, and environmental correlation matrices To investigate spatial and temporal patterns of the cortex we applied a hierarchical clustering algorithm to the phenotypic, genetic, and environmental correlation matrices obtained from the bivariate twin model using the cluster package in R (Maechler et al. 2016). Prior to clustering, the phenotypic and genetic correlation matrices were transformed into dissimilarity matrices using $1-r_{p h}$ for the phenotypic correlation matrix, $1-\left|r_{g}\right|$ for the genotypic correlation matrix, and $1-\left|r_{e}\right|$ for the environmental correlation matrix. For the phenotypic correlation matrix, this transformation ensured that highly correlated regions would have low dissimilarity whereas anti-correlated regions would have high dissimilarity. In contrast, for the genotypic and environmental correlation matrices, both highly correlated and anti-correlated regions (i.e. regions under the influence of the same genes or environmental factors but with opposing effects on the phenotype) would have low dissimilarity. The optimal number of clusters $k_{\text {opt }}$ was determined by selecting the minimum value of $k$ for which the average silhouette width was within one standard error of the maximum average silhouette width (Rousseeuw 1987).

\subsubsection{Post-hoc analyses}

To investigate the possible effects of confounds on the results of the analyses, we performed a qualitative post-hoc analysis using twin models where cortical thickness measurements were corrected for sex, age at scan (and thereby implicitly individual scan interval between ages), and handedness. Residuals after linear regression of covariates on the cortical thickness data were used as input to the structural equation models. We performed a qualitative evaluation of the effects of confounds by visual inspection of the cortical maps and correlation matrices with and without regression of covariates.

Since heteroscedasticity between groups can have greater influences on the results in twin modelling than correcting for mean effects, we performed a quantitative post-hoc analysis to investigate the effects of sex and handedness on the mean and variance of whole-brain cortical thickness estimates. We used a univariate saturated twin model with coefficients on the mean and (co)variance estimates to model effects of sex and handedness. The statistical significance of the effects was tested using a $\chi^{2}$ distribution with one degree of freedom on the difference in log-likelihood of the unconstrained model and a nested model with the coefficient constrained to a fixed value of 0 .

\subsection{RESULTS}

\subsubsection{Development of cortical thickness}

The mean global cortical thickness was 3.38, 3.31, and $3.07 \mathrm{~mm}$ at age 9,12 , and 17 years (Figure 2.3A; Supplementary Table A.3). A quadratic age curvature best described the 
trajectory of cortical thickness development (cubic versus quadratic: $p=0.7863$ ); quadratic versus linear: $p<0.001$ ). The linear approximation of the annual rate of change between the ages 9 and 12 years was $-0.023 \mathrm{~mm} /$ year $\left(\mathrm{CI}_{95}[-0.028\right.$ to $-0.018 \mathrm{~mm} /$ year]; $p<0.001)$ and doubled to $-0.049 \mathrm{~mm} /$ year $\left(\mathrm{CI}_{95}[-0.052\right.$ to $-0.045 \mathrm{~mm} /$ year $\left.] ; p<0.001\right)$ between the ages 12 and 17 years (Figure 2.3A; Supplementary Table A.3). No significant effects of sex $(p>0.302)$ or handedness ( $p>0.243)$ on mean global cortical thickness or changes in mean global cortical thickness (sex: $p>0.081$; handedness: $p>0.145$ ) were found (Figure 2.3A). No significant effects of sex $(p>0.469)$ or handedness $(p>0.107)$ on variance of mean global cortical thickness or changes in mean global cortical thickness (sex: $p>0.060$; handedness: $p>0.305$ ) were found.

As previously reported (van Soelen, Brouwer, van Baal, et al. 2012), at age 9 years, regions with highest cortical thickness are found at the insula, temporal pole, and medial frontal areas, with local thickness up to $4.58 \mathrm{~mm}$ (Figure 2.3B). Regions with lowest cortical thickness are found in the visual cortex and sensorimotor cortices, with local thickness down to $2.81 \mathrm{~mm}$ (Figure 2.3B). Between the ages 9 and 12 years there is a subtle decrease in cortical thickness for most parts of the cortex, whereas some parts of the cortex, such as the bilateral gyrus rectus, parahippocampal gyrus, and superior- and middle temporal poles, show non-significant changes in cortical thickness (Figure 2.3C). The decrease in cortical thickness is most prominent at the visual cortex, primary sensory and motor cortices, and frontal poles, with rate of change between -0.03 to $-0.05 \mathrm{~mm} /$ year (Figure 2.3C).

When including the third measurement at age 17 years, we found that the rate of changes in cortical thickness between the ages 12 and 17 years accelerates and expands to most regions of the cortex, with local maximum rate of change reaching up to $-0.16 \mathrm{~mm} /$ year in the superior frontal gyrus (Figure 2.3C). The least decrease in cortical thickness occurs bilaterally at parahippocampal gyrus, olfactory cortex, and cingulum, with rate of change between 0.01 to $0.02 \mathrm{~mm} /$ year (Figure $2.3 \mathrm{C}$ ).

\subsubsection{Heritability of cortical thickness}

Heritability of mean global cortical thickness was $62 \%\left(\mathrm{CI}_{95}[46 \%\right.$ to $\left.74 \%] ; p<0.001\right)$ at age 9, $80 \%\left(\mathrm{CI}_{95}[65 \%\right.$ to $\left.88 \%] ; p<0.001\right)$ at age 12 , and $54 \%\left(\mathrm{CI}_{95}[32 \%\right.$ to $\left.70 \%] ; p<0.001\right)$ at age 17 years; see Supplementary Figure A.3 for local heritability estimates of cortical thickness and Supplementary Table A.3 for regional heritability estimates. Differences in heritability of mean global cortical thickness was significant between ages 12 and 17 years $(p=0.036)$, but not significant between ages 9 and 12 years ( $p=0.104$ [n.s.]) or between ages 9 and 17 years $(p=0.495$ [n.s.]). 

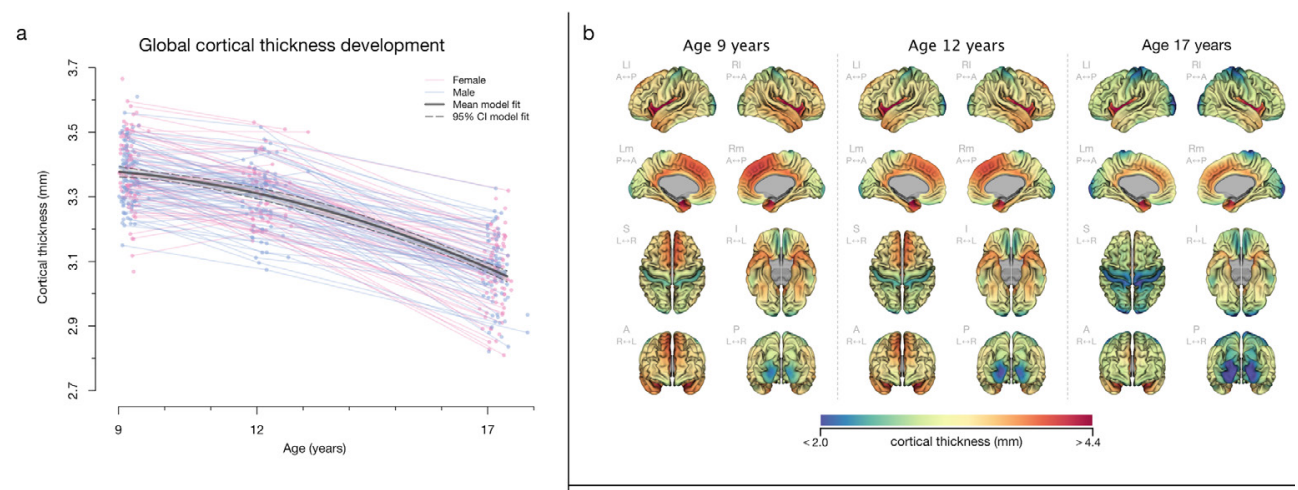

\begin{tabular}{|c|c|c|c|}
\hline Gex & Age 9 years & Age 12 years & Age 17 years \\
\hline Females & $3.38(3.36 ; 3.40)$ & $3.32(3.29 ; 3.34)$ & $3.07(3.04 ; 3.09)$ \\
\hline Males & $3.37(3.35 ; 3.38)$ & $3.30(3.27 ; 3.32)$ & $3.07(3.05 ; 3.09)$ \\
\hline Combined & $3.38(3.36 ; 3.39)$ & $3.31(3.29 ; 3.33)$ & $3.07(3.05 ; 3.08)$ \\
\hline
\end{tabular}

c

Annual change in global cortical thickness by age and sex

\begin{tabular}{|c|c|c|}
\hline Sex & Age $9 \rightarrow 12$ years & Age $12 \rightarrow 17$ years \\
\hline Females & $-0.021(-0.027 ;-0.015)$ & $-0.051(-0.055 ;-0.046)$ \\
\hline Males & $-0.022(-0.029 ;-0.016)$ & $-0.047(-0.053 ;-0.041)$ \\
\hline Combined & $-0.023(-0.028 ;-0.018)$ & $-0.049(-0.052 ;-0.045)$ \\
\hline
\end{tabular}

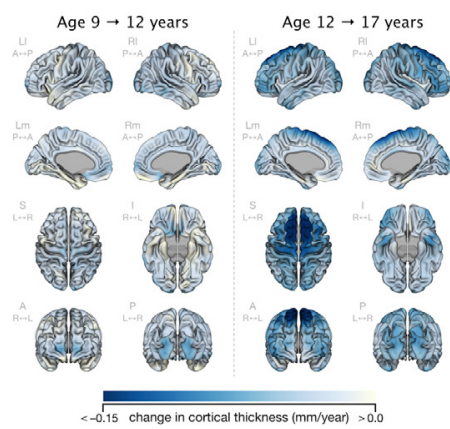

Figure 2.3. Developmental pattern of (A) global and (B,C) local cortical thickness during childhood and adolescence. (A) Global cortical thickness shows accelerated thinning during adolescence. Data points represent individual measurements, with lines connecting data points representing longitudinal measurements between age 9 and 12 years, and between age 12 and 17 years. A quadratic model best described overall thinning of mean global cortical thickness (thick solid line accompanied by $95 \%$ confidence interval). No significant sex effects were found for global cortical thickness at any age nor for annual change rates in global cortical thickness at either scan intervals. Values reported in tables are mean and 95\% confidence intervals. (B) Absolute cortical thickness across the ages 9 years (left panel), 12 years (middle panel), and 17 years (right panel) reveal regional effects of cortical thinning during adolescence. Cortical thickness ranges from less than $2.0 \mathrm{~mm}$ (blue) in the occipital cortex at age 17 years up to greater than $4.4 \mathrm{~mm}$ (red) in the insular cortex at age 9 years. (c) Annual development of cortical thickness between ages 9 and 12 years (left panel), and between ages 12 and 17 years (right panel) based on linear approximation emphasize regional differences in rate of cortical thinning, particularly in the medial frontal cortex. The rate of changes in cortical thickness ranges from non-significant changes in cortical thickness per year (white) in mostly medial temporal regions between age 9 and 12 years to a decrease in cortical thickness of $0.15 \mathrm{~mm} /$ year or greater (dark blue) in medial frontal cortex between age 12 and 17 years. The rate of cortical thinning doubles between the ages 12 and 17 years compared to the rate of change between the ages 9 and 12 years. (B,C) Order of views per age, from left to right, top to bottom: left lateral (Ll), right lateral (Rl), left medial (Lm), right medial $(\mathrm{Rm})$, superior $(\mathrm{S})$, inferior $(\mathrm{I})$, anterior $(\mathrm{A})$, and posterior $(\mathrm{P})$.

\subsubsection{Hierarchical clustering of phenotypic and genetic correlation matrices}

A highly correlated genetic factor was involved in mean global cortical thickness at ages 9 and 12 years $\left(r_{g}=0.92 ; \mathrm{CI}_{95}[0.75\right.$ to 1.00$\left.]\right)$. While there was significant genetic overlap 
between age 9 and age 17 years $\left(r_{g}=0.68 ; \mathrm{CI}_{95}\right.$ [0.40 to 0.94 ]) and age 12 and age 17 years $\left(r_{g}=0.64 ; \mathrm{CI}_{95}\right.$ [0.41 to 0.87]), the confidence intervals of the genetic correlations suggest additional genetic factors influencing cortical thickness at age 17 years.

We estimated the phenotypic, genetic, and environmental correlations of cortical thickness between the major lobes of the cortex across childhood and adolescent development (Figure 2.4). The phenotypic and environmental correlation matrices show strong similarities in pattern. There is a moderate to strong association between the major lobes within hemisphere (Figure 2.4AC), and a strong association between homologous regions across hemispheres within each age for phenotypic correlations that is absent for environmental correlations (Figure 2.4A-C). In addition, both phenotypic and environmental correlations show an association of the same regions over time, although this association is only weak to moderate for environmental correlations (Figure 2.4A-C). In contrast, genotypic correlations show strong association between all regions across all three ages. However, many of the associations with regions at age 17 years are absent due to non-significant associations (Figure 2.4B). Although many regions share a common genetic factor, a portion of these regions are influenced by an additional genetic factor unique for each region (i.e. incomplete pleiotropy), indicated by a white dot (Figure 2.4B). Of special note, regions within the same age are influenced by distinct genetic factors (i.e. spatial genetic differentiation of lobes), as well as the same regions across age (i.e. temporal genetic differentiation): left parietal cortex and right frontal cortex between age 9 and 17 years, and right parietal between age 12 and 17 years.

We performed hierarchical clustering analysis on the correlation matrices (Figure 2.4). The optimal number of clusters $k_{\text {opt }}$ was determined using the silhouette heuristic (Rousseeuw 1987); $k_{\text {opt }}=6$ for the phenotypic correlation matrix, $k_{\text {opt }}=7$ for the genetic correlation matrix, and $k_{\text {opt }}=6$ for the environmental correlation matrix (Supplementary Figure A.4).

The global pattern reveals the insular cortex and cingulate cortex form separate clusters from the frontal, temporal, and parietal lobes, with occipital lobe forming a third independent cluster. Clustering of the phenotypic and genetic correlation matrices separates the frontal, parietal, and temporal lobes at age 17 years from age 9 and 12 years (Figure 2.4A-B). In addition, clustering of the genetic correlation matrix pairs homotopic regions across hemisphere, whereas clustering of the phenotypic correlation matrix groups regions by hemisphere (Figure 2.4A-B). In contract, clustering of the environmental correlation matrix reveals a strong pattern where regions are first clustered by age followed 
a

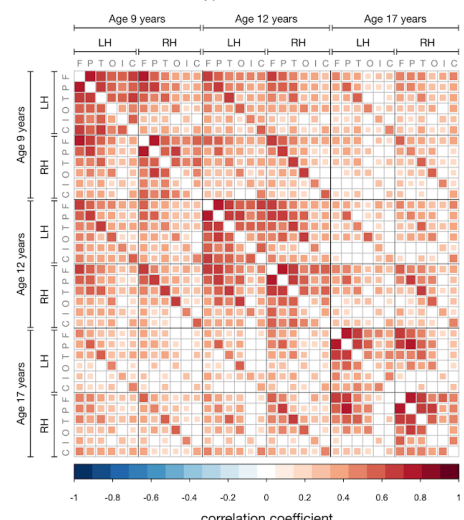

Dendogram of phenotypic correlation matrix

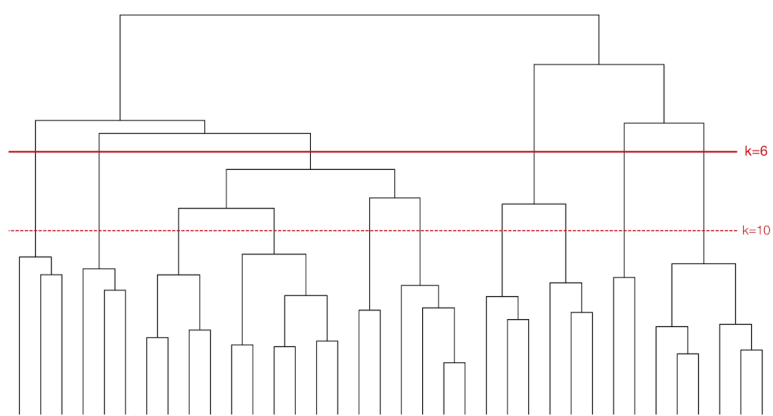

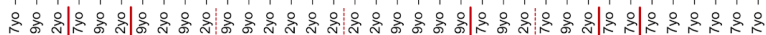

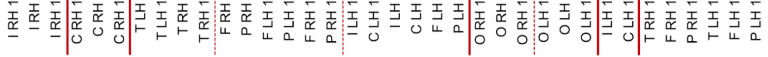
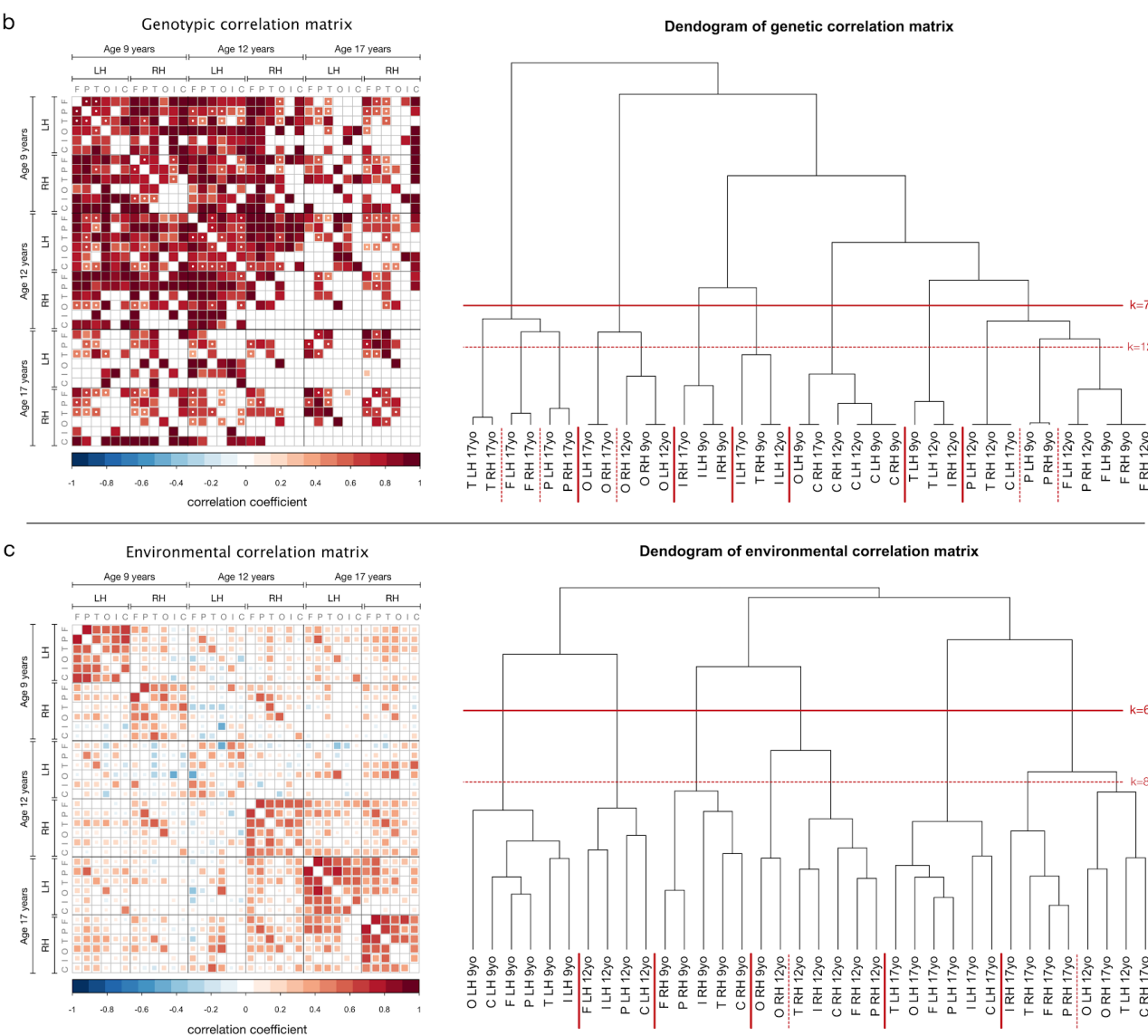

Dendogram of environmental correlation matrix

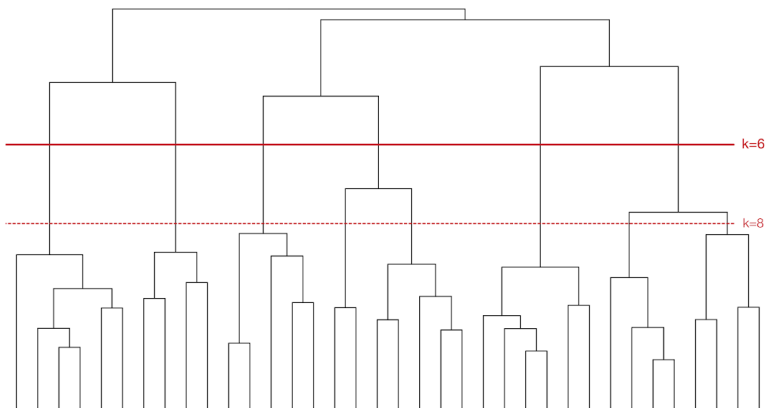

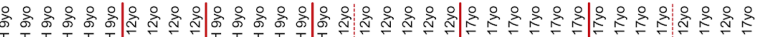

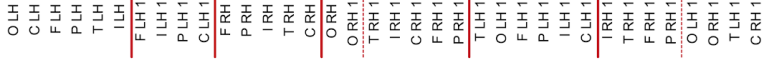

Figure 2.4 (continued on the next page). Hierarchical clustering of the phenotypic, genetic, and environmental correlation matrix of absolute cortical thickness across childhood and adolescent development. (A) The correlation matrices for phenotypic (top), genetic (middle), and environmental (bottom) correlation in cortical thickness 
Figure 2.4 (continued). between the major lobes of the cortex. Correlations range from -1 (blue) to +1 (red). Correlations that did not differ from zero $(p<0.001$; uncorrected) are left blank. Genetic correlations marked with a white dot indicate incomplete pleiotropy (i.e. unique genetic factors for each region in addition to a shared genetic factor). Regions are ordered from top-left to bottom-right, first by age (9, 12, and 17 years), then by hemisphere ( $\mathrm{LH}=$ left hemisphere, and $\mathrm{RH}=$ right hemisphere), and finally by lobe ( $\mathrm{F}=$ frontal, $P=$ parietal, $\mathrm{T}$ =temporal, $\mathrm{O}=$ occipital, $\mathrm{I}=$ insula, and $\mathrm{C}=$ cingulate). (B) Dendrogram for the hierarchical clustering of the distance-transformed phenotypic (top), genotypic (middle), and environmental (bottom) correlation matrices. Phenotypic correlations were transformed using one minus the phenotypic correlation, and genetic and environmental correlations were transformed using one minus the absolute of the genetic or environmental correlation. The optimal number of clusters was determined by the average silhouette (see Supplementary Figure A.4); $k_{\text {opt }}=6$ for the phenotypic correlation matrix, $k_{\text {opt }}=7$ for the genetic correlation matrix, and $k_{\text {opt }}=6$ for the environmental correlation matrix. Optimal clusters are separated by a solid red line, while global optimum uses a dotted red line. Labels for the regions are encoded as lobe ( $\mathrm{F}=$ frontal, $P=$ parietal, $\mathrm{T}=$ temporal, $\mathrm{O}=\mathrm{occipital}$, $\mathrm{I}=$ insula, and $\mathrm{C}=$ cingulate), followed by hemisphere ( $\mathrm{LH}=$ left hemisphere, and $\mathrm{RH}=$ right hemisphere), and finally age (9yo=age 9 years, 12 yo=age 12 years, and 17 yo=age 17 years).

by hemisphere (Figure 2.4C). The described patterns become more apparent when using regions from only one or two of the ages (Supplementary Figure A.5).

Post-hoc analysis on the effects of possible confounds revealed little effect from sex, age at scan, and handedness on the correlation matrices of cortical thickness between cerebral lobes (Supplementary Figure A.6).

\subsubsection{Heritability of changes in cortical thickness}

Heritability of changes in mean global cortical thickness is $21 \%$ ( $\mathrm{Cl}_{95}$ [0\% to $52 \%$ ]; $p=0.154$ [n.s.]) between the ages 9 and 12 years, and 53\% ( $\mathrm{CI}_{95}$ [26\% to 72\%]; $\left.p<0.001\right)$ between the ages 12 and 17 years; see Supplementary Table A.3 for regional heritability of changes in cortical thickness. Locally, heritability of changes in cortical thickness is most prominent at association cortices in the frontal, parietal, and temporal lobes (FDR-adjusted $p<0.05$ ), where it reaches up to $76 \%$ heritability between the ages 9 and 12 years, and up to $82 \%$ heritability between the ages 12 and 17 years (Figure 2.5A).

Post-hoc analysis on the effects of possible confounds reveals little effect from sex, age at scan and handedness on heritability of changes in cortical thickness (Supplementary Figure A.7).

\subsubsection{Decomposition of heritability of changes in cortical thickness}

Locally, we find several large clusters of genetic innovation in the right superior medial frontal gyrus, near the right calcarine sulcus, right superior medial frontal gyrus, left medial orbital frontal cortex, right parahippocampal gyrus, left fusiform gyrus, right Heschl gyrus, right middle frontal gyrus, bilateral postcentral gyrus, and right middle occipital gyrus between age 9 and 12 years (Figure 2.5B and Table 2.1). These clusters show strong heritability of changes in cortical thickness with estimates between $40 \%$ to $71 \%$ (FDRadjusted $p<0.023)$ and genetic innovation $(p<0.017)$. Between age 12 and 17 years, large 
a

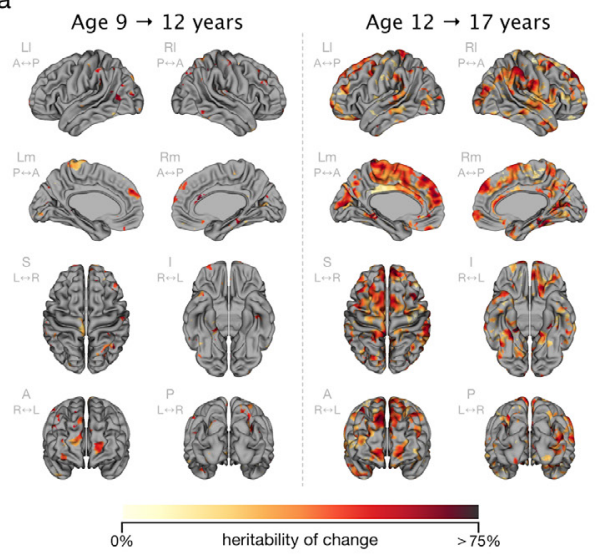

b

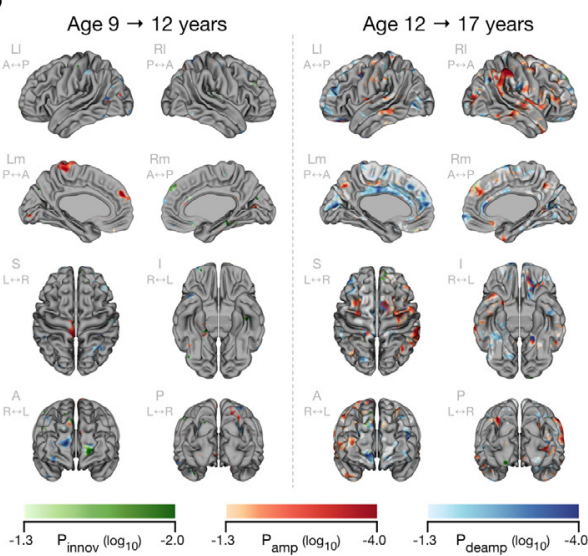

Figure 2.5. Estimated heritability of (A) changes in cortical thickness and (B) its decomposition into different genetic origins between the ages 9 and 12 years (left panel), and between the ages 12 and 17 years (right panel). (A) Heritability estimates that did not differ significantly from zero (FDR adjusted $p<0.05$ ) have been left grey. Approximately $3.1 \%$ of the vertices between age 9 and 12 years and $28.4 \%$ of the vertices between age 12 and 17 years are significant for heritability of changes in cortical thickness (FDR adjusted $p<0.05$ ). Heritability estimates range from $0 \%$ (light-yellow) up to $75 \%$ or greater (dark-red). (B) Heritability of changes in cortical thickness between the ages 9 and 12 years (left panel) and between the ages 12 and 17 years (right panel) was decomposed into sources of genetic innovation (green; $20.2 \%$ of the vertices significant for heritability of changes in cortical thickness between the ages 9 and 12 years, and $1.2 \%$ between the ages 12 and 17 years), and areas with amplification (red; $49.2 \%$ of the vertices between the ages 9 and 12 years, and $29.3 \%$ between the ages 12 and 17 years) or deamplification (blue; $28.5 \%$ of the vertices between the ages 9 and 12 years, and $28.4 \%$ between the ages 12 and 17 years) of a genetic factor across age. Areas with significant heritability of changes in cortical thickness for which these sources could not be disentangled are depicted in light grey (remaining $2.1 \%$ of the vertices significant for heritability of changes in cortical thickness between the ages 9 and 12 years, and $41.1 \%$ between the ages 12 and 17 years). Scale bars for significance start at $\log _{10}$-equivalent of $p=0.05$. (A,B) Order of views per age, from left to right, top to bottom: left lateral (Ll), right lateral (Rl), left medial (Lm), right medial (Rm), superior $(\mathrm{S})$, inferior $(\mathrm{I})$, anterior $(\mathrm{A})$, and posterior $(\mathrm{P})$.

clusters of genetic innovation were found at the right superior medial frontal cortex, right lingual gyrus, left supramarginal gyrus, left calcarine sulcus, and left superior parietal gyrus (Figure 2.5B and Table 2.1). These clusters show similarly strong heritability of changes in cortical thickness with estimates between $50 \%$ to $70 \%$ (FDR-adjusted $p<0.024$ ) and genetic innovation $(p<0.017)$. In addition to genetic innovation, we found clusters of genetic (de)amplification becoming more widespread throughout the cortex during later adolescence, with most evident amplification in the right supramarginal gyrus, and deamplification in the medial frontal cortex, cingulum, and occipital cortex between the ages 12 and 17 years (Figure 2.5B). 
Table 2.1. Overview of largest clusters with genetic innovation between age 9 and 12 years and between age 12 and 17 years; based on the size distribution of the clusters, only the largest clusters with 10 or more vertices in size are included in this table (approximately top 15\% largest clusters), ordered by anatomical position along the anterior-posterior axis.

\begin{tabular}{|c|c|c|c|c|c|c|c|c|}
\hline \multirow[t]{2}{*}{$\#$} & \multirow{2}{*}{$\begin{array}{l}\text { Region } \\
\text { Age } 9 \text { to } 12 \text { years }\end{array}$} & \multirow[t]{2}{*}{ Size } & \multicolumn{3}{|c|}{ MNI coordinates } & \multirow[t]{2}{*}{$h^{2}(\Delta C T)$} & \multirow[t]{2}{*}{$p\left(h^{2}(\Delta C T)\right)$} & \multirow[t]{2}{*}{$p($ Innov $)$} \\
\hline & & & & & & & & \\
\hline 1 & Med. Orb. Frontal LH & 40 & -17 & 69 & -2 & $53 \%$ & 0.020 & 0.004 \\
\hline 2 & Mid. Orb. Frontal RH & 10 & 26 & 55 & -15 & $48 \%$ & 0.033 & 0.003 \\
\hline 3 & Sup. Med. Frontal RH & 43 & 4 & 50 & 41 & $47 \%$ & 0.023 & 0.010 \\
\hline 4 & Ant. Cingulum RH & 12 & 3 & 24 & 22 & $71 \%$ & 0.027 & 0.017 \\
\hline 5 & Fusiform Gyrus LH & 28 & -39 & -14 & -34 & $54 \%$ & 0.010 & $<0.001$ \\
\hline 6 & Heschl Gyrus RH & 26 & 37 & -25 & 12 & $41 \%$ & 0.027 & 0.001 \\
\hline 7 & Heschl Gyrus LH & 10 & 35 & -29 & 17 & $41 \%$ & 0.036 & 0.003 \\
\hline 8 & Parahippocampal RH & 38 & 17 & -32 & -16 & $68 \%$ & 0.013 & $<0.001$ \\
\hline 9 & Calcarine Sulcus RH & 48 & 15 & -65 & 5 & $62 \%$ & 0.013 & 0.010 \\
\hline \multirow[t]{2}{*}{10} & Cuneus LH & 10 & -15 & -75 & 37 & $44 \%$ & 0.032 & 0.002 \\
\hline & Age 12 to 17 years & & & & & & & \\
\hline 1 & Sup. Med. Frontal RH & 55 & 11 & 53 & 5 & $70 \%$ & 0.003 & 0.013 \\
\hline 2 & Sup. Med. Frontal RH & 53 & 7 & 44 & 41 & $66 \%$ & 0.004 & 0.018 \\
\hline 3 & Calcarine Sulcus LH & 11 & -19 & -46 & -34 & $52 \%$ & 0.025 & 0.004 \\
\hline 4 & Lingual Gyrus RH & 39 & 14 & -60 & -2 & $67 \%$ & 0.002 & 0.006 \\
\hline 5 & Sup. Parietal LH & 10 & -4 & -71 & 12 & $57 \%$ & 0.011 & 0.015 \\
\hline 6 & Supramarginal Gyrus LH & 22 & -18 & -102 & -16 & $50 \%$ & 0.007 & 0.016 \\
\hline
\end{tabular}

Region are defined by the Automated Anatomic Labelling (AAL) Atlas (Tzourio-Mazoyer et al. 2002); size is reported as the number of connected vertices in the cluster; MNI coordinates are reported in $X Y Z$ format; $h^{2}(\triangle C T)=$ heritability of changes in cortical thickness; $p\left(h^{2}(\Delta C T)\right)=$ significance of heritability of changes in cortical thickness (FDR-adjusted $p<0.05) ; p($ Innov) $=$ significance of genetic innovation (uncorrected $p<$ $0.05)$

Post-hoc analysis on the effects of possible confounds revealed little effect from sex, age at scan and handedness on the decomposition of heritability of changes in cortical thickness (Supplementary Figure A.7). 


\subsection{DISCUSSION}

We applied twin modelling to a longitudinal cohort with three measurements to investigate the extent to which genetic influences drive changes in cortical thickness during childhood and adolescence. We find a single genetic factor that affects the acceleration of overall cortical thinning across childhood and adolescent development with increasing heritability of changes in mean global cortical thickness: $h_{\Delta_{12}}^{2}=21 \%(p=0.154$; [n.s.]) between ages 9 and 12 years, and $h_{\Delta_{23}}^{2}=53 \%(p<0.001)$ between ages 12 and 17 years. At age 17 years, a new genetic factor comes into play, separating cortical thickness development in late adolescence from early adolescence and childhood. Locally, we again find a core genetic factor influencing cortical thickness and a second genetic factor involved in innovation explaining changes in local cortical thickness during different stages of childhood and adolescent development; areas with the highest estimates include the anterior cingulate cortex $\left(h_{\Delta_{12}}^{2}=71 \%\right.$ in cortical thickness change between age 9 and 12 years) and the superior medial frontal cortex $\left(h_{\Delta_{23}}^{2}=70 \%\right.$ in cortical thickness change between age 12 and 17 years).

We report an accelerating decrease in cortical thickness in twins in this longitudinal design with up to three measurements (Figure 2.3 and Supplementary Table A.3) that is compatible with cortical development in healthy typically developing singletons (Gogtay et al. 2004; Sowell et al. 2007; Raznahan, Shaw, et al. 2011; Storsve et al. 2014; Schnack et al. 2015). The patterns in phenotypic correlation across time (Figure 2.4A), with strong correlations between homotopic regions and correlation of the same region across development, are comparable to those found by structural covariance analyses (Raznahan, Lerch, et al. 2011; Alexander-Bloch et al. 2013). We find moderate to high heritability estimates of cortical thickness for most of the cortex, corroborating the evidence that the cortex is under strong genetic control (Lenroot et al. 2009; Blokland et al. 2012; Schmitt et al. 2014). Estimating the genetic overlap in cortical thickness between lobar regions of the cortex revealed a strong core genetic factor affecting overall cortical thickness across childhood and adolescent development (Figure 2.4B). The evidence for a core genetic factor is in agreement with one of the earlier studies investigating distinct genetic influences on cortical thickness in a cross-sectional pediatric sample with age range from 5.4 to 18.7 years that found a single component explaining over $60 \%$ of the genetic variance (Schmitt et al. 2008). Another cross-sectional study in older male-only twins found moderate to strong genetic correlations ranging from 0.3 to 1.0 across most of the cortex, characteristic for an omnipresent genetic factor, for a seed placed in the middle frontal cortex (Rimol et al. 2010). We now add to these findings that the same genetic factor is responsible for cortical thinning during childhood and adolescence. 
In contrast to cross-sectional studies investigating regional genetic influences on cortical thickness after removing the effect of global cortical thickness (Schmitt et al. 2008; Eyler et al. 2012; Chen et al. 2013; Docherty et al. 2015; Fjell et al. 2015), we specifically wanted to investigate the temporal dynamics of the genetic factor affecting overall cortical thickness development during childhood and adolescence. Using the longitudinal twin model setup, we show there is a single genetic factor that dominates across childhood and adolescence which is involved in cortical thickness and cortical thickness change - and thus involved in cortical thinning. On top of this core genetic factor there is evidence for spatial genetic differentiation between the major lobes. This corroborates results from a recent publication in an extended longitudinal twin design that finds similar spatial patterns for genetic correlations of cortical thickness between the major lobes during childhood and adolescence (Schmitt et al. 2017). In addition, they show changes in genetic correlation across development, with some regions demonstrating an increase in genetic overlap towards the second decade of life. Their results could be related to the fluctuating influences of genes and genetic innovation found in our study and is characteristic for genetic differentiation. This genetic differentiation might be the result of continued areal specialization of the cortex, since it is well known that cortical areas continue to develop well into early adulthood and beyond, in particular the frontal cortex (Schnack et al. 2015).

During adolescence, areal specialization might be spurred by new genetic factors that could be related to the rapid cognitive and behavioral changes during adolescence. Indeed, decomposition of genetic influences on cortical plasticity revealed a second genetic factor, representing genetic innovation, that is influencing cortical thickness during childhood and adolescent development. The areas where genetic innovation occurred were most prominent in the frontal cortex, involving the anterior cingulate cortex (with a heritability of changes in cortical thickness of $71 \%$ between age 9 and 12 years) and superior medial frontal cortex (heritability of $70 \%$ between age 12 and 17 years). In addition, genetic innovation was found in other areas, such as the medial and middle orbital frontal cortices, the fusiform, Heschl's, and parahippocampal gyri and the cuneus between age 9 and 12 years and the calcarine, lingual, superior parietal and supramarginal cortices between age 12 and 17 years. Together, the two genetic factors involved in changes in cortical thickness explain the strong positive correlations between homotopic regions across the hemispheres and across age, evident from the diagonal banding in the correlation matrices (Figure 2.4) and as reported from cross-sectional twin studies at both lobar and local level (Chen et al. 2013; Wen et al. 2016).

Gene expression studies can reveal the spatiotemporal dynamics of individual genes expressed in the human brain across the lifespan (Naumova et al. 2013; Akbarian et al. 2015; 
Silbereis et al. 2016). It has been suggested that areal specialization of the neocortex is established during early development, and that later development is the result of more general maturational processes affecting the entire neocortex (Pletikos et al. 2014). Several studies report a remarkable homogeneity in gene expression profiles among neocortical areas despite their functional specialization (Roth et al. 2006; Kang et al. 2011; Hawrylycz et al. 2015; Jaffe et al. 2015). Results from our analysis show a similar strong overlap in genetic factors among neocortical areas while providing evidence for spatial differentiation among the major lobes. These results suggest that cortical thickness during childhood and adolescence is primarily driven by a core genetic component with secondary regionalspecific genetic influences. This result is in agreement with other twin studies that found distinct regional genetic influences on cortical thickness after removing global effects (Schmitt et al. 2008; Chen et al. 2013; Docherty et al. 2015). It suggests a majority of cortical thinning during childhood and adolescence might be part of a more generic developmental process affecting global cortical development (Jaffe et al. 2015), whereas secondary influences might be the result of gene-environment interactions.

Regarding temporal differential gene expression (i.e. increased or reduced expression of the same genes, or expression of novel genes over time), adolescence is a period during postnatal human development marked by the highest number of temporal differential expression of genes in the prefrontal cortex (Jaffe et al. 2015). During postnatal development, an hourglass model for spatially differential gene expression has been reported, where adolescence is identified by increased homogeneity in spatial gene expression among neocortical areas that is concluded by a second wave of changes in gene expression at the end of adolescence (Somel et al. 2010; Colantuoni et al. 2011; Pletikos et al. 2014). A similar conclusion can be made from our results where age 12 years shows increased complete genetic overlap between cortical regions compared to age 9 and 17 years, although this conclusion should be treated with caution as it might be the result of diminished statistical power to detect genetic differentiation due to reduced sample size at age 12 years. The more pronounced genetic (de)amplification and genetic innovation found in our analysis between age 12 and 17 years might be the first sign of the reported second wave of changes in gene expression at the end of adolescence. Thinning of the cortex during adolescence and early adulthood has been linked to increased myelination and associated gene expression (Whitaker et al. 2016). Gene co-expression network analyses have revealed modules enriched for genes associated with synaptic function, dendrite development and myelination emerging during late fetal development and reaching a plateau during early childhood (Kang et al. 2011). Spatiotemporal differentiation in gene expression profiles have been linked to differences in cellular composition of the neuropil rather than changes in gene expression of constituent cells (Jaffe et al. 2015). These gene 
expression findings support existing theories on the biological processes underlying the apparent cortical thinning observed during development (Huttenlocher 1979; Bourgeois and Rakic 1993; Huttenlocher and Dabholkar 1997; Paus et al. 2008; Paus 2010; Petanjek et al. 2011; Miller et al. 2012; Deoni et al. 2015). These theories anticipate a decrease in cortical grey matter content of the neuropil due to pruning of neuronal synapses and dendrites accompanied by a decrease in supporting glial cells, and a parallel increase of oligodendrocytes responsible for myelination of neuronal axons. The fluctuating influences of the core genetic factor from our results could represent a shift in balance of maturational processes. Another possible explanation of the fluctuating influences of the core genetic factor could be related to maturational timing of neocortical areas. Neocortical areas that mature during childhood and early adolescence might experience increased influences of a genetic factor associated with maturational genes around that age, followed by a decreased influence upon maturation during later adolescence. In contrast, neocortical areas that mature during late adolescence might initially experience a low or decreased influence of this genetic factor that suppresses or delays maturation until late adolescence. The genetic innovation found in our analysis could represent a novel genetic factor that arises upon maturation of neocortical, such as genes involved in maintaining matured neurons in good condition. On the other hand, the genetic innovation could also represent the disappearance of a genetic factor, something we cannot resolve with the current twin model design. This would mean the genetic "innovation" could be associated with the termination of maturational processes instead.

The brain is highly plastic and capable of adapting to new environments (Kramer et al. 2004; Zatorre et al. 2012). The environmental correlations estimated from our data could be due to true environmental influences unique to each individual, but are likely to be confounded by measurement errors from the MRI scans and image processing procedure. In particular, the strong correlations within ages will be confounded by measurement errors. Although there is a surprising laterality between hemispheres with each age, this may be the result of processing hemispheres independently during image processing. This can also explain the lack of environmental correlations between homologous regions across hemispheres. The strong environmental correlations of the same region across time are more likely to be caused by some unknown environmental influence unique to everyone (Figure 2.4C). Previous studies have shown that environmental influences from exercising (Voelcker-Rehage and Niemann 2013; López-Vicente et al. 2017), smoking and substance use (Jacobus et al. 2015; Karama et al. 2015), and prenatal exposure to aversive environment (Gautam et al. 2015; Marroun et al. 2016) can influence cortical thickness. 
With a similar number of females and males in the study it was possible to assess differences between the sexes. We found negligible influences of sex, handedness, and age at scan on heritability of (changes) in cortical thickness in qualitative post-hoc analyses. Quantitative evaluation of sex and handedness effects on the means and variance of (changes in) global cortical thickness confirmed the absence of sex or handedness effects. Although sex effects for mean and variance of changes in cortical thickness between ages 12 and 17 years were approaching significance, no discernible effects were observed in the qualitative evaluation. It remains unclear if sex differences in cortical thickness during development exist (Lenroot and Giedd 2010; Walhovd et al. 2017). Our longitudinal data on cortical thickness development reveals no sex differences in global cortical thickness across childhood and adolescent development (Figure 2.3).

This study has several limitations which should be taken into consideration when interpreting its findings. One, the bivariate twin analysis has a limited level of detail by using a lobar segmentation of the cortex. Although genetic clustering of the cortex appears to largely conform to anatomical boundaries defined by sulci and gyri, the genetically optimal configuration has a more refined subdivision of the cortical lobes (Chen et al. 2012; 2013; Peng et al. 2016; Wen et al. 2016). As a result, the use of a lobar segmentation may result in a mixture of different gene pools for each lobar region, which may impact our ability to detect distinct genetic factors between regions. Two, the statistical power of a twin study determines the effect sizes that can be detected (Posthuma and Boomsma 2000; Panizzon et al. 2009) and our sample is modest for twin modelling purposes. The longitudinal design of this study increases the power substantially for heritability estimates of measures correlated across time such as cortical thickness, while the ability to detect genetic innovation depends on non-shared genetic factors influencing the individual measures (i.e. genetic factors influences both measures are not perfectly correlated). Thus, there is a balance between being able to detect heritability and being able to detect genetic innovation depending on the amount of genetic overlap between the variables. Assuming a ground truth based on the phenotypic covariance matrix for mean global cortical thickness from our own data, a post-hoc simulation study detected significant heritability of cortical thickness at all three waves and heritability of cortical thickness change with a power between $90 \%$ to $100 \%$. However, it must be noted that we are underpowered to detect genetic innovation: genetic innovation at age 12 years was detected in $59 \%$ of the simulation runs, and at age 17 years in only in $26 \%$ of the simulation runs. Three, with a 5year interval between age 12 and 17 years compared to a 3-year interval between age 9 and 12 years, we expected to see increased sensitivity for heritability estimates of changes in cortical thickness since more time has passed to allow for changes in cortical thickness to occur and consequently an increased variance between individuals. This increased 
sensitivity is enhanced by the increased rate of changes in cortical thickness during the second half of adolescence. Indeed, we found larger areas significant for heritability of changes in cortical thickness between age 12 and 17 years than between age 9 and 12 years (Figure 2.5). With a five-fold increase in additive genetic variance for changes in cortical thickness between age 12 and 17 years compared to only two-fold increase in phenotypic variance and no changes to environmental variance, we believe the increased heritability of changes in cortical thickness between age 12 and 17 years can be attributed to increased rate of cortical thinning and not merely to the difference in time interval between scans. Despite the increase in sensitivity to detect changes between age 12 and 17 years, we found fewer and smaller clusters with genetic innovation than between age 9 and 12 years (Figure 2.5B and Table 2.1).

Despite its limitations, the longitudinal design of this study with its strict age range at each measurement is ideally suited for the longitudinal twin analysis investigating heritability of changes in cortical thickness since it permits exploiting rules of variance to readily obtain covariance matrices for change measures without having to consider a diverse range of ages of the participants. Slight variations in individuals' scan interval, implicitly modelled by incorporating age at scan as variable of no interest in the post-hoc analysis, did not have any discernible effect on the results.

In conclusion, cortical thickness development during childhood and adolescence is under strong genetic control and although it is largely driven by a single genetic factor, the influence exerted by this core genetic factor varies with age and its influence seems to decrease towards adulthood. In addition, new genetic factors influence regional cortical thickness development during different stages of childhood and adolescent development. These new genetic factors might explain the rapid cognitive and behavioral development during adolescence and could potentially be associated with the manifestation of psychiatric disorders during adolescence. 



\section{CHAPTER 3}

\section{RELIABILITY MODELLING OF RESTING-}

STATE FUNCTIONAL CONNECTIVITY

Original title: Reliability modelling of functional connectivity in resting-state functional MRI

Authors: Jalmar Teeuw ${ }^{1}$, Dorret I. Boomsma², Hilleke E. Hulshoff Pol $^{1}$, and Rachel M. Brouwer ${ }^{1}$

Affiliations: ${ }^{1}$ Department of Psychiatry, Brain Center Rudolf Magnus, University Medical Center Utrecht, Heidelberglaan 100, 5384 CX Utrecht, the Netherlands; ${ }^{2}$ Department of Biological Psychology, Vrije Universiteit Amsterdam, van der Boechorststraat 1, 1081 BT Amsterdam, the Netherlands 


\section{ABSTRACT}

Resting-state functional magnetic resonance imaging (rs-fMRI) has an inherently low signal-to-noise ratio largely due to thermal and physiological noise that attenuates the functional connectivity (FC) estimates. Such attenuation limits the reliability of FC and likely confounds its association with other traits. Low reliability also limits heritability estimates. Classical test theory can be used to obtain a true correlation estimate free of random measurement error from parallel tests, such as split-half sessions of a rs-fMRI scan.

We applied a measurement model to split-half FC estimates from the resting-state fMRI data of 1003 participants from the Human Connectome Project (HCP) to examine the benefit of reliability modelling of FC in association with traits from various domains. We evaluated the efficiency of the measurement model on extracting a stable and reliable component of FC and its association with several traits for various sample sizes and scan durations. In addition, we aimed to replicate our previous findings of increased heritability estimates when using a measurement model in a longitudinal adolescent twin cohort.

The split-half measurement model improved test-retest reliability of FC on average with +0.33 points (from +0.49 to +0.82 ), improved strength of associations between $\mathrm{FC}$ and various traits on average 1.2-fold (range 1.09 to 1.35 ), and increased heritability estimates on average with $+20 \%$ points (from $39 \%$ to $59 \%$ ) for the full HCP dataset. On average, about half of the variance in split-session FC estimates was attributed to the stable and reliable component of FC. Shorter scan durations showed greater benefit of reliability modelling (up to 1.6-fold improvement), with an additional gain for smaller sample sizes (up to 1.8-fold improvement). Reliability modelling of FC based on a split-half using a measurement model can benefit genetic and behavioral studies by extracting a stable and reliable component of FC that is free from random measurement error and improves genetic and behavioral associations.

Keywords: test-retest reliability; reliability modelling; functional connectivity; measurement model; measurement error; human connectome project 


\subsection{INTRODUCTION}

Resting-state functional connectivity has become a popular method to study the functional organization of the human brain (Biswal et al., 1995; Greicius et al., 2003; van den Heuvel and Hulshoff Pol, 2010). Functional connectivity has shown promise as a potential biomarker for its association with neuropsychiatric and neurological disorders (Hager and Keshavan, 2015; Hohenfeld et al., 2018; Whitfield-Gabrieli and Ford, 2012; Zhang and Raichle, 2010; Fox et al., 2014). In addition, it is associated with various cognitive and behavioral traits (Vaidya and Gordon, 2013; Basten et al., 2015; Shen et al., 2018; Smith et al., 2015; Toschi et al., 2018), and functional connectivity is heritable to a certain extent (Ge et al., 2017; Colclough et al., 2017; Adhikari et al., 2018; Teeuw et al., 2019). However, for resting-state functional connectivity to become a biomarker, it needs to be a reliable and state-independent endophenotype (Gottesman and Todd, 2003; Beauchaine and Constantino, 2017).

Resting-state functional magnetic resonance imaging (rs-fMRI) has an inherently low signal-to-noise ratio (SNR) primarily due to thermal noise of the magnetic resonance (MR) scanner and physiological noise from the subject, such as head motion, cardiovascular and respiratory activity (Logothetis and Wandell, 2004; Bianciardi et al., 2009; Murphy et al., 2013; Liu et al., 2016). These sources of noise are confounding the blood-oxygenation level dependent (BOLD) signal and lead to biased estimates of resting-state functional connectivity (Birn et al., 2014; Siegel et al., 2017). Numerous strategies have been proposed to clean the BOLD signal from these confounders and recover the signals related to neural activity (Burgess et al., 2016; Caballero-Gaudes and Reynolds, 2017; Power et al., 2015). However, no perfect solution exists (Bright and Murphy, 2015; Varikuti et al., 2017; Parkes et al., 2018; Krishnamurthy et al., 2018; Lindquist, 2020). Noise in the BOLD signal has a negative impact on the reliability of the signal and results in the attenuation of functional connectivity estimates based on temporal correlation between two BOLD signals (Spearman, 1904; Leigh Wang, 2010; Birn et al., 2014; Mueller et al., 2015). Even with stateof-the-art procedures, test-retest reliability of functional connectivity is only poor to moderate (Noble et al., 2019; Chen et al., 2015; Shah et al., 2016), with large variation in reliability for the different connections measured in a single individual over an extended period of time (Choe et al., 2015). Although reliability of functional connectivity can be improved by increasing the scan duration up to 1.5 hour (Birn et al., 2013; Laumann et al., 2015), this approach is not always feasible due to the burden on the subject or the cost and availability of MRI. The limited reliability of functional connectivity puts an upper bound on the heritability estimates of functional connectivity and its association with traits (Vul et al., 2009; Neale and Cardon, 1992; Ge et al., 2017). Thereby making it difficult to reliably 
identify functional connections in the brain that are associated with particular traits (Geerligs et al., 2017; Kruschwitz et al., 2018).

In Classical Test Theory, the true score of a measure can be obtained from the observed score if the error term is known: observed score = true score + error term (Streiner, 2003; Miller, 1995). The error term can be approximated from 'parallel scores' (i.e. repeated measures). When it is not feasible to acquire two full measurements, two parallel half-score measures might be an option; e.g. an odd-even split in an event-related study design (van Baal et al., 1998). Resting-state fMRI data is uniquely suited for 'split-half' reliability modelling because of the temporal nature of the BOLD signal to create two parallel halfscore measures by splitting scan session data into two or more parts (Brandmaier et al., 2018). For associations, such as functional connectivity based on the temporal correlation of two BOLD signals, the error term is defined by the reliability of the two measures (Spearman, 1904). The true association can be obtained by scaling the observed association with a factor inversely proportional to the reliability (Mueller et al., 2015; Golestani and Goodyear, 2011). However, classical disattenuation requires the correction factor to be known a priori and has the risk of overcorrecting the association (Leigh Wang, 2010). Instead, a structural equation measurement model can be applied to the half-score measures to derive a latent variable representing the trait of interest that is "free" of measurement error without the need of an a priori correction factor or the risk of overcorrection (Brandmaier et al., 2018; Cooper et al., 2019). Such measurement models have previously been applied in twin studies to separate the variance attributed to measurement error from genetic and environmental variance components to obtain a robust heritability estimate for the reliable part of the variation (van Baal et al., 1998; van Beijsterveldt et al., 2001; Ge et al. 2017; Teeuw et al., 2019). The measurement model is however suited for many types of measures and study designs, as long as some form of parallel scores can be obtained. This implies that reliability modelling can be useful for the typical cross-sectional resting-state fMRI dataset of unrelated individuals. However, little is known about the effectiveness of reliability modelling of resting-state MRI functional connectivity and its ability to uncover the true associations between functional connectivity and other traits.

Here, we examine the benefits of reliability modelling of functional connectivity in association with physiological, cognitive and behavioral traits. For that purpose, a measurement model was applied to functional connectivity estimates from the resting-state functional MRI scans of the Human Connectome Project Young Adult (HCP-YA) cohort (Van Essen et al., 2013). The efficiency of reliability modelling was evaluated for various sample sizes and scan durations on (i) the ability to extract a stable and reliable component 
of functional connectivity and (ii) the improvement in the associations of functional connectivity with various traits. We also aimed to replicate our previous findings of increased heritability estimates for the reliable component of functional connectivity (Teeuw et al., 2019).

\subsection{MATERIALS AND METHODS}

\subsubsection{Human connectome project}

We utilized data from the publicly available extensively processed fMRI data package that is part of the Human Connectome Project Young Adult cohort (Van Essen et al., 2013). The package provides data for 1003 related individuals (siblings, including monozygotic- and dizygotic twins; aged 22 to 37 years) from 429 families with four complete runs of restingstate fMRI scans (Table 3.1) and consists of precomputed denoised BOLD signal time series for nodes in the brain based on group-ICA decomposition of the data at various decomposition levels. The acquisition parameters and processing of this data have been described elsewhere (Smith et al., 2013; Glasser et al., 2013; see supplementary methods for summary). All analyses were performed using the group-ICA decomposition with 50 nodes (Supplementary Figure B.1).

Table 3.1. Demographics table for participants included in the extensively processed fMRI data package of the Human Connectome Project Young Adult cohort.

\begin{tabular}{ll}
\hline Measure & Statistic \\
\hline Participants $(N)$ & 1003 \\
Families $(N)$ & 429 \\
Age range (min; max $)$ & 22 to 37 years \\
Age (mean $\pm S D)$ & $28.71 \pm 3.71$ years \\
Sex ratio $($ female : male $)$ & $534: 469$ \\
\hline
\end{tabular}

Abbreviations: $\mathrm{SD}=$ standard deviation of the mean.

\subsubsection{Functional connectivity}

Functional connectivity estimates were obtained by calculating the temporal correlation coefficient between the BOLD time series of two nodes using Pearson correlation (Biswal et al., 1995; van den Heuvel and Hulshoff Pol, 2010). Functional connectivity was estimated for different temporal blocks of the time series data to provide full-, half-, and quarter-score estimates of functional connectivity for the purpose of reliability modelling (Figure 3.1A). 
All statistical and mathematical operations on functional connectivity were performed on Fisher's $r$-to- $Z$ transformed functional connectivity estimates.

\subsubsection{Reliability modelling of functional connectivity}

A measurement model is applied to half-score measures of functional connectivity to extract a reliable component of functional connectivity represented by the latent variable $F_{i}$ (Figure 3.1). Variance shared between half-score measures that can be attributed to the latent variable is quantified by the path coefficient $\left(f_{i}\right)$, which are constrained to be equal in proportion of variance for both half score measures. Any residual variance of the halfscore measures, which includes measurement error, is considered noise, and is represented by the measurement-specific latent variables $E s_{i}$ quantified by the path coefficients $e s_{i}$.

To demonstrate the ability of the measurement model to extract a stable and reliable component of functional connectivity, test-retest reliability is estimated between two latent variables $F_{1}$ and $F_{2}$ that each represent a reliable component of functional connectivity based on two half-score measures of functional connectivity from independent scan sessions acquired on different days (Figure 3.1B). The test-retest reliability ( $R p h f)$ is estimated as the correlation between the two latent variables. Test-retest reliability from the measurement model is compared to the standard test-retest reliability of functional connectivity estimated as the correlation between the two half-score measures of functional connectivity (Figure 3.1C). Improvement in test-retest reliability is determined by the pointwise difference compared to the standard test-retest reliability estimate $(R p h m)$.

The measurement model can be adapted to estimate the association between the reliable component of functional connectivity and a trait (Figure 3.1D). For comparison, the standard association between the full-score measure of functional connectivity and the trait is estimated and compared over all connections (Figure 3.1E). The overall improvement in association strength (i.e. the average improvement factor) is determined by the slope coefficient of the linear regression of the association strengths from the measurement model onto the association strengths from the standard association model. All models included fixed effects of sex, age, head motion and HCP processing pipeline version as covariates on the means of the functional connectivity estimates, and fixed effects of sex and age as covariates on the means of the trait. Head motion was approximated by mean framewise displacement (Power et al., 2012; Supplementary Figure B.2). All models were specified in the OpenMx structural equation modelling (SEM) software package (Neale et al., 2016; Boker et al., 2018) for $R$ (R Core Team, 2018). The definition of the measurement model in OpenMx is provided in the online supplementary data ${ }^{2}$.

\footnotetext{
${ }^{2}$ Supplementary data: https://dx.doi.org/https://doi.org/10.1093/cercor/bhy005
} 
(A) Partition of the BOLD time series into full-, half-, and quarter score estimates of functional connectivity

\begin{tabular}{|c|c|c|c|c|}
\hline Time series length & Session 1 - Day 1 & Session 2 - Day 1 & Session 3 - Day 2 & Session 4 - Day 2 \\
\hline $1 \times 4800$ frames $(1 \times 60 \mathrm{~min})$ & \multicolumn{4}{|c|}{ Full-score } \\
\hline $2 \times 2400$ frames $(2 \times 30 \mathrm{~min})$ & \multicolumn{2}{|c|}{ Half-score 1} & \multicolumn{2}{|c|}{ Half-score 2} \\
\hline $4 \times 1200$ frames $(4 \times 15 \mathrm{~min})$ & Quarter-score 1 & Quarter-score 2 & Quarter-score 3 & Quarter-score 4 \\
\hline
\end{tabular}

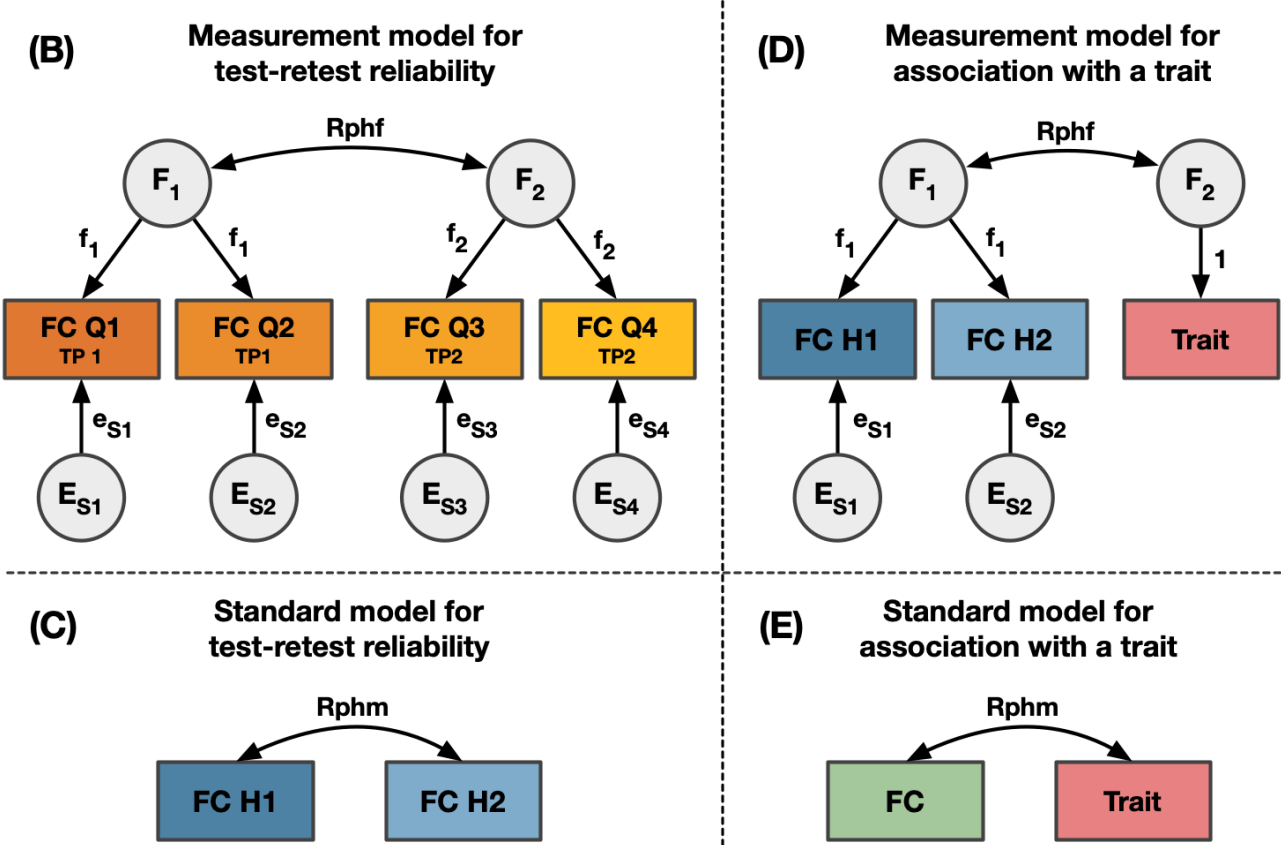

Figure 3.1. Partitioning of the BOLD time series and the measurement models used in the analyses. (A) Decomposition of the BOLD time series data into temporal blocks for the estimation of full-, half-, and quarterscore functional connectivity based on the original full-length time series with 4800 frames (approximately 1 hour) of resting-state functional MRI data. (B) A measurement model with two latent variables each representing a reliable component of functional connectivity across a set of half-score measures of functional connectivity. The model is used to estimate test-retest reliability between the two reliable components of functional connectivity at different scan sessions (Rphf) quantified by the correlation between the two latent variables. (C) A standard association model is used to estimate the test-retest reliability between the observed half-score measures of functional connectivity $(R p h m)$ quantified by the correlation between the two observed variables. (D) A measurement model with one latent variable representing the reliable component of functional connectivity of two half-score measures and another latent variable representing the trait. This model is used to estimate the association between the reliable component of functional connectivity and the trait (Rphf) quantified by the correlation between the two latent factors. (E) A standard association model is used to estimate the correlation between the observed full-score measure of functional connectivity and the trait. 
The suitability of applying a measurement model to the data was assessed with the goodness of fit metrics Comparative Fit Index (CFI) and root-mean-square error of approximation (RMSEA). Model fits with a CFI $>0.95$ and RMSEA $<0.05$ were deemed a good fit, model fits with a CFI $>0.90$ and RMSEA $<0.08$ were deemed an acceptable fit, and the remaining models $(\mathrm{CFI}<0.90$ or RMSEA $>0.08$ ) were deemed an unsuitable fit ( $\mathrm{Hu}$ and Bentler, 1999; Browne and Cudeck, 1993). All models with an unsuitable fit were excluded from statistical analyses.

\subsubsection{Physiological, cognitive, and behavioral traits}

The Human Connectome Project provides rich phenotypic information on the participants. Because of the computational complexity of the measurement models, we used the results from the standard association model (Figure 3.1E) applied to 110 continuous and normally distributed measures to identify seven representative traits from different domains for extensive analysis (see Supplementary Materials for details; Supplementary Table B.1): five traits (BPDiastolic - diastolic blood pressure levels; CogTotalComp_AgeAdj - total composite score on cognition adjusted for age; WM_Task_2bk_Acc - accuracy on all condition in the two-back working memory task; Emotion_Task_Median_RT - median response time for each condition in the emotion task; and PicVocab_AgeAdj - picture vocabulary test score adjusted for age) were among the most strongly associated measures with functional connectivity at any individual connection, and two traits (Gambling_Task_Reward_Perc_Larger-percentage of trials that received a 'larger' prediction in the gambling task; and Taste_AgeAdj - score on the taste intensity test adjusted for age) were chosen because they were only weakly associated with functional connectivity. For these seven traits, we estimated the association with all functional connectivity measures.

For the remaining 107 traits, measurement models were computed only for the top 20 connections most strongly associated with functional connectivity and the 5 connections with the weakest associated with functional connectivity (i.e. near zero association) based on the results from the standard association model. This sampling scheme provides a good approximation of the actual improvement factor in association strength for the seven fully sampled traits, with mean absolute difference in improvement factor $2 \%$ (range from $0 \%$ to $5 \%$; Supplementary Table B.2).

\subsubsection{Heritability of functional connectivity}

To emphasize that the reliability model can be applied to the typical dataset consisting of unrelated individuals, up to this point we assumed that the subjects were independent. However, the Human Connectome Project cohort includes families with monozygotic and dizygotic twins and their siblings, and families with non-twin siblings (Supplementary Table B.3). We aimed to replicate our previous findings of increased heritability estimates 
for the reliable and stable component of functional connectivity in a longitudinal adolescent twin cohort (Teeuw et al., 2019). In brief, genetic modelling of data from twins and siblings allows for the decomposition of the variance of a trait $(V)$ into genetic and environmental components. Often, three variance components representing additive genetic $(A)$, common environmental $(C)$ and unique environmental $(E)$ influences are considered (Boomsma et al., 2002; Posthuma et al., 2000; Neale and Cardon, 1992). Heritability is the standardized additive genetic component: $h^{2}=\frac{A}{V}=\frac{A}{A+C+E}$. The unique environmental influences are confounded by measurement error $(M)$ that can be separated from the "true" unique environmental $\left(E^{\prime}\right)$ influences by the measurement model: $E=E^{\prime}+M$. The variance of the reliable trait (i.e. the reliable component in the measurement model) becomes $V^{\prime}=V-M$, or $V^{\prime}=A+C+E^{\prime}$. Heritability of the reliable trait is estimated as the standardized additive genetic component after excluding measurement error: $h^{2}=\frac{A}{V^{\prime}}=\frac{A}{A+C+E^{\prime}}$. The heritability of the reliable component of functional connectivity was estimated for a measurement model on the half-score measures of functional connectivity (Supplementary Figure B.3). Heritability estimates of the reliable component of functional connectivity are compared to heritability estimates from the full (i.e. uncorrected) measure of functional connectivity (Supplementary Figure B.3). Full details on the heritability analysis are provided in the Supplementary Materials.

\subsubsection{Evaluation of the reliability model at different samples sizes and scan durations}

We performed a parameter sweep to empirically determine the efficiency of the measurement model on improving the strength of the association between traits and functional connectivity for various sample sizes and total scan duration (Table 3.2). Each combination of total scan duration and sample size was sampled 100 times per functional connection, with a random set of the desired number of participants drawn from the full

Table 3.2. Parameters and their values used in the evaluation of the minimal requirements on the input dataset for the reliability model.

\begin{tabular}{lll}
\hline Parameter & Values & Unit \\
\hline Total scan duration & $5,7.5,10,15,20,30,45,60$ & Minutes \\
& $--900,600,800,1200,1600,2400,3600,4800$ & Volumes \\
& $25,50,75,100,150,200,250,300,400,500$ & Count \\
$\begin{array}{l}\text { Number of participants } \\
\text { per sample }\end{array}$ & & \\
\hline
\end{tabular}

Total number of combinations of parameter values per connection: 8 scan durations x 10 sample sizes $\mathrm{x} 100$ samples $=8000$ models per connection. 
sample of participants at each iteration. To reduce the computational burden of evaluating the performance of the measurement model for all connections, the same 25 connections identified through the sparse sampling scheme previously described were used for each of the seven exemplar traits.

BOLD time series for the desired scan durations were extracted from the original fulllength BOLD time series data by distributing four time blocks equally across all four scan sessions, starting at the first volume of each scan session, and concatenating the time series where necessary to create full-, half-, or quarter-score measures of functional connectivity (Supplementary Figure B.4). This distributed approach was adopted to prevent half-score measures from crossing scan boundaries (e.g. for a total time series length of 1600 volumes, the second half-score measure would be computed across data from both scan \#1 [volumes 801:1200] and \#2 [volumes 1:400]).

\subsubsection{Minimal requirements on the input dataset}

The requirements, in terms of sample size and total scan duration, for a dataset suitable for reliability modelling was evaluated empirically to determine the threshold where the goodness of fit indices for the measurement model started to deteriorate. Since no universal absolute threshold exists, the proportion of bad fits over the hundred iterations for each combination of sample size and total scan duration is provided with a lower and upper boundary marked at $25 \%$ and $50 \%$ quantiles. The same goodness of fit indices Comparative Fit Index (CFI) and root-mean-squared-error of approximation (RMSEA) and their judgement criteria were used as described before.

\subsection{RESUltS}

\subsubsection{High consistency of group-level mean functional connectivity}

Group-level mean functional connectivity was highly consistent across the two half-score measures of functional connectivity ( $r h o=+0.996$; ICC $3,1=0.995)$, with functional connectivity estimates ranging from -0.52 to +0.66 (mean FC $=0.003$ ) (Supplementary Figure B.5), and absolute differences between the two half-score measure of less than 0.06 for individual connections. However, there is high variation within individuals.

\subsubsection{Reliability modelling improves test-retest reliability between scan sessions}

At the level of individual connection, test-retest reliability of functional connectivity between scan sessions acquired on different days improved substantially for 760 connections (62\% of all 1225 connections) with an acceptable or good fit of the measurement model (Figure 3.2; Supplementary Figure B.6). On average, the standard test-retest reliability estimate of functional connectivity was +0.49 (range $=+0.17$ to +0.82 ; 
Figure 3.2A) and the test-retest reliability estimates of the reliable component of functional connectivity was +0.83 (range $=+0.60$ to +1.00 ; Figure $3.2 \mathrm{~A}$ ). The test-retest reliability estimates improved on average with +0.33 points (range $=+0.13$ to +0.82 ; Figure $3.2 \mathrm{~B}$ ). Connections with lower standard test-retest reliability improved more than connections with already high test-retest reliability due to the ceiling effect of the upper bound of +1.00 on test-retest reliability estimates (Figure 3.2A). On average, $44 \%$ of the variance of the quarter-score measures of functional connectivity was explained by the reliable components of functional connectivity (range $=7 \%$ to $79 \%$; Figure $3.2 \mathrm{C}$ ).

\subsubsection{Reliability modelling improves the association between functional connectivity and traits}

The improvement in the strength of the associations between functional connectivity and the seven extensively tested traits ranged from $+17.0 \%$ to $+23.7 \%$ (Figure 3.3A; Supplementary Figure B.7). On average, the improvement in association strength for all 110 traits was $+20 \%$ (range $=+12$ to $+30 \%$; Figure $3.3 \mathrm{~B}$ ) using a sparse sampling scheme to approximate the improvement factor when the full set of connections would have been used (Supplementary

(A)

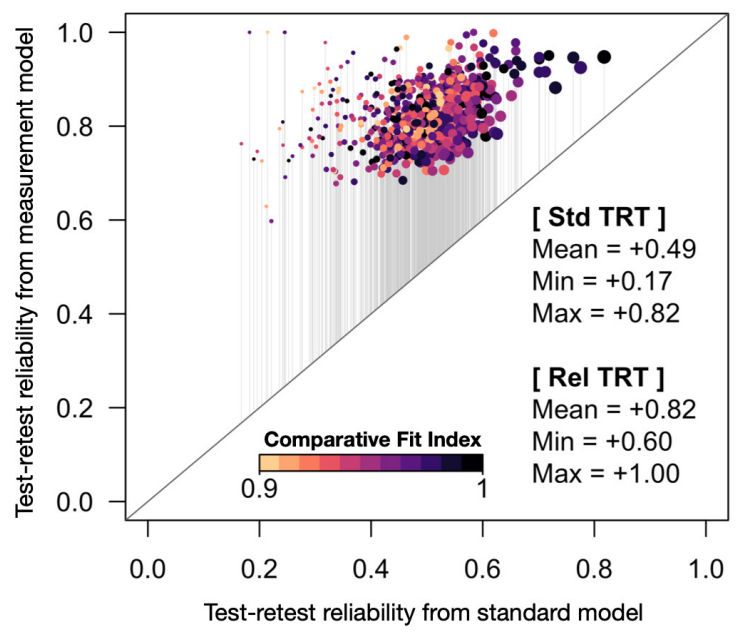

(B) Improvement in test-retest reliability

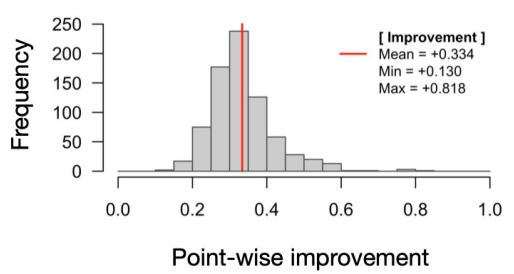

(C) Factor loadings reliable component

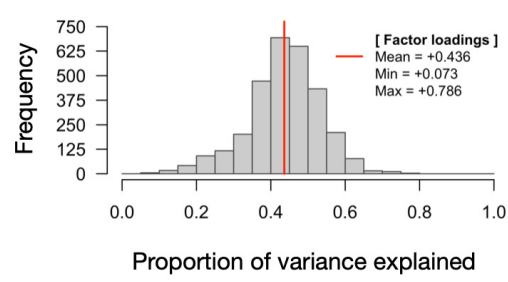

Figure 3.2. Test-retest reliability of functional connectivity estimates. (A) Improvement in test-retest reliability between the standard model (x-axis) and the measurement model (y-axis); data points are scaled by the average proportion of variance explained by the reliable component, thereby emphasizing the more reliable and stable connections, and color-coded by the Comparative Fit Index. (B) Point-wise improvement in test-retest reliability between standard model and measurement model. (C) Proportion of variance of the quarter-score measures explained by the reliable components of functional connectivity. For all panels, standard test-retest reliability was estimated as the association between half-score measures of functional connectivity. Test-retest reliability from the measurement model was estimated as the association between the two reliable components of functional connectivity based on the quarter-score measures of functional connectivity. The red lines indicate the mean of the distributions. 

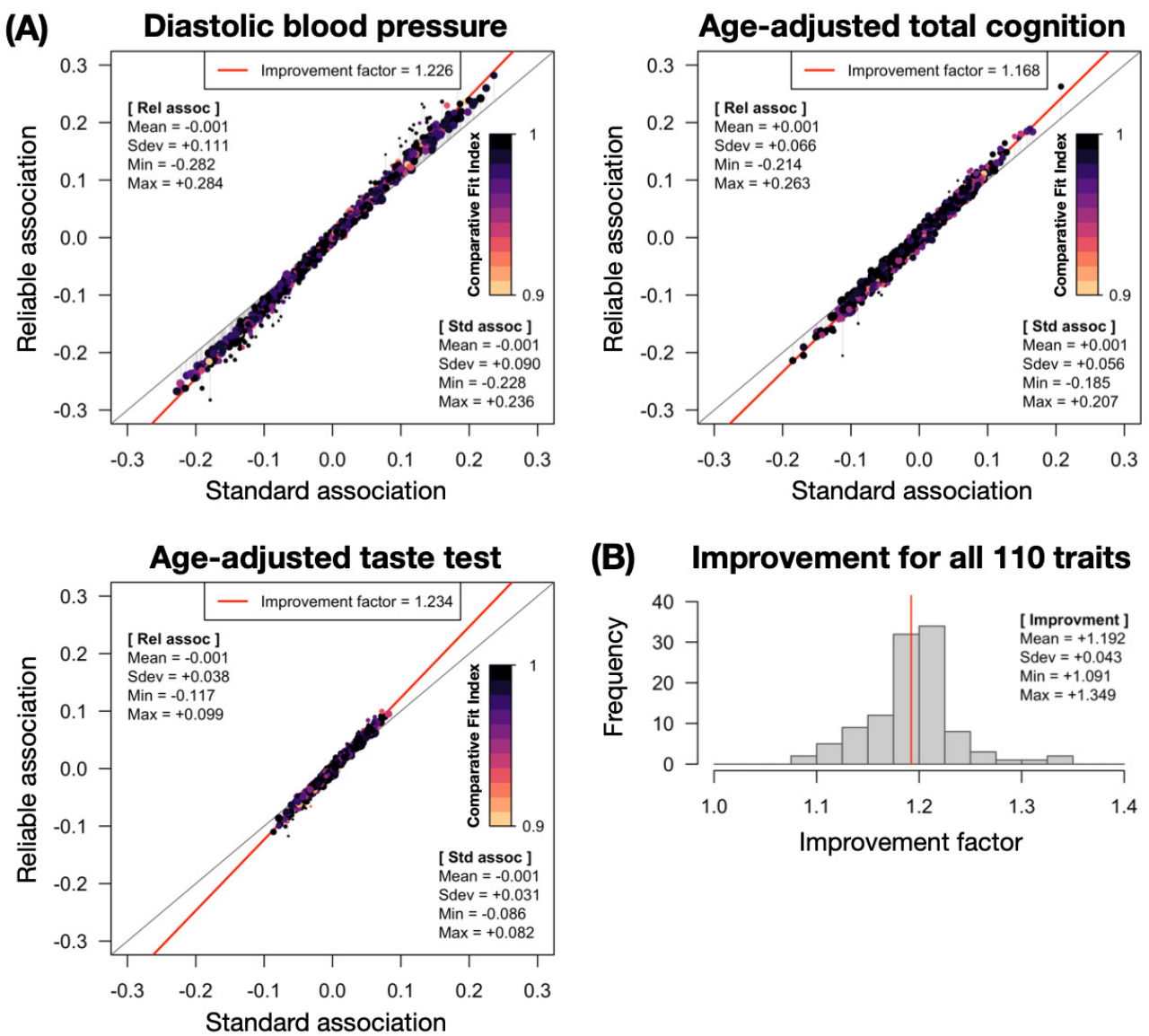

(B) Improvement for all 110 traits

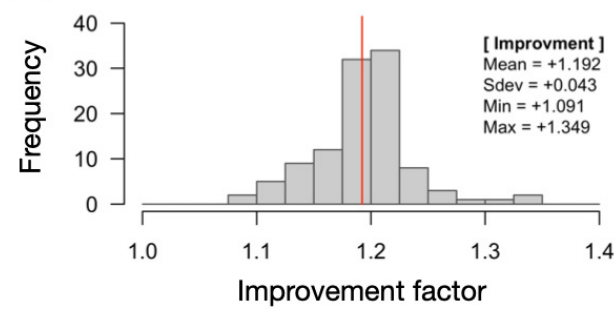

Figure 3.3. Association between functional connectivity and traits. (A) Improvement in association strength between functional connectivity and the traits diastolic blood pressure level (BPDiastolic), total composite score for cognition adjusted for age (CogTotalComp_AgeAdj), and taste intensity test adjusted for age (Taste_AgeAdj); with association strength from the standard association model on the $x$-axis and the association strength from the measurement model on the $y$-axis. The remaining four extensively tested traits show comparable results and are presented in the Supplement Materials (Supplementary Figure B.7). The red line represents the slope coefficient of the regression (i.e. the improvement factor). (B) Improvement factor for all 110 continuous and normally distributed traits. The red line represents the mean of the distribution. For all panels, a standard association model was used to estimate the association between the full-score measure of functional connectivity and the trait. A measurement model applied to the half-score measures of functional connectivity was used to estimate the association between the reliable component of functional connectivity and the trait.

Table B.2). On average, 1035 connections (84\% of all connections) passed the goodness of fit criteria, with highly similar distributions for the seven traits (Supplementary Figure B.6). On average, $50 \%$ of the variance of the half-score measures of functional connectivity is explained by the reliable component, with a range from $17 \%$ to $81 \%$. 

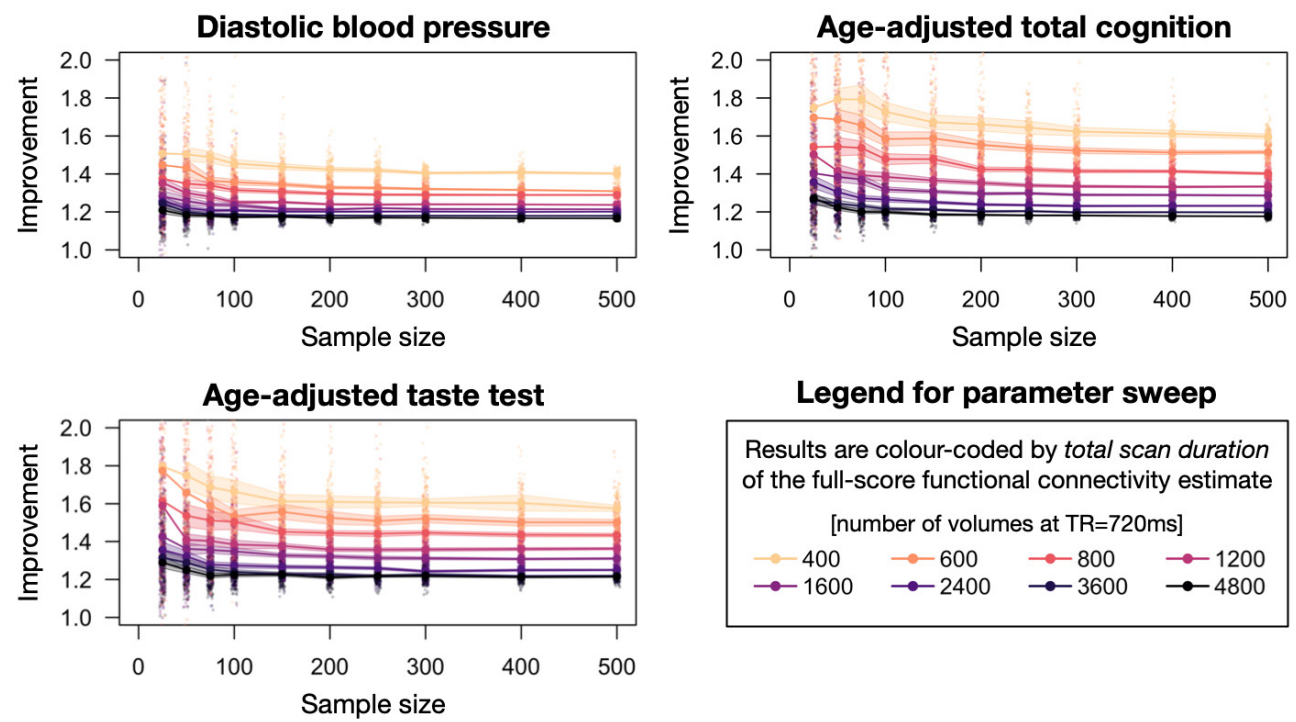

\section{Legend for parameter sweep}

Results are colour-coded by total scan duration of the full-score functional connectivity estimate

[number of volumes at TR=720ms]

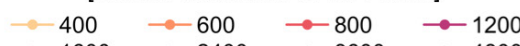

$\rightarrow 1600 \rightarrow 2400 \rightarrow 3600 \rightarrow 4800$

Figure 3.4. Improvement in association strength between functional connectivity and the traits diastolic blood pressure levels (BPDiastolic), age-adjusted total cognitive component (CogCompTotal_AgeAdj), and age-adjusted taste test score (Taste_AgeAdj) for various sample sizes (x-axis) and total scan duration (color-coding). Improvement factor (y-axis) is defined by the slope coefficient from the linear regression of reliable association strength onto standard association strength over all sparsely sampled connections. Color-shaded bands represent the $95 \%$ confidence interval of the means. For all panels, a standard association model was used to estimate the association between the full-score measure of functional connectivity and the traits. A measurement model applied to the half-score measures of functional connectivity was used to estimate the association between the reliable component of functional connectivity and the traits. The remaining four extensively tested measures showed similar patterns (Supplementary Figure B.9).

\subsubsection{Reliability modelling increases heritability estimates of functional connectivity}

The heritability estimates of full-score functional connectivity were on average $39 \%$ (range $=0 \%$ to $75 \%$; Supplementary Figure B.8). The heritability estimates of the reliable component of functional connectivity were on average 59\% (range $=0 \%$ to $93 \%$; Supplementary Figure B.8). On average, the heritability estimates increased with $+20 \%$ points (range $=-3 \%$ to $+54 \%$ points; Supplementary Figure B.8).

\subsubsection{Efficiency and minimal requirements for using reliability model}

All seven traits exhibit the same general pattern of improvement in the association strength, with greater benefit from reliability modelling for shorter scan durations and a slight increase for the smaller sample sizes up to 1.8-fold increase averaged over the 100 iterations per combination of total scan duration and sample size (Figure 3.4; Supplementary Figure B.9). Note that for the combinations of very low sample size (25 subjects) and short scan durations ( $\leq 1600$ volumes), model fits started to degrade. 


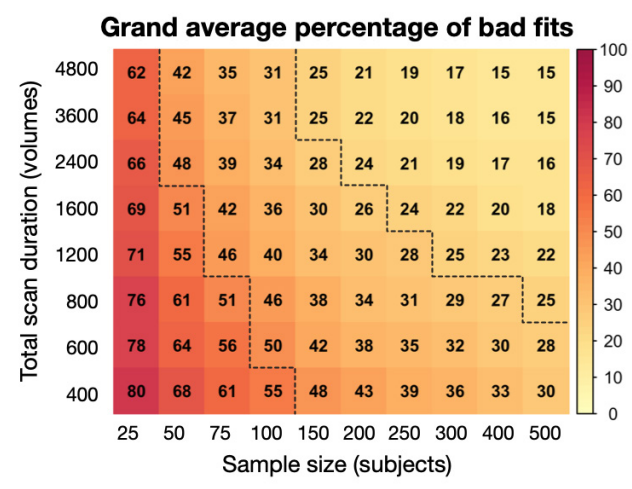

Figure 3.5. Percentage of sampled connections for each combination of sample size and total scan duration for which the goodness of fit for the behavioral association measurement models deteriorated below acceptable levels (CFI $<0.90$ or RMSEA $>0.08$ ), averaged across all seven measures (see Supplementary Figure B.10 for the profiles of the individual measures). Total scan duration is reported as the number of volumes at TR=720ms. Dotted lines mark the boundary where on average more than $25 \%$ and $50 \%$ of the model fits are considered bad.

The goodness of fit assessment from the parameter sweep was used to evaluate the minimal requirements on the input dataset in terms of sample size and total scan duration for reliability modelling. Although no clear boundary can be defined when a measurement model is no longer suitable or practical to use, the chance that the measurement model does not describe the data well for a random sample of participants starts to increase with lower sample size or shorter scan durations (Figure 3.5), with similar profiles for all seven measures (Supplementary Figure B.10).

\subsection{DISCUSSION}

We have shown that reliability modelling of functional connectivity using a measurement model on split-session half-score estimates of functional connectivity is able to extract a reliable component of functional connectivity with improved test-retest reliability between scan sessions acquired on separate days. Secondly, we found that the reliable component of functional connectivity is more strongly associated with traits than the full-score estimate of functional connectivity. Finally, we have empirically evaluated the minimal requirements of the dataset for reliability modelling of functional connectivity in terms of scan duration and sample size. We have previously reported increased heritability estimates for the stable and reliable component of functional connectivity in a longitudinal adolescent twin cohort (Teeuw et al., NeuroImage 2019). Here, we have replicated this finding in the Human Connectome Project. 
3.4.1 Reliability modelling is able to extract a stable and more reliable component of functional connectivity

The moderate standard test-retest reliability of functional connectivity (average rho $=+0.49$ ) that we found is at the higher spectrum compared to other studies (Noble et al., 2019), but comparable to other studies using the HCP Young Adult dataset (Shah et al., 2016; Ge et al., 2017; Noble et al., 2017; Meija et al., 2018; Elliot et al., 2019). There is a substantial improvement in test-retest reliability using a measurement model (average increase $=+0.33$ ), in some cases resulting in very good scores (average rho=+0.82). Other methods to obtain a more reliable measure, such as disattenuation (i.e. scaling a measure by its reliability to obtain a true estimate), shrinkage (i.e. gravitating unreliable measures towards a groupmean estimate), or combining multiple modalities (e.g. resting-state and task-based functional connectivity), are able to increase the reliability of functional connectivity from $+25 \%$ up to two-fold improvement (Mueller et al., 2015; Shou et al., 2014; Mejia et al., 2018; Elliot et al., 2019). Our results show that the split-session measurement model is able to extract a component of functional connectivity separately from independent scan sessions acquired on separate days that is more reliable than the individual half-score estimate of functional connectivity. This component represents a stable and reliable component of functional connectivity for a single link connection that explains on average about half (44\%) of the variance in the split-session measurements. The remaining half is attributed to random variation between split-session measurements and is considered "measurement error". In a 7-Tesla resting-state functional MRI study, a similar proportion (50\%) of the variance could be attributed to spontaneous neural activity, and the other half to nonthermal physiological noise (Bianciardi et al., 2009). Functional connectivity shows a strong state-like nature where it is influenced by intrinsic and extrinsic factors such as caffeine consumption, heart rate variability, circadian rhythm, daily mood, or attention (Wu et al., 2014; Choe et al., 2015; Hodkinson et al., 2014; Facer-Childs et al., 2019; Ismaylova et al., 2018; Geerligs et al., 2016). These short-term fluctuations in connectivity strength have been the topic of investigation for dynamic functional connectivity (Chang and Glover, 2010; Handwerker et al., 2012; Hutchison et al., 2013; Abrol et al., 2017). The residual variance that is specific to the individual half-score estimate of functional connectivity is considered "measurement error" in the measurement model, but is likely to represent both different sources of measurement error (Brandmaier et al., 2018) and relevant biological transients (Ge et al., 2017). Instead, the stable component of functional connectivity would be of particular interest for researchers in the pursuit of a reliable and state-independent endophenotype that could serve as a biomarker (Beauchaine and Constantino, 2017). 


\subsubsection{Reliability modelling improves association of functional connectivity with various} traits by revealing the true association in absence of measurement error

The stable and reliable core component of functional connectivity obtained by the measurement model on half-score estimates of functional connectivity is more strongly associated with all 110 traits compared to the association between full-score functional connectivity and the traits (average improvement factor 1.2) in the full-sized dataset of the Human Connectome Project ( $\mathrm{N}=1003$ participants; 1 hour of resting-state fMRI data). Overall, measures were only weakly associated with full-score measure of functional connectivity (maximum absolute rho<0.25) when measured by the connection with the strongest association. These low associations are typical for resting-state functional connectivity (Vaidya and Gordon, 2013; Kruschwitz et al., 2018; Geerligs et al., 2017; Noble et al., 2017; Basten et al., 2015; Toschi et al., 2018; Siegler et al., 2017). Even at connectome level, functional connectivity is only moderately associated with behavioral measures (Smith et al., 2015; Finn et al., 2015; Rosenberg et al., 2015). It was recently shown that individual variation in the spatial distribution of functional brain network organization are stable trait-like features that may be associated with behavior (Seitzman et al., 2019; Kong et al., 2019), and that general cognitive ability is associated with the stability of the dynamic functional connectome (Hilger et al., 2020). This could indicate that although the purpose of a measurement model is to provide a more reliable measure from parallel test scores, it could be the temporally stable component extracted from the split-session half-score measures of functional connectivity that provide the improved associations with behavioral measures.

\subsubsection{Studies with short scan duration and small samples size will experience greater benefits from reliability modelling}

The parameter sweep reveals that the improvement in association strength is dependent on scan duration and, to lesser extent, on sample size. Datasets with a shorter total scan duration (down to five minutes of resting-state fMRI data) show much greater benefit from reliability modeling (on average up to 1.8-fold increase in association strength, with a similar profile for all seven extensively tested traits). Previously, scan duration has been reported to influence reliability and reproducibility of functional connectivity estimates (Birn et al., 2003; Laumann et al., 2015; Noble et al., 2017; Meija et al., 2018; Elliot et al., 2019) but estimates on the recommended scan duration for maximal reliability vary from 5 to 90 minutes. With typical scan duration for resting-state fMRI studies anywhere between the minimum recommended 5 minutes (Birn et al., 2013) to 8 minutes (Waheed et al., 2016), equivalent to about 400 to 667 volumes in the Human Connectome Project dataset, suggests that most dataset can expect a decent boost in association strength with reliability modelling, despite the fact that the corrected associations remain modest. Datasets with 
smaller sample sizes show a slightly greater benefit of reliability modelling. However, smaller sample sizes are accompanied with increased variation in the improvement factor likely due to sampling bias that a measurement model cannot account for. For datasets with small sample sizes ( $\leq 50$ participants), the utility of the measurement model starts to drop, with $50 \%$ to $80 \%$ of the sampled connections resulting in a bad fit. Note that there is a baseline rejection rate of on average $15 \%$ of the connections with a bad fit of the measurement model in the full-sized dataset. This baseline is also present in the parameter sweep because we did not exclude connections with a bad fit prior to selecting the connections for sparse sampling. Previous studies have examined the sample size required for structural equation modelling, with initial estimates suggested that at least 200 participants are needed (Boomsma, 1985), or as a rule of thumb ten times the number of estimated parameters (Bentler and Chou, 1987; Wolf et al., 2013). Sample sizes as low as 50 participants might be enough to obtain satisfactory fits for task-based fMRI (Sideridis et al., 2014). Our parameter sweep shows that variation due to sampling bias goes down with a sample size around 100 to 150 participants, suggesting that, combined with a typical scan length of around 8 minutes, reliability modelling would be feasible for most contemporary resting-state fMRI studies.

\subsubsection{Stronger genetic signal for the reliable component of functional connectivity}

Measurement models have previously been used in the context of twin studies to obtain heritability estimates for the reliable portion of the variation (van Baal et al., 1998; van Beijsterveldt et al., 2001; Ge et al., 2015). We have previously applied a measurement model to functional connectivity in a longitudinal adolescent twin cohort (Teeuw et al., 2019). Here, we replicated our earlier finding that heritability estimates of functional connectivity can be increased substantially (from average $h^{2}=39 \%$ to $h^{2}=59 \%$ ) by using a measurement model on data from split-half scan sessions. Previous studies on the heritability of functional connectivity using the Human Connectome Project dataset have typically reported low heritability for single link connections (Ge et al., 2017; Colclough et al., 2017; Adhikari et al., 2018). One earlier study applied a custom linear mixed effects model to repeated measures of functional connectivity from the Human Connectome Project dataset (i.e. considered scans on Day one and Day two as repeated measures, similar to the two half-score measures for the full-sized dataset used in this study) and reported similar improvements for heritability estimates of around $+20 \%$ points when averaged across connections for the major functional networks (Ge et al., 2017).

\subsubsection{Methodological considerations for the application of a measurement model}

There are a few methodological considerations of measurement models in general that should be mentioned (Muchinsky, 1996). First, it is important to note that the ultimate 
purpose of the measurement model is to obtain estimates that are closer to the true value in the absence of error in the measurements. The measurement model will provide more accurate estimates that can guide future studies, but will not change the quality of the data. Secondly, while we discussed our results in terms of improvement factors, correlations obtained with the measurement model are associations between the stable components (i.e. reliable variation) rather than between the full traits (i.e. full variation) and those two are not directly comparable. In addition, there is no ground-truth available for resting-state functional connectivity of the human brain that could verify the correctness of the measurement model outputs. It is therefore good practice to always report the uncorrected results from standard association in addition to the corrected results from the measurement model, and important to assess the goodness of fit of the measurement model.

\subsubsection{Limitations to the current study}

There are some limitations specific to the current study. First, there is quite some variation in quality between resting-state fMRI datasets (Noble et al., 2019). Other datasets might see a shift in the performance and requirements curves based on the quality of the dataset. Secondly, reliability modelling was not applied to the traits and the reported corrected association might still be limited by the reliability of the trait. If multiple or repeated measures of the trait are available, a measurement model can be applied to both functional connectivity and the trait to obtain a more accurate estimate of the association between the two in the absence of measurement error (Beaty et al., 2015; Cooper et al., 2019).

\subsubsection{Conclusion}

In conclusion, reliability modelling of functional connectivity using a measurement model on split-half session resting-state fMRI data is an effective method to compensate for attenuation of the temporal correlation coefficient due to noise in the BOLD signal. The measurement model is able to extract a stable and reliable component of functional connectivity that can reveal the true associations with traits and increased heritability estimates compared to the analysis with full-score estimate of functional connectivity. The benefit of reliability modelling is greater for studies with short scan duration or a limited number of participants. 



\section{CHAPTER 4}

\section{LONGITUDINAL DEVELOPMENT OF}

\section{CORTICAL RESTING-STATE NETWORKS}

Original title: Genetic and environmental influences on functional connectivity within and between canonical cortical resting-state networks throughout adolescent development in boys and girls

Authors: Jalmar Teeuw ${ }^{1}$, Rachel M. Brouwer'1, João P.O.F.T. Guimarães ${ }^{3,1}$, Philip Brandner ${ }^{4,1}$, Marinka M.G. Koenis ${ }^{5,1}$, Suzanne C. Swagerman ${ }^{2}$, Maxime Verwoert ${ }^{1}$, Dorret I. Boomsma ${ }^{2}$, and Hilleke E. Hulshoff Pol ${ }^{1}$

Affiliations: ${ }^{1}$ University Medical Center Utrecht, UMC Brain Center, Department of Psychiatry, Utrecht, Netherlands; ${ }^{2}$ Vrije Universiteit Amsterdam, Department of Biological Psychology, Netherlands Twin Register, Amsterdam, Netherlands; ${ }^{3}$ Radboud Medical Center, Department of Cognitive Neuroscience, Nijmegen, Netherlands; ${ }^{4}$ Leiden University, Institute of Psychology, Leiden, Netherlands; ${ }^{5}$ Yale University School of Medicine, Department of Psychiatry, New Haven, CT, United States of America.

Published in 2019 in the journal NeuroImage 202, 116073. doi:10.1016/j.neuroimage.2019.116073

Full reference: Teeuw, J., Brouwer, R., Guimarães, J., Brandner, P., Koenis, M., Swagerman, S., Verwoert, M., Boomsma, D., Hulshoff Pol, H. (2019). Genetic and environmental influences on functional connectivity within and between canonical cortical resting-state networks throughout adolescent development in boys and girls NeuroImage 202, 116073. doi:10.1016/j.neuroimage.2019.116073 


\section{ABSTRACT}

The human brain is active during rest and hierarchically organized into intrinsic functional networks. These functional networks are largely established early in development, with reports of a shift from a local to more distributed organization during childhood and adolescence. It remains unknown to what extent genetic and environmental influences on functional connectivity change throughout adolescent development. We measured functional connectivity within and between eight cortical networks in a longitudinal resting-state fMRI study of adolescent twins and their older siblings on two occasions (mean ages 13 and 18 years). We modelled the reliability for these inherently noisy and head-motion sensitive measurements by analyzing data from split-half sessions. Functional connectivity between resting-state networks decreased with age whereas functional connectivity within resting-state networks generally increased with age, independent of general cognitive functioning. Sex effects were sparse, with stronger functional connectivity in the default mode network for girls compared to boys, and stronger functional connectivity in the salience network for boys compared to girls. Heritability explained up to $53 \%$ of the variation in functional connectivity within and between restingstate networks, and common environment explained up to $33 \%$. Genetic influences on functional connectivity remained stable during adolescent development. In conclusion, longitudinal age-related changes in functional connectivity within and between cortical resting-state networks are subtle but wide-spread throughout adolescence. Genes play a considerable role in explaining individual variation in functional connectivity with mostly stable influences throughout adolescence.

Keywords: longitudinal; twins; heritability; age effects; sex effects 


\subsection{INTRODUCTION}

The human brain is active during rest (Biswal et al., 1995; 1997). Data-driven approaches have been applied to resting-state functional MRI scans to obtain spatial patterns of temporally coherent signals that divide the brain into distinct intrinsic functional networks (DeLuca et al., 2005; Fox et al., 2005; Power et al., 2011; van den Heuvel and Hulshoff Pol, 2010; Yeo et al., 2011). The hierarchical organization of these functional networks is already present around birth. Primary functional networks, such as the sensorimotor, visual, and auditory networks are the first to develop in utero (Gilmore et al., 2018; Keunen et al., 2017; Thomason et al., 2015). After birth, the default mode network (DMN), dorsal mode network (DAN), and salience network (SN) mature into "adult-like" networks by the age of two years (Gao et al., 2011; Gilmore et al., 2018; Keunen et al., 2017). The executive control network $(E C N)$ matures later on in life, in line with the protracted development of executive functions during childhood and adolescence (Gilmore et al., 2018; Zhang et al., 2017). These functional networks can be reliably identified in children and adolescents aged 9 to 15 years for both short-term (i.e. consecutive scan sessions) and long-term repeated measures at 2.5 years interval (Thomason et al., 2011). Thus, by adolescence, these spatially distributed and functionally linked brain regions that share information already closely resemble their adult state.

Cross-sectional studies have provided indications that functional connectivity of the human brain is undergoing subtle alterations during childhood and adolescence (for reviews see Cao et al., 2016; Ernst et al., 2015; Grayson and Fair, 2017; Stevens, 2016). Based on these cross-sectional studies, it is generally believed that the functional brain shifts from a local to a more distributed organization (Cao et al., 2016; Ernst et al., 2015; Fair et al., 2009; Menon, 2013). This is supported by decreases in functional connectivity separating functionally distinct regions (i.e. segregation), and increases in functional connectivity improving communication between functionally related regions (i.e. integration) (Cao et al., 2014; Dosenbach et al., 2010; Fair et al., 2009; 2008; 2007; Gu et al., 2015; Kelly et al., 2009; Marek et al., 2015; Sato et al., 2014; Supekar et al., 2009; Uddin et al., 2011; Wig, 2017). The processes of segregation and integration are reflected in graph theoretical metrics by a decrease in local clustering coefficient, an increase in modularity, and an increase in global efficiency, and are furthermore accompanied by the emerging of hubs of increasing importance (i.e. consolidation of the network into rich-club networks) that shift from primary to higher order cortical regions (Cao et al., 2016; 2014; Grayson et al., 2014; Hwang et al., 2013; Sato et al., 2014; 2015; Supekar et al., 2009; Wu et al., 2013; Zuo et al., 2011). However, results are inconsistent regarding the direction of change and affected regions (Stevens, 2016). In part, this may be due to the limited ability of cross-sectional studies to 
control for inter-individual variation (i.e. the "cohort effect") and are thereby restricted in their interpretation of "true" development (i.e. within subject developmental trajectories). In contrast, longitudinal studies acquire repeated measures of the same individuals and can utilize these measures as control to measure development changes over time within the individual (Crone and Elzinga, 2015; Mills and Tamnes, 2014; Telzer et al., 2018). Longitudinal studies on resting-state or task-regressed functional connectivity in typically developing children and adolescents (aged 9 to 15 years) reveal high levels of consistency and stability of functional connectivity estimates within and between several cortical resting-state networks over a 2 to 3 years interval (Thomason et al., 2011). There are reports of longitudinal age-related increases in functional connectivity (or integration) within several networks (Bernard et al., 2016; Long et al., 2017; Sherman et al., 2014; Sylvester et al., 2018; Wendelken et al., 2017; 2016). However, age-related decrease in functional connectivity (or segregation) within (Sylvester et al., 2018; Wendelken et al., 2016) and between networks have also been reported (Sherman et al., 2014), as well as mixed results reported for cortical-subcortical connectivity (Jalbrzikowski et al., 2017; Peters et al., 2017; Strikwerda-Brown et al., 2015). Thus, although functional brain connectivity during childhood and adolescence is largely stable and adult-like, there are several indications from longitudinal studies of reorganization of functional cortical networks during childhood and adolescent development (Table 4.1).

Table 4.1 (continued on the next page). Studies on longitudinal development of cortical resting-state or taskregressed functional connectivity in typically developing children and adolescents; ordered by midrange age at baseline of each cohort.

\begin{tabular}{|c|c|c|c|}
\hline Study & Sample & Longitudinal & Age effects \\
\hline Long et al., 2017 & $\begin{array}{l}\mathrm{N}_{\text {subject }}=44(17 \mathrm{~F}) \\
\text { Age baseline }=2-6 \\
\text { years }\end{array}$ & $\begin{array}{l}\mathrm{N}_{\text {waves }}=5 \\
\text { Interval = } 1 \\
\text { years }\end{array}$ & $\begin{array}{l}\nearrow \text { Regional Homogeneity; } \\
\nearrow \text { Eigenvector Centrality; } \\
\nearrow \text { FC within FPN; } \\
+ \text { Local-to-global shift in FC for STG; } \\
+ \text { Global-to-local shift in FC for SPL } \\
\text { and FG }\end{array}$ \\
\hline Xiao et al., 2016 & $\begin{array}{l}\mathrm{N}_{\text {subject }}=53(26 \mathrm{~F}) \\
\text { Age baseline }=5-6 \\
\text { years }\end{array}$ & $\begin{array}{l}\mathrm{N}_{\text {waves }}=2 \\
\text { Interval = } 1 \text { year }\end{array}$ & $\begin{array}{l}\hookrightarrow \text { Degree Centrality within DMN; } \\
\nearrow \text { Degree Centrality for left STG: } \nearrow \\
\text { FC between left STG-left IFS and left } \\
\text { STG-left AG }\end{array}$ \\
\hline $\begin{array}{l}\text { Sherman et al., } \\
2014\end{array}$ & $\begin{array}{l}\mathrm{N}_{\text {subject }}=45(24 \mathrm{~F}) \\
\text { Age } \text { baseline }=10 \\
\text { years }\end{array}$ & $\begin{array}{l}\mathrm{N}_{\text {waves }}=2 \\
\text { Interval }=3 \\
\text { years }\end{array}$ & $\begin{array}{l}\text { Integration within DMN: } \nearrow \text { FC } \\
\text { between mPFC-PCC; } \\
\nearrow \text { FC within FPN; }\end{array}$ \\
\hline
\end{tabular}




\begin{tabular}{|c|c|c|c|}
\hline Study & Sample & Longitudinal & Age effects \\
\hline $\begin{array}{l}\text { Sylvester et al., } \\
2018\end{array}$ & $\begin{array}{l}\mathrm{N}_{\text {subject }}=147 \\
(71 \mathrm{~F}) \\
\text { Age baseline }=8-13 \\
\text { years }\end{array}$ & $\begin{array}{l}\mathrm{N}_{\text {waves }}=3 \\
\text { Interval }=1 \text { year }\end{array}$ & $\begin{array}{l}\text { Segregation (i.e. } \searrow \text { FC) between FPN- } \\
\text { DMN } \\
\hookrightarrow \text { FC within DMN; } \\
\iota \text { FC within FPN; } \\
\hookrightarrow \text { FC within SN; } \\
\searrow \text { FC within VAN }\end{array}$ \\
\hline $\begin{array}{l}\text { Wendelken et } \\
\text { al., } 2016[1]\end{array}$ & $\begin{array}{l}\mathrm{N}_{\text {subject }}=132 \\
(56 \mathrm{~F}) \\
\text { Agebaseline }=6-18 \\
\text { years }\end{array}$ & $\begin{array}{l}\mathrm{N}_{\text {waves }}=2 \\
\text { Interval = } 1.5 \\
\text { years }\end{array}$ & $\begin{array}{l}\text { Integration within FPN: } \nearrow \text { FC } \\
\text { between RLPFC-IPL; } \\
\text { Segregation within FPN: \FC within } \\
\text { frontal regions, and within parietal } \\
\text { regions }\end{array}$ \\
\hline $\begin{array}{l}\text { Wendelken et } \\
\text { al., } 2017 \text { [1] }\end{array}$ & $\begin{array}{l}\mathrm{N}_{\text {subject }}=523 \\
(254 \mathrm{~F}) \\
\text { Age baseline }=6-22 \\
\text { years }\end{array}$ & $\begin{array}{l}\mathrm{N}_{\text {waves }}=2,3, \\
\text { and } 2 \\
\text { Interval }=1.5, \\
1.3 \text {, and } 4 \text { years }\end{array}$ & $\begin{array}{l}\text { Integration within FPN: } \nearrow \text { FC } \\
\text { between RLPFC-IPL }\end{array}$ \\
\hline $\begin{array}{l}\text { Strikwerda- } \\
\text { Brown et al., } \\
2015 \text { [2] }\end{array}$ & $\begin{array}{l}\mathrm{N}_{\text {subject }}=56(25 \mathrm{~F}) \\
\text { Age baseline }=16 \\
\text { years }\end{array}$ & $\begin{array}{l}\mathrm{N}_{\text {waves }}=2 \\
\text { Interval = } 2 \\
\text { years }\end{array}$ & $\nearrow$ FC between sACC-VMPFC \\
\hline $\begin{array}{l}\text { Bernard et al., } \\
2016\end{array}$ & $\begin{array}{l}\mathrm{N}_{\text {subject }}=23(13 \mathrm{~F}) \\
\text { Age baseline }=12- \\
21 \text { years }\end{array}$ & $\begin{array}{l}\mathrm{N}_{\text {waves }}=2 \\
\text { Interval }=1 \text { year }\end{array}$ & $\begin{array}{l}\searrow \text { FC between lateral posterior } \\
\text { cerebellum and DLPFC; } \\
\hookrightarrow \text { FC for anterior cerebellum }\end{array}$ \\
\hline
\end{tabular}

Table 4.1 (continued). Symbols: $\searrow$ decreasing with age; $\nearrow$ increasing with age; $\hookrightarrow$ stable across ages. Abbreviations (in alphabetical order): $\mathrm{AMY}=$ amygdala; $\mathrm{DLPFC}=$ dorsolateral prefrontal cortex; DMN = default mode network; IFS = inferior frontal sulcus; $A G=$ angular gyrus; $F C=$ functional connectivity; FG = fusiform gyrus; FPN = frontoparietal network; IPL = inferior parietal lobule; $\mathrm{MDD}=$ major depressive disorder; $\mathrm{mOFC}=$ medial orbital frontal cortex; $\mathrm{mPFC}=$ medial prefrontal cortex; $\mathrm{PCC}=$ precuneus; RLPFC = rostrolateral prefrontal cortex; rs-fMRI = resting-state functional MRI; $\mathrm{sACC}=$ subgenual anterior cingulate cortex; $\mathrm{SN}=$ salience network; SPL = superior parietal lobule; STG = superior temporal gyrus; tb-fMRI = task-based functional MRI; $\mathrm{VAN}=$ ventral attention network; VMPFC = ventral medial prefrontal cortex. [1] collaboration between three cohorts to replicate original findings from Wendelken et al., 2016; [2] Same cohort.

Genes partly control individual differences in brain functioning, at least in adults (Blokland et al., 2012; Douet et al., 2014; Jansen et al., 2015; Martin, 2010; Richmond et al., 2016; Thompson et al., 2013). Heritability estimates for functional connectivity within the default mode network range from $10 \%$ to $80 \%$, depending on the population and methodology used, and typically identify connections involving the posterior cingulate cortex (PCC) and bilateral parietal cortices (LLP and RLP) as strongest heritable connections (Adhikari et al., 
2018a; Fu et al., 2015; Ge et al., 2017; Glahn et al., 2010; Korgaonkar et al., 2014; Meda et al., 2014; Sudre et al., 2017; Yang et al., 2016; Table 4.2). Findings in children and adolescents are still sparse. We were among the first to report that genes explain up to $40 \%$ of individual difference in brain activity during resting-state at the age of 12 years (van den Heuvel et al., 2013). These findings were confirmed and extended for cortical-subcortical connections in younger children, aged 7 to 9 years, with heritability ranging from $32 \%$ to $67 \%$ (Achterberg et al., 2018). And in 16-year-old adolescents reporting peaks of local clusters with heritability ranging between $55 \%$ to $83 \%$ - but note that several cortical resting-state networks revealed overall low heritability estimates $<10 \%$ (Fu et al., 2015). In the only longitudinal twin study on functional connectivity to date, in infants from birth to 2 years, age-dependent genetic effects on functional connectivity within cortical networks were found (Gao et al., 2014). It is unknown whether these age-dependent dynamic influences of genes on functional connectivity extend into childhood and adolescence in the absence of any longitudinal twin studies during this developmental period.

Table 4.2 (continued on the next page). Twin and family studies on heritability of functional connectivity; ordered by midrange age of each cohort.

\begin{tabular}{|c|c|c|c|c|}
\hline Study & Sample & Age & Phenotype & Heritability estimates \\
\hline Gao et al., 2014 & $\mathrm{~N}=288$ & $\begin{array}{l}1,12 \text {, and } \\
24 \text { months }\end{array}$ & $\begin{array}{l}\text { Longitudin } \\
\text { al FC }\end{array}$ & $\begin{array}{l}\text { Significant regression } \\
\text { coefficient for genetic effects } \\
\text { throughout the brain }\end{array}$ \\
\hline $\begin{array}{l}\text { Achterberg et } \\
\text { al., } 2018\end{array}$ & $\mathrm{~N}=220$ & $7-9$ years & FC & $\begin{array}{l}\text { VS-mPFC } h^{2}=67 \% \\
\text { VS-dACC } h^{2}=46 \% \\
\text { VS-AMY } h^{2}=42 \% \\
\text { VS-HPC } h^{2}=32 \%\end{array}$ \\
\hline $\begin{array}{l}\text { van den } \\
\text { Heuvel et al., } \\
2013\end{array}$ & $\begin{array}{l}\mathrm{N}=86 \\
(\text { BrainSCALE) }\end{array}$ & 12 years & GT & Global efficiency $h^{2}=42 \%$ \\
\hline Fu et al., 2015 & $\mathrm{~N}=112$ & $\begin{array}{l}16 \pm 1.5 \\
\text { years }\end{array}$ & FC & $\begin{array}{l}\text { Voxel-wise cluster peaks } h^{2}= \\
55-83 \% \text {, typically } h^{2} \cong 10 \%\end{array}$ \\
\hline Xu et al., 2016 & $\mathrm{~N}=92$ & $15-20$ years & $\begin{array}{l}\text { Effective } \\
\text { FC }\end{array}$ & Within DMN $h^{2}=54 \%$ \\
\hline $\begin{array}{l}\text { Sinclair et al., } \\
2015\end{array}$ & $\mathrm{~N}=592$ (QTIM) & $\begin{array}{l}23 \pm 2.5 \\
\text { years }\end{array}$ & GT & $\begin{array}{l}\text { Mean clustering } h^{2}=47 \%-59 \% \text {; } \\
\text { Modularity } h^{2}=38 \%-59 \% ; \\
\text { Rich-club } h^{2}=0 \%-29 \% \text { [n.s.]; } \\
\text { Global efficiency } h^{2}=52 \%-62 \% \text {; } \\
\text { Small-worldness } h^{2}=51 \%-59 \%\end{array}$ \\
\hline
\end{tabular}




\begin{tabular}{|c|c|c|c|c|}
\hline Study & Sample & Age & Phenotype & Heritability estimates \\
\hline $\begin{array}{l}\text { Yang et al., } \\
2016\end{array}$ & $\begin{array}{l}\mathrm{N}=272 \\
\mathrm{~N}=105 \\
\text { (QTIM) }\end{array}$ & $\begin{array}{l}18-28 \text { years } \\
19-29 \text { years }\end{array}$ & FC & $\begin{array}{l}\text { Within RSNs } h^{2}=23-65 \% \\
\text { Within SMN } c^{2}=35 \% \\
\text { Between }(8 / 21) \text { RSNs } h^{2}=26- \\
42 \% \text {; } \\
\text { Between (11/21) RSNs } c^{2}=18- \\
47 \%\end{array}$ \\
\hline $\begin{array}{l}\text { Moodie et al., } \\
2014\end{array}$ & $\begin{array}{l}\mathrm{N}=42(\mathrm{MZ} \\
\text { only) }\end{array}$ & 19-34 years & FC & $\begin{array}{l}\text { Familiality in several BrainMap } \\
\text { networks }\end{array}$ \\
\hline Ge et al., 2017 & $\begin{array}{l}\mathrm{N}=582(\mathrm{HCP}) \\
\mathrm{N}=809(\mathrm{GSP})\end{array}$ & $\begin{array}{l}22-36 \text { years } \\
18-35 \text { years }\end{array}$ & FC & Within RSNs $h^{2}=45-80 \%$ \\
\hline $\begin{array}{l}\text { Colclough et } \\
\text { al., } 2017\end{array}$ & $\mathrm{~N}=820(\mathrm{HCP})$ & $22-35$ years & FC & Mean FC $h^{2}=17-29 \%$ [n.s.] \\
\hline $\begin{array}{l}\text { Adhikari et al., } \\
\text { 2018a }\end{array}$ & $\begin{array}{l}\mathrm{N}=518(\mathrm{HCP}) \\
\mathrm{N}=334(\mathrm{GOBS})\end{array}$ & $\begin{array}{l}29 \pm 4 \text { years } \\
48 \pm 13 \\
\text { years }\end{array}$ & FC & Within RSN $=h^{2}=9-36 \%$ \\
\hline $\begin{array}{l}\text { Meda et al., } \\
2014\end{array}$ & $\mathrm{~N}=1305$ & $\begin{array}{l}35 \pm 14,35 \\
\pm 12,37 \pm \\
13,44 \pm 16 \\
40 \pm 16 \\
\text { years }\end{array}$ & FC & Within DMN $h^{2}=14-18 \%$ \\
\hline $\begin{array}{l}\text { Fornito et al., } \\
2011\end{array}$ & $\mathrm{~N}=58$ & $\begin{array}{l}38 \pm 14 \text { and } \\
43 \pm 10 \\
\text { years }\end{array}$ & GT & Global cost-efficiency $h^{2}=60 \%$ \\
\hline $\begin{array}{l}\text { Korgaonkar et } \\
\text { al., } 2014\end{array}$ & $\begin{array}{l}\mathrm{N}=250(\mathrm{TWIN}- \\
\mathrm{E})\end{array}$ & $18-65$ years & FC & Within DMN $h^{2}=9-41 \%$ \\
\hline $\begin{array}{l}\text { Sudre et al., } \\
2017\end{array}$ & $\begin{array}{l}\mathrm{N}=305 \\
\mathrm{~N}=132\end{array}$ & $\begin{array}{l}4-86 \text { years } \\
21 \pm 15 \\
\text { years }\end{array}$ & FC & $\begin{array}{l}\text { Within DMN } h^{2}=36-61 \% \\
\text { Within CCN } h^{2}=35-58 \% \\
\text { Within VAN } h^{2}=36-46 \%\end{array}$ \\
\hline $\begin{array}{l}\text { Glahn et al., } \\
2010\end{array}$ & $\mathrm{~N}=333$ (GOBS) & $26-86$ years & FC & Within DMN $h^{2}=42 \%$ \\
\hline
\end{tabular}

Table 4.2 (continued). Abbreviations (in alphabetical order): AMY = amygdala; $c^{2}=$ percentage variance explained by common environmental influences; $\mathrm{CCN}$ = cognitive control network; $\mathrm{dACC}=$ dorsal anterior cingulate cortex; DMN = default mode network; FC = functional connectivity; GOBS = Genetics of Brain Structure cohort; GSP = Brain Genomics Superstruct Project cohort; GT = graph theory; $h^{2}=$ heritability (percentage of phenotypic variance explained by additive genetic influences); HCP = Human Connectome Project cohort; HPC = hippocampus; $\mathrm{MZ}$ = monozygotic twins; n.s. = not significant; QTIM = Queensland Twin Imaging cohort; rs-fMRI = resting-state functional MRI; SMN = sensorimotor network; tb-fMRI = task-based functional MRI; TWIN-E = 
Twin study of Wellbeing using Integrative Neuroscience of Emotion cohort; VAN = ventral attention network; VS = ventral striatum.

Here we report on genetic and environmental influences on functional connectivity within and between eight canonical cortical resting-state networks in a longitudinal adolescent cohort of twins and their older siblings measured on two occasions (mean ages of twins 12 and 17 years; mean ages of siblings 15 and 20 years; mean ages combined 13 and 18 years). We utilize a model that accounts for measurement imprecision by analyzing data from split-half sessions to obtain a reliable component of functional connectivity. This is the first longitudinal study on cortical resting-state networks to estimate the importance of genetic factors for functional connectivity within and between cortical resting-state networks in adolescence. The longitudinal data allowed us to investigate possible dynamic influences of genetic factors throughout adolescence (Teeuw et al., 2018; van Soelen et al., 2012b). We investigated the effects of sex and age on functional connectivity while controlling for measurement imprecision and residual head motion. Finally, we investigated the relation between intelligence and functional development of resting-state networks.

\subsection{METHODS}

\subsubsection{Participants}

This project is part of the longitudinal BrainSCALE study on development of brain and cognition in twins and their older sibling (van Soelen et al., 2012a), a collaborative project between the Netherlands Twin Register (NTR; Boomsma et al., 2006; van Beijsterveldt et al., 2013) at the Vrije Universiteit (VU) Amsterdam and University Medical Center Utrecht (UMCU). The BrainSCALE cohort is a representative sample of typically-developing children from the Dutch population. A total of 112 families with twins and an older sibling participated in the study. The twins and siblings were assessed with a battery of cognitive and behavior tests and extensive neuroimaging protocol at baseline assessment when the twins were 9 years old (Peper et al., 2009). Two follow-up assessments were conducted when the twins were 12 and 17 years old (Koenis et al., 2017; Teeuw et al., 2018; van Soelen et al., 2012b; 2013). Here, we report results of the analysis of resting-state functional MRI scans that were acquired during the second and third assessment of the BrainSCALE study, when the twins and siblings were on average 13 and 18 years of age, hereafter referred to as time point 1 (TP1) and time point 2 (TP2). Intelligence was assessed using an abbreviated version of the Weschler Intelligence Scale for Children - Third edition (WISC-III; Wechsler, 1991) IQ test at age 13 years, and an abbreviated version of Weschler Adult Intelligence Scale - Third edition (WAIS-III; Wechsler, 1997) IQ test at age 18 years. The use of subtasks of the WISC-III as proxy for full WISC-III IQ test has previously been established as a valid construct (Koenis et al., 2015). 
The BrainSCALE study was approved by the Central Committee on Research Involving Human Subjects of The Netherlands (CCMO), and studies were performed in accordance with the Declaration of Helsinki. Children and their parents signed informed consent forms. Parents were financially compensated for travel expenses, and children received a present or gift voucher at the end of the testing days. In addition, a summary of cognition scores and a printed image of their T1 brain MRI scan, when available, were provided afterwards.

\subsubsection{MRI acquisition}

Whole-brain magnetic resonance imaging (MRI) scans were acquired on two identical 1.5 Tesla Philips Achieva scanners (Philips, Best, Netherlands) at the University Medical Center Utrecht (UMCU). Three-dimensional T1-weighted structural scans (Spoiled Gradient Echo; TE $=4.6 \mathrm{~ms} ; \mathrm{TR}=30 \mathrm{~ms}$; flip angle $=30^{\circ} ; 160$ to 180 contiguous coronal slices of $1.2 \mathrm{~mm}$; in-plane resolution of $1.0 \times 1.0 \mathrm{~mm}^{2}$; acquisition matrix of $256 \times 256$ voxels; field-of-view of $256 \mathrm{~mm}$ with $70 \%$ scan percentage (Peper et al., 2009; Teeuw et al., 2018) and resting-state functional MRI scans (PRESTO-SENSE; TE=31.1 ms; TR=21.1 ms; flip angle $=9^{\circ} ; 4.0 \mathrm{~mm}$ isotropic voxels; 900 volumes over 540 seconds; effective $\mathrm{TR}=600 \mathrm{~ms}$ ) of the whole head were acquired (van den Heuvel et al., 2013). The same scanners and scan sequence parameters were used to acquire MRI scans at both ages to limit possible effects of differences in scan acquisition. Subjects were instructed to lie still with their eyes closed and keep their mind free from thoughts while preventing them from falling asleep during acquisition of the resting-state functional MRI scans. Invited participants were excluded from the scanning protocol when contraindications for MRI were present at the time of examination. In particular, the presence of dental braces incompatible with the magnetic field of the MRI scanner resulted in a decline of participants for the neuroimaging assessment, specifically at age 13 years.

\subsubsection{Processing of resting-state functional MRI scans}

Processing of the MRI scans was performed using the CONN toolbox version 18a (Whitfield-Gabrieli and Nieto-Castanon, 2012; https://web.conn-toolbox.org/) and SPM toolbox version 12 (http://www.fil.ion.ucl.ac.uk/spm/) in MATLAB 2015b (The MathWorks Inc., Massachusetts, United States). The CONN toolbox is an open-source toolbox for processing and analysis of resting-state functional MRI scans. The toolbox is based on the aCompCor method for artefact correction that performs linear regression of undesired confounders, such as head motion and signal from white matter and cerebrospinal fluids, to recover the neuronal BOLD signal of interest (Behzadi et al., 2007). This artifact correction method has shown to reduce motion-related artifacts in resting-state fMRI in children (Muschelli et al., 2014). 
To obtain the signal from white matter and cerebrospinal fluid (CSF), brain tissue from the structural T1-weighted MRI scans was segmented into CSF, gray matter (GM), and white matter (WM) tissue maps using a partial volume segmentation algorithm that incorporates a non-uniform partial volume distribution (Brouwer et al., 2010). The structural T1weighted MRI scans were registered to MNI-152 space using non-linear transformation. The non-linear transformation was then applied to the tissue maps to warp them to MNI152 space and resampled to $3.0 \mathrm{~mm}$ isotropic voxels. The white matter and CSF tissue maps were threshold at $50 \%$ (i.e. selecting only voxels with $>50 \%$ of tissue proportion attributed to white matter or CSF) and binarized to create masks. The white matter tissue masks were eroded by two voxels to reduce the number of voxels at the white-gray matter tissue interface. No erosion was performed for the CSF tissue masks due to the occasional small volume of the lateral ventricles in children at age 13 years (Giedd et al., 1996; Lenroot and Giedd, 2006; Sowell et al., 2002). Instead, CSF tissue masks were constrained to contain only voxels inside the lateral ventricles.

The volumes within the resting-state functional MRI scans were first realigned to the mean image of the volumes using a rigid-body realignment procedure without reslicing the data. The rigid-body transformation parameters were used to retrospectively estimate head movement during scan acquisition using framewise displacement (Power et al., 2012). Mapping between resting-state functional MRI scans and structural MRI scans was determined by linear registration of the mean of the realigned resting-state functional MRI scan to the structural T1-weighted MRI scan. By concatenating all transformations (realignment, functional-to-T1 and T1-to-MNI), the mapping between individual functional space and MNI-152 space was obtained. The resulting transformation was used to warp the resting-state functional MRI scans into MNI space and resampled to $3.0 \mathrm{~mm}$ isotropic voxels. Global signal fluctuations time series were extracted from the warped functional MRI scans using the DVARS method (Power et al., 2012).

Correction of undesired confounders was performed using linear regression of the top ten principal components from the BOLD signal of white matter and (ventricular) cerebrospinal fluids (Behzadi et al., 2007; Chai et al., 2012), 24 head motion parameters (Friston et al., 1996; Yan et al., 2013), and scrubbing of subject-dependent number of high motion frames (Power et al., 2012). Linear regression was performed on the individual voxels of the brain after linear and quadratic detrending of the BOLD time series to reduce effects of scanner drift. The six rigid-body transformation parameters $(\mathrm{R})$ derived during realignment of resting-state volumes, its first-order temporal derivative ( $\left.R^{\prime}\right)$, and the squared product of all terms $\left(\mathrm{R}^{2}\right.$ and $\left.\mathrm{R}^{2}\right)$ were included as regressors to control for head motion during scan acquisition (Friston et al., 1996; Yan et al., 2013). In addition, scrubbing 
of frames with high motion (FD $>0.30 \mathrm{~mm}$ ) or unusually large whole-brain BOLD signal changes (DVARS Z-score > 3.0) was performed by including a regressor for each of the flagged frames, the frame immediately preceding the flagged frame, and the two frames following the flagged frame (Power et al., 2012); see supplementary information for more details of head motion and scrubbing. The average number of flagged frames is 79 (9\%) out of 900 frames at age 13 years, and 57 (6\%) out of 900 frames at age 18 years. By including frames surrounding the flagged frames the average number of scrubbed frames is $214(24 \%)$ out of 900 at age 13 years, and 154 (17\%) out of 900 frames at age 18 years. The residuals of the linear regression provided the voxel-wise denoised time series with a duration of 900 frames regardless of the number of frames scrubbing used in the regression. Temporal bandpass filtering was applied at the frequency range of 0.008 to $0.080 \mathrm{~Hz}$ after linear regression was performed that contained on average $39 \%$ of the total power spectral density after denoising (Biswal et al., 1995; Ciric et al., 2017; Waheed et al., 2016).

All resting-state functional MRI scans were processed independently from each other, including scans from subjects with longitudinal data.

\subsubsection{Functional connectivity estimates}

Functional connectivity matrices were obtained for the resting-state networks atlas provided by the CONN toolbox (Whitfield-Gabrieli and Nieto-Castanon, 2012; https://web.conn-toolbox.org/). The atlas is based on ICA analysis of resting-state scans of 497 unrelated young adults from the Human Connectome Project and provides regions of interest (ROI) for 7 canonical cortical resting-state networks and the cerebellum: the core Default Mode network (4 components), Sensorimotor (3), Visual (4), Salience (7), Dorsal Attention (4), Frontoparietal (4), Language (4), and Cerebellar (2); see supplementary information for details on the CONN atlas (Supplementary Figure C.1; Supplementary Table C.1), and for comparison, a group-ICA decomposition was performed on the BrainSCALE resting-state functional MRI scans used in this analysis (Supplementary Figure C.2).

The CONN toolbox atlas is based on a data-driven decomposition of resting-state functional data from the Human Connectome Project that consists of most large-scale canonical networks covering a large area of the cortical surface. Further decomposition of each network into separate regions that include homologous contra-lateral regions allows for studying global patterns of the developing functional brain within and between restingstate networks. Its limited number of regions makes it suitable for the computational complexity of twin modelling, and the moderate size of the regions provide the benefit of increased signal-to-noise ratio (SNR) through spatial averaging of the inherently noisy BOLD signal from neighboring voxels. 
Full-score and half-score measures of functional connectivity were obtained using full Pearson correlation between spatially averaged denoised time series of two regions of interest (ROI). Full-score measures were obtained by considering the entire denoised time series of the 900-volume resting-state functional MRI scan. Half-score measures were obtained by splitting the denoised time series into two independent halves of equal length; the first half-score measure (H1) corresponding to the first 450 volumes of the denoised time series, and the second half-score measure $(\mathrm{H} 2)$ consisting of the remaining 450 volumes. All functional connectivity correlations were transformed using Fisher's r-to-Z transformation prior to any statistical analysis. Mean functional connectivity for subsets of connections (e.g. mean functional connectivity between all resting-state networks) was calculated as the average of the r-to-Z-transformed correlations across the subset of connections.

\subsubsection{Quality control}

Incomplete resting-state fMRI scans and scans with discernable defects related to scanner artefacts or receiver coil malfunction were discarded beforehand, resulting in the exclusion of 18 of the 380 (5\%) scans from 17 subjects; 11 of the 152 (7\%) scans at age 13 years, and 7 of 228 (3\%) scans at age 18 years (Supplementary Table C.2). In addition, individual fullscore and half-score measures were excluded when the corresponding mean FD of the time series exceeded $0.30 \mathrm{~mm} /$ volume head motion or the number of scrubbed frames exceeded more than half the number of frames in the time series (i.e. more than 450 scrubbed frames for full-score measures, and more than 225 scrubbed frames for half-score measures). After filtering, 97 full-score measures remain at TP1 (age 13 years), 108 half-score measures at TP1 H1, 88 half-score measures at TP1 H2, 202 full-score measures at TP2 (age 18 years), 203 half-score measures at TP2 H1, and 200 half-score measures at TP2 H2 (Supplementary Table C.2; Supplementary Figure C4). See supplementary information for more details on quality control procedure and analysis of head motion.

\subsubsection{Genetic modelling}

Genetic modelling of twin and sibling data can provide information on the extent that variation of a trait in the population is explained by genetic factors (Boomsma et al., 2002; Posthuma et al., 2000). Monozygotic (MZ) twins share $100 \%$ of their genetic material and dizygotic twins and full siblings share on average $50 \%$ of their segregating genes. Inclusion of these relatives into an extended twin design enables decomposition of the phenotypic variance $\left(V_{P}\right)$ of a trait into three variance components: additive genetic $\left(V_{A}\right)$, common environmental $\left(V_{C}\right)$, and unique environmental $\left(V_{E}\right)$ components of variance. Additive genetic influences represent effects of multiple alleles at different loci across the genome that act upon the phenotypic trait. Common environment represents influences that are shared between twins and siblings from the same family and causes them to be more alike 
than children grown up in different families. Unique environmental influences are not shared between family members and may include measurement error (Boomsma et al., 2002; Falconer and Mackay, 1996). If monozygotic (MZ) twins resemble each other more than dizygotic (DZ) twins and siblings for a trait, then the hypothesis that the trait is influenced by genetic factors is supported. If both $\mathrm{MZ}$ and $\mathrm{DZ}$ twins are more alike in resemblance than expected based on genetics alone, common environmental influences are likely to play a role.

\subsubsection{Structural equation modeling}

Within genetic structural equation modelling (SEM), a phenotype can be modelled as influenced by latent additive genetic factors, and common and unique environmental factors. These factors are modelled as unobserved or latent variables with unit variance where path coefficients going from latent trait to phenotype and are symbolized by $a, c$, and $e$ quantify their respective influence on the phenotype. The model is made identifiable by putting constraints on the correlation $\rho_{A}$ between the latent variable $A$ of family members; $\rho_{A}=1.0$ for monozygotic twins, and $\rho_{A}=0.5$ for dizygotic twins and twin-sibling pairs. The correlation $\rho_{C}$ between latent variable $C$ of family members is constrained to $\rho_{C}=1.0$ for all twins and siblings from the same family. The latent variable $E$ is uncorrelated between individuals. The sum of the squared path coefficients $a^{2}, c^{2}$, and $e^{2}$, representing the variance components $A, C$, and $E$, is equal to the phenotypic variance $(V)$ of a trait; i.e. $V=A+C+E=a^{2}+c^{2}+e^{2}$. Heritability $\left(h^{2}\right)$ of the trait is estimated as the proportion of phenotypic variance $(V)$ that is due to additive genetic variance $(A)$; i.e. $h^{2}=$

$\frac{A}{V}=\frac{a^{2}}{a^{2}+c^{2}+e^{2}}$. Structural equation models were defined using OpenMx version 2.8.3 (Neale et al., 2015; https://openmx.ssri.psu.edu/), a package for structural equation modelling in $\mathrm{R}$ version 3.4.2 (R Core Team, 2017; https://www.r-project.org/). Model fitting was performed using full-information maximum likelihood (FIML) to take advantage of all available information in case of missing data.

\subsubsection{Modelling of twin and sibling data}

Longitudinal data from extended twin designs can be modeled by Cholesky decomposition (Supplementary Figure C.3) to estimate the genetic and environmental influences on repeated observations (Neale and Cardon, 1992). A longitudinal Cholesky decomposition, with multiple measurements of the same trait acquired at different ages within the same individuals, allows for estimating the dynamics of genetic and environmental influences on traits over age. This model provides estimates of heritability at the individual ages and of the genetic and environmental correlations that explain the sources of stability across ages. Genetic correlations represent the extent to which the same genes influence a trait at multiple ages. A longitudinal Cholesky decomposition can also provide indication of fluctuating influences of the same genes over time, or the presence of novel genetic 
influences (i.e. innovation) unique to a specific age (Teeuw et al., 2018; van Soelen et al., 2012b). Here, we modeled the split-half phenotypic information as a common factor at mean ages 13 and 18 years (van Baal et al., 1998; van Beijsterveldt et al., 2001) such that a latent phenotypic factor $F_{j}$ represented the reliable component of the two half-score measures at each age. Residual variance of a measurement $\left(E_{S}\right)$ that is not attributed to a latent phenotypic factor is considered to be measurement error due to imprecisions of the measurement instrument. Estimation of genetic and environmental components was carried out for two submodels: the model with two latent phenotypic factors loading on the half-scores at each age, and a second model with a single latent phenotypic factor loading on all four half-scores (Figure 4.1). Heritability of a latent phenotypic factor $F_{j}\left(h_{j}^{2}\right)$ is estimated as the proportion of additive genetic variance of the latent phenotypic factor $\left(A_{j}\right)$ over the variance of the latent phenotypic factor $\left(V_{j}\right): h_{j}^{2}=\frac{A_{j}}{V_{j}}$. The heritability estimate
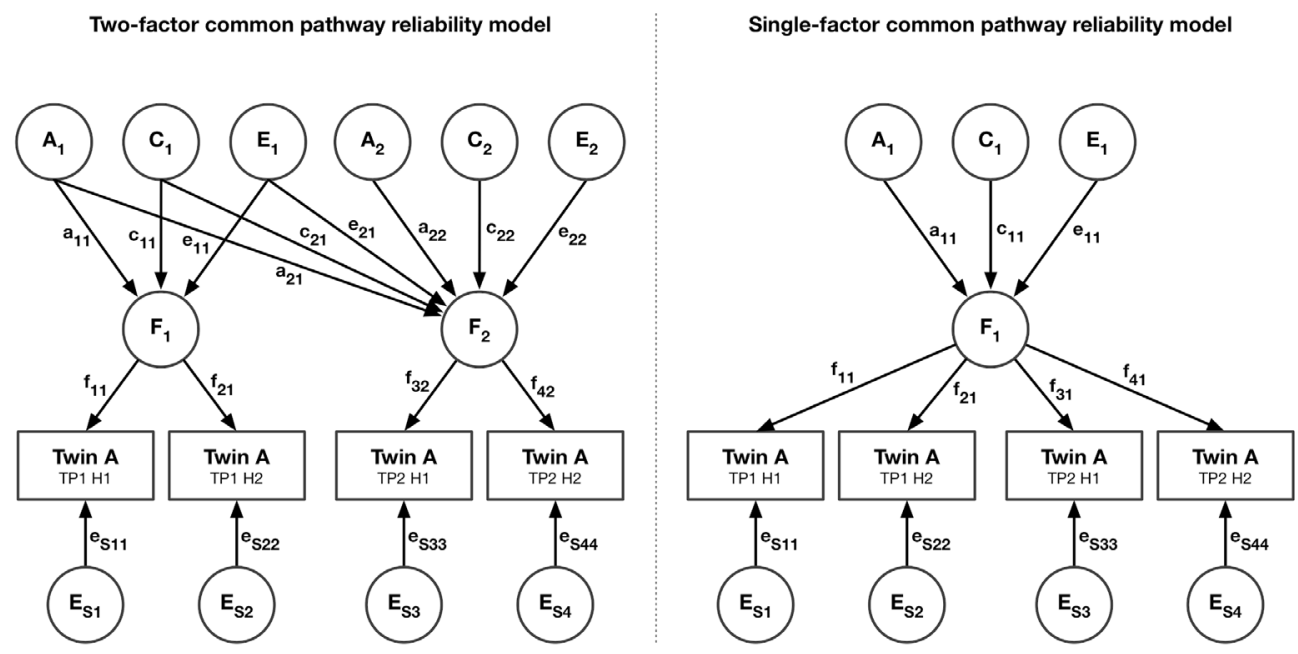

Figure 4.1. A common pathway reliability model with two (left) and one (right) latent phenotypic factor. Measurements are represented by rectangles for age 13 years (TP1) and age 18 years (TP2) for the first (H1) and second (H2) half-score measures. Latent variables are represented by circles. The variance of latent common factor $F_{j}$ represents the reliable component of the measurements and explains part of the variance of the measurement variables proportional to the square of the path coefficients on paths $f_{k j}$. Latent factors representing additive genetics $\left(A_{i}\right)$, common environment $\left(C_{i}\right)$, and unique environment $\left(E_{i}\right)$ together explain the variance of the common factor $F_{j}$ proportional to the square of their respective paths $a_{j i}, a_{c i}$, and $e_{j i}$. Measurement-specific variances (i.e. residual variances not attributed to the common factor) are explained by the latent variables $E_{S i}$ explaining the residual variances of measurements proportional to the square of the loadings on paths $e_{S k l}$. Family members are linked through bidirectional paths on their latent variance components with values constrained to 1.0 for the additive genetic factor(s) $\left(A_{i}\right)$ between monozygotic twins, 0.5 for the additive genetic factor(s) $\left(A_{i}\right)$ between dizygotic twins and siblings, 1.0 for common environmental factors $\left(C_{i}\right)$ for all pairs within one family, and 0.0 for unique environment $\left(E_{i}\right)$. 
of a latent phenotypic factor can be projected back to obtain heritability estimates for the individual half-score measures. According to path tracing rules, heritability of an individual measurement $M_{k}\left(h_{k}^{2}\right)$ due to heritability of the latent phenotypic factor is the sum of the multiplication of the path coefficients along all the paths that visit an additive genetic variance component; e.g. the heritability of the first half-score at age 13 years $\left(M_{1}\right)$ is $h_{1}^{2}=$ $f_{11} \cdot a_{11} \cdot a_{11} \cdot f_{11}$, or simplified, $h_{1}^{2}=f_{11}^{2} \cdot a_{11}^{2}$, and the heritability of the first half-score at age 18 years $\left(M_{1}\right)$ in the two-factor model is $h_{3}^{2}=\left(f_{32} \cdot a_{21} \cdot a_{21} \cdot f_{32}\right)+\left(\left(f_{32} \cdot a_{22} \cdot a_{22} \cdot f_{32}\right)\right.$, or simplified, $h_{3}^{2}=f_{32}^{2} \cdot\left(a_{21}^{2}+a_{22}^{2}\right)$ (see Figure 4.1). The genetic correlation $\left(r_{a}\right)$ between the latent phenotypic factors in the two-factor model is defined as the additive genetic covariance between the two factors over the square root product of the additive genetic variances of the two factors: $r_{g}=\frac{\operatorname{cov}\left(A_{1}, A_{2}\right)}{\sqrt{A_{1} \cdot A_{2}}}$.

\subsubsection{Associations between functional connectivity and intelligence}

The phenotypic associations between full-score functional connectivity (FC) estimates and intelligence (IQ) at each age, and the phenotypic associations between change in full-score functional connectivity with age $(\triangle \mathrm{FC})$ and change in IQ scores $(\triangle \mathrm{IQ})$ was assessed using a bivariate Cholesky model. Change scores were computed as the difference between the two ages. The bivariate Cholesky model included sex and age as fixed effects on each of the measures to account for possible mean differences between sexes and ages.

\subsubsection{Statistical testing}

Significance of parameters was determined using the log-likelihood ratio test by comparing the likelihood of the model with additional constraints on the parameters to the likelihood of the less constrained model. For bounded variance components (e.g. heritability estimates), the difference in -2 times the log likelihood (-2LL) between models with a single constraint follows a 50:50 mixture of $\chi^{2}$ distributions with zero and one degree of freedom, and a 50:45:5 mixtures of $\chi^{2}$ distributions with zero, one and two degrees of freedom for models with two constraints, etcetera; all effectively allow p-values to be cut in half (Dominicus et al., 2006).

Correction for multiple comparison was performed using FDR (Genovese et al., 2002) per condition (e.g. testing for effects of sex on functional connectivity) for all between and within resting-state network connections including mean functional connectivity estimates for a total of 92 tests per condition.

\subsubsection{Model selection}

The log-likelihood ratio test was used to determine the most parsimonious model amongst the models with different configuration of variance components on the latent phenotypic 
factors (i.e. ACE, AE, $\mathrm{CE}$ or E). The optimal number of latent phenotypic factors was determined using the log-likelihood ratio test on the models with ACE variance components. In advance of the results, note that in 24 of the 92 cases (26\%) a model with two factors was optimal (Supplementary Table C.4; Supplementary Table C.5) and that about half of these two-factor models do not have statistically significant heritability or common environmental influences at both ages (i.e. either $\mathrm{E}-\mathrm{AE}, \mathrm{E}-\mathrm{CE}$, or $\mathrm{E}-\mathrm{E}$ configuration; Supplementary Table C.6), and show mostly stable genetic influences primarily driven by the increased power at age 18 years. Although the presence of two factors might also be due to changes in unique environmental influence, we cannot distinguish between true unique environmental influences and age-specific measurement error in the common pathway reliability model with two factors. We therefore present our main analysis using the results of the common pathway reliability model with a single factor first, followed by the results from the two-factor model in a post-hoc fashion.

\subsubsection{Post-hoc analyses}

We performed a post-hoc analysis to validate the main findings from the CONN atlas when global signal regression (GSR) is applied during the preprocessing stage. We performed a second post-hoc analysis to validate the main findings using Yeo resting-state networks atlas (Yeo et al., 2011). This atlas has a slightly different parcellation of the cortical surface into networks, which includes an extended default mode network, i.e. the parahippocampal and temporal regions in addition to the regions of the core default mode network.

\subsection{RESULTS}

\subsubsection{Demographics}

Data from 240 participants with either one or two resting-state functional MRI scans that passed quality control were included in the analysis, providing a total of 315 scans (Table 4.3). The twins were on average $12.2 \pm 0.23$ and $17.2 \pm 0.17$ (mean $\pm \mathrm{SD}$ ) years old at time point 1 (TP1) and time point 2 (TP2), with their older siblings on average $2.7 \pm 1.2$ (mean $\pm \mathrm{SD}$ ) years older.

\subsubsection{Stability and reliability of functional connectivity}

Group-level mean full-score functional connectivity matrices between and within restingstate networks reveal minor changes in functional connectivity estimates between the two timepoints (Figure 4.2). Mean functional connectivity at group-level ranges from +0.25 to +0.71 at age 13 years and from +0.17 to +0.73 at age 18 years for connections between restingstate networks, and ranges from +0.32 to +0.76 at age 13 years and from +0.33 to +0.84 at age 18 years for connections within resting-state networks (Figure 4.2; Supplementary Table C.11; Supplementary Table C.12). Despite the appearance of "stable" functional connectivity 
Table 4.3. Demographics of participants in the BrainSCALE longitudinal study with resting-state fMRI scans.

\begin{tabular}{|c|c|c|c|c|}
\hline Measure & & Inclusion TP1 & Inclusion TP2 & Longitudinal sample \\
\hline \multirow[t]{7}{*}{ Individuals $(N)$} & Total: & 108 & 207 & 75 \\
\hline & $M Z M:$ & 17 & 31 & 13 \\
\hline & $M Z F:$ & 16 & 30 & 12 \\
\hline & $D Z M:$ & 8 & 32 & 7 \\
\hline & $D Z F:$ & 16 & 30 & 8 \\
\hline & DZOS: & 12 & 26 & 9 \\
\hline & Siblings: & 39 & 58 & 26 \\
\hline Age of twins & Range: & $11.8-12.7$ & $16.8-17.9$ & $11.8-17.9$ \\
\hline (years) & Mean $\pm S D$ : & $12.2 \pm 0.23$ & $17.2 \pm 0.17$ & $14.7 \pm 2.53$ \\
\hline Age of siblings & Range: & $13.0-17.8$ & $18.3-22.9$ & $13.2-22.9$ \\
\hline (years) & Mean $\pm S D$ : & $15.0 \pm 1.20$ & $19.8 \pm 1.13$ & $17.3 \pm 2.70$ \\
\hline Scan interval & Range: & N/A & $\mathrm{N} / \mathrm{A}$ & $4.1-5.7$ \\
\hline (years) & Mean $\pm S D$ : & $\mathrm{N} / \mathrm{A}$ & N/A & $5.0 \pm 0.29$ \\
\hline \multirow[t]{2}{*}{ Sex } & Females: & $62(57 \%)$ & $112(54 \%)$ & $42(56 \%)$ \\
\hline & Males: & $46(43 \%)$ & $95(46 \%)$ & $33(44 \%)$ \\
\hline \multirow[t]{2}{*}{ IQ scores } & Range: & $65-147$ & $75-152$ & $79-132.5$ \\
\hline & Mean $\pm S D$ : & $100.8 \pm 14.7$ & $104.2 \pm 12.6$ & $103.3 \pm 11.4$ \\
\hline Mean FD & Range: & $0.09-0.28$ & $0.09-0.27$ & $0.11-0.24$ \\
\hline$(\mathrm{mm} /$ volume $)$ & Mean $\pm S D$ : & $0.18 \pm 0.04$ & $0.17 \pm 0.03$ & $0.17 \pm 0.03$ \\
\hline Flagged frames & Range: & $3-234$ & $0-183$ & $\mathrm{~N} / \mathrm{A}$ \\
\hline$(N)$ & Mean $\pm S D$ : & $79 \pm 51$ & $57 \pm 42$ & \\
\hline Scrubbed frames & Range: & $12-450$ & $0-449$ & $\mathrm{~N} / \mathrm{A}$ \\
\hline$(N)$ & Mean $\pm S D$ : & $214 \pm 121$ & $154 \pm 106$ & \\
\hline
\end{tabular}

Number of complete twin pairs, or sibling paired with at least one of the twins, are reported between parenthesis in the participants row; IQ scores and mean FD for full-scores measures of participants with longitudinal data were averaged across both measurements in the longitudinal data column. Flagged frames are the number of frames within the scan that exceeded the threshold for head motion of FD $>0.30$. Scrubbed frames are the number of frames for which regressors were included in the preprocessing stage, and are derived from expansion of the flagged frames by also including one frame prior and the two frames following flagged frames. Abbreviations (in alphabetical order): DZF = dizygotic females; DZM = dizygotic males; DZOS = dizygotic opposite sex; FD = framewise displacement; IQ = intelligence quotient; $M Z F=$ monozygotic females; MZM = monozygotic males; SD $=$ standard deviation; $\mathrm{TP} 1=$ time point $1 ; \mathrm{TP} 2=$ time point 2 . 
with age, high individual variation exists with low to moderate short-term (i.e. within age) test-retest reliability $\left(r_{\mathrm{ph}}\right.$ between networks range $=[+0.13 ;+0.62] ; r_{\mathrm{ph}}$ within networks range $=[+0.20 ;+0.66]$; Supplementary Figure C.6; Supplementary Table C.11; Supplementary Table C.12) and slightly lower long-term (i.e. between ages) test-retest stability ( $r_{\text {ph }}$ between networks range $=[+0.06 ;+0.47] ; r_{\mathrm{ph}}$ within networks range $[+0.06 ;+0.55]$; Supplementary Figure C.6; Supplementary Table C.11; Supplementary Table C.12).

\subsubsection{Longitudinal age effects on functional connectivity}

Functional connectivity between resting-state networks decreases with age ( $\beta_{\text {age }}$ mean FC between RSNs $=-0.0060 ; p=0.032$; FDR-corrected $p=0.054$ [n.s.]; Figure 4.3; Supplementary Table C.9) whereas functional connectivity within resting-state networks increases with age $\left(\beta_{\text {age }}\right.$ mean FC within RSNs $=+0.0094 ; p<0.001$; FDR-corrected $p<0.001$; Figure 4.3; Supplementary Table C.9), except for several connections within the salience network (SN) involving the anterior cingulate cortex (ACC), left rostral prefrontal cortex (RPFC) and left supramarginal gyrus (SMG) that decrease with age (Figure 4.3; Supplementary Table C.7; Supplementary Table C.9). The strongest increases in functional connectivity occur mostly within the sensorimotor network ( $\beta_{\text {age }}$ mean FC within SMN $=+0.0256 ; p<0.001$; FDR-corrected $p<0.001$; Figure 4.3; Supplementary Table C.9) and the visual network ( $\beta_{\text {age }}$ mean FC within $\mathrm{VN}=+0.0190 ; p<0.001 ;$ FDR-corrected $p<0.001$; Figure 4.3; Supplementary Table C.9).

\section{Functional connectivity between resting-state networks

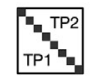

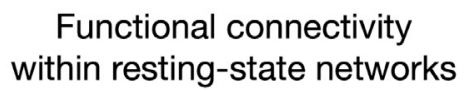
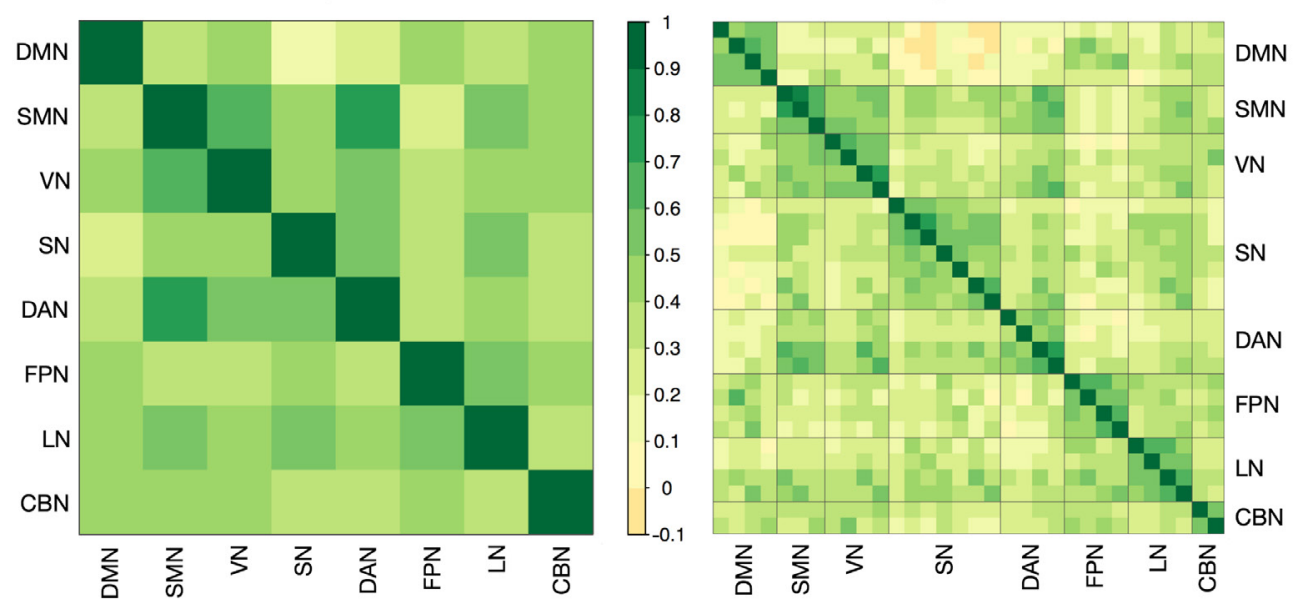

Figure 4.2. Group-level mean functional connectivity for connections between (left) and within canonical restingstate networks (right) at age 13 years (lower triangles; TP1) and 5 years later at age 18 years (upper triangles; TP2). For the order of regions within resting-state networks, see Supplementary Table C.1. Abbreviations (in alphabetical order): $\mathrm{CBN}$ = cerebellar network; $\mathrm{DAN}=$ dorsal attention network; $\mathrm{DMN}=$ default mode network; FPN = frontoparietal network; LN = language network; SMN = sensorimotor network; $\mathrm{SN}$ = salience network; $\mathrm{TP} 1=$ time point $1 ; \mathrm{TP} 2=$ time point $2 ; \mathrm{VN}=$ visual network . 


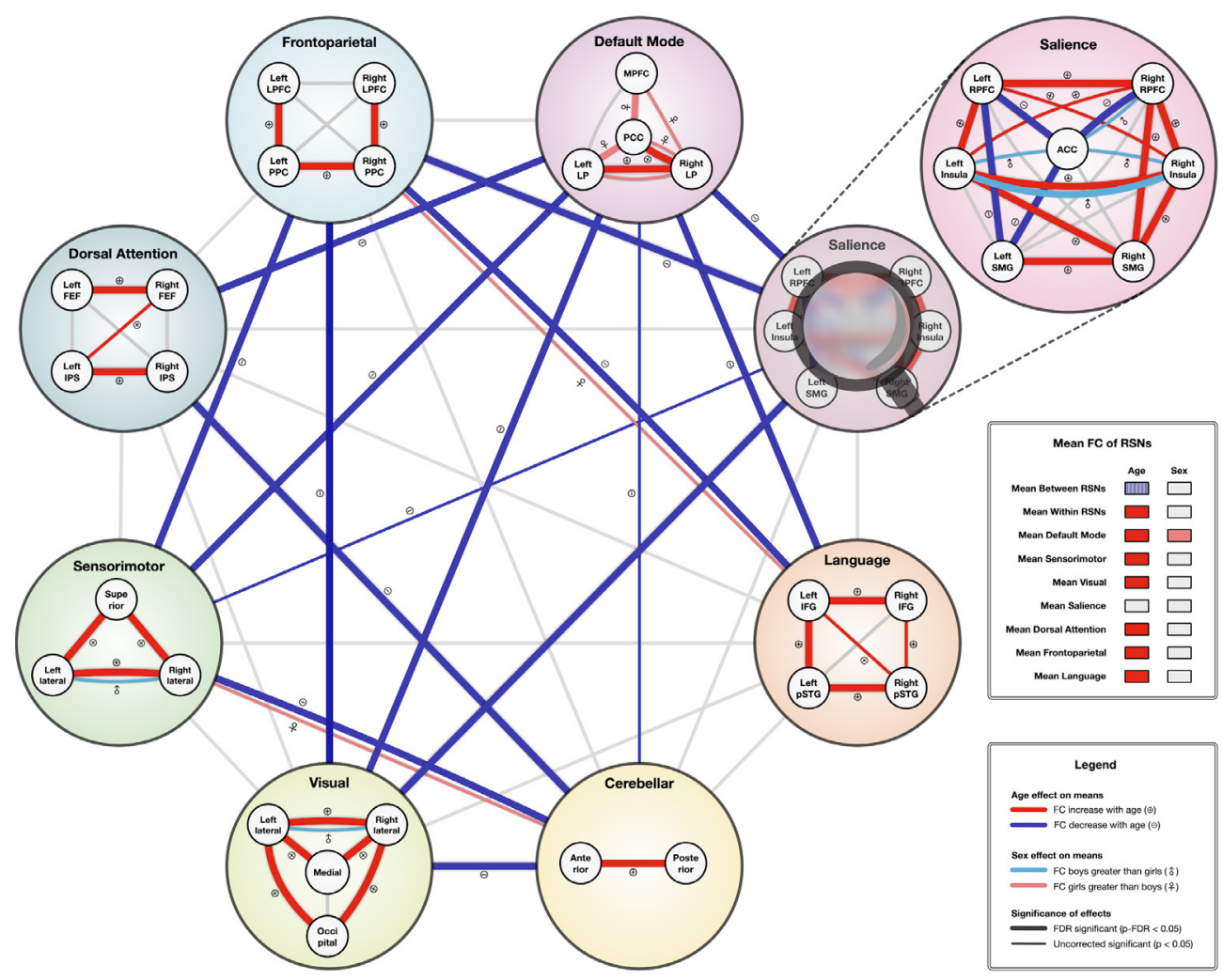

Figure 4.3. Sex and longitudinal age effects on functional connectivity between and within canonical resting-state networks. Thick lines represent effects significant after FDR correction for multiple comparison. Grey lines represent the absence of any significant effect for that particular connection. The salience network has been enlarged to accommodate the high number of connections within the network. Abbreviations (in alphabetical order): $\mathrm{ACC}=$ anterior cingulate cortex; FC = functional connectivity; FEF = frontal eye fields; IFG = inferior frontal gyrus; IPS = intraparietal sulcus; LP = lateral parietal; LPFC = lateral prefrontal cortex; MPFC = medial prefrontal cortex; $\mathrm{PCC}$ = posterior cingulate cortex; $\mathrm{PPC}=$ posterior parietal cortex; $\mathrm{pSTG}=$ posterior superior temporal gyrus; RPFC = rostral prefrontal cortex; RSN = resting-state network; SMG = supramarginal gyrus.

In addition, most contralateral connections between homotopic regions of the hemispheres are amongst the strongest to increase with age (Figure 4.3; Supplementary Table C.9). Several ipsilateral connections within the frontoparietal, salience, and language networks (e.g. the connection between lateral prefrontal cortex (LPFC) and posterior parietal cortex (PPC) show increase in functional connectivity (Figure 4.3; Supplementary Table C.9). Including mean framewise displacement as an additional covariate in the functional connectivity analysis does not alter the results. Moreover, the same pattern of age-related effects is found in a post-hoc analysis when global signal regression was included during the preprocessing stage, and when repeating the analysis with Yeo's resting-state networks atlas. 


\subsubsection{Sex and functional connectivity}

Sex effects on functional connectivity are sparse, and after multiple comparison correction found only for connections within the default mode network (DMN) and salience network (SN) (Figure 4.3; Supplementary Table C.7; Supplementary Table C.9). Increased functional connectivity for girls compared to boys is found within the default mode network (DMN) $\left(\beta_{\mathrm{sex}}=-0.0748 ; p<0.001\right.$; FDR-corrected $p=0.008$; Figure 4.3; Supplementary Table C.9), and is mostly due to the connections between the medial prefrontal cortex (MPFC) and the PCC $\left(\beta_{\text {sex }}=-0.0998 ; p<0.001\right.$; FDR-corrected $p=0.006$; Figure 4.3; Supplementary Table C.9) and between the left lateral parietal cortex (LLP) and the PCC $\left(\beta_{\text {sex }}=-0.0934 ; p=0.001\right.$; FDR-corrected $p=0.025$; Figure 4.3; Supplementary Table C.9). An opposing sex effect, where functional connectivity for boys is greater than for girls, is found for the connection between the left and right anterior insula (aIns) within the salience network (SN) $\left(\beta_{\text {sex }}=0.1018 ; p=0.001\right.$; FDR-corrected $p=0.025$; Figure 4.3; Supplementary Table C.9).

\subsubsection{IQ and functional connectivity}

None of the associations between functional connectivity and IQ test scores survived correction for multiple comparison (FDR-corrected $p \geq 0.3049$ [n.s.]; Supplementary Table C.11; Supplementary Table C.12).

\subsubsection{Genetic and environmental influences on functional connectivity}

About half of the connections between and within resting-state networks show influences of either additive genetics or common environmental influences on the reliable component of functional connectivity (i.e. the common factor), with heritability estimates ranging between $40 \%$ to $100 \%$ and common environment estimates ranging between $30 \%$ to $60 \%$ (Figure 4.4; Supplementary Table C.8; Supplementary Table C.10). In particular, connections involving the frontoparietal network, both within and between networks, show strong additive genetic influences (mean FC of connections within the frontoparietal network $h^{2}=97 \% ; p<0.001 ;$ FDR-corrected $p<0.001$ ). In addition, the visual network (mean FC within visual network $h^{2}=96 \%$; $p<0.001$; FDR-corrected $\left.p=0.002\right)$ the salience network (mean FC within salience network $h^{2}=59 \%$; $p<0.001$; FDR-corrected $p=0.008$ ), and the mean functional connectivity averaged over all within resting-state network connections $\left(h^{2}=73 \% ; p<0.001\right.$; FDR-corrected $\left.p=0.001\right)$ show strong additive genetic influences (Figure 4.4; Supplementary Table C.10). Common environmental influences are found in particular within the language (left pSTG-right IFG $c^{2}=50 \% ; p<0.001$; FDR-corrected $p=$ 0.001; and right pSTG-left IFG $c^{2}=50 \% ; p=0.013$; FDR-corrected $p=0.072$ [n.s. after FDR correction]; Figure 4.4; Supplementary Table C.10), sensorimotor (superior-left later $c^{2}$ $=31 \% ; p=0.006$; FDR-corrected $p=0.047$; Figure 4.4; Supplementary Table C.10), and 


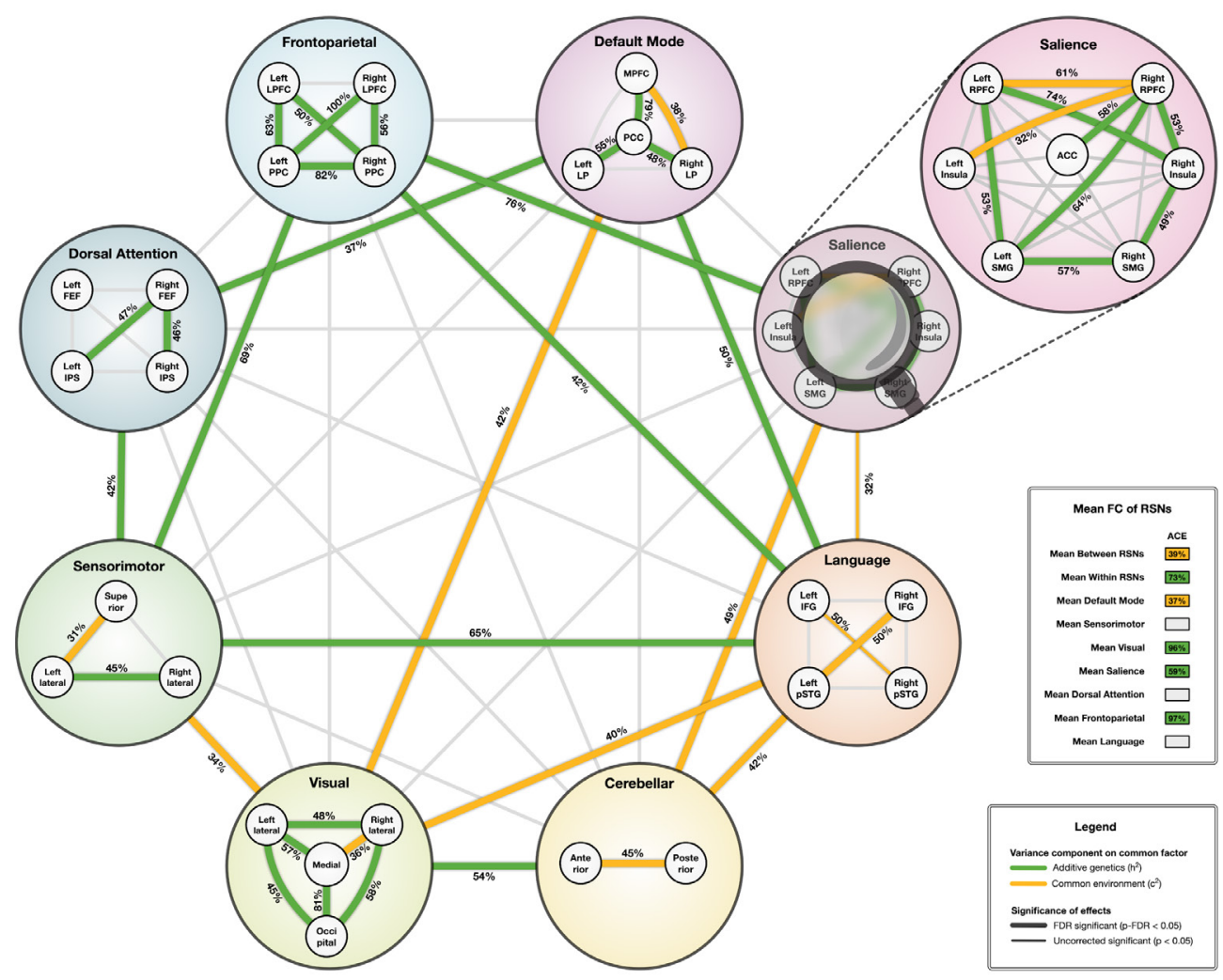

Figure 4.4. Genetic and environmental influences on the reliable component of functional connectivity between and within canonical resting-state networks. Thick lines represent effects significant after FDR correction for multiple comparison. Grey lines represent the absence of any significant effect for that particular connection. The salience network has been enlarged to accommodate the high number of connections within the network. Abbreviations (in alphabetical order): $\mathrm{ACC}=$ anterior cingulate cortex; $\mathrm{FC}=$ functional connectivity; FEF $=$ frontal eye fields; IFG = inferior frontal gyrus; IPS = intraparietal sulcus; LP = lateral parietal; LPFC = lateral prefrontal cortex; $\mathrm{MPFC}=$ medial prefrontal cortex $\mathrm{PCC}=$ posterior cingulate cortex $\mathrm{PPC}=$ posterior parietal cortex; $\mathrm{pSTG}=$ posterior superior temporal gyrus; RPFC = rostral prefrontal cortex; RSN = resting-state network; SMG = supramarginal gyrus.

cerebellar network (anterior-posterior cerebellar $c^{2}=45 \% ; p<0.001$; FDR-corrected $p=0.001$; Figure 4.4; Supplementary Table C.10), and for several between resting-state network connections (mean functional connectivity averaged over all between resting-state network connections $c^{2}=39 \% ; p=0.001$; FDR-corrected $p=0.013$; Figure 4.4; Supplementary Table C.8).

Within the core default mode network, connections involving the precuneus (PCC) show additive genetic influences (MPFC-PCC $h^{2}=79 \% ; p=0.002$; FDR-corrected $p=0.012$; and PCCLLP $h^{2}=55 \% ; p=0.011 ;$ FDR-corrected $p=0.031$; and PCC-RLP $h^{2}=48 \% ; p=0.015$; FDRcorrected $p=0.041$; Figure 4.4; Supplementary Table C.10), whereas connections between the medial prefrontal cortex (MPFC) right lateral parietal (RLP) are under common environmental 
influences (MPFC-RLP $c^{2}=38 \% ; p=0.002$; FDR-corrected $p=0.026$; Figure 4.4; Supplementary Table C.10). Mean functional connectivity averaged over all six connections within the default mode networks show significant influences of common environment $\left(c^{2}=37 \%\right.$; $p=$ 0.003; FDR-corrected $p=0.031$; Figure 4.4; Supplementary Table C.10).

By definition of the common factor model, heritability at the individual half-score measures depends on the proportion of variance explained by the common factor and the heritability of the reliable factor. Averaged standardized factor loading across the four half-scores of the individual connections ranged from $18 \%$ to $46 \%$ (mean standardized factor loadings $33 \%$ ), with higher loadings on measurements at age 18 years (mean standardized factor loadings: 41\%) than at age 13 years (mean standardized factor loadings: 24\%) (Supplementary Figure C.7; Supplementary Figure C.8). Heritability estimates at individual half-score measurements ranged from $5 \%$ to $53 \%$ and from $5 \%$ to $33 \%$ for common environment estimates (Supplementary Figure C.7; Supplementary Figure C.8; Supplementary Table C.8; Supplementary Table C.10).

We find a similar pattern of additive genetic and common environmental influences on the reliable components of functional connectivity in a post-hoc analysis when using Yeo's resting-state networks atlas. However, when applying global signal regression during the preprocessing stage, common environment estimates on the reliable component of functional connectivity, which no longer contains the global signal, is drastically reduced, and the reliable component of functional connectivity for connections previously influenced by common environment is now primarily influenced by additive genetics instead.

4.3.7 Dynamic genetic and environmental influences on functional connectivity throughout adolescence

A two-factor common pathway reliability model was a better fit to the data than a single factor for 24 of the 92 connections (26\%) (Supplementary Table C.4; Supplementary Table C.5). However, only half of these connections have statistically significant heritability or common environment estimates at both ages to warrant longitudinal investigation into dynamics of genetic and common environmental influences. For seven connections there is indication of possible dynamics in additive genetic or common environmental influences with age (Supplementary Table C.6). Two connections within the default mode network (DMN) show common environmental influences on changes in age-related functional connectivity, between the medial prefrontal cortex (MPFC) and right lateral parietal (RLP) with distinct genetic influences at each age due to innovation $\left(c^{2}(\Delta \mathrm{FC})=99 \%[61 \% ; 100 \%]\right.$; $p=0.003$; innovation $p=0.006$; Supplementary Table C.6) and between the MPFC and posterior cingulate cortex $(\mathrm{PCC})\left(c^{2}(\Delta \mathrm{FC})=98 \%[1 \% ; 100 \%] ; p=0.037\right.$; Supplementary Table C.6). The connection between medial and occipital regions within the visual network (VN) 
shows additive genetic influences on changes in functional connectivity due to increasing heritability originating from the same set of genes with age $\left(a^{2}(\Delta \mathrm{FC})=29 \%[2 \% ; 96 \%] ; p=\right.$ 0.015 ; fluctuating influences $p=0.028$; Supplementary Table C.6). The connection between occipital and left lateral regions of the visual network (VN) shows common environmental influences on change in functional connectivity with innovation in environment factors over time $\left(c^{2}(\Delta \mathrm{FC})=41 \%[17 \% ; 93 \%] ; p=0.012\right.$; innovation $p=0.019$; Supplementary Table C.6). Three connections within the frontoparietal network (FPN) show with additive genetic influences on changes in functional connectivity due to fluctuating influences from the same genes: between the left lateral prefrontal cortex (LPFC) and left posterior parietal cortex $(\mathrm{PPC})\left(a^{2}(\Delta \mathrm{FC})=58 \%[17 \% ; 91 \%] ; p=0.002\right.$; fluctuating influences $p=0.003$; Supplementary Table C.6), between the left LPFC and right PCC $\left(a^{2}(\Delta \mathrm{FC})=22 \%[5 \% ; 51 \%]\right.$; $\mathrm{p}=0.003$; fluctuating influences $p=0.006$; Supplementary Table C.6), and between the left PCC and right PCC $\left(a^{2}(\Delta \mathrm{FC})=48 \%\right.$ [4\%; 100\%]; $p=0.015$; fluctuating influences $p=0.029$; Supplementary Table C.6). The remaining connections with genetic or common environmental influences at both ages do not reveal any significant dynamics in heritability or common environment (Supplementary Table C.6). However, these results should be interpreted with caution due to limited power to detect significant genetic or environmental estimates at age 13 years, in part due to the reduced sample size at age 13 years.

\subsection{DISCUSSION}

With this longitudinal resting-state fMRI study, we measured the heritability of functional connectivity throughout adolescent development for the first time. Approximately half of the functional connections within and between canonical cortical resting-state networks are influenced by either additive genetic ( $h^{2}$ up to 53\%) or common environmental influences ( $c^{2}$ up to $33 \%$ ) during adolescence. During adolescence, functional connectivity between resting-state networks decreases with age, whereas functional connectivity within cortical resting-state networks increases with age, except for several connections within the salience network that decrease with age. There is limited evidence for dynamics in genetic or common environmental influences, suggesting mostly stable influences across adolescence. Girls had significantly stronger functional connectivity than boys within the default mode network between the precuneus and medial prefrontal cortex and between the precuneus and left lateral parietal cortex. Boys had significantly stronger functional connectivity than girls within the salience network for the connection between the bilateral insula. Associations between functional connectivity and intelligence did not survive multiple comparison correction. Head motion is heritable across the ages and shows a small but statistically significant decline with age (Supplementary Table C.3; Supplementary 
Figure C.5). The aCompCor method used by CONN is effective at removing head motion effect cross-sectionally, however, longitudinal changes in functional connectivity estimates between the canonical resting-state networks remain associated with the longitudinal changes in degree of head motion of individuals (Supplementary Table C.13; Supplementary Table C.14). The results remained consistent after including mean framewise displacement as an additional covariate in the functional connectivity analysis and after including global signal regression during the preprocessing stage.

We find significant heritability on functional connectivity in adolescence, $h^{2}$ ranging from $6 \%$ to $53 \%$ for 23 out of $55(42 \%)$ connections within resting-state networks, and common environment estimates $c^{2}$ ranging from $5 \%$ to $33 \%$ for 8 out of 55 (15\%) connections. Previous studies found heritability estimates ranging from $10 \%$ to $40 \%$, and occasionally up to $60 \%$ or $80 \%$ (Adhikari et al., 2018b; Colclough et al., 2017; Fu et al., 2015; Ge et al., 2017; Glahn et al., 2010; Korgaonkar et al., 2014; Meda et al., 2014; Sudre et al., 2017; Yang et al., 2016; Table 4.2), thus overall these findings are within the same range across the ages. The notable exception is the default mode network. In our cohort the default mode network is partially influenced by common environmental instead of additive genetics (mean FC within DMN $c^{2}=37 \%$ ). Previous studies have established the default mode network being influenced by additive genetics (Adhikari et al., 2018a; Fu et al., 2015; Ge et al., 2017; Glahn et al., 2010; Korgaonkar et al., 2014; Meda et al., 2014; Sudre et al., 2017; Xu et al., 2016; Yang et al., 2016). The discrepancy with previous studies may be due to increased sensitivity in finding common environmental effects in the current study because of three reasons. One, the extended twin design (i.e. including twin pairs and one of their singleton siblings) used in this study provides additional power to detect significant common environment estimates (Posthuma and Boomsma, 2000) allowing detection of statistically significant common environmental estimate at individual measures as low as $5 \%$ when separating measurement error from the common factor. However, most likely functional connections in the brain are influenced by both additive genetics and common environment. Two, since previous studies were conducted mostly in adults, it may be possible that heritability estimates on functional connectivity increase with age, as is the case with heritability of cognitive performance (Briley and Tucker-Drob, 2013). Indeed, the cohorts closest to our age range find similarly low estimates for heritability of functional connectivity within the default mode network (Fu et al., 2015; Yang et al., 2016). Finally, atlas choice and preprocessing strategies, including global mean signal regression, all varied between these studies, which could have introduced differences in heritability estimates. Although we were able to replicate our results using Yeo's resting-state atlas, including global signal regression during the preprocessing stage substantially decreased the common environment estimates in favor of additive genetics for some connections. 
Few other studies have investigated heritability of functional connectivity with restingstate networks beyond the default mode network (Adhikari et al., 2018; Ge et al., 2017; Sudre et al., 2017; Yang et al., 2016). Our heritability estimates for functional connectivity within the frontoparietal network of $h^{2} \cong 14 \%$ at around age 13 years and $h^{2} \cong 40 \%$ at around age 18 years are slightly lower than the estimates of $h^{2}=32 \%$ to $58 \%$ found across lifespan in families with ADHD family members (Sudre et al., 2017), and substantially less than the estimate of $h^{2}=65 \%$ reported in a sample of healthy young adults (Yang et al., 2016), but more in line with results from the Genetics of Brain Structure (GOBS) and the Human Connectome Project (HCP) studies (Adhikari et al., 2018a). The sensorimotor network in our cohort is influenced by a mixture of additive genetics $h^{2}=18 \%$ to $20 \%$ and common environment $c^{2}=5 \%$ to $16 \%$, with influences of common environment previously reported in young adults (Yang et al., 2016), and low to no heritability in the GOBS and HCP cohort, although they did not test for common environmental influences (Adhikari et al., 2018a). This is in stark contrast to the study performed on the Brain Genomics Superstruct Project (GSP) cohort and alternative analysis of the HCP cohort where they found high heritability estimates of $h^{2}=60 \%$ to $70 \%$ (Ge et al., 2017).

We find significant heritability of functional connectivity between resting-state networks in adolescence, with 8 out of $28(29 \%)$ connections influenced by additive genetics with $h^{2}$ $=5 \%$ to $50 \%$, and 6 out of $28(21 \%)$ connections influenced by common environment with $c^{2}=6 \%$ to $25 \%$. In particular, connections between the frontoparietal, dorsal attention, and salience networks, all involved in higher order cognitive control, were influenced primarily by additive genetics. Common environment played a considerable role for most sensory networks, including the language network and cerebellum. So far, the only other study that investigated heritability of functional connectivity between resting-state networks was performed in young adults aged 18 to 29 years (Yang et al., 2016), where 8 out of $21(38 \%)$ connections between resting-state networks were influenced by additive genetics with $h^{2}$ $=26 \%$ to $42 \%$, and 11 out of $21(52 \%)$ were influenced by common environment with $c^{2}=$ $18 \%$ to $47 \%$, showing overall similar connectivity profiles. Interestingly, synchronous resting-state activity in the brain has been associated with gene expression levels, where distal functionally connected regions show similar gene expression profiles (Hawrylycz et al., 2015), and the strength in functional connectivity was influenced by polymorphisms of a set of genes enriched for ion channels in healthy adolescents (Richiardi et al., 2015). Here, our study adds that functional connectivity within and between cortical resting-state networks is strongly influenced by genes and common environment throughout adolescent development. Including global signal regression during the preprocessing stage resulted in decreased estimates for common environment and increased heritability estimates on the reliable component of functional connectivity for some of the connections. This insight will 
have potential consequences for genetic studies that aim to find genetic variants implicated in functional connectivity.

Varying estimates of heritability or common environment between half-score measures of different sessions (i.e. between the two ages) may be an indication of dynamics in genes or common environment that was tested with a two-factor common pathway model; e.g. the change in heritability estimates for functional connectivity within the frontoparietal network increased significantly from $h^{2} \cong 14 \%$ at age 13 years to $h^{2} \cong 40 \%$ at age 18 years. However, the lower heritability estimates at age 13 years are likely due to the reduced sample size as a result of motion scrubbing and exclusion due to presence of dental braces incompatible with high magnetic fields or increased residual noise rather than represent "true" changes in additive genetic or common environmental variances. This effect is reflected in the two-factor common pathway model as "increasing" influences of the same additive genetic or common environmental factor over time, and is consequently found in the single factor common pathway model as varying estimates of heritability due to differences in factor loadings on the individual half-score measures. Therefore, the results from the two-factor model on dynamics of genetic and environmental influences are suggestive at best and generally demonstrate stable additive genetic or common environmental influences from a single source (Supplementary Table C.6). Varying estimates for half-score of a single session (i.e. within the same age) are very unlikely to represent short-term fluctuating genetic or environmental influences, but can most likely be attributed to fluctuating noise (e.g. slight increase in head motion or restlessness during second half of scan).

The longitudinal age effects that we found are subtle but wide-spread throughout the brain despite most resting-state networks already appearing "adult-like" by age 2 years (Gao et al., 2015; Gilmore et al., 2018). We found age-related decreases in functional connectivity for about half of the connections between cortical resting-state networks, which likely reflect segregation between functionally distinct modules of the brain. A previous longitudinal study during early adolescence reported segregation between the frontoparietal (FPN) and default mode network (DMN) (Sherman et al., 2014). Although our results are not statistically significant, it suggests a possible decrease between the FPN and $\operatorname{DMN}\left(\beta_{\text {age }}=-0.0076 ; p=0.061\right.$ [n.s.]; FDR-corrected $p=0.089$ [n.s.]) that is consistent with prior reports. Previously, a decrease in functional connectivity between the dorsolateral prefrontal cortex and posterior cerebellum, but not anterior cerebellum, was reported (Bernard et al., 2016). We do not find age-related changes in functional connectivity between the frontoparietal and cerebellar networks. However, we do not distinguish between subregions of the network for between-network connectivity. Nearly two-thirds 
of the connections within resting-state networks show age-related increase in functional connectivity that likely reflect integration within functional modules of the brain. Previously longitudinal studies have reported on integration within the default mode (DMN), frontoparietal (FPN), and language network (LN) during childhood and adolescence (Long et al., 2017; Sherman et al., 2014; Wendelken et al., 2017; 2016; Xiao et al., 2016). However, age-related changes in functional connectivity within the DMN, FPN, and SN are not always consistently found during early adolescence (Sylvester et al., 2018). Similar to previous reports, we find integration within the frontoparietal network (FPN) for the ipsilateral connections between the frontal and posterior regions of the FPN (Wendelken et al., 2017; 2016), and integration within the language network between the inferior frontal gyrus (IFG) and posterior superior temporal gyrus (pSTG) (Xiao et al., 2016). Better integration between frontal and parietal regions has been proposed to support better cognitive performance in the Parieto-Frontal Integration Theory (P-FIT; Deary et al., 2010; Jung and Haier, 2007). A notable exception to integration within resting-state networks is the age-related decrease in functional connectivity within the salience network for connections involving the anterior cingulate cortex (ACC), left rostral prefrontal cortex (RPFC) and left supramarginal gyrus (SMG). The only other longitudinal study that investigated the salience network in children and adolescents was between 8 and 13 years, preceding our age range, reporting absence of significant age-related effects (Sylvester et al., 2018). The anterior cingulate cortex plays an important role in motor control and cognition, in particular reward-based decision making and response inhibition (Bush et al., 2002; Stevens et al., 2011). A decreasing connectivity between the anterior cingulate cortex and insula could possibly reflect a decoupling between the integration of external sensory information and internal emotional and bodily state signals (Uddin et al., 2017) or indicate segregation of bottom-up stimuli processing and top-down cognitive control processing in the salience network that may coincide with improved self-control during adolescence (Casey, 2013). The decreasing connectivity strength between networks and increasing connectivity strength within networks that we find in this longitudinal study is the opposite pattern of what is typically reported in cross-sectional literature, where there is a shift from a local oriented (i.e. stronger functional connectivity between proximal regions) to a more distributed organization (i.e. stronger functional connectivity between distal regions) during childhood and adolescence (Cao et al., 2016; Ernst et al., 2015; Grayson and Fair, 2017; Stevens, 2016). This discrepancy could be due to residual head motion effects on changes in functional connectivity with age that are not always properly accounted for in studies predating 2012 (Power et al., 2012), although despite our stringent control for head motion there are still residual effects present. Secondly, cross-sectional studies do not always show consensus on the direction of change and affected regions (Stevens, 2016), 
which may be due to the cohort effect. Few longitudinal studies have been conducted to date (see Table 4.1), with even fewer conducting brain-wide analysis. Several longitudinal studies show increasing functional connectivity within functional networks or decreasing functional connectivity between functional networks with age consistent with our results (Bernard et al., 2016; Long et al., 2017; Sherman et al., 2014; Wendelken et al., 2017; 2016).

We find significant sex effects within the default mode (girls showing stronger functional connectivity) and salience network (boys showing stronger functional connectivity). Sex effects in functional connectivity analyses are typically discarded as covariate of no interest, despite extensive support for sex effects in behavior (Gur et al., 2012; Gur and Gur, 2016), brain gray matter (Giedd et al., 2012; Ruigrok et al., 2014) and white matter structure (Herting et al., 2012; Ingalhalikar et al., 2014), and function (Sacher et al., 2013; Stevens and Hamann, 2012). A few studies have reported sex effects for functional connectivity within the default mode network (stronger functional connectivity in females compared to males) and salience network (stronger functional connectivity in males compared to females) in adults (Biswal et al., 2010) and across the lifespan (Zuo et al., 2010). These previous reports are consistent with our findings, and corroborate that sex differences in brain functioning are already present during adolescence (Gur and Gur, 2016), although sex effects are not always found in these networks during development (Solé-Padullés et al., 2016; Sylvester et al., 2018). The default mode network plays an important role in auto-biography memory and emotion regulation (Mak et al., 2017; Raichle, 2015; Zhou et al., 2018). The increased functional connectivity within the default mode network for girls may therefore explain their better performance at memory and emotive tasks (Gur et al., 2012; Gur and Gur, 2016). In contrast, the salience network plays an important role in overt attention/stimuli processing, integration of multimodal sensory information, and switching between passive and active tasks (Marek et al., 2015; Seeley et al., 2007; Uddin, 2014; Zhou et al., 2018). The increased functional connectivity within the salience network for boys may therefore explain their better performance at visuospatial and motor tasks (Gur et al., 2012; Gur and Gur, 2016).

We do not find any association between functional connectivity and IQ scores that remain significant after multiple comparison correction. One of the leading theories on cognitive development postulates that better integration between frontal and parietal areas supports better cognitive performance (Deary et al., 2010; Jung and Haier, 2007). Previous studies on resting-state functional connectivity have provided support for this theory in both children and adults (Dubois et al., 2018; Langeslag et al., 2013; Santarnecchi et al., 2017; Song et al., 2008; Vakhtin et al., 2014). However, despite better integration in the frontoparietal network (FPN) with age, we found no association with IQ test scores that survived multiple comparison correction (Supplementary Table C.11; Supplementary Table C.12). 
Head motion is a major point of concern in resting-state fMRI measurements when studying functional connectivity during childhood and adolescent development (Power et al. 2012; Van Dijk et al. 2012; Satterthwaite, Wolf, et al. 2013). Heritability of head motion during childhood, adolescence, and young adulthood was previously established (Achterberg et al., 2018; Couvy-Duchesne et al., 2014; 2016; Engelhardt et al., 2017; Zhou et al., 2016), and is consistent with our findings during adolescence $\left(h^{2}=86 \%\right.$ at age 13 years; $p<0.0001$; and $h^{2}=43 \%$ at age 18 years; $p=0.0003$ ). Preprocessing the resting-state data with linear regression of white matter and cerebrospinal fluid signal components and derivatives of re-alignment parameters using CONN toolbox appears to be mostly effective to control for head motion at individual ages (i.e. cross-sectionally; Supplementary Table C.13; Supplementary Table C.14). However, in a longitudinal setting, change in the degree of head motion was still associated with changes in functional connectivity for more than half of the generally long-distance connections between resting-state networks, all with positive association (i.e. reduction in head motion results in smaller change in functional connectivity; Supplementary Table C.13; Supplementary Table C.14), with only the association between head motion and the connection between the sensorimotor and salience network surviving multiple comparison correction. Including global signal regression during the preprocessing stage - despite its controversy to introduce artificial negative correlations considered to be most effective at reducing head motion effects - did not substantially change the results in addition to the aCompCor method, as previously found (Ciric et al., 2017). This suggests that despite stringent control of head motion during preprocessing of the resting-state fMRI scans, not all variance due to head motion is accounted for, possible due to complex non-linear interaction of head motion with the BOLD signal (Satterthwaite et al., 2013). However, most connections within resting-state networks, including long-distance connections between frontal and parietal regions, and cross-hemisphere connections, showed few associations with head motion. Moreover, agerelated changes in functional connectivity were unaffected whether or not head motion was included as covariate. All scans, including longitudinal scans, were processed independently due to lack of publicly available tools that support longitudinal pipelines such as used for structural imaging (Reuter et al., 2012), although longitudinal methods for preprocessing of resting-state fMRI are becoming available (Hart et al., 2018).

A number of other limitations should be taken into consideration when interpreting these results. First, resting-state fMRI scans were acquired at 1.5T MRI scanner, using an at the time state-of-the-art fast repetition time (TR) T2-weighted PRESTO-SENSE acquisition protocol. The field strength was intentionally not upgraded to higher field strengths to minimize effects of scanner differences for longitudinal data acquisition. Although the fast TR aims to minimize head motion between acquired volumes, the $3 \mathrm{D}$ acquisition makes it 
more sensitive to abrupt motion resulting in ghosting and blurring of the BOLD signal that may require a different approach to denoising than traditional 2D single-shot EPI acquisition protocol (van Gelderen et al., 2012). In addition, effort was taken to reduce inscanner head motion using mock-scanner session before actual acquisition to acclimate the children during previous visitation at age 9 years when the children also experienced an MRI scan but no resting-state fMRI scan was acquired (Durston et al., 2009; van Soelen et al., 2012a). Secondly, although sample size is large for neuroimaging standard (i.e. $\mathrm{N}=108$ at age 13 years and $\mathrm{N}=207$ at age 18 years), it is modest in size for twin studies. In particular, the reduced sample size at age 13 years due to exclusion of high motion subjects has limited the number of subjects with longitudinal scans and will have an impact on the power of this study to detect age-related effects. Combining the data across the ages using common factor analysis with a single factor had the benefit to mitigate this reduction in power for detecting statistically significant heritability or common environment estimates under the assumption that these estimates remain stable across ages, which is the case for most connections. Thirdly, resting-state fMRI is inherently noisy with no optimal strategy to remove non-neuronal signal (e.g. due to head motion or vascular system) (Ciric et al., 2017; Parkes et al., 2018). Alternative approaches or more advanced methods, such as improving temporal signal-to-noise ratio using machine learning (Adhikari et al., 2018a) or the use of a longitudinal preprocessing pipeline (Hart et al., 2018) may prove beneficial for future studies. Finally, choice of atlas may influence the results as both the CONN and Yeo atlas consist of predefined regions that do not allow for individual variation in localization of brain function and the moderate size of the regions prevent analysis of possible localized effects with behavioral measures such as cognitive performance on IQ tests. In addition, both atlases do not include subcortical structures for which both longitudinal development and heritability has been reported. Unfortunately, we could not use more fine-grained atlases due to computational complexity of twin modelling and the limited spatial resolution of our scans. However, the comparable results for both the CONN toolbox atlas and Yeo's resting-state network atlas suggests at least reasonable robustness of findings independent of atlas choice.

In conclusion, there are wide-spread influences of additive genetics and common environment on the functioning of cortical resting-state networks during adolescent development that generally remain stable over time. Wide-spread subtle age-related changes in functional connectivity occur in the presence of sizable individual variation, with presence of sex effects to be taken into consideration in developmental studies on functional connectivity. 



\section{CHAPTER 5}

\section{PROGRESSIVE AGING IN SCHIZOPHRENIA}

Original title: Accelerated aging in the brain, epigenetic aging in blood, and polygenic risk for schizophrenia

Jalmar Teeuw [1], Anil P.S. Ori [2], Rachel M. Brouwer [1], Sonja M.C. de Zwarte [1], Hugo G. Schnack [1], Hilleke E. Hulshoff Pol [1], Roel A. Ophoff [2,3]

[1] Department of Psychiatry, UMC Brain Center, University Medical Center Utrecht, Utrecht, The Netherlands; [2] Center for Neurobehavioral Genetics, University of California, Los Angeles, United States of America; [3] Department of Psychiatry, Erasmus University Medical Center, Rotterdam, The Netherlands In review at the journal Schizophrenia Research. 


\section{ABSTRACT}

Schizophrenia patients show signs of progressive aging in cognitive and physiological domains, and the condition is associated with increased mortality. Accelerated aging, as measured by MRI brain images and epigenetic clocks, has been reported, but little is known about the interplay between these clocks in schizophrenia patients.

In schizophrenia patients and healthy subjects, accelerated aging was assessed in brain tissue using a longitudinal MRI ( $\mathrm{N}=715$ scans; mean scan interval 3.4 year) and in blood using two epigenetic age predictors $(\mathrm{N}=172)$. Differences ('gaps') between estimated, i.e., biological, ages and chronological ages were calculated, as well as the acceleration rate of brain age. Bivariate associations between these aging measures as well as with polygenic risk scores for schizophrenia (PRS; $\mathrm{N}=394$ ) were investigated.

Brain aging measures and epigenetic age predictors were not significantly associated. PRS was significantly associated with brain age gap, brain age acceleration rate, and, negatively, with Horvath's epigenetic age predictor, but not with Levine's epigenetic age predictor. After controlling for disease status and multiple comparisons correction, these effects were no longer significant. Our results could potentially imply that the (accelerated) aging observed in the different tissues reflect distinct biological processes.

Keywords: schizophrenia; DNA methylation; epigenetics; genetics; polygenic risk scores (PRS); structural magnetic resonance imaging (MRI); progressive aging; mediation analysis 


\subsection{INTRODUCTION}

Schizophrenia is a debilitating psychiatric disorder where the patients' expected lifespan is decreased on average by 15 to 20 years compared to the general population (Laursen et al., 2014; Hjorthøj et al., 2017). This shortened lifespan may be explained in terms of accelerated aging of the body (Kirkpatrick et al., 2008). In two separate studies we have previously reported on accelerated aging of the brain (Schnack et al., 2016) and epigenetic aging in blood (Ori et al., 2019) for schizophrenia patients. However, whether or not these different biological aging markers act in concert is not yet well understood.

Despite our current understanding of neuropsychiatric disorders, the etiology of schizophrenia remains largely unknown. There are several indications of aberrant brain development as early as the fetal period (Debnath et al., 2015; Kim et al., 2015; Faa et al., 2016), with progressive changes of the brain even after the onset of psychosis (van Haren et al., 2008; Hulshoff Pol and Kahn, 2008), which is characteristic for a progressive aging disorder (Olabi et al., 2011). Accelerated biological aging occurs when the rate of biological aging is increased as compared to chronological aging, and may in part explain the increase in early mortality rate within the patient population (Kirkpatrick et al., 2008; Shivakumar et al., 2014; Nguyen et al., 2017; Laursen, et al., 2014). Quantitative assessment of biological aging can be performed using advanced statistical techniques such as machine learning algorithms. These algorithms are trained to discover aging-related patterns in the properties of tissue from a subject or donor (Bzdok, 2017; Cole and Franke, 2017; Jylhävä et al., 2017). Using neuroimaging, the biological age of the brain can be predicted from gray matter distributions (Cole and Franke, 2017; Cole et al., 2017; Valizadeh et al., 2017), white matter properties (Mwangi et al, 2013), or brain-activity related properties (Dosenbach et al, 2010). For patients with schizophrenia, accelerated aging of the brain occurs around the onset of psychosis (Koutsouleris et al., 2014; Schnack et al., 2016; Nenadić et al., 2017; Kaufmann et al., 2019; Jonsson et al., 2019; Kolenic et al., 2018; Chung et al., 2018; Hajek et al., 2019; Shahab et al., 2019) before stabilizing several years after onset (Schnack et al., 2016). Accelerated brain age predicts all-cause mortality (Cole et al., 2018), is highly heritable and has a genetic overlap with common brain disorders, including schizophrenia (Cole et al., 2017; Kaufmann et al., 2019). For biological tissue samples, several molecular and phenotypic biomarkers of aging have been reported for research into proteomic, transcriptomics, metabolomics, telomere length, and DNA methylation levels (Jylhävä et al., 2017; Nguyen et al., 2017). The age of a tissue donor can be reliably estimated based on epigenetic methylation of DNA (Horvath et al., 2012; Horvath, 2013; Hannum et al., 2013; Levine et al., 2018). While no significant accelerated epigenetic aging has previously been observed schizophrenia, neither in postmortem brain nor blood tissue (Viana et al., 2017; Voisey et al., 2017; McKinney et al., 2017; 
McKinney et al., 2018), a recent large-scale DNA methylation study now robustly demonstrates that epigenetic age is accelerated in whole blood using an DNAm age predictor that strongly associates with mortality risk (Ori et al., 2019). The study furthermore reports that a subset of cases who carry high schizophrenia polygenic risk present the fastest age acceleration. This raises two intriguing questions; do cases who carry high schizophrenia polygenic risk also present faster brain age acceleration? And is aging in the brain associated with epigenetic aging in blood? Previously, no significant association between brain aging and epigenetic aging was reported in a population study of typical aging elderly subjects (Cole et al., 2018). However, little is known about the interplay of genetics, epigenetics, and brain morphology with regard to accelerated aging in schizophrenia patients.

\subsubsection{Current study}

Here we investigated the associations between brain aging, epigenetic aging, and polygenic risk for schizophrenia within a dataset of schizophrenia patients and healthy control subjects. MRI-derived brain ages were estimated from structural MRI scans using Schnack's brain age predictor (Schnack et al., 2016), and epigenetic ages were estimated from wholeblood array-based DNA samples profiles using Horvath's (Horvath et al., 2012) and Levine's (Levine et al., 2018) epigenetic age predictors. Genotype-based polygenic risk for schizophrenia was estimated using the schizophrenia GWAS summary statistics of the Psychiatric Genome Consortium (Ripke et al., 2014).

\subsection{MATERIALS AND METHODS}

\subsubsection{Cohort and sample description}

Subjects included in this study were part of two longitudinal schizophrenia cohorts (van Haren et al., 2007; Boos et al., 2012). Brain age in these cohorts has been described before (Schnack et al., 2016) and these cohorts were part of a study on epigenetic aging in schizophrenia (Ori et al., 2019). Here we included unrelated subjects that had imaging data and either epigenetic or genetic data available (Table 5.1; Figure 5.1), resulting in a dataset of 411 unrelated subjects (193 cases, 218 controls, 36\% female) of European descent spanning a wide range of the adult lifespan (mean $=32.7$ years, range $=[16.7$ to 67.5$]$ at baseline). For the majority of subjects (57\%) longitudinal imaging data was available (up to five scans), with a mean scanning interval of 3.4 years (range [0.9 to 7.0]). All patients met DSM-IV criteria for a nonaffective psychotic disorder (including schizophrenia, schizophreniform disorder or schizoaffective disorder). Written informed consent was obtained from all subjects, and both studies were approved by the Medical Ethics Committee for Research in Humans (METC) of the University Medical Center Utrecht. 
Table 5.1. Demographics table for individuals with data from the three modalities.

\begin{tabular}{|c|c|c|c|c|}
\hline Measure & Population & MRI brain age ${ }^{a}$ & Epigenetic ages & PRS schizophrenia \\
\hline Subjects & Total & $411[715]$ & 172 & 394 \\
\hline \multirow[t]{2}{*}{ (count) } & Controls & $218[345]$ & 63 & 212 \\
\hline & Patients & $193[370]$ & 109 & 182 \\
\hline Age at baseline & Total & $32.72 \pm 11.41$ & $32.31 \pm 13.01$ & $35.46 \pm 12.47$ \\
\hline$($ mean $\pm \mathrm{SD})$ & Controls & $34.92 \pm 12.10$ & $32.62 \pm 15.17$ & $37.55 \pm 13.17$ \\
\hline (year) & Patients & $30.24 \pm 10.05$ & $32.13 \pm 11.65$ & $33.02 \pm 11.16$ \\
\hline Sex & Total & $149: 262$ & $57: 115$ & $142: 252$ \\
\hline \multirow[t]{2}{*}{ (female:male) } & Controls & $115: 103$ & $37: 26$ & $110: 102$ \\
\hline & Patients & $34: 159$ & $20: 89$ & $32: 150$ \\
\hline
\end{tabular}

Abbreviations (in alphabetical order): $\mathrm{MRI}=$ magnetic resonance imaging; PRS = polygenic risk scores; $\mathrm{SD}=$

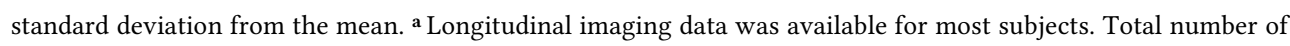
scans between brackets.

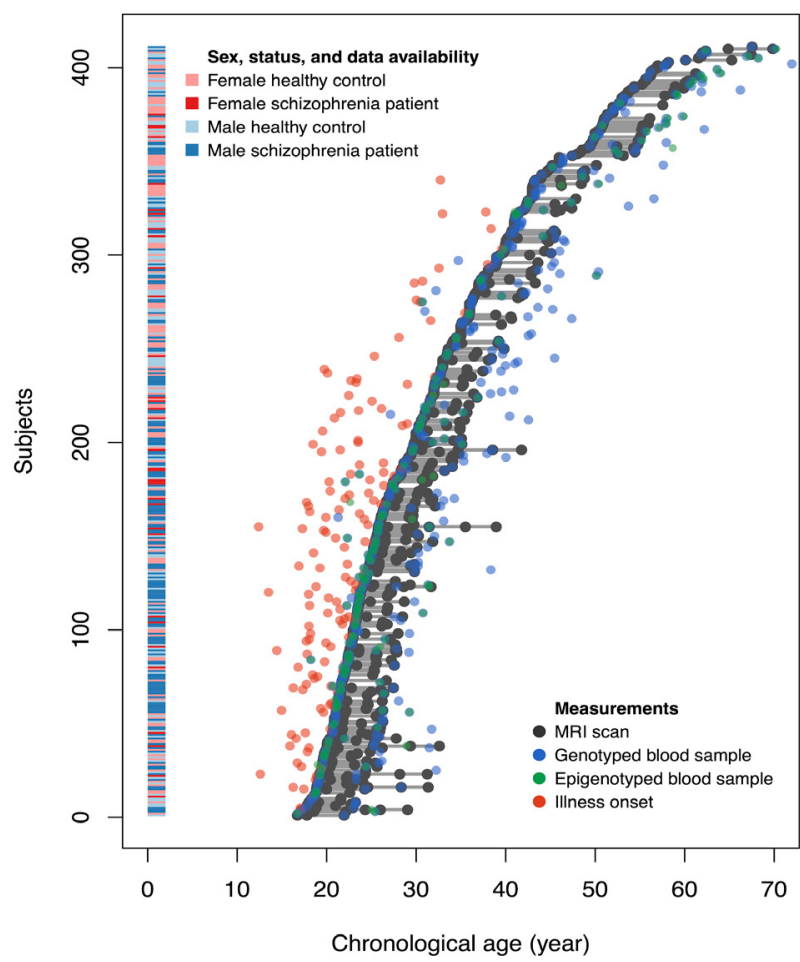

Figure 5.1. Overview of all subjects with their age at illness onset (not always available for older schizophrenia patients), and age at MRI scans and blood sample acquisition for DNA and epigenetics. 


\subsubsection{MRI brain age}

Structural magnetic resonance imaging (MRI) scans were acquired on a 1.5 tesla Philips scanner with a voxel resolution of $1 \times 1 \times 1.2 \mathrm{~mm}^{3}$. Images were processed using a validated in-house image-processing pipeline to produce gray matter density maps in standardized space and used to predict individuals' brain age. In brief, the predictor uses a model that predicts chronological age based on the weighted sum of whole brain voxel-wise gray matter densities. The model was trained on a sample of healthy control subjects and applied on schizophrenia patients. See (Schnack et al., 2016) for details.

\subsubsection{Blood-based epigenetic aging markers}

DNA methylation data was obtained from whole-blood DNA samples using the Illumina Infinium Human Methylation Beadchip technology according to manufacturer's guidelines. A total of 172 samples were assayed with either the $27 \mathrm{~K}(\mathrm{~N}=108$ samples $)$ or $450 \mathrm{~K}(\mathrm{~N}=64$ samples) platform, which interrogate 27,578 and 485,512 CpG sites across the genome, respectively. These data are a subset of previously published DNAm cohorts (Gene Expression Omnibus (GEO) ID: GSE41037 and GSE41169) for which brain age estimates from MRI scans were available. Blood-based DNAm age was estimated using two different predictors (Horvath, 2013; Levine et al., 2018). These two DNAm age predictors were designed for use with both the $27 \mathrm{~K}$ and $450 \mathrm{~K}$ platform allowing us to maximize our sample size. See Supplementary Methods for details.

\section{POLYGENIC RISK FOR SCHIZOPHRENIA}

Whole-blood DNA samples were processed on Illumina's HumanOmniExpressExome-8 v1.2 and Illumina's 550K platform. After quality control (see Supplementary Methods for details), SNPs were imputed on the Michigan server (Das et al., 2016) using the HRC r1.1 2016 reference panel with European samples after phasing with Eagle v2.3. Polygenic risk for schizophrenia was calculated from the SNP data using the schizophrenia GWAS summary statistics of the Psychiatric Genome Consortium excluding Dutch subjects (Ripke et al., 2014; see Supplementary Methods for details). Polygenic scores were calculated using PLINK's score function at ten GWAS p-value thresholds of significance of association: $\mathrm{p}<$ $5 \times 10^{-8}, 10^{-6}, 10^{-4}, 10^{-3}, 0.01,0.05,0.10,0.20,0.5$, and 1.0 .

Polygenic risk scores were then harmonized to reduce the variation due to acquisition on different platforms through principal component analysis on the full sample. The first principal component contained the majority of the differential disease risk. This component was standardized and used for subsequent analyses (Bergen et al., 2019). See Supplementary Methods for more information. 


\subsubsection{Data preparation}

We used a linear mixed-effects regression models to correct the MRI brain age and epigenetic age estimates for regression towards the mean (Le et al., 2018) and differences in acquisition platform within the non-psychiatric controls (Supplementary Table D.1; Supplementary Figure D.1). Age gaps were defined as the difference between the corrected age estimates and the chronological age, and brain age acceleration as the annual rate of change in corrected brain age estimates between consecutive scans. The age gaps and age acceleration were subsequently corrected for possible differences between the sexes by regression of the effect of sex within the healthy control population. The effects of sex were removed from all measures by linear regression regardless of statistical significance and prior to further statistical analyses (Supplementary Table D.2; Supplementary Figure D.2).

\subsubsection{Statistical analysis}

First, linear mixed-effects regression models were applied with each of the measures as the dependent variable and diagnosis status as independent fixed effect covariate to test for differences between the healthy controls and schizophrenia patients. The models included random intercepts to account for the repeated measures of the longitudinal MRI scans.

Secondly, the associations between brain aging (age gap and age acceleration), epigenetic aging (age gap from Horvath's and Levine's predictors), and polygenic risk for schizophrenia were determined using Spearman's correlation for all ten pairwise combinations. Since the MRI scans and the blood samples may have been acquired at different visitations, partial correlations were computed for the associations between brain age and epigenetic age measures to account for the difference in age of the participant at which the samples were acquired. No correction was applied for associations involving the polygenic risk for schizophrenia as this measure is time-invariant. To assess whether potential associations were driven by mean differences between patients and controls for both traits, we repeated these analyses correcting for disease status.

For most subjects more than one MRI scan was available. In the bivariate analyses, the brain age gap from the last MRI scan and the longitudinal brain age acceleration of the first two MRI scans were used. This choice was made based on previous results that show brain age acceleration for schizophrenia patients is maximal around the time of onset (i.e. typically around the acquisition date of the earliest MRI scan) and that its cumulative effect results in a maximal brain age gap for schizophrenia patients 5 years later before stabilizing (Schnack et al., 2016).

A Bonferroni correction was used to account for multiple testing. The corrected significance threshold was set at $p=0.05 / 5=0.01$ for group differences and $p=0.05 / 10=0.005$ for the tests of pairwise associations. 


\subsection{RESULTS}

Linear mixed-effects models with random intercepts revealed statistically significant effects of status for the MRI brain age gap, longitudinal MRI brain age acceleration, Levine's epigenetic age gap, and polygenic risk scores for schizophrenia indicating accelerated age or increased risk for schizophrenia patients, but not for Horvath's epigenetic age gap (Table 5.2; Figure 5.2).

Table 5.2. Group-differences between schizophrenia patients and non-patient controls in brain aging, epigenetic aging, and polygenic risk scores for schizophrenia.

\begin{tabular}{|c|c|c|c|}
\hline Measure & $\begin{array}{c}\text { Controls } \\
(\text { Mean } \pm \text { SD) }\end{array}$ & $\begin{array}{c}\text { Patients } \\
(\text { Mean } \pm \mathrm{SD})\end{array}$ & $\begin{array}{l}\text { Effect of } \\
\text { status }\end{array}$ \\
\hline $\begin{array}{l}\text { MRI brain age gap } \\
\text { (years } \pm S D)\end{array}$ & $-0.06 \pm 6.23$ & $+3.98 \pm 7.24$ & $\begin{array}{l}+4.03 \\
(p<0.0001)^{*}\end{array}$ \\
\hline $\begin{array}{l}\text { MRI brain age acceleration } \\
\text { (years/year } \pm S D)\end{array}$ & $1.01 \pm 1.38$ & $2.01 \pm 2.26$ & $\begin{array}{l}+1.00 \\
(p<0.0001)^{*}\end{array}$ \\
\hline $\begin{array}{l}\text { Horvath's epigenetic age gap } \\
\text { (years } \pm S D)\end{array}$ & $+0.00 \pm 3.56$ & $-0.52 \pm 5.69$ & $\begin{array}{l}-0.52 \\
(p=0.5103)\end{array}$ \\
\hline $\begin{array}{l}\text { Levine's epigenetic age gap } \\
\text { (years } \pm S D)\end{array}$ & $+0.00 \pm 6.95$ & $+2.27 \pm 6.74$ & $\begin{array}{l}+2.27 \\
(p=0.0354)\end{array}$ \\
\hline $\begin{array}{l}\text { Polygenic risk for schizophrenia } \\
(\text { mean } \pm S D)\end{array}$ & $+0.00 \pm 1.00$ & $+0.83 \pm 0.96$ & $\begin{array}{l}+0.83 \\
(p<0.0001)^{*}\end{array}$ \\
\hline
\end{tabular}

Nominal $p$-values are reported with a significance threshold of $p<0.05$ printed in boldface. Differences that survive Bonferroni correction for multiple testing are marked with * Abbreviations (in alphabetical order): SCZ = schizophrenia patients; $\mathrm{SD}=$ standard deviation from the mean.

Bivariate association between measures from the three modalities revealed statistically significant positive associations between polygenic risk score and MRI brain age gap and acceleration, and a negative association between polygenic risk for schizophrenia and Horvath's epigenetic age gap (Table 5.3A; Figure 5.3). Bivariate associations within modalities revealed strong positive correlations between MRI brain age gap and acceleration, and between Horvath's and Levine's epigenetic age gaps (Table 5.3A; Figure 5.3).

After including status as a covariate in the partial correlation, the association between the MRI brain age gap and acceleration with DNA polygenic risk scores are no longer significant (Table 5.3B). 


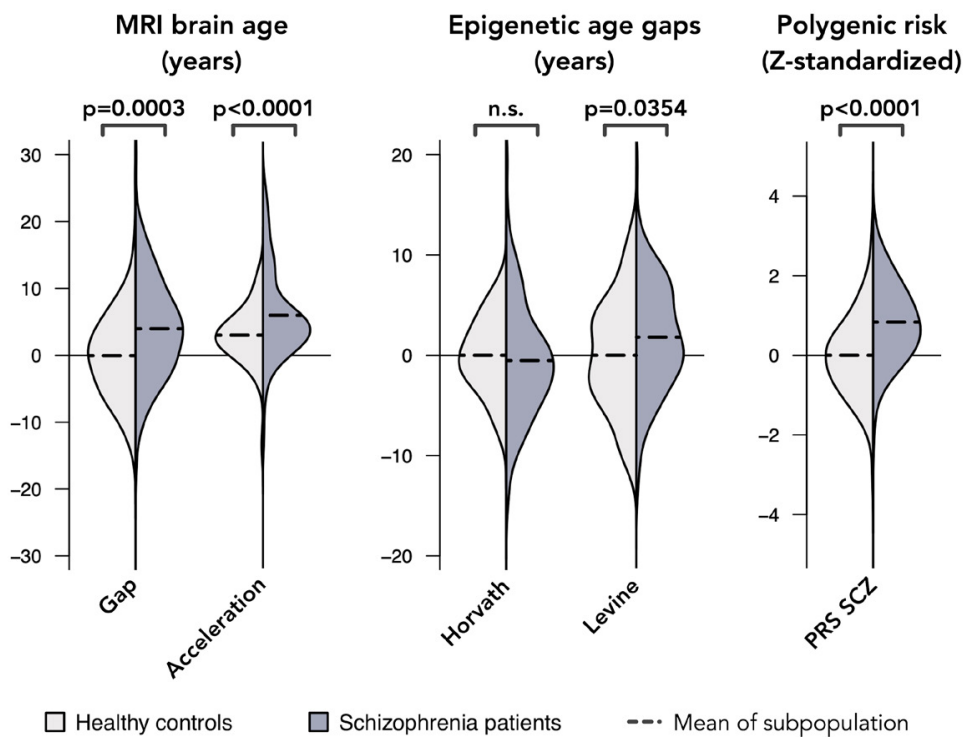

Figure 5.2. Status effect on the means for MRI brain age gap and acceleration, epigenetic age gaps, and polygenic risk for schizophrenia. Violin plots show an approximation of the distribution of scores within a subpopulation where density is smoothed by a Gaussian kernel and max height scaled to unit value. Abbreviations (in alphabetical order): PRS = polygenic risk score; $\mathrm{SCZ}=$ schizophrenia patients .

Table 5.3 (continued on the next page). Bivariate analysis between MRI brain age, epigenetic ages, and polygenic risk scores for schizophrenia.

\begin{tabular}{|c|c|c|c|c|c|}
\hline $\begin{array}{l}\text { [A] Full } \\
\text { correlations }\end{array}$ & $\begin{array}{l}\text { MRI brain } \\
\text { age gap }\end{array}$ & $\begin{array}{c}\text { MRI brain } \\
\text { age } \\
\text { acceleration }\end{array}$ & $\begin{array}{l}\text { Horvath's } \\
\text { epigenetic } \\
\text { age gap }\end{array}$ & $\begin{array}{l}\text { Levine's } \\
\text { epigenetic } \\
\text { age gap }\end{array}$ & $\begin{array}{c}\text { DNA } \\
\text { polygenic } \\
\text { risk scores }\end{array}$ \\
\hline $\begin{array}{r}\text { MRI brain age } \\
\text { gap }\end{array}$ & - & $\begin{array}{c}+0.36 \\
(p<0.0001)^{*}\end{array}$ & $\begin{array}{c}-0.08^{\mathrm{a}} \\
(p=0.3192)\end{array}$ & $\begin{array}{c}+0.02^{\mathrm{a}} \\
(p=0.8388)\end{array}$ & $\begin{array}{c}+0.10 \\
(p=0.0478)\end{array}$ \\
\hline $\begin{array}{r}\text { MRI brain age } \\
\text { acceleration }\end{array}$ & $\begin{array}{l}\text { HC: } 109 \\
\text { SCZ: } 126\end{array}$ & - & $\begin{array}{c}+0.02^{\mathrm{a}} \\
(p=0.8743)\end{array}$ & $\begin{array}{c}+0.03^{\mathrm{a}} \\
(p=0.7470)\end{array}$ & $\begin{array}{c}+0.15 \\
(p=0.0258)\end{array}$ \\
\hline $\begin{array}{l}\text { Horvath's epi- } \\
\text { genetic age gap }\end{array}$ & $\begin{array}{l}\text { HC: } 63 \\
\text { SCZ: } 109\end{array}$ & $\begin{array}{l}\text { HC: } 39 \\
\text { SCZ: } 68\end{array}$ & - & $\begin{array}{c}+0.42 \\
(p<0.0001)^{*}\end{array}$ & $\begin{array}{c}-0.20 \\
(p=0.0129)\end{array}$ \\
\hline $\begin{array}{l}\text { Levine's epi- } \\
\text { genetic age gap }\end{array}$ & $\begin{array}{l}\text { HC: } 63 \\
\text { SCZ: } 109\end{array}$ & $\begin{array}{l}\text { HC: } 39 \\
\text { SCZ: } 68\end{array}$ & $\begin{array}{l}\text { HC: } 63 \\
\text { SCZ: } 109\end{array}$ & - & $\begin{array}{c}+0.08 \\
(p=0.3261)\end{array}$ \\
\hline $\begin{array}{r}\text { DNA polygenic } \\
\text { risk scores }\end{array}$ & $\begin{array}{l}\text { HC: } 212 \\
\text { SCZ: } 182\end{array}$ & $\begin{array}{l}\text { HC: } 105 \\
\text { SCZ: } 121\end{array}$ & $\begin{array}{l}\text { HC: } 57 \\
\text { SCZ: } 98\end{array}$ & $\begin{array}{l}\text { HC: } 57 \\
\text { SCZ: } 98\end{array}$ & - \\
\hline
\end{tabular}




\begin{tabular}{|c|c|c|c|c|c|}
\hline $\begin{array}{l}\text { [B] Partial } \\
\text { correlations } \\
\text { accounting for } \\
\text { disease status }\end{array}$ & $\begin{array}{l}\text { MRI brain } \\
\text { age gap }\end{array}$ & $\begin{array}{l}\text { MRI brain } \\
\text { age } \\
\text { acceleration }\end{array}$ & $\begin{array}{l}\text { Horvath's } \\
\text { epigenetic } \\
\text { age gap }\end{array}$ & $\begin{array}{l}\text { Levine's } \\
\text { epigenetic } \\
\text { age gap }\end{array}$ & $\begin{array}{c}\text { DNA } \\
\text { polygenic } \\
\text { risk scores }\end{array}$ \\
\hline $\begin{array}{r}\text { MRI brain age } \\
\text { gap }\end{array}$ & - & $\begin{array}{c}+0.31 \\
(p<0.0001)^{*}\end{array}$ & $\begin{array}{c}-0.05^{\mathrm{a}} \\
(p=0.5333)\end{array}$ & $\begin{array}{c}-0.04^{\mathrm{a}} \\
(p=0.5744)\end{array}$ & $\begin{array}{c}-0.01 \\
(p=0.7781)\end{array}$ \\
\hline $\begin{array}{r}\text { MRI brain age } \\
\text { acceleration }\end{array}$ & $\begin{array}{l}\text { HC: } 109 \\
\text { SCZ: } 126\end{array}$ & - & $\begin{array}{c}+0.04^{\mathrm{a}} \\
(p=0.7000)\end{array}$ & $\begin{array}{c}+0.03 \mathrm{a} \\
(p=0.8458)\end{array}$ & $\begin{array}{c}+0.06 \\
(p=0.3466)\end{array}$ \\
\hline $\begin{array}{l}\text { Horvath's epi- } \\
\text { genetic age gap }\end{array}$ & $\begin{array}{l}\text { HC: } 63 \\
\text { SCZ: } 109\end{array}$ & $\begin{array}{l}\text { HC: } 39 \\
\text { SCZ: } 68\end{array}$ & - & $\begin{array}{c}+0.45 \\
(p<0.0001)^{*}\end{array}$ & $\begin{array}{c}-0.17 \\
(p=0.0313)\end{array}$ \\
\hline $\begin{array}{l}\text { Levine's epi- } \\
\text { genetic age gap }\end{array}$ & $\begin{array}{l}\text { HC: } 63 \\
\text { SCZ: } 109\end{array}$ & $\begin{array}{l}\text { HC: } 39 \\
\text { SCZ: } 68\end{array}$ & $\begin{array}{c}\text { HC: } 63 \\
\text { SCZ: } 109\end{array}$ & - & $\begin{array}{c}+0.03 \\
(p=0.7548)\end{array}$ \\
\hline $\begin{array}{r}\text { DNA polygenic } \\
\text { risk scores }\end{array}$ & $\begin{array}{l}\text { HC: } 212 \\
\text { SCZ: } 182\end{array}$ & $\begin{array}{l}\text { HC: } 105 \\
\text { SCZ: } 121\end{array}$ & $\begin{array}{l}\text { HC: } 57 \\
\text { SCZ: } 98\end{array}$ & $\begin{array}{l}\text { HC: } 57 \\
\text { SCZ: } 98\end{array}$ & - \\
\hline
\end{tabular}

Table 5.3 (continued). Spearman's correlation coefficients and their significant values are reported in the upper right triangle. Samples sizes are reported in the lower left triangle. Statistically significant associations (nominal $p<0.05$ ) are printed in boldface. Associations that survive Bonferroni correction are marked with * a Associations between MRI brain age gap or age acceleration and epigenetic age gaps were corrected for interval between acquisition of MRI scan and blood sample using partial correlations. Abbreviations (in alphabetical order): HC = healthy controls; $\mathrm{SCZ}=$ schizophrenia patients; $\mathrm{SD}=$ standard deviation from the mean.

\subsection{DISCUSSION}

We investigated the associations between different biological aging markers and with polygenic risk for schizophrenia. We found suggestive evidence of association between polygenic risk for schizophrenia with MRI-derived brain aging and with Horvath's epigenetic aging, but not between polygenic risk for schizophrenia and Levine's epigenetic aging or between brain aging and epigenetic aging.

\subsubsection{Brain aging and its association to polygenic risk for schizophrenia}

Diagnosis status (schizophrenia vs. healthy) had a highly significant effect on brain aging. The age gap, i.e., the difference between estimated age and chronological age, was 4 years in patients (and zero in healthy subjects), and the brain age acceleration rate was 2 year/year (and 1 in healthy subjects), consistent with the previously reported results for the broader sample (Schnack et al., 2016) and with other studies reporting accelerated aging 

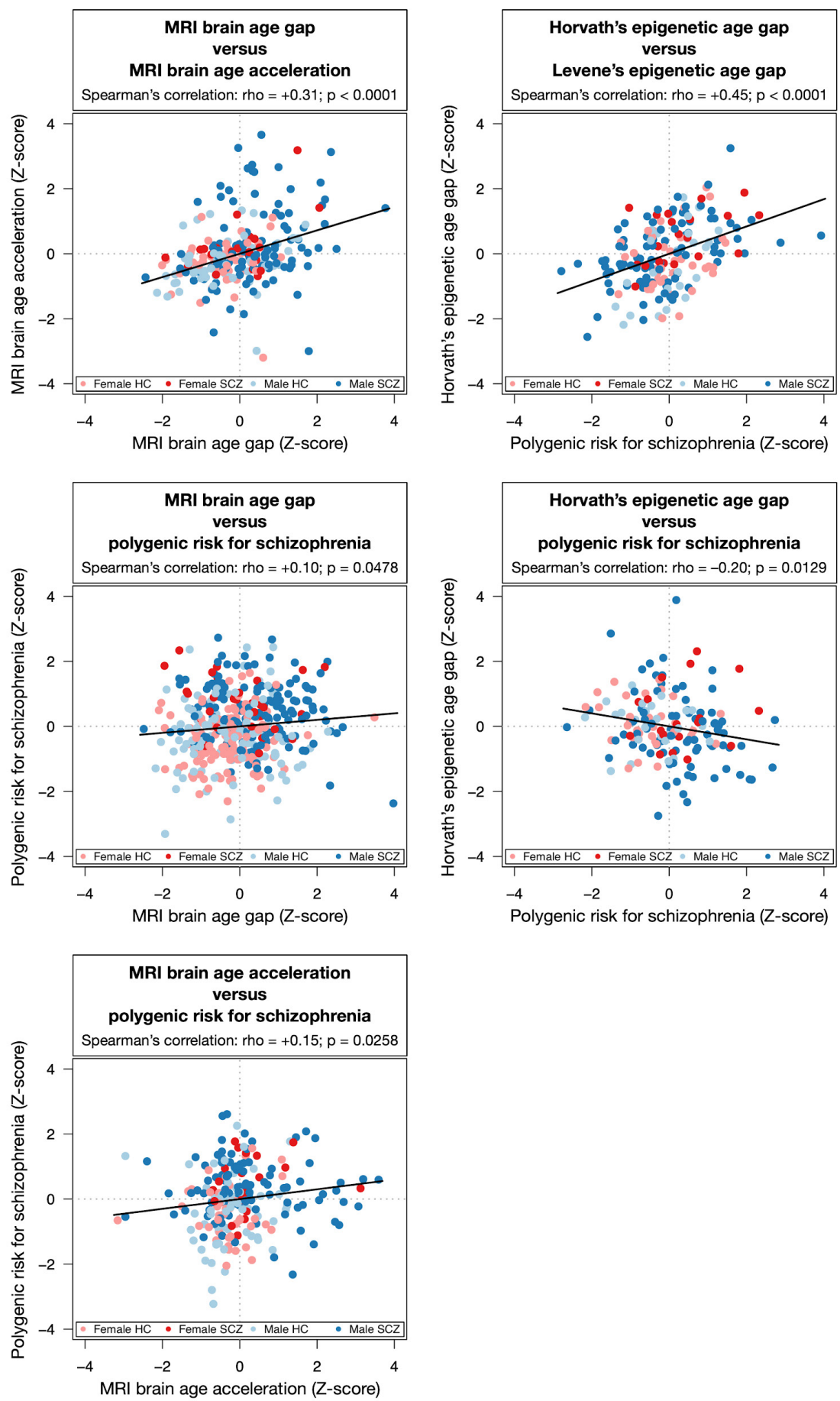

Figure 5.3. Statistically significant associations between MRI brain aging, epigenetic aging, and polygenic risk for schizophrenia. Abbreviations (in alphabetical order): $\mathrm{HC}=$ healthy controls; $\mathrm{SCZ}=$ schizophrenia. 
of the brain in schizophrenia patients (Koutsouleris et al., 2014; Nenadic et al., 2017; Kaufmann et al., 2019; Jonsson et al., 2019) and in subjects at clinically high-risk for psychosis and first-episode patients (Kolenic et al., 2018; Chung et al., 2018; Hajek et al., 2019; Shahab et al., 2019). Polygenic risk for schizophrenia (PRS), as expected, was significantly higher in the patients as compared to the control subjects $(\mathrm{p}<0.0001)$. We found nominal significant associations between polygenic risk for schizophrenia and brain age gap and acceleration. These associations were largely moderated by diagnosis status, indicating that cases who carry higher polygenic risk for schizophrenia display faster brain age acceleration. Our results are in line with recent work that observed an overlap between common genetic variants associated with brain aging and common variants associated with schizophrenia in the population (Kaufmann et al., 2019). Here, we observe a direct association between brain aging and schizophrenia polygenic risk within individuals. While these findings provide evidence for a shared mechanism between genetic risk of schizophrenia and aging in the brain, replication and further work in a larger sample should be a first priority for future work. This study nevertheless reports the first efforts and value of such analyses. Structural brain aging could thus be used as an intermediate phenotype for psychosis (Palaniyappan et al., 2017; Dukart et al., 2017) and may show promise in predicting transition to psychosis in at-risk populations (Koutsouleris et al., 2014).

\subsubsection{Epigenetic aging and its association to polygenic risk for schizophrenia}

We found that age was significantly accelerated by +2.3 year in schizophrenia patients as compared to healthy controls for Levine's epigenetic clock, but not for Horvath's epigenetic clock. Previous reports suggest no accelerated epigenetic aging or association with premature mortality for schizophrenia in blood or post-mortem brain samples (McKinney et al., 2017; Voisey et al., 2017; McKinney et al., 2018; Kowalec et al., 2019), with some exceptions (Okazaki et al., 2019; Ori et al., 2019).

In addition, we found a significant negative association $(r h o=-0.20)$ between polygenic risk for schizophrenia and Horvath's epigenetic age gap regardless of diagnosis status, but not for Levine's epigenetic age gap ( $r h o=+0.08$; [n.s.]). We previously reported on the association between polygenic risk and epigenetic age in a larger sample of schizophrenia patients (Ori et al., 2019); the associations turned out to be age- and sex-specific, with female patients above age 36 showing an increase in epigenetic age of 3 years. However, the current sample is too small to stratify by age group or sex. In another study, polygenic risk for schizophrenia was associated with mortality predictions based on Levine's epigenetic age and suicidal behavior (Laursen et al., 2017). This warrants caution interpreting our findings and further emphasizes the need for follow-up studies. 
Accelerated epigenetic aging is heritable (Marioni et al., 2015; Li et al., 2015), with an important role for the TERT locus related to telomerase and aging and nine other loci related to metabolism and immune system pathways (Lu et al., 2017; Gibson et al., 2019). No overlap between genetic variants identified for schizophrenia and epigenetic aging is reported in a relatively small sample (Lu et al., 2018). However, there is an indication for colocalization of genetic and epigenetic loci implicated in schizophrenia (Hannon et al., 2016); epigenetic loci used to predict epigenetic age do not overlap with known epigenetic loci implicated in schizophrenia (Mill et al., 2008; Hannon et al., 2016). Instead, it is possible that the association between epigenetic age and polygenic risk for schizophrenia is mediated by other factors, e,g, a shared pathway that increases risk of early mortality (Marioni et al., 2015; Levine et al, 2018; Laursen et al., 2014) such a genetic predisposition to smoking (Boardman et al., 2010) or stressful life events (Wolf et al., 2018).

\subsubsection{Brain aging and its association with epigenetic aging}

We did not find any significant association between the MRI brain age gap or age acceleration with the DNAm age gap of either Horvath's or Levine's predictor (range of $r h o=[-0.08 ;+0.03]$; nominal $p>0.32$ ). An absence of association between the MRI-derived brain age gap and Horvath's epigenetic age gap in blood samples has previously been reported (Cole et al., 2018). Here we complement the previous finding by not only replicating the null result for Horvath's epigenetic age predictor (that is a reliable predictor of chronological age regardless of tissue type or disease), but also investigating the association between MRI brain aging and Levine's epigenetic age predictor in blood, (which might be more sensitive to aberrant biological aging due to the inclusion of extrinsic factors more representative of apparent phenotypic aging) (Levine et al., 2018). However, the association remains absent despite the effects of diagnostic status and associations with polygenic risk for schizophrenia in the individual measures. As previously reported, epigenetic aging in schizophrenia shows an age- and sex-specific effect in a larger study that included this cohort (Ori et al., 2019), and accelerated aging of the brain is already present in the first years following the onset of psychosis before it stabilizes several years afterward (Schnack et al., 2016). The absence of an association between MRI brain aging and epigenetic aging might be due to distinct aging processes. Epigenetic aging, in particular Horvath's epigenetic age predictor, is a measure of cellular aging rather than cellular senescence (Lowe et al., 2016; Kabacik et al., 2018). In contrast, the aging of the brain, in our study reflecting decreases in gray matter tissue, is likely due to cell senescence rather than cellular aging (Fernandez-Egea, 2017), and it reflects changes in the morphology of the cells or composition of the neuropil. This argument is used to explain the absence of epigenetic aging in post-mortem samples of the brains of schizophrenia patients (McKinney et al., 2017; Voisey et al., 2017; McKinney et al., 2018). The possibility 
of two independent aging processes was previously suggested given the lack of association between the age gaps and the fact that combining information from the two 'clocks' improved mortality predictions (Cole et al., 2018). The absence of association with Levine's epigenetic age predictor, one that takes into account extrinsic factors of typical aging, from our results affirms the conclusion that aging of the brain and epigenetic aging in blood might be two distinct processes in the aetiology of schizophrenia, despite their commonality in predicting mortality (Cole et al., 2018; Marioni et al., 2015; Chen et al., 2016). A similar conclusion on the dissociation between brain aging and epigenetic aging can be concluded for other psychiatric disorders based on the reports from several independent studies. For bipolar disorder, accelerated epigenetic aging (Nenadić et al., 2017) but not aging of the brain (Fries et al., 2017; Shahab et al., 2019; Nenadić et al., 2017) has been reported, although lithium use may have confounded these results, since patients who were not treated with lithium have been found to show increased brain age (Van Gestel et al., 2919). For major depressive disorder, a large $(\mathrm{N}=1689)$ international multicenter study (Han et al., 2020) has found accelerated aging of the brain, but within smaller samples results are inconclusive (Koutsouleris et al., 2014; Besteher et al., 2019; Kaufmann et al., 2019). Epigenetic aging in blood (Han et al., 2018) but not epigenetic aging in post-mortem brain samples ( $\mathrm{Li}$ et al., 2018) has been reported.

\subsubsection{Limitations and future directions}

There are a few limitations to this study that should be taken into account. First, the sample size of this study, whilst large for a longitudinal neuroimaging study, is very modest for a genetic or epigenetic study. Secondly, the cross-sectional design for epigenetics limits our ability to detect a possible age accelerating rate in the blood and its association to accelerating brain age (Nelson et al., 2020). Depending on the time lag between illness onset and accelerated epigenetic aging, and because of the fact that most of the blood sample were acquired at baseline, the effects of the disease on DNA methylation may have yet to occur, especially in the younger adolescent population when onset of psychosis typically occurs (Paus et al., 2008). Future studies, measuring both brain aging and epigenetic aging in large, longitudinal, studies should further elucidate the possible (dynamic) relationships between these different measures of biological aging. 



\section{CHAPTER 6}

\section{HIGHLIGHTS AND GENERAL DISCUSSION}




\subsection{HighLIGHTS}

The research in this thesis has brought forward several findings of interest to the field of adolescent brain development:

> The thinning of the cerebral cortex during adolescence is heritable and can be described by a brain-wide genetic factor with fluctuating influences across the ages that acts in addition to secondary localized influences from novel genetic factors at the different ages (Chapter 2).

Reliability modelling of the inherently noisy BOLD functional MRI signal can reveal the "true" (i.e. unconfounded by measurement error) resting-state functional connectivity between brain regions; this is reflected by the improved strength in the associations of functional connectivity with various traits and by the increased heritability estimates for the reliable component of functional connectivity (Chapter 3).

There is a subtle but wide-spread developmental pattern of resting-state functional connectivity during adolescence; this is described by mostly increased functional connectivity within brain networks and by decreased functional connectivity between brain networks, and in addition to sex-specific effects for the functional connectivity within the default mode network and within the salience network (Chapter 4).

Resting-state functional connectivity in the adolescent brain is partially influenced by a mixture of stable genetic and common environmental factors; i.e. there is no clear evidence for fluctuating influence of a core genetic or environmental factor or indication for novel factors at the different ages (Chapter 4).

Progressive aging of the brain in patients with schizophrenia is accelerated around the time of onset for the disorder, which typically occurs during adolescence, but does not appear to be associated with progressive aging measured with epigenetics in blood, although separately both are associated with polygenic risk for schizophrenia (Chapter 5).

\subsection{GENERAL DISCUSSION}

\subsubsection{Maturation and sex effects on the adolescent brain}

\subsubsection{Development of cerebral cortical thickness in adolescence}

In Chapter 2, we investigated the development of cortical thickness in the longitudinal BrainSCALE study of children and adolescents at 9,12 and 17 years of age. We report a gradual decrease in thickness of the cerebral cortex between 9 and 12 years of age that is accelerated between 12 and 17 years of age, with the most prominent changes occurring in the sensorimotor regions (visual, sensor, and motor cortices) and the frontal pole (Chapter 2). Moreover, we 
reported that the accelerated thinning of the cortex that occurs between the ages 12 and 17 years makes the frontal, parietal, and temporal lobes at the age of 17 years distinct from those at the ages of 9 and 12 years in hierarchical clustering analysis (Chapter 2).

The developmental pattern of cortical thinning is typically observed during adolescence (Gogtay et al. 2004; Sowell et al., 2007; Raznahan et al., 2011; Wierenga et al., 2014; Zhou et al., 2015), and was now revealed to accelerate and differentiate the cortex during late adolescence based on three assessments per individual in the BrainScale cohort (van Soelen et al, 2012; van Soelen et al, 2012). Despite the numerous studies on the development of the cerebral cortex, no clear consensus has been reached whether cortical gray matter peaks during childhood or shows a continuous decline from infancy onward (Walhovd et al., 2017; Wierenga et al., 2014; Fjell et al., 2015). Between the ages 9 and 12 years, we reported that a majority of the children in the BrainSCALE cohort showed only a slight decrease in global cortical thickness, with several local cortical regions showing no significant changes in thickness (Chapter 2; van Soelen et al., 2012; Brouwer et al., 2014). These findings would suggest a possible plateau or peak in local cortical thickness before adolescence. For average cortical thickness, the thinning of the cortex continues beyond adolescence, and does not reach a plateau until adulthood between 30 and 60 years of age (Schnack et al, 2015). The rapid changes in the cortex during adolescence that results in phenotypic separation of the cortex in late adolescence from that in late childhood and early adolescence makes the frontal, parietal, and temporal lobe prominent candidates for studying behavioral changes during adolescence from a neuroscience perspective.

\subsubsection{Sexual differentiation in cortical thickness development in adolescence}

In Chapter 2, we investigated possible sex effects on the development of cortical thickness in the BrainSCALE cohort. However, we did not find any significant sex effects on the development of global cortical thickness throughout adolescence (Chapter 2).

In previous research on the BrainSCALE cohort, the onset of secondary sexual characteristics of puberty have been associated with decreased frontal and parietal gray matter densities at the age of 9 years (Peper et al., 2009). Changes in the levels of follicle stimulating hormone (FSH) in girls has been associated with changes in grey matter density between the ages 9 and 12 years, and levels of estradiol have been associated with grey matter density at age 12 years (Brouwer et al., 2015). This suggests that puberty may trigger the reorganization of brain structures such as the thinning of the cortex during adolescence (Peper et al., 2011). Despite the influence of puberty and pubertal hormones on the development of the cortex and the difference in pubertal timing of about 1 to 2 years between the sexes (Fredriks et al., 2000; Koenis et al., 2013), no differences between the sexes were present for the thickness of the cerebral cortex (Chapter 2). Sex effects play a prominent role in explaining variation of 
volumetric and surface area measures of the brain structures (Lenroot et al., 2007; Ruigrok et al, 2014; Herting et al., 2018), and could explain differences observed in behavior and cognition between the sexes (Gur and Gur, 2016). However, the presence of sex effects on cortical thickness is still disputed (Walhovd et al., 2017; Koolschijn and Crone, 2013; Sowell et al., 2007; Lenroot and Giedd, 2010; Raznahan et al., 2011). Cortical thickness and surface area are two largely independent measures that make up cortical gray matter volume (Lenroot et al., 2007; Pannizon et al., 2009). Sex effects in cortical thickness might be explained by interaction with the development of surface area in childhood and early adolescence as the brain still grows in volume (Wierenga et al., 2014; Schnack et al., 2015). Alternatively, sex effects may not necessarily be present on the means, but rather on the variances; with larger variation in males compared to females (Wieringa et al., 2018). However, we performed a qualitative analysis of sex effects in cortical thickness in Chapter 2, and we not find any perceivable differences in phenotypic between regions of the cortex that precludes the possibility of sex effects on the means or variances of cortical thickness.

\subsubsection{Development of functional connectivity in adolescence}

In Chapter 4, we investigated the development of brain-wide functional connectivity within and between major cortical resting-state networks. We reported that there are changes in functional connectivity during adolescence that are characterized by a remarkable developmental pattern of increasing functional connectivity within cortical resting-state networks and decreasing functional connectivity between networks with age (Chapter 4).

Although resting-state networks may already be established by early age (Turk et al., 2019; Gao et al., 2015), our results show that there are still wide-spread but subtle changes in the functional coupling strength between brain regions during adolescence. Only a few longitudinal studies have been performed on functional connectivity in typically developing adolescents, with most studies focusing on specific connections (see overview of literature in Table 4.1 from Chapter 4). These studies generally report increasing functional connectivity within functional networks or decreasing functional connectivity between functional networks with age that is consistent with our results (Bernard et al., 2016; Long et al., 2017; Sherman et al., 2014; Wendelken et al., 2017; Wendelken et al., 2016). These developmental changes in brain connectivity may explain cognitive and behavioral changes during adolescence by tighter integration of functionally related brain regions and segregation of unrelated regions (Wig, 2017). For example, the better integration within the frontoparietal network we reported for the BrainSCALE cohort (Chapter 4) may support better cognitive performance (Jung and Haier, 2007). The segregation between the anterior cingulate cortex and insula of the salience network we reported for the BrainSCALE cohort (Chapter 4) could reflect segregation of bottom-up stimuli processing and top-down 
cognitive control processing that might coincide with improved self-control and response inhibition during adolescence in reward-based decision making (Casey, 2013; Uddin et al., 2017; Bush et al., 2002; Stevens et al., 2011).

\subsubsection{Sexual differentiation in functional connectivity development in adolescence}

In Chapter 4, we investigated the effect of sex on functional connectivity within and between the major cortical resting-state networks. We reported that there is increased functional connectivity within the default mode network for girls compared to boys, and an opposite sex effect with increased functional connectivity within the salience network for boys compared to girls (Chapter 4).

Sex effects on functional connectivity have also been reported for adults and across the lifespan (Biswal et al., 2010; Zuo et al., 2010), and corroborate that sex differences in brain functioning are present during childhood and adolescence (Gur and Gur, 2016), with recent reports that sex difference in functional connectivity are already present during pregnancy in the fetal brains (Wheelock et al., 2019). These sex effects in functional connectivity could have potential relevance to behavioral differences in adolescent development. The increased functional connectivity within the default mode network for girls may explain their better performance at memory and emotive tasks for its role in auto-biography memory and emotion regulation (Gur et al., 2012; Raichle, 2015). Whereas the increased functional connectivity within the salience network for boys may explain their better performance at visuospatial and motor tasks for its role in overt attention/stimuli processing, integration of multimodal sensory information (Gur et al., 2012; Uddin, 2014).

6.2.1.5 Necessity of stringent control for head motion effects on functional connectivity development In addition to developmental finding of functional connectivity in the BrainSCALE cohort reported in Chapter 4, we also report a sharp decline in the amount of in-scanner head motion during the acquisition of the resting-state fMRI scans between the ages 13 and 18 years (Chapter 4).

The issue of head motion during scan acquisition has raised concerns for developmental findings of functional connectivity (Power et al., 2012). Despite best efforts to control for the effect of head motion during preprocessing of the resting-state scans, significant residual effects of head motion on functional connectivity within and between resting-state networks was present and was subsequently removed with additional regression during statistical analysis (Chapter 4). Although head motion will likely remain an issue in developmental studies, our results should be robust due to rigorous control for the effects of head motion on functional connectivity in a longitudinal study design. 


\subsubsection{Necessity of longitudinal studies on functional connectivity development}

Cross-sectional studies on the development of functional connectivity do not always show consensus on the direction of change and affected regions (Stevens, 2016). In contrast to longitudinal studies, cross-sectional findings are confounded by possible cohort effects (Mills and Tamnes, 2014; Crone and Elzinga, 2014; Telzer et al., 2018). This concern is particularly true for the development of functional connectivity given the subtle changes in functional connectivity we report in Chapter 4 in the presence of large inter-individual variation typically present in functional connectivity and the sharp decline in head motion with age. With only a few longitudinal studies that focus on the development of specific connections for comparison, this emphasizes the need for more longitudinal studies in brain functioning to replicate the developmental findings for functional connectivity we report in Chapter 4 for the BrainSCALE cohort.

\subsubsection{Genetic and environmental influences on brain development}

\subsubsection{Genetic and environmental influences on cortical thickness in adolescence}

In Chapter 2, we investigated the dynamic influences of genes and environment on the development of cerebral cortical thickness in the BrainSCALE cohort. We reported that the thinning of the cortex is driven by a core genetic factor with fluctuating influences from late childhood throughout adolescence, and novel genetic influences at the regional level that may reflect differential expression of genes marking distinct stages of cortical development in adolescence (Chapter 2).

Earlier genetic studies of cerebral cortex characteristics have reported evidence that cortical thickness for regions across the brain, including homotopic regions on each hemisphere, are influenced by a common genetic factor in both children and in adults (Schmitt et al., 2008; Rimol et al., 2010; Chen et al., 2013; Wen et al., 2016; Schmitt et al., 2017). The genetic correlation between some of these regions showed increased towards the second decade of life (Schmitt et al., 2017), that could be related to an increasing influence of the core genetic factor found in the BrainSCALE cohort (Chapter 2).

Moreover, we found that the core genetic factor influences both the cortical thickness measured at a specific age as well as the rate of change for the accelerated thinning of the cortex throughout adolescence (Chapter 2). Previous studies have reported genetic influences on the change rate of brain structures (Brans et al., 2008; van Soelen et al., 2012; van Soelen et al., 2013; Brouwer et al., 2014; Bootsman et al., 2015; Hedman et al., 2016; Brouwer et al., 2017), and how genes that drive these changes in brain volumes and cortex thickness are also responsible for intelligence (Brans et al., 2008; Brouwer et al., 2014), thinning of the cortex in schizophrenia patients (Hedman et al., 2016), and growth of cerebellar volume is related to stature during development (van Soelen et al., 2013). In a prior study of the BrainSCALE 
cohort, novel genetic influences were reported for local regions of the cortex at age 12 years (van Soelen et al., 2012). In Chapter 2, we extended these findings with the third assessment of the BrainSCALE cohort at age 17 years, and we reported that the novel genetic influences expanded to additional regions of the cortex (Chapter 2). This genetic differentiation of the cortex during adolescence might be the result of continued areal specialization of the cortex that continues to develop well into early adulthood and beyond, in particular the frontal cortex (Schnack et al., 2015; Fjell et al, 2015).

It has been suggested that areal specialization of the neocortex is established during early development, and that later development is the result of more general maturational processes affecting the entire neocortex (Pletikos et al., 2014). Several studies reported a remarkably strong homogeneity in gene expression profiles among neocortical areas despite their functional specialization (Roth et al., 2006; Kang et al., 2011; Hawrylycz et al., 2015; Jaffe et al., 2015). These studies match the findings we reported from the BrainSCALE cohort of a core genetic factor that influences the cortical thickness throughout the entire brain and across childhood and adolescence (Chapter 2; van Soelen et al., 2012). However, there is a distinct temporal differential gene expression during adolescence that is marked by a second wave of substantial changes in gene expression at the end of adolescence (Somel et al., 2010; Colantuoni et al., 2011; Pletikos et al., 2014; Jaffe et al., 2015). This temporal differential gene expression of the cortex coincides with the novel genetic influences and the more pronounced waxing and waning influences of the core genetic factor we found in the BrainSCALE cohort during the accelerated thinning of the cortex between the ages 12 and 17 years (Chapter 2). The thinning of the cortex during adolescence has been linked to gene expression of genes related to synaptic function, dendrite development, myelination, and cellular composition of the neuropil (Whitaker et al., 2016; Kang et al., 2011; Jaffe et al., 2015). These studies support the idea that the biological processes underlying the apparent cortical thinning observed during development is due to pruning of neuronal synapses and dendrites accompanied by a decrease in supporting glial cells, and a parallel increase of oligodendrocytes responsible for myelination of neuronal axons (Huttenlocher, 1979; Bourgeois and Rakic, 1993; Huttenlocher and Dabholkar, 1997; Paus et al., 2008; Paus, 2010; Petanjek et al., 2011; Miller et al., 2012; Deoni et al., 2015). Together, the ongoing areal specialization of the cortex during adolescence might be the result of shifting balances in ongoing maturational processes and spurred by new genetic factors that could be related to the rapid cognitive and behavioral changes during adolescence. 


\subsubsection{Reliability modelling of functional connectivity for genetic studies}

In Chapter 3, we investigated the application of a measurement model for improving the reliability of noisy measures such as functional connectivity. We reported that reliability modelling of functional connectivity can benefit genetic studies by detecting the genetic signal for the stable and reliable component of functional connectivity that is free from random measurement error (Chapter 3 and Chapter 4).

Twin studies have reported genetic control over brain activity at rest with generally low heritability estimates (see overview of literature in Table 4.2 from Chapter 4 ). Heritability estimates are influenced by the presence of measurement error (Posthuma et al., 2000). The reliable component of functional connectivity can be derived by controlling for measurement error using repeated measures or split-session measures in a measurement model (van Baal et al., 1998; van Beijsterveldt et al., 2001; Brandmaier et al., 2018; Cooper et al., 2019). An empirical evaluation of the utility of the measurement model revealed the ability to extract a reliable component of functional connectivity that had increased heritability (Chapter 3). This increase is due to standardizing genetic variances over the variance of the reliable component rather than the full phenotypic variance that contains the measurement error that would otherwise be attributed to an individual's unique environmental influences (Posthuma et al., 2000). The use of a measurement model resulted in similar increases in heritability estimates as previous study using a custom linear mixed effects model on repeated measures (Ge et al., 2017). This method has been published in a public repository on Github ${ }^{3}$ and can be applied in other studies including in existing resting-state studies on fMRI brain measurements.

\subsubsection{Genetic and environmental influences on functional connectivity in adolescence}

In Chapter 4 we applied the reliability modelling detailed in Chapter 3 to the resting-state functional connectivity measures of the BrainSCALE cohort. We investigated the genetic and environmental influences on brain-wide functional connectivity within and between eight major cortical resting-state networks: the default mode, frontoparietal, dorsal attention, salience, sensorimotor, visual, language, and cerebellar networks. We reported that the reliable component of functional connectivity within and between the major resting-state networks is influenced by additive genetics and common environmental factors during adolescent brain development (Chapter 4).

So far, only a few studies have investigated genetic influences on networks beyond the default mode network. These studies were mostly performed in adults and did not always investigate influences from common environment that cannot be directly dismissed in a

\footnotetext{
${ }^{3}$ https://github.com/jalmar/openmx-models/reliability/
} 
study of children living in the same household (Adhikari et al., 2018; Ge et al., 2017; Sudre et al., 2017; Yang et al., 2016). One study in particular was close to the age range of the BrainSCALE cohort and reported similar mixed influences of both additive genetics and common environment on function connectivity of resting-state networks during early adulthood (Yang et al., 2016). In both studies, connections from the frontoparietal, dorsal attention, and salience networks, all involved in higher order cognitive control, were influenced primarily by additive genetics (Chapter 4; Yang et al., 2016). Common environment plays a considerable role for most sensory networks, including the sensorimotor network, language network and cerebellum in the BrainSCALE cohort (Chapter 4) that has previously been reported only for the sensorimotor network (Yang et al., 2016). A notable exception is the default mode network that is partially influenced by an additive genetic factor or a common environmental factor in the BrainSCALE cohort instead of only an additive genetic factor typically reported by the other studies (Chapter 4; Adhikari et al., 2018; Ge et al., 2017; Sudre et al., 2017; Yang et al., 2016). The application of a measurement model and the use of an extended twin design may have contributed to the improved detection of common environmental influences. Although most likely a combination of both genetic and environmental influences play a role with possible interaction between the two that could not be modeled with the current limitation on sample sizes.

There was no strong indication for distinct genetic influences at the different ages or heritability of the changes in functional connectivity, suggesting mostly stable influences across adolescence (Chapter 4). This is in contrast to the dynamic genetic influences on the structural brain of the BrainSCALE cohort we reported in Chapter 2 for the same ages. A longitudinal study of functional brain development during infancy reported age-dependent genetic effects on functional connectivity within resting-state networks (Gao et al., 2014). Although it is possible that genetic influences of brain function at rest may have stabilized during adolescence, additional longitudinal twin studies with larger sample sizes would be needed to verify these results.

Where twin studies can tell if a trait is influenced by genetic factors, a gene discovery study is needed to identify possible causal variants. In a genome-wide association study, the strength in functional coupling between brain regions in healthy adolescents was dependent on polymorphisms of a set of genes enriched for ion channels (Richiardi et al., 2015). The synchronous resting-state activity in the brain has been associated with gene expression levels in the cortex based on similarity in gene expression profiles for distal functionally connected brain regions (Hawrylycz et al., 2015). The oscillatory activity of the brain measured with electroencephalography (EEG) is associated with tissue-specific expression of genes that also play a role in psychiatric disorders (Smit et al., 2018) These 
studies confirm that functional connectivity of the human brain is indeed partially determined by genetics.

\subsubsection{Brain development and cognitive performance}

\subsubsection{Individual differences in intellect, cortical thickness, and functional connectivity}

In Chapter 4, we investigated the association between intelligence and the coupling strength within and between the major cortical resting-state networks in the BrainSCALE cohort, including the frontoparietal network that has been implicated to support a distributed network of cognitive performance (Jung and Haier, 2007). However, no significant association between intelligence and functional connectivity was found for individual connections within or between the major cortical resting-state networks (Chapter 4). Moreover, preliminary investigation of the association between intelligence and cortical thickness in the BrainSCALE cohort revealed that the association previously reported to appear around 12 years of age (Brouwer et al., 2014) started to diminish again at age 17 years (not shown).

Previous research on the BrainSCALE cohort has reported an association between cognitive abilities and global brain volumes already at the age of 9 years that is partially determined by a genetic correlation (van Leeuwen et al., 2009). However, the phenotypic and genetic association between (verbal) intelligence and cortical thickness does not emerge until the age of 12 years (Brouwer et al., 2014). At the age of 17 years, the association between intelligence and cortical thickness started to disappear again, and is most likely explained by the reversal of the association between intelligence and cortical thickness (Schnack et al., 2015). This reversal of the association has implications for studying cognitive development in late adolescence and emerging adulthood. Other developmental studies have reported similar associations between intelligence and cortical development during childhood and adolescence that is both dependent on age with diminishing strength during late adolescence and show strong genetic overlap between the two measures (Shaw et al., 2006; Burgaleta et al., 2014; Schmitt et al., 2019).

Previously research on the BrainSCALE cohort has linked cognitive performance to increases in global network efficiency of the structural brain (Koenis et al., 2015; Koenis et al., 2018). However, no association between intelligence and functional connectivity was found for individual connections within or between the major cortical resting-state networks (Chapter 4). Other studies have typically reported on increased functional connectivity between regions that are part of the fronto-parietal network in association with intelligence in adults, but extends to other regions depending on the type of the cognitive process (Song et al., 2008; Hearne et al., 2016; Basten et al., 2015; Shearer et al., 2020). Contemporary theories on human intelligence suggest that cognitive performance 
is attributed to the integration of a brain-wide regions that extend beyond the frontoparietal network, rather than supported by individual regions or structural or functional connections (Jung and Haier, 2007; Deary et al., 2010; Barbey, 2017). Although, in contrast to global efficiency of the structural brain, evidence that global efficiency of the functional brain supports intelligence has been disputed (Kruschwitz et al., 2018). In general, there have been sparse number of reports on associations between functional connectivity and behavioral traits that has been attributed to unreliable measurements at the individual functional connections of the brain (Vaidya and Gordon, 2013; Geerligs et al., 2017; Noble et al., 2019). The application of a reliability model in the association between functional connectivity and traits may reveal the "true" association in absence of measurement error (Leigh Wang, 2010; Cooper et al., 2019).

\subsubsection{Improving the associations between functional connectivity and traits with reliability modelling}

In Chapter 3, we investigated the application of a measurement model for improving the reliability of noisy measures such as functional connectivity. Empirical evaluation of the utility of a measurement model in the Human Connectome Project Young Adult cohort for different sample sizes and scan durations revealed improvement in association strengths up to 1.8 -fold (Chapter 3).

Associations between functional connectivity and behavioral measures are typically low for resting-state functional connectivity (Vaidya and Gordon, 2013; Smith et al., 2015). The sparse and conflicting reports of functional connectivity with measures such as intelligence (Kruschwitz et al., 2018), might be the result of unreliable measurements that puts an upper limit on the association (Vul et al., 2009; Leigh Wang, 2010). However, despite the improvements in association strength using a measurement model to obtain estimates of functional connectivity "free" of random measurement error, most traits remained only weakly associated for individual connections (Chapter 3). Functional connectivity might simply not be sensitive enough for detecting associations with behavioral traits at individual connections but instead requires a holistic approach (e.g. multivariate or connectome-based modelling) to find robust associations with behavior (Geerligs et al., 2017; Smith et al., 2015; Finn et al., 2015; Rosenberg et al., 2015). Alternatively, the highly adaptive and flexible nature of human behavior might be better supported by dynamic properties of functional connectivity (Hutchison et al., 2013; Hilger et al., 2020). The measurement model and example code are available on a public repository on Github ${ }^{4}$ and can be applied in other studies including in existing resting-state studies on fMRI brain measurements.

\footnotetext{
${ }^{4}$ https://github.com/jalmar/openmx-models/reliability
} 


\subsubsection{Brain development and progressive aging in schizophrenia}

\subsubsection{Accelerated aging in the brain of schizophrenia patients}

In Chapter 5, we reported on accelerated aging of brain structure for patients with schizophrenia that is nominally associated with polygenic risk for schizophrenia. The presence of accelerated aging in schizophrenia is consistent with other studies reporting accelerated aging of the brain in schizophrenia patients (Koutsouleris et al., 2014; Nenadić et al., 2017; Kaufmann et al., 2019; Jonsson et al., 2019). We reported that individuals with higher polygenic risk for schizophrenia displayed faster aging of the brain (Chapter 5) that is in line with reported overlap between common genetic variants associated with brain aging and common variants associated with schizophrenia in the population (Kaufmann et al., 2019). Typical development provides a baseline for aberrant development that is usually associated with psychiatric disorders such as schizophrenia (de Wit et al., 2016; Smieskova et al., 2010; Rapoport et al., 2012). The combination of polygenic risk for schizophrenia, longitudinal brain changes, and clinical measures could be used to better predict transition to psychosis (Perkins et al., 2019). However, discordance for the disorder in monozygotic twins suggests that despite the high heritability of the disorder and brain structures in general, the onset of the disorder might be a result of an interaction with the environment that is unique to each individual (Smith, 1970; van Os et al., 2008).

\subsubsection{Epigenetic aging in schizophrenia patients}

In Chapter 5, we reported on epigenetic aging in blood that was significantly accelerated in schizophrenia patients, but was not associated with accelerated aging in the brain. Previous reports have suggested no accelerated epigenetic aging or association with premature mortality for schizophrenia in blood or post-mortem brain samples (McKinney et al., 2017; Voisey et al., 2017; McKinney et al., 2018; Kowalec et al., 2019), with some exceptions (Okazaki et al., 2019; Ori et al., 2019). An absence of association between the MRI-derived brain age gap and Horvath's epigenetic age gap in blood samples has previously been reported in elderly population (Cole et al., 2018). No overlap between genetic variants identified for schizophrenia and epigenetic aging is reported in a relatively small sample despite indication for colocalization of genetic and epigenetic loci implicated in schizophrenia (Lu et al., 2018; Hannon et al., 2016). Moreover, epigenetic loci used to predict epigenetic age do not overlap with known epigenetic loci implicated in schizophrenia (Mill et al., 2008; Hannon et al., 2016). The absence of an association between MRI brain aging and epigenetic aging has been reason to suspect distinct aging processes are involved (McKinney et al., 2017; Voisey et al., 2017; McKinney et al., 2018; Cole et al., 2018). The absence of association with Levine's epigenetic age predictor, one that also takes into account extrinsic factors of typical aging, from the results of the schizophrenia cohort 
affirms the conclusion that aging of the brain and epigenetic aging in blood might be two distinct processes in the etiology of schizophrenia, despite their commonality in predicting mortality (Cole et al., 2018; Marioni et al., 2015; Chen et al., 2016).

\subsubsection{Methodological considerations}

There are some methodological limitations that should be considered when interpreting the findings of this thesis. First, the BrainSCALE cohort was acquired on 1.5 Tesla MRI scanners that, in contrast to the 3.0 Tesla MRI scanners used in the schizophrenia cohort and the Human Connectome Project Young Adult cohort, produce images with lower signal-to-noise ratio (Frayne et al., 2003). Despite the availability of 3.0 Tesla MRI scanners at follow-up assessments, a deliberate choice was made to continue using the same 1.5 Tesla MRI scanners to minimize the impact of scanner variations on the brain measures. Second, although the BrainSCALE cohort has a decent sample size for neuroimaging, it is relatively modest for twin studies. The sample size limits the power of the genetic analyses in the ability to detect weak genetic signals, especially for noisy measurements such as resting-state functional connectivity (Boomsma et al., 2000). However, restrictions on the power of a study due to unreliable measurements can be partially lifted by the application of reliability modelling. Similarly, the progressive aging study in the schizophrenia cohort is limited in the availability of genetic and epigenetic samples. It should be noted that the cohort was not used to identify genetic or epigenetic loci related to progressive brain aging. Instead, the summary scores from large discovery samples were used to obtain the polygenic risk for schizophrenia and epigenetic aging scores used in the analyses. Third, although longitudinal statistical analyses were employed to take advantage of the repeated measurements in the BrainSCALE and the schizophrenia cohorts, the data were not processed using longitudinal pipelines. Longitudinal pipelines can help to minimize interscan variation that may arise during processing (Reuter et al., 2012; Hart et al., 2018). Finally, no longitudinal epigenetic samples were available in the schizophrenia cohort. Although epigenetic modifications are believed to be relatively stable over time (Talens et al., 2010; Shah et al., 2014), the timing of the epigenetic modifications can still play a crucial role in the manifestation of psychiatric disorders (Kofink et al., 2013).

\subsubsection{General conclusion}

Understanding typical brain development in children and adolescents is important because it provides a baseline for what is to be considered aberrant development, and helps to understand what brain structures or functioning is responsible for adolescent behavior. The inclusion of twins can determine to what extent variation in adolescent brain characteristics and behavior are determined by genetic and environmental influences. Together, these 
studies can inform what makes some children thrive and others don't, and aid in the development of diagnostic tools and interventions to assist children who are struggling.

In this thesis, it has been established that the thickness of the cerebral cortex and its rate of development during adolescence is largely under genetic control. A new wave of influences from genetic origin marks the transition during adolescence. Aberrant development is associated with psychiatric disorders such as schizophrenia. Accelerated aging of the structural brain is in part genetically determined by genes implicated for schizophrenia, but does not show a dependable association with epigenetic modifications associated with aging phenotypes. Functional connectivity in the resting brain is also under genetic control, but to a lesser extent than brain structures, and we found indication of environmental influences from familial origin. This suggests that, while the development of brain structures might be preordained based on the genetic markup inherited from the parents, brain functioning might be more flexible to extrinsic influences that could be more easily targeted by interventions.

Future studies are needed not only to extend research on adolescent brain development and behavior, but also to validate and replicate existing research findings. Large population-based cohorts can help by prospectively including subjects that have yet to develop problems. Their statistical power will help to more reliably detect associations between the adolescent brain and behavior of smaller effect sizes. Combined with a longitudinal design and twin design, these studies could validate results from this thesis, and be used to extend the results by investigating gene-environment interactions and longitudinal statistical causal modelling that typically require large sample sizes. 



\section{APPENDIX A}

Supplementary information

to chapter 2 


\section{APPENDIX A.1 SUPPLEMENTARY INFORMATION FOR CHAPTER 2}

\section{A.1.1 Exclusion criteria participants}

The BrainSCALE cohort consists of healthy typically developing children. Exclusion criteria for participation in the study were: a known major medical or psychiatric history; chronic use of medication; participation in special education; and physical or sensory disabilities; having a pacemaker; metal materials in the head with the exception for dental braces (van Soelen et al. 2012).

\section{A.1.2 Demographics table}

The demographics and zygosity of the twins for which structural MRI scans were available for use in this study is presented in Supplementary Table A.1. Zygosity of same-sex twins was determined by DNA polymorphisms. Handedness of participants was determined using the Edinburgh Handedness Inventory (Oldfield 1971) followed by classification into right-handed and non-right-handed (i.e. left-handed or ambidextrous) groups. Approximately $16 \%$ of the participants are non-right-handed.

\section{A.1.3 Longitudinal repeated measurements}

The BrainSCALE cohort is a longitudinal study with three measurements at age 9, 12 and 17 years. For this study, based on a subsample of the BrainSCALE cohort consisting of

Supplementary Table A.1. Demographics of the longitudinal twin sample from the BrainSCALE cohort based on participants included in the analyses (i.e. after exclusion of substandard structural MRI scans).

\begin{tabular}{llll}
\hline & Wave 1 & Wave 2 & Wave 3 \\
\hline $\begin{array}{l}\text { Number of participants (fe male; } \\
\text { male) }\end{array}$ & $192(99 ; 93)$ & $125(59 ; 66)$ & $165(86 ; 79)$ \\
MZ male twins (complete pairs) & $39(17)$ & $30(13)$ & $31(15)$ \\
MZ female twins (complete pairs) & $43(21)$ & $26(10)$ & $39(18)$ \\
DZ-SS male twins (complete pairs) & $39(17)$ & $23(8)$ & $33(16)$ \\
DZ-SS female twins (complete pairs) & $37(16)$ & $22(10)$ & $33(15)$ \\
DZ-OS twins (complete pairs) & $34(15)$ & $24(10)$ & $39(13)$ \\
Mean age at scan in years (range) & $9.2(9.0$ to 9.7) & $12.2(11.7$ to 13.1$)$ & $17.2(16.8$ to 17.9) \\
Handedness (right; non-right) & $160 ; 32$ & $105 ; 20$ & $140 ; 23$ \\
& & & \\
\hline
\end{tabular}

Abbreviations: $M Z$ = monozygotic; DZ-SS = same-sex dizygotic; DZ-OS = opposite-sex dizygotic. 
twins only, 192 participants had good quality structural MRI scans available at baseline measurement, 125 participants at the first follow-up, and 165 participants at the second follow-up (Figure A.1). The temporary decline in available structural MRI scans for the second wave is largely due to exclusion of participants with dental braces incompatible with the magnetic field of the MR scanner; see Image Processing section from Chapter 2 for details on exclusion of substandard MRI scans.

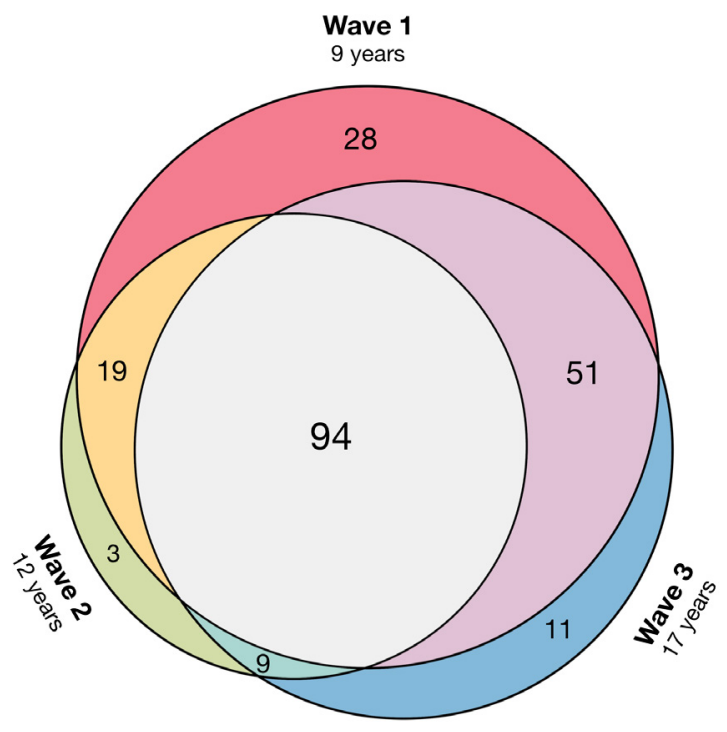

Supplementary Figure A.1. Venn diagram of overlap in participants with good quality structural MRI scans between the three waves. Numbers reported are individual participants; e.g., the number 94 at the center of the overlap between the three circles indicates that 94 individuals for which good quality structural MRI scans were available in all three waves, and $19+94=113$ individuals with good quality structural MRI scans available for both wave 1 and wave 2, etcetera. Total number of unique individuals across the three waves is 215 .

\section{A.1.4 Path diagram of bivariate twin model}

The path diagram for the bivariate model used to determine the overlap between genetic and environmental factors of cortical regions is presented in Supplementary Figure A.2. 


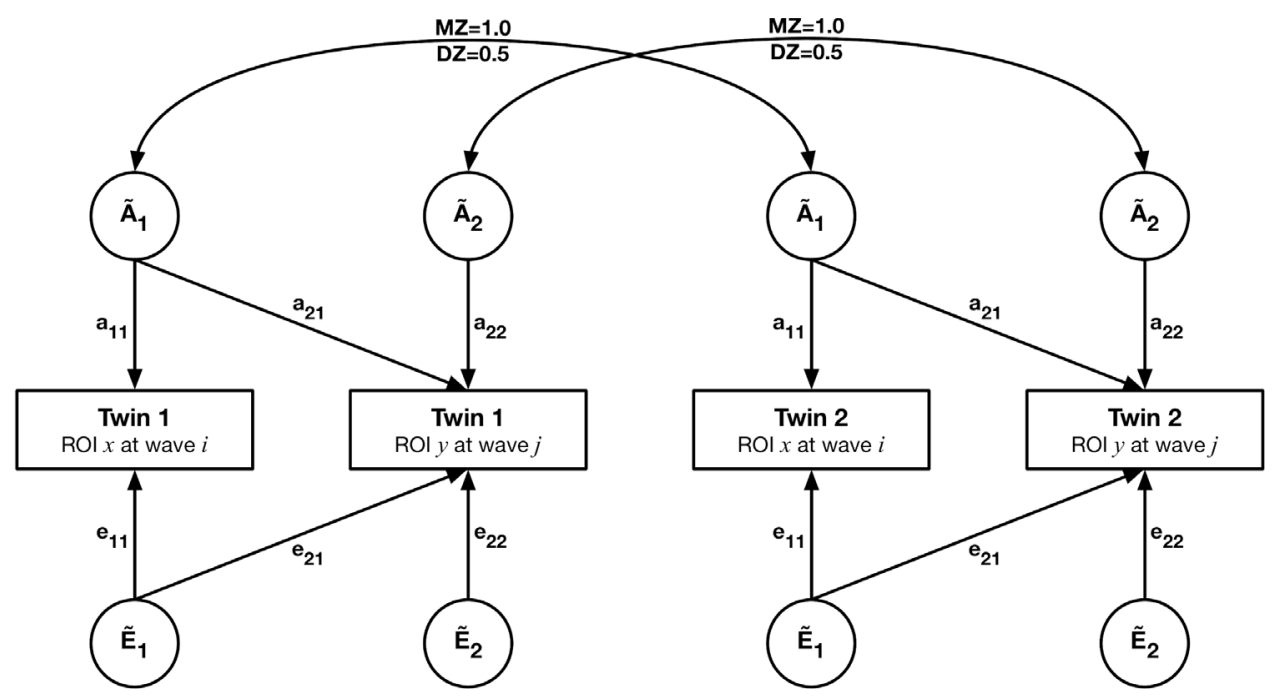

Supplementary Figure A.2. Path diagram of bivariate $A E$ model used to determine the overlap between genetic factors of cortical regions across space and time. Cortical thickness measurements for two regions of interest $x$ at wave $i$ and region $y$ at wave $j$ are used as observed variables (rectangular boxes) for the first (left half of path diagram) and the second twin (right half of path diagram). Independent genetic factors $\widehat{A_{1}}$ and $\widehat{A_{2}}$ (circles) load onto the cortical thickness measurements through path coefficients. The genetic factor $\widehat{A_{1}}$ represents genetic influences shared between both regions through path coefficients $a_{11}$ and $a_{21}$. Genetic factor $\widehat{A_{2}}$ represents genetic influences specific for one of the regions through path coefficient $a_{22}$. The same motif applies for the unique environmental factors $\widehat{E_{1}}$ and $\widehat{E_{2}}$, and path coefficients $e_{11}, e_{21}$, and $e_{22}$. The model is made identifiable by constraining the correlation between genetic factors of both twins to 1.0 in case of monozygotic twins and 0.5 in case of dizygotic twins.

\section{A.1.5 Interpolation of cortical thickness at intermediate ages}

We fitted a cubic spline to local cortical thickness measurements at each vertex on the surface mesh of the 3D model brain. Anchor points for the cubic spline were defined at the mean age of the three measurements (i.e. at age 9.2, 12.2, and 17.2 years). The cubic spline models were used to estimate local cortical thickness measurements between the ages 9.0 and 18.0 years at 0.01 -year increments.

\section{APPENDIX A.2 SUPPLEMENTARY RESULTS FROM CHAPTER 2}

\section{A.2.1 Development of cortical thickness across adolescence}

We fitted cubic spline models to local cortical thickness at each vertex to reconstruct estimates of local mean cortical thickness at intermediate ages (Video S1 ${ }^{5}$ ). It reveals a gradual decline in cortical thickness for most of the cortex with age, and accelerated cortical thinning at medial frontal cortical regions from around age 12 to 13 years onward.

\footnotetext{
${ }^{5}$ Online supplementary data: https://dx.doi.org/10.1093/cercor/bhy005
} 


\section{A.2.2 Heritability of cortical thickness}

We obtained estimates for heritability of cortical thickness by fitting a longitudinal twin model to the cortical thickness measurements (Supplementary Figure A.3). Regionally, heritability of cortical thickness varies throughout the cortex, with $72.6 \%$ of the vertices showing significant heritability for age 9 years (FDR-corrected $p<0.05$ ), and $66.1 \%$ and $69.5 \%$ of the vertices for ages 12 and 17 years respectively (Supplementary Figure A.3). Heritability of cortical thickness is highest at the primary sensory and motor cortices, temporal cortices, and midsagittal areas, including frontal poles, cingulum, and occipital cortex across the age range, with local maxima up to $84 \%$ heritability (Supplementary Figure A.3).

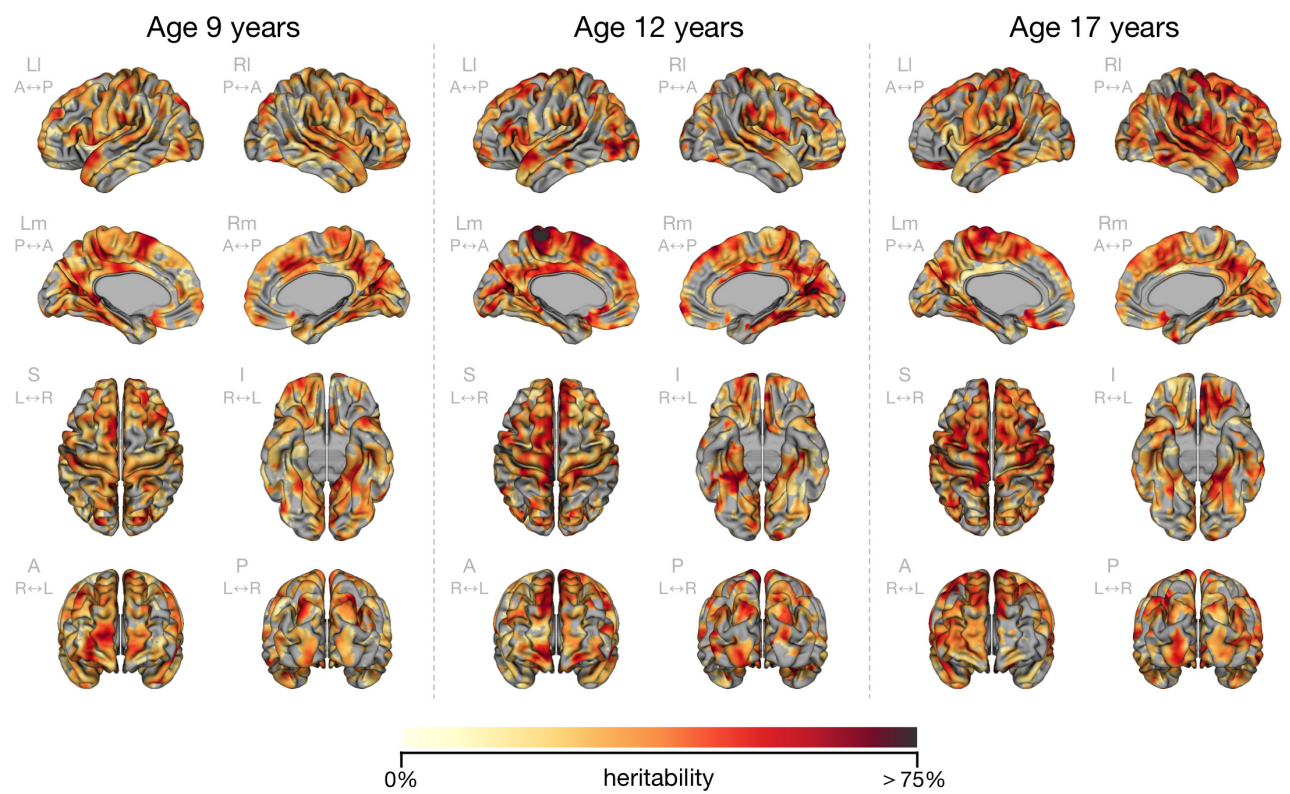

Supplementary Figure A.3. Estimated heritability of cortical thickness across the three ages 9 years (left panel), 12 years (middle panel), and 17 years (right panel). Heritability estimates that did not differ significantly from zero (FDR adjusted $p>0.05$ ) have been left grey. Approximately $72.6 \%$ of the vertices at age 9 years, $66.1 \%$ of the vertices at age 12 years, and $69.5 \%$ of the vertices at age 17 years are significant for heritability of cortical thickness (FDR adjusted $p<0.05$ ). Heritability ranges from $0 \%$ (white-yellow) up to $75 \%$ or greater (black-red). Order of views per age, from left to right, top to bottom: left lateral (Ll), right lateral (Rl), left medial (Lm), right medial $(\mathrm{Rm})$, superior $(\mathrm{S})$, inferior $(\mathrm{I})$, anterior $(\mathrm{A})$, and posterior $(\mathrm{P})$.

\section{A.2.3 Development and heritability of cortical thickness at regional level}

We applied the longitudinal twin model described in the Methods sections of Chapter 2 to the observed cortical thickness measurements at whole-brain and lobar level. In general, the model with additive $(\hat{A})$ and unique environmental $(\hat{E})$ factors fitted the data best, with exception for the right temporal lobe, right occipital lobe, right cingulate cortex, and bilateral insula for which the model with only unique environmental $(\widehat{E})$ factors fitted the 
data best (Supplementary Table A.2). Augmented by the fact that evidence for common environmental influences on cortical thickness in the literature is limited, we adopted the $A E$ model in all our analyses. The estimated cortical thickness, heritability, and changes in cortical thickness and heritability of changes in cortical thickness at the whole-brain and lobar level are presented in Supplementary Table A.3.

\section{A.2.4 Determining the optimal number of clusters for hierarchical clustering}

We applied hierarchical clustering to the phenotypic, genetic, and environmental correlation matrices using the cluster package version 2.0.4 in R (Maechler et al. 2016). Correlation matrices were transformed to distance matrices using $1-r_{p h}$ for the phenotypic correlation matrix, $1-\left|r_{g}\right|$ for the genotypic correlation matrix, and $1-\left|r_{e}\right|$ for the environmental correlation matrix. The hierarchical ordering of the regions into clusters was obtained using the "ward.D2" linkage method for both the phenotypic and genetic correlation matrices.

The average silhouette width (Rousseeuw 1987) was computed for each configuration of clusters obtained from hierarchical clustering of the phenotypic and genetic correlation matrices (Supplementary Figure A.4). A typical heuristic to determine the number of clusters $k_{\max }$ is to maximize the average silhouette width. For clustering of the phenotypic correlation matrix, the maximal number of clusters is $k_{\max }=10$, for the genetic correlation matrix $k_{\max }=12$, and for the environmental correlation matrix $k_{\max }=8$ (Supplementary Figure A.4). However, to account for model complexity, the optimal number of clusters $k_{\text {opt }}$ used in the analysis is determined as the lowest value of $k$ with average silhouette width within one standard error below the average silhouette width for the maximal number of clusters $k_{\max }$. For clustering of the phenotypic correlation matrix, the optimal number of clusters is $k_{o p t}=6$, for the genetic correlation matrix $k_{o p t}=7$, and for the environmental correlation matrix $k_{o p t}=6$ (Supplementary Figure A.4). Although we select an optimal number of clusters based on a widely-accepted selection criteria, we believe the sequence in which clusters are formed presents a more meaningful insight into the relation between regions than a single representation of an optimal number of clusters. We have therefore provided the full dendrogram of the clustering algorithm from which the clustering at any value of $k$ can be determined (Figure 2.4 from Chapter 2). 


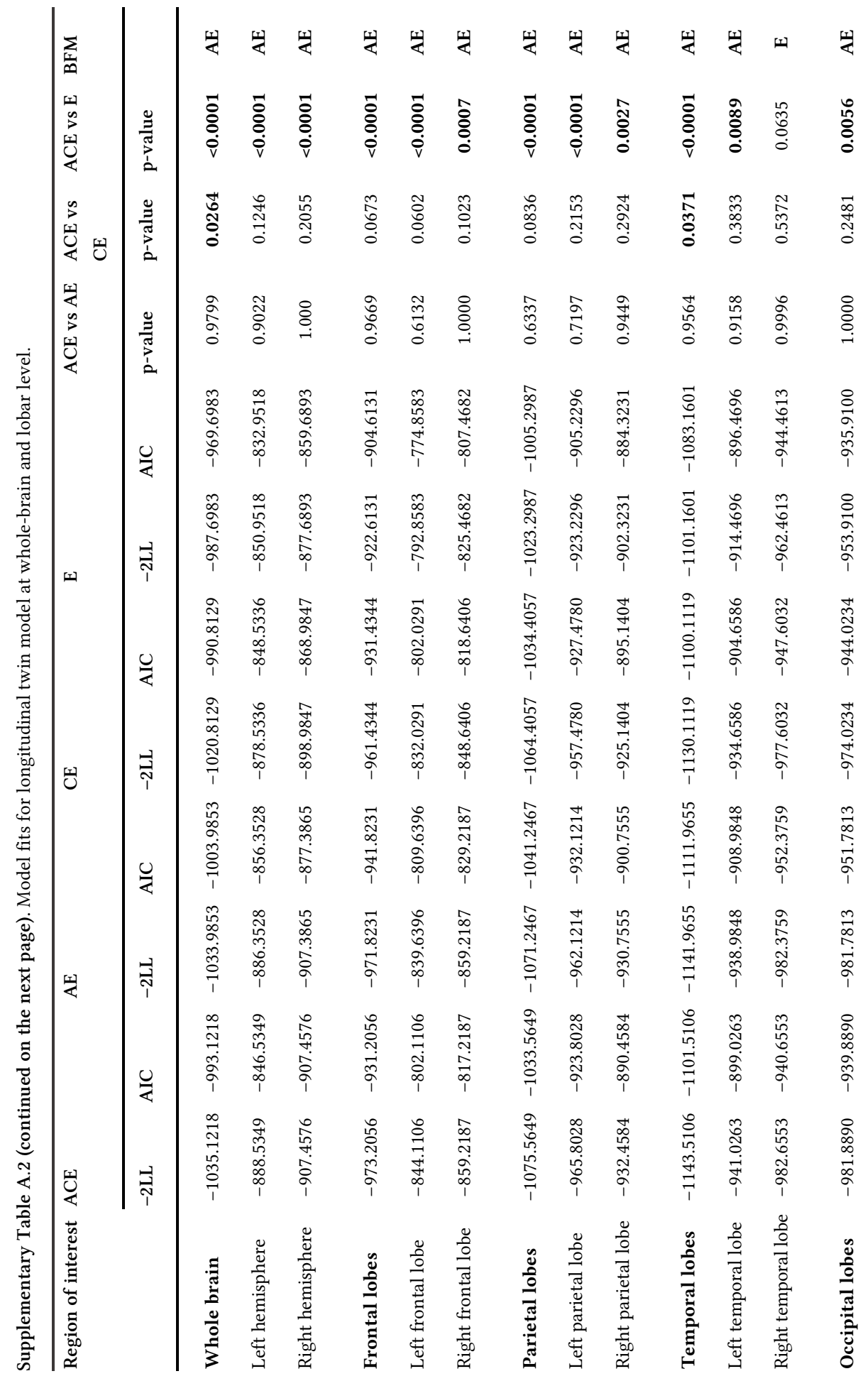




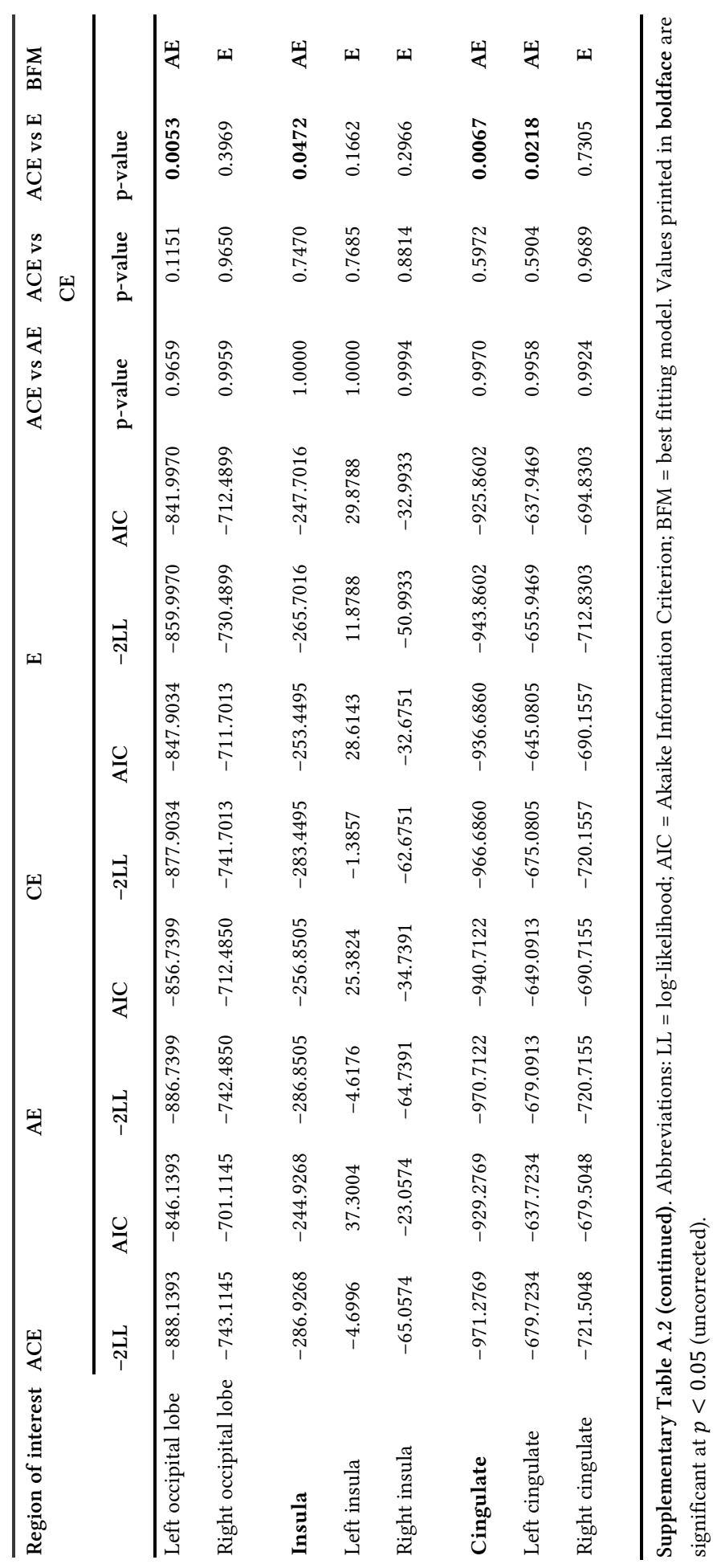




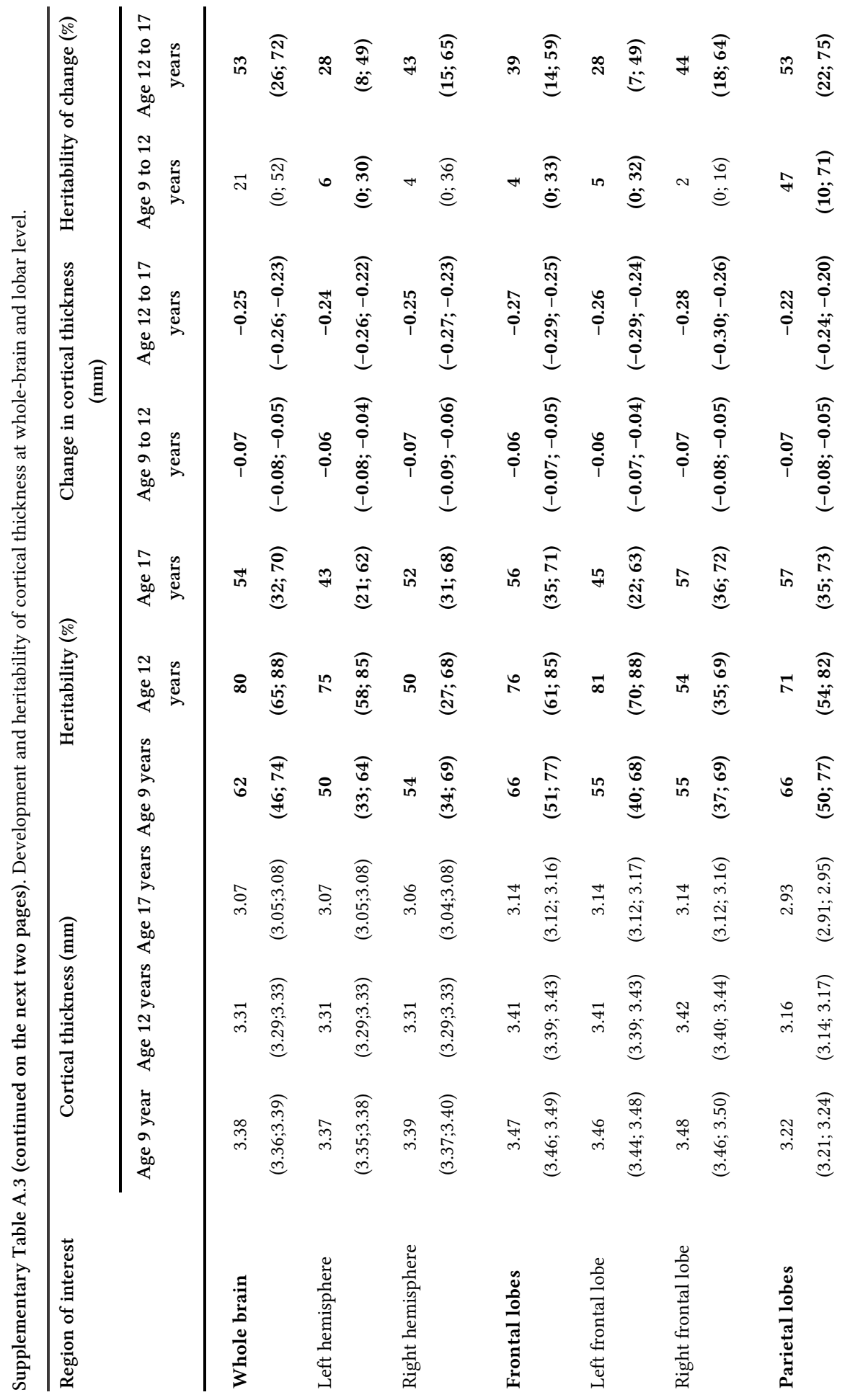




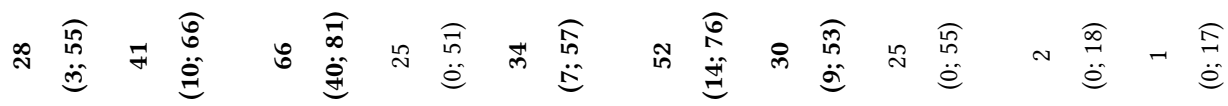

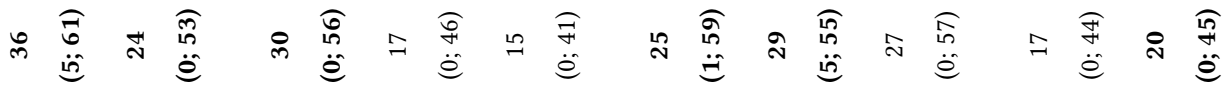

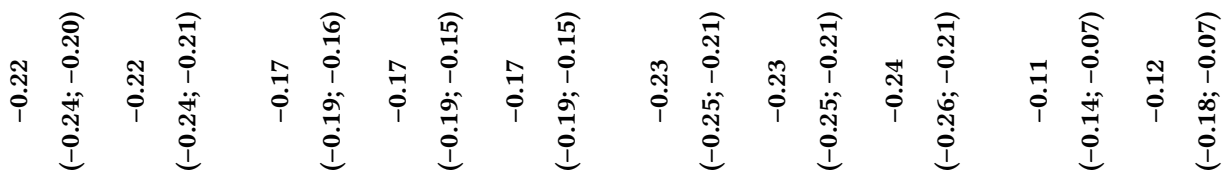

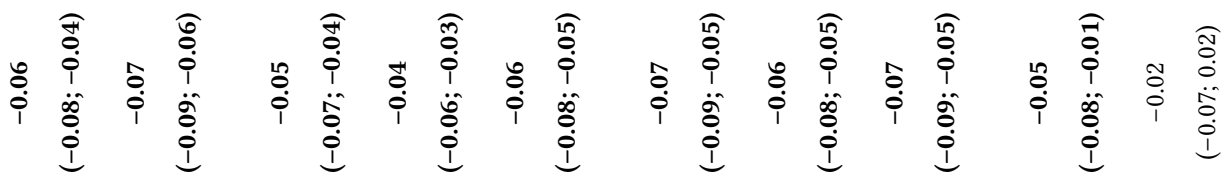

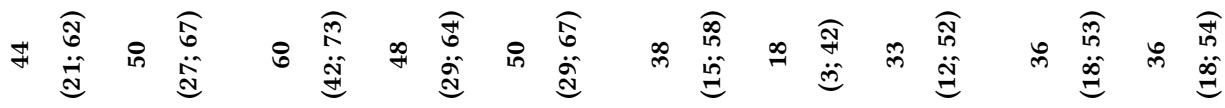

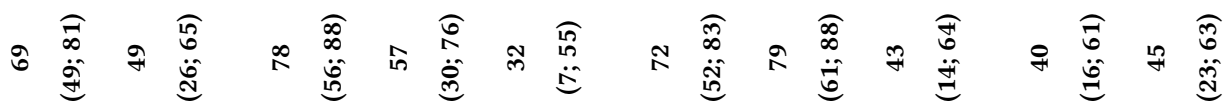

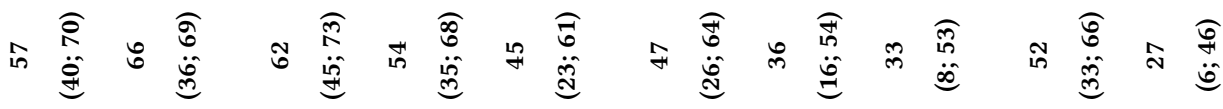

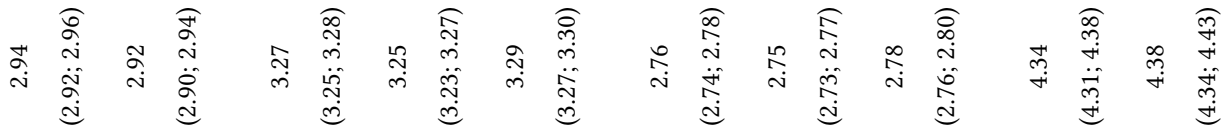

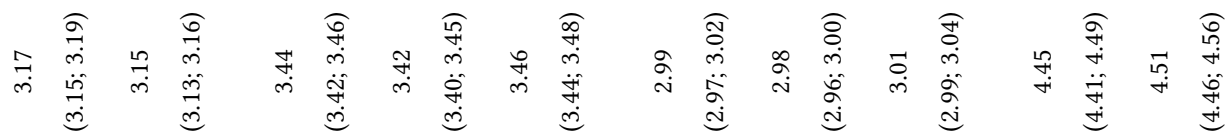

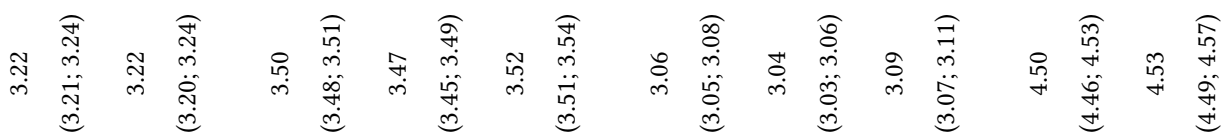

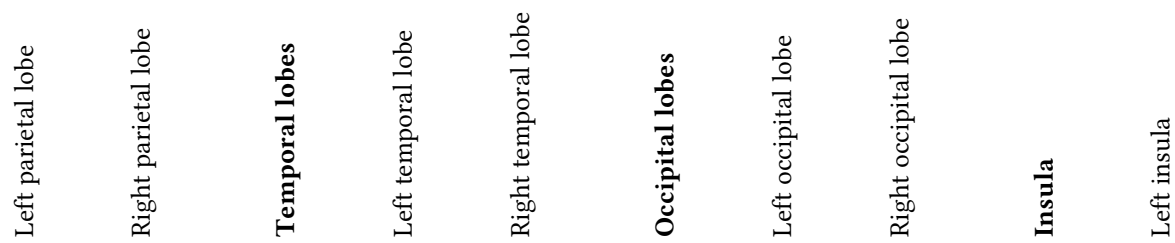




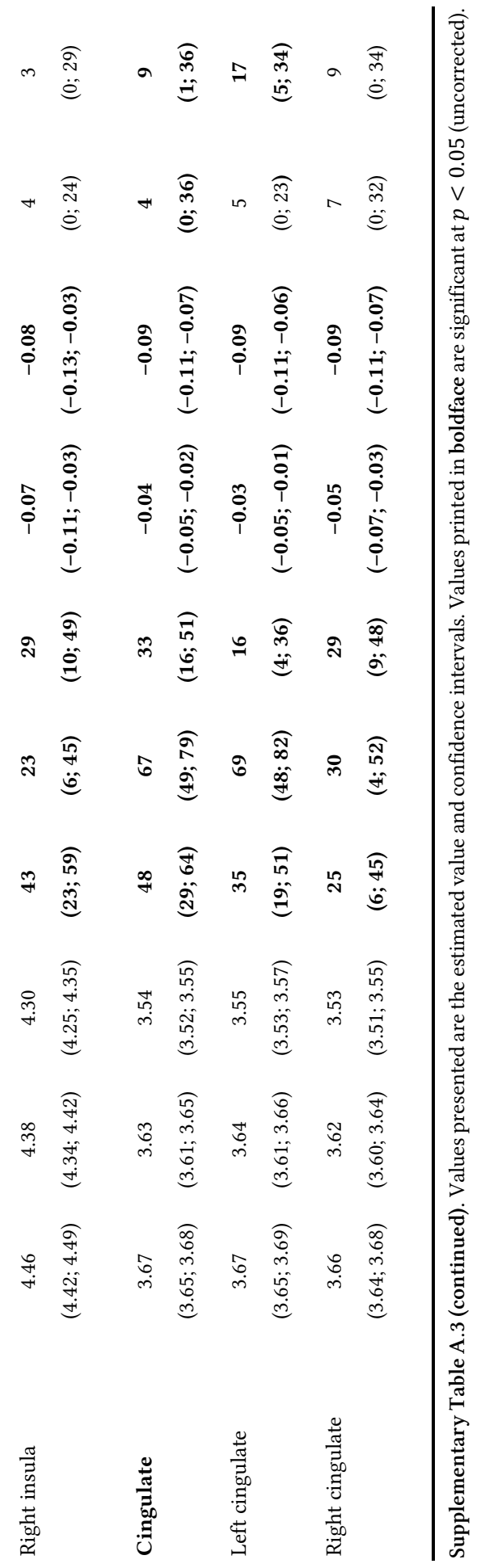


Silhouette coefficients for clustering of phenotypic correlation matrix

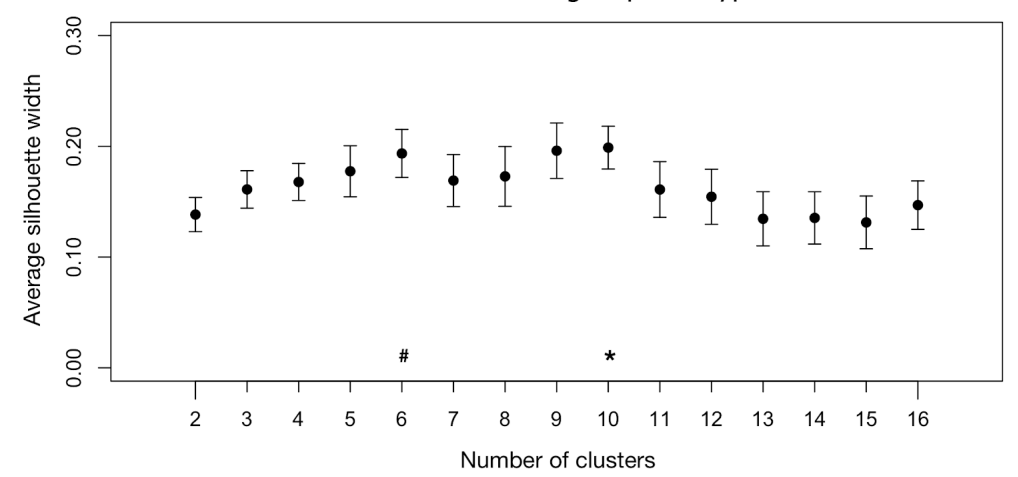

Silhouette coefficients for clustering of genetic correlation matrix

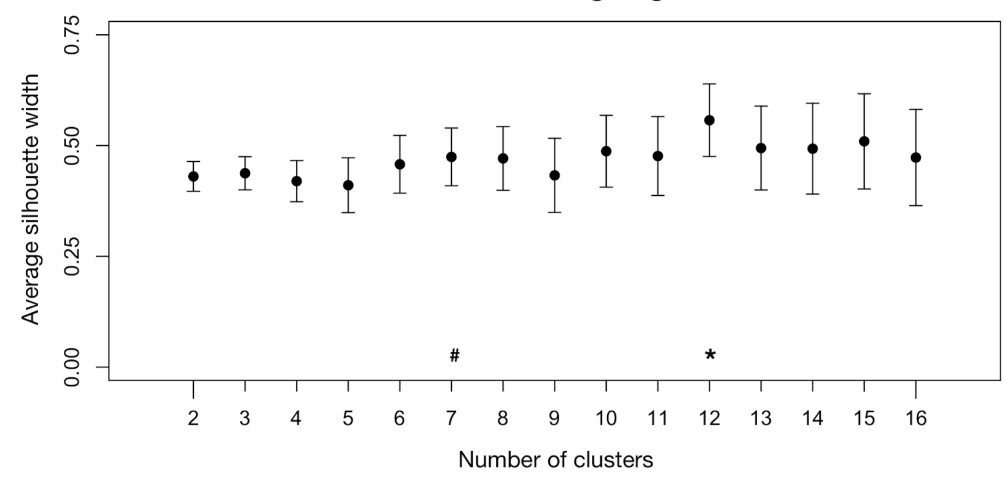

Silhouette coefficients for clustering of environmental correlation matrix

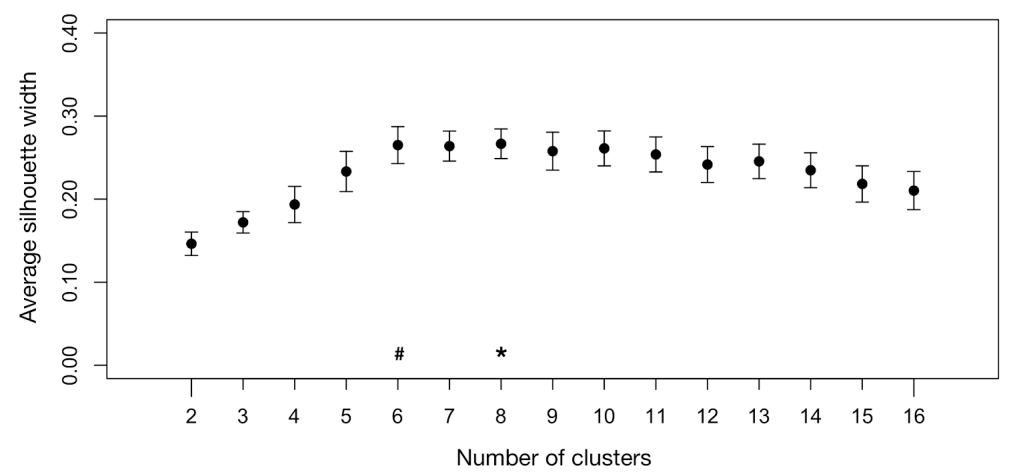

Supplementary Figure A.4. The average silhouette width for each configuration of clusters obtained from hierarchical clustering of the phenotypic, genetic, and environmental correlation matrices. Solid dots represent the average silhouette width, and the error bars represent the standard error of the mean. The number of clusters $k_{\max }$ (marked with an asterisk *) is determined by the number of clusters that maximizes the average silhouette width. The number of clusters $k_{\text {opt }}$ used in the analysis (marked with a hash mark \#) is determined by the minimum value of $k$ for which the average silhouette width is within one standard error below the average silhouette width of $k_{\max }$ to account for model complexity due to the increasing number of clusters. 


\section{A.2.5 Hierarchical clustering of correlation matrices at select ages}

We repeated the hierarchical clustering of the phenotypic, genetic, and environmental correlation matrices using different combinations of ages. Clustering of correlation matrices at each individual age reveals clear patterns where phenotypic and environmental correlations are initially clustered by hemisphere (Supplementary Figure A.5).
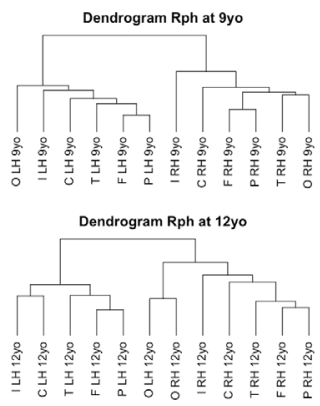

Dendrogram Rph at 17 yo

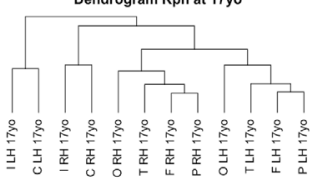

Dendrogram Rph at 9\&12yo

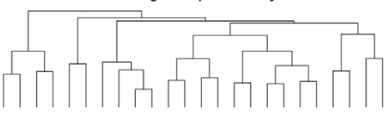

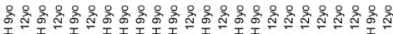

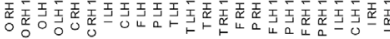

Dendrogram Rph at 9\&.17yo

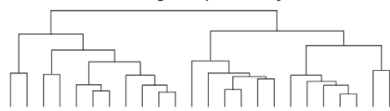

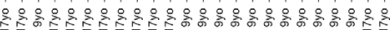

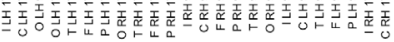

Dendrogram Rph at 12817 yo

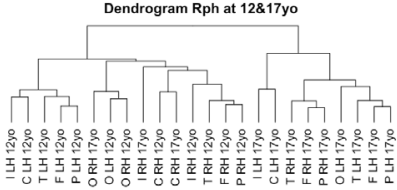

Dendrogram Rg at 9yo

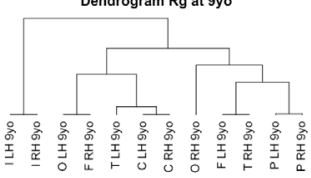

Dendrogram $\mathrm{Rg}$ at $12 \mathrm{yo}_{0}$

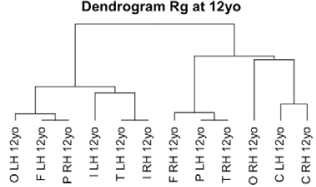

Dendrogram Rg at $17 y 0$

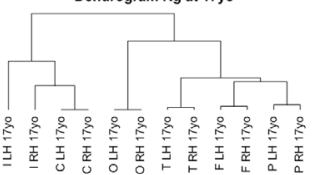

Dendrogram Rg at $9 \& 12 y \circ$
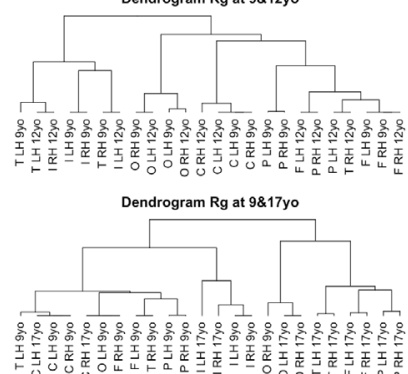

Dendrogram Rg at $12 \& 17$ yo

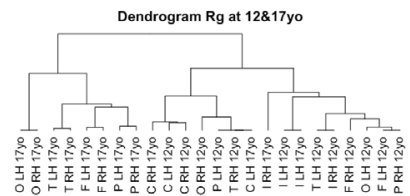

Dendrogram Re at 9yo

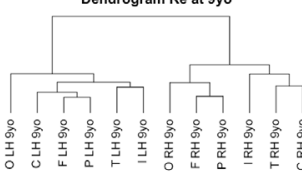

Dendrogram Re at $12 y o$

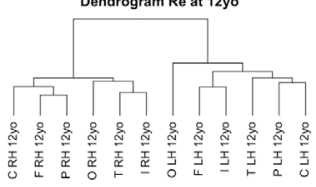

Dendrogram Re at 17yo

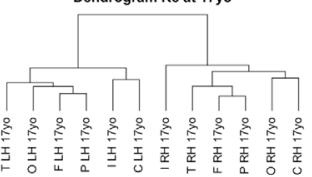

Dendrogram Re at $9 \& 12 y \circ$

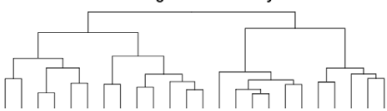

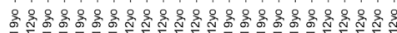

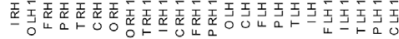
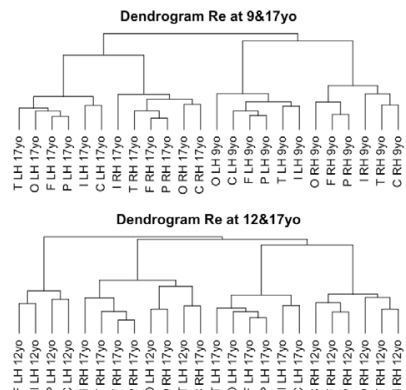

Supplementary Figure A.5. Hierarchical clustering of the phenotypic $\left(r_{p h}\right.$; left), genetic $\left(r_{g}\right.$; middle), and environmental $\left(r_{e} ;\right.$ right) correlation matrices at select ages. Phenotypic correlations were transformed using one minus the phenotypic correlation, and genetic and environmental correlations were transformed using one minus the absolute of the genetic or environmental correlation. Labels for the regions are encoded as lobe $(\mathrm{F}=$ frontal; $\mathrm{P}=$ parietal; $\mathrm{T}=$ temporal; $\mathrm{O}=$ occipital; $\mathrm{I}=$ insula; and $\mathrm{C}=$ cingulate), followed by hemisphere $(\mathrm{LH}=$ left hemisphere; and $\mathrm{RH}=$ right hemisphere), and finally age (9yo = age 9 years; 12 yo = age 12 years; and 17yo = age 17 years). 
Subsequent divisions result in clusters where the frontal, parietal, and temporal lobes are grouped together, the insula and cingulate are grouped together, and the occipital lobe is shared between these two groups. Clustering of the genetic correlation matrix reveals a similar grouping of the lobar regions, where a high number of clusters homologous regions between the two hemispheres form a cluster rather than being separated in the first step of the clustering algorithm (Supplementary Figure A.5). Clustering of correlation matrices including two ages reveal similar patterns where regions of the same age are grouped together (Supplementary Figure A.5). Most notable exceptions regarding clustering of regions of the same age occur mostly for phenotypic and genetic correlations between age 9 and 12 years when changes in phenotype and genotype are subtle, as is reflected by the high correlation between regions and across the two ages (Figure 2.4 from Chapter 2).

\section{A.2.6 Qualitative evaluation of sex, handedness, and age at scan}

We performed qualitative evaluation of the effects of sex, handedness, and age at scan on estimates from the bivariate model for phenotypic, genetic, and environmental correlation matrices (Supplementary Figure A.6), and estimates from the longitudinal model for heritability of changes in cortical thickness and its decomposition into genetic innovation and (de)amplification (Supplementary Figure A.7). The covariates were regressed from the cortical thickness measures prior to modelling. Overall, the correction for covariates slightly reduces the estimates for heritability of changes in cortical thickness accompanied by a slight reduction in size of clusters, but the larger, most prominent clusters remain present (Supplementary Figure A.7 compared to Figure 2.5 from Chapter 2).
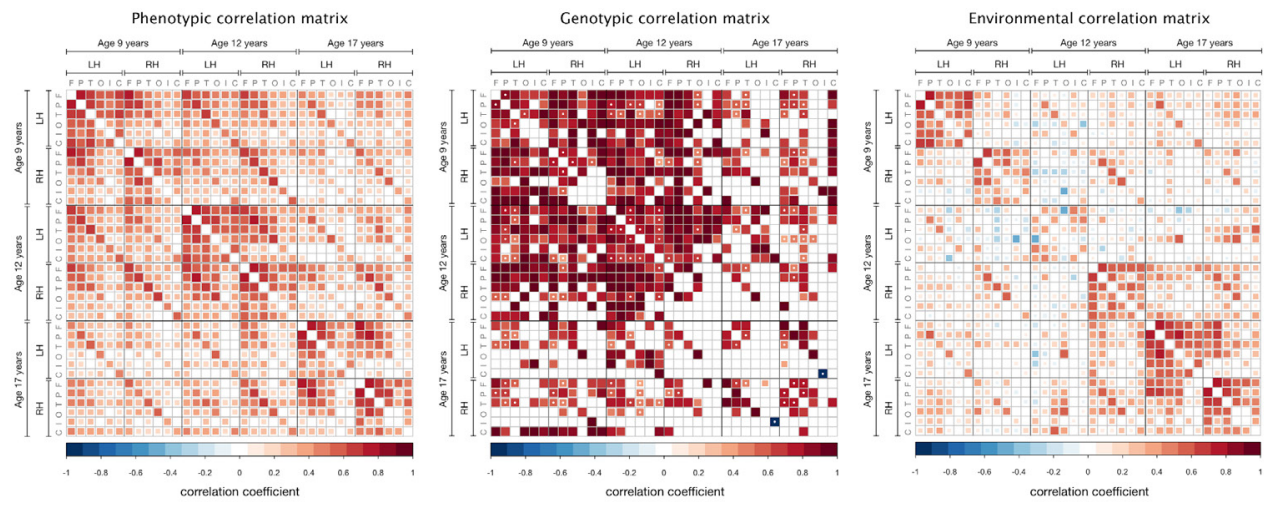

Supplementary Figure A.6. Phenotypic (left), genetic (middle), and environmental (right) correlation matrices of absolute cortical thickness after correction for sex, handedness, and age at scan between major lobes of the cortex. Correlations range from -1 (blue) to +1 (red). Correlations that did not significantly differ from zero $(p>0.05$; uncorrected) are left blank. Genetic correlations marked with a white dot indicate incomplete pleiotropy (i.e. unique genetic factor for either region in addition to a shared genetic factor between both regions). Regions are ordered from top-left to bottom-right, first by age $(9,12$, and 17 years), then by hemisphere ( $\mathrm{LH}=$ left hemisphere; and $\mathrm{RH}=\mathrm{right}$ hemisphere), and finally by lobe $(\mathrm{F}=$ frontal; $\mathrm{P}=$ parietal; $\mathrm{T}=$ temporal; $\mathrm{O}=$ occipital; $\mathrm{I}=$ insula; and $\mathrm{C}=$ cingulate $)$. 
a

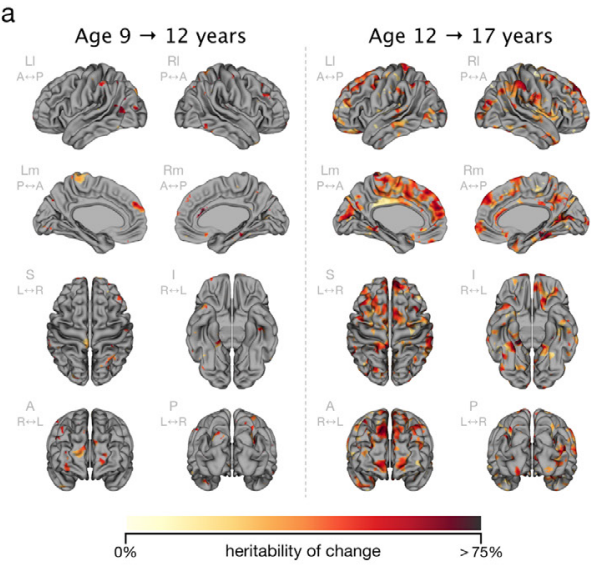

b

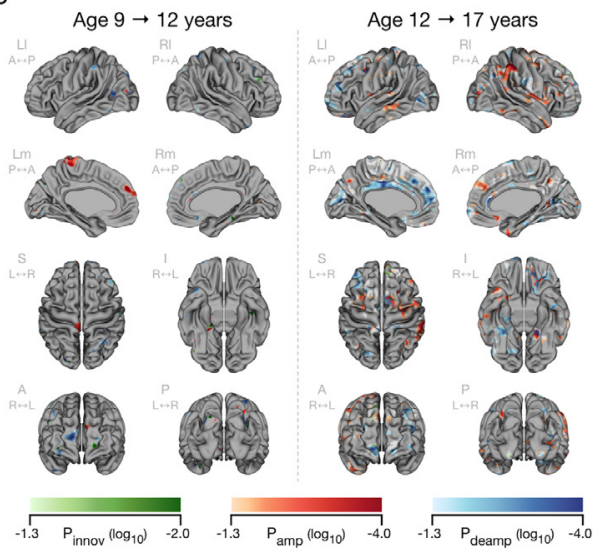

Supplementary Figure A.7. Estimated heritability of (a) changes in cortical thickness and (b) its decomposition into different genetic origins between the ages 9 and 12 years (left panel), and between the ages 12 and 17 years (right panel) after correction for sex, handedness, and age at scan. (a) Heritability estimates that did not differ significantly from zero (FDR adjusted $p>0.05$ ) have been left grey. Approximately $2.4 \%$ of the vertices between age 9 and 12 years and $20.8 \%$ of the vertices between age 12 and 17 years are significant for heritability of changes in cortical thickness (FDR adjusted $p<0.05$ ). Heritability estimates range from $0 \%$ (light-yellow) up to $75 \%$ or greater (dark-red). (b) Heritability of changes in cortical thickness between the ages 9 and 12 years (left panel) and between the ages 12 and 17 years (right panel) was decomposed into sources of genetic innovation (green; $13.5 \%$ of the vertices significant for heritability of changes in cortical thickness between the ages 9 and 12 years, and $0.8 \%$ between the ages 12 and 17 years), and areas with amplification (red; $25.0 \%$ of the vertices between the ages 9 and 12 years, and 30.6\% between the ages 12 and 17 years) or deamplification (blue; $60.7 \%$ of the vertices between the ages 9 and 12 years, and 33.0\% between the ages 12 and 17 years) of a genetic factor across age. Areas with significant heritability of changes in cortical thickness for which these sources could not be disentangled are depicted in light grey (remaining $0.8 \%$ of the vertices significant for heritability of changes in cortical thickness between the ages 9 and 12 years, and 35.6\% between the ages 12 and 17 years). Scale bars for significance start at $\log _{10}$-equivalent of $p=0.05$. $(\mathbf{a}, \mathbf{b})$ Order of views per age, from left to right, top to bottom: left lateral (Ll), right lateral (Rl), left medial (Lm), right medial (Rm), superior (S), inferior (I), anterior (A), and posterior (P).

The effect of covariates on the correlation matrices is subtle resulting in minor shifts in correlation estimates between regions across age (Supplementary Figure A.6 compared to Figure 2.4 from Chapter 2). Particularly the environmental correlations between the frontal, parietal, temporal, and occipital lobes across hemispheres at 17 years become more pronounced. For the genetic correlation matrix, some associations disappear as they drop below the significance threshold (e.g. for the insular and cingulate cortex), whereas other associations appear as they now pass the significance threshold. The overall picture remains the same, including the pattern for incomplete pleiotropy between regions and across time, with only subtle changes due to the inclusion of the covariates. 



\section{APPENDIX B}

\section{Supplementary information to chapter 3}




\section{APPENDIX B.1 SUPPLEMENTARY METHODS FROM CHAPTER 3}

\section{B.1.1 MRI scan acquisition parameters of Human Connectome Project dataset}

The MRI acquisition parameters of the Human Connectome Project (van Essen et al., 2013) have been described elsewhere (Glasser et al., 2013). In brief, high-resolution structural MRI scans were acquired on a 3 Tesla MRI scanner using a 3D MPRAGE pulse sequence (TR = $2400 \mathrm{~ms} ; \mathrm{TE}=2.14 \mathrm{~ms} ; \mathrm{TI}=1000 \mathrm{~ms} ;$ flip angle $=8^{\circ} ; \mathrm{FOV}=224 \mathrm{x} 224 \mathrm{~mm}$; reconstructed voxel size $=0.7 \mathrm{~mm}$ isotropic; bandwidth $=210 \mathrm{~Hz} / \mathrm{Px}$, iPAT $=2$; acquisition time 7 minutes and 40 seconds). Resting-state functional MRI scans were acquired on the same MRI scanner using a multiband gradient-echo EPI pulse sequence $(\mathrm{TR}=720 \mathrm{~ms}$; $\mathrm{TE}=33.1 \mathrm{~ms}$; flip angle $=52^{\circ} ; \mathrm{FOV}=208 \times 180 \mathrm{~mm} ; 72$ slices; reconstructed voxel size $=2.0 \mathrm{~mm}$ isotropic; multiband factor $=8$; echo spacing $=0.58 \mathrm{~ms}$; bandwidth $=2290 \mathrm{~Hz} / \mathrm{Px}$ ). Four runs of 1200 volumes per run (approximately 15 minutes per run) were acquired for each subject across two sessions. Each session, the phase encoding direction was alternated between right-toleft for the first run and left-to-right for the second run. More details on the resting-state MRI acquisition protocol are provided in Smith et al., 2013; Ugurbil et al., 2013).

\section{B.1.2 MRI processing pipeline for resting-state fMRI of the Human Connectome Project} The extensively processed $\mathrm{FMRI}$ data package provides precomputed denoised BOLD signal time series for nodes in the brain based on group-ICA decomposition of the data at various decomposition levels for the resting-state functional MRI scans of the Human Connectome Project. The acquisition parameters and processing of this data have been described elsewhere (Smith et al., 2013; Glasser et al., 2013). Briefly, one hour of resting-state fMRI data was collected for each participant in four runs of 1200 volumes per run (effective TR = $720 \mathrm{~ms}$; total run length approximately 15 minutes per run) during two separate scan sessions across two days. Each 15-minute run was preprocessed (Glasser et al., 2013) and had artefacts removed using ICA+FIX (Salimi-Khorshidi et al., 2014; Griffanti et al., 2014). Cortical surfaces were aligned to a common template using areal-feature-based alignment and the Multimodal Surface Matching algorithm (MSMAll; Robinson et al., 2014; Glasser et al., 2016). MELODIC's Incremental Group-PCA (MIGP; Smith et al., 2014) was applied to temporally demeaned and variance normalised datasets (Beckmann and Smith, 2004) to generate the top 4500 weighted spatial eigenvectors. The group-PCA output was then decomposed into spatially independent components at several different dimensionalities (d=15, 25, 50, 100, 200, or 300) by group-ICA using FSL's MELODIC tool (Hyvärinen et al., 1999, Beckmann and Smith, 2004). The ICA decomposition was performed on grayordinates that consist of cortical grey matter vertices and subcortical grey matter voxels (Glasser et al., 2013). Finally, individual BOLD time series were obtained for each ICA component (considered a 'node' in the network) using the standard "dual regression-stage-1" 
approach (Filippini et al., 2009). Note that two versions of the HCP processing pipeline were used on this dataset.

B.1.3 Overview of the 50 independent components from the decomposition of the resting-state data

The extensively processed fMRI data package of the Human Connectome Project provides BOLD time series data for 'nodes' in the brain. These nodes were determined by groupICA decomposition of the resting-state functional MRI data that was performed for several dimensionalities $(d=25,50,100,200,300)$. In the analyses, the decomposition at $d=50$ was used. Nodes were organized into their Louvain community order based on the similarity in group-level mean functional connectivity patterns between nodes, thereby clustering related nodes together (Supplementary Figure B.1). The same order of the nodes is used in the matrix visualizations presented in this supplementary information document.

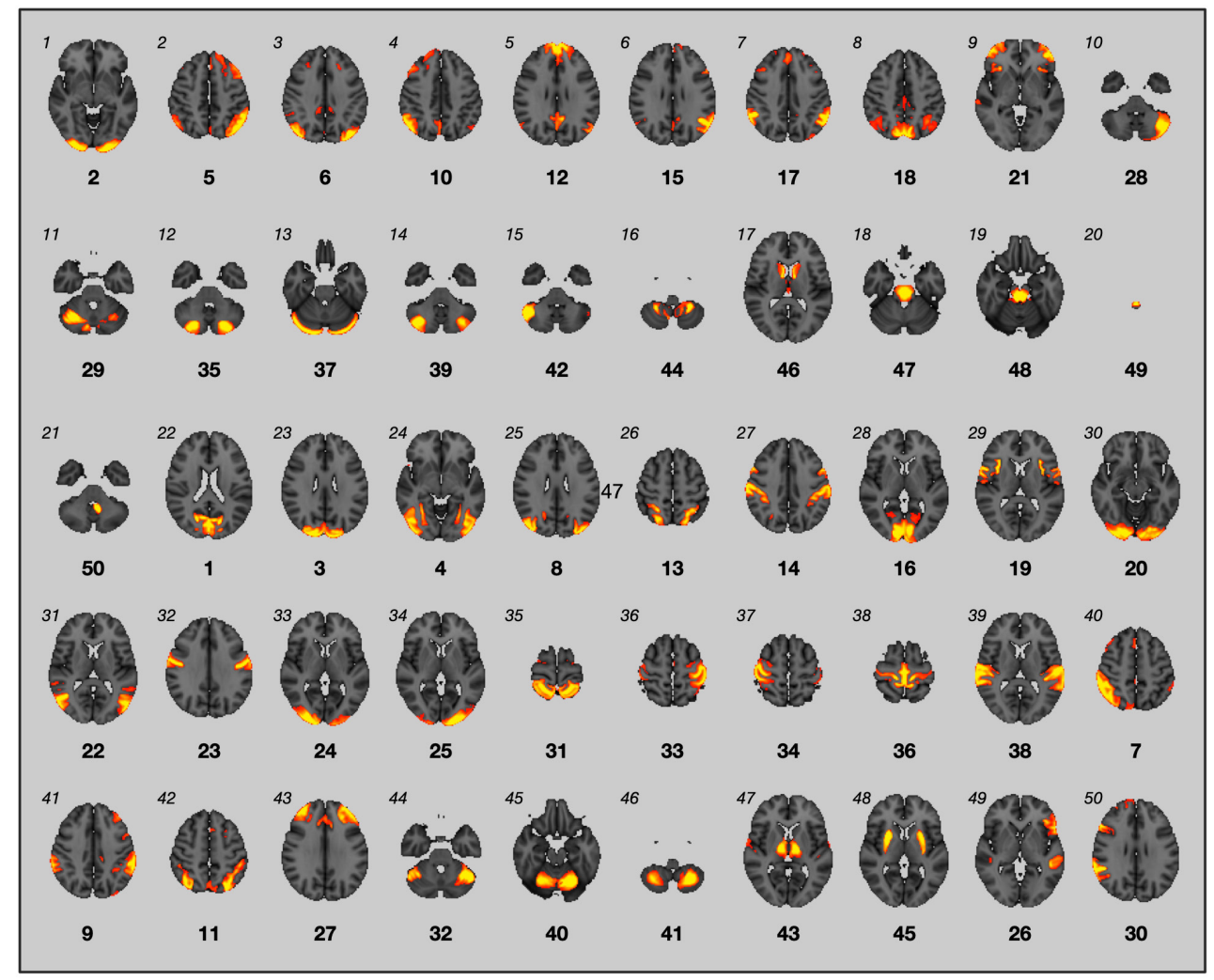

Supplementary Figure B.1. Overview of the 50 components derived by independent component analysis of restingstate functional MRI data. Components are ordered (left-to-right, top-to-bottom) based on their Louvain community structure. Components are visualized on the axial slice with highest likelihood (yellow color) for the localization of the component. Images of components were provided with the extensively preprocessed fMRI data package. 


\section{B.1.4 Approximation of in-scanner head motion}

We approximated in-scanner head motion of participants using the six registration parameters from the intra-subject re-alignment procedure applied during minimal preprocessing. Mean framewise displacement was calculated by averaging the sum of absolute transformation parameters across the length of the time series (Power et al., 2012).

\section{Distribution of in-scanner head motion}

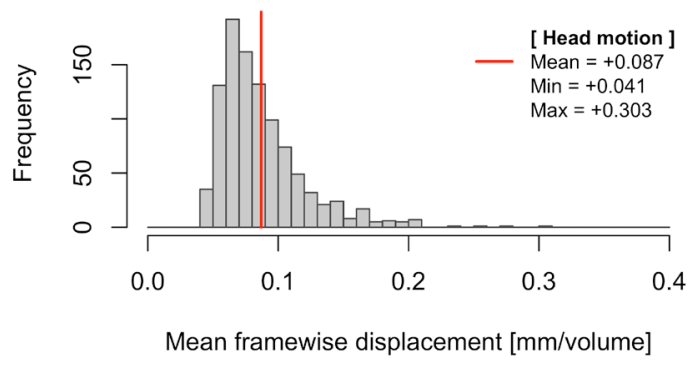

Supplementary Figure B.2. Distribution of in-scanner head motion.

B.1.5 Group-level mean functional connectivity matrices and hierarchical organization Group mean functional connectivity matrices were computed by averaging functional connectivity estimates between all pairs of nodes over all subjects for each pair of nodes. The matrices were reorganized into Louvain community structures using the igraph software package (Csardi and Nepusz, 2006) and visualized using the corrplot software package (Wei and Simko, 2017). The hierarchical ordering of the nodes at full-score functional connectivity was subsequently applied to all further visualizations for ease of comparison. All statistical and mathematical operations on functional connectivity are performed on Fisher's r-to-Z transformed functional connectivity estimates. Consistency of group-mean functional connectivity is estimated by the Pearson correlation coefficient (rho) and the intraclass correlation coefficient with a two-way mixed effects model for a single rater (ICC3,1; Shrout and Fleiss, 1979) computed using the R package irr (Gamer et al., 2019).

\section{B.1.6 Selection of the seven representative traits}

The Human Connectome Project dataset provides an extensive set of traits across various domains of human behavior. Endocrinological, sociodemographic, family history of neurological or psychiatric disorders, substance usage, raw test scores (including test scores prior to age adjustment), answers to questionnaires, measures with highly skewed distribution (e.g. due to boundary effects) and measures with fewer respondents than $50 \%$ of the total sample size have been excluded. In addition, only measures that were continuous or ordinal in nature were selected, providing a total of 110 traits. No transformations have been applied to address possible non-normality in the distributions of the traits. 
A preliminary sweep was performed on a set of 110 of the traits to determine the extent of their association with full-score functional connectivity using Pearson's correlation after regression of sex, age, head motion, and processing pipeline version as covariates of no interest on the means of the functional connectivity estimate and sex and age on the means of the trait in the standard behavioral association model (Figure 3.1E from Chapter 3).

Seven of the 110 traits from various domains were selected for extensive analysis based on their association strength with functional connectivity: five traits (BPDiastolic - diastolic blood pressure levels; CogTotalComp_AgeAdj - total composite score on cognition adjusted for age; WM_Task_2bk_Acc - accuracy on all condition in the 2-back working memory task; Emotion_Task_Median_RT-median response time for each condition in the emotion task; and PicVocab_AgeAdj - picture vocabulary test score adjusted for age) were among the most strongly associated measures with functional connectivity at any individual connection, and two traits (Gambling_Task_Reward_Perc_Larger - percentage of trials that received a 'larger' prediction in the gambling task; and Taste_AgeAdj - score on the taste intensity test adjusted for age) were chosen because they were only weakly associated with functional connectivity (Supplementary Table B.1).

Supplementary Table B.1 (continued on the next page). Summary statistics for the seven extensively tested traits and their association with functional connectivity from the standard behavioral association model.

\begin{tabular}{|c|c|c|c|}
\hline Measure & $\begin{array}{l}\text { Summary } \\
\text { statistics }\end{array}$ & $\begin{array}{l}\text { Distribution of } \\
\text { measure }^{\mathrm{a}}\end{array}$ & $\begin{array}{c}\text { Distribution of } \\
\text { associations with FC }\end{array}$ \\
\hline \multirow{4}{*}{$\begin{array}{l}\text { BPDiastolic } \\
\text { Diastolic blood pressure levels } \\
\text { Physiological domain }\end{array}$} & \multirow{4}{*}{$\begin{array}{c}N=991 \\
\text { Mean } \pm S D= \\
76.5 \pm 10.4\end{array}$} & & \\
\hline & & & \\
\hline & & $T_{+3}$ & $+0.2 !$ \\
\hline & & Range $=[41.6 ; 115.2]$ & Range $=[-0.228 ;+0.236]$ \\
\hline \multirow{4}{*}{$\begin{array}{l}\text { CogTotalComp_AgeAdj } \\
\text { Total composite score on } \\
\text { cognition adjusted for age } \\
\text { Cognitive domain }\end{array}$} & \multirow{4}{*}{$\begin{array}{c}N=991 \\
\text { Mean } \pm S D= \\
114.2 \pm 20.1\end{array}$} & & \\
\hline & & & \\
\hline & & & $\begin{array}{r}\ulcorner \\
-0.25\end{array}$ \\
\hline & & Range $=[61.4 ; 156.1]$ & Range $=[-0.185 ;+0.207]$ \\
\hline \multirow{4}{*}{$\begin{array}{l}\text { WM_Task_2bk_Acc } \\
\text { Accuracy on all conditions in the } \\
\text { 2-back working memory task } \\
\text { Working memory domain }\end{array}$} & \multirow{4}{*}{$\begin{array}{c}N=995 \\
\text { Mean } \pm S D= \\
83.9 \pm 10.3\end{array}$} & & \\
\hline & & & \\
\hline & & -3 & ]$_{+0.25}$ \\
\hline & & Range $=[40.1 ; 101.4]$ & Range $=[-0.175 ;+0.196]$ \\
\hline \multirow{4}{*}{$\begin{array}{l}\text { Emotion_Task_Median_RT } \\
\text { Median response time from each } \\
\text { condition in the emotion task } \\
\text { Emotion domain }\end{array}$} & \multirow{4}{*}{$\begin{array}{c}N=995 \\
\text { Mean } \pm S D= \\
775.0 \pm 114.0\end{array}$} & & \\
\hline & & & \\
\hline & & $-3 \quad \pm 0$ & -0.25 \\
\hline & & Range $=[517.4 ; 1414.7]$ & Range $=[-0.193 ;+0.182]$ \\
\hline
\end{tabular}




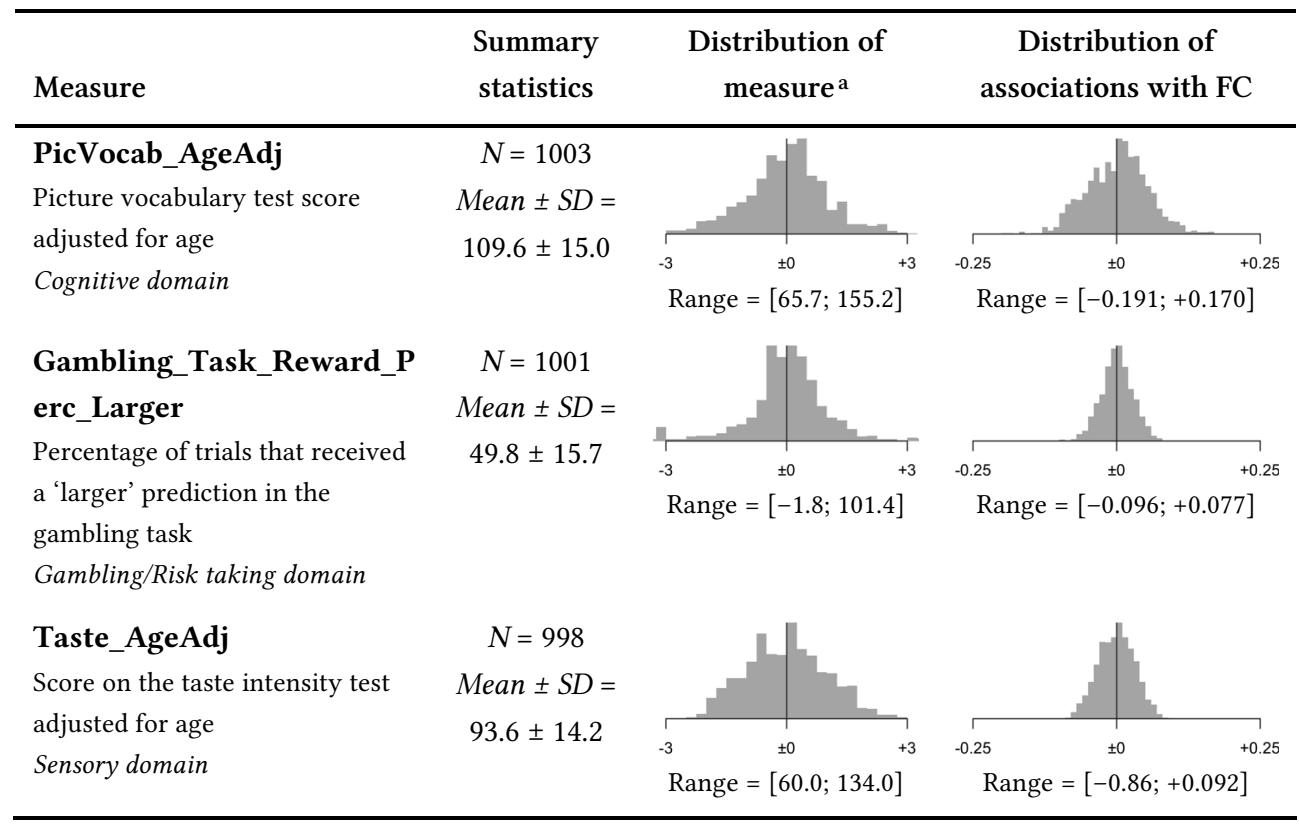

Supplementary Table B.1 (continued). a Histograms of the distribution of behavioral measures are shown after $\mathrm{Z}$-standardization of the sex- and age-regressed measure.

\section{B.1.7 Sparse sampling of connections to approximate improvement factor in behavioral association strength}

Due to the computational complexity of the reliability model, the improvement factor in association strength between functional connectivity and the traits is estimated using a sparse sampling scheme for all but seven traits. With the sparse sampling scheme, only the top 20 connections with the strongest associations to functional connectivity from the standard association model (both positive and negative) and the 5 connections with weakest association (i.e. near zero) were sampled (representing only $2 \%$ of all connections, thereby reducing computation complexity 50 -fold). The seven fully-sampled traits were used to verify if the sparse sampling scheme provided a representative estimate of the improvement factor. This sampling scheme provided a good approximation of the improvement factor compared to the actual improvement factor when all 1225 connections were sampled, with mean absolute difference in improvement factor $2 \%$ (range from $0 \%$ to 5\%; Supplementary Table B.2). 
Supplementary Table B.2. Improvement in association between functional connectivity and traits using the reliability model on the full-sized dataset (i.e. all connections with an acceptable or good fit; max. 1225) and sparsely sampling for only 25 of the connections.

\begin{tabular}{|c|c|c|c|}
\hline \multirow[b]{2}{*}{ Measure } & \multirow{2}{*}{$\begin{array}{c}\text { Full sampling } \\
\begin{array}{l}\text { Improvement } \\
\text { factor }\end{array}\end{array}$} & \multicolumn{2}{|c|}{ Sparse sampling scheme ${ }^{a}$} \\
\hline & & $\begin{array}{l}\text { Improvement } \\
\text { factor }\end{array}$ & Difference \\
\hline $\begin{array}{l}\text { BPDiastolic } \\
\text { Diastolic blood pressure levels } \\
\text { Physiological domain }\end{array}$ & 1.226 & 1.165 & $\begin{array}{l}-0.061 \\
(-5 \%)\end{array}$ \\
\hline $\begin{array}{l}\text { CogTotalComp_AgeAdj } \\
\text { Total composite score on cognition adjusted for age } \\
\text { Cognitive domain }\end{array}$ & 1.168 & 1.177 & $\begin{array}{l}+0.009 \\
(+1 \%)\end{array}$ \\
\hline $\begin{array}{l}\text { WM_Task_2bk_Acc } \\
\text { Accuracy on all conditions in 2-back working } \\
\text { memory task } \\
\text { Working } \text { memory domain }\end{array}$ & 1.170 & 1.202 & $\begin{array}{l}+0.032 \\
(+3 \%)\end{array}$ \\
\hline $\begin{array}{l}\text { Emotion_Task_Median_RT } \\
\text { Median response time from each condition in } \\
\text { emotion tasks } \\
\text { Emotion domain }\end{array}$ & 1.162 & 1.133 & $\begin{array}{l}-0.029 \\
(-2 \%)\end{array}$ \\
\hline $\begin{array}{l}\text { PicVocab_AgeAdj } \\
\text { Picture vocabulary test adjusted for age } \\
\text { Cognitive domain }\end{array}$ & 1.197 & 1.186 & $\begin{array}{l}-0.011 \\
(-1 \%)\end{array}$ \\
\hline $\begin{array}{l}\text { Gambling_Task_Reward_Perc_Larger } \\
\text { Percentage of trials that received a 'larger' } \\
\text { prediction in the gambling task } \\
\text { Gambling/Risk taking domain }\end{array}$ & 1.227 & 1.233 & $\begin{array}{l}+0.006 \\
(+0 \%)\end{array}$ \\
\hline $\begin{array}{l}\text { Taste_AgeAdj } \\
\text { Score on taste intensity test adjusted for age } \\
\text { Sensory domain }\end{array}$ & 1.234 & 1.218 & $\begin{array}{l}-0.016 \\
(-1 \%)\end{array}$ \\
\hline
\end{tabular}

The sparse sampling scheme uses only the top 20 strongest associated connections and the top 5 weakest associated connections ( $2 \%$ of all connections) to approximate the improvement factor (i.e. average improvement ratio in association strength). 


\section{B.1.8 Heritability analysis of functional connectivity and its reliable component}

A subset of this dataset, consisting of 311 monozygotic and 202 dizygotic twins, their 238 full-blood siblings and 225 full-blood siblings from non-twin families up to four family members per family, was used for heritability analysis (Supplementary Table B.3). Zygosity was determined by genotypic data or, when genotypic data was not available, by self-report. Two participants were excluded due to missing zygosity, 20 participants due to half-blood relationships, and 5 full-blood sibling participants due to exceeding a family size of four with family members excluded at random.

Supplementary Table B.3. Demographics table for the subset of participants from the extensively processed fMRI data package of the Human Connectome Project Young Adult cohort consisting of monozygotic and dizygotic twins and full-blood siblings used for heritability analysis.

\begin{tabular}{ll}
\hline Measure & Twins and full-blood siblings \\
\hline Participants $(N)$ & 976 \\
Families $(N)$ & 429 \\
Age range (min; max $)$ & 22 to 36 years \\
Age (mean $\pm S D)$ & $28.71 \pm 3.72$ years \\
Sex ratio (female : male $)$ & $518: 458$ \\
\hline
\end{tabular}

Abbreviations: $\mathrm{SD}=$ standard deviation of the mean.

Genetic modelling of twins and siblings can provide information on the extent to what proportion of variation of a trait in the population is explained by genetic and environmental factors (Boomsma et al., 2002; Posthuma et al., 2000). Based on the assumption that monozygotic (MZ) twins share $100 \%$ of their genetic material and that dizygotic (DZ) twins and full-blood siblings share on average $50 \%$ of their segregating genes, the phenotypic variance $\left(V_{P}\right)$ of a trait are typically decomposed into three variance components: additive genetic $\left(V_{A}\right)$, common environmental $\left(V_{C}\right)$, and unique environmental $\left(V_{E}\right)$ components. Using structural equation modelling (SEM), these variance components can be modelled by latent variables with unit variance for additive genetic $(A)$, common environment $(C)$, and unique environment $(E)$. The path coefficients $a$, $c$, and e quantify the influence of their respective latent variable on the phenotype. The sum of the squared path coefficients $a^{2}, c^{2}$, and $e^{2}$, representing the variance components $V_{A}, V_{C}$, and $V_{E}$, is equal to the phenotypic variance $\left(V_{P}\right)$ of a trait; i.e. $V_{P}=V_{A}+V_{C}+V_{E}=a^{2}+c^{2}+e^{2}$. Heritability $\left(h^{2}\right)$ of the trait is defined as the proportion of phenotypic variance $\left(V_{P}\right)$ that is due to additive 


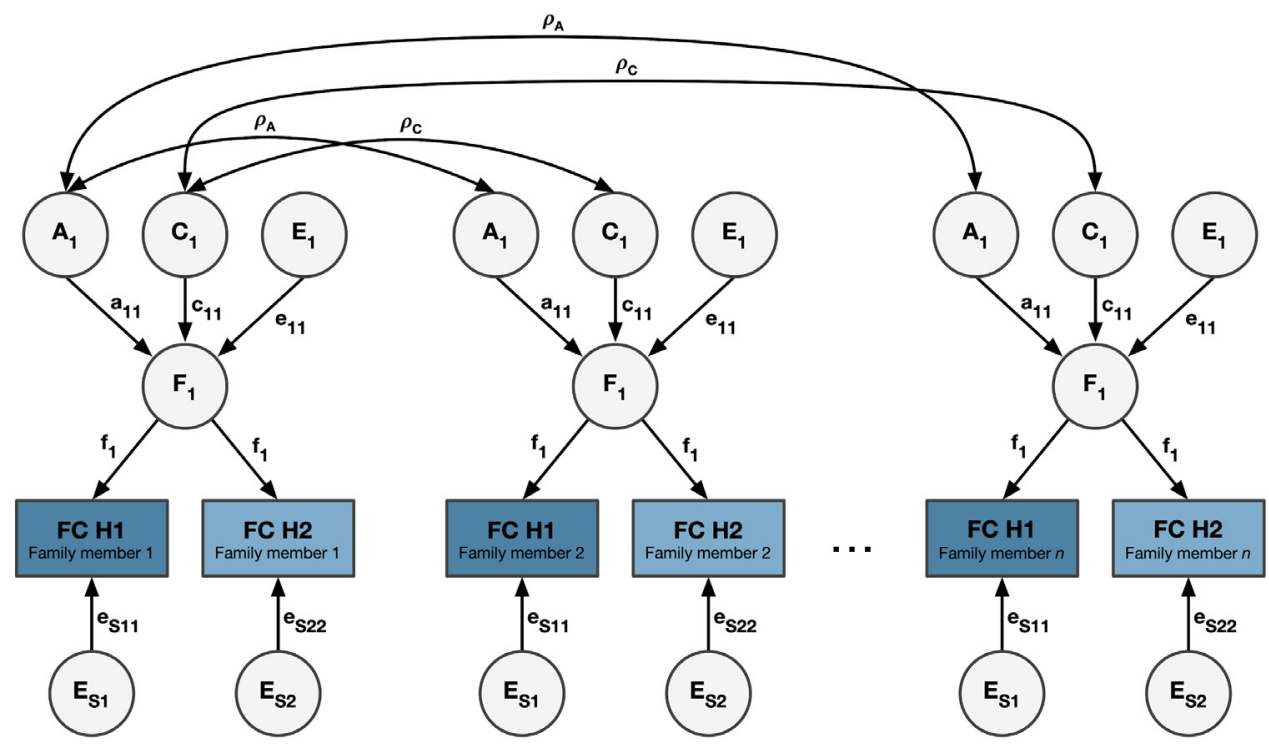

$\rho_{\mathrm{A}}=\mathbf{1 . 0}$ between $\mathrm{MZ}$ twins and $\rho_{\mathrm{A}}=\mathbf{0 . 5}$ between $\mathrm{DZ}$ twins or full-blood siblings; $\rho_{\mathrm{C}}=\mathbf{1 . 0}$ irrespective of kinship

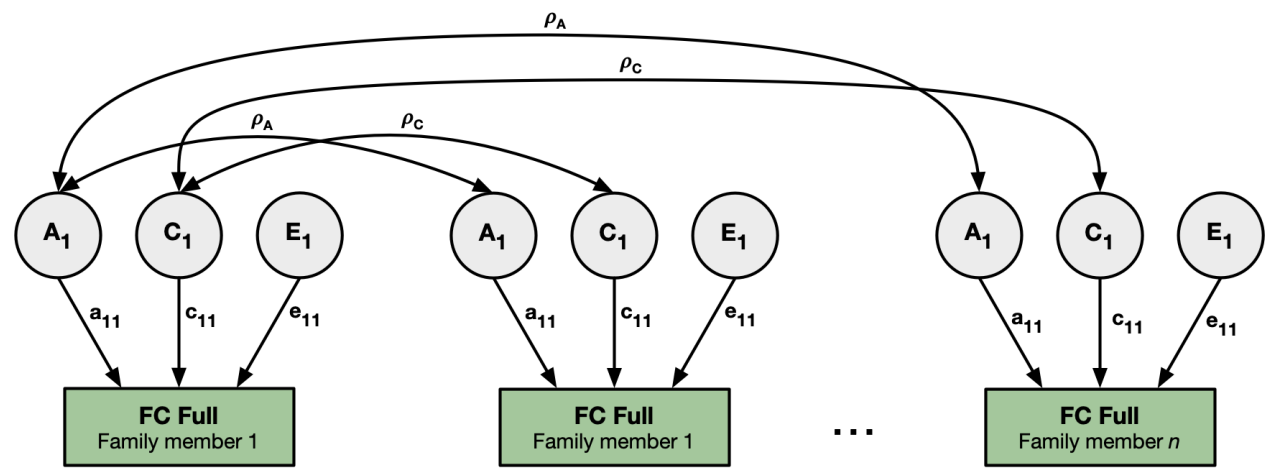

Supplementary Figure B.3. A measurement model with a single factor across two half-score measures of functional connectivity extended to accommodate the family structure of the HCP data to estimate the genetic and environmental influences on the reliable component of functional connectivity, and a traditional univariate Cholesky decomposition model on the full-score measure of functional connectivity.

genetic variance $\left(\mathrm{V}_{\mathrm{A}}\right)$; i.e. $h^{2}=V_{A} / V_{P}=a^{2} /\left(a^{2}+c^{2}+e^{2}\right)$. The model is made identifiable by constraints on the correlation between the latent variables of the family members based on kinship; $\boldsymbol{\rho}_{\mathrm{A}}=1.0$ for monozygotic twins, $\boldsymbol{\rho}_{\mathrm{A}}=0.5$ for dizygotic twins and twin-sibling pairs, and $\boldsymbol{\rho}_{\mathrm{C}}=1.0$ for all twins and siblings from the same family. The latent variable $\mathrm{E}$ is uncorrelated between individuals (i.e. $\boldsymbol{\rho}_{\mathrm{E}}=0.0$ ). The reliability model was extended to estimate the heritability of the reliable component of functional connectivity (Supplementary Figure B.3). Heritability estimates from the reliability model were compared to heritability estimates from a standard Cholesky decomposition of the full-score measure of functional 
connectivity (Neale and Cardon, 1992; Supplementary Figure B.3). Structural equation models were defined using OpenMx version 2.11.5 (Neale et al., 2016; Boker et al., 2018; https://openmx.ssri.psu.edu/), a package for structural equation modelling in $\mathrm{R}$ version 3.5.1 (R Core Team, 2018; https://www.r-project.org/). Model fitting was performed using full-information maximum likelihood (FIML) to take advantage of all available information in case of missing or incomplete data.

\section{B.1.9 Partitioning of the BOLD time series for various total scan durations used in the parameter sweep}

BOLD time series for the desired scan durations were extracted from the original fulllength BOLD time series data by distributing four time blocks equally across all four scan sessions, starting at the first volume of each scan session, and concatenating the time series where necessary to create full-, half-, or quarter-score measures of functional connectivity (Supplementary Figure B.4).

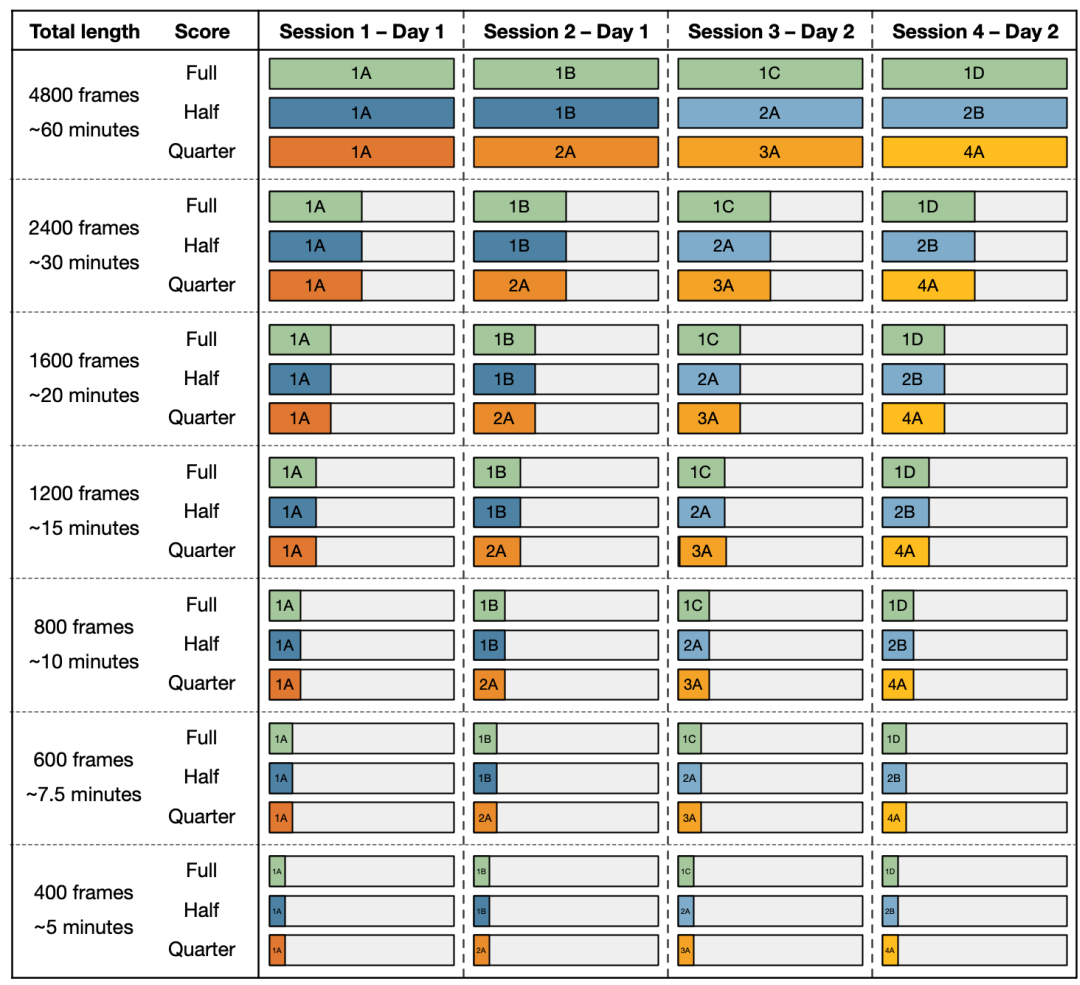

Supplementary Figure B.4. Distribution of time blocks used to extract full-, half-, and quarter scores measures of functional connectivity for various total scan duration. A distributed approach across all four scans was adopted to prevent half-score measures from crossing scan boundaries (e.g. for a total time series length of 1600 volumes, the second half-score measure would be computed across data from both scan \#1 [volumes 801:1200] and \#2 [volumes 1:400]). 


\section{APPENDIX B.2 SUPPLEMENTARY RESULTS FROM CHAPTER 3}

\section{B.2.1 Stability of group-level mean functional connectivity}

At group level, mean functional connectivity shows highly consistent estimates between the two half-score measures of functional connectivity ( $r h o=+0.996$; ICC $3,1=0.995$ ), with functional connectivity estimates ranging from -0.52 to +0.66 for the individual connections (mean FC = 0.003) (Supplementary Figure B.5), and absolute differences between the two half-score measures of less than 0.06 for individual connections.

Group-level mean functional connectivity

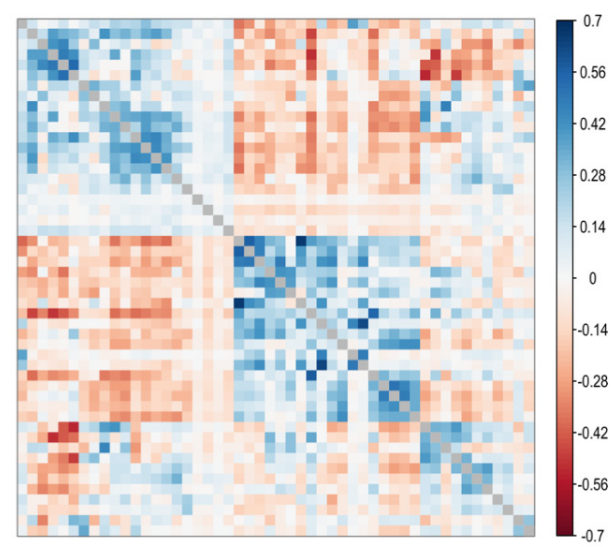

Group-level mean functional connectivity beween half-score measures

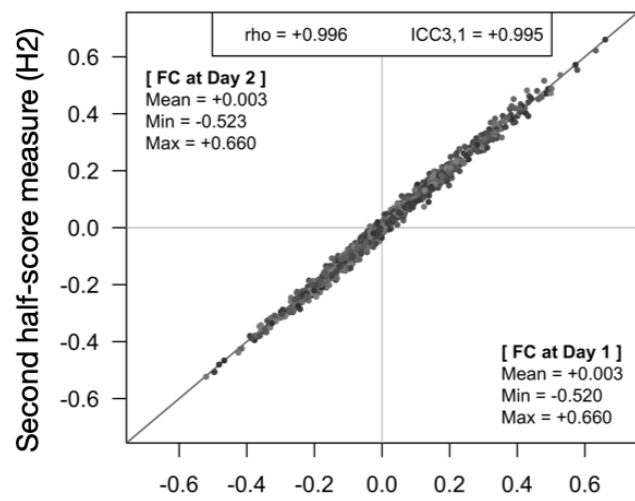

First half-score measure $(\mathrm{H} 1)$

Supplementary Figure B.5. Group-level mean functional connectivity matrix and consistency between two halfscore sessions. (A) Functional connectivity estimates for the first half-score measure at Day 1 (lower-left triangle) and the second half-score measure at Day 2 (upper-right triangle) of functional connectivity organized into their Louvain communities (see Supplementary Figure B.1 for order of nodes). (B) Consistency between the functional connectivity estimates of the first half-score measure at Day 1 and the second half-score measure at Day 2.

\section{B.2.2 Goodness of fit assessment of measurement models}

The applicability of the measurement model to the data was assessed with the two goodness of fit metrics Comparative Fit Index (CFI) and the root-mean-squared error of approximation (RMSEA). Model fits with a CFI $>0.95$ and RMSEA $<0.05$ were deemed a good fit, model fits with a CFI $>0.90$ and RMSEA $<0.08$ were deemed an acceptable fit, and the remaining models $(\mathrm{CFI}<0.90$ or RMSEA $>0.08)$ were deemed an unsuitable fit. For the test-retest measurement model, there were 760 connections ( $62 \%$ of all 1225 connections) with an acceptable or good fit of the model to the data (Supplementary Figure B.6A). For the seven extensively sampled traits, on average 1035 connections ( $84 \%$ of all connections) passed the goodness of fit criteria, with highly similar distributions for the seven traits (Supplementary Figure B.6B). 
(A) Goodness of fit assessment for the test-retest measurement model

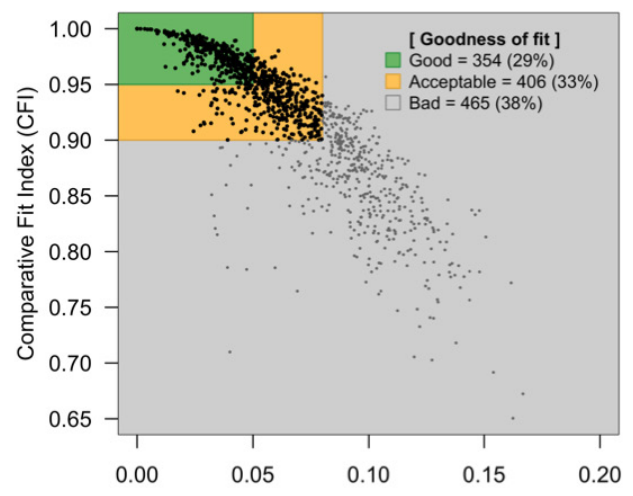

Root-mean-square error of approximation (RMSEA)
(B) Goodness of fit assessment for the trait association measurement model

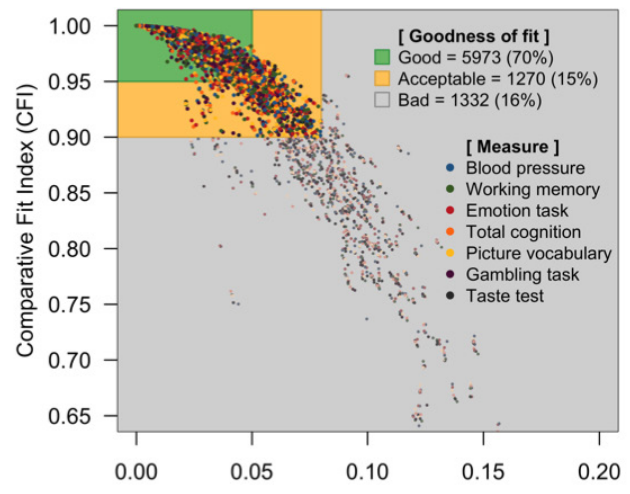

Root-mean-square error of approximation (RMSEA)

Supplementary Figure B.6. Assessment of goodness of fit for the measurement models in the full dataset. (A) For the measurement models used to estimate test-retest reliability between the two reliable components of functional connectivity. (B) And for the measurement models used to estimate the association between the reliable component of functional connectivity and traits. For both panels, models with CFI $>0.95$ and RMSEA $<0.05$ are judged to be a good fit, CFI $>0.90$ and RMSEA $<0.08$ are judged to be acceptable, and CFI $<0.90$ and RMSEA $>0.08$ are bad fits.

\section{B.2.3 Improvement in association between functional connectivity and traits}

The improvement factor in association strength for the three traits diastolic blood pressure level (BPDiastolic), age-adjusted total cognitive composite score (CogTotalComp_AgeAdj), and age-adjusted taste test score (Taste_AgeAdj) are presented in Figure 3.3 from Chapter 3. The improvement factor in association strength for the remaining four traits accuracy on the 2-back working memory test (WM_Task_2bk_Acc), median response time to the emotion tasks (Emotion_Task_Median_RT), age-adjusted picture vocabulary (PicVocab_AgeAdj), and percentage of trials that received a 'larger' prediction in the gambling task (Gambling_Task_Rweard_Perc_Larger) showed similar performance in the full-length full-sized sample (Supplementary Figure B.7). 

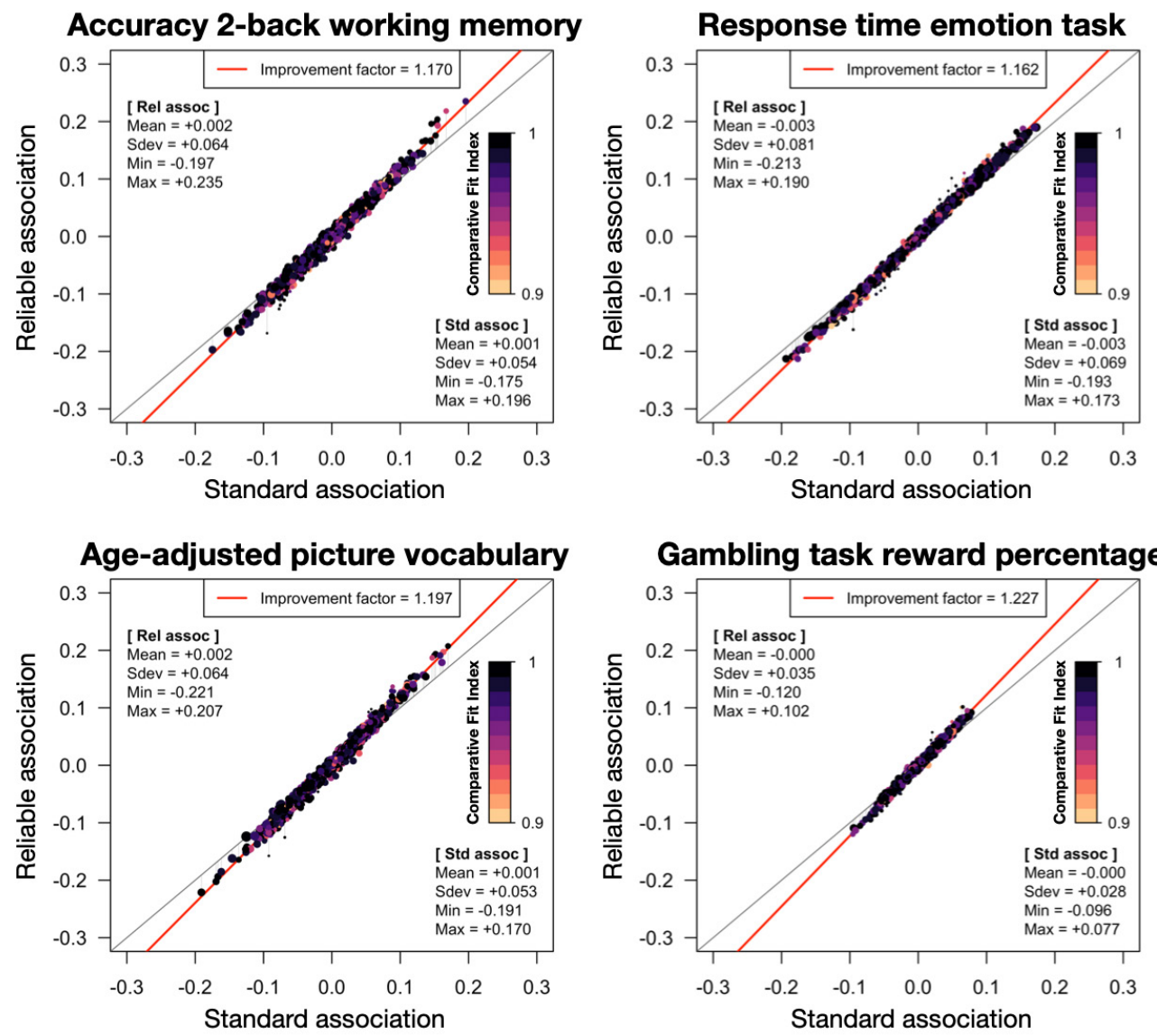

Supplementary Figure B.7. Improvement in association strength between functional connectivity and the traits accuracy on 2-back working memory task ( $W M_{-}$Task_2bk_Acc), median response time to emotion task (Emotion_Task_Median_RT), picture vocabulary test adjusted for age (PicVocab_AgeAdj), and percentage of trials received a 'larger' prediction in the gambling task (Gambling_Task_Reward_Perc_Larger). 


\section{Heritability estimate of \\ functional connectivity}

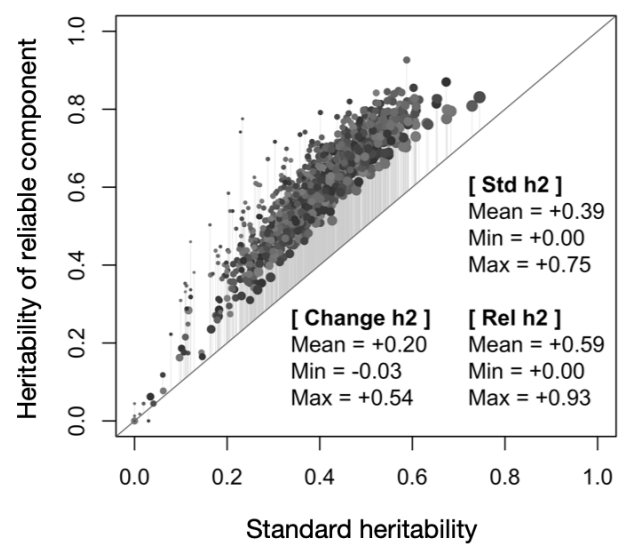

Supplementary Figure B.8. Increased heritability estimates of functional connectivity. Standard heritability is estimated using a Cholesky decomposition of the full-score measure of functional connectivity (x-axis). A measurement model applied to the half-score measures of functional connectivity was used to estimate heritability of the reliable component of functional connectivity (y-axis). Data points are scaled by the average proportion of variance explained by the reliable component, thereby emphasizing the more reliable and stable connections, and color-coded by random grey values to allow for better distinction between individual data points.

\section{B.2.4 Increased heritability estimates of functional connectivity}

The heritability estimates of full-score functional connectivity using a standard Cholesky decomposition was on average $39 \%$ (range $=0 \%$ to $75 \%$; Supplementary Figure B.8). The heritability estimates of the reliable component of functional connectivity was on average $59 \%$ (range $=0 \%$ to $93 \%$; Supplementary Figure B.8). On average, the heritability estimates improved with $+0.20 \%$ points (range $=-0.03$ to +0.54 ; Supplementary Figure B.8).

\section{B.2.5 Parameter sweep for association between functional connectivity and traits}

The improvement in strength of the association between the reliable component of functional connectivity and behtraits (i.e. the improvement factor) is greater for shorter scan duration and smaller sample size for all seven extensively sampled traits (Figure 3.4; Supplementary Figure B.9).

\section{B.2.6 Minimal requirements of dataset for reliability modelling}

The goodness of fit assessment from the parameter sweep was used to determine the minimal requirements on the input dataset in terms of sample size and total scan duration for reliability modelling. Although no clear boundary can be defined when a measurement model is no longer suitable or practical to use, the chance that the measurement model does not describe the data well for a random sample of participants starts to increase with lower sample size or shorter scan durations (Supplementary Figure B.10). 

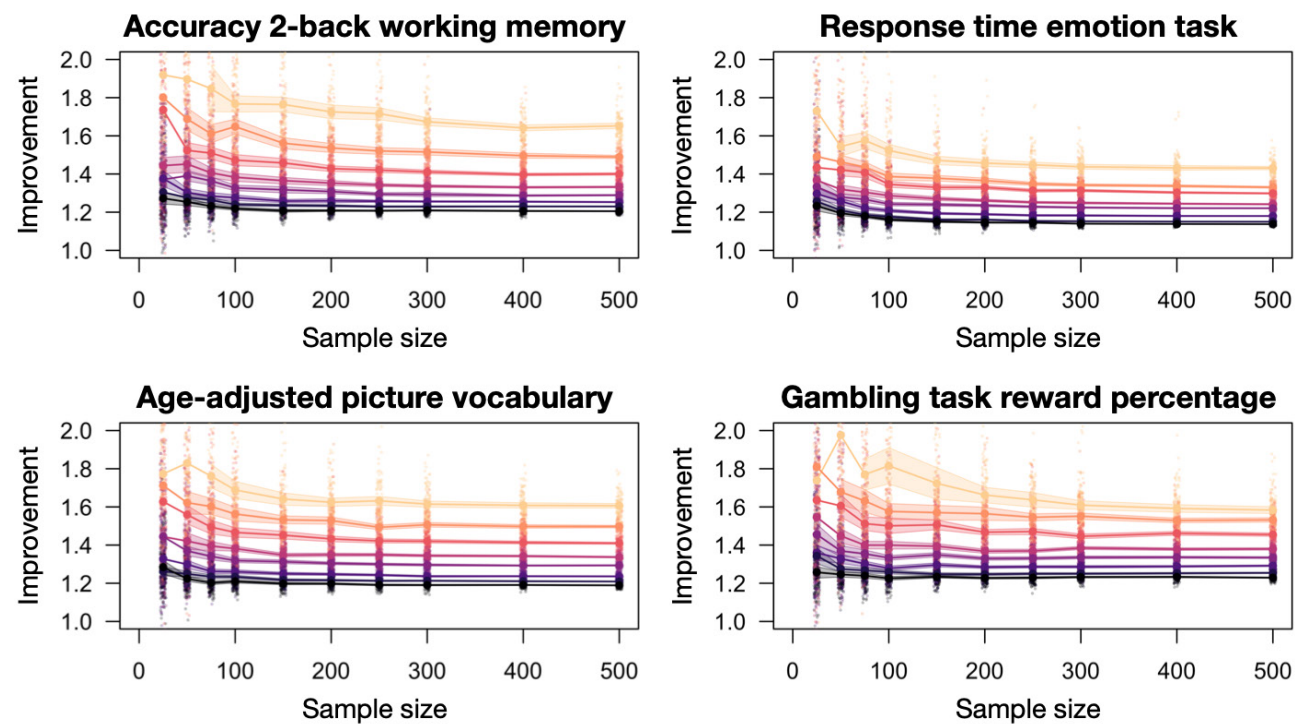

Supplementary Figure B.9. Improvement in association strength between functional connectivity and the traits accuracy on 2-back working memory task (WM_Task_2bk_Acc), median response time to emotion task (Emotion_Task_Median_RT), picture vocabulary test adjusted for age (PicVocab_AgeAdj), and percentage of trials received a 'larger' prediction in the gambling task (Gambling_Task_Reward_Perc_Larger) for various sample sizes (x-axis) and total scan duration (color-coding). Improvement factor (y-axis) is defined by the slope coefficient from the linear regression of reliable association strength onto standard association strength over all sparsely sampled connections. Color-shaded bands represent the $95 \%$ confidence interval of the means. For all panels, a standard association model was used to estimate the association between the full-score measure of functional connectivity and the trait. A measurement model applied to the half-score measures of functional connectivity was used to estimate the association between the reliable component of functional connectivity and the trait. The other three extensively tested measures showed similar patterns (Figure 3.4 from Chapter 3). 

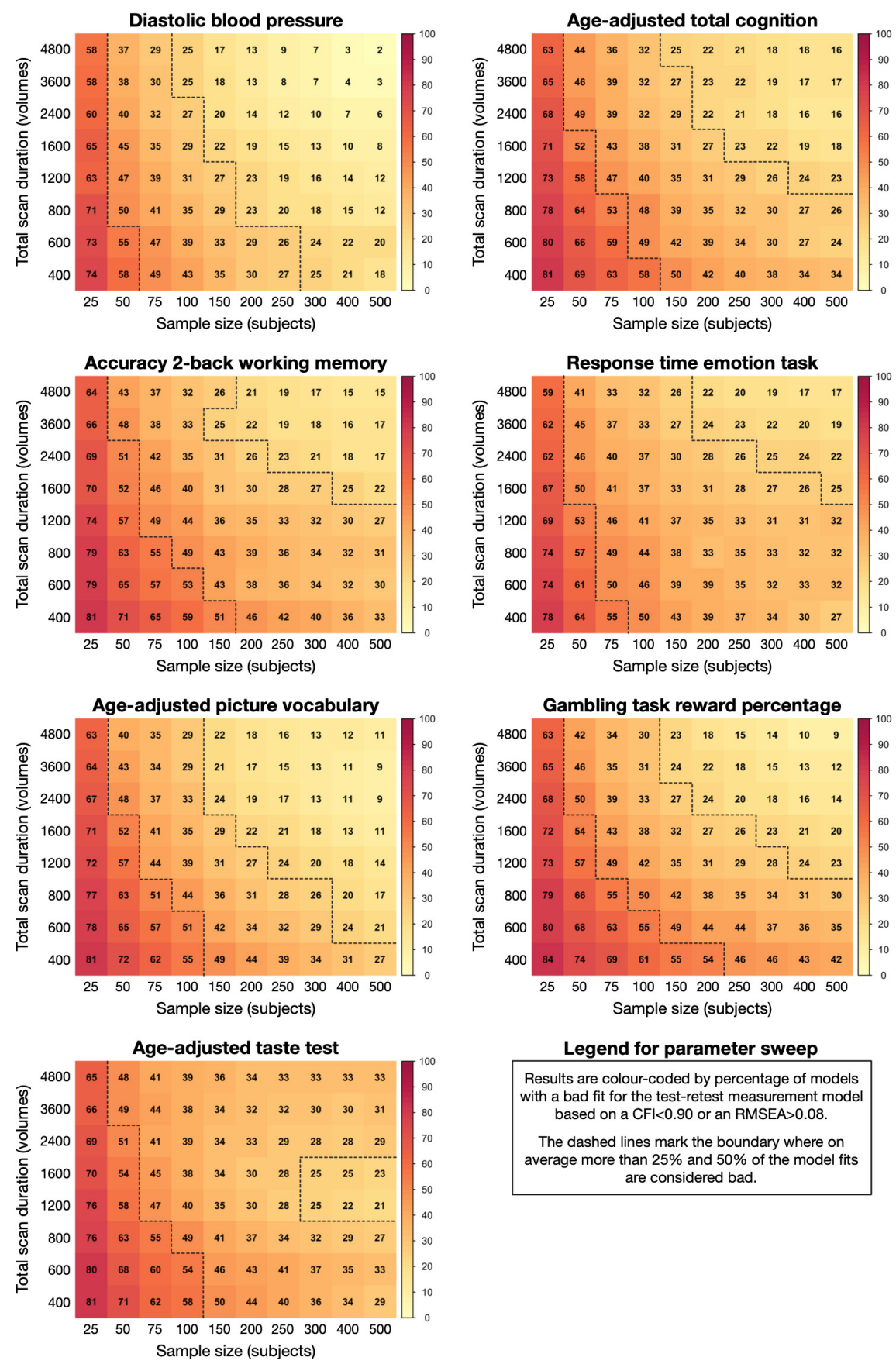

\section{Legend for parameter sweep}

Results are colour-coded by percentage of models with a bad fit for the test-retest measurement model based on a $\mathrm{CFI}<0.90$ or an RMSEA $>0.08$.

The dashed lines mark the boundary where on average more than $25 \%$ and $50 \%$ of the model fits are considered bad.

Supplementary Figure B.10. Percentage of sampled connections for each combination of sample size and total scan duration for which the goodness of fit for the measurement models deteriorated below acceptable levels (CFI $<0.90$ or RMSEA > 0.08). Total scan duration is reported as the number of volumes at TR $=720 \mathrm{~ms}$. Dotted lines mark the boundary where on average more than $25 \%$ and $50 \%$ of the model fits are considered bad. 



\section{APPENDIX C}

Supplementary information

to chapter 4 


\section{APPENDIX C.1 SUPPLEMENTARY INFORMATION FROM CHAPTER 4}

\section{C.1.1 Resting-state networks atlas}

Regions of interests (ROI) of canonical resting-state networks and their decomposition into spatially separated components are extracted from the networks atlas provided by the CONN toolbox (Whitfield-Gabrieli and Nieto-Castanon, 2012; https://web.conntoolbox.org/). Networks were identified in resting-state MRI scans of 497 independent young adults from the Human Connectome Project (Marcus et al., 2013) using the independent component analysis pipeline built into the CONN toolbox. The atlas provides regions of interest (ROI) for 7 canonical cortical resting-state networks and the cerebellum: the core Default Mode network (4 components), Sensorimotor (3), Visual (4), Salience (7), Dorsal Attention (4), Frontoparietal (4), Language (4), and Cerebellar (2), for a total of 32 components for all networks combined (Supplementary Figure C.1; Supplementary Table C.1). The choice to use an atlas based on subjects of older age (young adults aged 22 to 35 years instead of adolescents) is warranted by the fact that spatial organization of canonical resting-state networks have been reported to be "adult-like" by the second year after birth (Gao et al., 2015). Moreover, group-level mean functional connectivity matrices of our sample using the atlas shows satisfactory distinction between nodes of different restingstate networks (i.e. stronger functional connectivity for connections within resting-state networks than for connections between resting-state networks; Figure 4.2 from Chapter 4).

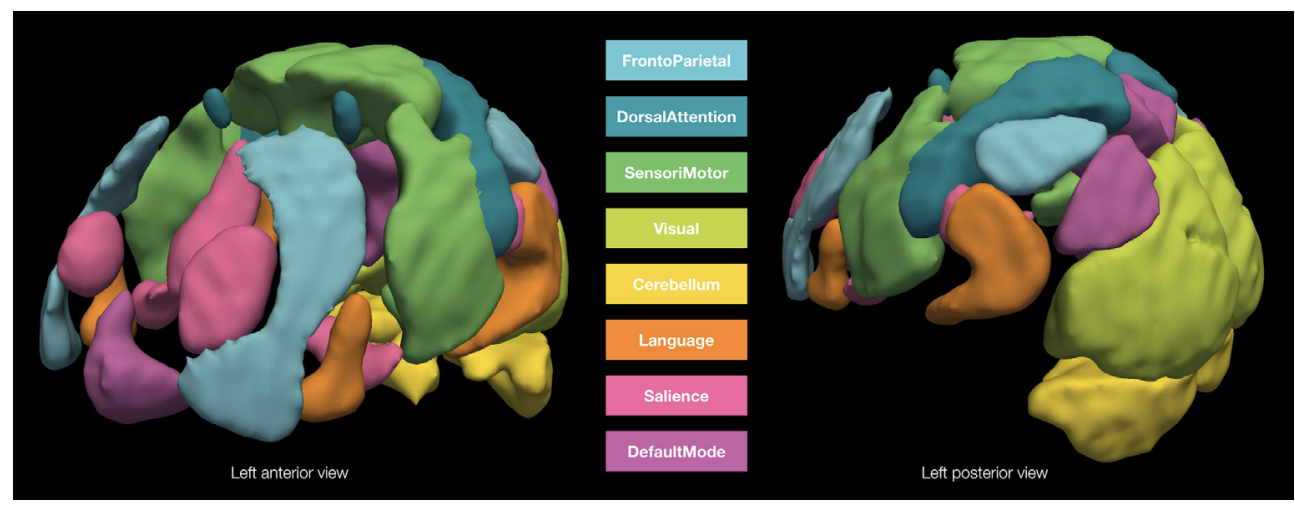

Supplementary Figure C.1. Atlas of canonical resting-state networks and their spatially distinct components provided by the CONN toolbox version 18.a based on ICA decomposition of 497 young adults from the Human Connectome Project. 
Supplementary Table C.1 (continued on the next two pages). The canonical resting-state networks and their spatially distinct subcomponents.

\begin{tabular}{|c|c|c|c|c|c|c|c|c|}
\hline Network & Region & $\mathrm{X}$ & $\mathrm{Y}$ & $\mathrm{Z}$ & Volume & Voxels & Abbr. & \# \\
\hline \multirow[t]{4}{*}{ Default Mode } & $\begin{array}{l}\text { Medial pre- } \\
\text { frontal cortex }\end{array}$ & 1 & 55 & -3 & 10768 & 397 & MPFC & 1 \\
\hline & $\begin{array}{l}\text { Left lateral } \\
\text { parietal }\end{array}$ & -39 & -77 & 33 & 8328 & 302 & LLP & 2 \\
\hline & $\begin{array}{l}\text { Right lateral } \\
\text { parietal }\end{array}$ & 47 & -67 & 29 & 10608 & 382 & RLP & 3 \\
\hline & $\begin{array}{l}\text { Posterior } \\
\text { cingulate cortex }\end{array}$ & 1 & -61 & 39 & 38664 & 1446 & PCC & 4 \\
\hline \multirow[t]{3}{*}{ Sensorimotor } & Left lateral & -55 & -12 & 29 & 30272 & 1145 & LLSMC & 5 \\
\hline & Right lateral & 56 & -10 & 29 & 30968 & 1132 & RLSMC & 6 \\
\hline & Superior & 0 & -31 & 67 & 57016 & 2166 & SSMC & 7 \\
\hline \multirow[t]{4}{*}{ Visual } & Medial & 2 & -79 & 12 & 79224 & 2921 & MVC & 8 \\
\hline & Occipital & 0 & -93 & -4 & 48712 & 1761 & OVC & 9 \\
\hline & Left lateral & -37 & -79 & 10 & 24832 & 917 & LLVC & 10 \\
\hline & Right lateral & 38 & -72 & 13 & 33968 & 1249 & RLVC & 11 \\
\hline \multirow[t]{5}{*}{ Salience } & $\begin{array}{l}\text { Anterior } \\
\text { cingulate cortex }\end{array}$ & 0 & 22 & 35 & 8504 & 315 & $\mathrm{ACC}$ & 12 \\
\hline & $\begin{array}{l}\text { Left anterior } \\
\text { insula }\end{array}$ & -44 & 13 & 1 & 3568 & 132 & LAIns & 13 \\
\hline & $\begin{array}{l}\text { Right anterior } \\
\text { insula }\end{array}$ & 47 & 14 & 0 & 3104 & 121 & RAIns & 14 \\
\hline & $\begin{array}{l}\text { Left rostral } \\
\text { prefrontal cortex }\end{array}$ & -32 & 45 & 27 & 9328 & 353 & LRPFC & 15 \\
\hline & $\begin{array}{l}\text { Right rostral } \\
\text { prefrontal cortex }\end{array}$ & 32 & 46 & 28 & 4648 & 172 & RRPFC & 16 \\
\hline
\end{tabular}




\begin{tabular}{|c|c|c|c|c|c|c|c|c|}
\hline Network & Region & $\mathrm{X}$ & $\mathrm{Y}$ & $\mathrm{Z}$ & Volume & Voxels & Abbr. & $\#$ \\
\hline & $\begin{array}{l}\text { Left supra- } \\
\text { marginal gyrus }\end{array}$ & -60 & -39 & 31 & 1864 & 71 & LSMG & 17 \\
\hline & $\begin{array}{l}\text { Right supra- } \\
\text { marginal gyrus }\end{array}$ & 62 & -35 & 32 & 2272 & 90 & RSMG & 18 \\
\hline \multirow[t]{4}{*}{$\begin{array}{l}\text { Dorsal } \\
\text { Attention }\end{array}$} & $\begin{array}{l}\text { Left frontal eye } \\
\text { fields }\end{array}$ & -27 & -9 & 64 & 704 & 27 & LFEF & 19 \\
\hline & $\begin{array}{l}\text { Right frontal eye } \\
\text { fields }\end{array}$ & 30 & -6 & 64 & 432 & 19 & RFEF & 20 \\
\hline & $\begin{array}{l}\text { Left intraparietal } \\
\text { sulcus }\end{array}$ & -39 & -43 & 52 & 26280 & 992 & LIPS & 21 \\
\hline & $\begin{array}{l}\text { Right intra- } \\
\text { parietal sulcus }\end{array}$ & 39 & -42 & 54 & 25096 & 935 & RIPS & 22 \\
\hline \multirow[t]{4}{*}{ Frontoparietal } & $\begin{array}{l}\text { Left lateral } \\
\text { prefrontal cortex }\end{array}$ & -43 & 33 & 28 & 13624 & 524 & LLPFC & 23 \\
\hline & $\begin{array}{l}\text { Left posterior } \\
\text { parietal cortex }\end{array}$ & -46 & -58 & 49 & 6656 & 245 & LPPC & 24 \\
\hline & $\begin{array}{l}\text { Right lateral } \\
\text { prefrontal cortex }\end{array}$ & 41 & 38 & 30 & 14064 & 526 & RLPFC & 25 \\
\hline & $\begin{array}{l}\text { Right posterior } \\
\text { parietal cortex }\end{array}$ & 52 & -52 & 45 & 6696 & 243 & $\mathrm{RPPC}$ & 26 \\
\hline \multirow[t]{4}{*}{ Language } & $\begin{array}{l}\text { Left inferior } \\
\text { frontal gyrus }\end{array}$ & -51 & 26 & 2 & 3856 & 145 & LIFG & 27 \\
\hline & $\begin{array}{l}\text { Right inferior } \\
\text { frontal gyrus }\end{array}$ & 54 & 28 & 1 & 4296 & 156 & RIFG & 28 \\
\hline & $\begin{array}{l}\text { Left posterior } \\
\text { superior } \\
\text { temporal gyrus }\end{array}$ & -57 & -47 & 15 & 18104 & 676 & LPSTG & 29 \\
\hline & $\begin{array}{l}\text { Right posterior } \\
\text { superior } \\
\text { temporal gyrus }\end{array}$ & 58 & -42 & 13 & 13496 & 503 & RPSTG & 30 \\
\hline
\end{tabular}




\begin{tabular}{llccccccc}
\hline Network & Region & X & Y & Z & Volume & Voxels & Abbr. & $\#$ \\
\hline Cerebellar & Anterior & 0 & -63 & -30 & 18672 & 696 & ACER & 31 \\
& Posterior & 0 & -79 & -32 & 43264 & 1591 & PCER & 32 \\
\hline
\end{tabular}

Supplementary Table C.1 (continued). Coordinates of ROIs are reported in MNI space for the center of mass of the region. Volumes of ROIs are reported in $\mathrm{mm}^{3}$ for the atlas at $1.0 \mathrm{~mm}$ isotropic resolution. The number of voxels in ROIs are reported for the atlas at $3.0 \mathrm{~mm}$ isotropic resolution (i.e. analysis space). Abbreviations: RSN = resting-state network; $\mathrm{ROI}=$ region of interest.

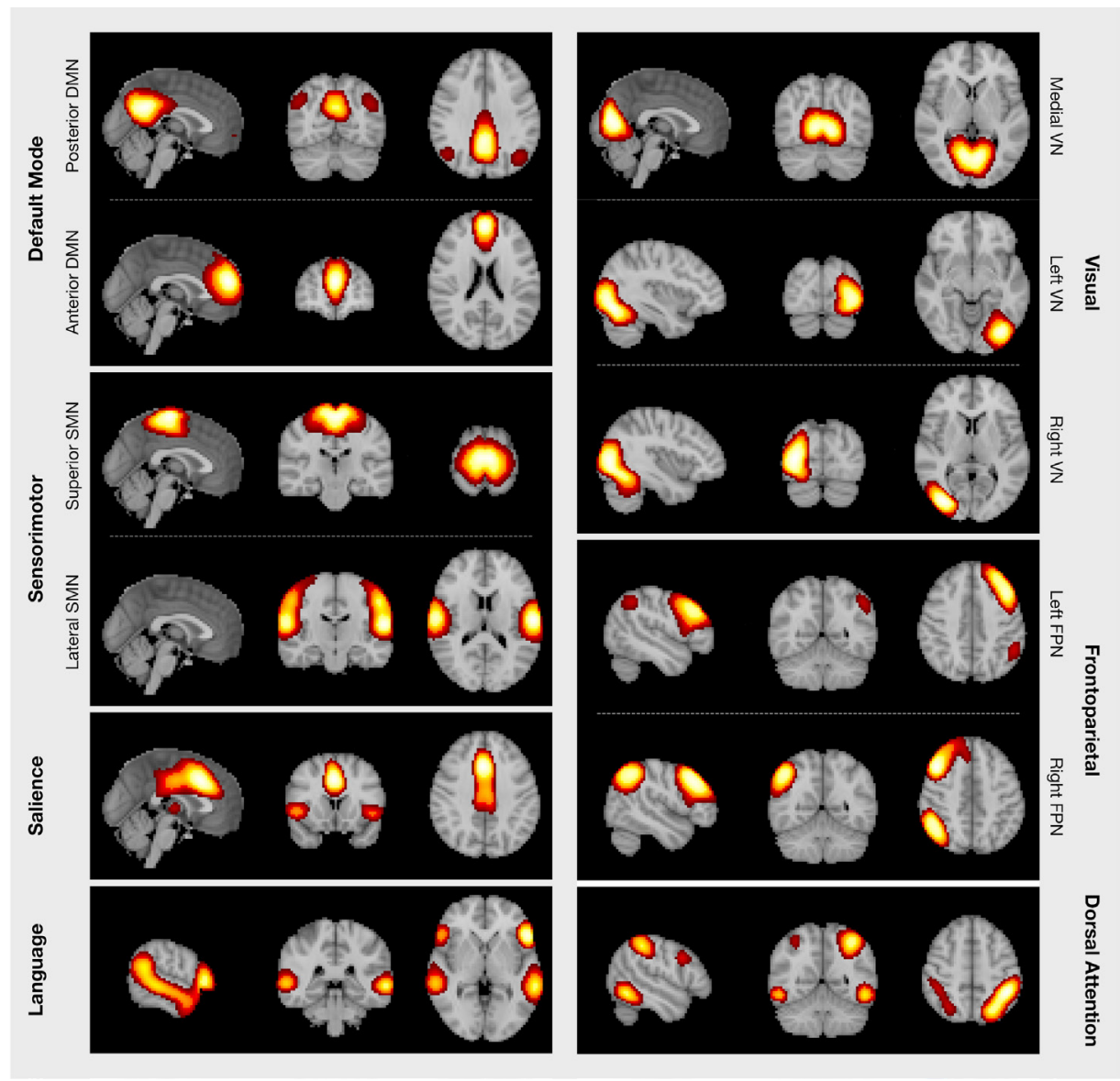

Supplementary Figure C.2. Group-ICA decomposition of the BrainSCALE resting-state functional MRI data into components representing (parts of) the canonical cortical resting-state networks used in the analysis. Abbreviations: $\mathrm{DMN}$ = default mode networks; $\mathrm{VN}$ = visual network; FPN = frontoparietal network. 


\section{C.1.2 Group-ICA decomposition}

We performed a group-ICA decomposition of the denoised scans using Melodic from FSL version 5.0.10 (Beckmann and Smith, 2004). First, denoised scans were smoothed within the brain mask using a $6 \mathrm{~mm}$ Gaussian kernel to increase voxel-wise SNR (Smith and Brady, 1997). Group-ICA decomposition was performed by concatenating scans for all subjects across both ages using Incremental Group-PCA (Smith et al., 2014). The number of components to extract was set to 20 . This number was chosen from a range of values because it resulted in the decomposition of the brain signal into the canonical resting-state networks or its major constituents without breaking the networks apart in small fragments. Statistical maps of the components are visualized on top of the structural MNI152 atlas at a threshold of $Z>5$.

\section{C1.3 Cholesky twin model}

A Cholesky decomposition of twin and family data can be used to determine genetic and environmental influences of one or multiple traits (Neale and Cardon, 1992). A multivariate Cholesky model allows for estimating of genetic and environmental influences on individual traits and overlap in shared variances between traits by genetic and environmental correlations (Supplementary Figure C.3).

In this study, a Cholesky decomposition is used to account for non-independence between family members of phenotypic correlation estimates in the association analyses between intelligence and full-score functional connectivity, and in the reliability estimates between

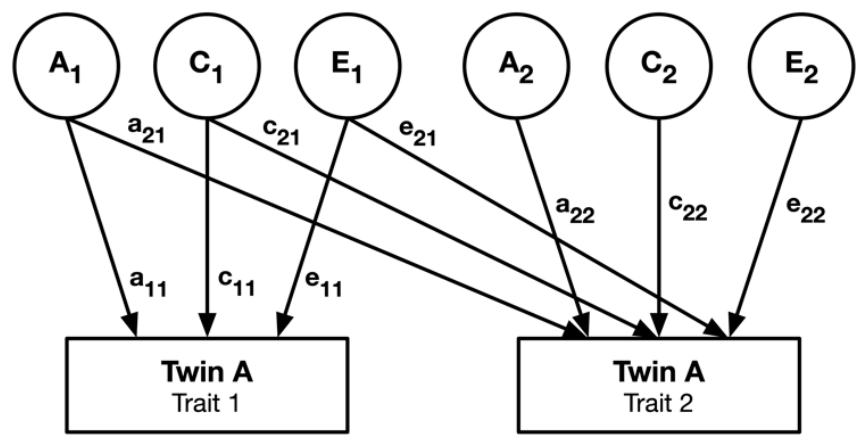

Supplementary Figure C.3. A graphical representation of a generalized bivariate Cholesky model used to estimate heritability of individual traits and the phenotypic and genetic correlation between the two traits. Latent twin factors represented by circles load onto the individual measurements, with one set of variance components loading on both measurements as shared components and one set of variance components unique to one of the measures. Family members are linked through bidirectional paths on their latent variance components with values constrained to 1.0 for the additive genetic factor(s) $\left(A_{i}\right)$ between monozygotic twins, 0.5 for the additive genetic factor(s) $\left(A_{i}\right)$ between dizygotic twins and siblings, 1.0 for common environmental factors $\left(C_{i}\right)$ for all pairs within one family, and 0.0 for unique environment $\left(E_{i}\right)$. 
full-scores (long-term stability) and half-scores (short-term reliability). Sex and age coefficients on the means of the individual traits were included as fixed effects to account for possible difference between the sexes or ages. For longitudinal models (i.e. models with measurements of the same trait at different ages), the sex and age coefficient are shared between measurements.

\section{C.1.4 Quality control based on head motion and scrubbing of high-motion volumes}

The amount of head motion during acquisition of the resting-state functional MRI scan was retrospectively estimated as framewise displacement derived from the rigid-body transformations of the realignment registration procedure. Framewise displacement (FD) is defined as the sum of the absolute values of the first-order derivative of the realignment parameters between volumes (Power et al., 2012), where rotational parameters are converted from radians to arc length equivalent to motion of a point travelling on a circle with a radius of 50 millimeter:

$$
F D=\left|\Delta t_{x}\right|+\left|\Delta t_{y}\right|+\left|\Delta t_{z}\right|+\left|\Delta r_{x}\right|+\left|\Delta r_{y}\right|+\left|\Delta r_{z}\right|
$$

Mean framewise displacement is calculated as the framewise displacement averaged along the entire time series of a subject. Split-half scores of head motion were calculated as the mean framewise displacement along the independent first and second half of the subject's time series.

Incomplete resting-state scans and scans with discernable scanner-related artefacts were discarded beforehand, resulting in the exclusion of 11 scans at age 13 years and 7 scans at age 18 years (Supplementary Table C.2; Supplementary Figure C.4). In addition, individual full and half-score measures of functional connectivity were excluded from statistical analysis if the mean framewise displacement for the corresponding measure was greater than $0.30 \mathrm{~mm} /$ volume or the number of scrubbed frames exceeded $50 \%$ of the volumes on which the functional connectivity measure was based (i.e. $>450$ volumes for full-score measures, or $>225$ volumes for half-score measures). This resulted in an additional 33 to 53 full-score or half-score measures being excluded from statistical analysis at age 13 years, and between 18 and 21 at age 18 years (Supplementary Table C.2; Supplementary Figure C.4). Typically, subjects showed a slight increase in mean framewise displacement during the second half of the scan compared to the first half of the scan, especially at age 13 years, and consequently showed an increased number of scrubbed frames (Supplementary Figure C.4). It is therefore possible that only one of the half scores was included in the statistical 

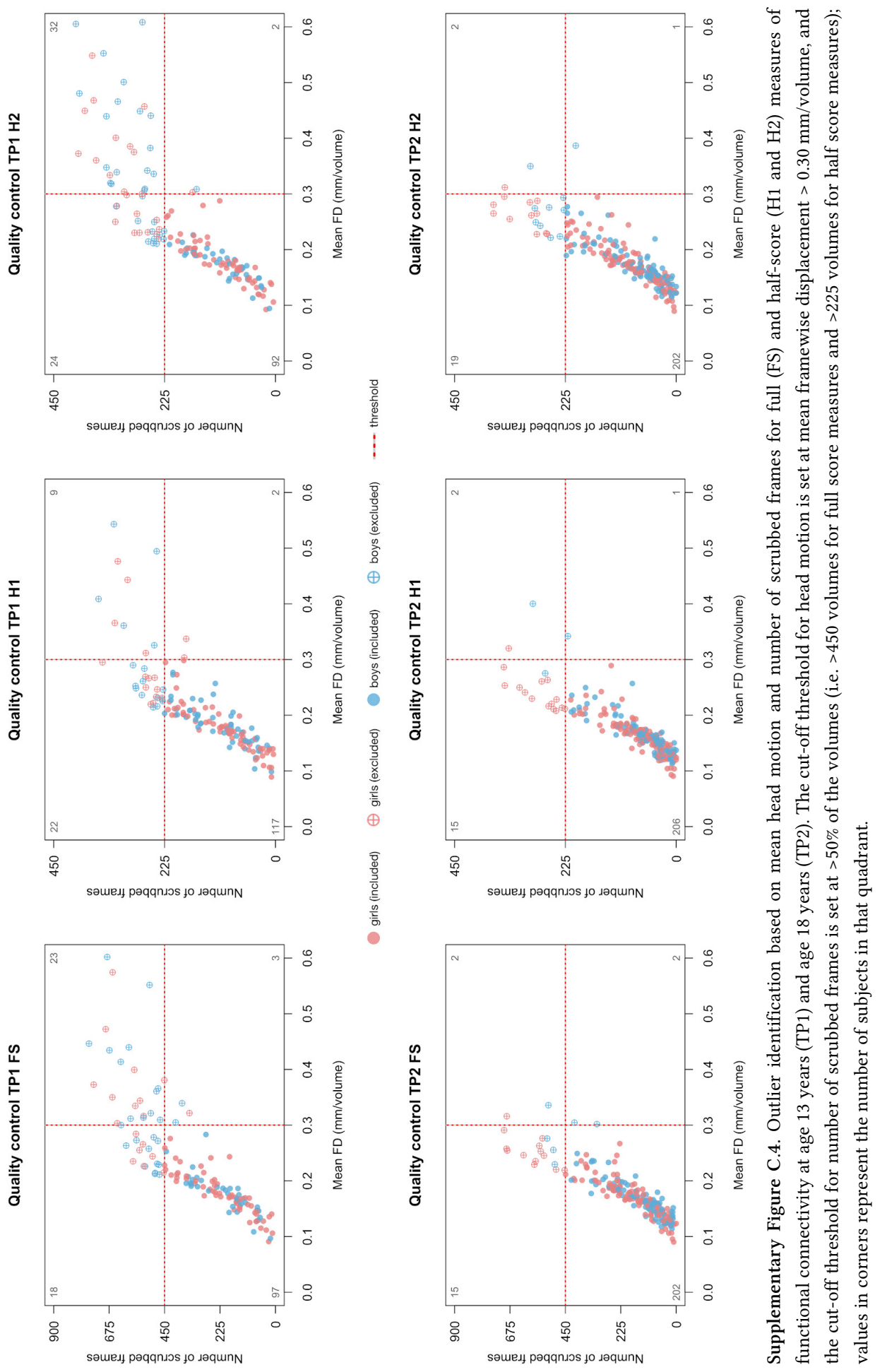
Supplementary Table C.2. Sample sizes during various stages of quality control.

\begin{tabular}{llll}
\hline Stage in quality control & Split session score & Age 13 years & Age 18 years \\
\hline Original sample & & $152(100 \%)$ & $228(100 \%)$ \\
Post manual inspection & & $141(93 \%)$ & $221(97 \%)$ \\
& & & \\
Post quality control & Full scores (FS) & $95(63 \%)$ & $200(88 \%)$ \\
& Half scores (H1) & $106(70 \%)$ & $201(88 \%)$ \\
& Half scores (H2) & $86(57 \%)$ & $198(87 \%)$
\end{tabular}

Percentages are reported with sample size of original dataset as baseline (i.e. $100 \%$ ).

analysis. However, single half score measures may still contribute information to the overall fit of the twin models because the full-information maximum likelihood (FIML) estimator of OpenMx used in the twin analysis can operate on missing or incomplete data.

\section{C.1.5 Analysis of head motion}

A longitudinal bivariate Cholesky model with sex and age beta coefficients on the means was used to investigate genetic and environmental influences on the $\log _{10}$-transformed fullscore measurements of mean framewise displacement after quality control (i.e. excluding scans with artefacts and excessive head motion). The most parsimonious twin model included additive genetic and unique environmental factors (i.e. a twin model with only $\mathrm{AE}$ variance components loading on the measurements). There is a non-significant phenotypic correlation between measurements of mean framewise displacement over time for subjects with longitudinal measurements $\left(r_{\mathrm{ph}}=+0.14 ; p=0.1279\right.$ [n.s.]; Supplementary Table C.3). There is a significant genetic influence on head motion at both ages $\left(h^{2}=43 \%\right.$ to $86 \%$; $p<0.003$; Supplementary Table C.3) from the same genes pool $\left(r_{\mathrm{a}}>+0.37 ; p=\right.$ 0.0691; Supplementary Table C.3) that decreases significantly with age $\left(\Delta h^{2}=-43 \% ; p=\right.$ 0.0137). No effect of sex on head motion was found $\left(\beta_{\text {sex }}\left[\log _{10}\right]=+0.0009 ; p=0.9392\right.$; Supplementary Table C.3; Supplementary Figure C.5). Despite stringent criteria for exclusion of subjects with excessive head motion, a small but significant effect of age on head motion was found $\left(\beta_{\text {age }}\left[\log _{10}\right]=-0.0172 ; p<0.0001\right.$; Supplementary Table C.3; Supplementary Figure C.5). A significant negative association between mean framewise displacement and IQ scores was found only at age 18 years $\left(r_{\mathrm{ph}}=-0.20 ; p=0.0160\right.$; Supplementary Table C.3). 
Supplementary Table C.3. The genetic influences and the effects of sex and age on full-score head motion estimates.

\begin{tabular}{lll}
\hline Measure & Age 13 years & Age 18 years \\
\hline Mean FD & $0.18 \pm 0.04$ & $0.16 \pm 0.03$ \\
Mean FD $\left(\log _{10}\right)$ & $-0.68 \pm 0.16$ & $-0.78 \pm 0.09$ \\
Association with IQ score & $-0.23[-0.53 ; 0.14] ; p=0.2287$ & $\mathbf{- 0 . 2 0}[-\mathbf{0 . 3 5} ; \mathbf{- 0 . 0 4}] ;$ \\
& & $\boldsymbol{p}=\mathbf{0 . 0 1 6 0}$ \\
Heritability $\left(h^{2}\right)$ & $\mathbf{8 6 \%}[\mathbf{6 8 \%} ; \mathbf{9 3 \%}] ; \boldsymbol{p}<\mathbf{0 . 0 0 0 1}$ & $\mathbf{4 3 \%}[\mathbf{2 1 \%} \mathbf{6 3 \%}] ; \boldsymbol{p}=\mathbf{0 . 0 0 0 3}$ \\
Phenotypic correlation $\left(r_{\mathrm{ph}}\right)$ & $+0.14[-0.04 ; 0.33] ; p=0.1279$ & \\
Genetic correlation $\left(r_{\mathrm{a}}\right)$ & $+0.37[-0.06 ; 1.00] ; p=0.0691$ & \\
Effect of sex $\left(\beta_{\text {sex }}\right)$ & $+0.0009[-0.0234 ;+0.0253] ; p=0.9392$ \\
Effect of age $\left(\beta_{\text {age }}\right)$ & $-\mathbf{0 . 0 1 7 2}[-\mathbf{0 . 0 2 2 6} ;-\mathbf{0 . 0 1 1 8}] ; \boldsymbol{p}<\mathbf{0 . 0 0 0 1}$ \\
\hline
\end{tabular}

Effects on head motion were assessed after quality control when subjects with excessive head motion have been excluded from analysis based on their mean framewise displacement (FD) and number of scrubbed high motion frames. Reported p-values are uncorrected for multiple comparison; beta coefficients are in $\log _{10}$-transformed space.

\section{Head motion post quality control}

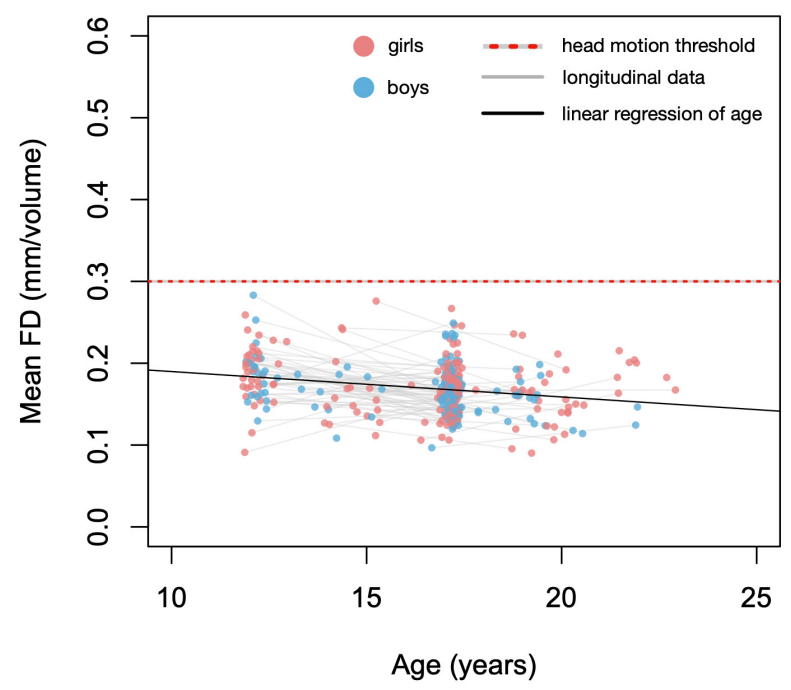

Supplementary Figure C.5. Estimated full-score head motion by age of subjects after quality control when subjects with excessive head motion have been excluded from analysis based on their mean framewise displacement (FD) and number of scrubbed high motion frames. 


\section{C.1.6 Reliability and stability of functional connectivity}

We use the phenotypic correlation as a proxy for short-term test-retest reliability and longterm test-retest stability assessment as it reveals coherence in individual variation within split-session and between session estimates of functional connectivity. The phenotypic correlation between full-scores measures and the two half-score measures at each age was obtained using a bivariate Cholesky decomposition with $A C E$ variance components to account for non-independence between family members and included sex and age fixed effects on the means.

Short-term reliability of functional connectivity is low to moderate for connections between resting-state networks $\left(\mathrm{r}_{\mathrm{ph}}=+0.36 \pm 0.09\right.$ at age 13 years; and $r_{\mathrm{ph}}=+0.40 \pm 0.09$ at age 18 years; Supplementary Figure C.6) and within resting-state networks $\left(r_{\mathrm{ph}}=+0.41 \pm\right.$ 0.12 at age 13 years; and $r_{\mathrm{ph}}=+0.44 \pm 0.07$ at age 18 years; Supplementary Figure C.6). Long-term stability of functional connectivity is low for connections between resting-state networks $\left(r_{\mathrm{ph}}=+0.26 \pm 0.12\right.$; Supplementary Figure C.6; Supplementary Table C.11) and within resting-state networks $\left(r_{\mathrm{ph}}=+0.35 \pm 0.11\right.$; Supplementary Figure C.6; Supplementary Table C.11).

\section{C.1.7 Variance explained by the reliable component of functional connectivity}

A proportion of the variance of each half-score measure can be explained by the reliable factor that is determined by its standardized factor loadings. Standardized factor loadings for a connection can vary from balanced (i.e. loading approximately equally on all four half-score measures; e.g. the bilateral connection of the sensorimotor network; Supplementary Figure C.8) to favoring the half-score measures of one of the ages (e.g. the medial - occipital connection of the visual network (Supplementary Figure C.7) and the sensorimotor - frontoparietal connection favoring age 18 years; Supplementary Figure C.7) to favoring a single half-score measure (e.g. the visual - cerebellum, and salience frontoparietal connections; Supplementary Figure C.7; and the right IFG - left poster STG connection of the language network favoring first half of age 18 years; Supplementary Figure C.8). Variation in standardized factor loadings between half-scores may be considered an indication for the level of noise (i.e. unique variance per half-score). On average $33 \%$ of the variances can be explained by the reliable factor, with higher standardized factor loadings on measurements at age 18 years (mean standardized factor loadings: 41\%) than at age 13 years (mean standardized factor loadings: 24\%) (Supplementary Figure C.7; Supplementary Figure C.8). 


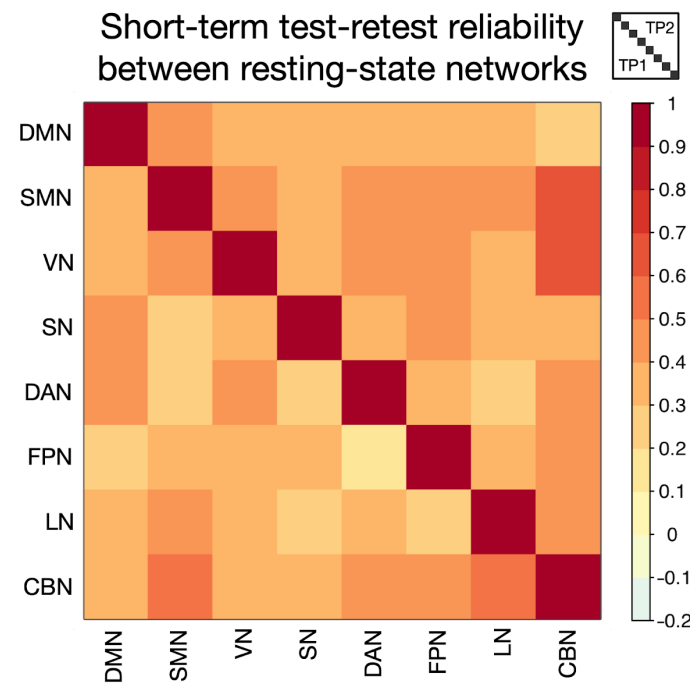

\section{Short-term test-retest reliability within resting-state networks}

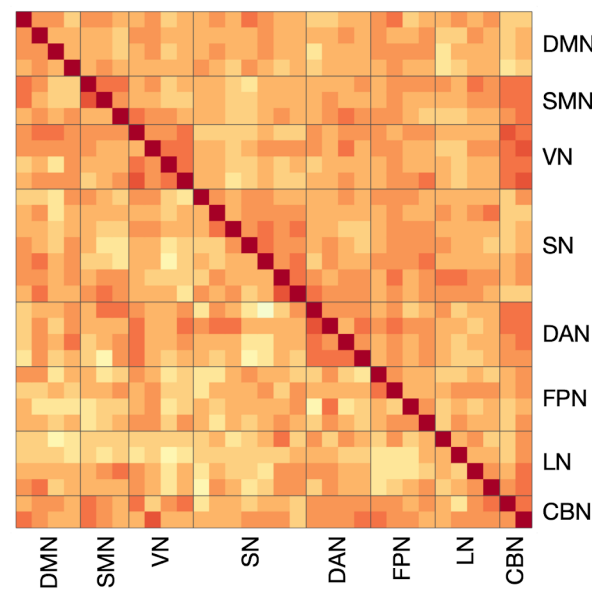

\section{Long-term test-retest reliability between resting-state networks}

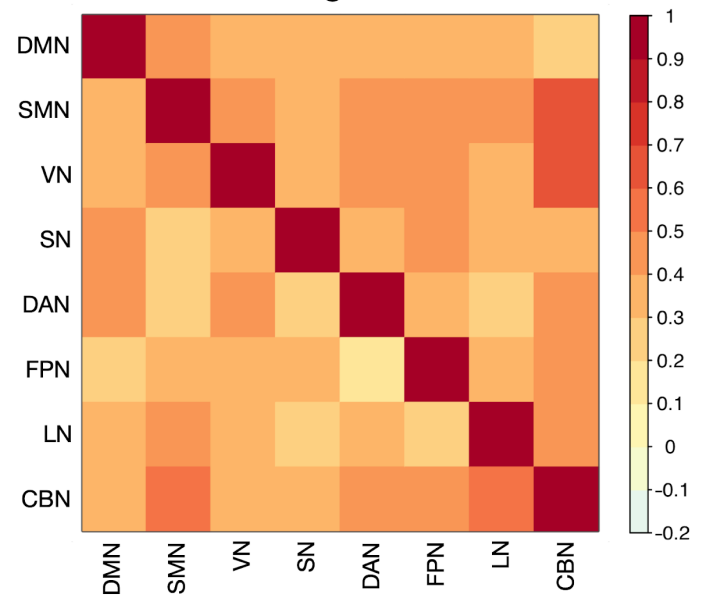

\section{Long-term test-retest reliability within resting-state networks}

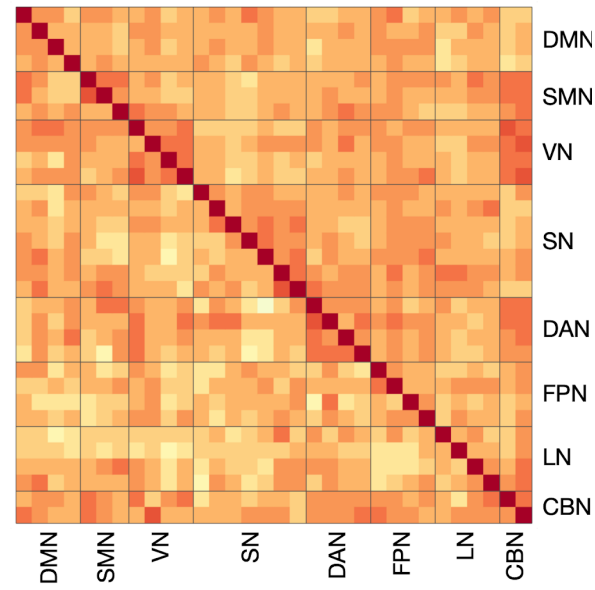

Supplementary Figure C.6. Phenotypic correlation of individuals functional connectivity estimates for connections between canonical resting-state networks (left) and with resting-state networks (right) for half-score estimates (top; approximation of short-term test-retest reliability within sessions) and full-score estimates (bottom; approximation of long-term test-retest stability between sessions). The phenotypic correlation coefficients between half-score measures of the same scan session at age 13 years (TP1) are located in the lowerleft triangle, and correlation coefficients at age 18 years (TP2) are located in the upper-right triangle. For the order of regions within resting-state networks, see Supplementary Table C.1. Abbreviations (in alphabetical order): $\mathrm{CBN}$ = cerebellar network; DAN = dorsal attention network; DMN = default mode network; FPN = frontoparietal network; LN = language network; SMN = sensorimotor network; $\mathrm{SN}$ = salience network; TP1 = time point 1 ; $\mathrm{TP} 2$ = time point $2 ; \mathrm{VN}=$ visual network . 


\begin{tabular}{|c|c|c|c|c|c|c|c|c|c|c|c|c|c|c|}
\hline \multirow{3}{*}{$\begin{array}{c}\text { Between RSNs } \\
\text { Connection } \\
\text { Default Mode - Sensorimotor }\end{array}$} & \multicolumn{4}{|c|}{ Common Factor } & \multicolumn{4}{|c|}{ Time point 1} & \multicolumn{6}{|c|}{ Time point 2} \\
\hline & \multirow{3}{*}{$\begin{array}{c}\text { Model } \\
\text { E }\end{array}$} & \multicolumn{2}{|c|}{ Factor } & \multirow{3}{*}{$\begin{array}{l}\text { Var. component } \\
\text { N/A }\end{array}$} & \multicolumn{2}{|c|}{ TP1 H1 } & \multicolumn{2}{|c|}{ TP1 H2 } & \multicolumn{3}{|c|}{ TP2 $\mathrm{H} 1$} & \multicolumn{3}{|c|}{ TP2 $\mathrm{H} 2$} \\
\hline & & & & & 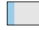 & & & & & & & & & \\
\hline & & $0 \%$ & $100 \%$ & & N/A & $6 \% 94 \%$ & $N / A$ & $3 \% 97 \%$ & N/A & $56 \%$ & $44 \%$ & N/A & $28 \%$ & $72 \%$ \\
\hline \multirow[t]{2}{*}{ Default Model - Visual } & CE & & & $42 \%[14 \% ; 70 \%]$ & L & & L & & 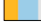 & & & 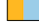 & & \\
\hline & & $42 \%$ & $58 \%$ & $\mathrm{p}=0.0052^{*}$ & $5 \%$ & $7 \% 88 \%$ & $7 \%$ & $9 \% 84 \%$ & $12 \%$ & $18 \%$ & $70 \%$ & $14 \%$ & $20 \%$ & $65 \%$ \\
\hline Default Mode - Salience & $\mathrm{E}$ & & & N/A & & 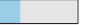 & L & & 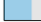 & & & & & \\
\hline & & $0 \%$ & $100 \%$ & & N/A & $49 \% 51 \%$ & $\mathrm{~N} / \mathrm{A}$ & $15 \% 85 \%$ & $\mathrm{~N} / \mathrm{A}$ & $24 \%$ & $76 \%$ & $\mathrm{~N} / \mathrm{A}$ & $37 \%$ & $63 \%$ \\
\hline efault Mode - Dorsal Attention & $\mathrm{AE}$ & & & $37 \%[15 \% ; 96 \%]$ & & 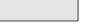 & L & 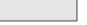 & 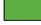 & & & 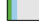 & & \\
\hline & & $37 \%$ & $63 \%$ & $\mathrm{p}=0.00933^{*}$ & $0 \%$ & $0 \% 100 \%$ & $1 \%$ & $2 \% 98 \%$ & $37 \%$ & $63 \%$ & $0 \%$ & $3 \%$ & $5 \%$ & \\
\hline Default Mode - Frontoparietal & E & & & N/A & 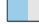 & - & $\mathrm{D}^{\mathrm{L}}$ & - & 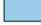 & 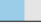 & & 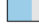 & 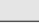 & \\
\hline & & $0 \%$ & $100 \%$ & & N/A & $18 \% 82 \%$ & $N / A$ & $24 \% \quad 76 \%$ & N/A & $56 \%$ & $44 \%$ & N/A & $21 \%$ & $79 \%$ \\
\hline Default Mode - Language & AE & & & $50 \%[18 \% ; 85 \%]$ & 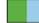 & $=$ & 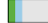 & 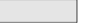 & 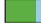 & L & 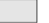 & 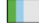 & & \\
\hline & & $50 \%$ & $50 \%$ & $\mathrm{p}=0.00368^{*}$ & $17 \%$ & $17 \% \quad 66 \%$ & $6 \%$ & $6 \% 89 \%$ & $27 \%$ & $27 \%$ & $46 \%$ & $7 \%$ & $7 \%$ & $85 \%$ \\
\hline Default Mode - Cerebellum & E & & & N/A & 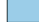 & 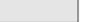 & 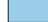 & 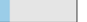 & 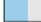 & 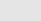 & & 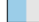 & & \\
\hline & & $0 \%$ & $100 \%$ & & N/A & $31 \% 69 \%$ & $N / A$ & $41 \% 59 \%$ & N/A & $21 \%$ & $79 \%$ & N/A & $16 \%$ & $84 \%$ \\
\hline Sensorimotor - Visual & CE & 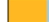 & & \%; 56\%] & 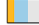 & 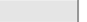 & 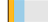 & & 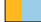 & 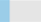 & & 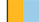 & 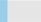 & \\
\hline & & $34 \%$ & $66 \%$ & $\mathrm{p}=0.00$ & $6 \%$ & $12 \% 82 \%$ & $5 \%$ & $9 \% \quad 87 \%$ & $14 \%$ & $28 \%$ & $58 \%$ & $14 \%$ & $27 \%$ & $59 \%$ \\
\hline Sensorimotor - Salience & $\mathrm{E}$ & & & N/A & 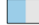 & & 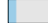 & & & & & 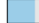 & & \\
\hline & & $0 \%$ & $100 \%$ & & N/A & $16 \% 84 \%$ & $\mathrm{~N} / \mathrm{A}$ & $7 \% \quad 93 \%$ & N/A & $35 \%$ & $65 \%$ & N/A & $24 \%$ & $76 \%$ \\
\hline Sensorimotor - Dorsal Attention & AE & & & $42 \%[15 \% ; 72 \%]$ & L & 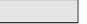 & L & & ( & 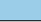 & & 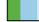 & & \\
\hline & & $42 \%$ & $58 \%$ & $\mathrm{p}=0.0$ & $5 \%$ & $7 \% 88 \%$ & $1 \%$ & $1 \% \quad 99 \%$ & $32 \%$ & $44 \%$ & $24 \%$ & $11 \%$ & $15 \%$ & $73 \%$ \\
\hline Sensorimotor - Frontoparietal & $\mathrm{AE}$ & & 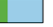 & $69 \%[32 \% ; 100 \%]$ & $\square$ & 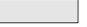 & 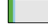 & & - & 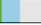 & & 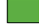 & 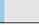 & \\
\hline & & $69 \%$ & $31 \%$ & $\mathrm{p}=0.00088^{\circ *}$ & $7 \%$ & $3 \% 89 \%$ & $4 \%$ & $2 \% 95 \%$ & $35 \%$ & $16 \%$ & $49 \%$ & $28 \%$ & $12 \%$ & $60 \%$ \\
\hline Sensorimotor - Language & $A E$ & & 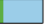 & $65 \%[24 \% ; 99 \%]$ & $\square$ & - & D & 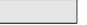 & - & 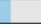 & & 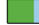 & & \\
\hline & & $65 \%$ & $35 \%$ & $\mathrm{p}=0.00442^{*}$ & $20 \%$ & $11 \% \quad 69 \%$ & $12 \%$ & $7 \% 81 \%$ & $28 \%$ & $15 \%$ & $57 \%$ & $23 \%$ & $12 \%$ & $65 \%$ \\
\hline Sensorimotor - Cerebellum & E & & & N/A & L & & L & 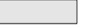 & 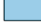 & 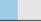 & & 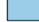 & & \\
\hline & & & $100 \%$ & & N/A & $29 \% 71 \%$ & $N / A$ & $24 \% 76 \%$ & N/A & $49 \%$ & $51 \%$ & N/A & $72 \%$ & $28 \%$ \\
\hline Visual - Salience & E & & & N/A & \begin{tabular}{|r} 
\\
\end{tabular} & 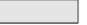 & $\square$ & & 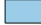 & & & 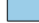 & & \\
\hline & & & $100 \%$ & & N/A & $27 \% 73 \%$ & N/A & $12 \% 88 \%$ & $\mathrm{~N} / \mathrm{A}$ & $33 \%$ & $67 \%$ & N/A & $29 \%$ & $71 \%$ \\
\hline Visual - Dorsal Attention & $\mathrm{E}$ & & & N/A & 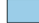 & 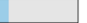 & $\square$ & 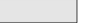 & - & 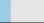 & & - & 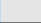 & \\
\hline & & & $100 \%$ & & N/A & $39 \% \quad 61 \%$ & N/A & $15 \% 85 \%$ & N/A & $41 \%$ & $59 \%$ & N/A & $36 \%$ & $64 \%$ \\
\hline Visual - Frontoparietal & $\mathrm{E}$ & & & $\mathrm{N} / \mathrm{A}$ & 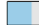 & 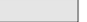 & 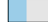 & 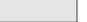 & 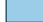 & 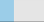 & & 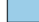 & 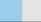 & \\
\hline & & & $100 \%$ & & N/A & $21 \% 79 \%$ & $\mathrm{~N} / \mathrm{A}$ & $16 \% 84 \%$ & $\mathrm{~N} / \mathrm{A}$ & $44 \%$ & $56 \%$ & N/A & $55 \%$ & $45 \%$ \\
\hline Visual - Language & CE & & & $40 \%[15 \% ; 65 \%]$ & 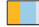 & & \begin{tabular}{|l} 
\\
\end{tabular} & 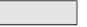 & - & & & 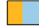 & & \\
\hline & & $40 \%$ & $60 \%$ & $\mathrm{p}=0.00748^{*}$ & $10 \%$ & $15 \% 76 \%$ & $9 \%$ & $14 \% 77 \%$ & $11 \%$ & $17 \%$ & $72 \%$ & $13 \%$ & $19 \%$ & $68 \%$ \\
\hline Visual - Cerebellum & $A E$ & & & $54 \%[29 \% ; 75 \%]$ & $\mathrm{T}$ & 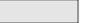 & 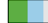 & & 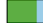 & 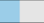 & & +2 & 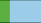 & \\
\hline & & $54 \%$ & $46 \%$ & $\mathrm{p}=0.00035^{* *}$ & $6 \%$ & $5 \% \quad 89 \%$ & $17 \%$ & $14 \% 69 \%$ & $26 \%$ & $22 \%$ & $51 \%$ & $44 \%$ & $37 \%$ & $19 \%$ \\
\hline Salience-Dorsal & E & & & $\mathrm{N} / \mathrm{A}$ & 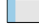 & & L & & 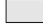 & 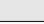 & & 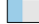 & - & \\
\hline & & & $100 \%$ & & N/A & $7 \% 93 \%$ & $N / A$ & $0 \% 100 \%$ & N/A & $0 \%$ & $100 \%$ & N/A & $13 \%$ & $87 \%$ \\
\hline Salience - Fr & $A E$ & & 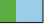 & ; 100\%] & 品 & - & 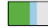 & 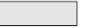 & 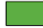 & 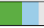 & + & 工 & 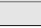 & \\
\hline & & $76 \%$ & $24 \%$ & $p=0.00002^{* * *}$ & $11 \%$ & $3 \% 85 \%$ & $19 \%$ & $6 \% 75 \%$ & $50 \%$ & $15 \%$ & $35 \%$ & $20 \%$ & $6 \%$ & $74 \%$ \\
\hline Salience - Language & $C E$ & L & & $32 \%[10 \% ; 62 \%]$ & L & & $L_{0}$ & 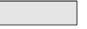 & 然 & - & & $\square$ & - & \\
\hline & & $32 \%$ & $68 \%$ & $\mathrm{p}=0.00945$ & $0 \%$ & $0 \% \quad 99 \%$ & $0 \%$ & $0 \% \quad 99 \%$ & $25 \%$ & $53 \%$ & $22 \%$ & $4 \%$ & $10 \%$ & $86 \%$ \\
\hline Salience-Cerebellum & CE & & & $49 \%[18 \% ; 83 \%]$ & $\square$ & - & 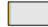 & 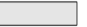 & 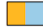 & 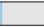 & & 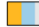 & & \\
\hline & & $49 \%$ & $51 \%$ & $\mathrm{p}=0.0 \mathrm{c}$ & $8 \%$ & $9 \% 83 \%$ & $1 \%$ & $1 \% 98 \%$ & $16 \%$ & $17 \%$ & $68 \%$ & $12 \%$ & $12 \%$ & $75 \%$ \\
\hline rsal Attention - Frontoparietal & E & & & N/A & 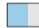 & - & 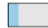 & & - & 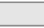 & & 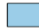 & & \\
\hline & & & $100 \%$ & & N/A & $18 \% 82 \%$ & $\mathrm{~N} / \mathrm{A}$ & $9 \% 91 \%$ & N/A & $18 \%$ & $82 \%$ & N/A & $63 \%$ & $37 \%$ \\
\hline Dorsal Attention - Language & E & & & $\mathrm{N} / \mathrm{A}$ & & & $\square$ & & 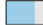 & & & - & & \\
\hline & & & $100 \%$ & & N/A & $33 \% \quad 67 \%$ & $\mathrm{~N} / \mathrm{A}$ & $26 \% 74 \%$ & N/A & $25 \%$ & $75 \%$ & N/A & $20 \%$ & $80 \%$ \\
\hline Dorsal Attention-Cerebellum & E & & & $\mathrm{N} / \mathrm{A}$ & 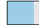 & & 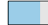 & & & & & & & \\
\hline & & & $100 \%$ & & N/A & $23 \% 77 \%$ & $N / A$ & $28 \% 72 \%$ & N/A & $36 \%$ & $64 \%$ & N/A & $60 \%$ & $40 \%$ \\
\hline Frontoparietal - Language & $A E$ & & & $42 \%$ & 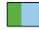 & & E & & ( & 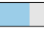 & & 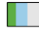 & 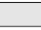 & \\
\hline & & $42 \%$ & $58 \%$ & $\mathrm{p}=\mathrm{c}$ & $12 \%$ & $16 \% 72 \%$ & $6 \%$ & $8 \% 85 \%$ & $24 \%$ & $33 \%$ & $43 \%$ & $7 \%$ & $10 \%$ & $83 \%$ \\
\hline Frontoparietal - Cerebellum & E & & & $\mathrm{N} / \mathrm{A}$ & 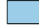 & 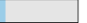 & 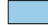 & & 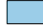 & 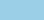 & & - & & \\
\hline & & & $100 \%$ & & N/A & $36 \% 64 \%$ & $N / A$ & $36 \% 64 \%$ & N/A & $73 \%$ & $27 \%$ & N/A & $26 \%$ & $74 \%$ \\
\hline Language - Cerebellum & CE & 1 & & \%; 63\% & 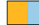 & - & $\square$ & & ( & T & & 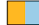 & & \\
\hline & & $42 \%$ & $58 \%$ & $\mathrm{p}=0.00058^{*}$ & $18 \%$ & $25 \% 58 \%$ & $12 \%$ & $16 \% 72 \%$ & $22 \%$ & $30 \%$ & $48 \%$ & $15 \%$ & $21 \%$ & $63 \%$ \\
\hline Mean FC Between All RSNs & CE & & & $\%[19 \% ; 60 \%]$ & 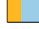 & 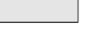 & 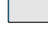 & . & 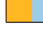 & 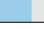 & & 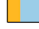 & & \\
\hline & & & $61 \%$ & $\mathrm{p}=0.00069^{*}$ & $12 \%$ & $19 \% \quad 69 \%$ & $0 \%$ & $0 \%$ & $23 \%$ & $36 \%$ & & $1 \%$ & $18 \%$ & \\
\hline
\end{tabular}

Supplementary Figure C.7. Variance components of the common factor mapped back to individual measures for between resting-state networks connections with significant additive genetic or common environmental variance components loading on the reliable common factor (after FDR correction). The confidence intervals and uncorrected p-values are reported for the additive genetic or common environmental variance component; $\mathrm{p}-$ values typeset in boldface are significant at uncorrected $p$-value $<0.05 ; p$-values marked with a symbol are significant after FDR correction at $\mathrm{Q}=0.05$ ( ${ }^{*}$ FDR-corrected $p$-value $<0.05$; ${ }^{* *}$ FDR-corrected $p$-value $<0.01$; *** FDR-corrected $p$-value $<0.001)$. Abbreviations: TP1 = time point 1 (at mean age 13 years); TP2 = time point 2 (at mean age 18 years); $\mathrm{H} 1$ = first half-score measure; $\mathrm{H} 2$ = second half-score measure; RSNs = resting-state networks. See Supplementary figure C.8 for legend. 


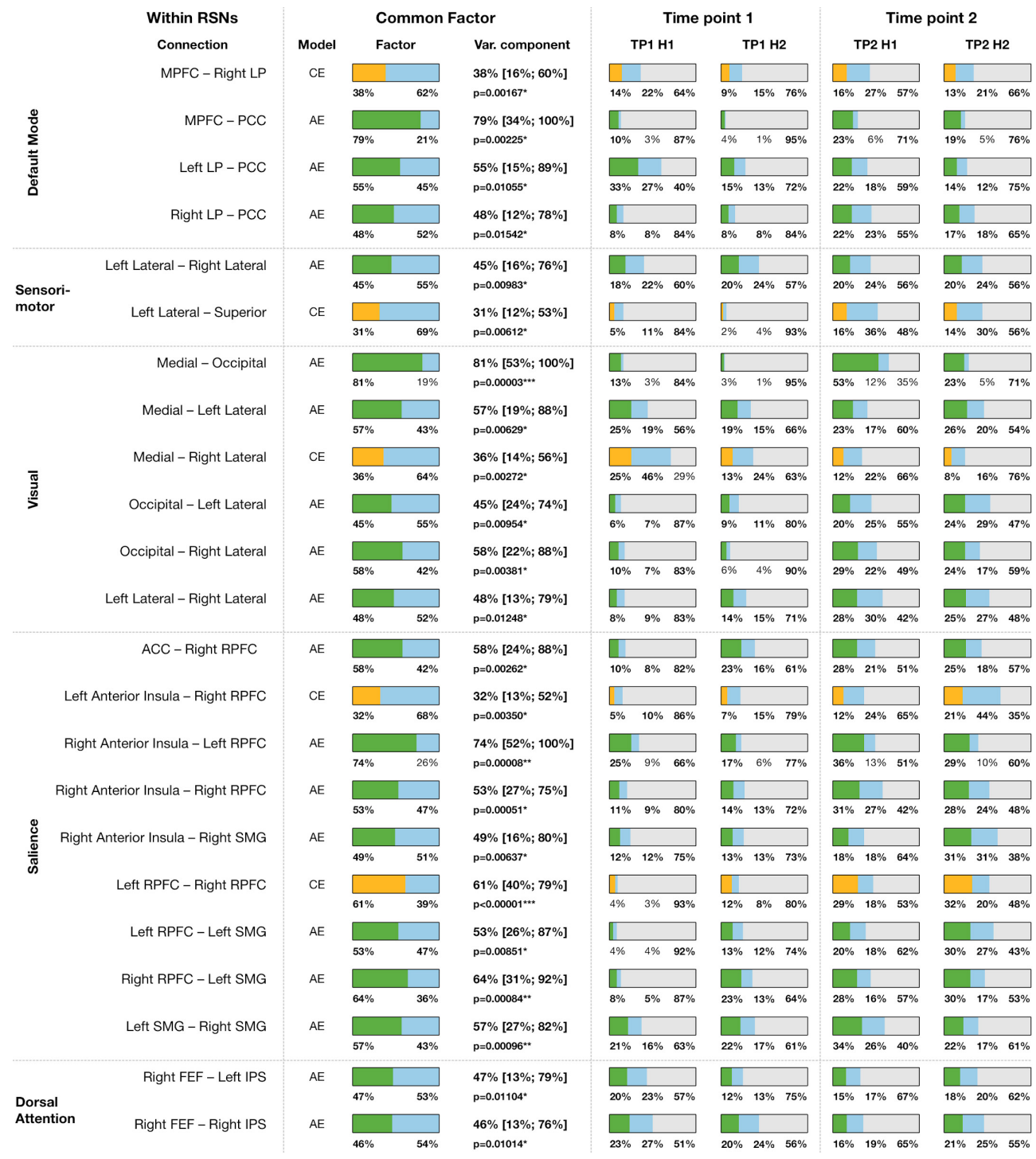

Supplementary Figure C.8 (continued on next page). Variance components of the common factor mapped back to individual measures for within resting-state networks connections with significant additive genetic or common environmental variance components loading on the reliable common factor (after FDR correction). The confidence intervals and uncorrected $\mathrm{p}$-values are reported for the additive genetic or common environmental variance component; $\mathrm{p}$-values typeset in boldface are significant at uncorrected $p$-value $<0.05 ; p$-values marked with a symbol are significant after FDR correction at $Q=0.05$ (* FDR-corrected $p$-value $<0.05$; ${ }^{* *}$ FDR-corrected $p-$ value $<0.01$; $^{* * *}$ FDR-corrected $p$-value $<0.001$ ). Abbreviations: TP1 = time point 1 (at mean age 13 years); TP2 = time point 2 (at mean age 18 years); $\mathrm{H} 1$ = first half-score measure; $\mathrm{H} 2$ = second half-score measure; RSNs = resting-state networks. 


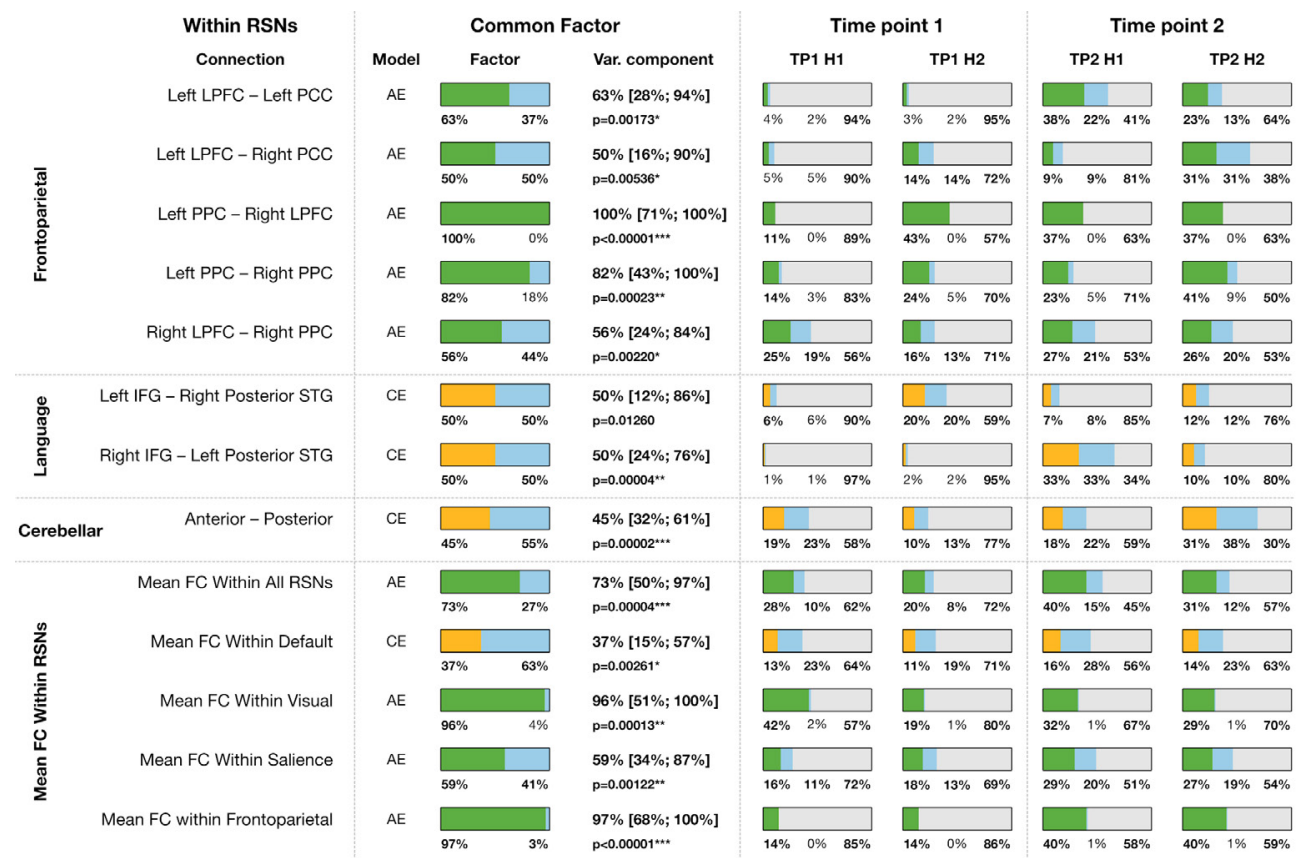

\begin{tabular}{||l|c||}
\hline \multicolumn{1}{|c||}{ Legend } & Details \\
Variance explained by common factor & Variance of individual measures \\
$\square$ Additive genetics & $\begin{array}{c}\text { Variance of individual measure } \\
\text { explained by common factor } \\
V_{c}=X+Y\end{array}$ \\
$\square$ Common environment & \\
$\square$ Unique environment & $\mathrm{X} \% \quad \mathrm{Y} \% \quad \mathrm{Z} \%$ \\
Measurement specific variance & $\begin{array}{c}\text { Total variance of individual measure } \\
\mathrm{V}_{\mathrm{t}}=\mathrm{X}+\mathrm{Y}+\mathrm{Z}=\mathrm{V}_{\mathrm{c}}+\mathrm{V}_{\mathrm{s}}=100 \%\end{array}$ \\
$\square$ Residual variance (i.e. noise) & \\
\hline
\end{tabular}

Supplementary Figure C.8 (continued). Variance components of the common factor mapped back to individual measures for within resting-state networks connections with significant additive genetic or common environmental variance components loading on the reliable common factor (after FDR correction). The confidence intervals and uncorrected p-values are reported for the additive genetic or common environmental variance component; $\mathrm{p}$-values typeset in boldface are significant at uncorrected $p$-value $<0.05$; $\mathrm{p}$-values marked with a symbol are significant after FDR correction at $\mathrm{Q}=0.05$ ( ${ }^{*}$ FDR-corrected $p$-value $<0.05 ;{ }^{* *}$ FDR-corrected $p-$ value $<0.01 ;{ }^{* * *}$ FDR-corrected $p$-value $<0.001$ ). Abbreviations: TP1 = time point 1 (at mean age 13 years); TP2 = time point 2 (at mean age 18 years); $\mathrm{H} 1$ = first half-score measure; $\mathrm{H} 2$ = second half-score measure; RSNs = resting-state networks. 


\section{C.1.8 Supplementary data tables}

A number of supplementary data tables (Supplementary Tables C.4 through C.14) are available that contain estimated model parameters, including their confidence intervals and significance levels, and model fit parameters, that were used to create figures and support statements in this text. Below is a brief description of each table.

\section{Table Description}

Table C.4 Model fit parameters for both single and two-factor models to determine which model best described the data - for connections between resting-state networks.

Table C.5 Same as Table C.4, but for connections within resting-state networks.

Table C.6 Heritability estimates of the two-factor model, with estimates for changes in heritability, and heritability of changes, including tests for genetic innovation and fluctuation of genes across the ages, and model fit parameters - for connections, both within and between resting-state networks, where a two-factor model best described the data (see Supplementary Tables C.4 and C.5).

Table C.7 Phenotypic parameter estimates from the single factor model, including group mean functional connectivity (Figure 4.2 from Chapter 4 ), and beta coefficients for sex, age, and head motion (Figure 4.3 from Chapter 4 ) - for connections between resting-state networks.

Table C.8 Genotypic parameter estimates from the single factor model, including standardized estimates of individual measures (Supplementary Figures C.7 and C8), and standardized estimates of the common factor (Figure 4.4 from Chapter 4) - for connections between resting-state networks.

Table C.9 Same as Table C.7, but for connections within resting-state networks.

Table C.10 Same as Table C.8, but for connections within resting-state networks.

Table C.11 Phenotypic association between functional connectivity and IQ test-scores, and association between longitudinal change in functional connectivity and longitudinal change in IQ test scores - for connections between resting-state networks.

Table C.12 Same as Table C.11, but for connections within resting-state networks.

Table C.13 Same as Table C.11, but for mean framewise displacement instead of IQ test scores.

Table C.14 Same as Table C.12, but for mean framewise displacement instead of IQ test scores. 


\section{C.1.9 Model fit criteria for one and two factor common pathway models of functional connectivity between resting-state networks}

Supplementary Table C.4 contains the model fit parameters for models with a single common factor $(1 \mathrm{~F})$ across both ages or two common factors $(2 \mathrm{~F})$, one for each age, for connections between resting-state networks. Model fit parameter were used to determine the model that best described the data using the log-likelihood (LL) ratio test.

Supplementary Table C.4 (continued on the next page). Model fit criteria for common pathway reliability twin model for functional connectivity between resting-state networks.

\begin{tabular}{|c|c|c|c|}
\hline Connection & One factor & Two factors & Optimal model \\
\hline Default Mode - & LL: -49.466 & LL: -44.712 & $1 \mathrm{~F}(p=0.090)$ \\
\hline Sensorimotor & AIC: 126.932 & AIC: 127.424 & \\
\hline Default Mode - & LL: -60.517 & LL: -55.725 & $1 \mathrm{~F}(p=0.088)$ \\
\hline Visual & AIC: 149.034 & AIC: 149.450 & \\
\hline Default Mode - & LL: -71.974 & LL: -67.967 & $1 \mathrm{~F}(p=0.156)$ \\
\hline Salience & AIC: 171.948 & AIC: 173.934 & \\
\hline Default Mode - & LL: -47.762 & LL: -41.256 & $2 \mathrm{~F}(p=0.023)$ \\
\hline Dorsal Attention & AIC: 123.524 & AIC: 120.512 & \\
\hline Default Mode - & LL: 5.029 & LL: 5.673 & $1 \mathrm{~F}(p=0.936)$ \\
\hline Frontoparietal & AIC: 17.942 & AIC: 26.654 & \\
\hline Default Mode - & LL: -37.274 & LL: -35.157 & $1 \mathrm{~F}(p=0.516)$ \\
\hline Language & AIC: 102.548 & AIC: 108.314 & \\
\hline Default Mode - & LL: 47.208 & LL: 47.405 & $1 \mathrm{~F}(p=0.995)$ \\
\hline Cerebellum & AIC: -66.416 & AIC: -56.810 & \\
\hline Sensorimotor - & LL: -121.349 & LL: -116.315 & $1 \mathrm{~F}(p=0.073)$ \\
\hline Visual & AIC: 270.698 & AIC: 270.630 & \\
\hline Sensorimotor - & LL: -63.770 & LL: -61.703 & $1 \mathrm{~F}(p=0.530)$ \\
\hline Salience & AIC: 155.54 & AIC: 161.406 & \\
\hline Sensorimotor - & LL: -108.293 & LL: -106.557 & $1 \mathrm{~F}(p=0.628)$ \\
\hline Dorsal Attention & AIC: 244.586 & AIC: 251.114 & \\
\hline Sensorimotor - & LL: -54.829 & LL: -52.172 & $1 \mathrm{~F}(p=0.379)$ \\
\hline Frontoparietal & AIC: 137.658 & AIC: 142.344 & \\
\hline Sensorimotor - & LL: -74.587 & LL: -70.926 & $1 \mathrm{~F}(p=0.198)$ \\
\hline Language & AIC: 177.174 & AIC: 179.852 & \\
\hline Sensorimotor - & LL: -113.738 & LL: -107.469 & $2 \mathrm{~F}(p=0.028)$ \\
\hline Cerebellum & AIC: 255.476 & AIC: 252.938 & \\
\hline
\end{tabular}




\begin{tabular}{|c|c|c|c|}
\hline Connection & One factor & Two factors & Optimal model \\
\hline \multirow[t]{2}{*}{ Visual - Salience } & LL: -49.001 & LL: -44.371 & $1 \mathrm{~F}(p=0.099)$ \\
\hline & AIC: 126.002 & AIC: 126.742 & \\
\hline Visual - Dorsal & LL: -98.375 & LL: -94.364 & $1 \mathrm{~F}(p=0.155)$ \\
\hline Attention & AIC: 224.750 & AIC: 226.728 & \\
\hline Visual - & LL: -13.782 & LL: -10.188 & $1 \mathrm{~F}(p=0.207)$ \\
\hline Frontoparietal & AIC: 55.564 & AIC: 58.376 & \\
\hline \multirow[t]{2}{*}{ Visual - Language } & LL: -80.492 & LL: -78.803 & $1 \mathrm{~F}(p=0.642)$ \\
\hline & AIC: 188.984 & AIC: 195.606 & \\
\hline Visual - & LL: -95.041 & LL: -91.136 & $1 \mathrm{~F}(p=0.167)$ \\
\hline Cerebellum & AIC: 218.082 & AIC: 220.272 & \\
\hline Salience - Dorsal & LL: -11.376 & LL: -9.377 & $1 \mathrm{~F}(p=0.550)$ \\
\hline Attention & AIC: 50.752 & AIC: 56.754 & \\
\hline Salience - & LL: -55.419 & LL: -52.757 & $1 \mathrm{~F}(p=0.378)$ \\
\hline Frontoparietal & AIC: 138.838 & AIC: 143.514 & \\
\hline Salience - & LL: -79.333 & LL: -77.517 & $1 \mathrm{~F}(p=0.604)$ \\
\hline Language & AIC: 186.666 & AIC: 193.034 & \\
\hline Salience - & LL: -1.346 & LL: 3.174 & $1 \mathrm{~F}(p=0.108)$ \\
\hline Cerebellum & AIC: 30.692 & AIC: 31.652 & \\
\hline Dorsal Attention - & LL: -38.645 & LL: -38.617 & $1 \mathrm{~F}(p=1.000)$ \\
\hline Frontoparietal & AIC: 105.290 & AIC: 115.234 & \\
\hline Dorsal Attention - & LL: -138.999 & LL: -137.039 & $1 \mathrm{~F}(p=0.561)$ \\
\hline Language & AIC: 305.998 & AIC: 312.078 & \\
\hline Dorsal Attention - & LL: -31.692 & LL: -28.019 & $1 \mathrm{~F}(p=0.196)$ \\
\hline Cerebellum & AIC: 91.384 & AIC: 94.038 & \\
\hline Frontoparietal - & LL: -37.516 & LL: -37.197 & $1 \mathrm{~F}(p=0.986)$ \\
\hline Language & AIC: 103.032 & AIC: 112.394 & \\
\hline Frontoparietal - & LL: -0.882 & LL: 1.023 & $1 \mathrm{~F}(p=0.577)$ \\
\hline Cerebellum & AIC: 29.764 & AIC: 35.954 & \\
\hline Language - & LL: -42.555 & LL: -32.025 & $2 \mathrm{~F}(p=0.001)$ \\
\hline Cerebellum & AIC: 113.110 & AIC: 102.050 & \\
\hline Mean FC Between & LL: 250.871 & LL: 253.621 & $1 \mathrm{~F}(p=0.358)$ \\
\hline All RSNs & AIC: -473.742 & AIC: -469.242 & \\
\hline
\end{tabular}

Supplementary Table C.4 (continued). The log-likelihood ratio test was used to determines the optimal number of factors. Abbreviations: $\mathrm{LL}=\log$-likelihood; AIC = Akaike Information Criteria; $1 \mathrm{~F}=$ model with one common factor on all four split-half measures of functional connectivity; $2 \mathrm{~F}=$ model with two common factors, each loading on the two split-half measures of functional connectivity within a time point. 
C.1.10 Model selection criteria for one and two factor common pathway models of functional connectivity within resting-state networks

Supplementary Table C.5 contains the model fit parameters for models with a single common factor $(1 \mathrm{~F})$ across both ages or two common factors (2F), one for each age, for connections within resting-state networks. Model fit parameter were used to determine the model that best described the data using the log-likelihood (LL) ratio test.

Supplementary Table C.5 (continued on the next four pages). Model fit criteria for common pathway reliability twin model for functional connectivity within resting-state networks.

\begin{tabular}{|c|c|c|c|}
\hline Connection & One factor & Two factors & Optimal model \\
\hline \multicolumn{4}{|l|}{ Default mode } \\
\hline MPFC - Left LP & $\begin{array}{l}\text { LL: } 26.734 \\
\text { AIC: }-25.468\end{array}$ & $\begin{array}{l}\text { LL: } 27.653 \\
\text { AIC: }-17.306\end{array}$ & $1 \mathrm{~F}(p=0.871)$ \\
\hline MPFC - Right LP & $\begin{array}{l}\text { LL: } 1.771 \\
\text { AIC: } 24.458\end{array}$ & $\begin{array}{l}\text { LL: } 7.501 \\
\text { AIC: } 22.998\end{array}$ & $2 \mathrm{~F}(p=0.043)$ \\
\hline MPFC - PCC & $\begin{array}{l}\text { LL: } 1.445 \\
\text { AIC: } 25.11\end{array}$ & $\begin{array}{l}\text { LL: } 7.741 \\
\text { AIC: } 22.518\end{array}$ & $2 \mathrm{~F}(p=0.028)$ \\
\hline Left LP - Right LP & $\begin{array}{l}\text { LL: }-20.47 \\
\text { AIC: } 68.94\end{array}$ & $\begin{array}{l}\text { LL: }-16.502 \\
\text { AIC: } 71.004\end{array}$ & $1 \mathrm{~F}(p=0.160)$ \\
\hline Left LP - PCC & $\begin{array}{l}\text { LL: }-14.742 \\
\text { AIC: } 57.484\end{array}$ & $\begin{array}{l}\text { LL: }-10.884 \\
\text { AIC: } 59.768\end{array}$ & $1 \mathrm{~F}(p=0.173)$ \\
\hline Right LP - PCC & $\begin{array}{l}\text { LL: }-23.362 \\
\text { AIC: } 74.724\end{array}$ & $\begin{array}{l}\text { LL: }-20.263 \\
\text { AIC: } 78.526\end{array}$ & $1 \mathrm{~F}(p=0.287)$ \\
\hline \multicolumn{4}{|l|}{ Sensorimotor } \\
\hline $\begin{array}{l}\text { Left Lateral - Right } \\
\text { Lateral }\end{array}$ & $\begin{array}{l}\text { LL: }-101.748 \\
\text { AIC: } 231.496\end{array}$ & $\begin{array}{l}\text { LL: }-91.811 \\
\text { AIC: } 221.622\end{array}$ & $2 F(p=0.001)$ \\
\hline $\begin{array}{l}\text { Left Lateral - } \\
\text { Superior }\end{array}$ & $\begin{array}{l}\text { LL: }-70.092 \\
\text { AIC: } 168.184\end{array}$ & $\begin{array}{l}\text { LL: }-65.585 \\
\text { AIC: } 169.170\end{array}$ & $1 \mathrm{~F}(p=0.109)$ \\
\hline $\begin{array}{l}\text { Right Lateral - } \\
\text { Superior }\end{array}$ & $\begin{array}{l}\text { LL: }-76.612 \\
\text { AIC: } 181.224\end{array}$ & $\begin{array}{l}\text { LL: }-73.066 \\
\text { AIC: } 184.132\end{array}$ & $1 \mathrm{~F}(p=0.214)$ \\
\hline \multicolumn{4}{|l|}{ Visual } \\
\hline Medial - Occipital & $\begin{array}{l}\text { LL: }-133.890 \\
\text { AIC: } 295.780\end{array}$ & $\begin{array}{l}\text { LL: }-126.359 \\
\text { AIC: } 290.718\end{array}$ & $2 \mathrm{~F}(p=0.010)$ \\
\hline Medial - Left & LL: -111.304 & LL: -101.518 & $2 \mathrm{~F}(p=0.002)$ \\
\hline Lateral & AIC: 250.608 & AIC: 241.036 & \\
\hline
\end{tabular}




\begin{tabular}{|c|c|c|c|}
\hline Connection & One factor & Two factors & Optimal model \\
\hline Medial - Right & LL: -91.045 & LL: -75.266 & $2 \mathrm{~F}(p<0.001)$ \\
\hline Lateral & AIC: 210.09 & AIC: 188.532 & \\
\hline Occipital - Left & LL: -123.151 & LL: -116.770 & $2 \mathrm{~F}(p=0.026)$ \\
\hline Lateral & AIC: 274.302 & AIC: 271.540 & \\
\hline Occipital - Right & LL: -129.103 & LL: -121.779 & $2 \mathrm{~F}(p=0.012)$ \\
\hline Lateral & AIC: 286.206 & AIC: 281.558 & \\
\hline Left Lateral - Right & LL: -152.271 & LL: -144.463 & $2 \mathrm{~F}(p=0.008)$ \\
\hline Lateral & AIC: 332.542 & AIC: 326.926 & \\
\hline \multicolumn{4}{|l|}{ Salience } \\
\hline ACC - Left Anterior & LL: -26.947 & LL: -26.114 & $1 \mathrm{~F}(p=0.893)$ \\
\hline Insula & AIC: 81.894 & AIC: 90.228 & \\
\hline ACC - Right & LL: -21.391 & LL: -14.485 & $2 \mathrm{~F}(p=0.017)$ \\
\hline Anterior Insula & AIC: 70.782 & AIC: 66.970 & \\
\hline \multirow[t]{2}{*}{ ACC - Left RPFC } & LL: -12.366 & LL: -11.367 & $1 \mathrm{~F}(p=0.849)$ \\
\hline & AIC: 52.732 & AIC: 60.734 & \\
\hline \multirow[t]{2}{*}{ ACC - Right RPFC } & LL: -26.544 & LL: -22.631 & $1 \mathrm{~F}(p=0.166)$ \\
\hline & AIC: 81.088 & AIC: 83.262 & \\
\hline \multirow[t]{2}{*}{ ACC - Left SMG } & LL: 1.775 & LL: 1.879 & $1 \mathrm{~F}(p=0.999)$ \\
\hline & AIC: 24.450 & AIC: 34.242 & \\
\hline \multirow[t]{2}{*}{ ACC - Right SMG } & LL: 19.679 & LL: 21.898 & $1 \mathrm{~F}(p=0.488)$ \\
\hline & AIC: -11.358 & AIC: -5.796 & \\
\hline Left Anterior Insula & LL: -65.583 & LL: -58.684 & $2 \mathrm{~F}(p=0.017)$ \\
\hline - Right Anterior & AIC: 159.166 & AIC: 155.368 & \\
\hline \multicolumn{4}{|l|}{ Insula } \\
\hline Left Anterior Insula & LL: 8.095 & LL: 9.783 & $1 \mathrm{~F}(p=0.642)$ \\
\hline - Left RPFC & AIC: 11.810 & AIC: 18.434 & \\
\hline Left Anterior Insula & LL: 15.868 & LL: 20.113 & $1 \mathrm{~F}(p=0.131)$ \\
\hline - Right RPFC & AIC: -3.736 & AIC: -2.226 & \\
\hline Left Anterior Insula & LL: -23.246 & LL: -20.835 & $1 \mathrm{~F}(p=0.438)$ \\
\hline - Left SMG & AIC: 74.492 & AIC: 79.670 & \\
\hline Left Anterior Insula & LL: -5.830 & LL: -5.783 & $1 \mathrm{~F}(p=1.000)$ \\
\hline - Right SMG & AIC: 39.660 & AIC: 49.566 & \\
\hline Right Anterior & LL: 33.946 & LL: 37.938 & $1 \mathrm{~F}(p=0.157)$ \\
\hline Insula - Left RPFC & AIC: -39.892 & AIC: -37.876 & \\
\hline Right Anterior & LL: -4.724 & LL: -1.717 & $1 \mathrm{~F}(p=0.305)$ \\
\hline Insula - Right RPFC & AIC: 37.448 & AIC: 41.434 & \\
\hline
\end{tabular}




\begin{tabular}{|c|c|c|c|}
\hline Connection & One factor & Two factors & Optimal model \\
\hline Right Anterior & LL: -26.664 & LL: -25.687 & $1 \mathrm{~F}(p=0.856)$ \\
\hline Insula - Left SMG & AIC: 81.328 & AIC: 89.374 & \\
\hline Right Anterior & LL: -26.793 & LL: -22.068 & $1 \mathrm{~F}(p=0.092)$ \\
\hline Insula - Right SMG & AIC: 81.586 & AIC: 82.136 & \\
\hline Left RPFC - Right & LL: -24.760 & LL: -14.094 & $2 \mathrm{~F}(p=0.001)$ \\
\hline RPFC & AIC: 77.520 & AIC: 66.188 & \\
\hline Left RPFC - Left & LL: 23.278 & LL: 26.101 & $1 \mathrm{~F}(p=0.342)$ \\
\hline SMG & AIC: -18.556 & AIC: -14.202 & \\
\hline Left RPFC - Right & LL: 12.200 & LL: 14.230 & $1 \mathrm{~F}(p=0.541)$ \\
\hline SMG & AIC: 3.600 & AIC: 9.540 & \\
\hline Right RPFC - Left & LL: 2.212 & LL: 6.481 & $1 \mathrm{~F}(p=0.129)$ \\
\hline SMG & AIC: 23.576 & AIC: 25.038 & \\
\hline Right RPFC - Right & LL: -39.683 & LL: -37.539 & $1 \mathrm{~F}(p=0.509)$ \\
\hline SMG & AIC: 107.366 & AIC: 113.078 & \\
\hline Left SMG - Right & LL: -61.609 & LL: -46.602 & $2 \mathrm{~F}(p<0.001)$ \\
\hline SMG & AIC: 151.218 & AIC: 131.204 & \\
\hline \multicolumn{4}{|l|}{ Dorsal Attention } \\
\hline Left FEF - Right & LL: -29.274 & LL: -24.921 & $1 \mathrm{~F}(p=0.121)$ \\
\hline FEF & AIC: 86.548 & AIC: 87.842 & \\
\hline \multirow[t]{2}{*}{ Left FEF - Left IPS } & LL: -13.481 & LL: -10.767 & $1 \mathrm{~F}(p=0.366)$ \\
\hline & AIC: 54.962 & AIC: 59.534 & \\
\hline \multirow[t]{2}{*}{ Left FEF - Right IPS } & LL: -9.122 & LL: -4.650 & $1 \mathrm{~F}(p=0.111)$ \\
\hline & AIC: 46.244 & AIC: 47.300 & \\
\hline \multirow[t]{2}{*}{ Right FEF - Left IPS } & LL: -57.588 & LL: -52.736 & $1 \mathrm{~F}(p=0.084)$ \\
\hline & AIC: 143.176 & AIC: 143.472 & \\
\hline Right FEF - Right & LL: -104.318 & LL: -93.235 & $2 \mathrm{~F}(p<0.001)$ \\
\hline IPS & AIC: 236.636 & AIC: 224.47 & \\
\hline \multirow[t]{2}{*}{ Left IPS - Right IPS } & LL: -40.069 & LL: -31.766 & $2 \mathrm{~F}(p=0.005)$ \\
\hline & AIC: 108.138 & AIC: 101.532 & \\
\hline \multicolumn{4}{|l|}{ Frontoparietal } \\
\hline Left LPFC - Left & LL: -39.561 & LL: -25.094 & $2 \mathrm{~F}(p<0.001)$ \\
\hline PPC & AIC: 107.122 & AIC: 88.188 & \\
\hline Left LPFC - Right & LL: -18.438 & LL: -15.469 & $1 \mathrm{~F}(p=0.312)$ \\
\hline LPFC & AIC: 64.876 & AIC: 68.938 & \\
\hline
\end{tabular}




\begin{tabular}{|c|c|c|c|}
\hline Connection & One factor & Two factors & Optimal model \\
\hline Left LPFC - Right & LL: 52.982 & LL: 60.209 & $2 \mathrm{~F}(p=0.013)$ \\
\hline PPC & AIC: -77.964 & AIC: -82.418 & \\
\hline Left PPC - Right & LL: 30.636 & LL: 30.636 & $1 \mathrm{~F}(p=1.000)$ \\
\hline LPFC & AIC: -33.272 & AIC: -23.272 & \\
\hline Left PPC - Right & LL: -0.786 & LL: 5.603 & $2 \mathrm{~F}(p=0.026)$ \\
\hline PPC & AIC: 29.572 & AIC: 26.794 & \\
\hline Right LPFC - Right & LL: -6.964 & LL: -4.982 & $1 \mathrm{~F}(p=0.554)$ \\
\hline PPC & AIC: 41.928 & AIC: 47.964 & \\
\hline \multicolumn{4}{|l|}{ Language } \\
\hline \multirow[t]{2}{*}{ Left IFG - Right IFG } & LL: 20.243 & LL: 23.890 & $1 \mathrm{~F}(p=0.200)$ \\
\hline & AIC: -12.486 & AIC: -9.780 & \\
\hline Left IFG - Left & LL: 1.421 & LL: 3.116 & $1 \mathrm{~F}(p=0.640)$ \\
\hline Posterior STG & AIC: 25.158 & AIC: 31.768 & \\
\hline Left IFG - Right & LL: -3.204 & LL: -1.670 & $1 \mathrm{~F}(p=0.689)$ \\
\hline Posterior STG & AIC: 34.408 & AIC: 41.340 & \\
\hline Right IFG - Left & LL: 44.773 & LL: 46.697 & $1 \mathrm{~F}(p=0.572)$ \\
\hline Posterior STG & AIC: -61.546 & AIC: -55.394 & \\
\hline Right IFG - Right & LL: 2.694 & LL: 4.778 & $1 \mathrm{~F}(p=0.525)$ \\
\hline Posterior STG & AIC: 22.612 & AIC: 28.444 & \\
\hline Left Posterior STG - & LL: -57.990 & LL: -57.074 & $1 \mathrm{~F}(p=0.872)$ \\
\hline Right Posterior STG & AIC: 143.980 & AIC: 152.148 & \\
\hline \multicolumn{4}{|l|}{ Cerebellar } \\
\hline \multirow[t]{2}{*}{ Anterior - Posterior } & LL: -45.773 & LL: -42.455 & $1 \mathrm{~F}(p=0.249)$ \\
\hline & AIC: 119.546 & AIC: 122.910 & \\
\hline \multicolumn{4}{|l|}{ Mean FC Within RSN } \\
\hline \multirow[t]{2}{*}{ All RSNs } & LL: 456.201 & LL: 457.709 & $1 \mathrm{~F}(p=0.550)$ \\
\hline & AIC: -884.402 & AIC: -877.418 & \\
\hline \multirow[t]{2}{*}{ Default Mode } & LL: 444.530 & LL: 446.529 & $1 \mathrm{~F}(p=0.302)$ \\
\hline & AIC: -861.060 & AIC: -855.058 & \\
\hline \multirow[t]{2}{*}{ Sensorimotor } & LL: 203.990 & LL: 207.011 & $2 \mathrm{~F}(p=0.036)$ \\
\hline & AIC: -379.980 & AIC: -376.022 & \\
\hline \multirow[t]{2}{*}{ Visual } & LL: 5.089 & LL: 11.046 & $2 \mathrm{~F}(p=0.003)$ \\
\hline & AIC: 17.822 & AIC: 15.908 & \\
\hline \multirow[t]{2}{*}{ Salience } & LL: 51.818 & LL: 60.850 & $1 \mathrm{~F}(p=0.765)$ \\
\hline & AIC: -75.636 & AIC: -83.700 & \\
\hline
\end{tabular}




\begin{tabular}{llll}
\hline Connection & One factor & Two factors & Optimal model \\
\hline Dorsal Attention & LL: 250.402 & LL: 251.691 & $2 \mathrm{~F}(\boldsymbol{p}=\mathbf{0 . 0 1 3})$ \\
& AIC: -472.804 & AIC: -465.382 & \\
Frontoparietal & LL: 126.590 & LL: 133.800 & \multirow{2}{*}{$1 \mathrm{~F}(p=0.248)$} \\
& AIC: -225.180 & AIC: -229.600 & \\
Language & LL: 194.572 & LL: 197.900 & 1F $(p=0.977)$ \\
& AIC: -361.144 & AIC: -357.800 & \\
\hline
\end{tabular}

Supplementary Table C.5 (continued). The log-likelihood ratio test was used to determines the optimal number of factors. Abbreviations: LL = log-likelihood; AIC = Akaike Information Criteria; $1 \mathrm{~F}=$ model with one common factor on all four split-half measures of functional connectivity; $2 \mathrm{~F}=$ model with two common factors, each loading on the two split-half measures of functional connectivity within a time point. 
C.1.11 Dynamics of genetic and environmental influences on functional connectivity throughout adolescence

Supplementary Table C.6 contains estimated parameters and model fit parameters for the common factor model with two factors for connections between resting-state networks. The columns "Factor 1" and "Factor 2" contain the heritability $\left(a^{2}\right)$, common environment $\left(c^{2}\right)$, and unique environment $\left(e^{2}\right)$ estimates and their confidence intervals for that factor. The dynamics column contains the change in heritability $\left(\Delta\left(a^{2}\right)\right)$ or common environment $\left(\Delta\left(c^{2}\right)\right)$ estimates from age 13 years to age 18 years, and heritability $\left(a^{2}(\Delta)\right)$ or common environment $\left(c^{2}(\Delta)\right)$ estimates of change in functional connectivity; both with confidence intervals and statistical significance whether change is different from zero. In addition, significance is reported on testing for innovation (innov), used to test whether unique genes or common environment influence one of the common factors, and testing for fluctuating influences (flux), used to test whether the same genes or common environment exerts a different amount of influence on one of the common factors compared to the other. Statistically significant effects are printed in boldface. The last column contains the loglikelihood model fit parameters of the full model with ACE latent variance components loadings on each of the common factors, and its nested models with only AE, CE, or E; these parameters are used to determine which of the models (full or nested) best described the data using the log-likelihood (LL) ratio test, with the best fitting model printed in bold. Estimates without data because they do not apply are marked with N/A (e.g. no estimate for $c^{2}, \Delta\left(c^{2}\right)$, or $c^{2}(\Delta)$ are available in models for which an AE-model best described the data). 


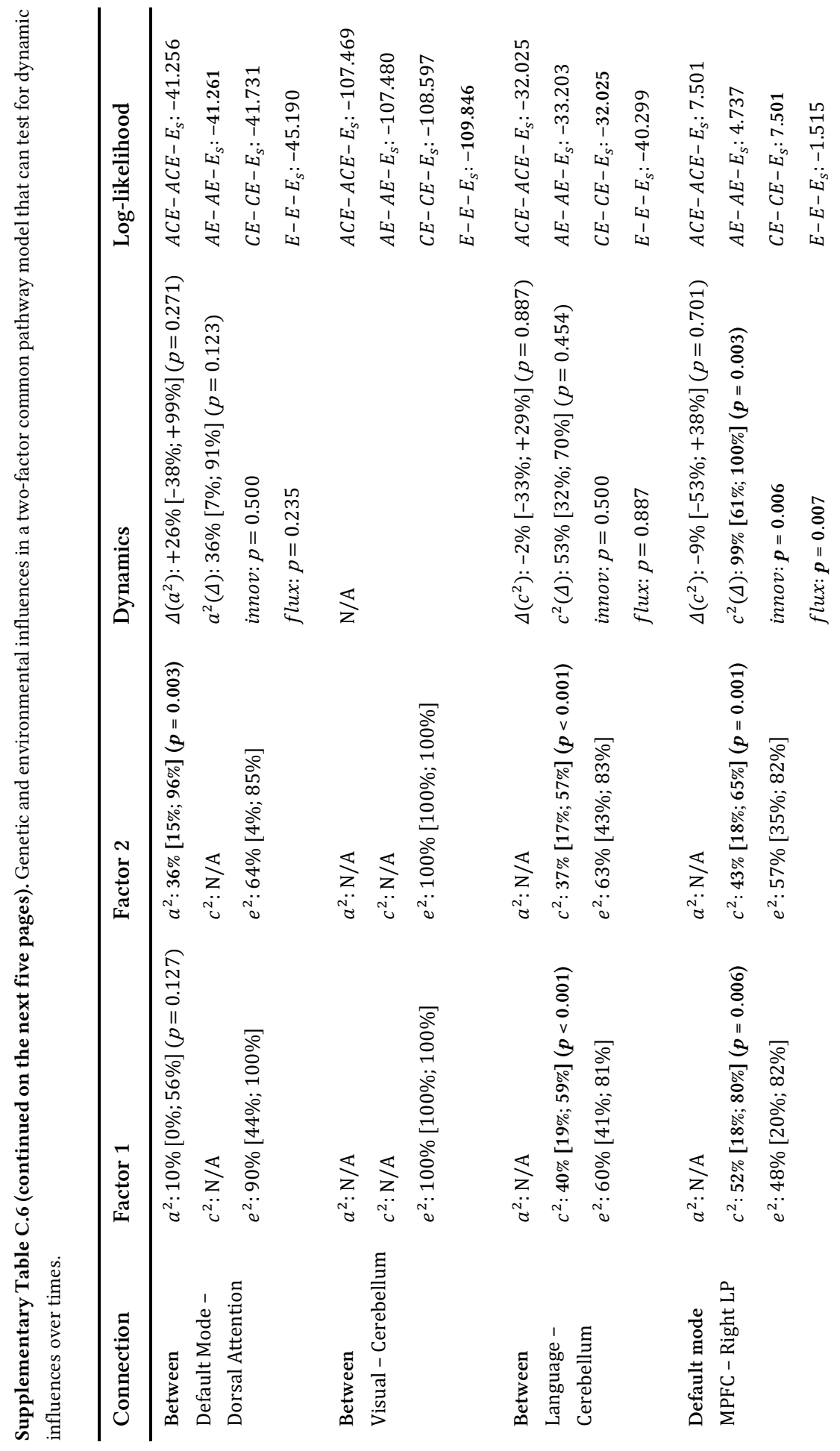




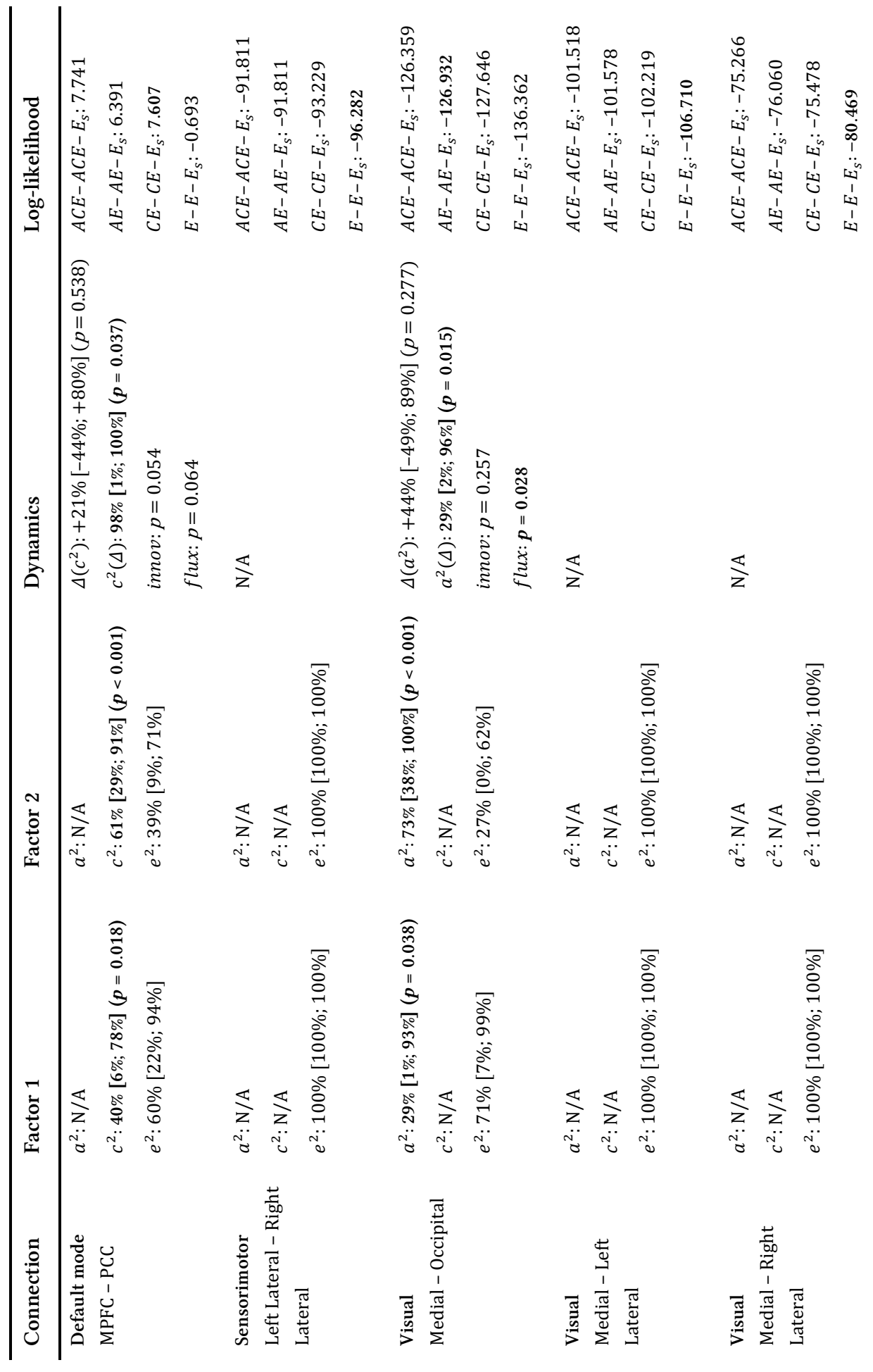




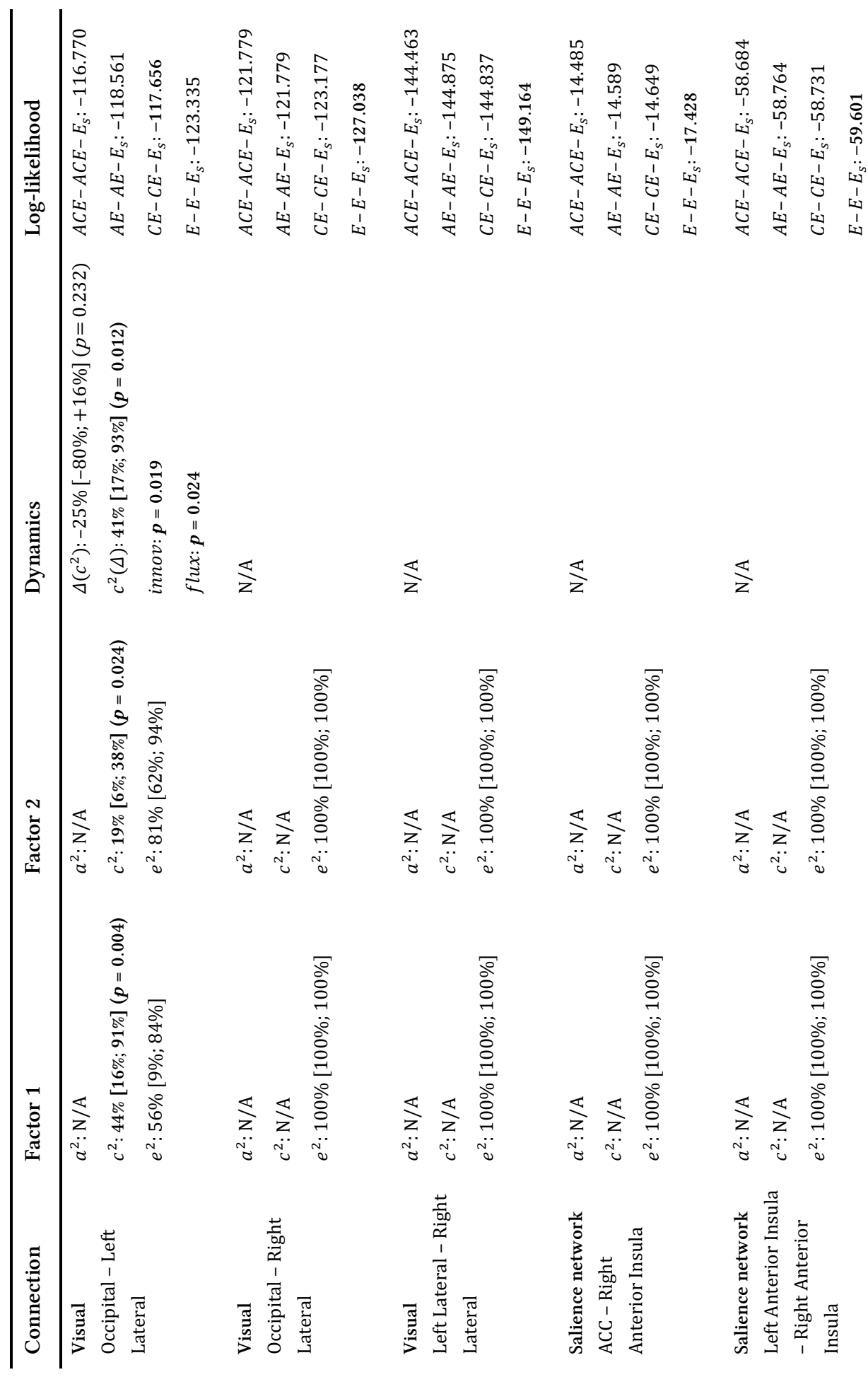




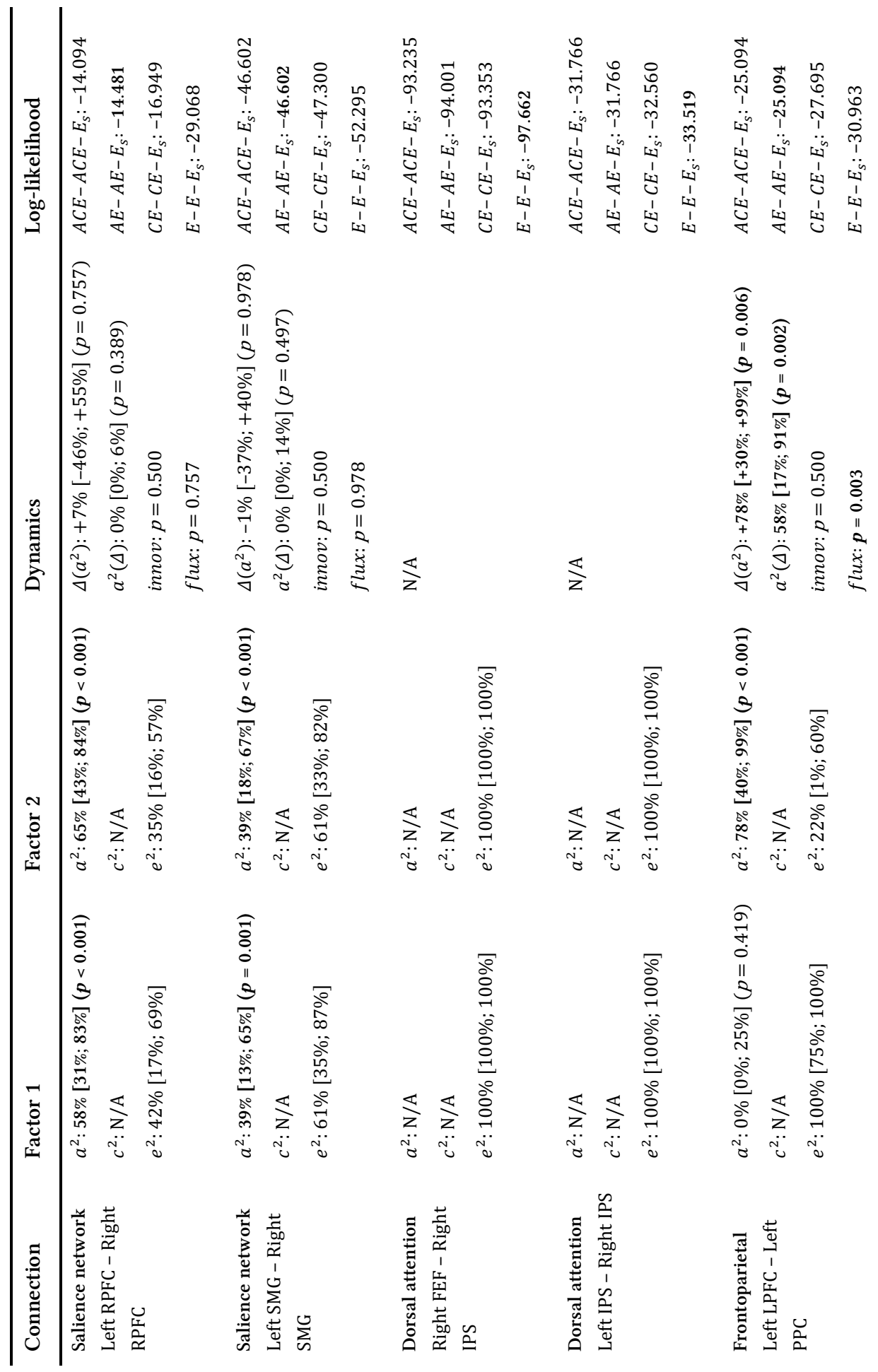




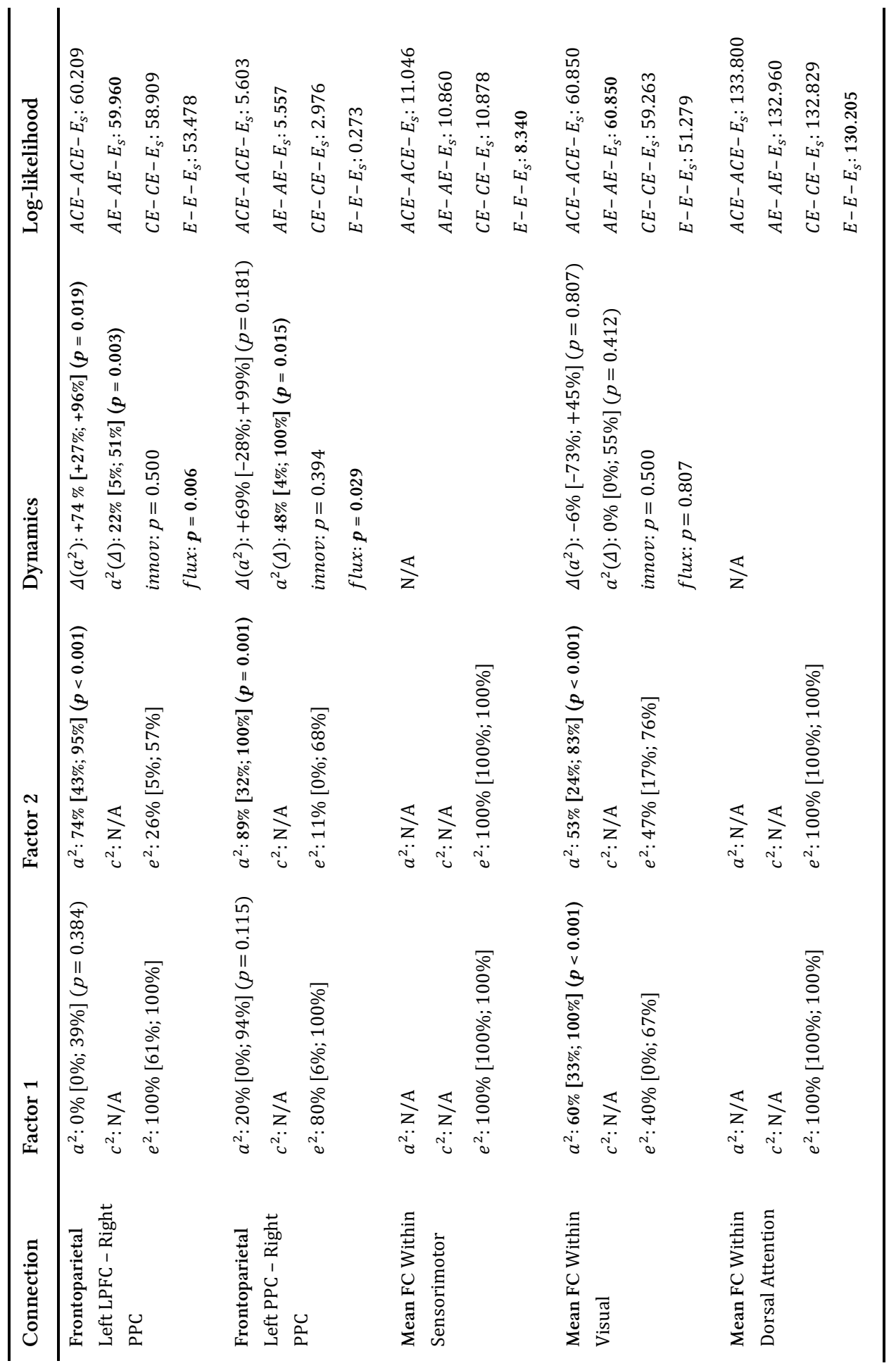




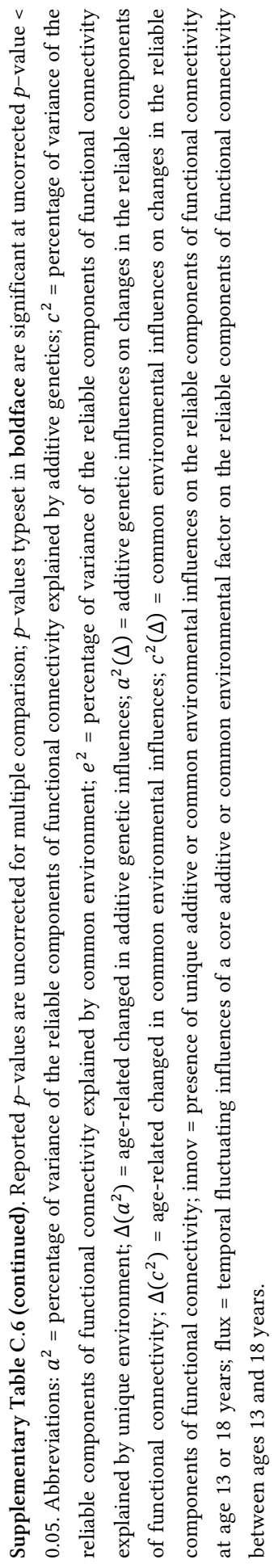




\section{C.1.12 Phenotypic effects on functional connectivity between resting-state networks} Supplementary Table C.7 contains the estimated phenotypic parameters and model fit parameters for the common factor model with a single factor for connections between resting-state networks. The "Half-score" columns contain the group-mean and standard deviation (M) of the functional connectivity estimates (see Figure 4.2 from Chapter 4), the standardized estimates for the proportion of variance explained by the common factor $\left(V_{c}\right)$ and measurement-specific variance $\left(V_{s}\right.$; i.e. considered noise), and their confidence intervals (see Supplementary Figures C.7 and C.8). The last three columns contain the beta coefficients and their significance for sex $\left(\beta_{\text {sex }}\right)$, age $\left(\beta_{\text {age }}\right)$, and head motion $\left(\beta_{F D}\right)$ measured as mean framewise displacement (mean FD); see Figure 4.3 from Chapter 4. Statistically significant effects are printed in bold; with effects significant after FDR-correction marked with symbols $\left({ }^{*}\right.$ FDR-p $<0.05 ;{ }^{* *}$ FDR-p $<0.01 ;{ }^{* * *}$ FDR-p $\left.<0.001\right)$. 


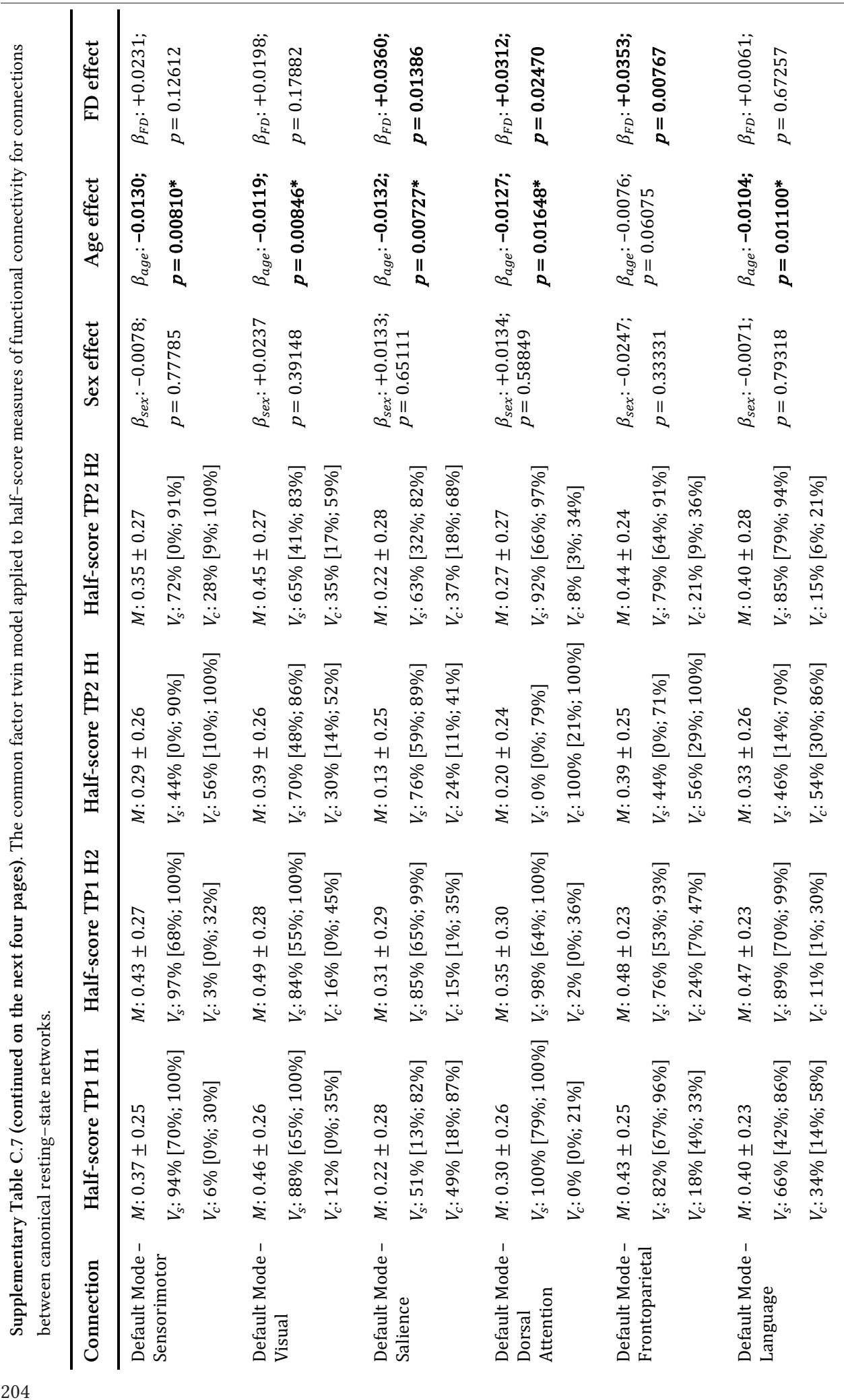




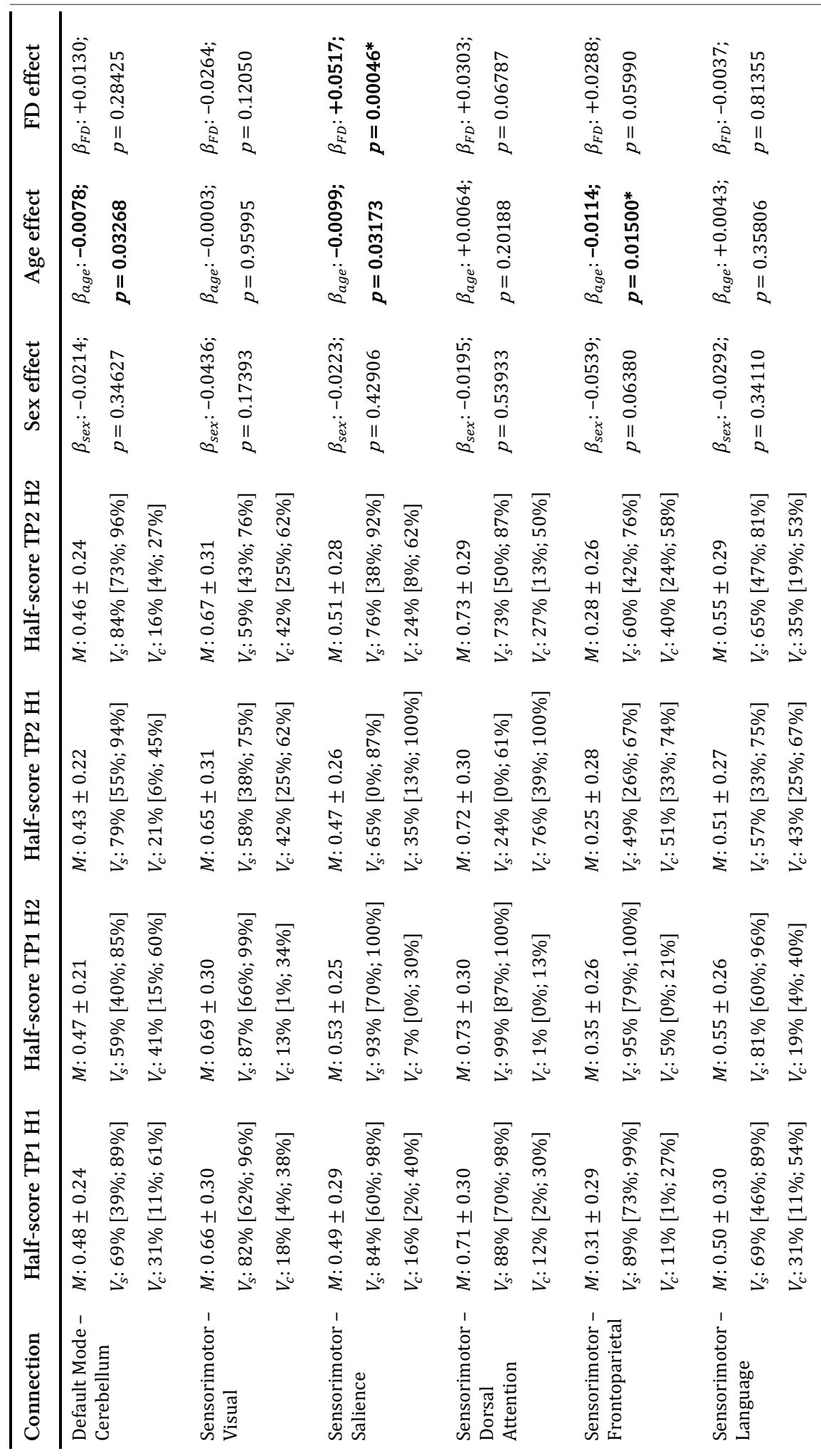




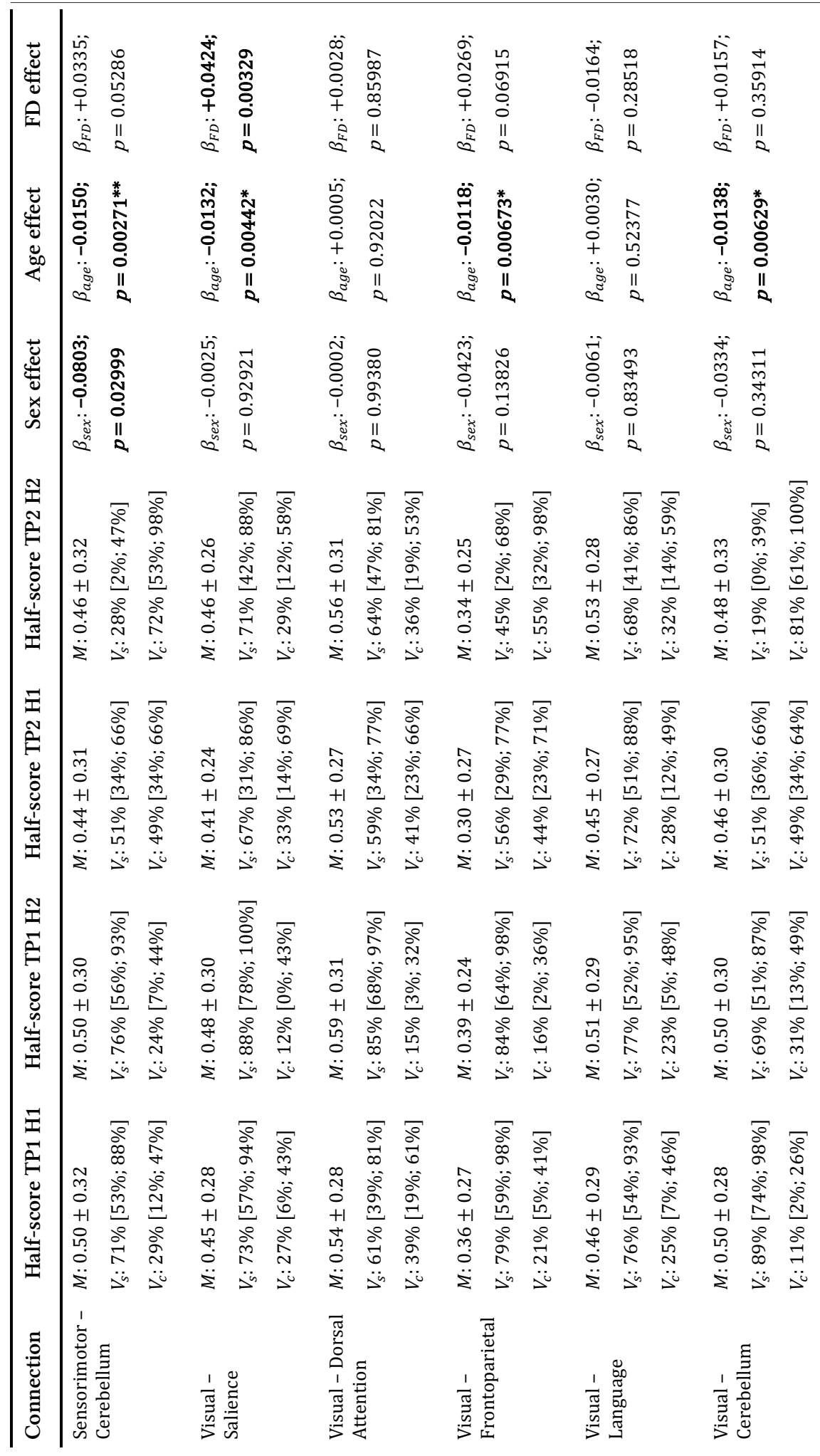




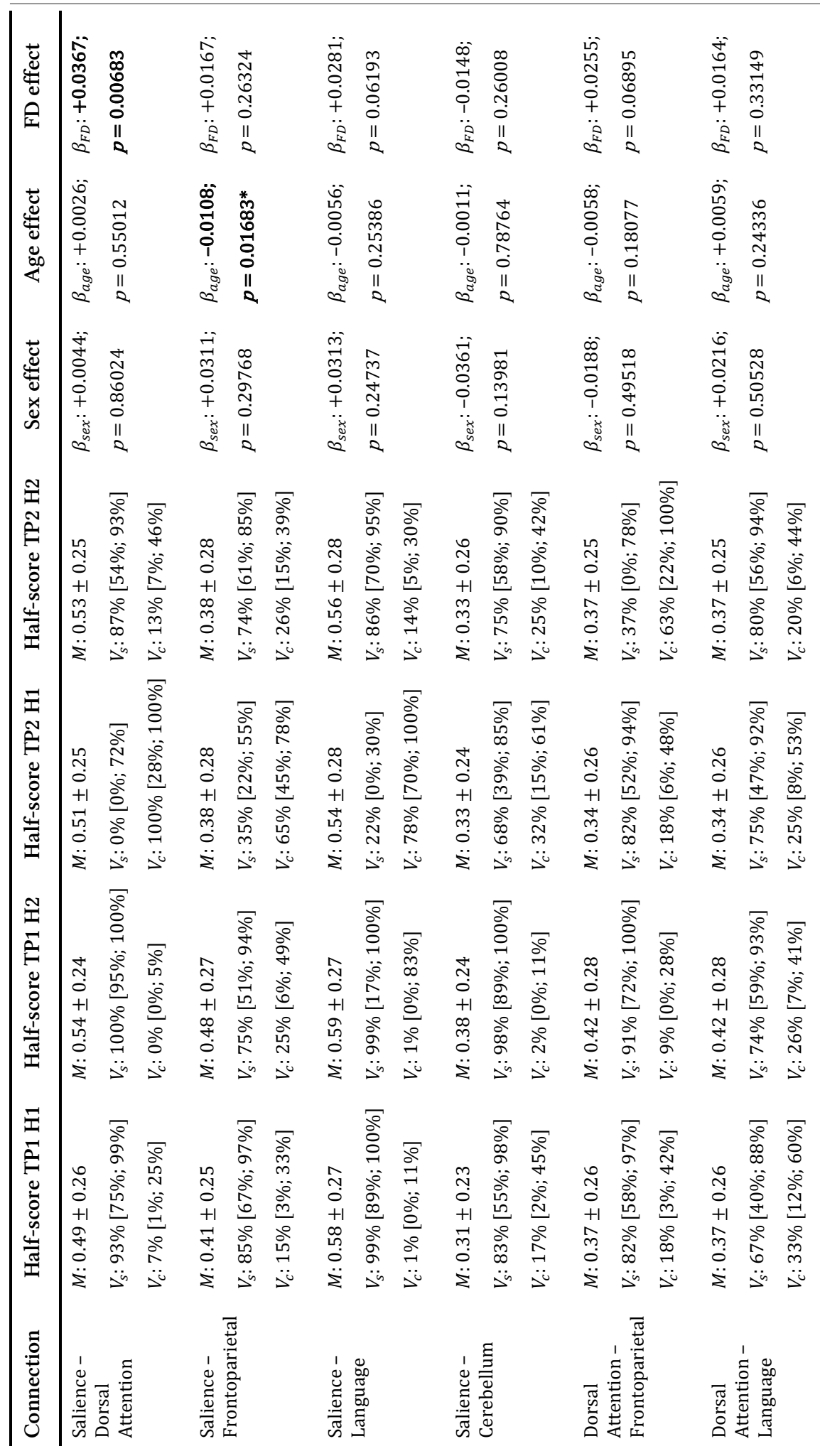




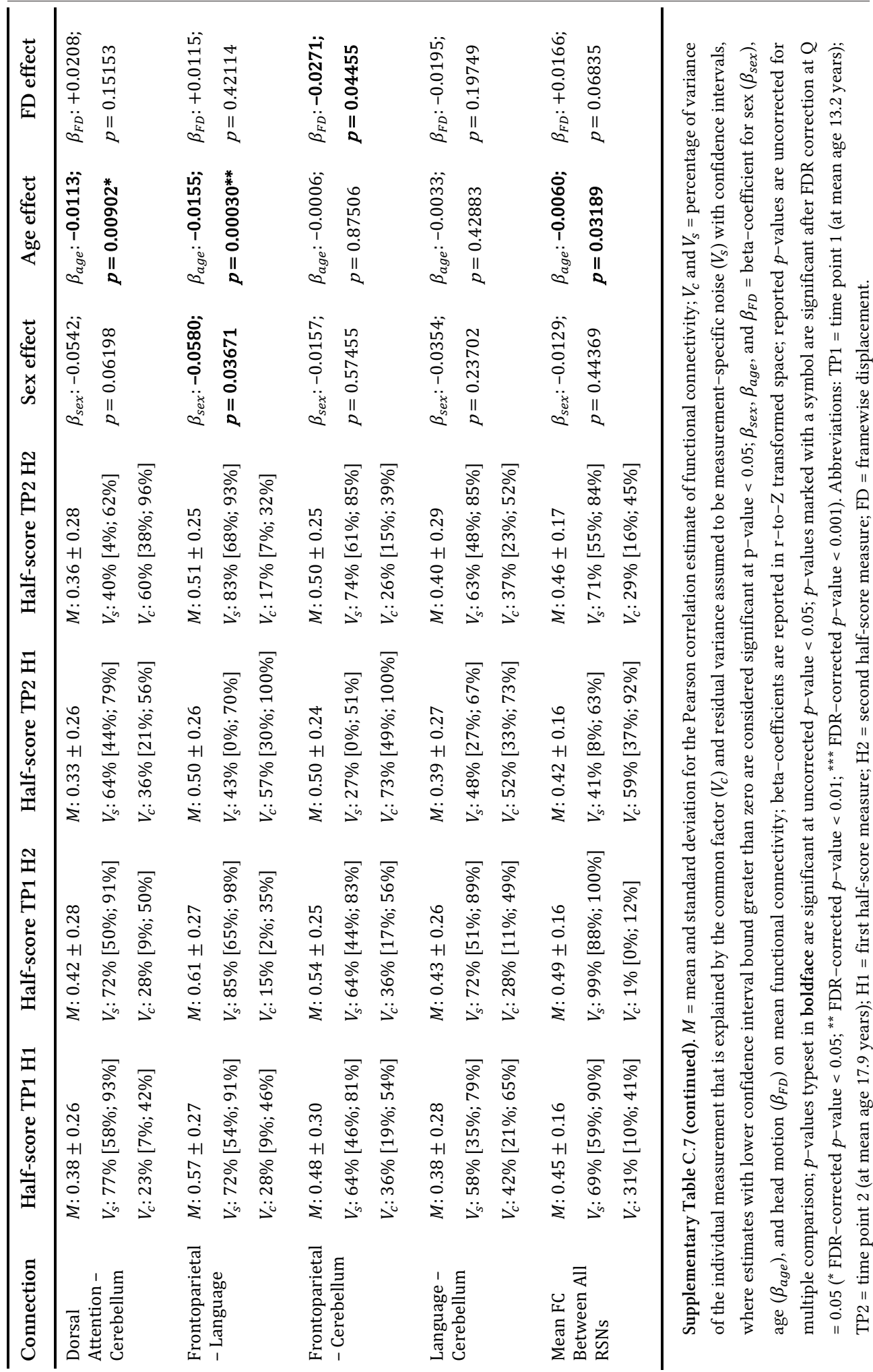


C.1.13 Genotypic effects on the reliable component of functional connectivity between resting-state networks

Supplementary Table C.8 contains the estimated genetic parameters and model fit parameters for the common factor model with a single factor for connections between resting-state networks. The "Half-score" columns contain the standardized estimates (i.e. the proportion of variance of the individual half-scores explained by each component) for heritability $\left(a_{c}^{2}\right)$, common environment $\left(c_{c}^{2}\right)$, unique environment $\left(e_{c}^{2}\right)$, and the measurement-specific contribution $\left(e_{s}^{2}\right)$, including their confidence intervals, for each of the half-score measures (see Supplementary Figures C.7 and C.8). The "Factor" column contains the standardized estimates for heritability $\left(a_{f}^{2}\right)$, common environment $\left(c_{f}^{2}\right)$, and unique environment $\left(e_{f}^{2}\right)$ on the common factor, including their confidence intervals (see Figure 4.4 from Chapter 4; Supplementary Figures C.7 and C.8). Statistically significant effects are printed in bold; with effects significant after FDR-correction marked with symbols ( ${ }^{*}$ FDR-corrected $p<0.05 ;{ }^{* *}$ FDR-corrected $p<0.01 ;{ }^{* * *}$ FDR-corrected $p<0.001$ ). The last column contains the log-likelihood model fit parameters of the full model with ACE latent variance components loadings on each of the common factors, and its nested models with only AE, CE, or E; these parameters are used to determine which of the models (full or nested) best described the data using the log-likelihood (LL) ratio test, with the best fitting model printed in bold. Estimates without data because they do not apply are marked with N/A (e.g. no estimate for $c_{f}^{2}$ or $c_{c}^{2}$ are available in models for which an AE-model best described the data). 


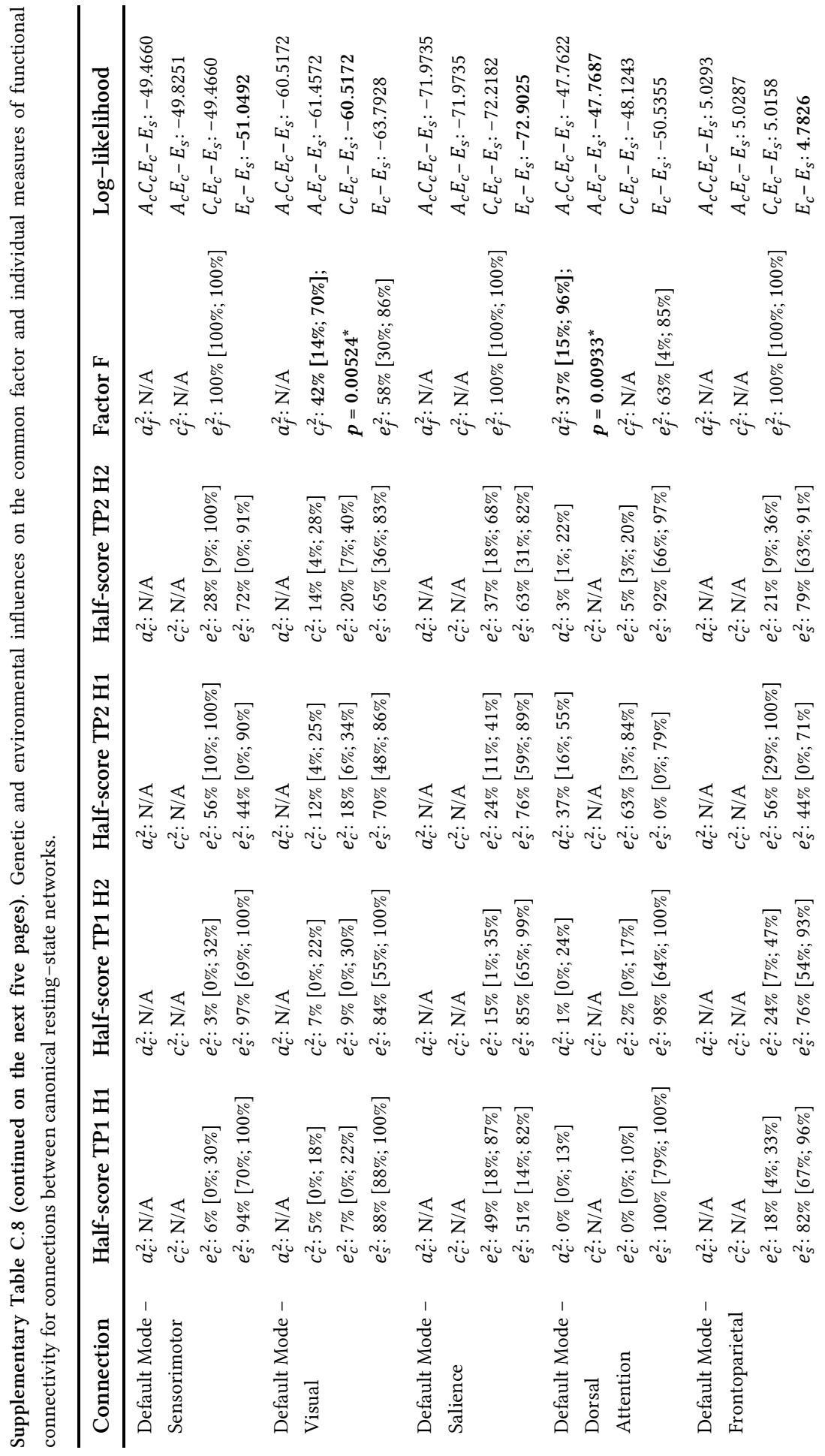




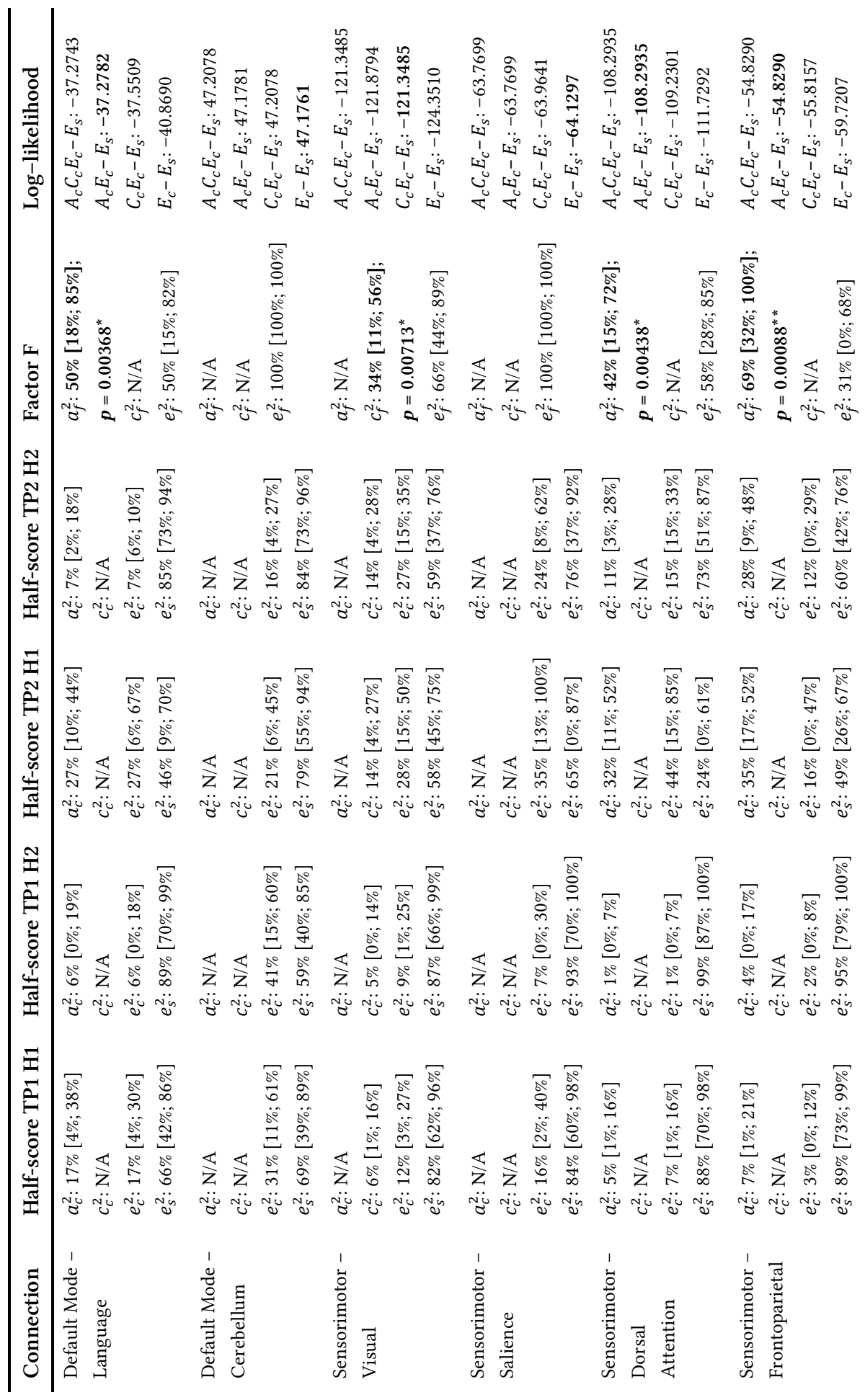




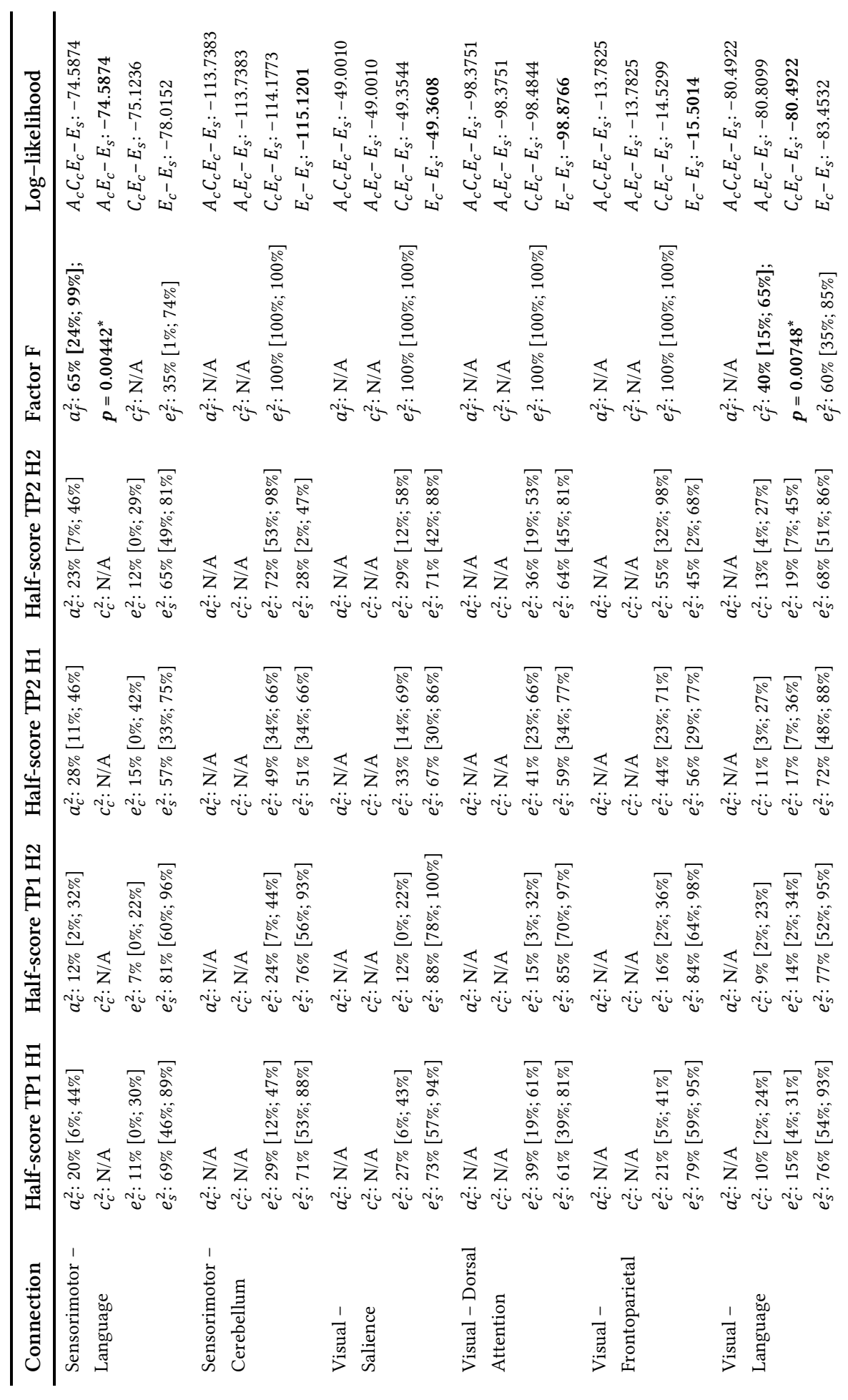




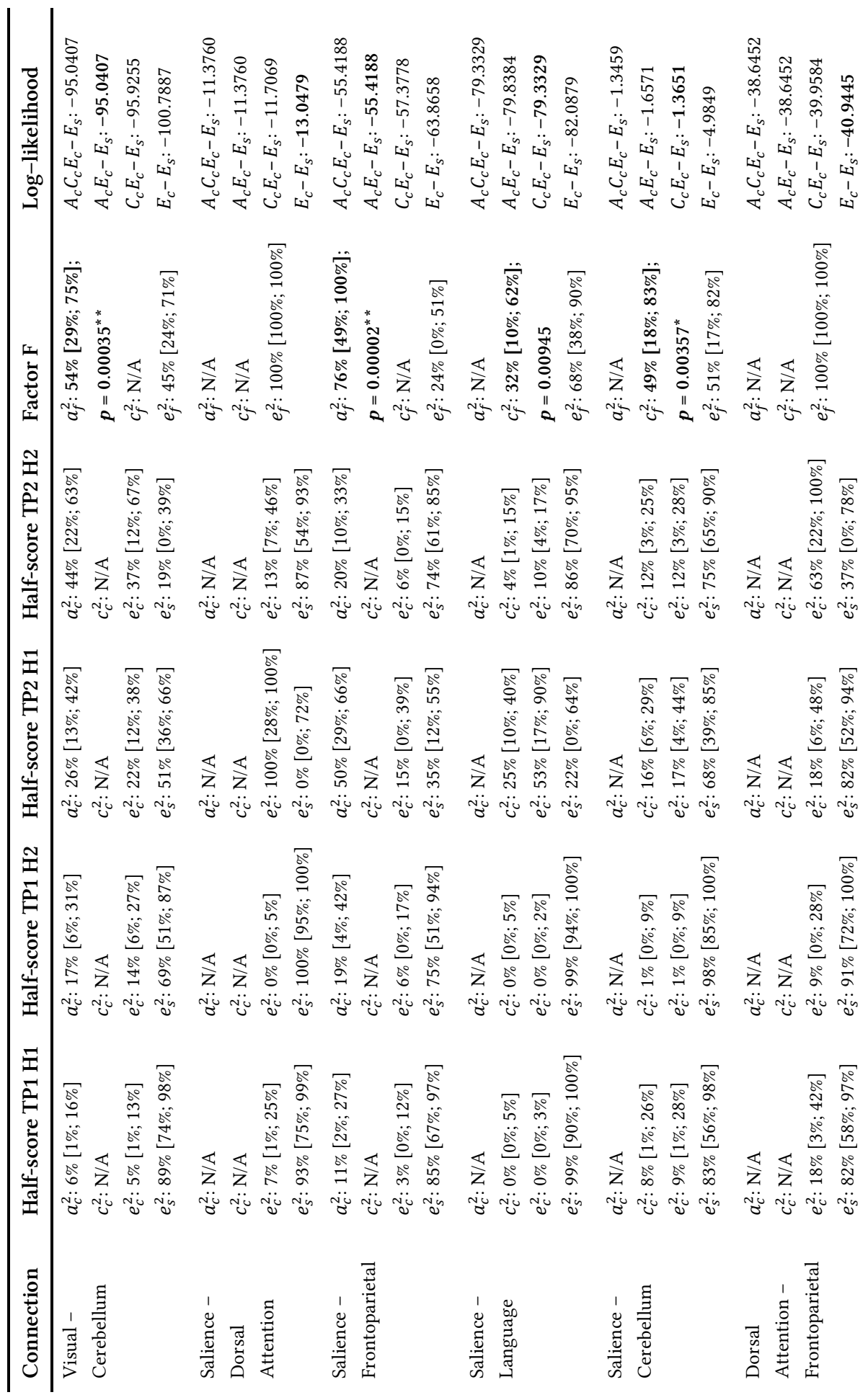




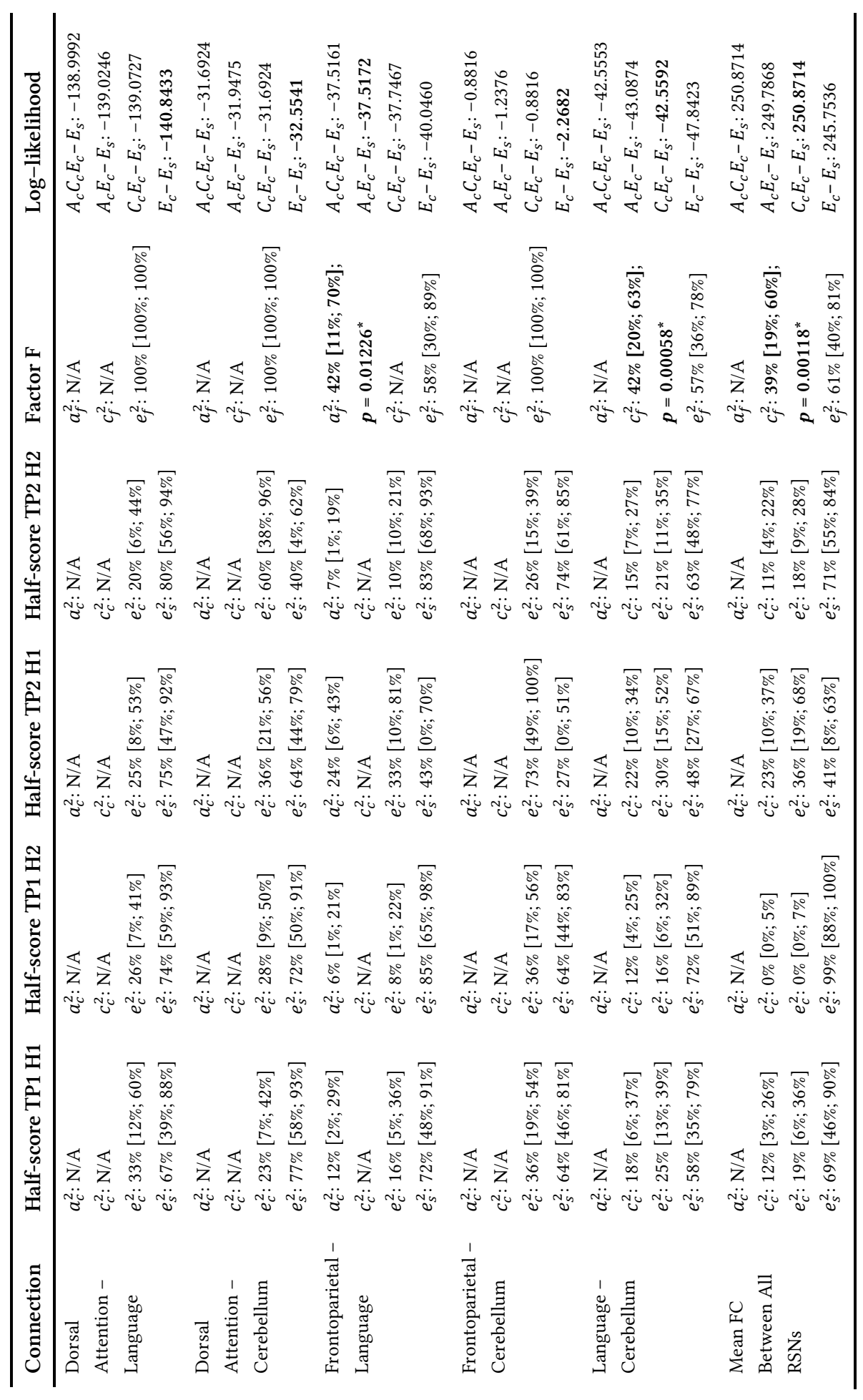




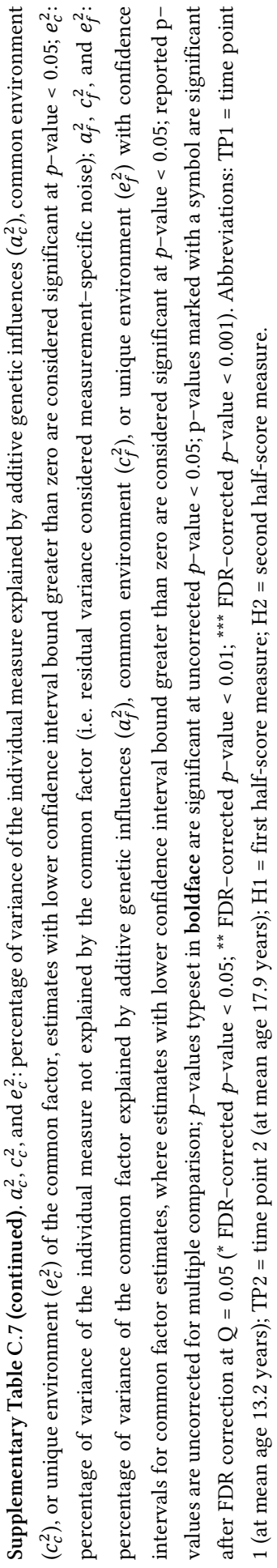




\section{C.1.14 Phenotypic effects on functional connectivity within resting-state networks} Supplementary Table C.9 contains the estimated phenotypic parameters and model fit parameters for the common factor model with a single factor for connections within resting-state networks. The "Half-score" columns contain the group-mean and standard deviation (M) of the functional connectivity estimates (see Figure 4.2 from Chapter 4), the standardized estimates for the proportion of variance explained by the common factor $\left(V_{c}\right)$ and measurement-specific variance $\left(V_{s}\right.$; i.e. considered noise), and their confidence intervals (see Supplementary Figures C.7 and C.8). The last three columns contain the beta coefficients and their significance for sex $\left(\beta_{\text {sex }}\right)$, age $\left(\beta_{\text {age }}\right)$, and head motion $\left(\beta_{F D}\right)$ measured as mean framewise displacement (mean FD); see Figure 4.3 from Chapter 4. Statistically significant effects are printed in bold; with effects significant after FDR-correction marked with symbols ( ${ }^{*}$ FDR-corrected $p<0.05 ;{ }^{* *}$ FDR-corrected $p<0.01 ;{ }^{* * *}$ FDR-corrected $p<0.001$ ). 


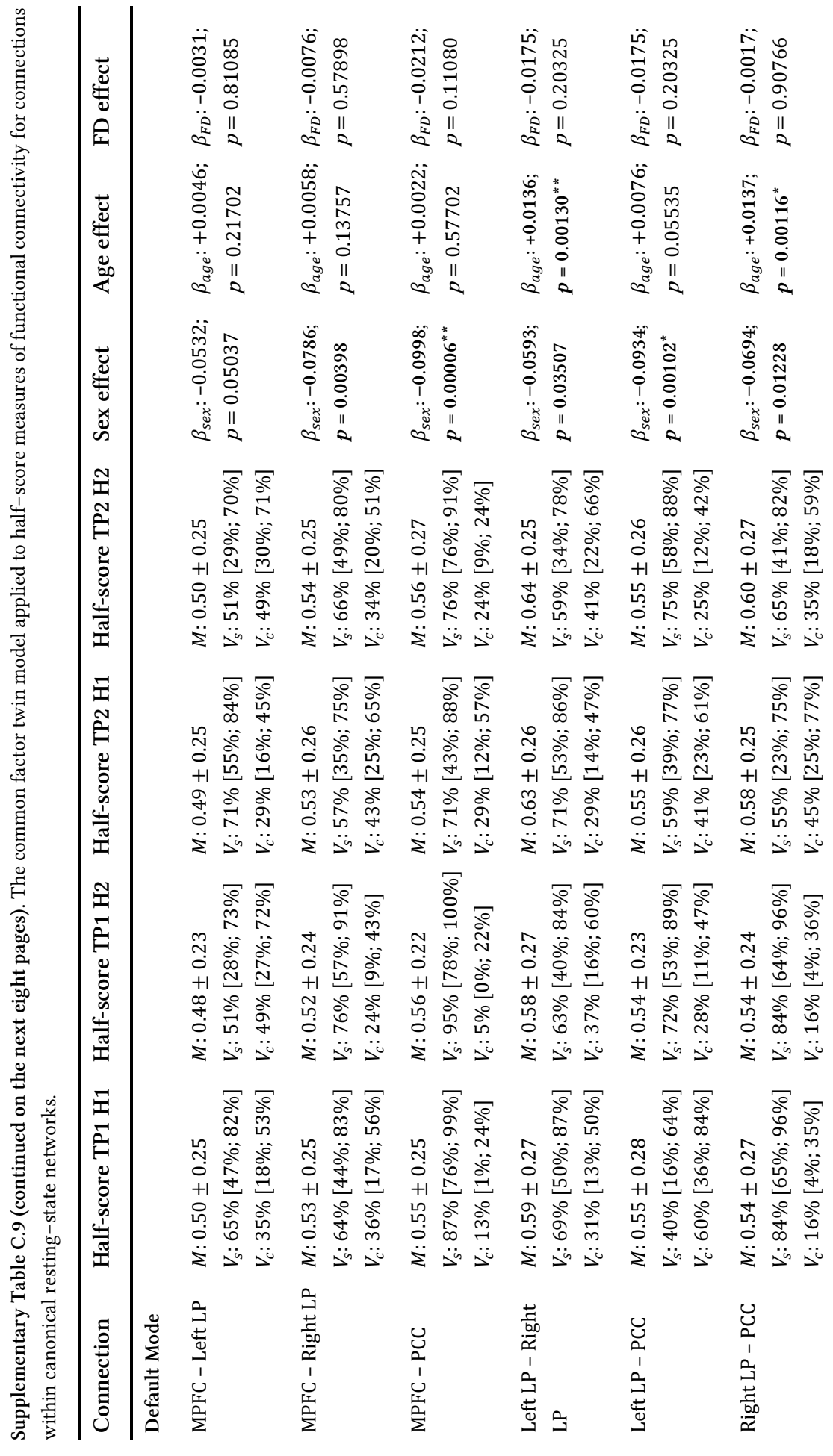




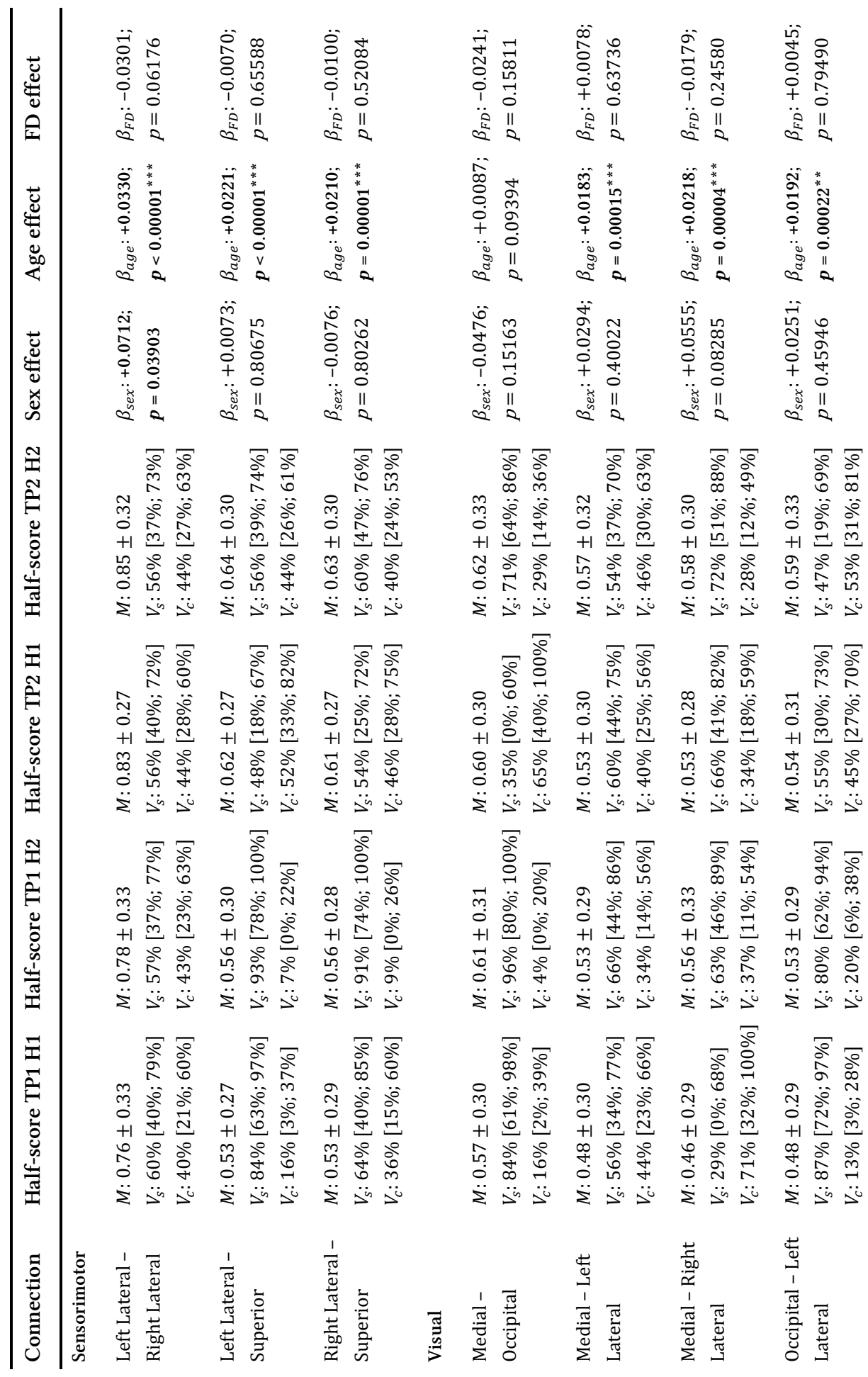




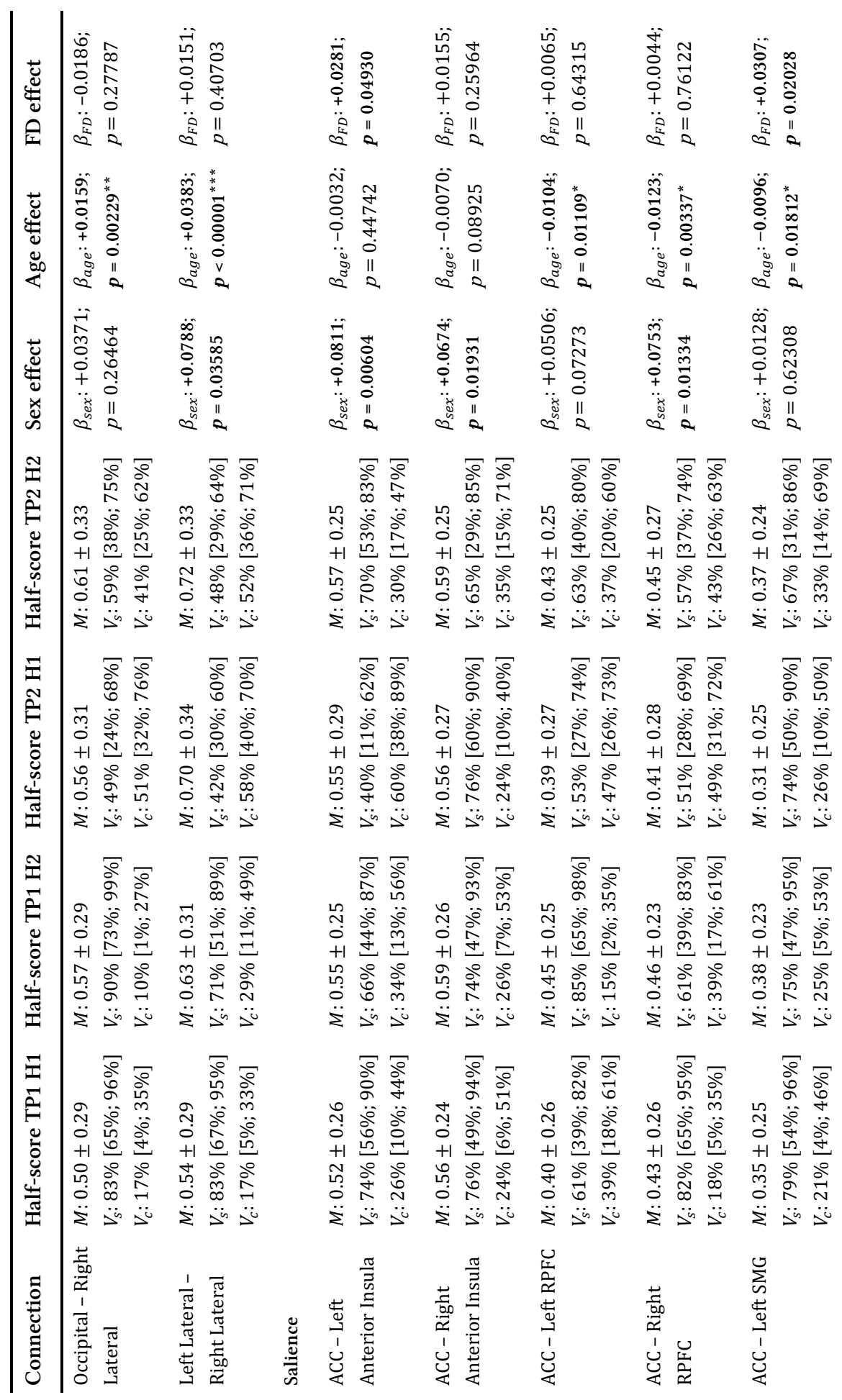




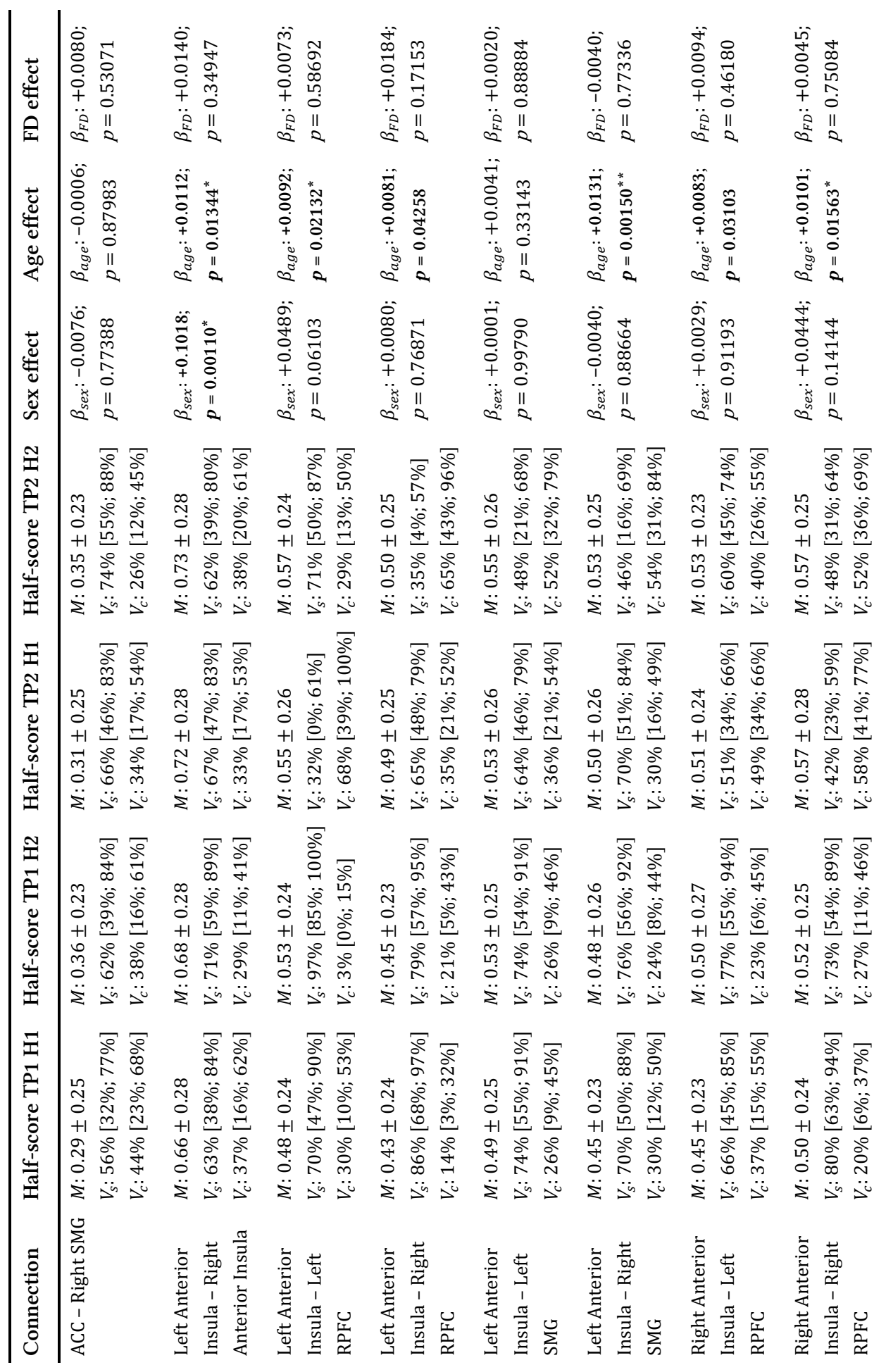




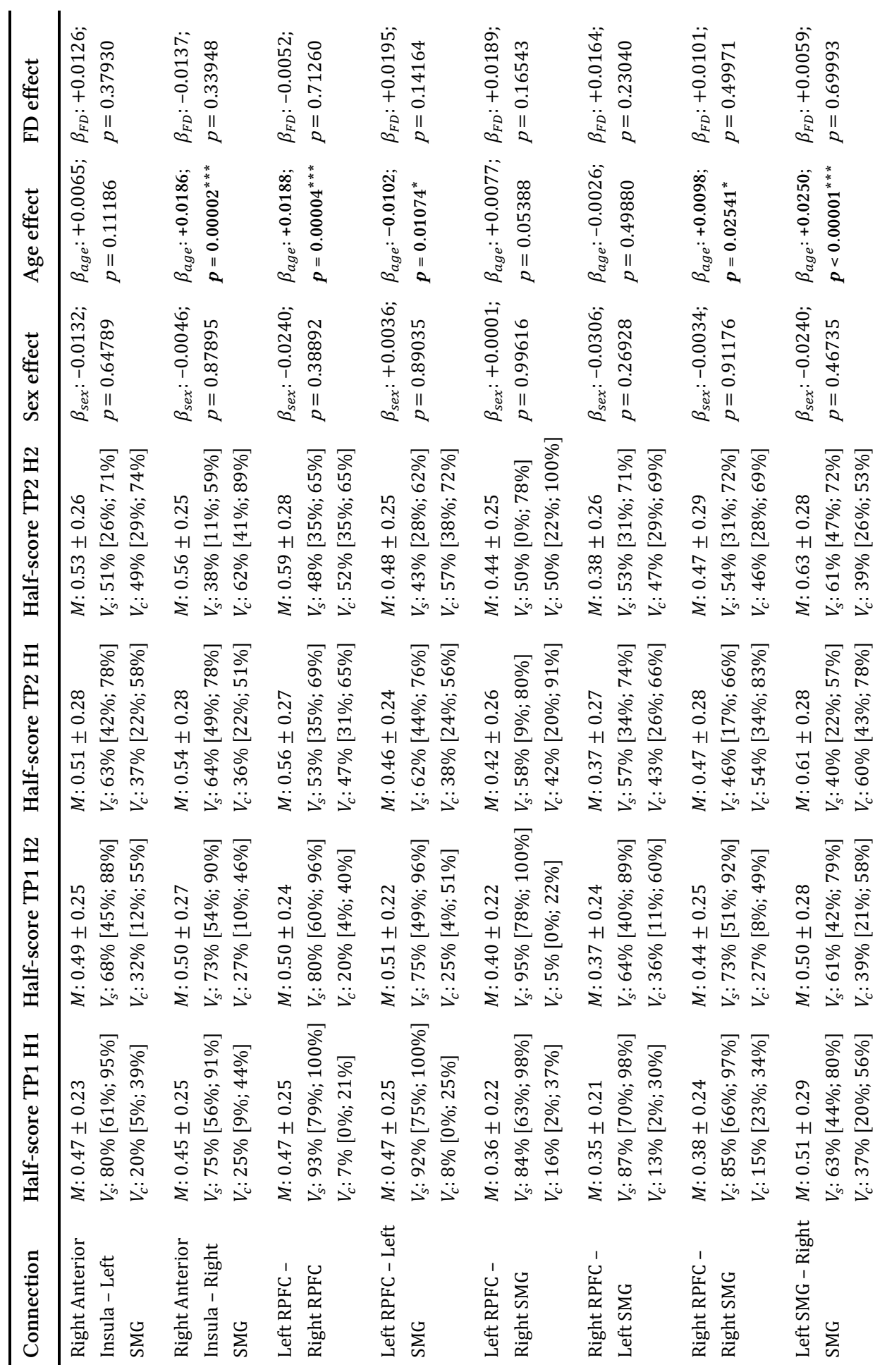




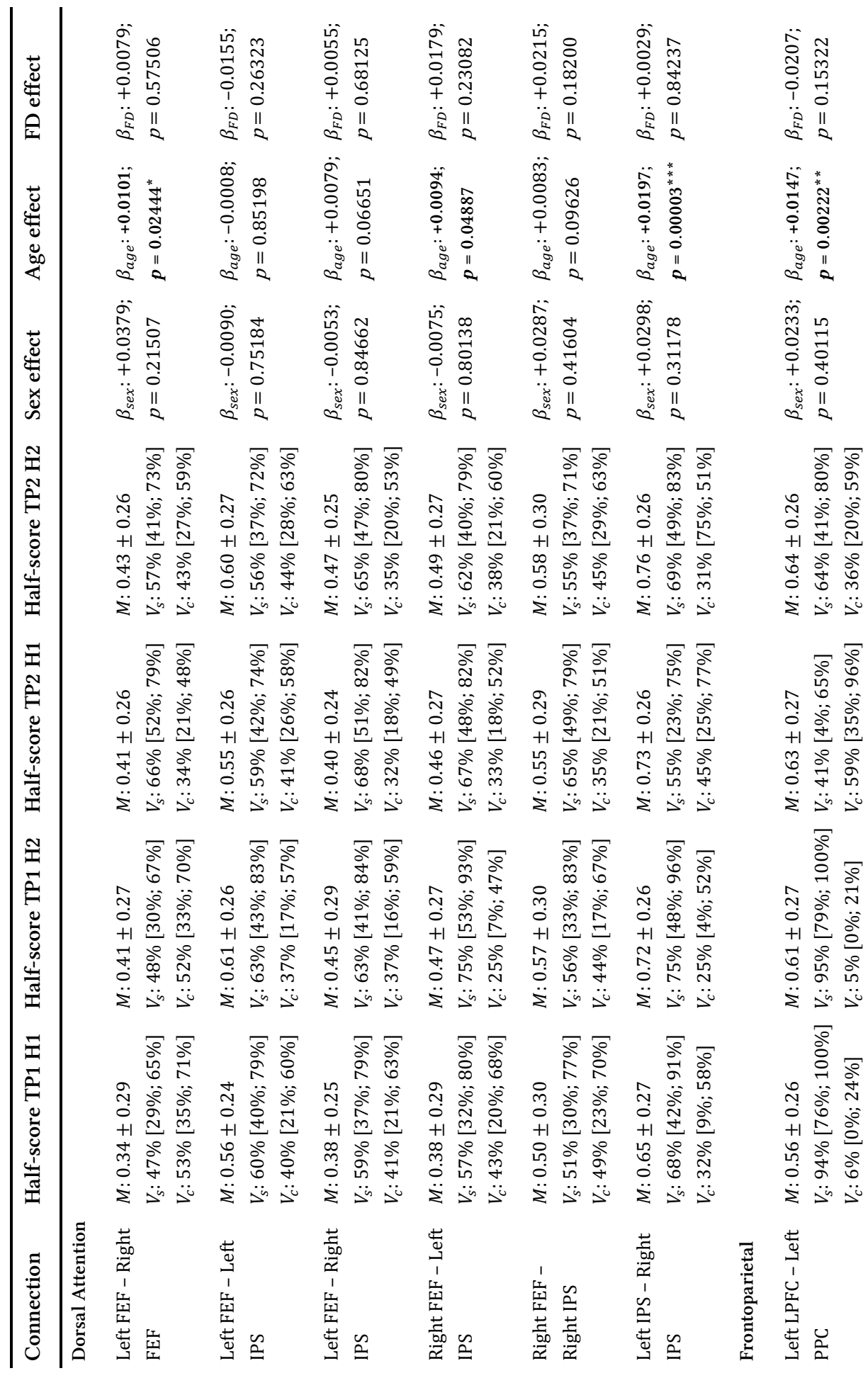




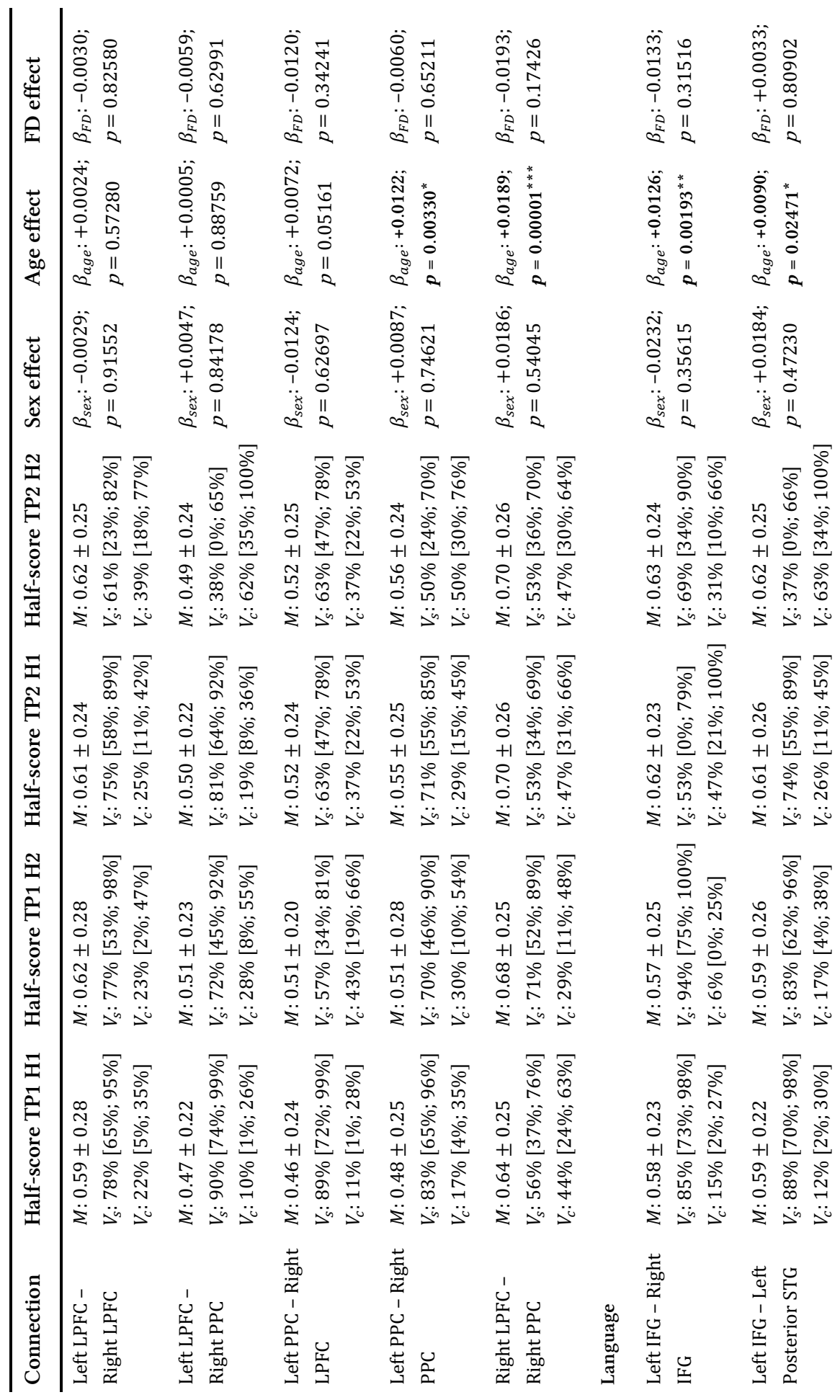




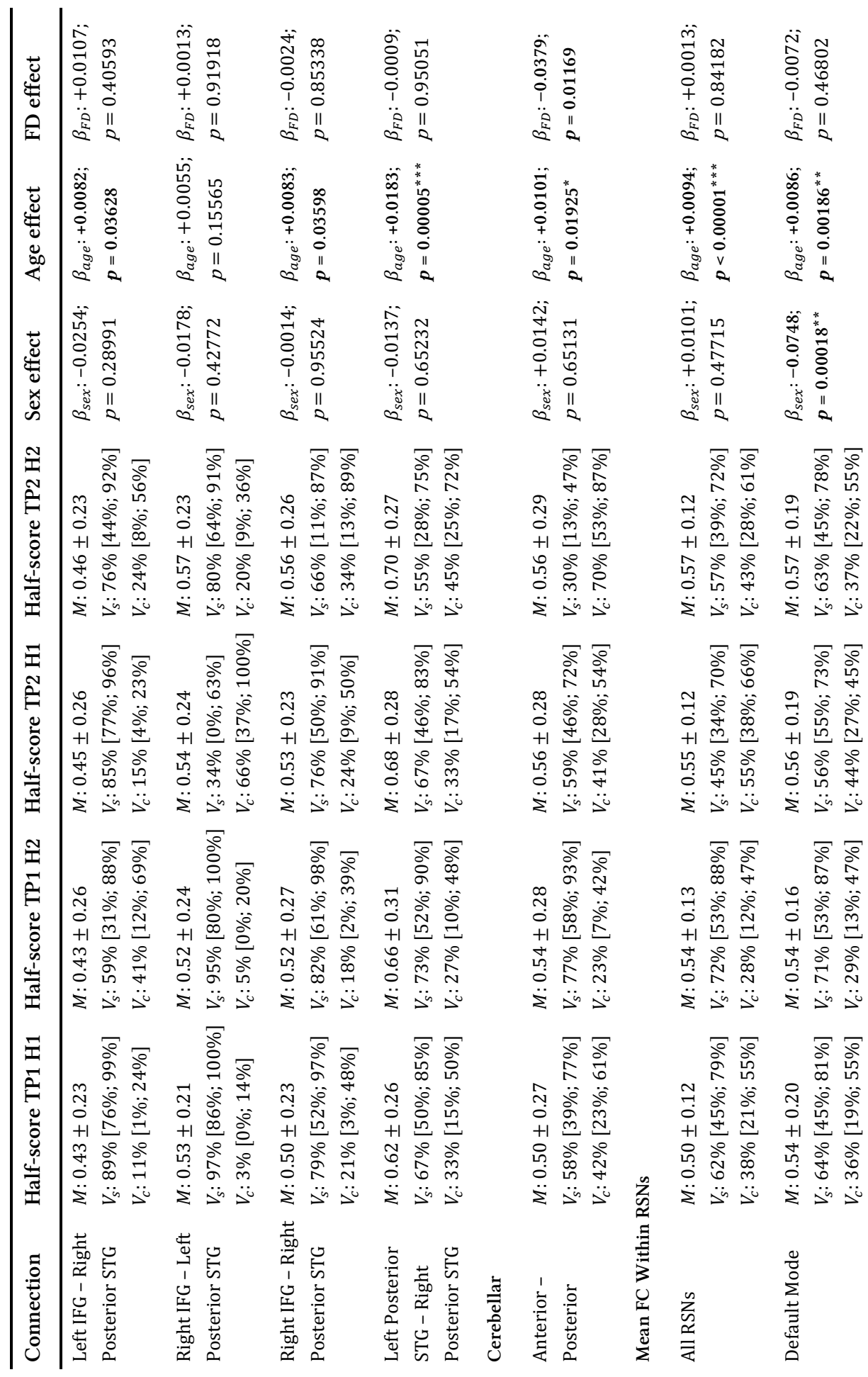




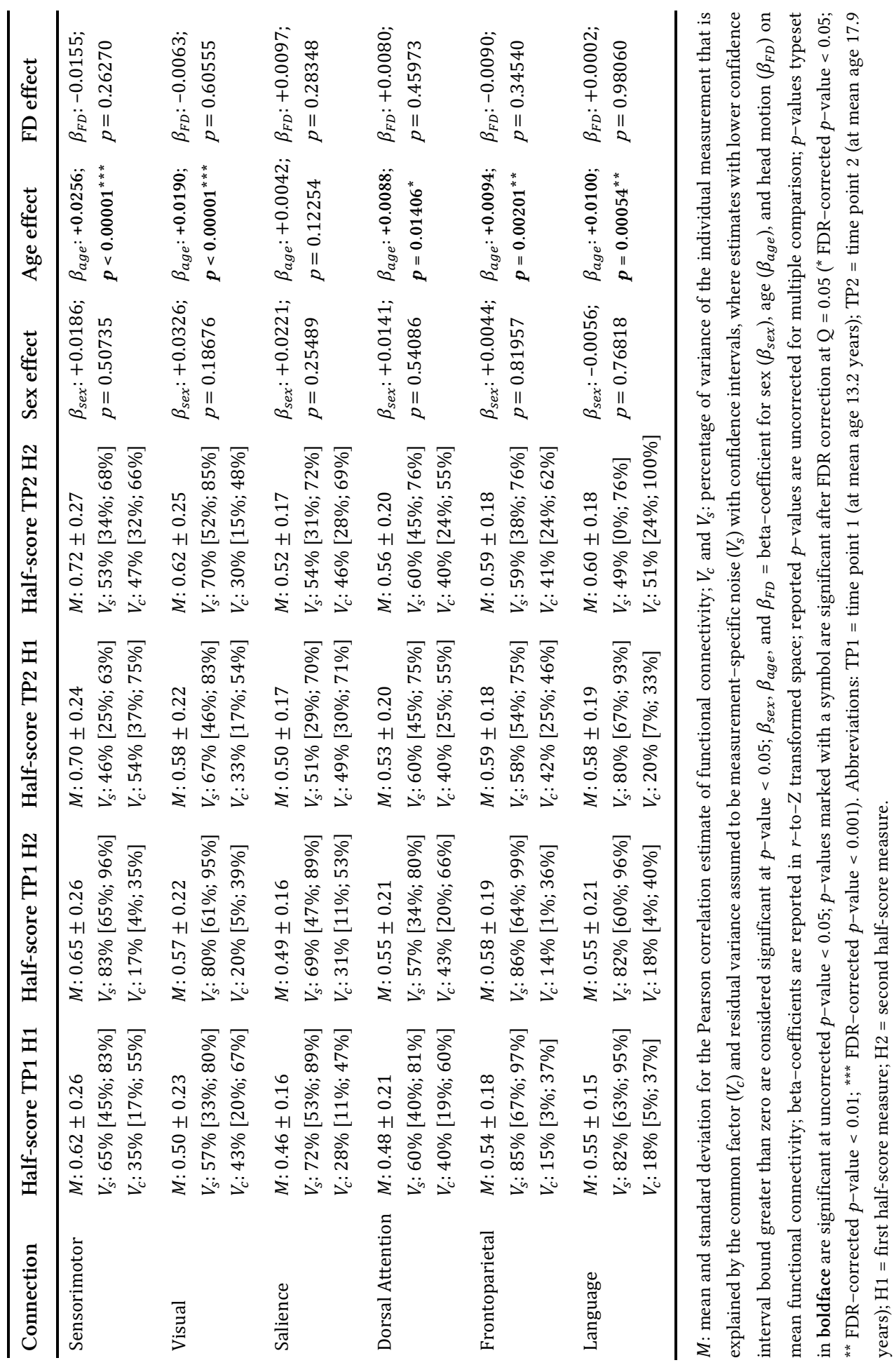


C.1.15 Genotypic effects on the reliable component of functional connectivity between resting-state networks

Supplementary Table C.10 contains the estimated genetic parameters and model fit parameters for the common factor model with a single factor for connections within resting-state networks. The "Half-score" columns contain the standardized estimates (i.e. the proportion of variance of the individual half-scores explained by each component) for heritability $\left(a_{c}^{2}\right)$, common environment $\left(c_{c}^{2}\right)$, unique environment $\left(e_{c}^{2}\right)$, and the measurement-specific contribution $\left(e_{s}^{2}\right)$, including their confidence intervals, for each of the half-score measures (see Supplementary Figures C.7 and C.8). The "Factor" column contains the standardized estimates for heritability $\left(a_{f}^{2}\right)$, common environment $\left(c_{f}^{2}\right)$, and unique environment $\left(e_{f}^{2}\right)$ on the common factor, including their confidence intervals (see Figure 4.4 from Chapter 4; Supplementary Figures C.7 and C.8). Statistically significant effects are printed in bold; with effects significant after FDR-correction marked with symbols ( ${ }^{*}$ FDR-corrected $p<0.05 ;{ }^{* *}$ FDR-corrected $p<0.01 ;{ }^{* * *}$ FDR-corrected $\left.p<0.001\right)$. The last column contains the log-likelihood model fit parameters of the full model with ACE latent variance components loadings on each of the common factors, and its nested models with only AE, CE, or E; these parameters are used to determine which of the models (full or nested) best described the data using the log-likelihood (LL) ratio test, with the best fitting model printed in bold. Estimates without data because they do not apply are marked with N/A (e.g. no estimate for $c_{f}^{2}$ or $c_{c}^{2}$ are available in models for which an AE-model best described the data). 


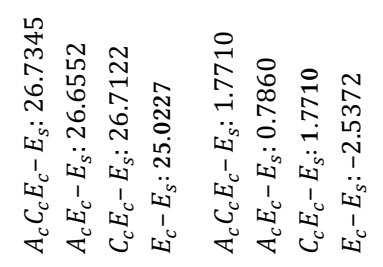

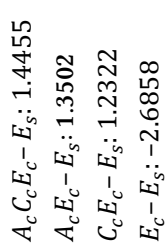

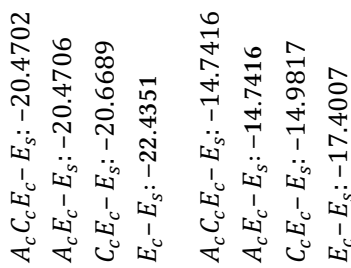

ஓे

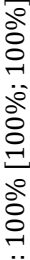

तु लुं

\section{更}

高

$\ddot{0}$

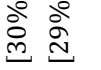

这造

के

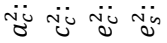

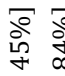

jे

空

¿ «

సี

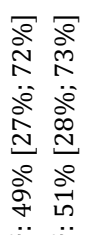

نं نูं نูं

ले ले

$\ddot{\circ} \ddot{\circ}$

$\stackrel{\infty}{\stackrel{5}{+}}$

«

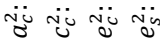

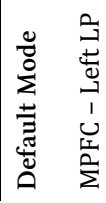

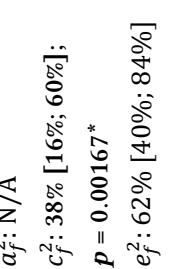

范

ปั่

ूั

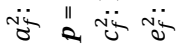

$\sigma e$

के

旅要过

« ेे

نं

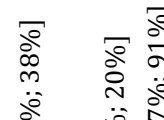

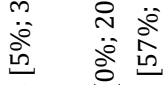

के $\frac{1}{2}$ वे

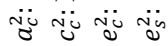

ঃे

高

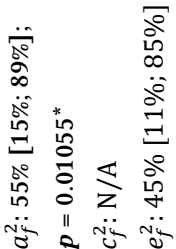

ivi ivis

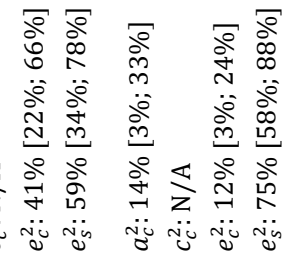

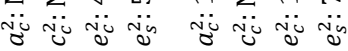

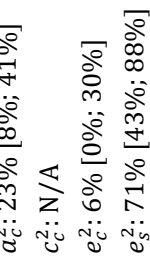

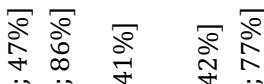

产芒

ป

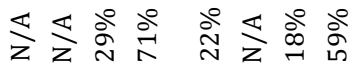

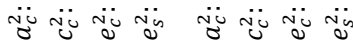

نं

$\circ$ o

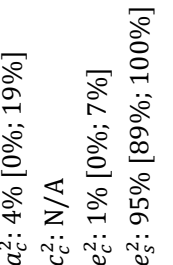

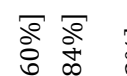

के के ने

ले

䒽这

उ

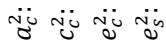

نं نูن Nंن

z z

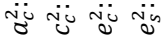

\section{उ}

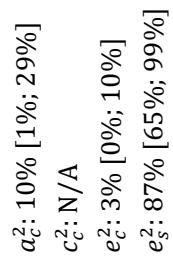

कृ

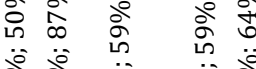

ลे

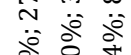

in

उ过高

m.

$\varangle \ll$ वें

亏े ڤें

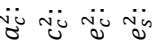

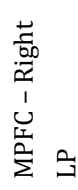

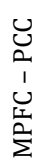

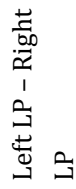

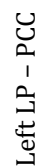

$\therefore \ddot{j}$

స్ ठे

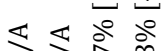




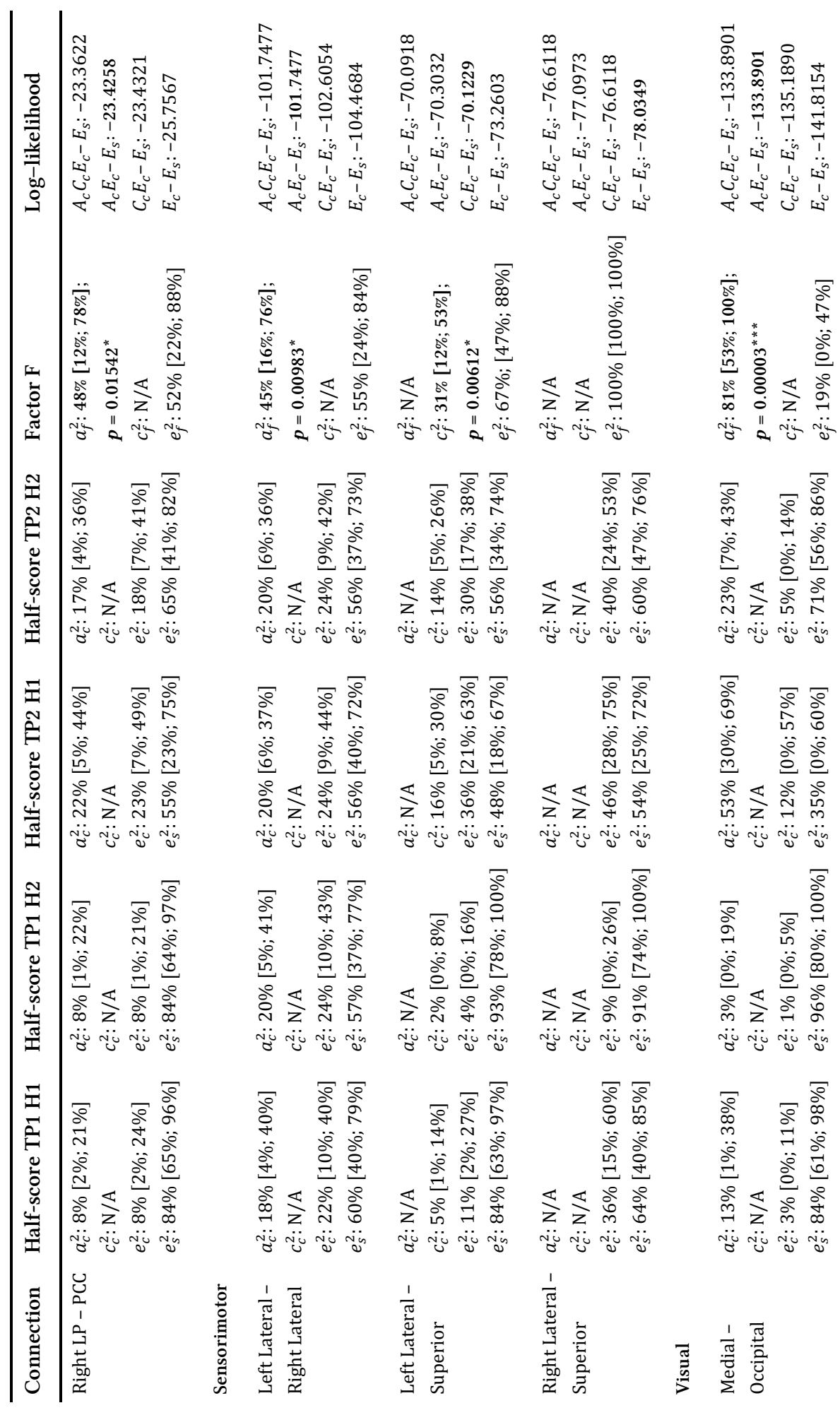




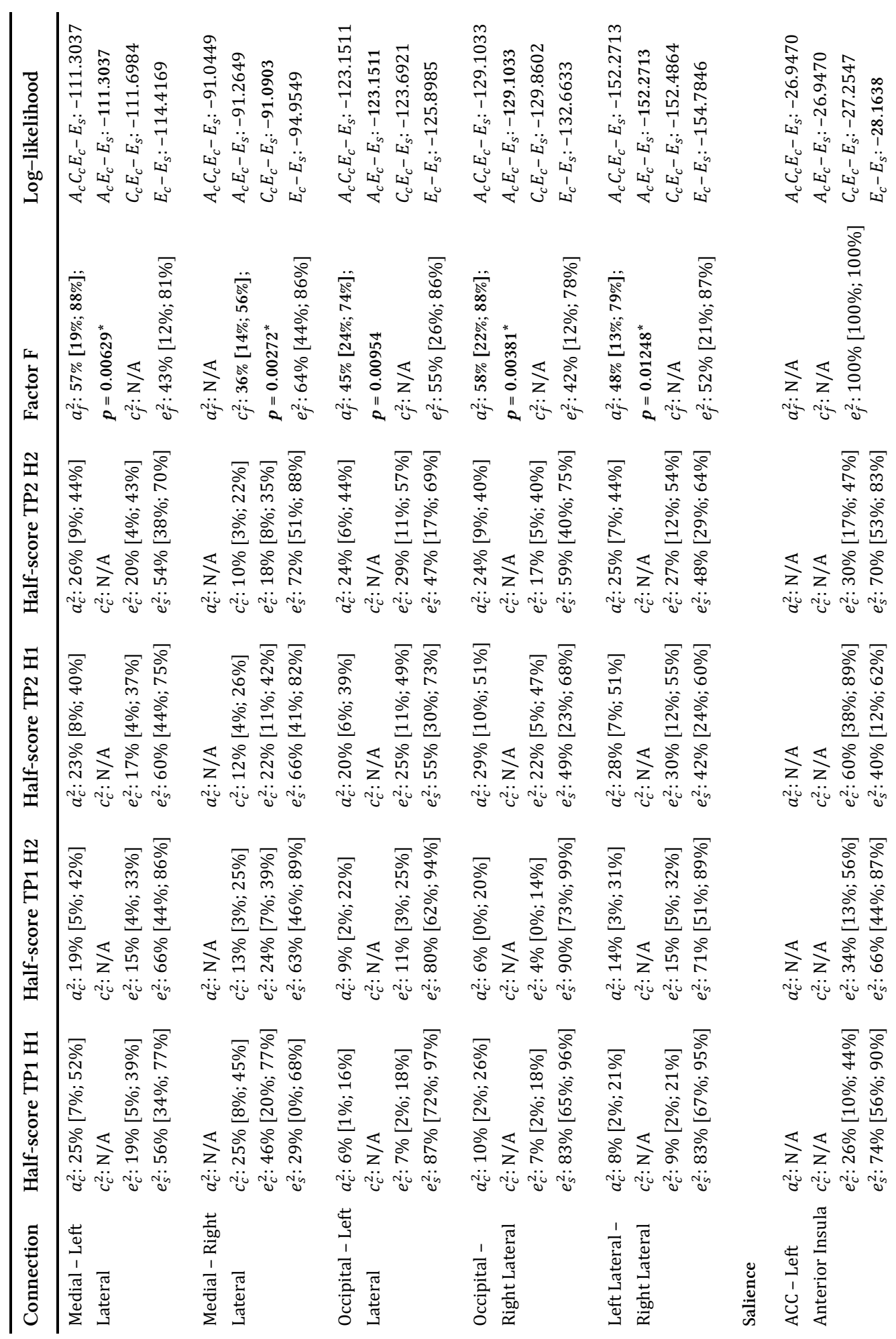




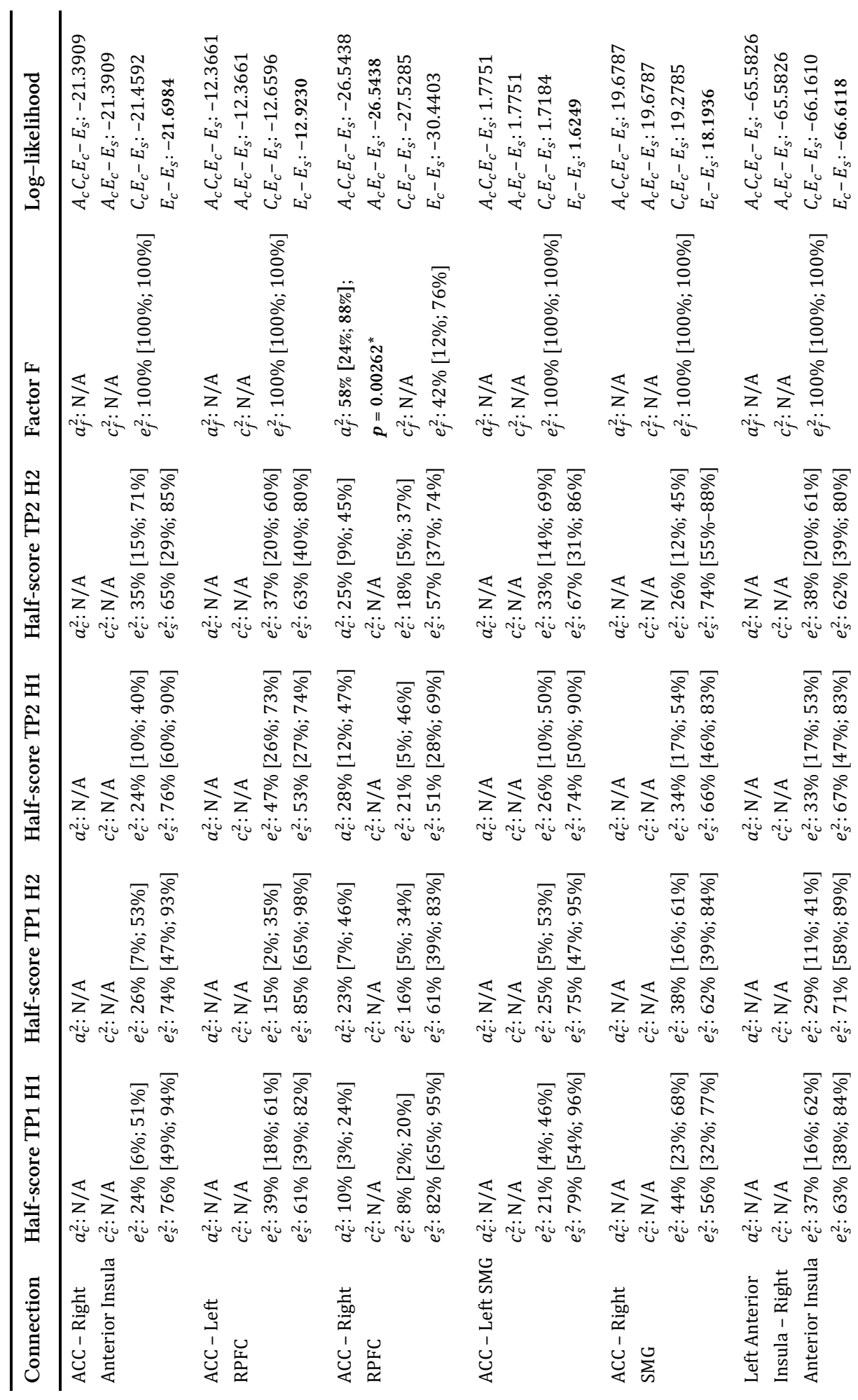




\begin{tabular}{|c|c|c|c|c|c|c|}
\hline 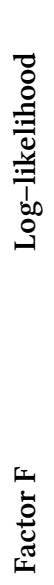 & 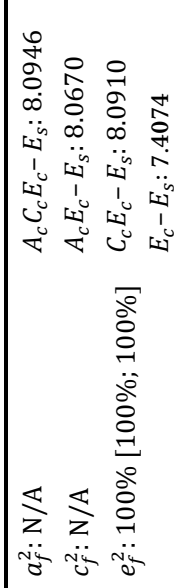 & 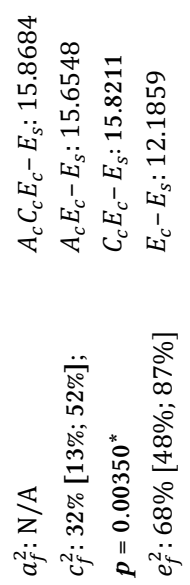 & 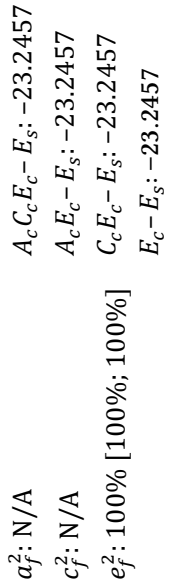 & 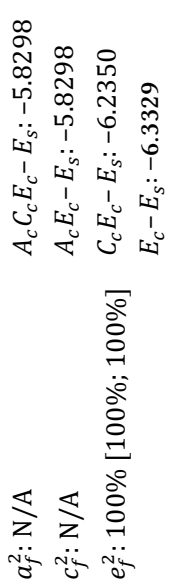 & 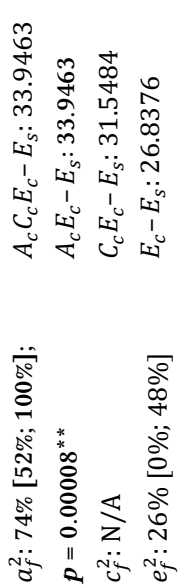 & 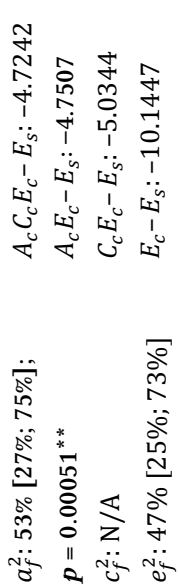 \\
\hline $\begin{array}{l}F \\
0 \\
0 \\
0 \\
0 \\
.1\end{array}$ & 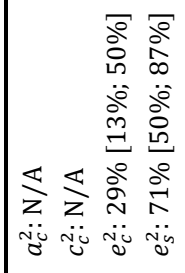 & 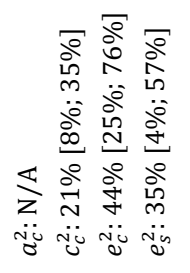 & 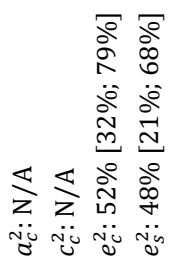 & 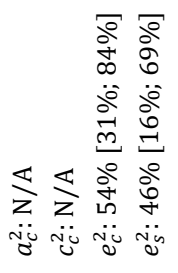 & 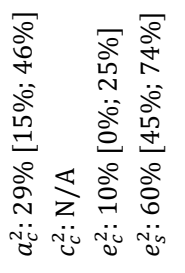 & 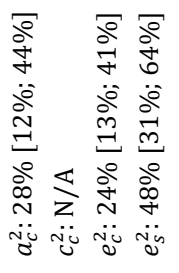 \\
\hline 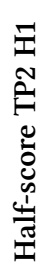 & 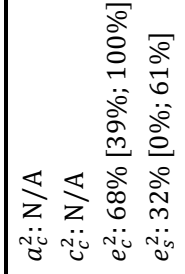 & 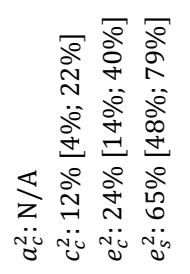 & 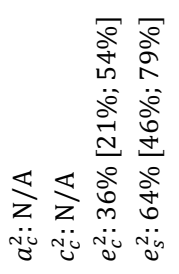 & 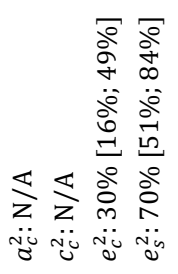 & 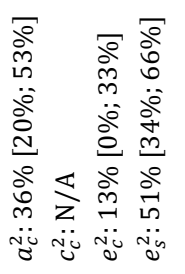 & 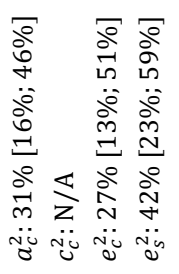 \\
\hline 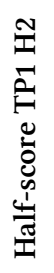 & 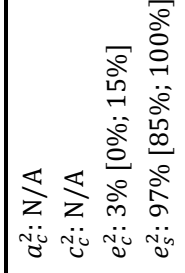 & 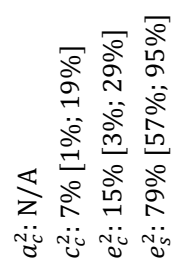 & 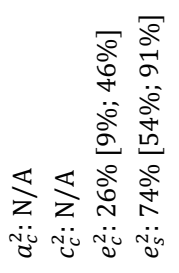 & 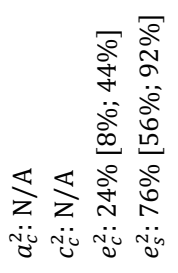 & 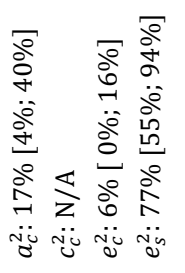 & 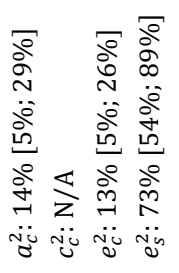 \\
\hline 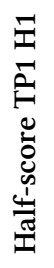 & 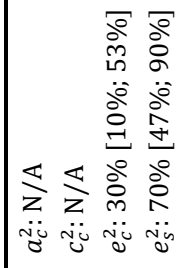 & 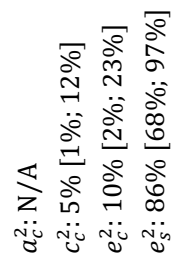 & 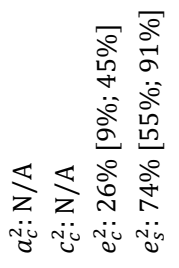 & 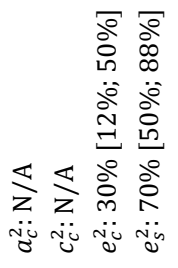 & 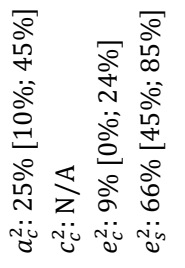 & 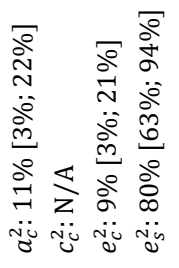 \\
\hline 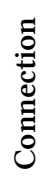 & 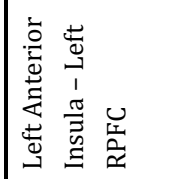 & 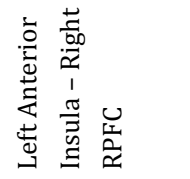 & 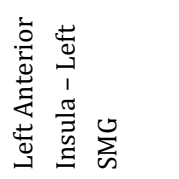 & 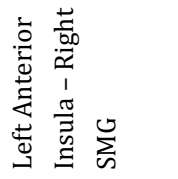 & 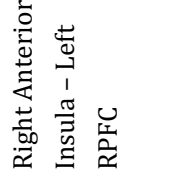 & 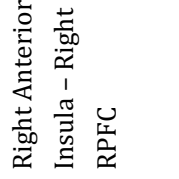 \\
\hline
\end{tabular}




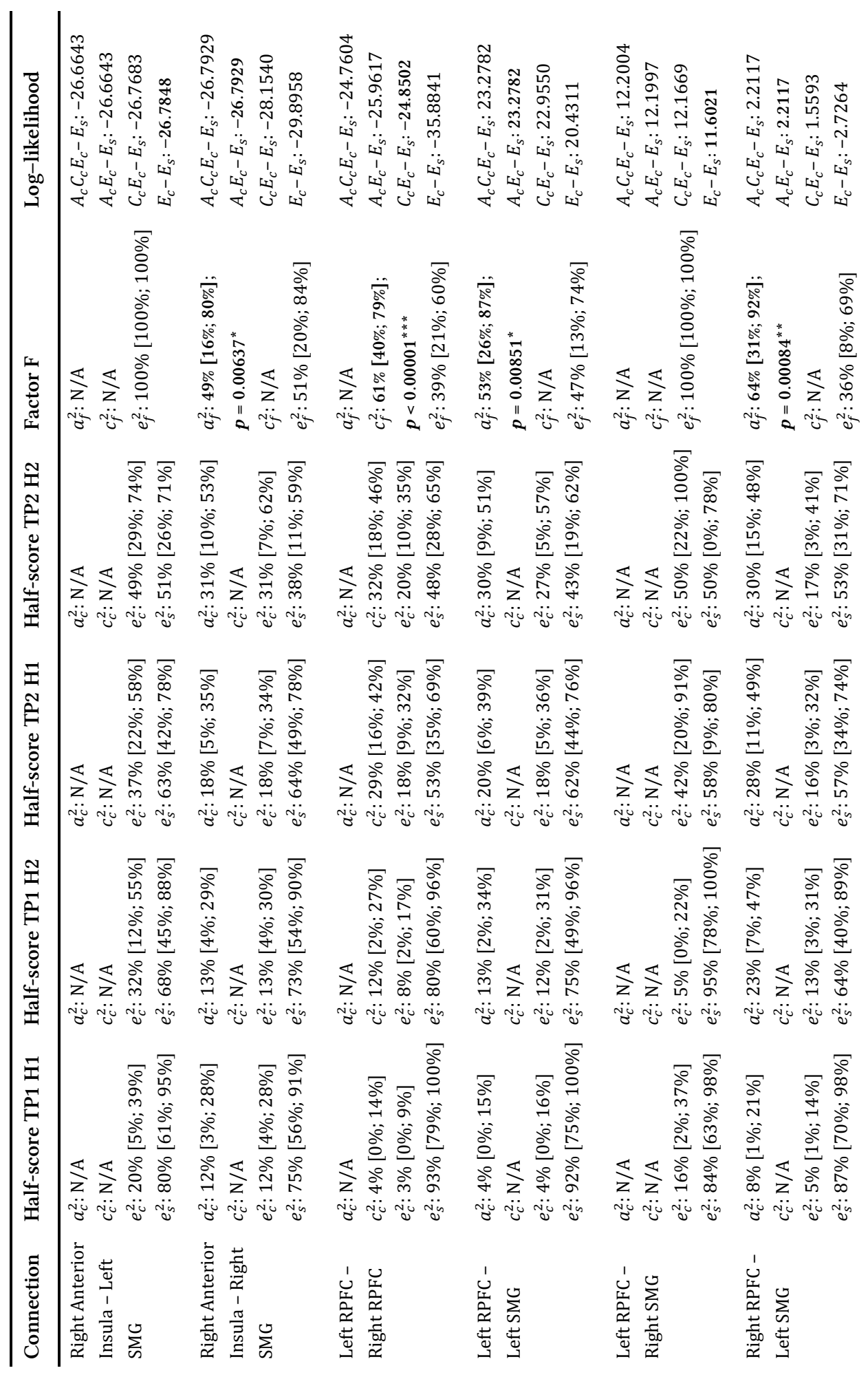




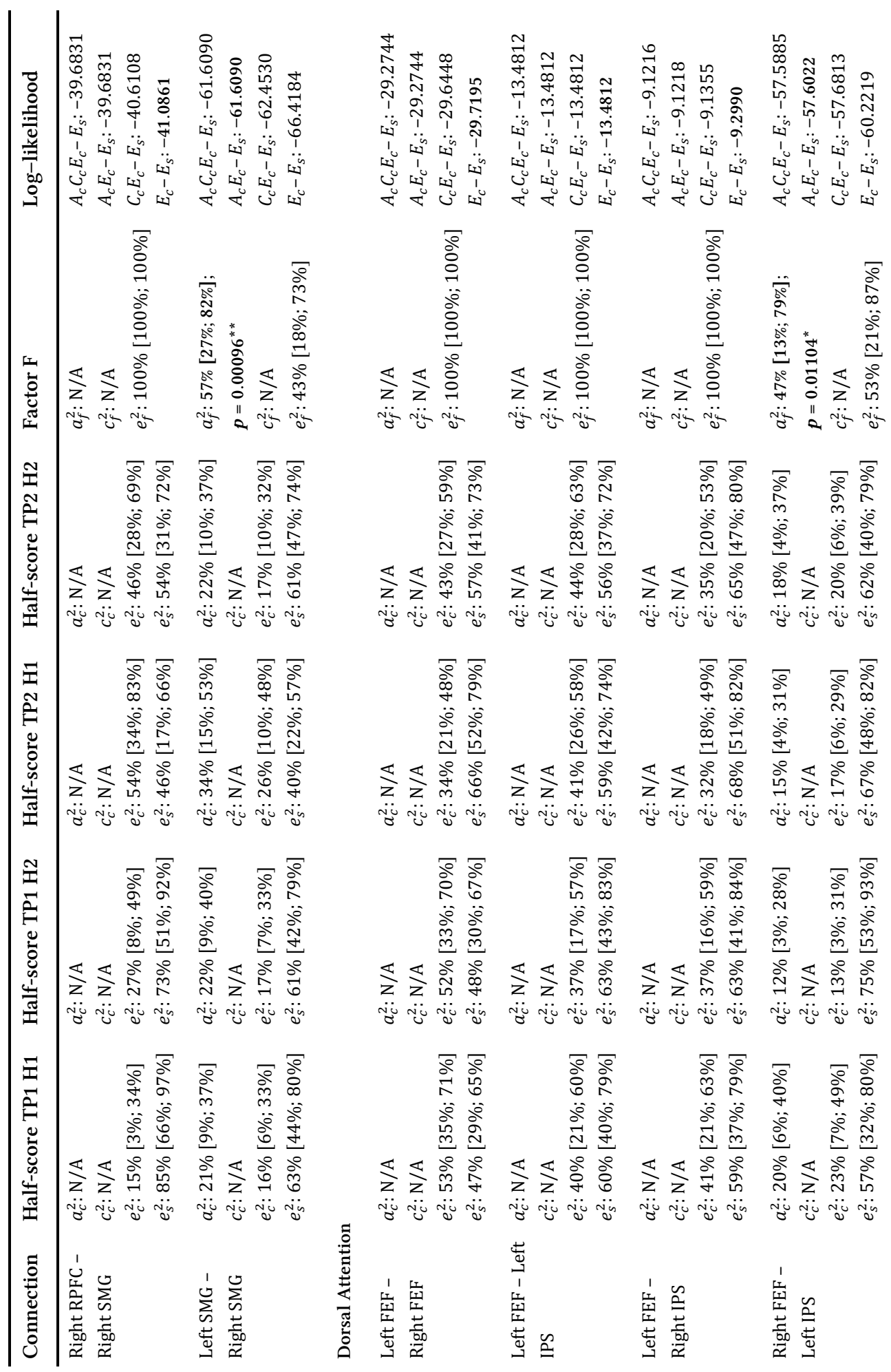




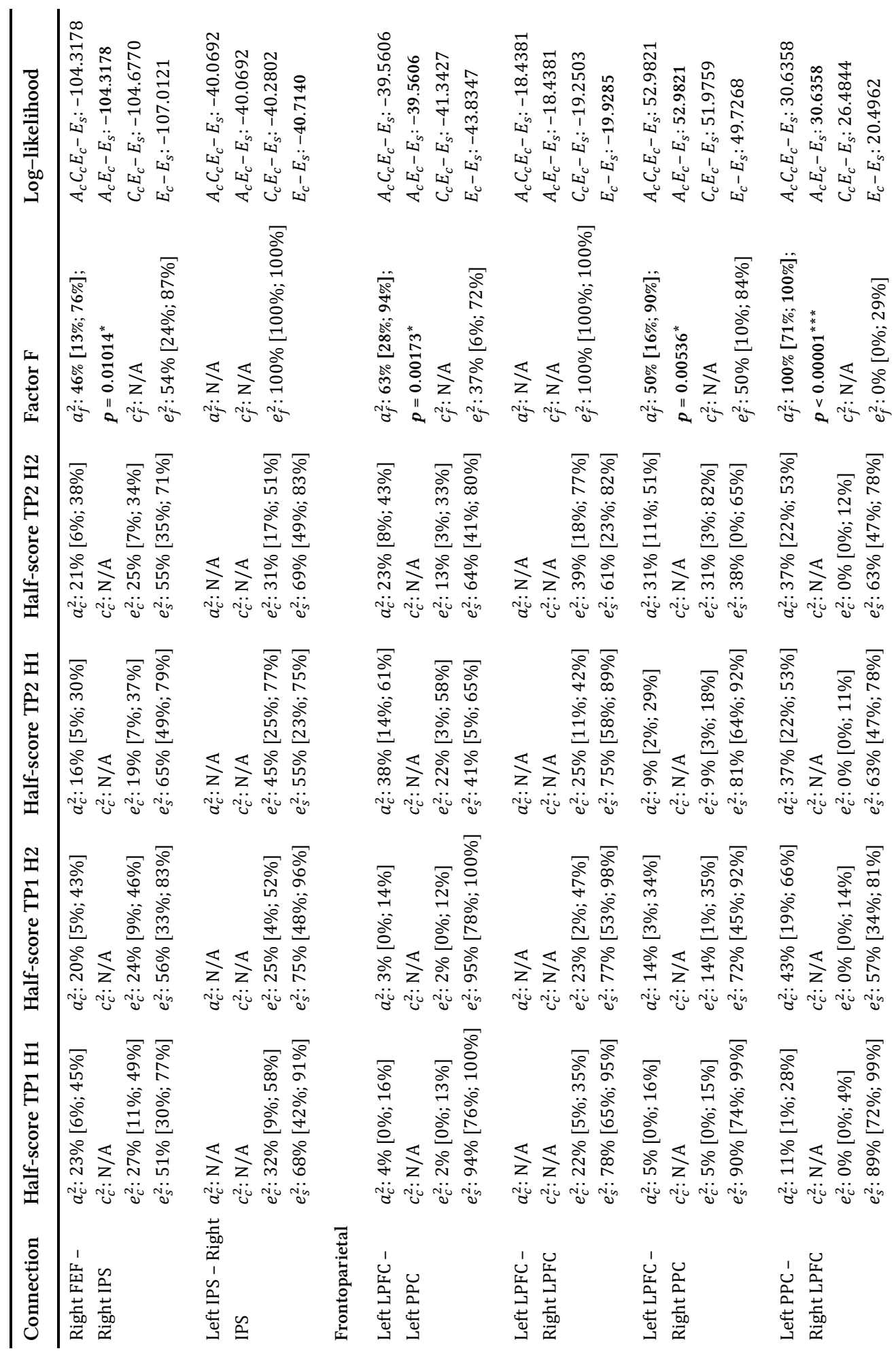




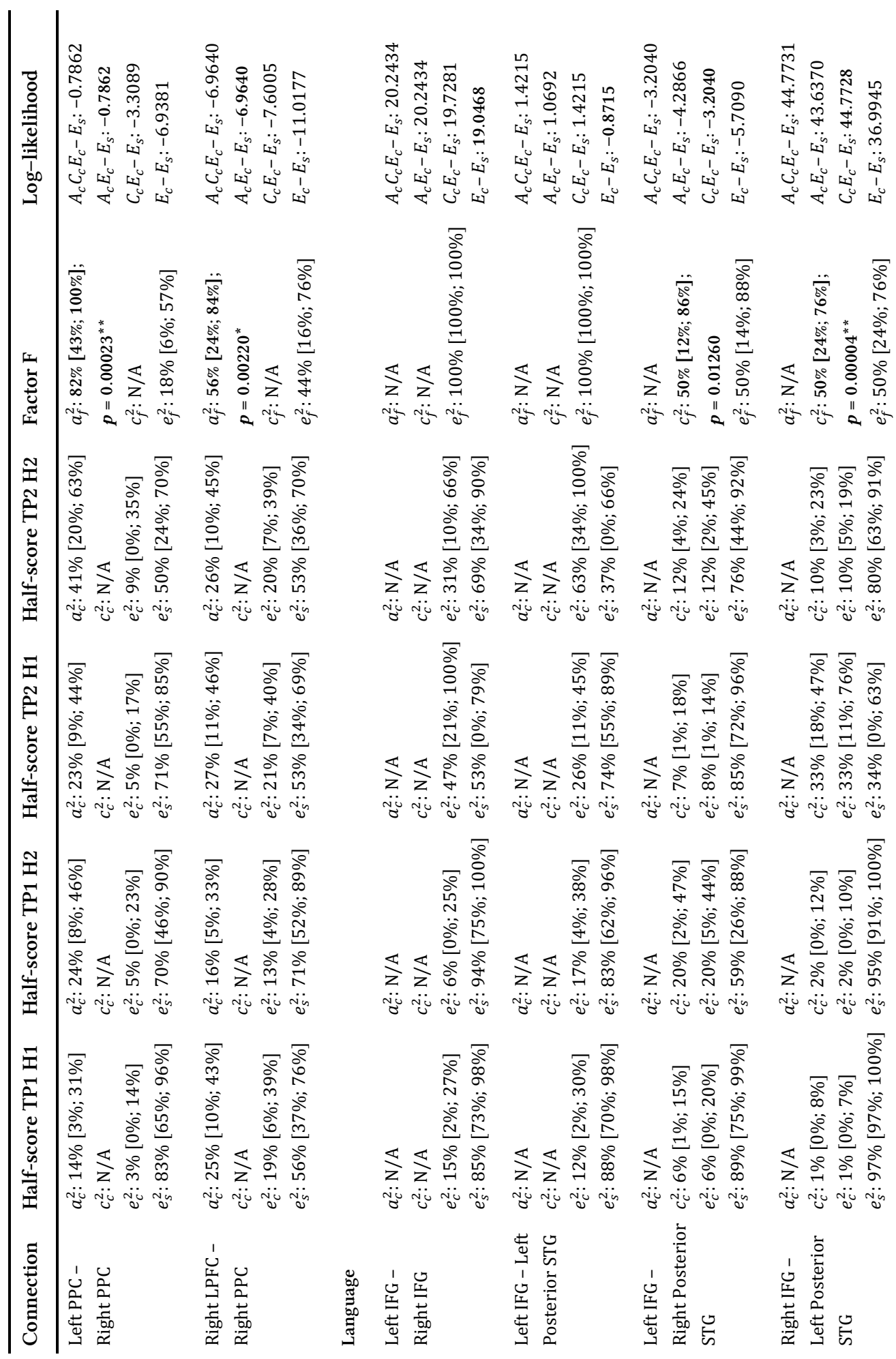




\begin{tabular}{|c|c|c|c|c|c|c|c|}
\hline ¿ & 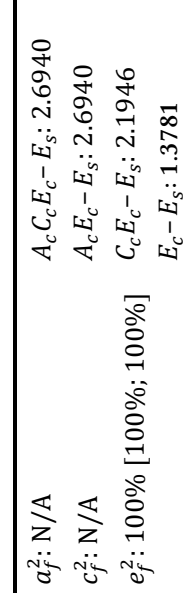 & 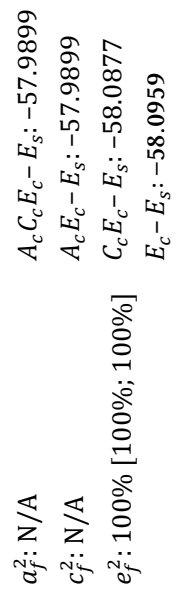 & & 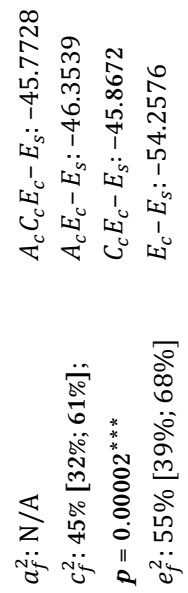 & & 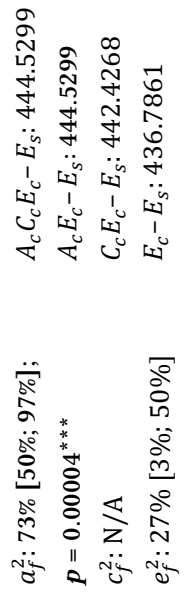 & 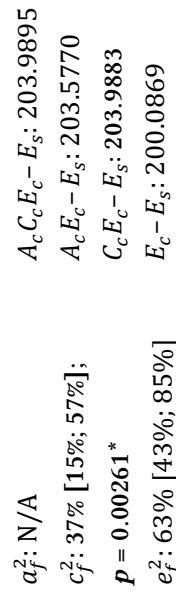 \\
\hline 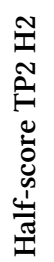 & 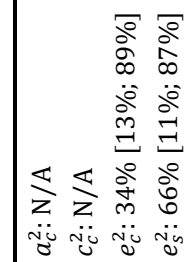 & 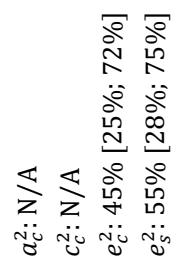 & & 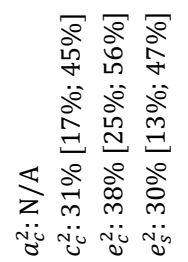 & & 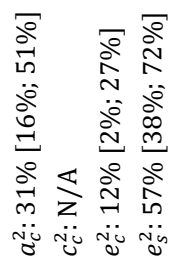 & 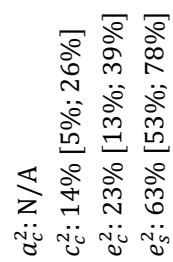 \\
\hline : & 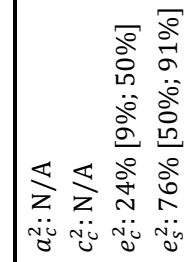 & 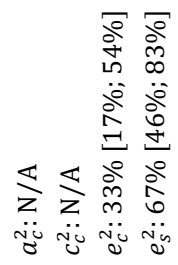 & & 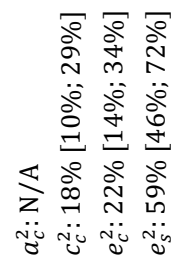 & & 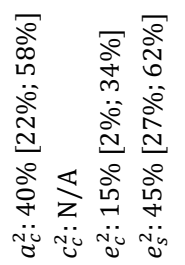 & 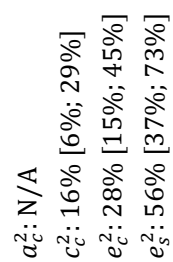 \\
\hline 0 & 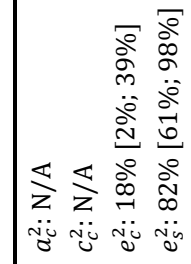 & 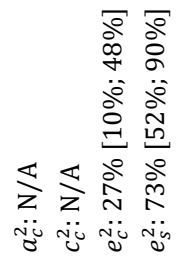 & & 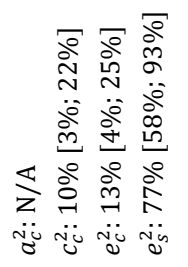 & & 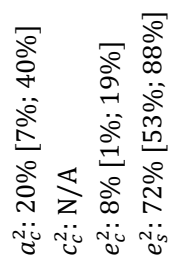 & 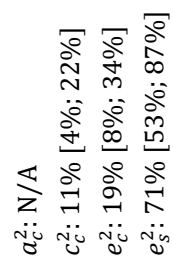 \\
\hline 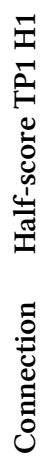 & 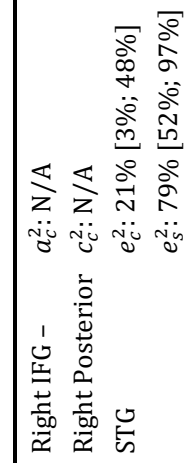 & 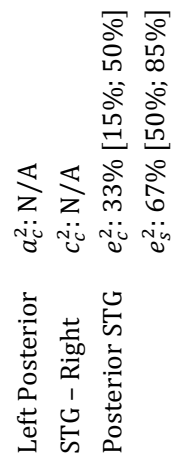 & 0 & 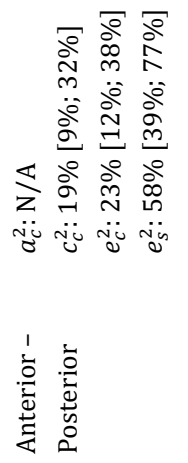 & $\begin{array}{l}\mathbf{3} \\
0 \\
0\end{array}$ & 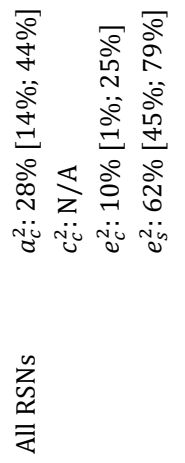 & 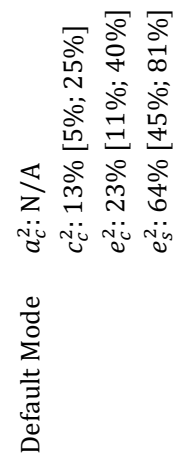 \\
\hline
\end{tabular}




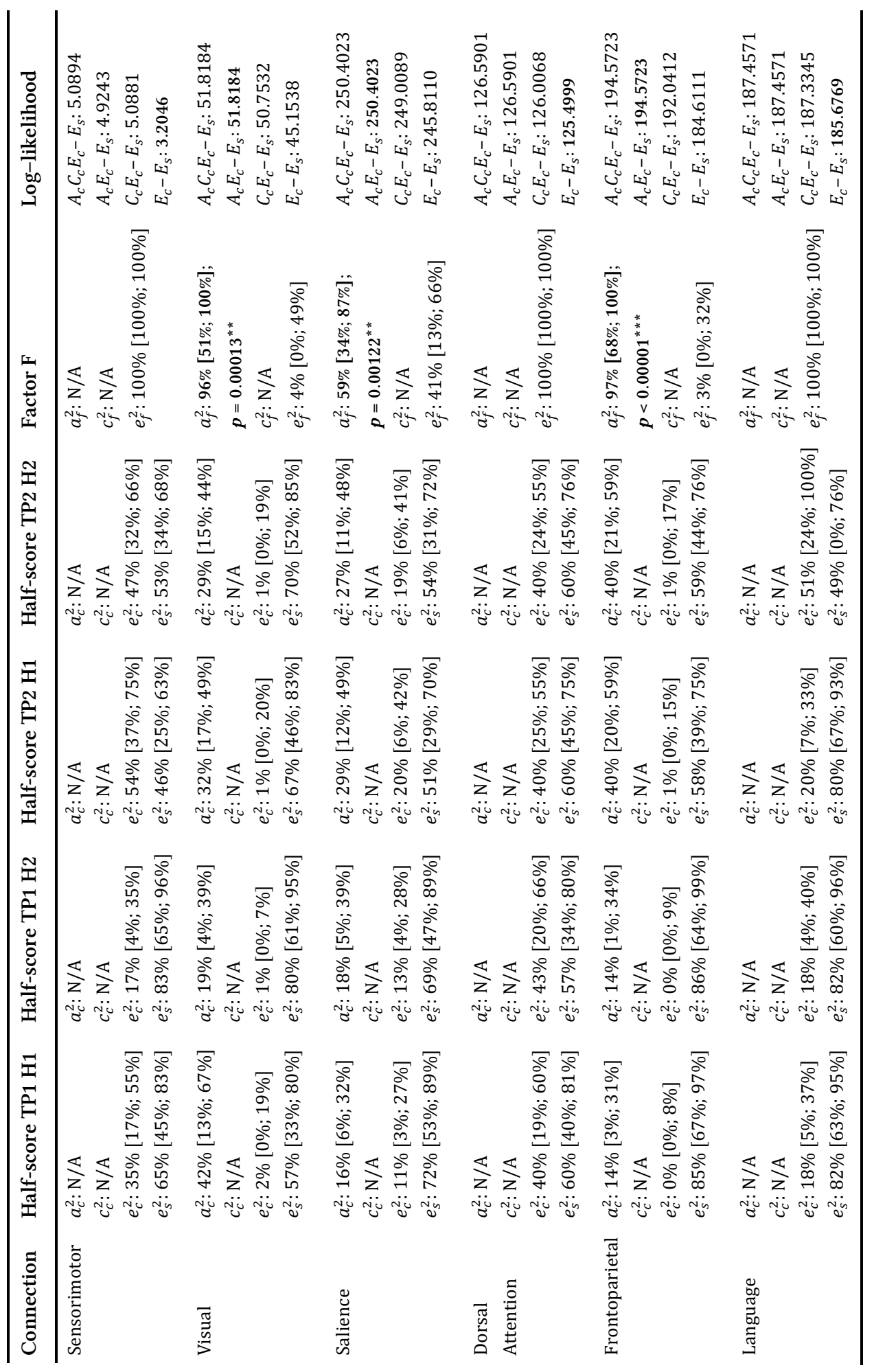




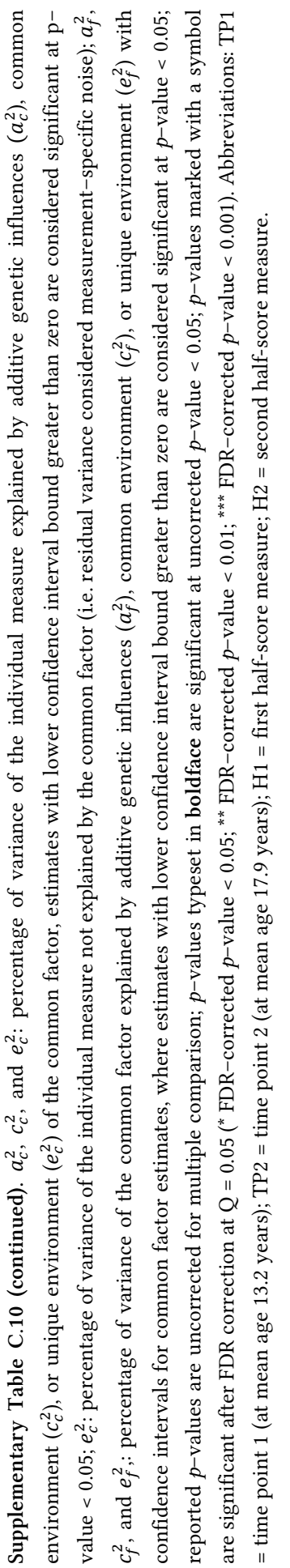


C.1.16 Associations between intelligence and functional connectivity between restingstate networks

Supplementary Table C.11 contains the association coefficients between functional connectivity and IQ test scores at age 13 years $\left(\mathrm{FC}_{\mathrm{TP} 1} \times \mathrm{IQ}_{\mathrm{TP} 1}\right)$ and age 18 years $\left(\mathrm{FC}_{\mathrm{TP} 2} \times\right.$ IQTP2), and the association between the longitudinal change in functional connectivity and longitudinal change in IQ test scores between ages 13 and 18 years $\left(\Delta \mathrm{FC}_{\mathrm{TP} 1, \mathrm{TP} 2} \times \Delta \mathrm{IQ}_{\mathrm{TP} 1, \mathrm{TP} 2}\right)$ for connections between resting-state networks. In addition, the table contains group-mean and standard deviation of the full-score measures at age 13 years $\left(\mathrm{FC}_{\mathrm{TP} 1}\right)$ and age 18 years ( $\mathrm{FC}_{\mathrm{TP} 2}$ ) (see Figure 4.2 from Chapter 4 ), the longitudinal change in functional connectivity from age 13 years to age 18 years $\left(\triangle \mathrm{FC}_{\mathrm{TP} 1, \mathrm{TP} 2}\right)$, and the phenotypic correlation between the full-scores of functional connectivity at age 13 and 18 years $\left(\mathrm{FC}_{\mathrm{TP} 1} \times \mathrm{FC}_{\mathrm{TP} 2}\right)$ used as estimate for long-term test-retest reliability (see Supplementary Figure C.6). Statistically significant associations are printed in bold; with association that are significant after FDR-correction marked with symbols ( ${ }^{*}$ FDR-corrected $p<0.05$; ${ }^{* *}$ FDR-corrected $p<0.01$; ${ }^{* *}$ FDRcorrected $p<0.001)$. 


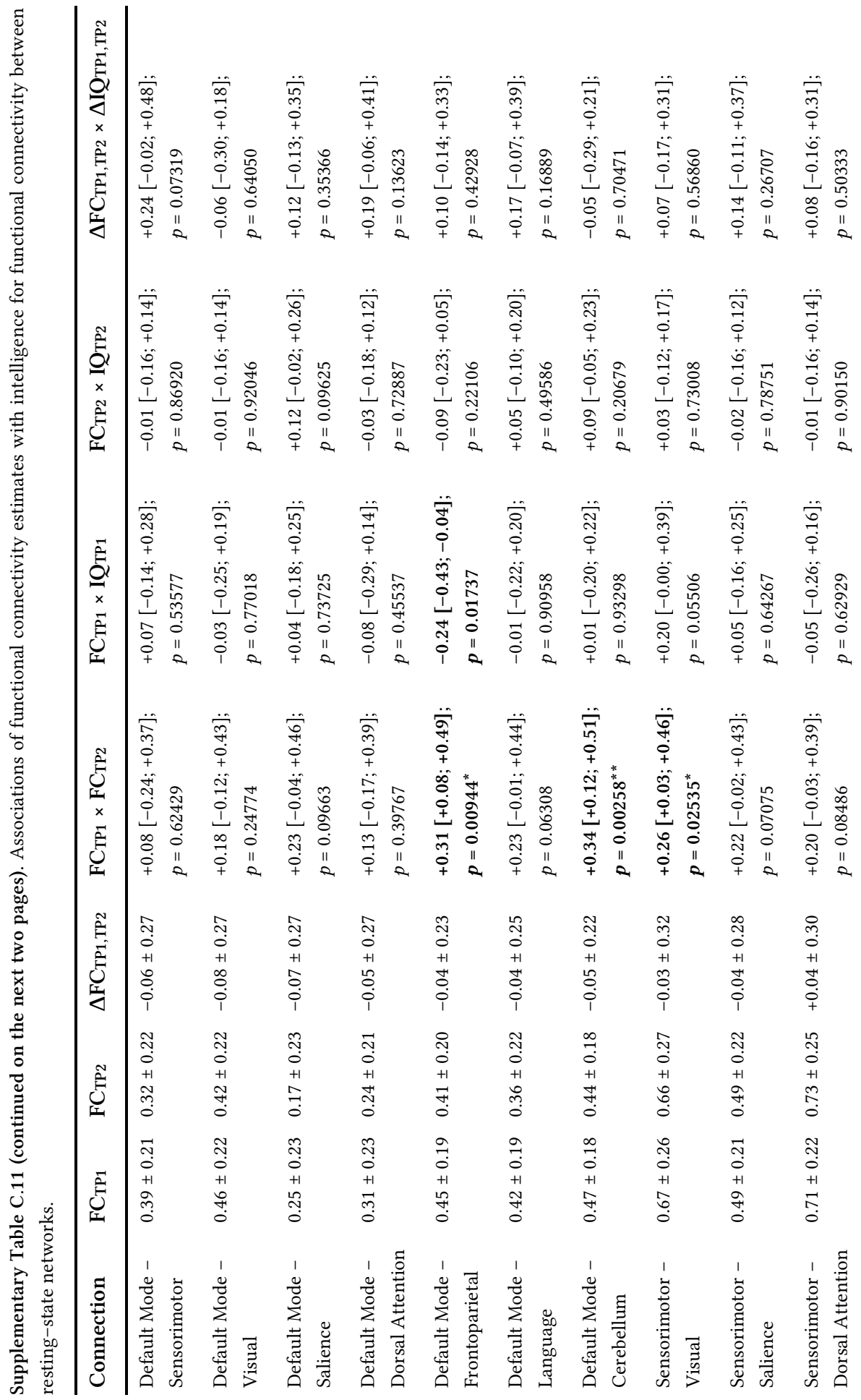




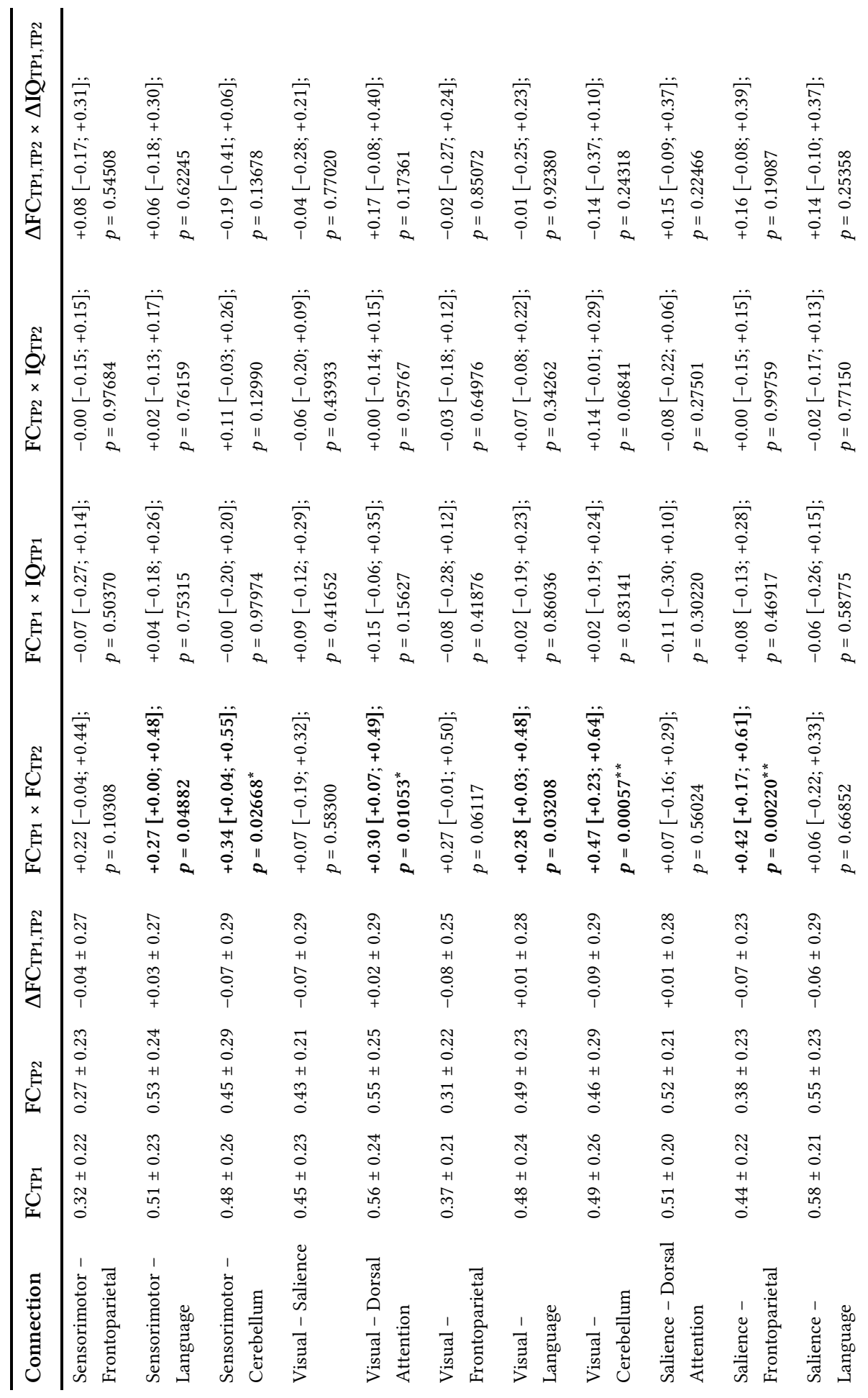




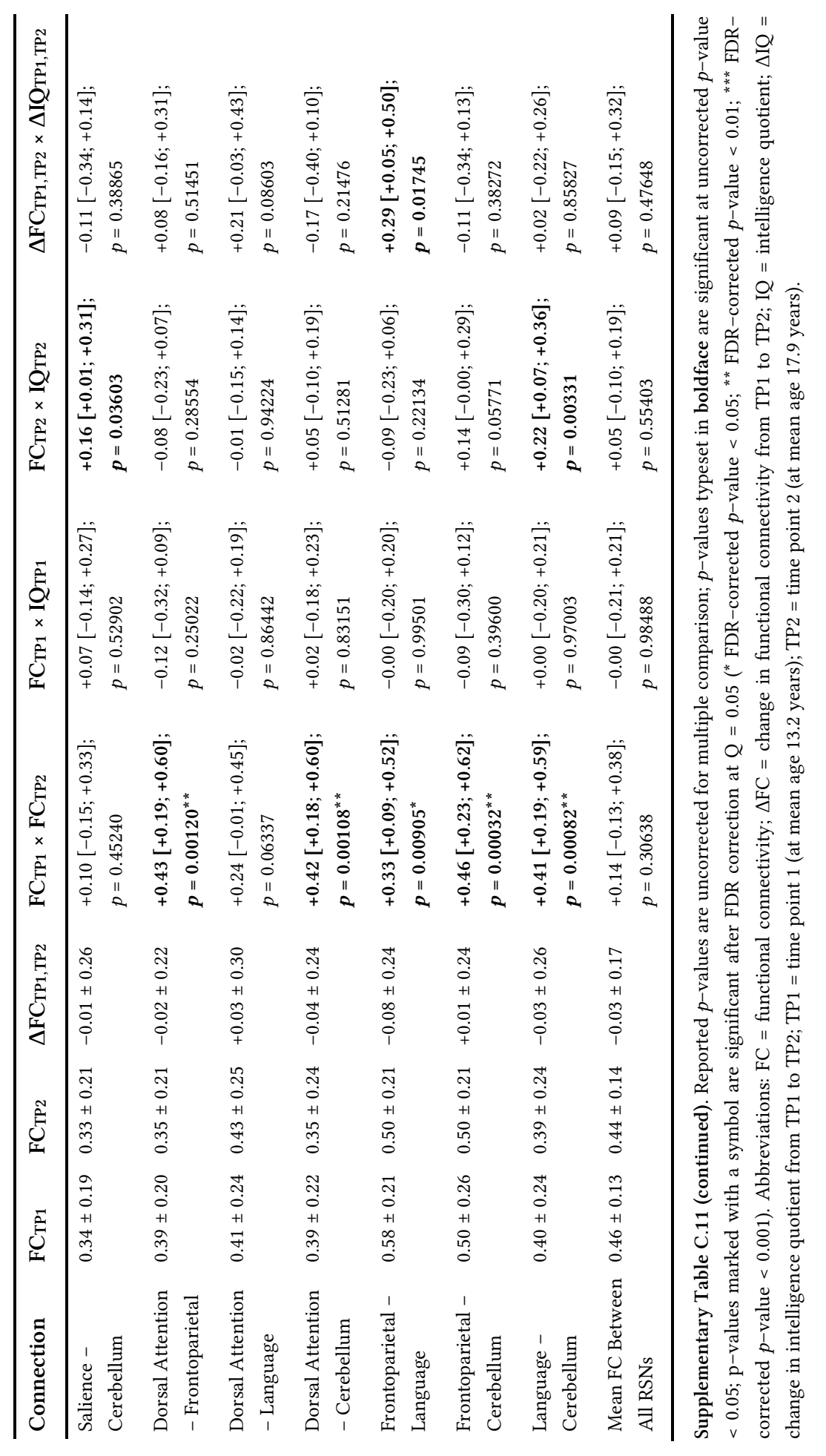


C.1.17 Association between intelligence and functional connectivity within restingstate networks

Supplementary Table C.12 contains the association coefficients between functional connectivity and IQ test scores at age 13 years $\left(\mathrm{FC}_{\mathrm{TP} 1} \times \mathrm{IQ}_{\mathrm{TP} 1}\right)$ and age 18 years $\left(\mathrm{FC}_{\mathrm{TP} 2} \times\right.$ IQTP2), and the association between the longitudinal change in functional connectivity and longitudinal change in IQ test scores between ages 13 and 18 years $\left(\Delta \mathrm{FC}_{\mathrm{TP} 1, \mathrm{TP} 2} \times \Delta \mathrm{IQ} \mathrm{QP}_{\mathrm{TP} 2 \mathrm{~T} 2}\right)$ for connections within resting-state networks. In addition, the table contains group-mean and standard deviation of the full-score measures at age 13 years $\left(\mathrm{FC}_{\mathrm{TP} 1}\right)$ and age 18 years $\left(\mathrm{FC}_{\mathrm{TP} 2}\right.$ ) (see Figure 4.2 from Chapter 4 ), the longitudinal change in functional connectivity from age 13 years to age 18 years $\left(\triangle \mathrm{FC}_{\mathrm{TP} 1, \mathrm{TP} 2}\right)$, and the phenotypic correlation between the full-scores of functional connectivity at age 13 and 18 years $\left(\mathrm{FC}_{\mathrm{TP} 1} \times \mathrm{FC}_{\mathrm{TP} 2}\right)$ used as estimate for long-term test-retest reliability (see Supplementary Figure C.6). Statistically significant associations are printed in bold; with association that are significant after FDR-correction marked with symbols ( ${ }^{*}$ FDR-corrected $p<0.05$; ${ }^{* *}$ FDR-corrected $p<0.01$; ${ }^{* *}$ FDRcorrected $p<0.001)$. 


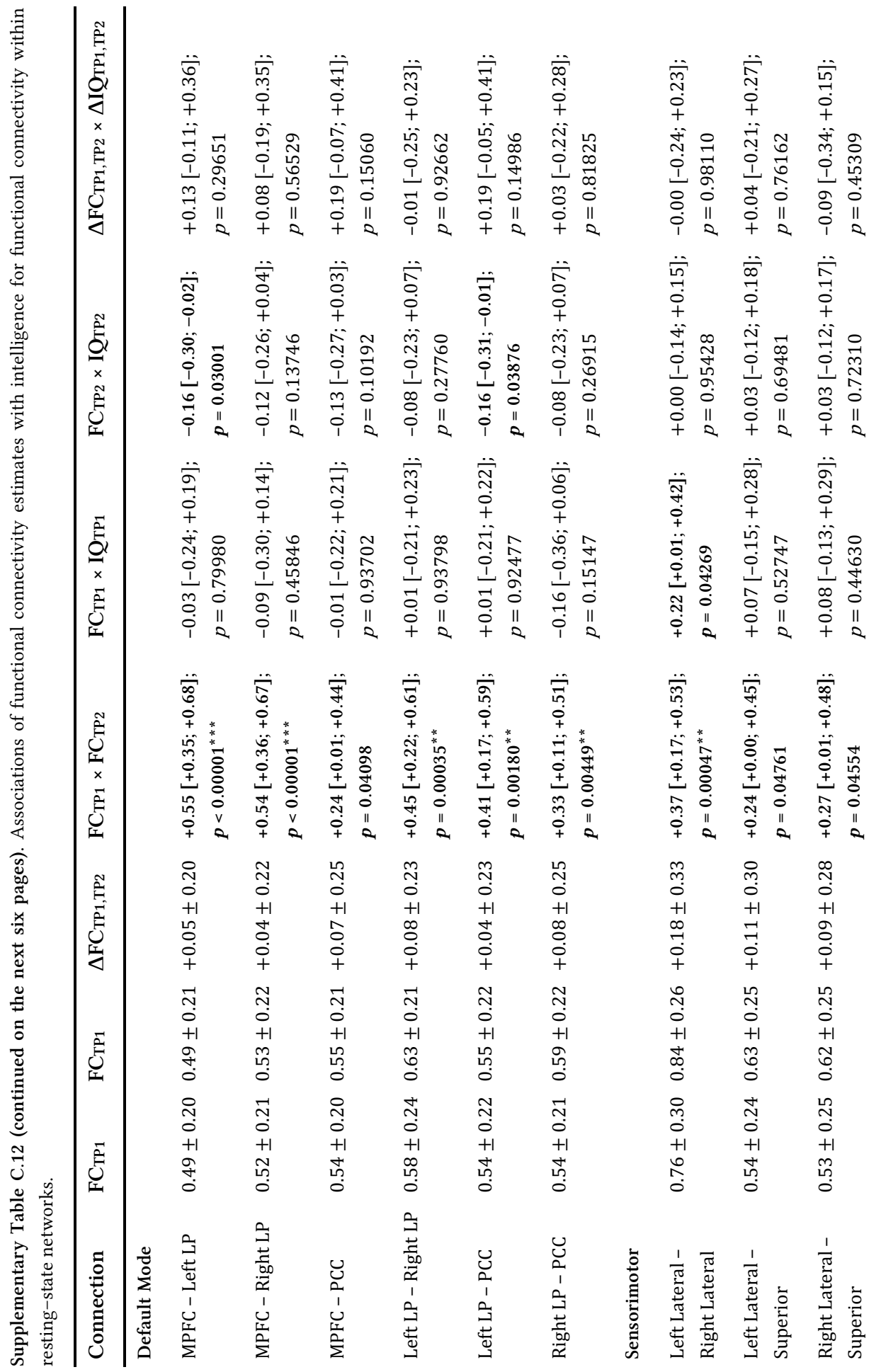




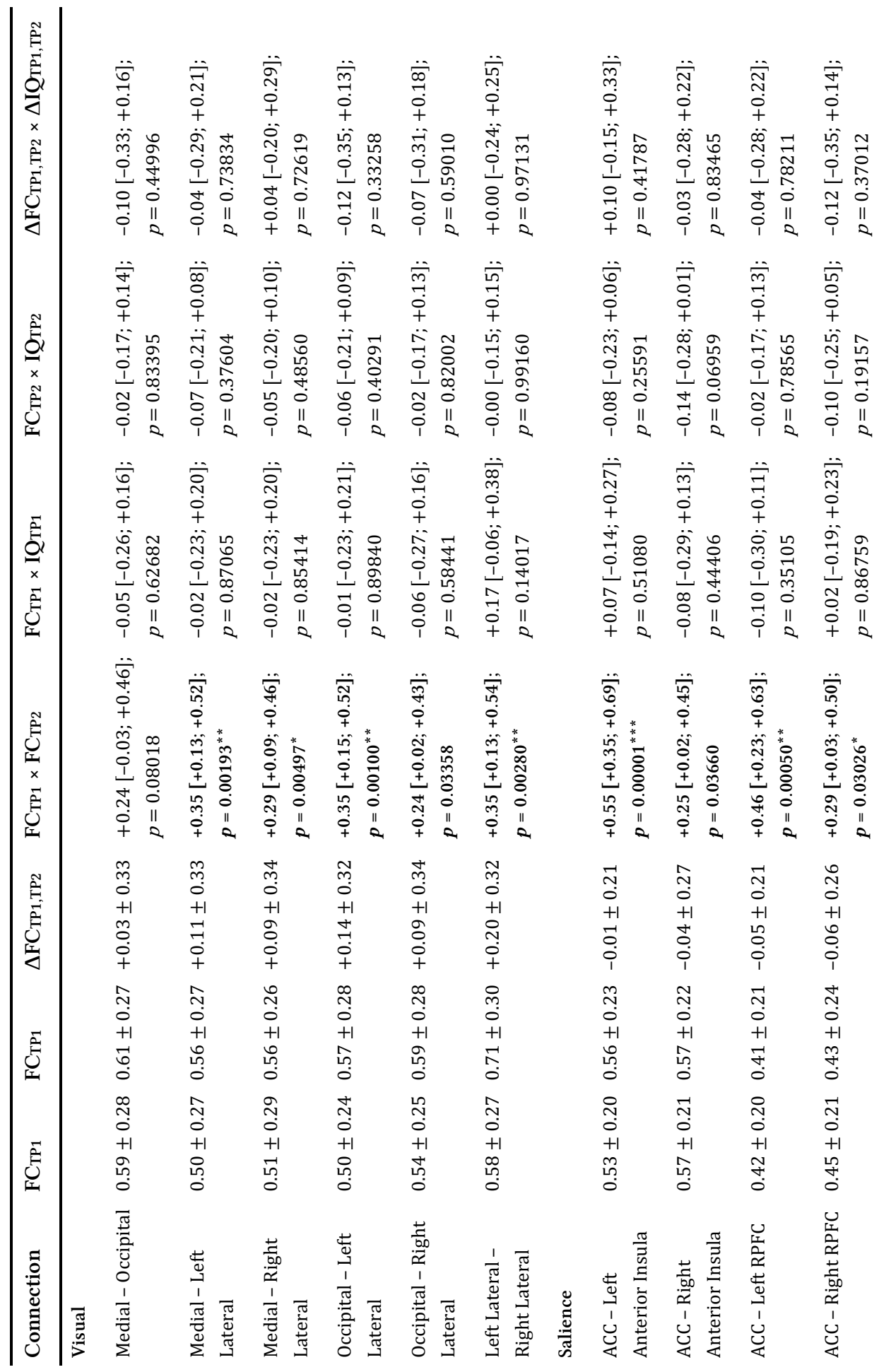




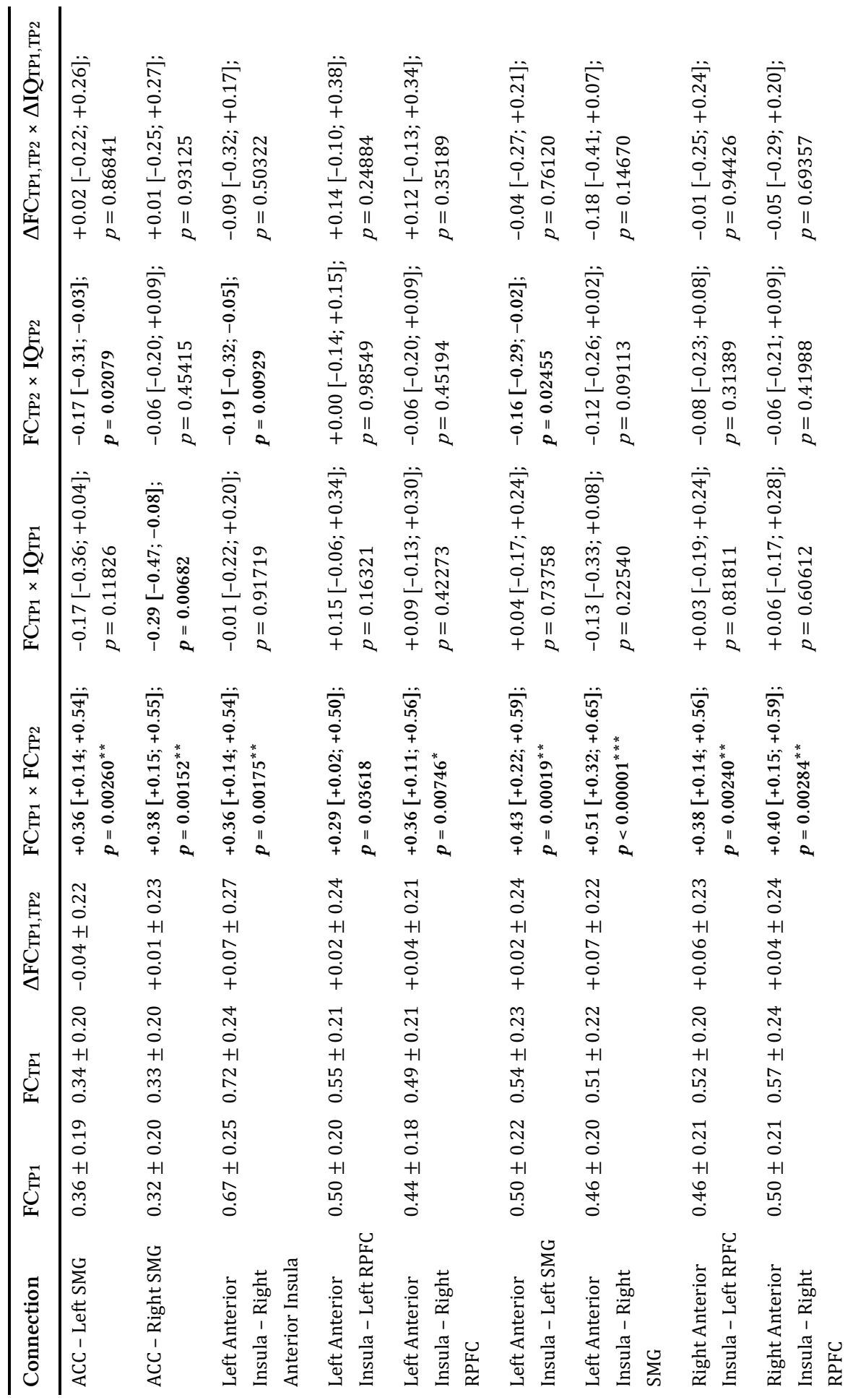




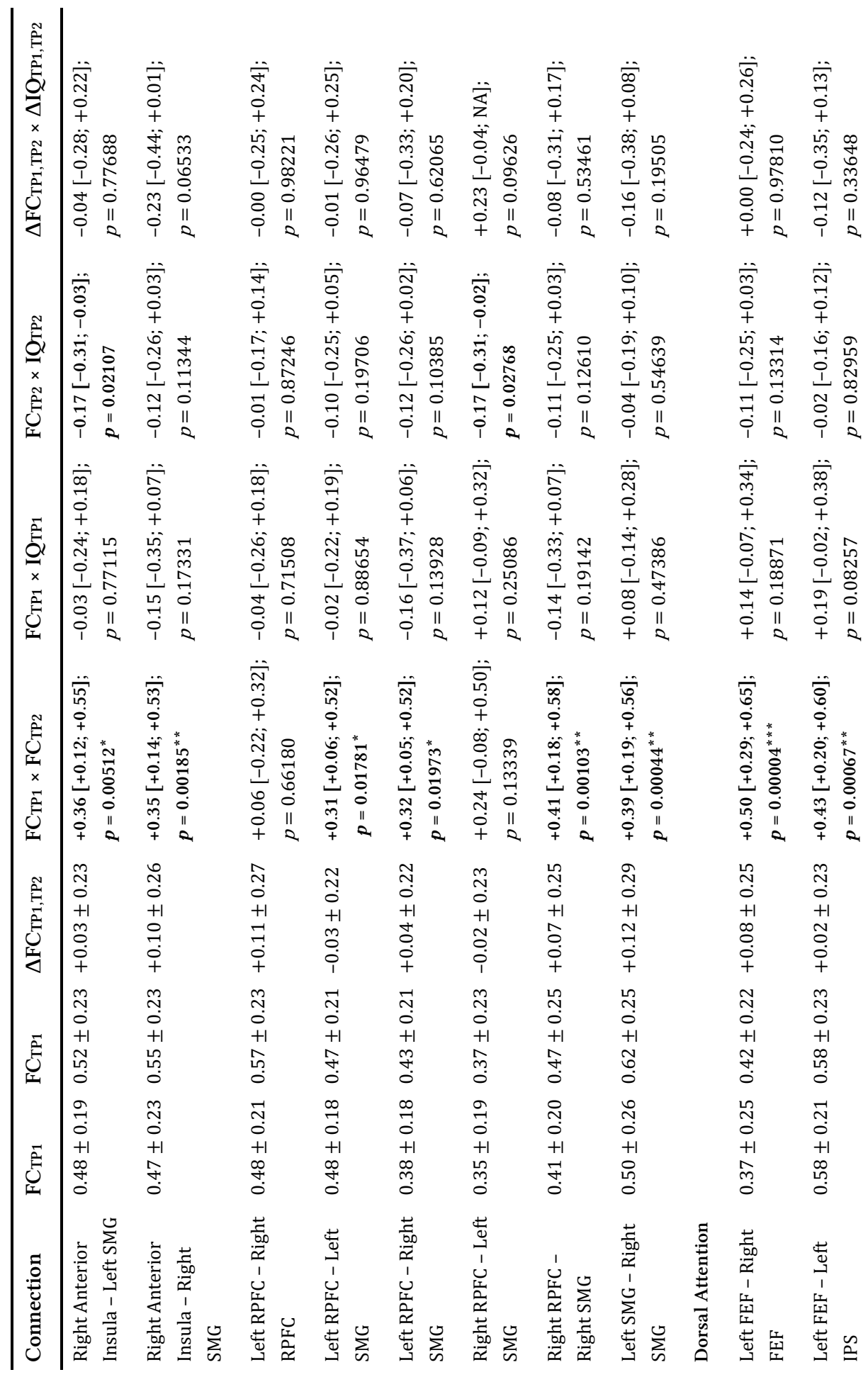




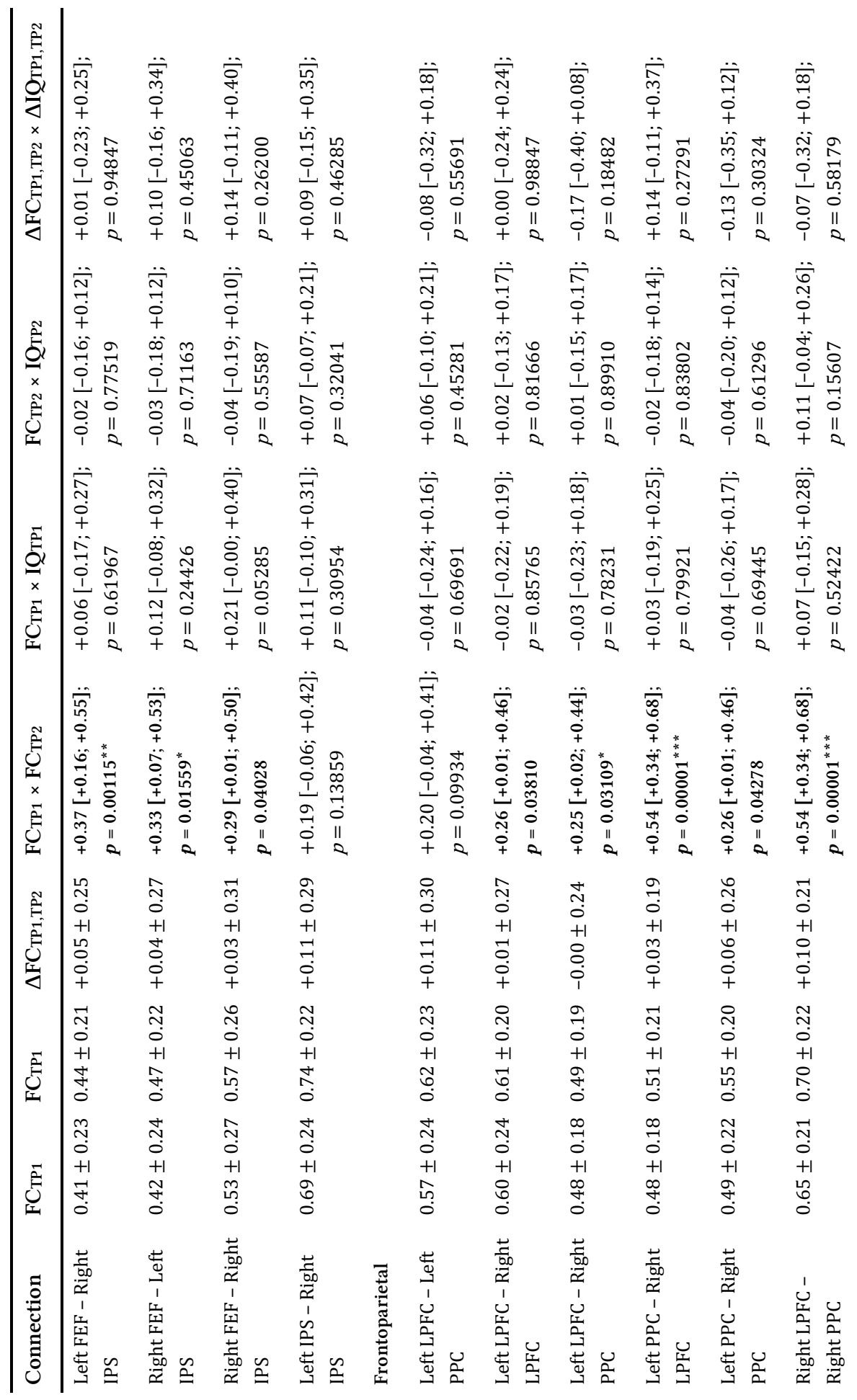




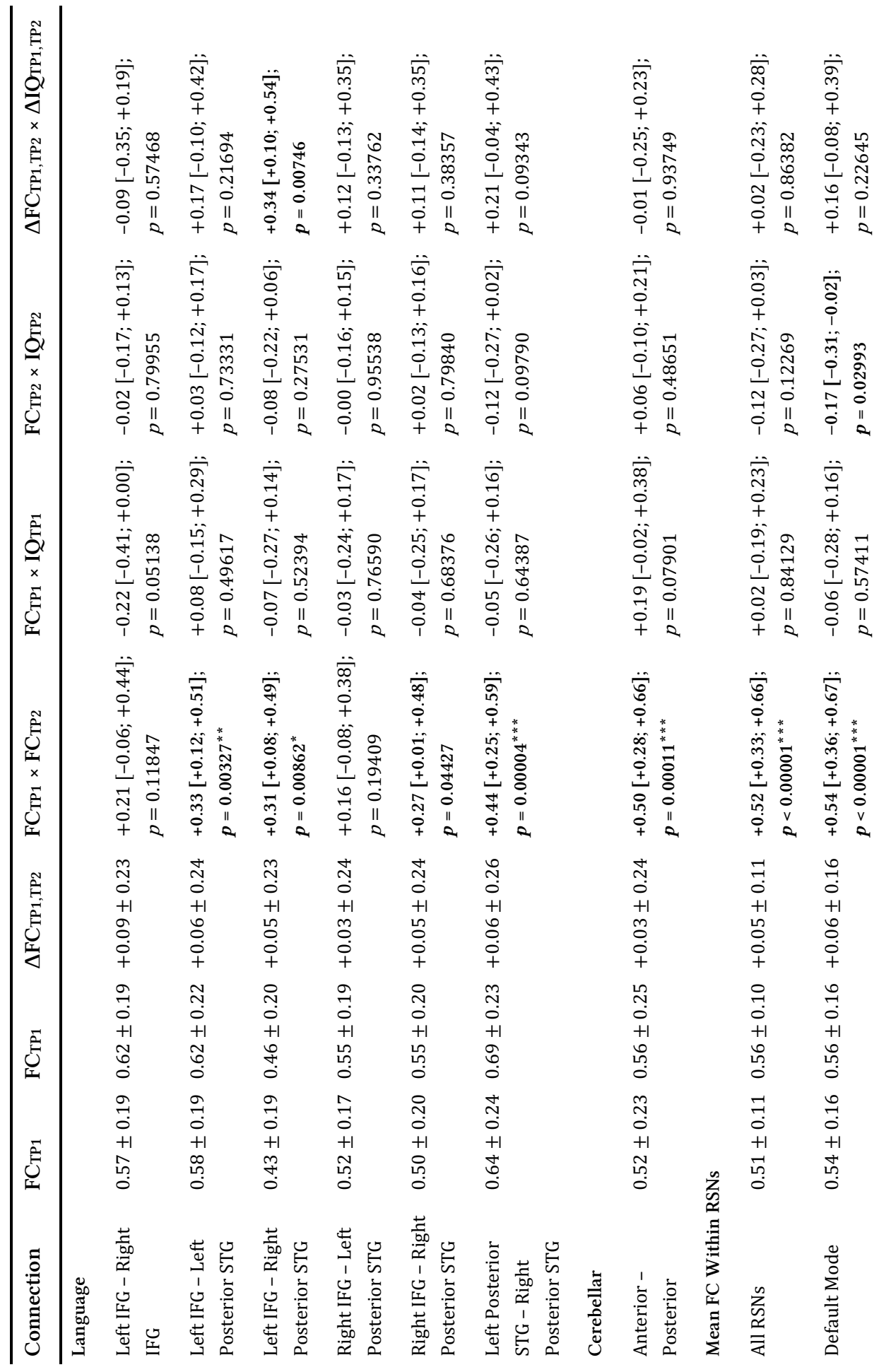




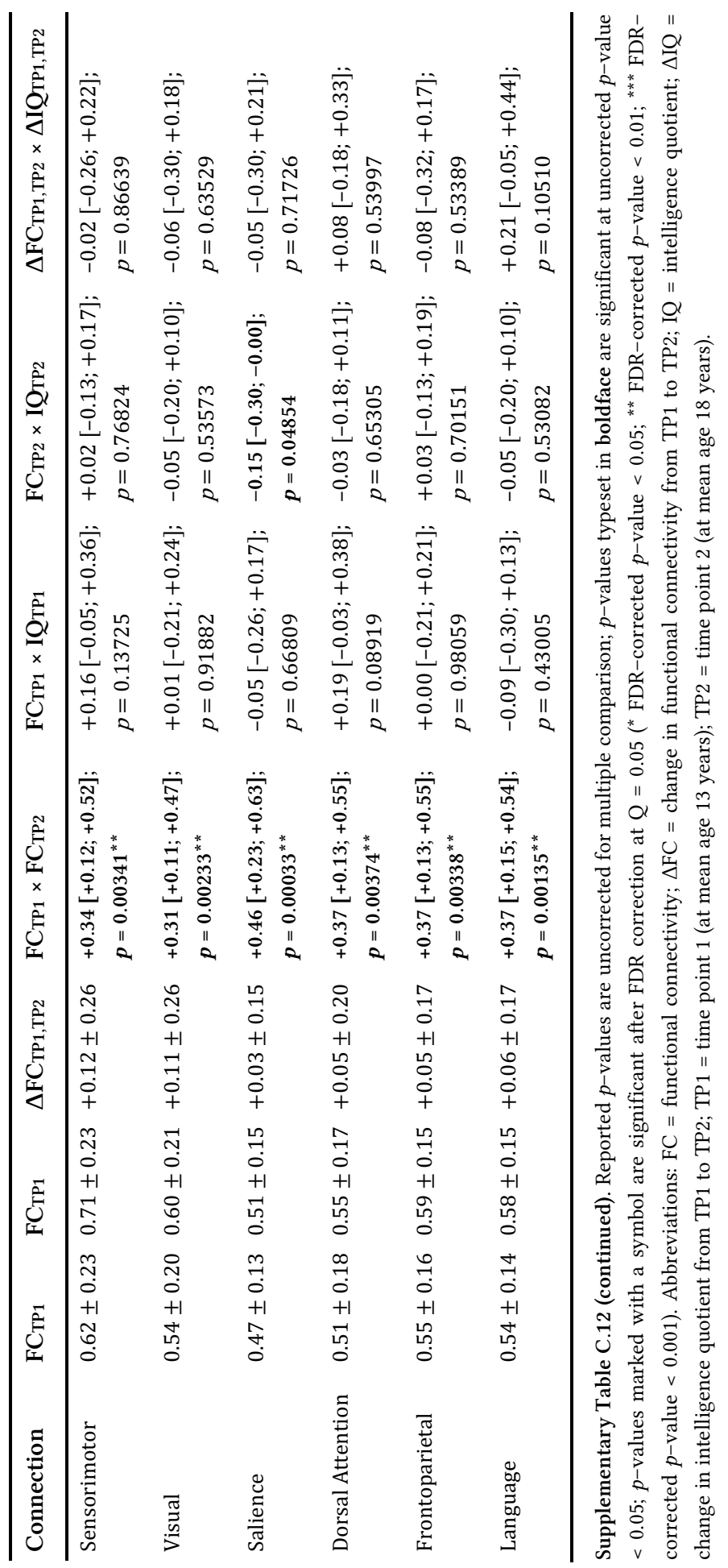


C.1.18 Associations between head motion and functional connectivity between restingstate networks

Supplementary Table C.13 contains the association coefficients between functional connectivity and mean framewise displacement at age 13 years $\left(\mathrm{FC}_{\mathrm{TP} 1} \times \mathrm{FD}_{\mathrm{TP} 1}\right)$ and age 18 years $\left(\mathrm{FC}_{\mathrm{TP} 2} \times \mathrm{FD}_{\mathrm{TP} 2}\right)$, and the association between the longitudinal change in functional connectivity and longitudinal change in mean framewise displacement between ages 13 and 18 years $\left(\triangle \mathrm{FC}_{\mathrm{TP} 1, \mathrm{TP} 2} \times \Delta \mathrm{FD}_{\mathrm{TP} 1, \mathrm{TP} 2}\right)$ for connections between resting-state networks. In addition, the table contains group-mean and standard deviation of the full-score measures at age 13 years $\left(\mathrm{FC}_{\mathrm{TP} 1}\right)$ and age 18 years $\left(\mathrm{FC}_{\mathrm{TP} 2}\right)$ (see Figure 4.2 from Chapter 4 ), the longitudinal change in functional connectivity from age 13 years to age 18 years $\left(\triangle \mathrm{FC}_{\mathrm{TP} 1, \mathrm{TP} 2}\right)$, and the phenotypic correlation between the full-scores of functional connectivity at age 13 and 18 years $\left(\mathrm{FC}_{\mathrm{TP} 1} \times \mathrm{FC}_{\mathrm{TP} 2}\right)$ used as estimate for long-term testretest reliability (see Supplementary Figure C.6). Statistically significant associations are printed in bold; with association that are significant after FDR-correction marked with symbols ( ${ }^{*}$ FDR-corrected $p<0.05 ;{ }^{* *}$ FDR-corrected $p<0.01 ;{ }^{* * *}$ FDR-corrected $p<0.001$ ). 


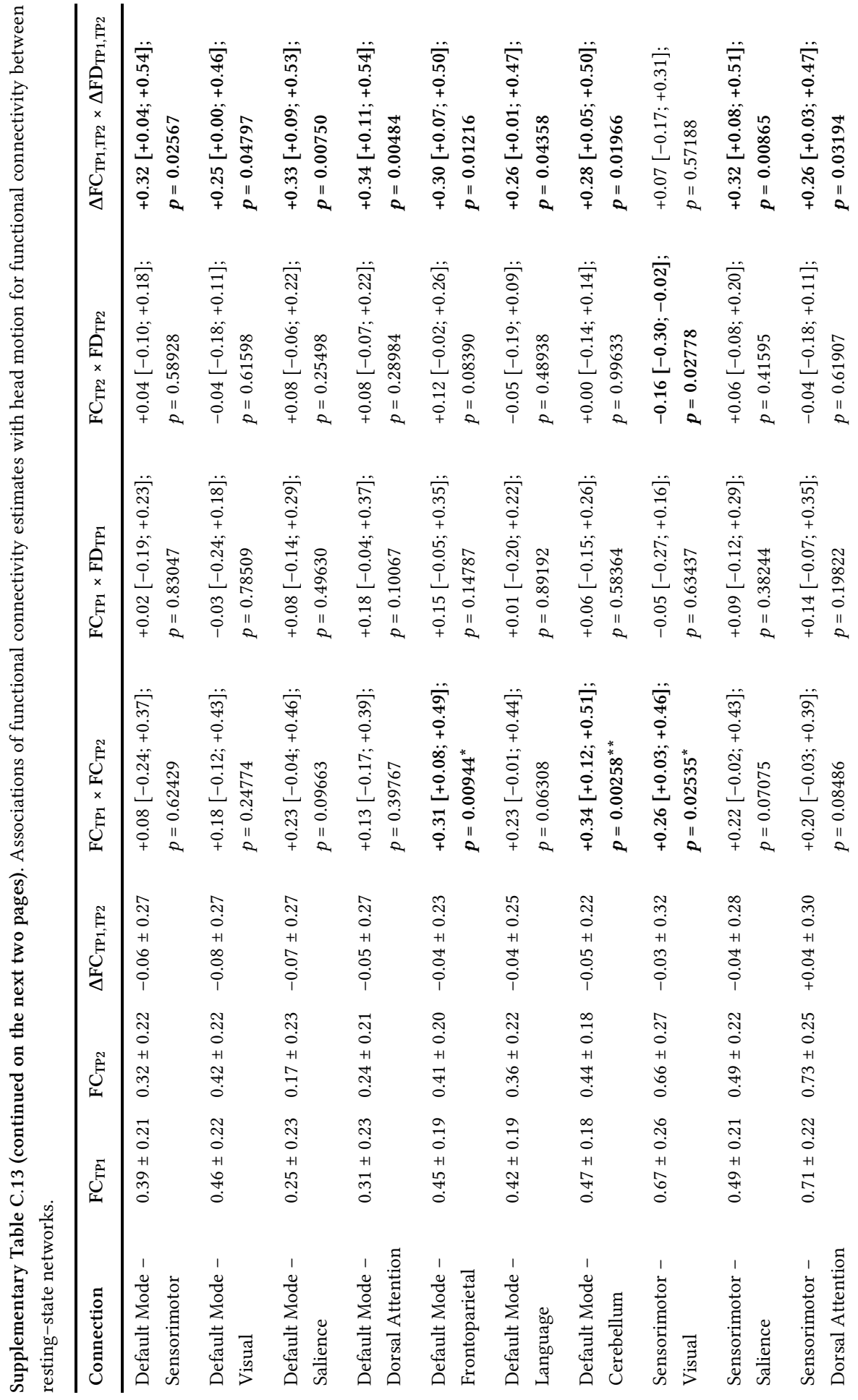




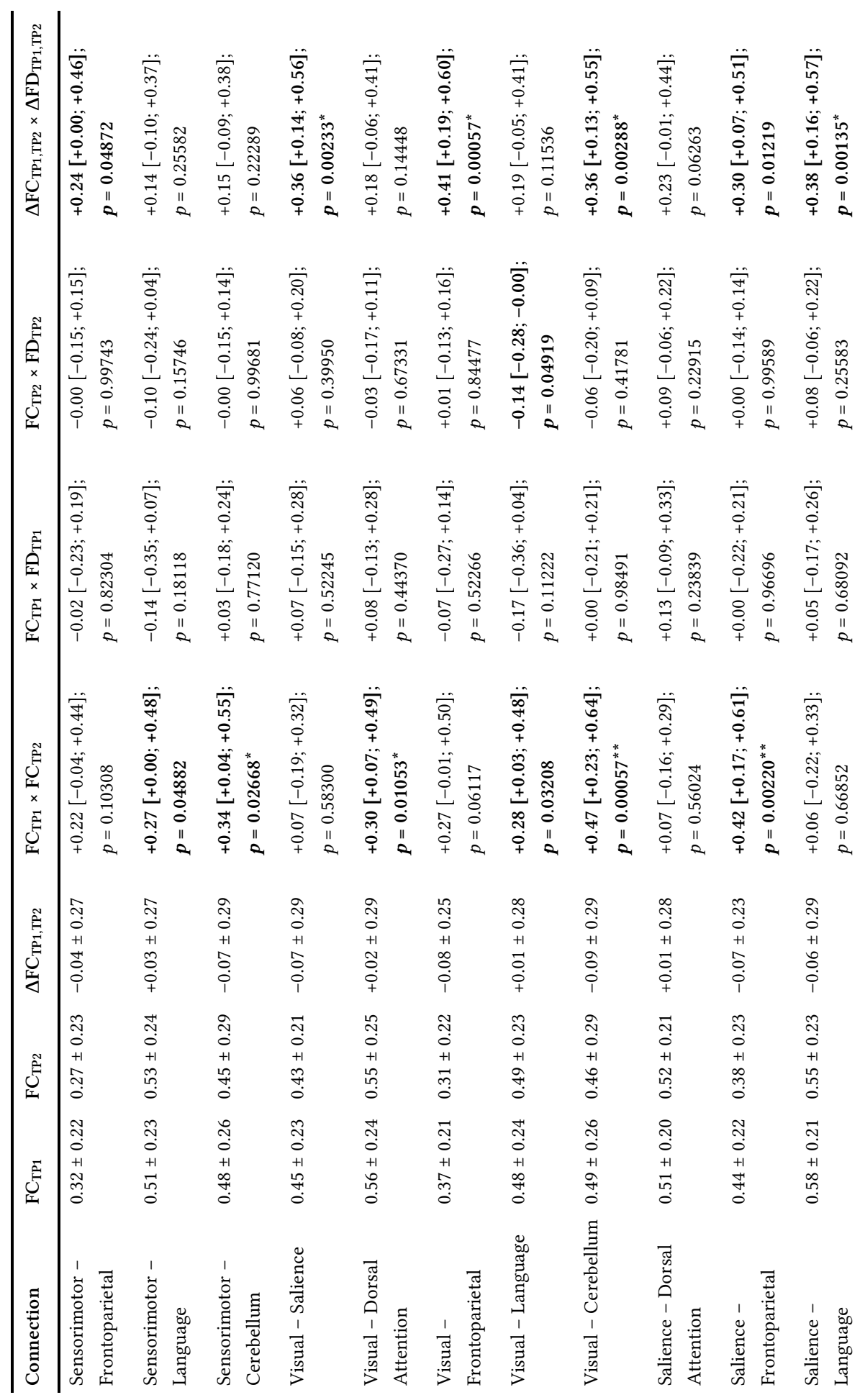




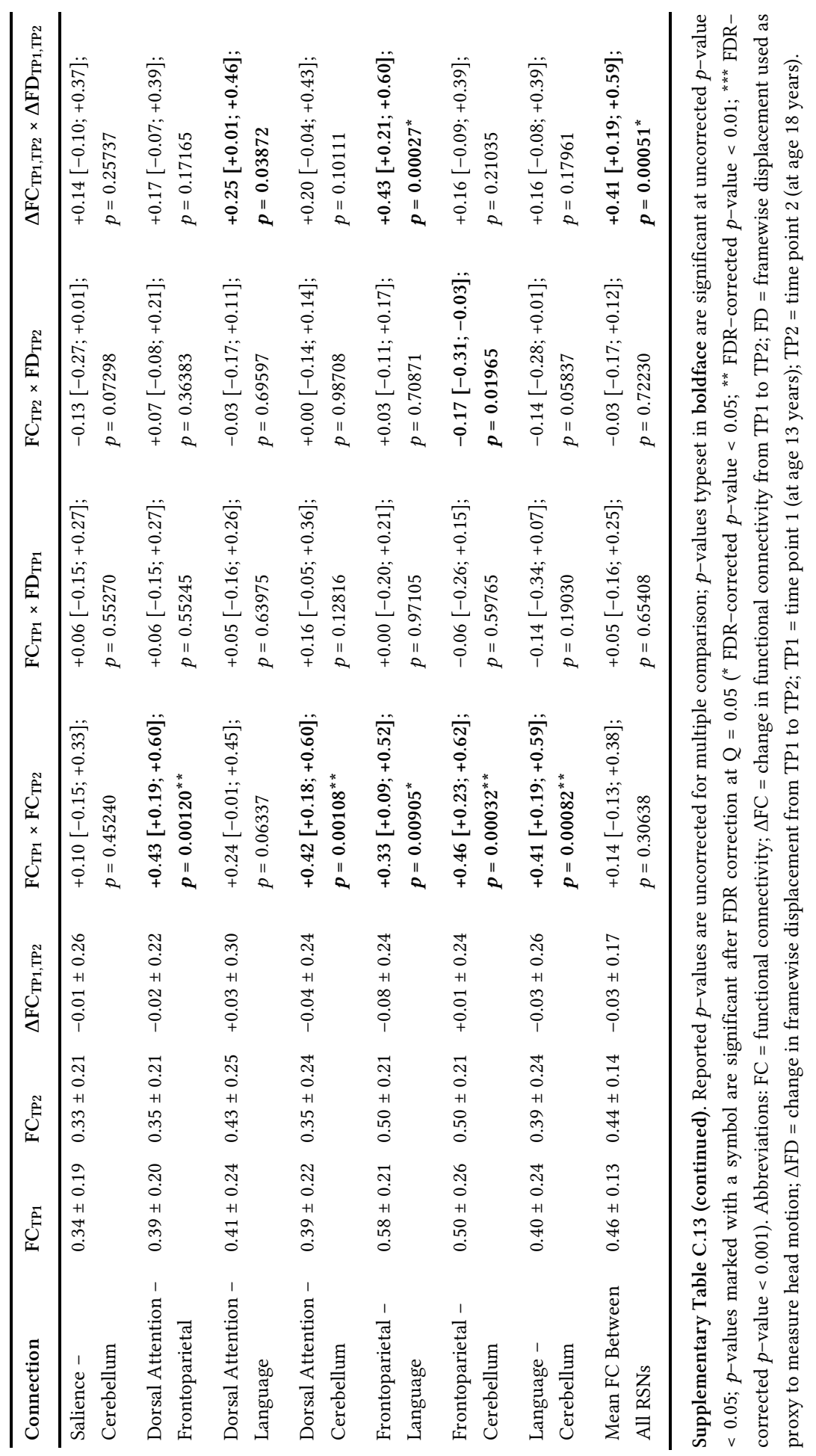


C.1.19 Associations between head motion and functional connectivity within restingstate networks

Supplementary Table C.14 contains the association coefficients between functional connectivity and mean framewise displacement at age 13 years $\left(\mathrm{FC}_{\mathrm{TP} 1} \times \mathrm{FD}_{\mathrm{TP} 1}\right)$ and age 18 years $\left(\mathrm{FC}_{\mathrm{TP} 2} \times \mathrm{FD}_{\mathrm{TP} 2}\right)$, and the association between the longitudinal change in functional connectivity and longitudinal change in mean framewise displacement between ages 13 and 18 years $\left(\triangle \mathrm{FC}_{\mathrm{TP} 1, \mathrm{TP} 2} \times \Delta \mathrm{FD}_{\mathrm{TP} 1, \mathrm{TP} 2}\right)$ for connections within resting-state networks. In addition, the table contains group-mean and standard deviation of the full-score measures at age 13 years $\left(\mathrm{FC}_{\mathrm{TP} 1}\right)$ and age 18 years $\left(\mathrm{FC}_{\mathrm{TP} 2}\right)$ (see Figure 4.2 from Chapter 4 ), the longitudinal change in functional connectivity from age 13 years to age 18 years $\left(\triangle \mathrm{FC}_{\mathrm{TP} 1, \mathrm{TP} 2}\right)$, and the phenotypic correlation between the full-scores of functional connectivity at age 13 and 18 years $\left(\mathrm{FC}_{\mathrm{TP} 1} \times \mathrm{FC}_{\mathrm{TP} 2}\right)$ used as estimate for long-term testretest reliability (see Supplementary Figure C.6). Statistically significant associations are printed in boldface; with association that are significant after FDR-correction marked with symbols ( ${ }^{*}$ FDR-corrected $p<0.05 ;{ }^{* *}$ FDR-corrected $p<0.01 ;{ }^{* * *}$ FDR-corrected $p<0.001$ ). 


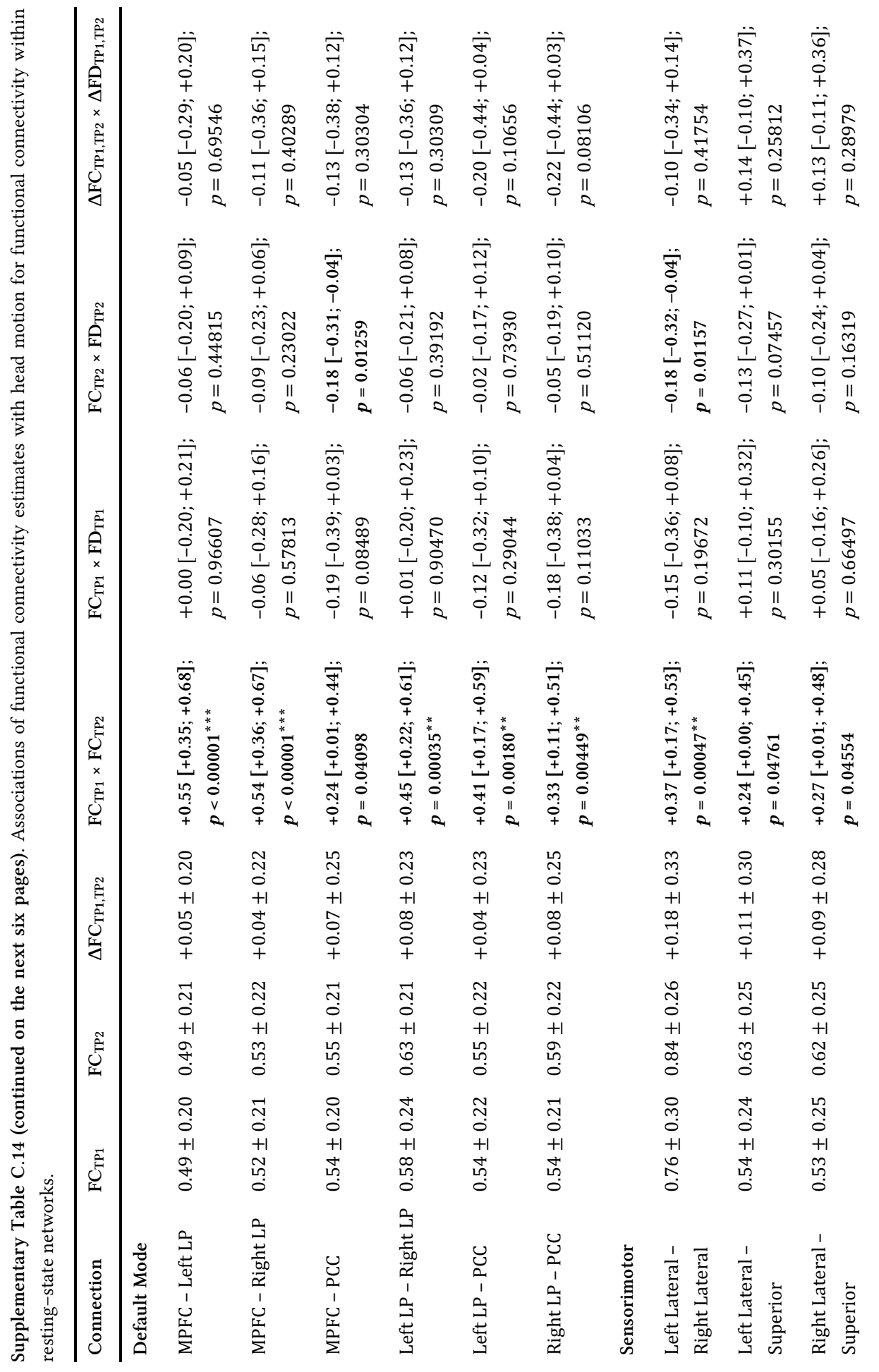




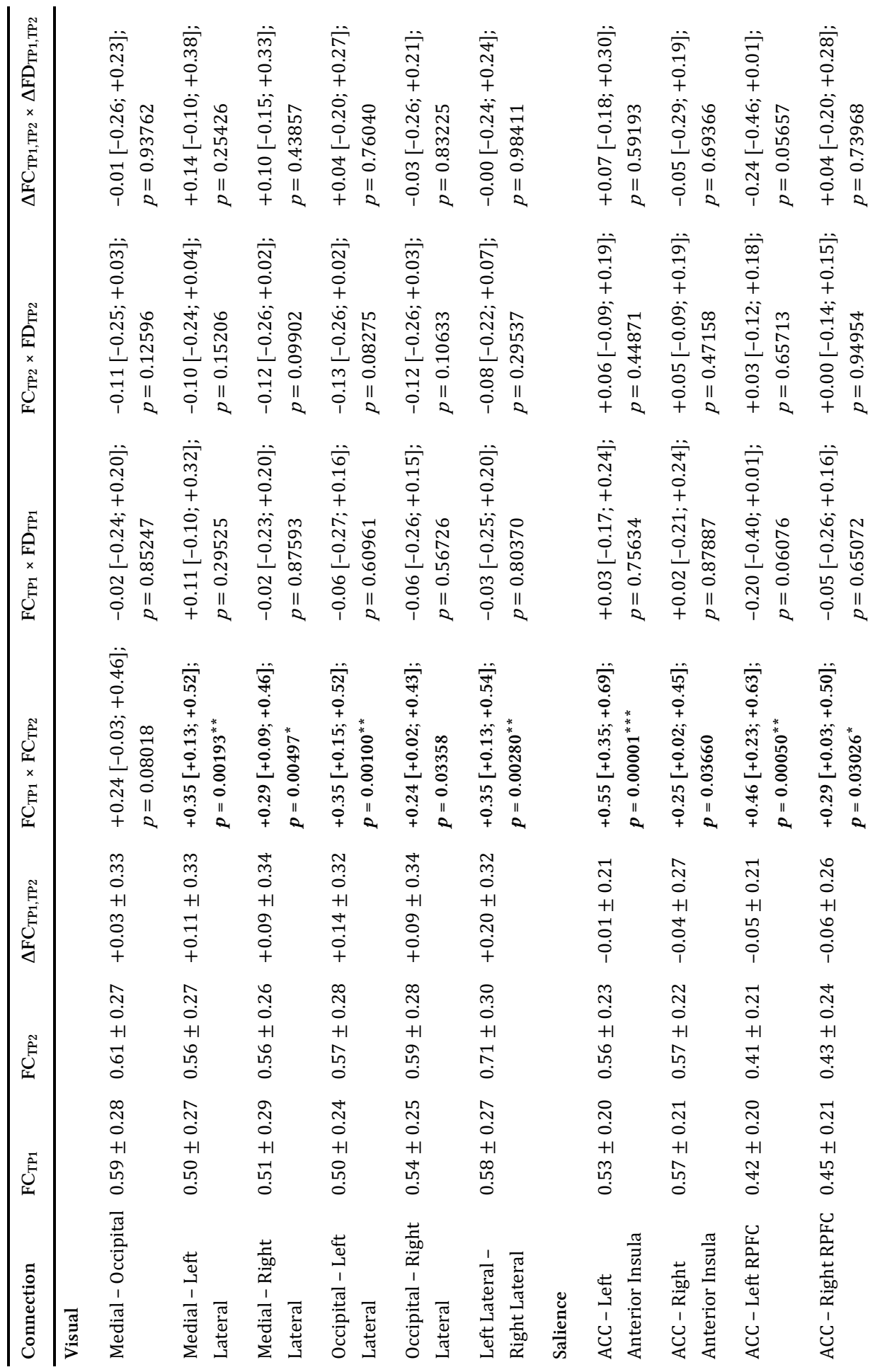




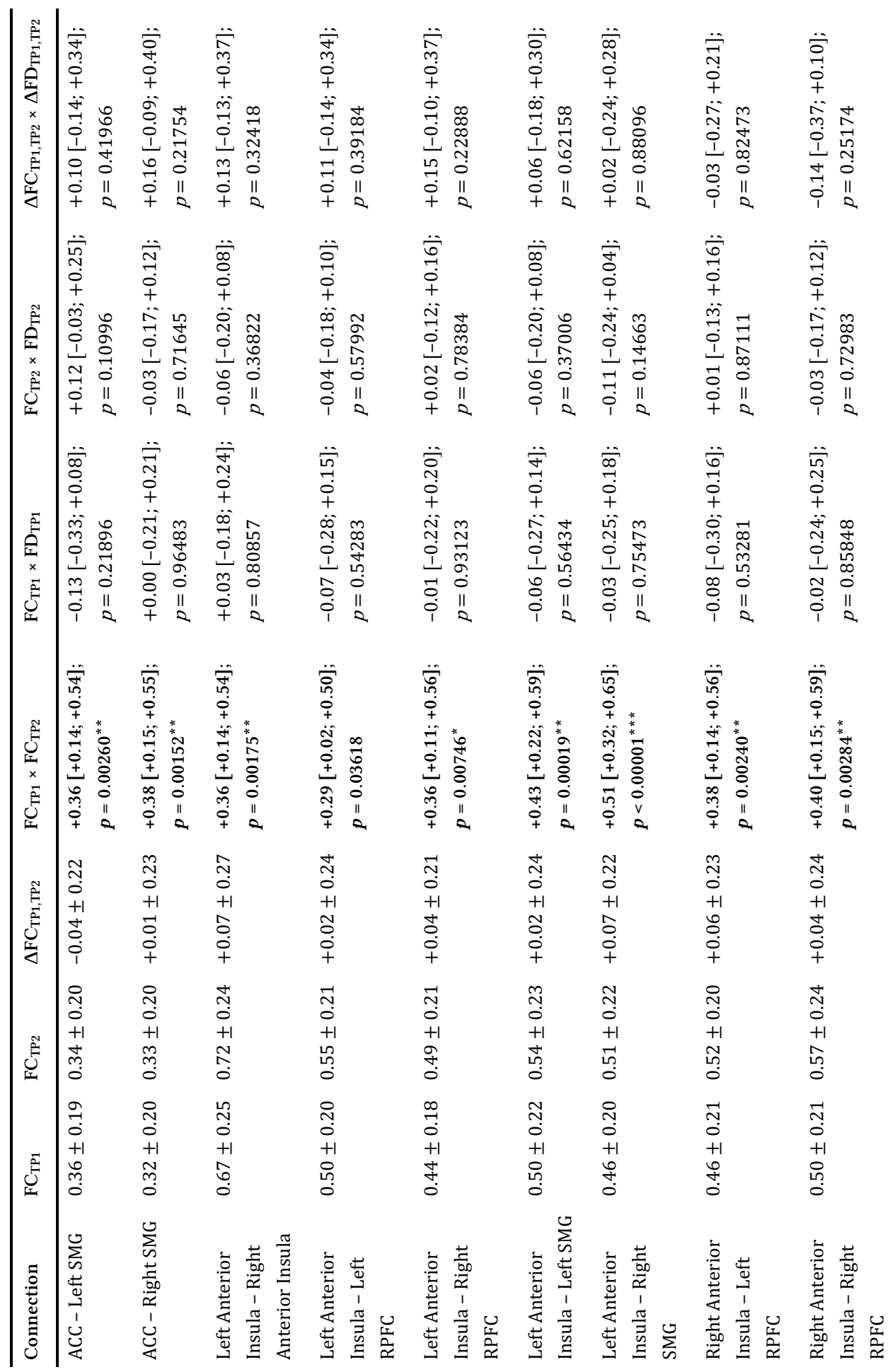




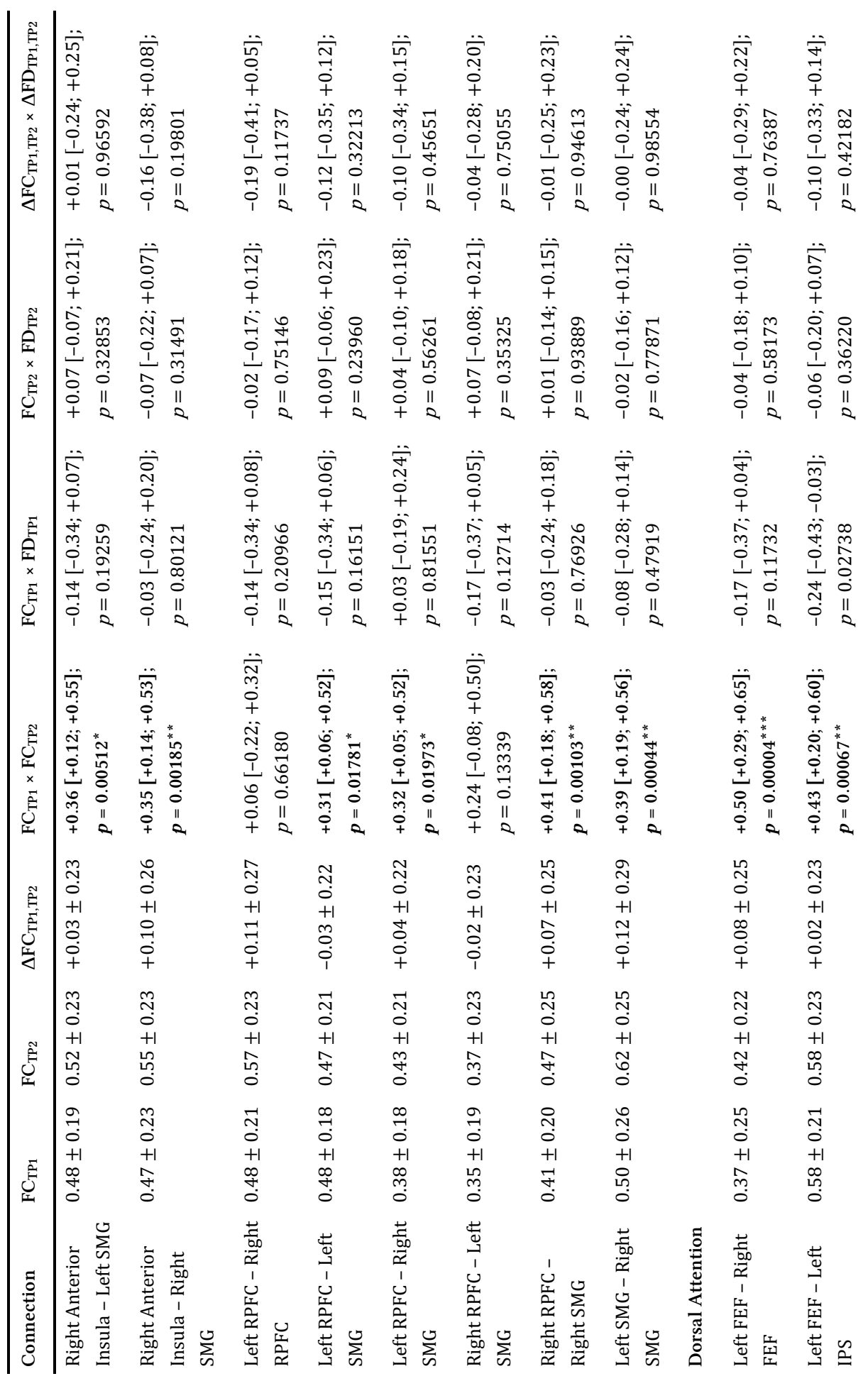




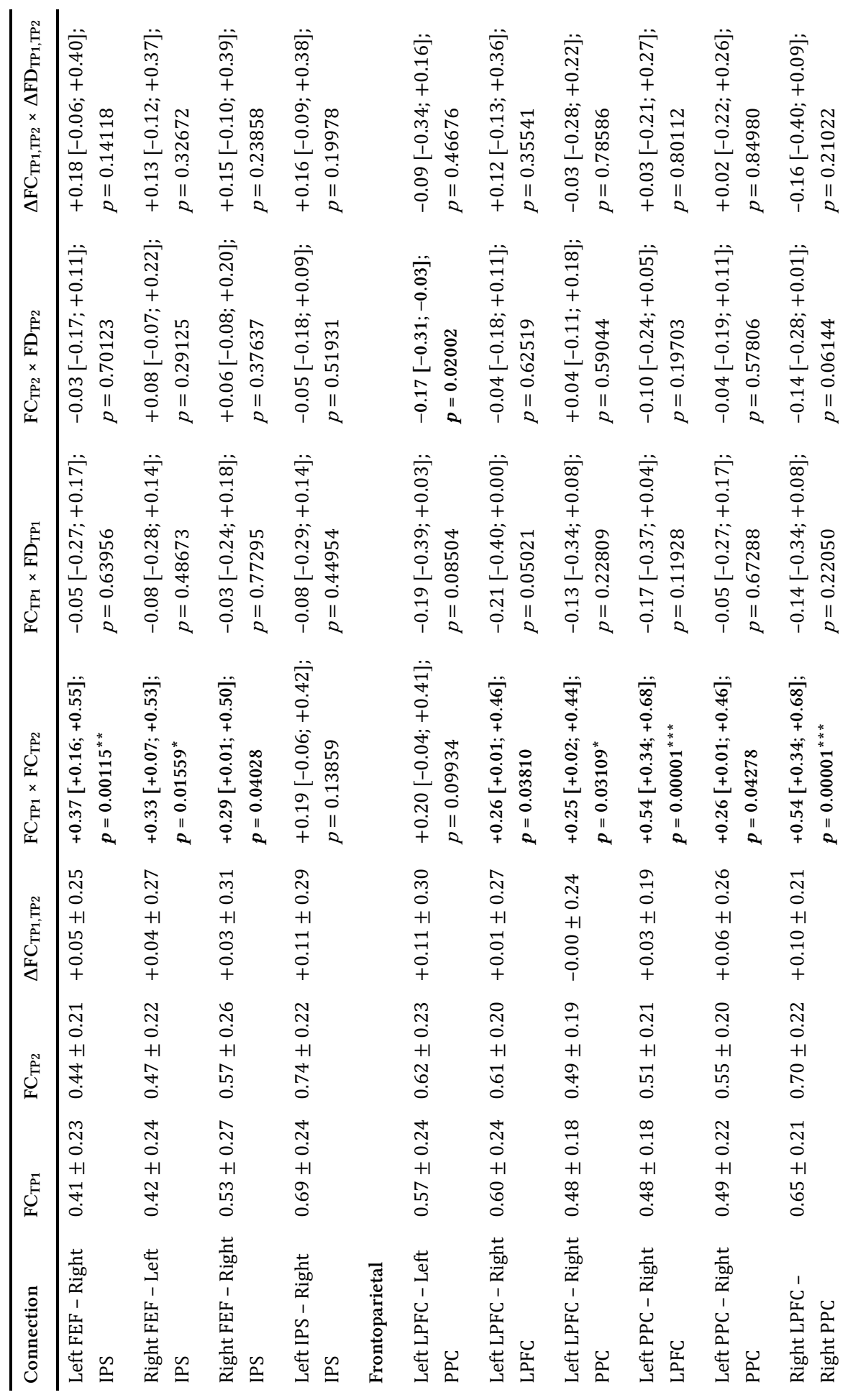




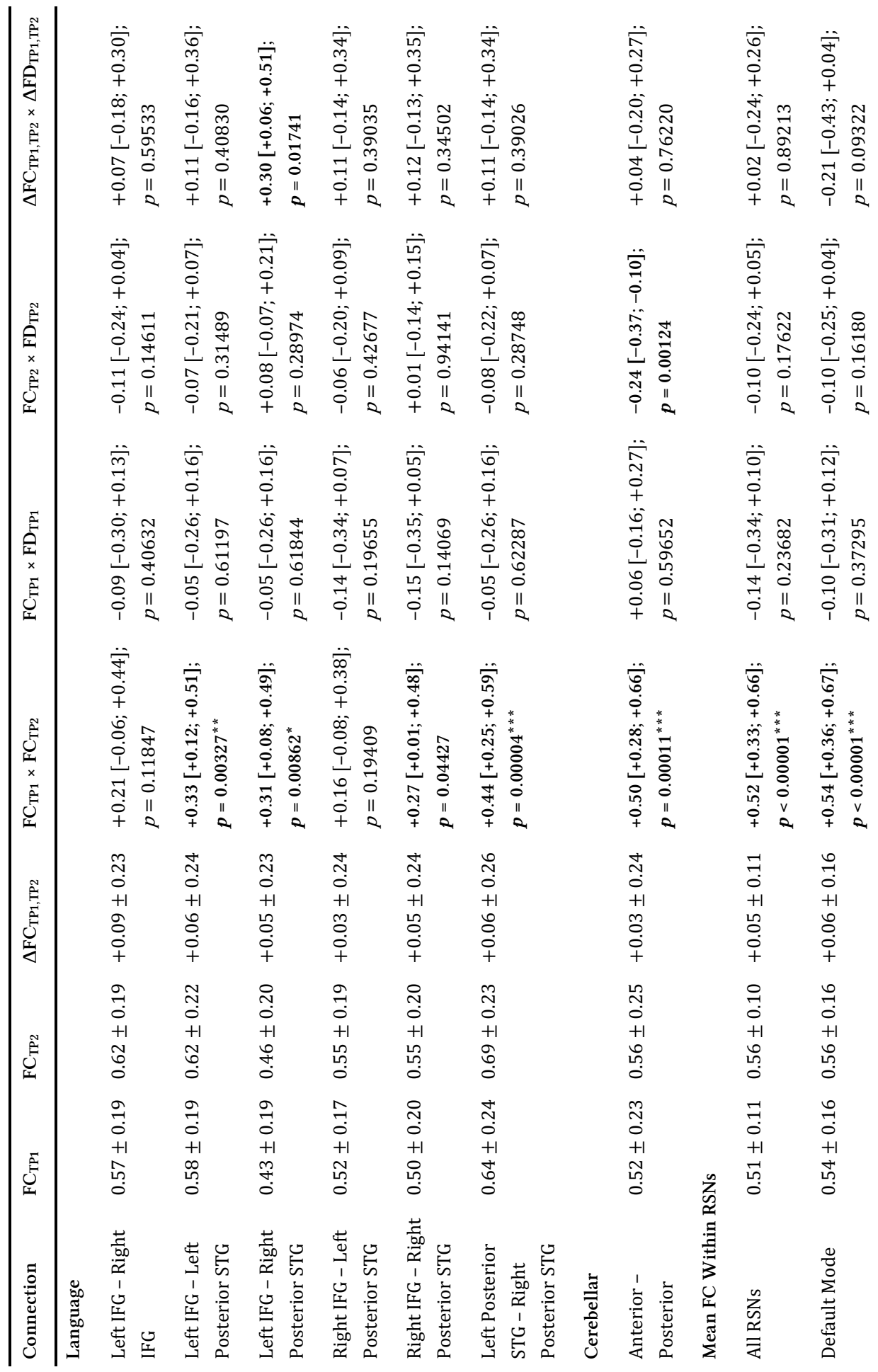




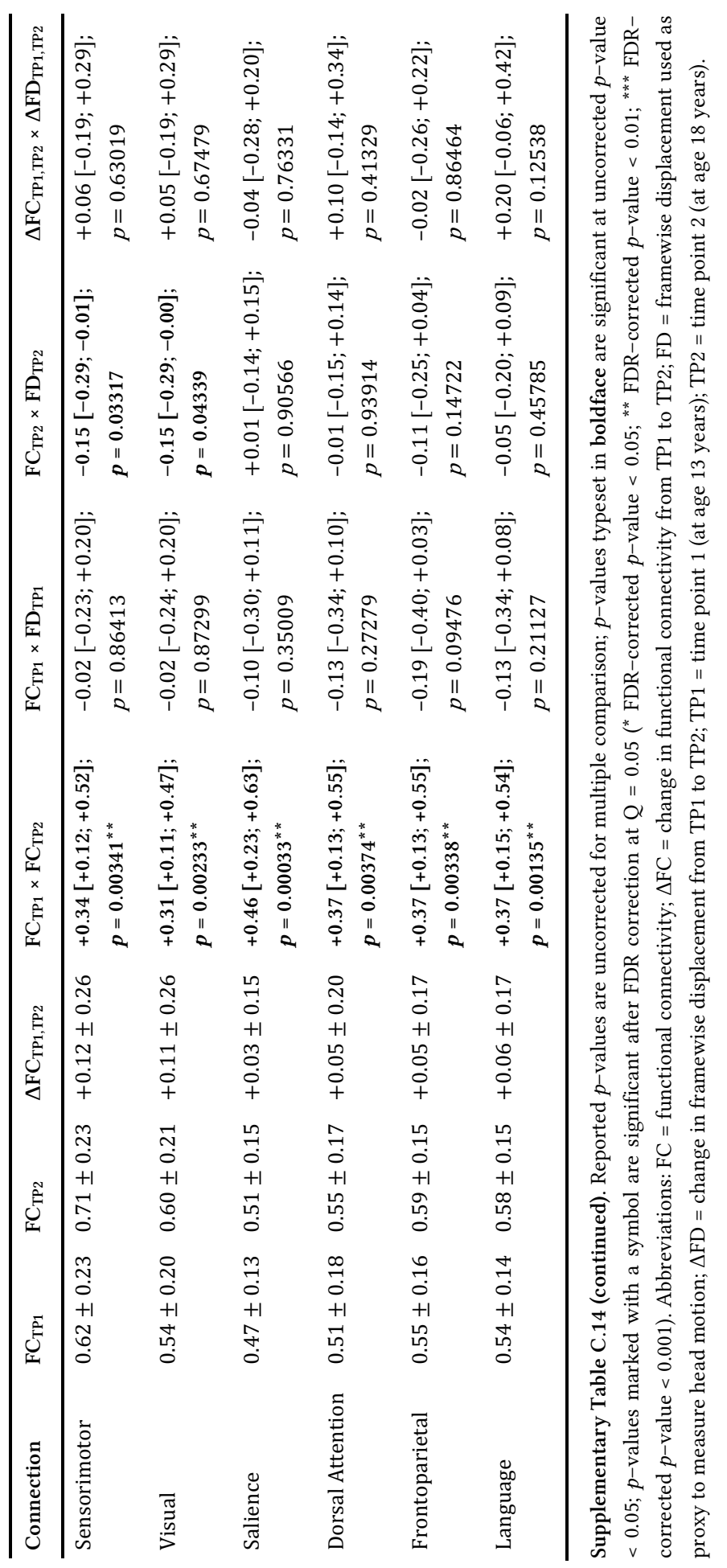



APPENDIX D

\section{Supplementary information to chapter 5}




\section{APPENDIX D.1 SUPPLEMENTARY INFORMATION FROM CHAPTER 5}

\section{D.1.1 Correcting for regression toward the mean, and platform and sex effects}

Regression toward the mean occurs when a statistical model trained on a trait of a set of subjects for which the outcome is dependent on a large number of factors, is applied to an independent set of subjects. The extremes of the distribution, in this case the youngest and oldest participants in the study, will have scores (i.e. predicted age) that congregate toward the mean of the original study population (Cutter, 1976), and needs to be corrected before age gaps are computed by including age as covariate to avoid potential misleading findings (Khoury et al., 2018; Le et al., 2018).

To correct for regression toward the mean, a linear mixed-effects regression model with random intercept is fitted to the healthy controls subpopulation to re-establish the baseline of typical aging in the current sample. The predicted age is used as the dependent variable, and the true chronological age is used as the independent variable. Predicted ages $\left(\widehat{a g} e_{\text {pred,i }}\right)$ for all subjects, including the patient subpopulation, are then projected to their corrected estimates $\left(\right.$ age $\left._{\text {pred }, i}\right)$ using the slope $\left(\beta_{1}\right)$ and intercept $\left(\beta_{0}\right)$ from the fit of the linear regression model

$$
\operatorname{age}_{\text {pred }, i}=\left(\widehat{\operatorname{ag} e_{\text {pred }, i}}-\beta_{0}\right) / \beta_{1} \text {. }
$$

For the epigenetic ages, an additional beta coefficient for the microarray platform was included in the model for the epigenetic ages to account for possible differences between the two Illumina platforms used to acquire the methylation data. After corrections, healthy controls are centered around mean error of zero with an average aging rate of 1.0 predicted year per chronological year (Supplementary Figure D.1; Supplementary Table D.1).
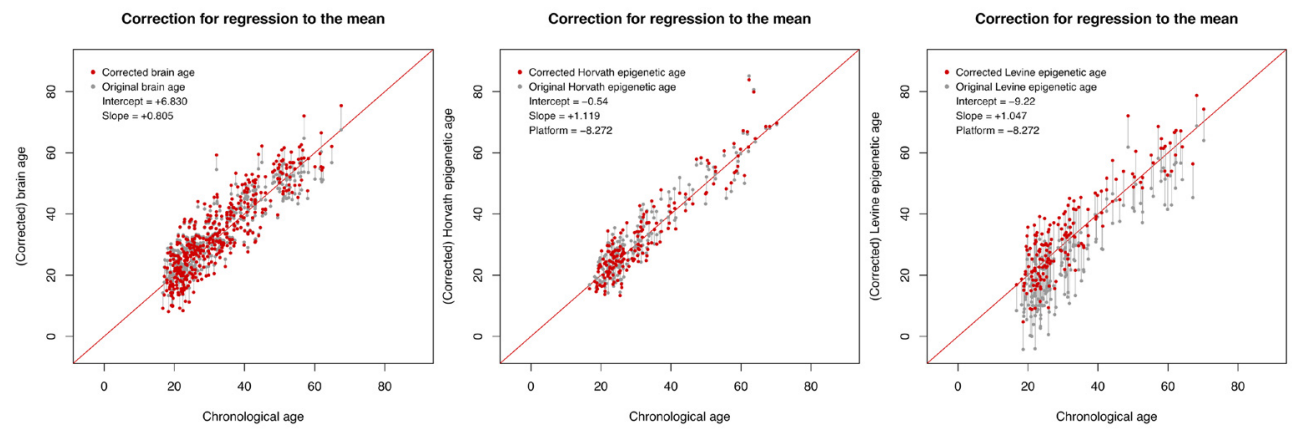

Supplementary Figure D.1. Projections of the predicted MRI brain and epigenetic age estimates to their corrected age estimates after correction for regression toward the mean and platform effects. 
Supplementary Table D.1. Regression coefficients used to correct the predicted MRI brain and epigenetic ages from regression toward the mean.

\begin{tabular}{llll}
\hline & MRI brain age & Horvath epigenetic age & Levine epigenetic age \\
\hline Healthy controls $(N)$ & 218 & 63 & 63 \\
Samples $(N)$ & 345 & 63 & 63 \\
Illumina platform & N/A & $51: 12$ & $51: 12$ \\
ratio $(27 K: 450 K)$ & & & +1.119 \\
Slope $($ age effect $)$ & +0.805 & -0.544 & -9.224 \\
Intercept & +6.830 & $-8.272(p=0.0116)$ & $-4.399(p=0.3968)$ \\
Platform effect ${ }^{\mathrm{a}}$ & N/A & &
\end{tabular}

Estimates were based on the healthy subpopulation. a Negative platform effect indicates predicted ages were lower on Illumina's $27 \mathrm{~K}$ platform compared to predicted ages on Illumina's $450 \mathrm{~K}$ platform.

The age gap (also referred to in literature as "age acceleration") of the subjects is then defined as the difference between their corrected age prediction and chronological age. For MRI brain age, longitudinal age acceleration (or "age gap acceleration") is defined as the annual rate of change in [corrected] predicted brain ages between two consecutive MRI scans; i.e. values greater than 1.0 suggest the brain ages faster than typical in healthy controls, and values less than 1.0 suggest the brain ages slower than typical in healthy controls. The same approach as before is used to correct for possible differences in the age gap and the age acceleration between sexes: a linear mixed-effects regression model with random intercept is fitted to the healthy controls subpopulation with the age gap or age acceleration as the dependent variable and sex of the subjects as the independent variable (Supplementary Table D.2; Supplementary Figure D.2), followed by the removal of the sex effect from all subjects to a gender-neutral level. For age acceleration, the distribution is centered to mean 1.0 such that the unit at predicted year per chronological year is retained.

\section{D.1.2 Epigenetic aging markers}

Blood-based DNAm age was estimated using two different predictors (Horvath, 2013; Levine et al., 2018). These two DNAm age predictors were designed for use with both the $27 \mathrm{~K}$ and $450 \mathrm{~K}$ platform allowing us to maximize our sample size. Other blood-based age predictors, like the Hannum clock (Hannum et al., 2013), were not analyzed as these are not applicable to the older $27 \mathrm{~K}$ array. 
Supplementary Table D.2. Regression coefficients used to correct the corrected MRI brain and epigenetic age gaps from possible sex effects.

\begin{tabular}{|c|c|c|c|c|}
\hline & $\begin{array}{l}\text { MRI brain } \\
\text { age gap }\end{array}$ & $\begin{array}{c}\text { MRI brain } \\
\text { age acceleration }\end{array}$ & $\begin{array}{c}\text { Horvath epigenetic } \\
\text { age gap }\end{array}$ & $\begin{array}{c}\text { Levine epigenetic } \\
\text { age gap }\end{array}$ \\
\hline $\begin{array}{l}\text { Healthy } \\
\text { controls }(N)\end{array}$ & 218 & 109 & 63 & 63 \\
\hline Samples $(N)$ & 345 & 127 & 63 & 63 \\
\hline $\begin{array}{l}\text { Sex ratio } \\
(\text { female : male })\end{array}$ & $115: 103$ & $50: 77$ & $37: 26$ & $37: 26$ \\
\hline Intercept & -0.035 & +0.958 & +0.325 & -0.040 \\
\hline Sex effect ${ }^{a}$ & $\begin{array}{c}-1.523 \\
(p=0.0618)\end{array}$ & $\begin{array}{c}+0.278 \\
(p=0.2452)\end{array}$ & $\begin{array}{c}+3.721 \\
(p=0.0001)\end{array}$ & $\begin{array}{c}-0.456 \\
(p=0.7999)\end{array}$ \\
\hline
\end{tabular}

Estimates were based on the healthy subpopulation. a A positive effect of brain age gap indicates that males' brain age is estimated older than those of females with respect to their true age. A positive brain age acceleration means faster acceleration in males.
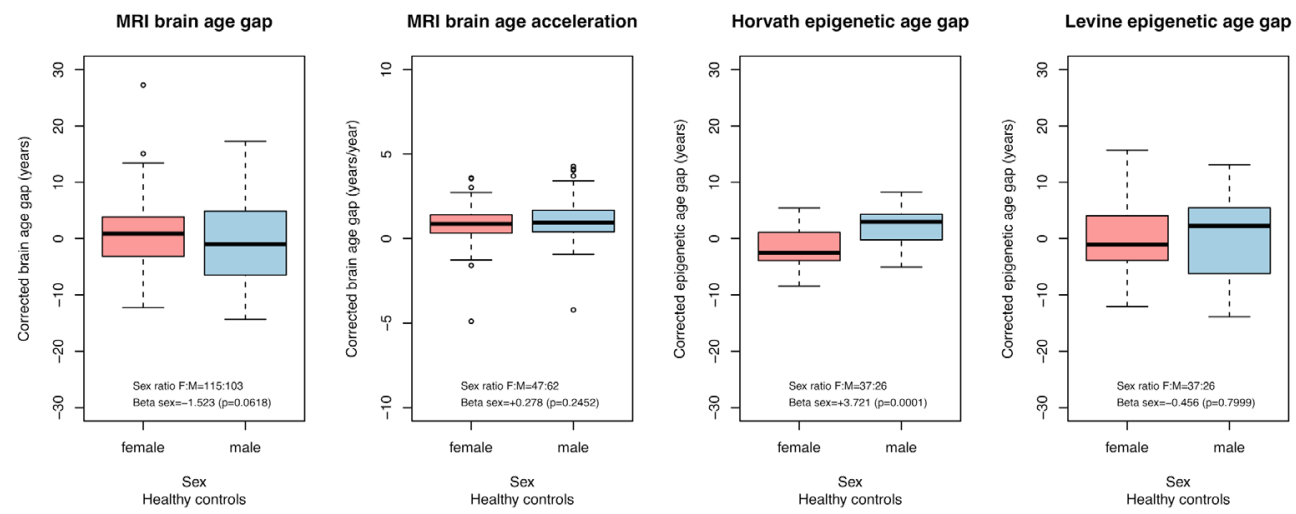

Supplementary Figure D.2. Sex effects on MRI brain and epigenetic age gaps and MRI brain age acceleration within the healthy controls subpopulation.

DNA methylation data was obtained from whole-blood DNA samples using the Illumina Infinium Human Methylation Beadchip technology according to manufacturer's guidelines. A total of 172 samples were assayed with either the $27 \mathrm{~K}$ ( $\mathrm{n}=108$ samples) or 450K (N=64 samples) platform, which interrogate 27,578 and 485,512 CpG sites across the genome, respectively. These data are a subset of previously published DNAm cohorts (Gene Expression Omnibus (GEO) ID: GSE41037 and GSE41169) for which brain age estimates 
from MRI scans were available. For all samples, IDAT files and thus raw fluorescence intensity values were available and read into an RGChannelSetExtended object in the $\mathrm{R}$ programming environment (R Core Team, 2017) using the read.metharray function in the minfi package (v1.20.2) (Aryee et al., 2014) for analysis.

We first applied data processing and normalization strategies separately for each platform. For $27 \mathrm{~K}$ arrays, normal-exponential out-of-band (noob) correction was applied using the preprocessNoob function in minfi (Aryee et al., 2014). For 450K arrays, background and dyebias correction were applied using the preprocessENmix function in the ENmix package (Xu et al., 2016). Probe design type correction was then applied by Regression on Correlated Probes (RCP) through the $r c p$ function of the ENmix framework and processed beta values generated for downstream analyses. Levine phenotypic age was then estimated based on the weighted sum of methylation beta values of 511 probes. Levine phenotypic age is a compound index score based on chronological age and nine other phenotypic biomarkers characteristic for aging (Levine et al., 2018). Horvath's epigenetic age predictor (Horvath, 2013) was applied to the raw methylation data and uses a custom internal BMIQ normalization procedure to normalize the methylation beta values of each sample to a 'golden standard' and predict the chronological age of the sample based on the weighted sum of methylation beta values of 353 probes.

\section{D.1.3 Computation and harmonization of polygenic risk scores}

Whole-blood DNA samples were processed on Illumina's HumanOmniExpressExome-8 v1.2 and Illumina's 550K platform. Quality control procedures were performed using PLINK v1.9 (Purcell et al., 2007). SNPs and samples with call rates below 95\% and 98\%, respectively, were removed. Sex errors, heterozygosity $(\mathrm{F}<3$ standard deviation (SD)), homozygosity ( $\mathrm{F}>3 \mathrm{SD}$ ) and relatedness by pairwise identity by descent (IBD) values were assessed from a subset of SNPs that satisfied a strict SNP QC (minor allele frequency (MAF) threshold $>0.10$, Hardy-Weinberg equilibrium (HWE) $p>1 \mathrm{e}-05$, linkage disequilibrium (LD) based SNP pruning $\left(\mathrm{R}^{2}<0.2\right)$ ). After removing failing samples, a regular SNP QC was performed (SNP call rate $>0.98$, HWE $p>1 \mathrm{e}-06, \mathrm{MAF}>0.01$ ). Ethnicity was assessed by MDS clustering with Hapmap Phase 3 and samples that deviated more than 3 SD were removed. Strand ambiguous SNPs and duplicate SNPs were removed. Mendelian errors were set to missing followed by another missingness check ( 0.02 threshold) for samples and SNPs, and SNPs with a differential missingness between cases and controls were removed.

After quality control, SNPs were imputed on the Michigan server (Das et al., 2016) using the HRC r1.1 2016 reference panel with European samples after phasing with Eagle v2.3. Post-imputation QC steps included: removing SNPs with INFO score $<0.8, \mathrm{MAF}<0.01$, SNPs that had a discordant MAF compared to the reference panel, strand ambiguous and multi-allelic SNPs. 
Overlapping SNPs between the schizophrenia GWAS summary statistics, 1000 reference Genome, and dataset of interest were selected. The following SNPs were excluded: i) insertion, deletion or ambiguous SNPs, 2) MAF $<0.01$ and SNPs with imputation quality $\left.\left(\mathrm{R}^{2}\right)<0.8,3\right)$ SNPs located in complex-LD regions (Price et al., 2008). The remaining SNPs were clumped in two rounds using PLINK: round 1 (physical distance threshold 250kb, LD threshold $\mathrm{R}^{2}<0.5$ ), round 2 (physical distance threshold 5,000kb, LD threshold $\mathrm{R}^{2}<0.2$ ). The resulting SNPs were used for polygenic risk score calculation. Odds ratios in the schizophrenia summary statistics were log-converted to beta values.

To reduce possible effects of cross-platform variation, all polygenic risk scores were harmonized using a published method to reduce between-cohort variation (Bergen et al., 2019). The procedure involves normalizing the polygenic risk scores for all subjects, including schizophrenia patients, based on the mean within the healthy controls subpopulation for each platform and threshold independently and then re-scaling the data back to the means and variances of the original distribution. Since polygenic risk scores at different thresholds are correlated, a principal component analysis is applied to the normalized polygenic risk scores of the combined datasets across all thresholds to obtain a single component that contains the majority of the disease-differential variance. The first principal component (PC1) explains $69.9 \%$ of the variance in polygenic risk scores across the thresholds and its factor loadings show a strong disease differentiating power at an odds ratio of 2.50 for schizophrenia (Supplementary Table D.3) that is consistent with previously reported results (Bergen et al., 2019).

The factor loadings of the PC1 were Z-standardized based on the distribution of the healthy controls subpopulation, such that the mean score of the healthy subpopulation is zero, and that a positive score represents an increased risk for schizophrenia compared to the norm within healthy controls. 
Supplementary Table D.3. Principal component analysis on the harmonized polygenic risk scores for schizophrenia.

\begin{tabular}{lcc}
$\begin{array}{l}\text { Principal } \\
\text { component }\end{array}$ & $\begin{array}{c}\text { Percentage variance } \\
\text { explained }\end{array}$ & $\begin{array}{c}\text { Odds ratio for } \\
\text { schizophrenia }\end{array}$ \\
\hline PC1 & $69.9 \%$ & 2.50 \\
PC2 & $17.6 \%$ & 1.05 \\
PC3 & $5.8 \%$ & 1.04 \\
PC4 & $2.5 \%$ & 1.00 \\
PC5 & $1.8 \%$ & 1.02 \\
PC6 & $1.3 \%$ & 1.02 \\
PC7 & $0.6 \%$ & 1.02 \\
PC8 & $0.3 \%$ & 1.02 \\
PC9 & $0.2 \%$ & 1.03 \\
PC10 & $0.0 \%$ & 1.05
\end{tabular}



REFERENCES 
Abreu, A., Kaiser, U. (2016). Pubertal development and regulation. The Lancet Diabetes \& Endocrinology 4(3), $254-264$. doi:10.1016/s2213-8587(15)00418-0

Abrol, A., Damaraju, E., Miller, R., Stephen, J., Claus, E., Mayer, A., Calhoun, V. (2017). Replicability of time-varying connectivity patterns in large resting state fMRI samples. NeuroImage 163, $160-176$. doi:10.1016/j.neuroimage.2017.09.020

Achterberg, M., Bakermans-Kranenburg, M., Ijzendoorn, M., Meulen, M., Tottenham, N., Crone, E. (2018). Distinctive heritability patterns of subcortical-prefrontal cortex resting state connectivity in childhood: A twin study. NeuroImage 175, 138-149. doi:10.1016/j.neuroimage.2018.03.076

Adhikari, B., Jahanshad, N., Shukla, D., Glahn, D., Blangero, J., Fox, P., Reynolds, R., Cox, R., Fieremans, E., Veraart, J., Novikov, D., Nichols, T., Hong, L., Thompson, P., Kochunov, P. (2018). Comparison of heritability estimates on resting state fMRI connectivity phenotypes using the ENIGMA analysis pipeline. Human Brain Mapping 39(12), 4893-4902. doi:10.1002/hbm.24331

Adhikari, B.M., Jahanshad, N., Shukla, D., Glahn, D.C., Blangero, J., Reynolds, R.C., Cox, R.W., Fieremans, E., Veraart, J., Novikov, D.S., Nichols, T.E., Hong, L.E., Thompson, P.M., Kochunov, P.V. (2018). Heritability estimates on resting state fMRI data using ENIGMA analysis pipeline. Pac Symp Biocomput 23, 307-318.

Akbarian S, Liu C, Knowles JA, Vaccarino FM, Farnham PJ, Crawford GE, Jaffe AE, Pinto D, Dracheva S, Geschwind DH, Mill J, Nairn AC, Abyzov A, Pochareddy S, Prabhakar S, Weissman S, Sullivan PF, State MW, Weng Z, Peters MA, White KP, Gerstein MB, Amiri A, Armoskus C, Ashley-Koch AE, Bae T, Beckel-Mitchener A, Berman BP, Coetzee GA, Coppola G, Francoeur N, Fromer M, Gao R, Grennan K, Herstein J, Kavanagh DH, Ivanov NA, Jiang Y, Kitchen RR, Kozlenkov A, Kundakovic M, Li M, Li Z, Liu S, Mangravite LM, Mattei E, Markenscoff-Papadimitriou E, Navarro FCP, North N, Omberg L, Panchision D, Parikshak N, Poschmann J, Price AJ, Purcaro M, Reddy TE, Roussos P, Schreiner S, Scuderi S, Sebra R, Shibata M, Shieh AW, Skarica M, Sun W, Swarup V, Thomas A, Tsuji J, van Bakel H, Wang D, Wang Y, Wang K, Werling DM, Willsey AJ, Witt H, Won H, Wong CCY, Wray GA, Wu EY, Xu X, Yao L, Senthil G, Lehner T, Sklar P, Sestan N. (2015). The PsychENCODE project. Nat Hum Behav. 18:1707-1712.

Alexander-Bloch AF, Giedd JN, Bullmore ET. (2013). Imaging structural co-variance between human brain regions. Nat Rev Neurosci. 14:322-336.

Alnæs D, Kaufmann T, van der Meer D, Córdova-Palomera A, Rokicki J, Moberget T, Bettella F, Agartz I, Barch DM, Bertolino A, Brandt CL, Cervenka S, Djurovic S, Doan NT, Eisenacher S, Fatouros-Bergman H, Flyckt L, Di Giorgio A, Haatveit B, Jönsson EG, KaSP Consortium, Kirsch P, Lund MJ, Meyer-Lindenberg A, Pergola G, Schwarz E, Smeland OB, Quarto T, Zink M, Andreassen OA, Westlye LT. (2019). The dark side of the mean: brain structural heterogeneity in schizophrenia and its polygenic risk. bioRxiv. 8:12.

Alzheimer's Disease Neuroimaging Initiative, CHARGE Consortium, EPIGEN Consortium, IMAGEN Consortium, SYS Consortium, Hibar, D., Stein, J., Renteria, M., Arias-Vasquez, A., Desrivières, S., Jahanshad, N., Toro, R., Wittfeld, K., Abramovic, L., Andersson, M., Aribisala, B., Armstrong, N., Bernard, M., Bohlken, M., Boks, M., Bralten, J., Brown, A., Chakravarty, M., Chen, Q., Ching, C., Cuellar-Partida, G., den Braber, A., Giddaluru, S., Goldman, A., Grimm, O., Guadalupe, T., Hass, J., Woldehawariat, G., Holmes, A., Hoogman, M., Janowitz, D., Jia, T., Kim, S., Klein, M., Kraemer, B., Lee, P., Loohuis, L., Luciano, M., Macare, C., Mather, K., Mattheisen, M., Milaneschi, Y., Nho, K., Papmeyer, M., Ramasamy, A., Risacher, S., Roiz-Santiañez, R., Rose, E., Salami, A., Sämann, P., Schmaal, L., Schork, A., Shin, J., Strike, L., Teumer, A., Donkelaar, M., Eijk, K., Walters, R., Westlye, L., Whelan, C., Winkler, A., Zwiers, M., Alhusaini, S., Athanasiu, L., Ehrlich, S., Hakobjan, M., Hartberg, C., Haukvik, U., Heister, A., Hoehn, D., Kasperaviciute, D., Liewald, D., Lopez, L., Makkinje, R., Matarin, M., Naber, M., McKay, D., Needham, M., Nugent, A., Pütz, B., Royle, N., Shen, L., Sprooten, E., Trabzuni, D., Marel, S., Hulzen, K., Walton, E., Wolf, C., Almasy, L., Ames, D., Arepalli, S., Assareh, A., Bastin, M., Brodaty, H., Bulayeva, K., Carless, M., Cichon, S., Corvin, A., Curran, J., Czisch, M., Zubicaray, G., Dillman, A., Duggirala, R., Dyer, T., Erk, S., Fedko, I., Ferrucci, L., Foroud, T., Fox, P., Fukunaga, M., Gibbs, J., Göring, H., Green, R., Guelfi, S., Hansell, N., Hartman, C., Hegenscheid, K., Heinz, A., Hernandez, D., Heslenfeld, D., Hoekstra, P., Holsboer, F., Homuth, G., Hottenga, J., Ikeda, M., Jack, C., Jenkinson, M., Johnson, R., Kanai, R., Keil, M., Kent, J., 
Kochunov, P., Kwok, J., Lawrie, S., Liu, X., Longo, D., McMahon, K., Meisenzahl, E., Melle, I., Mohnke, S., Montgomery, G., Mostert, J., Mühleisen, T., Nalls, M., Nichols, T., Nilsson, L., Nöthen, M., Ohi, K., Olvera, R., Perez-Iglesias, R., Pike, G., Potkin, S., Reinvang, I., Reppermund, S., Rietschel, M., Romanczuk-Seiferth, N., Rosen, G., Rujescu, D., Schnell, K., Schofield, P., Smith, C., Steen, V., Sussmann, J., Thalamuthu, A., Toga, A., Traynor, B., Troncoso, J., Turner, J., Hernández, M., Ent, D., Brug, M., Wee, N., Tol, M., Veltman, D., Wassink, T., Westman, E., Zielke, R., Zonderman, A., Ashbrook, D., Hager, R., Lu, L., McMahon, F., Morris, D., Williams, R., Brunner, H., Buckner, R., Buitelaar, J., Cahn, W., Calhoun, V., Cavalleri, G., Crespo-Facorro, B., Dale, A., Davies, G., Delanty, N., Depondt, C., Djurovic, S., Drevets, W., Espeseth, T., Gollub, R., Ho, B., Hoffmann, W., Hosten, N., Kahn, R., Hellard, S., Meyer-Lindenberg, A., Müller-Myhsok, B., Nauck, M., Nyberg, L., Pandolfo, M., Penninx, B., Roffman, J., Sisodiya, S., Smoller, J., Bokhoven, H., van Haren, N., Völzke, H., Walter, H., Weiner, M., Wen, W., White, T., Agartz, I., Andreassen, O., Blangero, J., Boomsma, D., Brouwer, R., Cannon, D., Cookson, M., Geus, E., Deary, I., Donohoe, G., Fernández, G., Fisher, S., Francks, C., Glahn, D., Grabe, H., Gruber, O., Hardy, J., Hashimoto, R., Hulshoff Pol, H.E., Jönsson, E., Kloszewska, I., Lovestone, S., Mattay, V., Mecocci, P., McDonald, C., McIntosh, A., Ophoff, R., Paus, T., Pausova, Z., Ryten, M., Sachdev, P., Saykin, A., Simmons, A., Singleton, A., Soininen, H., Wardlaw, J., Weale, M., Weinberger, D., Adams, H., Launer, L., Seiler, S., Schmidt, R., Chauhan, G., Satizabal, C., Becker, J., Yanek, L., Lee, S., Ebling, M., Fischl, B., Longstreth, W., Greve, D., Schmidt, H., Nyquist, P., Vinke, L., Duijn, C., Xue, L., Mazoyer, B., Bis, J., Gudnason, V., Seshadri, S., Ikram, M., Martin, N., Wright, M., Schumann, G., Franke, B., Thompson, P., Medland, S. (2015). Common genetic variants influence human subcortical brain structures. Nature 520(7546), 224-229. doi:10.1038/nature14101

Andrews-Hanna, J. (2012). The Brain's Default Network and Its Adaptive Role in Internal Mentation. The Neuroscientist 18(3), 251-270. doi:10.1177/1073858411403316

Anticevic, A., Hu, X., Xiao, Y., Hu, J., Li, F., Bi, F., Cole, M., Savic, A., Yang, G., Repovs, G., Murray, J., Wang, X., Huang, X., Lui, S., Krystal, J., Gong, Q. (2015). Early-Course Unmedicated Schizophrenia Patients Exhibit Elevated Prefrontal Connectivity Associated with Longitudinal Change The Journal of Neuroscience 35(1), 267-286. doi:10.1523/jneurosci.2310-14.2015

Arion D, Corradi JP, Tang S, Datta D, Boothe F, He A, Cacace AM, Zaczek R, Albright CF, Tseng G, Lewis DA. (2015). Distinctive transcriptome alterations of prefrontal pyramidal neurons in schizophrenia and schizoaffective disorder. Mol Psychiatry. 20:1397-1405.

Aryee, M., Jaffe, A., Corrada-Bravo, H., Ladd-Acosta, C., Feinberg, A., Hansen, K., Irizarry, R. (2014). Minfi: a flexible and comprehensive Bioconductor package for the analysis of Infinium DNA methylation microarrays. Bioinformatics 30(10), 1363-1369.

Baaré, W., Oel, C., Hulshoff Pol, H.E., Schnack, H., Durston, S., Sitskoorn, M., Kahn, R. (2001). Volumes of Brain Structures in Twins Discordant for Schizophrenia. Archives of General Psychiatry 58(1), 33. doi:10.1001/archpsyc.58.1.33

Bakulski KM, Halladay A, Hu VW, Mill J, Fallin MD. (2016). Epigenetic Research in Neuropsychiatric Disorders: the "Tissue Issue". Curr Behav Neurosci Rep. 3:264-274.

Bandettini, P. (2020). fMRI, 1st edition. MIT Press

Barbey, A. (2018). Network Neuroscience Theory of Human Intelligence. Trends in Cognitive Sciences 22(1), 8-20. doi:10.1016/j.tics.2017.10.001

Barkhuizen, W., Pain, O., Dudbridge, F., Ronald, A. (2020). Genetic overlap between psychotic experiences in the community across age and with psychiatric disorders. Translational Psychiatry 10(1), 86. doi:10.1038/s41398-020-0765-2

Bartels, M., Rietveld, M., Baal, G., Boomsma, D. (2002). Genetic and Environmental Influences on the Development of Intelligence. Behavior Genetics 32(4), 237-249. doi:10.1023/a:1019772628912

Basser, P., Pajevic, S., Pierpaoli, C., Duda, J., Aldroubi, A. (2000). In vivo fiber tractography using DT-MRI data. Magnetic Resonance in Medicine 44(4), 625-632. doi:10.1002/1522-2594(200010)44:4<625::aid-mrm17>3.0.co;2-o

Basser, P., Pierpaoli, C. (1996). Microstructural and Physiological Features of Tissues Elucidated by Quantitative-DiffusionTensor MRI. Journal of Magnetic Resonance, Series B 111(3), 209-219. doi:10.1006/jmrb.1996.0086 
Basten, U., Hilger, K., Fiebach, C. (2015). Where smart brains are different: A quantitative meta-analysis of functional and structural brain imaging studies on intelligence. Intelligence 51, 10-27. doi:10.1016/j.intell.2015.04.009

Bayer TA, Falkai P, Maier W. (1999). Genetic and non-genetic vulnerability factors in schizophrenia: The basis of the "Two hit hypothesis.”. Journal of Psychiatric Research. 33:543-548.

Beauchaine, T., Constantino, J. (2017). Redefining the endophenotype concept to accommodate transdiagnostic vulnerabilities and etiological complexity. Biomarkers in Medicine 11(9), 769-780. doi:10.2217/bmm-2017-0002

Beaulieu, C. (2002). The basis of anisotropic water diffusion in the nervous system - a technical review. NMR in Biomedicine 15(7-8), 435-455. doi:10.1002/nbm.782

Beck, S., Rakyan, V. (2008). The methylome: approaches for global DNA methylation profiling. Trends in Genetics 24(5), 231-237. doi:10.1016/j.tig.2008.01.006

Beckmann, C., DeLuca, M., Devlin, J., Smith, S. (2005). Investigations into resting-state connectivity using independent component analysis. Philosophical Transactions of the Royal Society B: Biological Sciences 360(1457), 1001-1013. doi:10.1098/rstb.2005.1634

Beckmann, C., Smith, S. (2004). Probabilistic Independent Component Analysis for Functional Magnetic Resonance Imaging. IEEE Transactions on Medical Imaging 23(2), 137-152. doi:10.1109/tmi.2003.822821

Behzadi, Y., Restom, K., Liau, J., Liu, T.T., (2007). A component based noise correction method (CompCor) for BOLD and perfusion based fMRI. NeuroImage 37, 90-101. doi:10.1016/j.neuroimage.2007.04.042

Belsky DW, Moffitt TE, Cohen AA, Corcoran DL, Levine ME, Prinz JA, Schaefer J, Sugden K, Williams B, Poulton R, Caspi A. (2018). Eleven Telomere, Epigenetic Clock, and Biomarker-Composite Quantifications of Biological Aging: Do They Measure the Same Thing? Am J Epidemiol. 187:1220-1230.

Bentler, P., Chou, C. (1987). Practical Issues in Structural Modeling. Sociological Methods \& Research 16(1), 78-117. doi:10.1177/0049124187016001004

Bergen, S., Ploner, A., Howrigan, D., Psychiatric Genomics Consortium, O’Donovan, M., Smoller, J., Sullivan, P., Sebat, J., Neale, B., Kendler, K. (2019). Joint Contributions of Rare Copy Number Variants and Common SNPs to Risk for Schizophrenia. American Journal of Psychiatry 176(1), 29-35.

Bernard, J.A., Orr, J.M., Mittal, V.A., (2016). Differential motor and prefrontal cerebello-cortical network development: Evidence from multimodal neuroimaging. NeuroImage 124, 591-601. doi:10.1016/j.neuroimage.2015.09.022

Besteher B, Gaser C, Nenadić I. (2019). Machine-learning based brain age estimation in major depression showing no evidence of accelerated aging. Psychiatry Research: Neuroimaging. 290, 1-4.

Bianciardi, M., Fukunaga, M., Gelderen, P., Horovitz, S., Zwart, J., Shmueli, K., Duyn, J. (2009). Sources of functional magnetic resonance imaging signal fluctuations in the human brain at rest: a 7 T study. Magnetic Resonance Imaging 27(8), 1019-1029. doi:10.1016/j.mri.2009.02.004

Bijsterbosch, J., Smith, S., Beckmann, C. (2017). Introduction to Resting State fMRI Functional Connectivity, 1st edition. Oxford University Press

Binder AM, Corvalan C, Mericq V, Pereira A, Santos JL, Horvath S, Shepherd J, Michels KB. (2018). Faster ticking rate of the epigenetic clock is associated with faster pubertal development in girls. Epigenetics. 13:85-94.

Birn, R., Cornejo, M., Molloy, E., Patriat, R., Meier, T., Kirk, G., Nair, V., Meyerand, M., Prabhakaran, V. (2014). The Influence of Physiological Noise Correction on Test-Retest Reliability of Resting-State Functional Connectivity. Brain Connectivity 4(7), 511-522. doi:10.1089/brain.2014.0284

Birn, R., Molloy, E., Patriat, R., Parker, T., Meier, T., Kirk, G., Nair, V., Meyerand, M., Prabhakaran, V. (2013). The effect of scan length on the reliability of resting-state fMRI connectivity estimates. NeuroImage 83, 550-558. doi:10.1016/j.neuroimage.2013.05.099

Biswal, B., Yetkin, F., Haughton, V., Hyde, J. (1995). Functional connectivity in the motor cortex of resting human brain using echo-planar MRI. Magnetic Resonance in Medicine 34(4), 537-541. doi:10.1002/mrm.1910340409

Biswal, B.B., Mennes, M., Zuo, X.-N., Gohel, S., Kelly, A.M.C., Smith, S.M., Beckmann, C.F., Adelstein, J.S., Buckner, R.L., Colcombe, S., Dogonowski, A.-M., Ernst, M., Fair, D.A., Hampson, M., Hoptman, M.J., Hyde, J.S., Kiviniemi, V.J., Kötter, 
R., Li, S.-J., Lin, C.-P., Lowe, M.J., Mackay, C.E., Madden, D.J., Madsen, K.H., Margulies, D.S., Mayberg, H.S., McMahon, K.L., Monk, C.S., Mostofsky, S.H., Nagel, B.J., Pekar, J.J., Peltier, S.J., Petersen, S.E., Riedl, V., Rombouts, S.A.R.B., Rypma, B., Schlaggar, B.L., Schmidt, S., Seidler, R.D., Siegle, G.J., Sorg, C., Teng, G.-J., Veijola, J., Villringer, A., Walter, M., Wang, L., Weng, X.-C., Whitfield-Gabrieli, S., Williamson, P., Windischberger, C., Zang, Y.-F., Zhang, H.-Y., Castellanos, F.X., Milham, M.P. (2010). Toward discovery science of human brain function. Proc. Natl. Acad. Sci. U.S.A. 107, 4734-4739. doi:10.1073/pnas.0911855107

Biswal, B.B., Van Kylen, J., Hyde, J.S. (1997). Simultaneous assessment of flow and BOLD signals in resting-state functional connectivity maps. NMR Biomed 10, 165-170.

Blakemore, S. (2012). Imaging brain development: The adolescent brain. NeuroImage 61(2), 397-406. doi:10.1016/j.neuroimage.2011.11.080

Blokland, G.A.M., de Zubicaray, G.I., McMahon, K.L., Wright, M.J. (2012). Genetic and environmental influences on neuroimaging phenotypes: a meta-analytical perspective on twin imaging studies. Twin Res Hum Genet 15, 351-371. doi:10.1017/thg.2012.11

Blokland GAM, de Zubicaray GI, McMahon KL, Wright MJ. (2012). Genetic and environmental influences on neuroimaging phenotypes: a meta-analytical perspective on twin imaging studies. Twin Res Hum Genet. 15:351-371.

Boardman JD, Blalock CL, Pampel FC. (2010). Trends in the genetic influences on smoking. J Health Soc Behav. 51:108-123.

Bocklandt S, Lin W, Sehl ME, Sánchez FJ, Sinsheimer JS, Horvath S, Vilain E. (2011). Epigenetic predictor of age. PLoS ONE. 6:e14821.

Boekel, W., Wagenmakers, E., Belay, L., Verhagen, J., Brown, S., Forstmann, B. (2015). A purely confirmatory replication study of structural brain-behavior correlations. Cortex 66, 115-133. doi:10.1016/j.cortex.2014.11.019

Bohlken, M., Brouwer, R., Mandl, R., Kahn, R., Hulshoff Pol, H.E. (2016). Genetic Variation in Schizophrenia Liability is Shared With Intellectual Ability and Brain Structure. Schizophrenia Bulletin 42(5), 1167-1175. doi:10.1093/schbul/sbw034

Boker, S., Neale, M., Maes, H., Wilde, M., Spiegel, M., Brick, T., Estabrook, R., Bates, T., Mehta, P., Oertzen, T., Gore, R., Hunter, M., Hackett, D., Karch, J., Brandmaier, A., Pritikin, J., Zahery, M., Kirkpatrick, R., Wang, Y., Driver, C., Massachusetts Institute of Technology, Johnson, S., Machinery, A., Kraft, D., Wilhelm, S., Medland, S., Falk, C., Keller, M., Manjunath, B., The Regents of the University of California, Ingber, L., Voon, W. (2018). OpenMx version 2.11.5. https://openmx.ssri.psu.edu

Boker SM, Neale MC, Maes HH, Wilde MJ, Spiegel M, Brick TR, Estabrook R, Bates TC, Mehta P, Oertzen von T, Gore RJ, Hunter MD, Hackett DC, Karch J, Brandmaier AM, Pritikin JN, Zahery M, Kirkpatrick RM, Wang Y, Driver C. (2015). OpenMX 2.2.6 User Guide.

Boomsma, A. (1985). Nonconvergence, improper solutions, and starting values in lisrel maximum likelihood estimation. Psychometrika 50(2), 229-242. doi:10.1007/bf02294248

Boomsma, D., Busjahn, A., Peltonen, L. (2002). Classical twin studies and beyond. Nature Reviews Genetics 3(11), 872-882. doi:10.1038/nrg932

Boomsma, D.I., de Geus, E.J.C., Vink, J.M., Stubbe, J.H., Distel, M.A., Hottenga, J.-J., Posthuma, D., van Beijsterveldt, C.E.M., Hudziak, J.J., Bartels, M., Willemsen, G. (2006). Netherlands Twin Register: from twins to twin families. Twin Res Hum Genet 9, 849-857. doi:10.1375/183242706779462426

Boos, H.B., Cahn, W., van Haren, N.E., Derks, EM.., Brouwer, R.M., Schnack, H.G., Hulshoff Pol, H.E., Kahn, R.S. (2012). Focal And Global Brain Measurements in Siblings of Patients With Schizophrenia. Schizophrenia Bulletin 38(4), 814-825. doi:10.1093/schbul/sbq147

Boos, H., Mandl, R., van Haren, N., Cahn, W., Baal, G., Kahn, R., Hulshoff Pol, H.E. (2013). Tract-based diffusion tensor imaging in patients with schizophrenia and their non-psychotic siblings. European Neuropsychopharmacology 23(4), 295-304. doi:10.1016/j.euroneuro.2012.05.015

Bootsman, F., Brouwer, R., Kemner, S., Schnack, H., Schot, A., Vonk, R., Hillegers, M., Boomsma, D., Hulshoff Pol, H.E., Nolen, W., Kahn, R., van Haren, N. (2015). Contribution of genes and unique environment to cross-sectional and 
longitudinal measures of subcortical volumes in bipolar disorder. European Neuropsychopharmacology 25(12), 21972209. doi:10.1016/j.euroneuro.2015.09.023

Bourgeois JP, Rakic P. (1993). Changes of synaptic density in the primary visual cortex of the macaque monkey from fetal to adult stage. J Neurosci. 13:2801-2820.

Braber den A, Bohlken MM, Brouwer RM, van 't Ent D, Kanai R, Kahn RS, de Geus EJC, Hulshoff Pol HE, Boomsma DI. (2013). Heritability of subcortical brain measures: A perspective for future genome-wide association studies. NeuroImage. 83:98-102.

Brandmaier, A., Wenger, E., Bodammer, N., Kühn, S., Raz, N., Lindenberger, U. (2018). Assessing reliability in neuroimaging research through intra-class effect decomposition (ICED). eLife 7, e35718. doi:10.7554/elife.35718

Brans RGH, Kahn RS, Schnack HG, van Baal GCM, Posthuma D, van Haren NEM, Lepage C, Lerch JP, Collins DL, Evans AC, Boomsma DI, Hulshoff Pol HE. (2010). Brain Plasticity and Intellectual Ability Are Influenced by Shared Genes. J Neurosci. 30(16), 5519-5524. doi:10.1523/jneurosci.5841-09.2010

Bright, M., Murphy, K. (2015). Is fMRI "noise” really noise? Resting state nuisance regressors remove variance with network structure. NeuroImage 114, 158-169. doi:10.1016/j.neuroimage.2015.03.070

Briley, D.A., Tucker-Drob, E.M., 2013. Explaining the Increasing Heritability of Cognitive Ability Across Development: A Meta-Analysis of Longitudinal Twin and Adoption Studies. Psychol Sci 24, 1704-1713. doi:10.1177/0956797613478618

Brouwer, R., Hedman, A., van Haren, N., Schnack, H., Brans, R., Smit, D., Kahn, R., Boomsma, D., Hulshoff Pol, H.E. (2014). Heritability of brain volume change and its relation to intelligence. NeuroImage 100, 676-683. doi:10.1016/j.neuroimage.2014.04.072

Brouwer, R., Klein, M., Grasby, K., Schnack, H., Jahanshad, N., Teeuw, J., Thomopoulos, S., Sprooten, E., Franz, C., Gogtay, N., Kremen, W., Panizzon, M., Loohuis, L., Whelan, C., Aghajani, M., Alloza, C., Alnæs, D., Artiges, E., Ayesa-Arriola, R., Barker, G., Blok, E., Bøen, E., Breukelaar, I., Bright, J., Buimer, E., Bülow, R., Cannon, D., Ciufolini, S., Crossley, N., Damatac, C., Dazzan, P., Mol, C., de Zwarte, S., Desrivières, S., Díaz-Caneja, C., Doan, N., Dohm, K., Fröhner, J., Goltermann, J., Grigis, A., Grotegerd, D., Han, L., Hartman, C., Heany, S., Heindel, W., Heslenfeld, D., Hohmann, S., Ittermann, B., Jansen, P., Janssen, J., Jia, T., Jiang, J., Jockwitz, C., Karali, T., Keeser, D., Koevoets, M., Lenroot, R., Malchow, B., Mandl, R., Medel, V., Meinert, S., Morgan, C., Mühleisen, T., Nabulsi, L., Opel, N., Foz, V., Overs, B., Martinot, M., Quinlan, E., Redlich, R., Marques, T., Repple, J., Roberts, G., Roshchupkin, G., Setiaman, N., Shumskaya, E., Stein, F., Sudre, G., Takahashi, S., Thalamuthu, A., Tordesillas-Gutiérrez, D., Lugt, A., van Haren, N., Wen, W., Westeneng, H., Wittfeld, K., Zugman, A., Armstrong, N., Bralten, J., Dalvie, S., Forti, M., Ding, L., Donohoe, G., Forstner, A., Gonzalez-Peñas, J., Guimaraes, J., Homuth, G., Hottenga, J., Knol, M., Kwok, J., Hellard, S., Mather, K., Milaneschi, Y., Morris, D., Nöthen, M., Papiol, S., Rietschel, M., Santoro, M., Steen, V., Stein, J., Streit, F., Tankard, R., Teumer, A., Ent, D., Meer, D., Eijk, K., Vassos, E., Vázquez-Bourgon, J., Witt, S., Alzheimer's Disease Neuroimaging Initiative, Adams, H., Agartz, I., Ames, D., Amunts, K., Andreassen, O., Arango, C., Banaschewski, T., Baune, B., Belangero, S., Bokde, A., Boomsma, D., Bressan, R., Brodaty, H., Buitelaar, J., Cahn, W., Caspers, S., Cichon, S., Facorro, B., Dannlowski, U., Elvsåshagen, T., Espeseth, T., Falkai, P., Fisher, S., Flor, H., Fullerton, J., Garavan, H., Gowland, P., Grabe, H., Hahn, T., Heinz, A., Hillegers, M., Hoare, J., Hoekstra, P., Ikram, M., Jackowski, A., Jansen, A., Jönsson, E., Kahn, R., Kircher, T., Korgaonkar, M., Krug, A., Lemaitre, H., Malt, U., Martinot, J., McDonald, C., Mitchell, P., Muetzel, R., Murray, R., Nees, F., Nenadic, I., Oosterlaan, J., Ophoff, R., Pan, P., Penninx, B., Poustka, L., Sachdev, P., Salum, G., Schofield, P., Schumann, G., Shaw, P., Sim, K., Smolka, M., Stein, D., Trollor, J., Berg, L., Veldink, J., Walter, H., Westlye, L., Whelan, R., White, T., Wright, M., Medland, S., Franke, B., Thompson, P., Hulshoff Pol, H.E. (2020). Dynamics of Brain Structure and its Genetic Architecture over the Lifespan. bioRxiv doi:10.1101/2020.04.24.031138

Brouwer, R., Koenis, M., Schnack, H., Baal, G., van Soelen, I., Boomsma, D., Hulshoff Pol, H.E. (2015). Longitudinal Development of Hormone Levels and Grey Matter Density in 9 and 12-Year-Old Twins. Behavior Genetics 45(3), 313-323. doi:10.1007/s10519-015-9708-8

Brouwer, R., Mandl, R., Peper, J., Baal, G., Kahn, R., Boomsma, D., Hulshoff Pol, H.E. (2010). Heritability of DTI and MTR in nine-year-old children. NeuroImage 53(3), 1085-1092. doi:10.1016/j.neuroimage.2010.03.017 
Brouwer, R., Mandl, R., Schnack, H., van Soelen, I., Baal, G., Peper, J., Kahn, R., Boomsma, D., Hulshoff Pol, H.E. (2012). White Matter Development in Early Puberty: A Longitudinal Volumetric and Diffusion Tensor Imaging Twin Study. PLoS ONE 7(4), e32316. doi:10.1371/journal.pone.0032316

Brouwer, R., Panizzon, M., Glahn, D., Hibar, D., Hua, X., Jahanshad, N., Abramovic, L., Zubicaray, G., Franz, C., Hansell, N., Hickie, I., Koenis, M., Martin, N., Mather, K., McMahon, K., Schnack, H., Strike, L., Swagerman, S., Thalamuthu, A., Wen, W., Gilmore, J., Gogtay, N., Kahn, R., Sachdev, P., Wright, M., Boomsma, D., Kremen, W., Thompson, P., Hulshoff Pol, H.E. (2017). Genetic influences on individual differences in longitudinal changes in global and subcortical brain volumes: Results of the ENIGMA plasticity working group. Human Brain Mapping 38(9), 4444-4458. doi:10.1002/hbm.23672

Brouwer, R., van Soelen, I., Swagerman, S., Schnack, H., Ehli, E., Kahn, R., Hulshoff Pol, H.E, Boomsma, D. (2014). Genetic associations between intelligence and cortical thickness emerge at the start of puberty. Human Brain Mapping 35(8), 37603773. doi:10.1002/hbm.22435

Brouwer, R.M., Hulshoff Pol, H.E., Schnack, H.G., (2010). Segmentation of MRI brain scans using non-uniform partial volume densities. NeuroImage 49, 467-477. doi:10.1016/j.neuroimage.2009.07.041

Brown, T., Jernigan, T. (2012). Brain Development During the Preschool Years. Neuropsychology Review 22(4), $313-333$. doi:10.1007/s11065-012-9214-1

Brown, T., Kuperman, J., Chung, Y., Erhart, M., McCabe, C., Hagler, D., Venkatraman, V., Akshoomoff, N., Amaral, D., Bloss, C., Casey, B., Chang, L., Ernst, T., Frazier, J., Gruen, J., Kaufmann, W., Kenet, T., Kennedy, D., Murray, S., Sowell, E., Jernigan, T., Dale, A. (2012). Neuroanatomical Assessment of Biological Maturity. Current Biology 22(18), $1693-1698$. doi:10.1016/j.cub.2012.07.002

Browne, M., Cudeck, R. (1992). Alternative Ways of Assessing Model Fit. Sociological Methods \& Research 21(2), $230-258$. doi:10.1177/0049124192021002005

Bullmore, E., Sporns, O. (2009). Complex brain networks: graph theoretical analysis of structural and functional systems. Nature Reviews Neuroscience 10(3), 186-198. doi:10.1038/nrn2575

Burgaleta, M., Johnson, W., Waber, D., Colom, R., Karama, S. (2014). Cognitive ability changes and dynamics of cortical thickness development in healthy children and adolescents. NeuroImage 84, 810-819. doi:10.1016/j.neuroimage.2013.09.038

Burgess, G., Kandala, S., Nolan, D., Laumann, T., Power, J., Adeyemo, B., Harms, M., Petersen, S., Barch, D. (2016). Evaluation of Denoising Strategies to Address Motion-Correlated Artifacts in Resting-State Functional Magnetic Resonance Imaging Data from the Human Connectome Project. Brain Connectivity 6(9), 669-680. doi:10.1089/brain.2016.0435

Bush, G., Vogt, B.A., Holmes, J., Dale, A.M., Greve, D.N., Jenike, M.A., Rosen, B.R., (2002). Dorsal anterior cingulate cortex: A role in reward-based decision making. Proc. Natl. Acad. Sci. U.S.A. 99, 523-528. doi:10.1073/pnas.012470999

Button, K., Ioannidis, J., Mokrysz, C., Nosek, B., Flint, J., Robinson, E., Munafò, M. (2013). Power failure: why small sample size undermines the reliability of neuroscience. Nature Reviews Neuroscience 14(5), 365-376. doi:10.1038/nrn3475

Bzdok D. (2017). Classical statistics and statistical learning in imaging neuroscience. Front Neurosci. 11:543.

Caballero-Gaudes, C., Reynolds, R. (2017). Methods for cleaning the BOLD fMRI signal. NeuroImage 154, $128-149$. doi:10.1016/j.neuroimage.2016.12.018

Cabezas, M., Oliver, A., Lladó, X., Freixenet, J., Cuadra, M. (2011). A review of atlas-based segmentation for magnetic resonance brain images. Computer Methods and Programs in Biomedicine 104(3), e158-e177. doi:10.1016/j.cmpb.2011.07.015

Cao, M., Huang, H., Peng, Y., Dong, Q., He, Y. (2016). Toward Developmental Connectomics of the Human Brain. Frontiers in Neuroanatomy 10, 25. doi:10.3389/fnana.2016.00025

Cao, M., Wang, J.-H., Dai, Z.-J., Cao, X.-Y., Jiang, L.-L., Fan, F.-M., Song, X.-W., Xia, M.-R., Shu, N., Dong, Q., Milham, M.P., Castellanos, F.X., Zuo, X.-N., He, Y., (2014). Topological organization of the human brain functional connectome across the lifespan. Dev Cogn Neurosci 7, 76-93. doi:10.1016/j.dcn.2013.11.004 
Cariaga-Martinez A, Alelú-Paz R. (2016). False data, positive results in neurobiology: Moving beyond the epigenetics of blood and saliva samples in mental disorders. J Negat Results Biomed. 15:21.

Casey, B.J., (2013). The Teenage Brain. Curr Dir Psychol Sci 22, 80-81. doi:10.1177/0963721413486971

Caspi, Y., Brouwer, R., Schnack, H., Nieuwenhuijzen, M., Cahn, W., Kahn, R., Niessen, W., Lugt, A., Hulshoff Pol, H.E. (2020). Changes in the intracranial volume from early adulthood to the sixth decade of life: A longitudinal study. NeuroImage 220, 116842. doi:10.1016/j.neuroimage.2020.116842

Cevenini E, Invidia L, Lescai F, Salvioli S, Tieri P, Castellani G, Franceschi C. (2008). Human models of aging and longevity. Expert Opin Biol Ther. 8:1393-1405.

Chai, X.J., Castañón, A.N., Óngür, D., Whitfield-Gabrieli, S., (2012). Anticorrelations in resting state networks without global signal regression. NeuroImage 59, 1420-1428. doi:10.1016/j.neuroimage.2011.08.048

Chang, C., Glover, G. (2010). Time-frequency dynamics of resting-state brain connectivity measured with $\mathrm{fMRI}$. NeuroImage 50(1), 81-98. doi:10.1016/j.neuroimage.2009.12.011

Chen, B., Xu, T., Zhou, C., Wang, L., Yang, N., Wang, Z., Dong, H., Yang, Z., Zang, Y., Zuo, X., Weng, X. (2015). Individual Variability and Test-Retest Reliability Revealed by Ten Repeated Resting-State Brain Scans over One Month. PLOS ONE 10(12), e0144963. doi:10.1371/journal.pone.0144963

Chen BH, Marioni RE, Colicino E, Peters MJ, Ward-Caviness CK, Tsai P-C, Roetker NS, Just AC, Demerath EW, Guan W, Bressler J, Fornage M, Studenski S, Vandiver AR, Moore AZ, Tanaka T, Kiel DP, Liang L, Vokonas P, Schwartz J, Lunetta KL, Murabito JM, Bandinelli S, Hernandez DG, Melzer D, Nalls M, Pilling LC, Price TR, Singleton AB, Gieger C, Holle R, Kretschmer A, Kronenberg F, Kunze S, Linseisen J, Meisinger C, Rathmann W, Waldenberger M, Visscher PM, Shah S, Wray NR, McRae AF, Franco OH, Hofman A, Uitterlinden AG, Absher D, Assimes T, Levine ME, Lu AT, Tsao PS, Hou L, Manson JE, Carty CL, LaCroix AZ, Reiner AP, Spector TD, Feinberg AP, Levy D, Baccarelli A, van Meurs J, Bell JT, Peters A, Deary IJ, Pankow JS, Ferrucci L, Horvath S. (2016). DNA methylation-based measures of biological age: meta-analysis predicting time to death. Aging (Albany NY). 8:1844-1865.

Chen C-H, Fiecas MJA, Gutiérrez ED, Panizzon MS, Eyler LT, Vuoksimaa E, Thompson WK, Fennema-Notestine C, Hagler DJ, Jernigan TL, Neale MC, Franz CE, Lyons MJ, Fischl B, Tsuang MT, Dale AM, Kremen WS. (2013). Genetic topography of brain morphology. Proc Natl Acad Sci USA. 110:17089-17094.

Chen C-H, Gutiérrez ED, Thompson W, Panizzon MS, Jernigan TL, Eyler LT, Fennema-Notestine C, Jak AJ, Neale MC, Franz CE, Lyons MJ, Grant MD, Fischl B, Seidman LJ, Tsuang MT, Kremen WS, Dale AM. (2012). Hierarchical genetic organization of human cortical surface area. Science. 335:1634-1636.

Chen, G., Taylor, P., Haller, S., Kircanski, K., Stoddard, J., Pine, D., Leibenluft, E., Brotman, M., Cox, R. (2018). Intraclass correlation: Improved modeling approaches and applications for neuroimaging. Human Brain Mapping 39(3), 1187-1206. doi:10.1002/hbm.23909

Chiapponi, C., Piras, F., Fagioli, S., Piras, F., Caltagirone, C., Spalletta, G. (2013). Age-related brain trajectories in schizophrenia: A systematic review of structural MRI studies. Psychiatry Research: Neuroimaging 214(2), 83-93. doi:10.1016/j.pscychresns.2013.05.003

Chinta SJ, Woods G, Rane A, Demaria M, Campisi J, Andersen JK. (2015). Cellular senescence and the aging brain. Exp Gerontol. 68:3-7.

Choe, A., Jones, C., Joel, S., Muschelli, J., Belegu, V., Caffo, B., Lindquist, M., Zijl, P., Pekar, J. (2015). Reproducibility and Temporal Structure in Weekly Resting-State fMRI over a Period of 3.5 Years. PLOS ONE 10(10), e0140134. doi:10.1371/journal.pone.0140134

Chouinard-Decorte, F., McKay, D., Reid, A., Khundrakpam, B., Zhao, L., Karama, S., Rioux, P., Sprooten, E., Knowles, E., Kent, J., Curran, J., Göring, H., Dyer, T., Olvera, R., Kochunov, P., Duggirala, R., Fox, P., Almasy, L., Blangero, J., Bellec, P., Evans, A., Glahn, D. (2014). Heritable changes in regional cortical thickness with age. Brain Imaging and Behavior 8(2), 208-216. doi:10.1007/s11682-014-9296-x 
Christensen, B., Houseman, E., Marsit, C., Zheng, S., Wrensch, M., Wiemels, J., Nelson, H., Karagas, M., Padbury, J., Bueno, R., Sugarbaker, D., Yeh, R., Wiencke, J., Kelsey, K. (2009). Aging and Environmental Exposures Alter Tissue-Specific DNA Methylation Dependent upon CpG Island Context. PLoS Genetics 5(8), e1000602. doi:10.1371/journal.pgen.1000602

Chung Y, Addington J, Bearden CE, Cadenhead K, Cornblatt B, Mathalon DH, McGlashan T, Perkins D, Seidman LJ, Tsuang M, Walker E, Woods SW, McEwen S, van Erp TGM, Cannon TD. (2018). Use of machine learning to determine deviance in neuroanatomical maturity associated with future psychosis in youths at clinically high risk. JAMA Psychiatry. 75:960-968.

Ciric, R., Wolf, D., Power, J., Roalf, D., Baum, G., Ruparel, K., Shinohara, R., Elliott, M., Eickhoff, S., Davatzikos, C., Gur, R., Gur, R., Bassett, D., Satterthwaite, T. (2017). Benchmarking of participant-level confound regression strategies for the control of motion artifact in studies of functional connectivity. NeuroImage 154, 174-187. doi:10.1016/j.neuroimage.2017.03.020

Cohn, M., Veltman, D., Pape, L., Lith, K., Vermeiren, R., Brink, W., Doreleijers, T., Popma, A. (2015). Incentive Processing in Persistent Disruptive Behavior and Psychopathic Traits: A Functional Magnetic Resonance Imaging Study in Adolescents. Biological Psychiatry 78(9), 615-624. doi:10.1016/j.biopsych.2014.08.017

Colantuoni C, Lipska BK, Ye T, Hyde TM, Tao R, Leek JT, Colantuoni EA, Elkahloun AG, Herman MM, Weinberger DR, Kleinman JE. (2011). Temporal dynamics and genetic control of transcription in the human prefrontal cortex. Nature. 478:519-523.

Colclough, G.L., Smith, S.M., Nichols, T.E., Winkler, A.A.M., Sotiropoulos, S.N., Glasser, M.F., Van Essen, D.C., Woolrich, M.W., (2017). The heritability of multi-modal connectivity in human brain activity. Elife $6, \mathrm{e} 01867$. doi:10.7554/eLife.20178

Cole JH, Franke K. (2017). Predicting Age Using Neuroimaging: Innovative Brain Ageing Biomarkers. Trends in Neurosciences 40(12), 681-690. doi:10.1016/j.tins.2017.10.001

Cole JH, Marioni RE, Harris SE, Deary IJ. (2019). Brain age and other bodily 'ages': implications for neuropsychiatry. Molecular Psychiatry 24(2), 266-281. doi:10.1038/s41380-018-0098-1

Cole JH, Poudel RPK, Tsagkrasoulis D, Caan MWA, Steves C, Spector TD, Montana G. (2017). Predicting brain age with deep learning from raw imaging data results in a reliable and heritable biomarker. NeuroImage 163(Nature 475 2011), 115-124. doi:10.1016/j.neuroimage.2017.07.059

Cole JH, Ritchie SJ, Bastin ME, Valdés Hernández MC, Muñoz Maniega S, Royle N, Corley J, Pattie A, Harris SE, Zhang Q, Wray NR, Redmond P, Marioni RE, Starr JM, Cox SR, Wardlaw JM, Sharp DJ, Deary IJ. (2018). Brain age predicts mortality. Molecular psychiatry 23(5), 1385-1392. doi:10.1038/mp.2017.62

Cole, M., Bassett, D., Power, J., Braver, T., Petersen, S. (2014). Intrinsic and Task-Evoked Network Architectures of the Human Brain. Neuron 83(1), 238-251. doi:10.1016/j.neuron.2014.05.014

Cooper, S., Jackson, J., Barch, D., Braver, T. (2019). Neuroimaging of Individual Differences: A Latent Variable Modeling Perspective. Neuroscience \& Biobehavioral Reviews 98(Biological Psychiatry $70 \quad 2011)$, 29-46. doi:10.1016/j.neubiorev.2018.12.022

Couvy-Duchesne, B., Blokland, G.A.M., Hickie, I.B., Thompson, P.M., Martin, N.G., de Zubicaray, G.I., McMahon, K.L., Wright, M.J., (2014). Heritability of head motion during resting state functional MRI in 462 healthy twins. NeuroImage 102 Pt 2, 424-434. doi:10.1016/j.neuroimage.2014.08.010

Couvy-Duchesne, B., Ebejer, J.L., Gillespie, N.A., Duffy, D.L., Hickie, I.B., Thompson, P.M., Martin, N.G., de Zubicaray, G.I., McMahon, K.L., Medland, S.E., Wright, M.J., (2016). Head Motion and Inattention/Hyperactivity Share Common Genetic Influences: Implications for fMRI Studies of ADHD. PLoS ONE 11, e0146271. doi:10.1371/journal.pone.0146271

Crone, E., Dahl, R. (2012). Understanding adolescence as a period of social-affective engagement and goal flexibility. Nature Reviews Neuroscience 13(9), 636-650. doi:10.1038/nrn3313

Crone, E.A., Elzinga, B.M., (2015). Changing brains: how longitudinal functional magnetic resonance imaging studies can inform us about cognitive and social-affective growth trajectories. Wiley Interdisciplinary Reviews: Cognitive Science 6, 53-63. doi:10.1002/wcs.1327 
Csárdi, G., Nepusz, T. (2006). The igraph software package for complex network research. InterJournal Complex Systems, 1695. http://igraph.org

Cullen, K., Westlund, M., Klimes-Dougan, B., Mueller, B., Houri, A., Eberly, L., Lim, K. (2014). Abnormal Amygdala Resting-State Functional Connectivity in Adolescent Depression. JAMA Psychiatry 71(10), 1138-1147. doi:10.1001/jamapsychiatry.2014.1087

Cutter GR. (1976). Some Examples for Teaching Regression Toward the Mean from a Sampling Viewpoint. The American Statistician. 30:194-195.

Dale, B., Brown, M., Semelka, R. (2015). MRI: Basic Principles and Applications, 5th edition. John Wiley and Sons

Damoiseaux, J. (2017). Effects of aging on functional and structural brain connectivity. NeuroImage 160, 32-40. doi:10.1016/j.neuroimage.2017.01.077

Darmanis, S., Sloan, S., Zhang, Y., Enge, M., Caneda, C., Shuer, L., Gephart, M., Barres, B., Quake, S. (2015). A survey of human brain transcriptome diversity at the single cell level. Proceedings of the National Academy of Sciences 112(23), 7285-7290. doi:10.1073/pnas.1507125112

Das S, Forer L, Schönherr S, Sidore C, Locke AE, Kwong A, Vrieze SI, Chew EY, Levy S, McGue M, Schlessinger D, Stambolian D, Loh P-R, Iacono WG, Swaroop A, Scott LJ, Cucca F, Kronenberg F, Boehnke M, Abecasis GR, Fuchsberger C. (2016). Next-generation genotype imputation service and methods. Nat Genet. 48:1284-1287.

de Nooij L, Harris MA, Hawkins EL, Shen X, Clarke T-K, Chan SWY, Ziermans TB, McIntosh AM, Whalley HC. (2019). Longitudinal trajectories of brain age in young individuals at familial risk of mood disorder. bioRxiv. 27:3.

de Wit, S., Wierenga, L., Oranje, B., Ziermans, T., Schothorst, P., Engeland, H., Kahn, R., Durston, S. (2016). Brain development in adolescents at ultra-high risk for psychosis: Longitudinal changes related to resilience. NeuroImage: Clinical 12, 542-549. doi:10.1016/j.nicl.2016.08.013

de Zwarte, S., Brouwer, R., Agartz, I., Alda, M., Aleman, A., Alpert, K., Bearden, C., Bertolino, A., Bois, C., Bonvino, A., Bramon, E., Buimer, E., Cahn, W., Cannon, D., Cannon, T., Caseras, X., Castro-Fornieles, J., Chen, Q., Chung, Y., Serna, E., Giorgio, A., Doucet, G., Eker, M., Erk, S., Fears, S., Foley, S., Frangou, S., Frankland, A., Fullerton, J., Glahn, D., Goghari, V., Goldman, A., Gonul, A., Gruber, O., Haan, L., Hajek, T., Hawkins, E., Heinz, A., Hillegers, M., Hulshoff Pol, H.E., Hultman, C., Ingvar, M., Johansson, V., Jönsson, E., Kane, F., Kempton, M., Koenis, M., Kopecek, M., Krabbendam, L., Krämer, B., Lawrie, S., Lenroot, R., Marcelis, M., Marsman, J., Mattay, V., McDonald, C., MeyerLindenberg, A., Michielse, S., Mitchell, P., Moreno, D., Murray, R., Mwangi, B., Najt, P., Neilson, E., Newport, J., van Os, J., Overs, B., Ozerdem, A., Picchioni, M., Richter, A., Roberts, G., Aydogan, A., Schofield, P., Simsek, F., Soares, J., Sugranyes, G., Toulopoulou, T., Tronchin, G., Walter, H., Wang, L., Weinberger, D., Whalley, H., Yalin, N., Andreassen, O., Ching, C., van Erp, T., Turner, J., Jahanshad, N., Thompson, P., Kahn, R., van Haren, N. (2019). The association between familial risk and brain abnormalities is disease-specific: an ENIGMA-Relatives study of schizophrenia and bipolar disorder. Biological Psychiatry 86(7), 545-556. doi:10.1016/j.biopsych.2019.03.985

DeLuca, M., Devlin, J.T., Smith, S.M., (2005). Investigations into resting-state connectivity using independent component analysis. Philosophical Transactions of the Royal Society B: Biological Sciences 360, 1001-1013. doi:10.1098/rstb.2005.1634

den Braber, A., Bohlken, M., Brouwer, R., Ent, D., Kanai, R., Kahn, R., Geus, E., Hulshoff Pol, H.E., Boomsma, D. (2013). Heritability of subcortical brain measures: A perspective for future genome-wide association studies. NeuroImage 83, 98102. doi:10.1016/j.neuroimage.2013.06.027

Deary, I.J., Penke, L., Johnson, W., (2010). The neuroscience of human intelligence differences. Nat Rev Neurosci 11, 201211. doi: $10.1038 / \mathrm{nrn} 2793$

Debnath, M., Venkatasubramanian, G., Berk, M. (2015). Fetal programming of schizophrenia: Select mechanisms. Neuroscience \& Biobehavioral Reviews 49, 90-104. doi:10.1016/j.neubiorev.2014.12.003

Dekaban AS, Sadowsky D. (1978). Changes in brain weights during the span of human life: Relation of brain weights to body heights and body weights. Annals of Neurology 4(4), 345-356. doi:10.1002/ana.410040410 
Dennis, E., Thompson, P. (2014). Functional Brain Connectivity Using fMRI in Aging and Alzheimer's Disease. Neuropsychology Review 24(1), 49-62. doi:10.1007/s11065-014-9249-6

Deoni SCL, Dean DC, Remer J, Dirks H, O'Muircheartaigh J. (2015). Cortical maturation and myelination in healthy toddlers and young children. NeuroImage. 115:147-161.

Despotović, I., Goossens, B., Philips, W. (2015). MRI Segmentation of the Human Brain: Challenges, Methods, and Applications. Computational and Mathematical Methods in Medicine 2015, 1-23. doi:10.1155/2015/450341

Dick, D. (2011). Gene-Environment Interaction in Psychological Traits and Disorders. Annual Review of Clinical Psychology 7(1), 383-409. doi:10.1146/annurev-clinpsy-032210-104518

Docherty AR, Sawyers CK, Panizzon MS, Neale MC, Eyler LT, Fennema-Notestine C, Franz CE, Chen C-H, McEvoy LK, Verhulst B, Tsuang MT, Kremen WS. (2015). Genetic network properties of the human cortex based on regional thickness and surface area measures. Front Hum Neurosci. 9:1-14.

Dominicus, A., Skrondal, A., Gjessing, H.K., Pedersen, N.L., Palmgren, J., (2006). Likelihood ratio tests in behavioral genetics: problems and solutions. Behav Genet 36, 331-340. doi:10.1007/s10519-005-9034-7

Dosenbach, N.U.F., Nardos, B., Cohen, A.L., Fair, D.A., Power, J.D., Church, J.A., Nelson, S.M., Wig, G.S., Vogel, A.C., Lessov-Schlaggar, C.N., Barnes, K.A., Dubis, J.W., Feczko, E., Coalson, R.S., Pruett, J.R., Barch, D.M., Petersen, S.E., Schlaggar, B.L., (2010). Prediction of Individual Brain Maturity Using fMRI. Science 329(5997), 1358-1361. doi:10.1126/science.1194144

Douet, V., Chang, L., Cloak, C., Ernst, T.M., (2014). Genetic influences on brain developmental trajectories on neuroimaging studies: from infancy to young adulthood. Brain Imaging Behav 8, 234-250. doi:10.1007/s11682-013-9260-1

Dubois, J. (2016). Brain Age: A State-Of-Mind? On the Stability of Functional Connectivity across Behavioral States. The Journal of Neuroscience 36(8), 2325-2328. doi:10.1523/jneurosci.4312-15.2016

Dubois, J., Galdi, P., Paul, L.K., Adolphs, R., (2018). A distributed brain network predicts general intelligence from restingstate human neuroimaging data. Philosophical Transactions of the Royal Society B: Biological Sciences 373, 20170284. doi:10.1098/rstb.2017.0284

Dukart J, Smieskova R, Harrisberger F, Lenz C, Schmidt A, Walter A, Huber C, Riecher-Rössler A, Simon A, Lang UE, Fusar-Poli P, Borgwardt S. (2017). Age-related brain structural alterations as an intermediate phenotype of psychosis. JPN. 42:307-319.

Durston, S., Hulshoff Pol, H.E., Casey, B., Giedd, J., Buitelaar, J., England, H. (2001). Anatomical MRI of the Developing Human Brain: What Have We Learned? Journal of the American Academy of Child \& Adolescent Psychiatry 40(9), 1012-1020. doi:10.1097/00004583-200109000-00009

Durston, S., Nederveen, H., Dijk, S., Belle, J., Zeeuw, P., Langen, M., Dijk, A. (2009). Magnetic resonance simulation is effective in reducing anxiety related to magnetic resonance scanning in children. Journal of the American Academy of Child and Adolescent Psychiatry 48(2), 206-7. doi:10.1097/chi.0b013e3181930673

Edwards, S., Beesley, J., French, J., Dunning, A. (2013). Beyond GWASs: Illuminating the Dark Road from Association to Function. The American Journal of Human Genetics 93(5), 779-797. doi:10.1016/j.ajhg.2013.10.012

Elliott, M., Knodt, A., Cooke, M., Kim, M., Melzer, T., Keenan, R., Ireland, D., Ramrakha, S., Poulton, R., Caspi, A., Moffitt, T., Hariri, A. (2019). General functional connectivity: Shared features of resting-state and task fMRI drive reliable and heritable individual differences in functional brain networks. NeuroImage 189, 516-532. doi:10.1016/j.neuroimage.2019.01.068

Engelhardt, L.E., Roe, M.A., Juranek, J., DeMaster, D., Harden, K.P., Tucker-Drob, E.M., Church, J.A., (2017). Children's Head Motion During fMRI Tasks is Heritable and Stable over Time. Dev Cogn Neurosci. doi:10.1016/j.dcn.2017.01.011

Ernst, M., Mueller, S. (2008). The adolescent brain: Insights from functional neuroimaging research. Developmental Neurobiology 68(6), 729-743. doi:10.1002/dneu.20615

Ernst, M., Torrisi, S., Balderston, N., Grillon, C., Hale, E.A., (2015). fMRI functional connectivity applied to adolescent neurodevelopment. Annu Rev Clin Psychol 11, 361-377. doi:10.1146/annurev-clinpsy-032814-112753 
Essen, D., Smith, S., Barch, D., Behrens, T., Yacoub, E., Ugurbil, K., WU-Minn HCP Consortium. (2013). The WU-Minn Human Connectome Project: An overview. NeuroImage 80, 62-79. doi:10.1016/j.neuroimage.2013.05.041

Eyler LT, Chen C-H, Panizzon MS, Fennema-Notestine C, Neale MC, Jak AJ, Jernigan TL, Fischl B, Franz CE, Lyons MJ, Grant MD, Prom-Wormley EC, Seidman LJ, Tsuang MT, Fiecas MJA, Dale AM, Kremen WS. (2012). A comparison of heritability maps of cortical surface area and thickness and the influence of adjustment for whole brain measures: a magnetic resonance imaging twin study. Twin Res Hum Genet. 15:304-314.

Faa, G., Manchia, M., Pintus, R., Gerosa, C., Marcialis, M.A., Fanos, V. (2016). Fetal programming of neuropsychiatric disorders. Birth Defects Research Part C: Embryo Today: Reviews 108(3), 207-223. doi:10.1002/bdrc.21139

Facer-Childs, E., Campos, B., Middleton, B., Skene, D., Bagshaw, A. (2019). Circadian phenotype impacts the brain's resting state functional connectivity, attentional performance and sleepiness. Sleep 42(5), zsz033-. doi:10.1093/sleep/zsz033

Fair, D.A., Cohen, A.L., Dosenbach, N.U.F., Church, J.A., Miezin, F.M., Barch, D.M., Raichle, M.E., Petersen, S.E., Schlaggar, B.L., (2008). The maturing architecture of the brain's default network. Proc Natl Acad Sci USA 105, 4028-4032. doi:10.1073/pnas.0800376105

Fair, D.A., Cohen, A.L., Power, J.D., Dosenbach, N.U.F., Church, J.A., Miezin, F.M., Schlaggar, B.L., Petersen, S.E., (2009). Functional Brain Networks Develop from a "Local to Distributed" Organization. PLoS Computational Biology 5(5), e1000381. doi:10.1371/journal.pcbi.1000381

Fair, D.A., Dosenbach, N.U.F., Church, J.A., Cohen, A.L., Brahmbhatt, S., Miezin, F.M., Barch, D.M., Raichle, M.E., Petersen, S.E., Schlaggar, B.L., (2007). Development of distinct control networks through segregation and integration. Proceedings of the National Academy of Sciences 104(33), 13507-13512. doi:10.1073/pnas.0705843104

Falconer, D.S., Mackay, T.F.C., (1996). Introduction to quantitative genetics, 4th edition. Pearson.

Farré P, Jones MJ, Meaney MJ, Emberly E, Turecki G, Kobor MS. (2015). Concordant and discordant DNA methylation signatures of aging in human blood and brain. Epigenetics Chromatin. 8:19.

Fernandez-Egea E, Kirkpatrick B. (2017). Correspondence regarding two recent publications in npj:schizophrenia about DNAm and accelerated aging in schizophrenia. npj Schizophrenia. 3:38.

Ferreira, L., Busatto, G. (2013). Resting-state functional connectivity in normal brain aging. Neuroscience \& Biobehavioral Reviews 37(3), 384-400. doi:10.1016/j.neubiorev.2013.01.017

Filippini, N., MacIntosh, B., Hough, M., Goodwin, G., Frisoni, G., Smith, S., Matthews, P., Beckmann, C., Mackay, C. (2009). Distinct patterns of brain activity in young carriers of the APOE - 44 allele. Proceedings of the National Academy of Sciences 106(17), 7209-7214. doi:10.1073/pnas.0811879106

Finn, E., Shen, X., Scheinost, D., Rosenberg, M., Huang, J., Chun, M., Papademetris, X., Constable, R. (2015). Functional connectome fingerprinting: identifying individuals using patterns of brain connectivity. Nature Neuroscience 18(11), 1664-1671. doi:10.1038/nn.4135

Fischl, B., Dale, A. (2000). Measuring the thickness of the human cerebral cortex from magnetic resonance images. Proceedings of the National Academy of Sciences 97(20), 11050-11055. doi:10.1073/pnas.200033797

Fischl, B., Kouwe, A., Destrieux, C., Halgren, E., Ségonne, F., Salat, D., Busa, E., Seidman, L., Goldstein, J., Kennedy, D., Caviness, V., Makris, N., Rosen, B., Dale, A. (2004). Automatically Parcellating the Human Cerebral Cortex. Cerebral Cortex 14(1), 11-22. doi:10.1093/cercor/bhg087

Fischl, B., Salat, D., Busa, E., Albert, M., Dieterich, M., Haselgrove, C., Kouwe, A., Killiany, R., Kennedy, D., Klaveness, S., Montillo, A., Makris, N., Rosen, B., Dale, A. (2002). Whole Brain Segmentation Automated Labeling of Neuroanatomical Structures in the Human Brain. Neuron 33(3), 341-355. doi:10.1016/s0896-6273(02)00569-x

Fjell, A., Grydeland, H., Krogsrud, S., Amlien, I., Rohani, D., Ferschmann, L., Storsve, A., Tamnes, C., Sala-Llonch, R., DueTønnessen, P., Bjørnerud, A., Sølsnes, A., Håberg, A., Skranes, J., Bartsch, H., Chen, C., Thompson, W., Panizzon, M., Kremen, W., Dale, A., Walhovd, K. (2015). Development and aging of cortical thickness correspond to genetic organization patterns. Proceedings of the National Academy of Sciences 112(50), 15462-15467. doi:10.1073/pnas.1508831112

Fjell, A., Grydeland, H., Wang, Y., Amlien, I., Bartrés-Faz, D., Brandmaier, A., Düzel, S., Elman, J., Franz, C., Håberg, A., Kietzmann, T., Kievit, R., Kremen, W., Krogsrud, S., Kühn, S., Lindenberger, U., Macià, D., Mowinckel, A., Nyberg, L., 
Panizzon, M., Solé-Padullés, C., Sørensen, Ø., Westerhausen, R., Walhovd, K. (2020). The genetic organization of subcortical volumetric change is stable throughout the lifespan. bioRxiv doi:10.1101/2020.06.12.143834

Fjell AM, Grydeland H, Krogsrud SK, Amlien IK, Rohani DA, Ferschmann L, Storsve AB, Tamnes CK, Sala-Llonch R, Due-Tønnessen P, Bjørnerud A, Sølsnes AE, Håberg AK, Skranes JS, Bartsch H, Chen C-H, Thompson WK, Panizzon MS, Kremen WS, Dale AM, Walhovd KB. (2015). Development and aging of cortical thickness correspond to genetic organization patterns. Proc Natl Acad Sci USA. 112:15462-15467.

Fornito, A., Zalesky, A., Bassett, D.S., Meunier, D., Ellison-Wright, I., Yücel, M., Wood, S.J., Shaw, K., O'Connor, J., Nertney, D.A., Bullmore, E.T., (2011). Genetic influences on cost-efficient organization of human cortical functional networks. J Neurosci 31, 3261-3270. doi:10.1523/JNEUROSCI.4858-10.2011

Fox, M.D., Buckner, R., Liu, H., Chakravarty, M., Lozano, A., Pascual-Leone, A. (2014). Resting-state networks link invasive and noninvasive brain stimulation across diverse psychiatric and neurological diseases. Proceedings of the National Academy of Sciences 111(41), E4367-E4375. doi:10.1073/pnas.1405003111

Fox, M., Raichle, M. (2007). Spontaneous fluctuations in brain activity observed with functional magnetic resonance imaging. Nature Reviews Neuroscience 8(9), 700-711. doi:10.1038/nrn2201

Fox, M.D., Snyder, A.Z., Vincent, J.L., Corbetta, M., Van Essen, D.C., Raichle, M.E., (2005). The human brain is intrinsically organized into dynamic, anticorrelated functional networks. Proc. Natl. Acad. Sci. U.S.A. 102, 9673-9678. doi:10.1073/pnas.0504136102

Fraga, M., Ballestar, E., Paz, M., Ropero, S., Setien, F., Ballestar, M., Heine-Suner, D., Cigudosa, J., Urioste, M., Benitez, J., Boix-Chornet, M., Sanchez-Aguilera, A., Ling, C., Carlsson, E., Poulsen, P., Vaag, A., Stephan, Z., Spector, T., Wu, Y., Plass, C., Esteller, M. (2005). From The Cover: Epigenetic differences arise during the lifetime of monozygotic twins. Proceedings of the National Academy of Sciences 102(30), 10604-10609. doi:10.1073/pnas.0500398102

Franke, K., Gaser, C. (2019). Ten Years of BrainAGE as a Neuroimaging Biomarker of Brain Aging: What Insights Have We Gained? Frontiers in Neurology 10, 789. doi:10.3389/fneur.2019.00789

Frayne, R., Goodyear, B., Dickhoff, P., Lauzon, M., Sevick, R. (2003). Magnetic Resonance Imaging at 3.0 Tesla: Challenges and Advantages in Clinical Neurological Imaging. Investigative Radiology 38(7), 385-402. doi:10.1097/01.rli.0000073442.88269.c9

Fredriks, A., Buuren, S., Burgmeijer, R., Meulmeester, J., Beuker, R., Brugman, E., Roede, M., Verloove-Vanhorick, S., Wit, J. (2000). Continuing Positive Secular Growth Change in the Netherlands 1955-1997. Pediatric Research 47(3), 316-323. doi:10.1203/00006450-200003000-00006

Friedrich, P., Fraenz, C., Schlüter, C., Ocklenburg, S., Mädler, B., Güntürkün, O., Genç, E. (2020). The Relationship Between Axon Density, Myelination, and Fractional Anisotropy in the Human Corpus Callosum. Cerebral Cortex 30(4), $2042-2056$. doi:10.1093/cercor/bhz221

Fries GR, Bauer IE, Scaini G, Wu M-J, Kazimi IF, Valvassori SS, Zunta-Soares G, Walss-Bass C, Soares JC, Quevedo J. (2017). Accelerated epigenetic aging and mitochondrial DNA copy number in bipolar disorder. Translational Psychiatry. 7:1283.

Friston, K.J., Williams, S., Howard, R., Frackowiak, R.S., Turner, R., (1996). Movement-related effects in fMRI time-series. Magnetic Resonance in Medicine 35, 346-355. https://doi.org/10.1002/mrm.1910350312

Fu, Y., Ma, Z., Hamilton, C., Liang, Z., Hou, X., Ma, X., Hu, X., He, Q., Deng, W., Wang, Y., Zhao, L., Meng, H., Li, T., Zhang, N. (2015). Genetic influences on resting-state functional networks: A twin study. Human Brain Mapping 36(10), 3959-3972. doi:10.1002/hbm.22890

GERAD1 Consortium, CRESTAR Consortium, Pardiñas, A., Holmans, P., Pocklington, A., Escott-Price, V., Ripke, S., Carrera, N., Legge, S., Bishop, S., Cameron, D., Hamshere, M., Han, J., Hubbard, L., Lynham, A., Mantripragada, K., Rees, E., MacCabe, J., McCarroll, S., Baune, B., Breen, G., Byrne, E., Dannlowski, U., Eley, T., Hayward, C., Martin, N., McIntosh, A., Plomin, R., Porteous, D., Wray, N., Caballero, A., Geschwind, D., Huckins, L., Ruderfer, D., Santiago, E., Sklar, P., Stahl, E., Won, H., Agerbo, E., Als, T., Andreassen, O., Bækvad-Hansen, M., Mortensen, P., Pedersen, C., Børglum, A., Bybjerg-Grauholm, J., Djurovic, S., Durmishi, N., Pedersen, M., Golimbet, V., Grove, J., Hougaard, D., 
Mattheisen, M., Molden, E., Mors, O., Nordentoft, M., Pejovic-Milovancevic, M., Sigurdsson, E., Silagadze, T., Hansen, C., Stefansson, K., Stefansson, H., Steinberg, S., Tosato, S., Werge, T., Collier, D., Rujescu, D., Kirov, G., Owen, M., O’Donovan, M., Walters, J. (2018). Common schizophrenia alleles are enriched in mutation-intolerant genes and in regions under strong background selection. Nature Genetics 50(3), 381-389. doi:10.1038/s41588-018-0059-2

Gao, W., Alcauter, S., Smith, J.K., Gilmore, J.H., Lin, W., (2015). Development of human brain cortical network architecture during infancy. Brain Structure and Function 220, 1173-1186. doi:10.1007/s00429-014-0710-3

Gao, W., Elton, A., Zhu, H., Alcauter, S., Smith, J.K., Gilmore, J.H., Lin, W., (2014). Intersubject Variability of and Genetic Effects on the Brain's Functional Connectivity during Infancy. The Journal of Neuroscience 34(34), 11288-11296. doi:10.1523/jneurosci.5072-13.2014

Gao, W., Gilmore, J.H., Giovanello, K.S., Smith, J.K., Shen, D., Zhu, H., Lin, W., (2011). Temporal and Spatial Evolution of Brain Network Topology during the First Two Years of Life. PLoS ONE 6(9), e25278. doi:10.1371/journal.pone.0025278

Gao X, Zhang Y, Breitling LP, Brenner H. (2016). Tobacco smoking and methylation of genes related to lung cancer development. Oncotarget. 7:59017-59028.

Gautam P, Warner TD, Kan EC, Sowell ER. (2015). Executive function and cortical thickness in youths prenatally exposed to cocaine, alcohol and tobacco. Dev Cogn Neurosci. 16:155-165.

Ge, T., Holmes, A.J., Buckner, R.L., Smoller, J.W., Sabuncu, M.R., (2017). Heritability analysis with repeat measurements and its application to resting-state functional connectivity. Proceedings of the National Academy of Sciences 114(21), 5521-5526. doi:10.1073/pnas.1700765114

Geerligs, L., Tsvetanov, K., Cam-CAN, Henson, R. (2017). Challenges in measuring individual differences in functional connectivity using fMRI: The case of healthy aging. Human Brain Mapping 38(8), 4125-4156. doi:10.1002/hbm.23653

Genovese, C.R., Lazar, N.A., Nichols, T.E., (2002). Thresholding of Statistical Maps in Functional Neuroimaging Using the False Discovery Rate. NeuroImage 15, 870-878. doi:10.1006/nimg.2001.1037

Giedd, J., Rapoport, J. (2010). Structural MRI of Pediatric Brain Development: What Have We Learned and Where Are We Going? Neuron 67(5), 728-734. doi:10.1016/j.neuron.2010.08.040

Giedd JN, Blumenthal JD, Jeffries NO, Castellanos FX, Liu H, Zijdenbos AP, Paus T, Evans AC, Rapoport JL. (1999). Brain development during childhood and adolescence: a longitudinal MRI study. Nature Neuroscience 2(10), 861-863. doi: $10.1038 / 13158$

Giedd JN, Raznahan A, Alexander-Bloch AF, Schmitt E, Gogtay N, Rapoport JL. (2015). Child psychiatry branch of the National Institute of Mental Health longitudinal structural magnetic resonance imaging study of human brain development. Neuropsychopharmacology. 40:43-49.

Giedd, J.N., Raznahan, A., Mills, K.L., Lenroot, R.K., (2012). Review: magnetic resonance imaging of male/female differences in human adolescent brain anatomy. Biology of Sex Differences 3, 19. doi:10.1186/2042-6410-3-19

Giedd, J.N., Snell, J.W., Lange, N., Rajapakse, J.C., Casey, B.J., Kozuch, P.L., Vaituzis, A.C., Vauss, Y.C., Hamburger, S.D., Kaysen, D., Rapoport, J.L., (1996). Quantitative magnetic resonance imaging of human brain development: ages 4-18. Cereb. Cortex 6, 551-560.

Giedd JN, Stockman M, Weddle C, Liverpool M, Alexander-Bloch AF, Wallace GL, Lee NR, Lalonde F, Lenroot RK. (2010). Anatomic magnetic resonance imaging of the developing child and adolescent brain and effects of genetic variation. Neuropsychol Rev. 20:349-361.

Gilmore, J.H., Knickmeyer, R.C., Gao, W., (2018). Imaging structural and functional brain development in early childhood Nature Reviews Neuroscience 19(3), 123-137. doi:10.1038/nrn.2018.1

Glahn, D., Thompson, P., Blangero, J. (2007). Neuroimaging endophenotypes: Strategies for finding genes influencing brain structure and function. Human Brain Mapping 28(6), 488-501. doi:10.1002/hbm.20401

Glahn, D.C., Winkler, A.A.M., Kochunov, P.V., Almasy, L., Duggirala, R., Carless, M.A., Curran, J.C., Olvera, R.L., Laird, A.R., Smith, S.M., Beckmann, C.F., Fox, P.T., Blangero, J., (2010). Genetic control over the resting brain. Proc Natl Acad Sci USA 107, 1223-1228. doi:10.1073/pnas.0909969107 
Glasser, M., Coalson, T., Robinson, E., Hacker, C., Harwell, J., Yacoub, E., Ugurbil, K., Andersson, J., Beckmann, C., Jenkinson, M., Smith, S., Essen, D. (2016). A multi-modal parcellation of human cerebral cortex. Nature 536(7615), 171178. doi:10.1038/nature18933

Glasser, M., Sotiropoulos, S., Wilson, J., Coalson, T., Fischl, B., Andersson, J., Xu, J., Jbabdi, S., Webster, M., Polimeni, J., Essen, D., Jenkinson, M., WU-Minn HCP Consortium. (2013). The minimal preprocessing pipelines for the Human Connectome Project. NeuroImage 80, 105-124. doi:10.1016/j.neuroimage.2013.04.127

Glausier JR, Lewis DA. (2013). Dendritic spine pathology in schizophrenia. Neuroscience. 251:90-107.

Gogtay N, Giedd JN, Lusk L, Hayashi KM, Greenstein DK, Vaituzis AC, Nugent TF, Herman DH, Clasen LS, Toga AW, Rapoport JL, Thompson PM. (2004). Dynamic mapping of human cortical development during childhood through early adulthood. Proc Natl Acad Sci USA. 101:8174-8179.

Goldner, E., Hsu, L., Waraich, P., Somers, J. (2002). Prevalence and Incidence Studies of Schizophrenic Disorders: A Systematic Review of the Literature. The Canadian Journal of Psychiatry 47(9), 833-843. doi:10.1177/070674370204700904

Golestani, A., Goodyear, B. (2011). A Resting-State Connectivity Metric Independent of Temporal Signal-to-Noise Ratio and Signal Amplitude. Brain Connectivity 1(2), 159-167. doi:10.1089/brain.2011.0003

Gottesman, I., Gould, T. (2003). The Endophenotype Concept in Psychiatry: Etymology and Strategic Intentions. American Journal of Psychiatry 160(4), 636-645. doi:10.1176/appi.ajp.160.4.636

Grasby, K., Jahanshad, N., Painter, J., Colodro-Conde, L., Bralten, J., Hibar, D., Lind, P., Pizzagalli, F., Ching, C., McMahon, M., Shatokhina, N., Zsembik, L., Thomopoulos, S., Zhu, A., Strike, L., Agartz, I., Alhusaini, S., Almeida, M., Alnæs, D., Amlien, I., Andersson, M., Ard, T., Armstrong, N., Ashley-Koch, A., Atkins, J., Bernard, M., Brouwer, R., Buimer, E., Bülow, R., Bürger, C., Cannon, D., Chakravarty, M., Chen, Q., Cheung, J., Couvy-Duchesne, B., Dale, A., Dalvie, S., Araujo, T., Zubicaray, G., de Zwarte, S., den Braber, A., Doan, N., Dohm, K., Ehrlich, S., Engelbrecht, H., Erk, S., Fan, C., Fedko, I., Foley, S., Ford, J., Fukunaga, M., Garrett, M., Ge, T., Giddaluru, S., Goldman, A., Green, M., Groenewold, N., Grotegerd, D., Gurholt, T., Gutman, B., Hansell, N., Harris, M., Harrison, M., Haswell, C., Hauser, M., Herms, S., Heslenfeld, D., Ho, N., Hoehn, D., Hoffmann, P., Holleran, L., Hoogman, M., Hottenga, J., Ikeda, M., Janowitz, D., Jansen, I., Jia, T., Jockwitz, C., Kanai, R., Karama, S., Kasperaviciute, D., Kaufmann, T., Kelly, S., Kikuchi, M., Klein, M., Knapp, M., Knodt, A., Krämer, B., Lam, M., Lancaster, T., Lee, P., Lett, T., Lewis, L., Lopes-Cendes, I., Luciano, M., Macciardi, F., Marquand, A., Mathias, S., Melzer, T., Milaneschi, Y., Mirza-Schreiber, N., Moreira, J., Mühleisen, T., Müller-Myhsok, B., Najt, P., Nakahara, S., Nho, K., Loohuis, L., Orfanos, D., Pearson, J., Pitcher, T., Pütz, B., Quidé, Y., Ragothaman, A., Rashid, F., Reay, W., Redlich, R., Reinbold, C., Repple, J., Richard, G., Riedel, B., Risacher, S., Rocha, C., Mota, N., Salminen, L., Saremi, A., Saykin, A., Schlag, F., Schmaal, L., Schofield, P., Secolin, R., Shapland, C., Shen, L., Shin, J., Shumskaya, E., Sønderby, I., Sprooten, E., Tansey, K., Teumer, A., Thalamuthu, A., Tordesillas-Gutiérrez, D., Turner, J., Uhlmann, A., Vallerga, C., Meer, D., Donkelaar, M., Eijk, L., van Erp, T., van Haren, N., Rooij, D., Tol, M., Veldink, J., Verhoef, E., Walton, E., Wang, M., Wang, Y., Wardlaw, J., Wen, W., Westlye, L., Whelan, C., Witt, S., Wittfeld, K., Wolf, C., Wolfers, T., Wu, J., Yasuda, C., Zaremba, D., Zhang, Z., Zwiers, M., Artiges, E., Assareh, A., Ayesa-Arriola, R., Belger, A., Brandt, C., Brown, G., Cichon, S., Curran, J., Davies, G., Degenhardt, F., Dennis, M., Dietsche, B., Djurovic, S., Doherty, C., Espiritu, R., Garijo, D., Gil, Y., Gowland, P., Green, R., Häusler, A., Heindel, W., Ho, B., Hoffmann, W., Holsboer, F., Homuth, G., Hosten, N., Jack, C., Jang, M., Jansen, A., Kimbrel, N., Kolskår, K., Koops, S., Krug, A., Lim, K., Luykx, J., Mathalon, D., Mather, K., Mattay, V., Matthews, S., Son, J., McEwen, S., Melle, I., Morris, D., Mueller, B., Nauck, M., Nordvik, J., Nöthen, M., O’Leary, D., Opel, N., Martinot, M., Pike, G., Preda, A., Quinlan, E., Rasser, P., Ratnakar, V., Reppermund, S., Steen, V., Tooney, P., Torres, F., Veltman, D., Voyvodic, J., Whelan, R., White, T., Yamamori, H., Adams, H., Bis, J., Debette, S., Decarli, C., Fornage, M., Gudnason, V., Hofer, E., Ikram, M., Launer, L., Longstreth, W., Lopez, O., Mazoyer, B., Mosley, T., Roshchupkin, G., Satizabal, C., Schmidt, R., Seshadri, S., Yang, Q., Alzheimer's Disease Neuroimaging Initiative, CHARGE Consortium, EPIGEN Consortium, IMAGEN Consortium, SYS Consortium, Parkinson's Progression Markers Initiative, Alvim, M., Ames, D., Anderson, T., Andreassen, O., Arias-Vasquez, A., Bastin, M., Baune, B., Beckham, J., Blangero, J., Boomsma, D., Brodaty, H., Brunner, H., Buckner, R., Buitelaar, J., Bustillo, J., Cahn, W., Cairns, M., Calhoun, V., Carr, V., Caseras, X., Caspers, S., 
Cavalleri, G., Cendes, F., Corvin, A., Crespo-Facorro, B., Dalrymple-Alford, J., Dannlowski, U., Geus, E., Deary, I., Delanty, N., Depondt, C., Desrivières, S., Donohoe, G., Espeseth, T., Fernández, G., Fisher, S., Flor, H., Forstner, A., Francks, C., Franke, B., Glahn, D., Gollub, R., Grabe, H., Gruber, O., Håberg, A., Hariri, A., Hartman, C., Hashimoto, R., Heinz, A., Henskens, F., Hillegers, M., Hoekstra, P., Holmes, A., Hong, L., Hopkins, W., Hulshoff Pol, H.E., Jernigan, T., Jönsson, E., Kahn, R., Kennedy, M., Kircher, T., Kochunov, P., Kwok, J., Hellard, S., Loughland, C., Martin, N., Martinot, J., McDonald, C., McMahon, K., Meyer-Lindenberg, A., Michie, P., Morey, R., Mowry, B., Nyberg, L., Oosterlaan, J., Ophoff, R., Pantelis, C., Paus, T., Pausova, Z., Penninx, B., Polderman, T., Posthuma, D., Rietschel, M., Roffman, J., Rowland, L., Sachdev, P., Sämann, P., Schall, U., Schumann, G., Scott, R., Sim, K., Sisodiya, S., Smoller, J., Sommer, I., Pourcain, B., Stein, D., Toga, A., Trollor, J., Wee, N., Ent, D., Völzke, H., Walter, H., Weber, B., Weinberger, D., Wright, M., Zhou, J., Stein, J., Thompson, P., Medland, S., group, E. (2020). The genetic architecture of the human cerebral cortex. Science 367(6484), eaay6690. doi:10.1126/science.aay6690

Grayson, D.S., Fair, D.A., (2017). Development of large-scale functional networks from birth to adulthood: A guide to the neuroimaging literature. NeuroImage 160, 15-31. doi:10.1016/j.neuroimage.2017.01.079

Grayson, D.S., Ray, S., Carpenter, S., Iyer, S., Dias, T.G.C., Stevens, C., Nigg, J.T., Fair, D.A., (2014). Structural and functional rich club organization of the brain in children and adults. PLoS ONE 9, e88297. doi:10.1371/journal.pone.0088297

Green, M. (1996). What are the functional consequences of neurocognitive deficits in schizophrenia? American Journal of Psychiatry 153(3), 321-330. doi:10.1176/ajp.153.3.321

Greenstein DK, Lerch JP, Shaw PW, Clasen LS, Giedd JN, Gochman P, Rapoport JL, Gogtay N. (2006). Childhood onset schizophrenia: cortical brain abnormalities as young adults. Journal of Child Psychology and Psychiatry 47(10), 10031012. doi:10.1111/j.1469-7610.2006.01658.x

Greicius, M., Krasnow, B., Reiss, A., Menon, V. (2002). Functional connectivity in the resting brain: A network analysis of the default mode hypothesis. Proceedings of the National Academy of Sciences 100(1), 253-258. doi:10.1073/pnas.0135058100

Griffanti, L., Salimi-Khorshidi, G., Beckmann, C., Auerbach, E., Douaud, G., Sexton, C., Zsoldos, E., Ebmeier, K., Filippini, N., Mackay, C., Moeller, S., Xu, J., Yacoub, E., Baselli, G., Ugurbil, K., Miller, K., Smith, S. (2014). ICA-based artefact removal and accelerated fMRI acquisition for improved resting state network imaging. NeuroImage 95, 232-247. doi:10.1016/j.neuroimage.2014.03.034

Gu, S., Satterthwaite, T.D., Medaglia, J.D., Yang, M., Gur, R.E., Gur, R.C., Bassett, D.S., (2015). Emergence of system roles in normative neurodevelopment. Proc Natl Acad Sci USA 201502829. doi:10.1073/pnas.1502829112

Gur, R., Gur, R. (2017). Complementarity of sex differences in brain and behavior: From laterality to multimodal neuroimaging. Journal of Neuroscience Research 95(1-2), 189-199. doi:10.1002/jnr.23830

Gur, R.C., Richard, J., Calkins, M.E., Chiavacci, R., Hansen, J.A., Bilker, W.B., Loughead, J., Connolly, J.J., Qiu, H., Mentch, F.D., Abou-Sleiman, P.M., Hakonarson, H., Gur, R.E., (2012). Age group and sex differences in performance on a computerized neurocognitive battery in children age 8-21. Neuropsychology 26, 251-265. doi:10.1037/a0026712

Gur, R.E., Gur, R.C., (2016). Sex differences in brain and behavior in adolescence: Findings from the Philadelphia Neurodevelopmental Cohort. Neuroscience \& Biobehavioral Reviews 70, 159-170. doi:10.1016/j.neubiorev.2016.07.035

Hager, B., Keshavan, M. (2015). Neuroimaging Biomarkers for Psychosis. Current Behavioral Neuroscience Reports 2(2), 102-111. doi:10.1007/s40473-015-0035-4

Haijma, S., van Haren, N., Cahn, W., Koolschijn, P., Hulshoff Pol, H.E., Kahn, R. (2013). Brain Volumes in Schizophrenia: A Meta-Analysis in Over 18000 Subjects. Schizophrenia Bulletin 39(5), 1129-1138. doi:10.1093/schbul/sbs118

Hajek T, Franke K, Kolenic M, Capkova J, Matejka M, Propper L, Uher R, Stopkova P, Novak T, Paus T, Kopecek M, Spaniel F, Alda M. (2019). Brain Age in Early Stages of Bipolar Disorders or Schizophrenia. Schizophr Bull. 45:190-198.

Haller, S., Mills, K., Hartwright, C., David, A., Kadosh, K. (2018). When change is the only constant: The promise of longitudinal neuroimaging in understanding social anxiety disorder. Developmental Cognitive Neuroscience 33, 73-82. doi:10.1016/j.den.2018.05.005 
Han LKM, Aghajani M, Clark SL, Chan RF, Hattab MW, Shabalin AA, Zhao M, Kumar G, Xie LY, Jansen R, Milaneschi Y, Dean B, Aberg KA, van den Oord EJCG, Penninx BWJH. (2018). Epigenetic aging in major depressive disorder. AJP. 175(8), 774-782. doi:10.1176/appi.ajp.2018.17060595

Han, L., Dinga, R., Hahn, T., Ching, C., Eyler, L., Aftanas, L., Aghajani, M., Aleman, A., Baune, B., Berger, K., Brak, I., Filho, G., Carballedo, A., Connolly, C., Couvy-Duchesne, B., Cullen, K., Dannlowski, U., Davey, C., Dima, D., Duran, F., Enneking, V., Filimonova, E., Frenzel, S., Frodl, T., Fu, C., Godlewska, B., Gotlib, I., Grabe, H., Groenewold, N., Grotegerd, D., Gruber, O., Hall, G., Harrison, B., Hatton, S., Hermesdorf, M., Hickie, I., Ho, T., Hosten, N., Jansen, A., Kähler, C., Kircher, T., Klimes-Dougan, B., Krämer, B., Krug, A., Lagopoulos, J., Leenings, R., MacMaster, F., MacQueen, G., McIntosh, A., McLellan, Q., McMahon, K., Medland, S., Mueller, B., Mwangi, B., Osipov, E., Portella, M., Pozzi, E., Reneman, L., Repple, J., Rosa, P., Sacchet, M., Sämann, P., Schnell, K., Schrantee, A., Simulionyte, E., Soares, J., Sommer, J., Stein, D., Steinsträter, O., Strike, L., Thomopoulos, S., Tol, M., Veer, I., Vermeiren, R., Walter, H., Wee, N., Werff, S., Whalley, H., Winter, N., Wittfeld, K., Wright, M., Wu, M., Völzke, H., Yang, T., Zannias, V., Zubicaray, G., ZuntaSoares, G., Abé, C., Alda, M., Andreassen, O., Bøen, E., Bonnin, C., Canales-Rodriguez, E., Cannon, D., Caseras, X., Chaim-Avancini, T., Elvsåshagen, T., Favre, P., Foley, S., Fullerton, J., Goikolea, J., Haarman, B., Hajek, T., Henry, C., Houenou, J., Howells, F., Ingvar, M., Kuplicki, R., Lafer, B., Landén, M., Machado-Vieira, R., Malt, U., McDonald, C., Mitchell, P., Nabulsi, L., Otaduy, M., Overs, B., Polosan, M., Pomarol-Clotet, E., Radua, J., Rive, M., Roberts, G., Ruhe, H., Salvador, R., Sarró, S., Satterthwaite, T., Savitz, J., Schene, A., Schofield, P., Serpa, M., Sim, K., Soeiro-de-Souza, M., Sutherland, A., Temmingh, H., Timmons, G., Uhlmann, A., Vieta, E., Wolf, D., Zanetti, M., Jahanshad, N., Thompson, P., Veltman, D., Penninx, B., Marquand, A., Cole, J., Schmaal, L. (2020). Brain aging in major depressive disorder: results from the ENIGMA major depressive disorder working group. Molecular Psychiatry doi:10.1038/s41380-020-0754-0

Handwerker, D., Roopchansingh, V., Gonzalez-Castillo, J., Bandettini, P. (2012). Periodic changes in fMRI connectivity. NeuroImage 63(3), 1712-1719. doi:10.1016/j.neuroimage.2012.06.078

Hannon, E., Dempster, E., Viana, J., Burrage, J., Smith, A., Macdonald, R., Clair, D., Mustard, C., Breen, G., Therman, S., Kaprio, J., Toulopoulou, T., Hulshoff Pol, H.E., Bohlken, M., Kahn, R., Nenadic, I., Hultman, C., Murray, R., Collier, D., Bass, N., Gurling, H., McQuillin, A., Schalkwyk, L., Mill, J. (2016). An integrated genetic-epigenetic analysis of schizophrenia: evidence for co-localization of genetic associations and differential DNA methylation. Genome Biology 17(1), 176. doi:10.1186/s13059-016-1041-x

Hannon E, Dempster E, Viana J, Burrage J, Smith AR, Macdonald R, St Clair D, Mustard C, Breen G, Therman S, Kaprio J, Toulopoulou T, Hulshoff Pol HE, Bohlken MM, Kahn RS, Nenadić I, Hultman CM, Murray RM, Collier DA, Bass N, Gurling H, McQuillin A, Schalkwyk L, Mill J. (2016). An integrated genetic-epigenetic analysis of schizophrenia: Evidence for co-localization of genetic associations and differential DNA methylation. Genome Biol. 17:176.

Hannon, E., Lunnon, K., Schalkwyk, L., Mill, J. (2015). Interindividual methylomic variation across blood, cortex, and cerebellum: implications for epigenetic studies of neurological and neuropsychiatric phenotypes. Epigenetics 10(11), 10241032. doi:10.1080/15592294.2015.1100786

Hannum G, Guinney J, Zhao L, Zhang L, Hughes G, Sadda SV, Klotzle B, Bibikova M, Fan J-B, Gao Y, Deconde R, Chen M, Rajapakse I, Friend S, Ideker T, Zhang K. (2013). Genome-wide Methylation Profiles Reveal Quantitative Views of Human Aging Rates. Molecular Cell 49(2), 359-367. doi:10.1016/j.molcel.2012.10.016

Hart, B., Cribben, I., Fiecas, M.J.A., Alzheimer's Disease Neuroimaging Initiative. (2018). A longitudinal model for functional connectivity networks using resting-state fMRI. NeuroImage 178, 687-701. doi:10.1016/j.neuroimage.2018.05.071

Hawrylycz, M., Miller, J.A., Menon, V., Feng, D., Dolbeare, T., Guillozet-Bongaarts, A.L., Jegga, A.G., Aronow, B.J., Lee, C.-K., Bernard, A., Glasser, M.F., Dierker, D.L., Menche, J., Szafer, A., Collman, F., Grange, P., Berman, K.A., Mihalas, S., Yao, Z., Stewart, L., Barabási, A.-L., Schulkin, J., Phillips, J.P., Ng, L., Dang, C., Haynor, D.R., Jones, A., Van Essen, D.C., Koch, C., Lein, E.S., (2015). Canonical genetic signatures of the adult human brain. Nat Neurosci 18, $1832-1844$. doi:10.1038/nn.4171 
He J, Kong J, Tan Q-R, Li X-M. (2009). Neuroprotective effect of atypical antipsychotics in cognitive and non-cognitive behavioral impairment in animal models. Cell Adh Migr. 3:129-137.

Hearne, L., Mattingley, J., Cocchi, L. (2016). Functional brain networks related to individual differences in human intelligence at rest. Scientific Reports 6(1), 32328. doi:10.1038/srep32328

Hedman AM, van Haren NEM, Schnack HG, Kahn RS, Hulshoff Pol HE. (2012). Human brain changes across the life span: A review of 56 longitudinal magnetic resonance imaging studies. Human Brain Mapping 33(8), 1987-2002. doi:10.1002/hbm.21334

Hedman AM, van Haren NEM, van Baal GCM, Brouwer RM, Brans RGH, Schnack HG, Kahn RS, Hulshoff Pol HE. (2016). Heritability of cortical thickness changes over time in twin pairs discordant for schizophrenia. Schizophrenia Research 173(3), 192-199. doi:10.1016/j.schres.2015.06.021

Heidemann, R., Özsarlak, Ò., Parizel, P., Michiels, J., Kiefer, B., Jellus, V., Müller, M., Breuer, F., Blaimer, M., Griswold, M., Jakob, P. (2003). A brief review of parallel magnetic resonance imaging. European Radiology 13(10), $2323-2337$. doi:10.1007/s00330-003-1992-7

Heinrichs, R., Zakzanis, K. (1998). Neurocognitive Deficit in Schizophrenia: A Quantitative Review of the Evidence. Neuropsychology 12(3), 426-445. doi:10.1037/0894-4105.12.3.426

Herting, M., Johnson, C., Mills, K., Vijayakumar, N., Dennison, M., Liu, C., Goddings, A., Dahl, R., Sowell, E., Whittle, S., Allen, N., Tamnes, C. (2018). Development of subcortical volumes across adolescence in males and females: A multisample study of longitudinal changes. NeuroImage 172, 194-205. doi:10.1016/j.neuroimage.2018.01.020

Herting, M.M., Maxwell, E.C., Irvine, C., Nagel, B.J., (2012). The impact of sex, puberty, and hormones on white matter microstructure in adolescents. Cereb. Cortex 22, 1979-1992. doi:10.1093/cercor/bhr246

Hilger, K., Fukushima, M., Sporns, O., Fiebach, C. (2020). Temporal stability of functional brain modules associated with human intelligence. Human Brain Mapping 41(2), 362-372. doi:10.1002/hbm.24807

Hilker, R., Helenius, D., Fagerlund, B., Skytthe, A., Christensen, K., Werge, T., Nordentoft, M., Glenthøj, B. (2018). Heritability of Schizophrenia and Schizophrenia Spectrum Based on the Nationwide Danish Twin Register. Biological Psychiatry 83(6), 492-498. doi:10.1016/j.biopsych.2017.08.017

Hillary RF, Stevenson AJ, Cox SR, McCartney DL, Harris SE, Seeboth A, Higham J, Sproul D, Taylor AM, Redmond P, Corley J, Pattie A, Valdés Hernández MDC, Muñoz-Maniega S, Bastin ME, Wardlaw JM, Horvath S, Ritchie CW, Spires-Jones TL, McIntosh AM, Evans KL, Deary IJ, Marioni RE. (2019). An epigenetic predictor of death captures multimodal measures of brain health. bioRxiv. 10:573.

Hirschhorn, J., Daly, M. (2005). Genome-wide association studies for common diseases and complex traits. Nature Reviews Genetics 6(2), 95-108. doi:10.1038/nrg1521

Hjorthøj, C., Stürup, A., McGrath, J., Nordentoft, M. (2017). Years of potential life lost and life expectancy in schizophrenia: a systematic review and meta-analysis. The Lancet Psychiatry 4(4), 295-301. doi:10.1016/s2215-0366(17)30078-0

Hodkinson, D., O'Daly, O., Zunszain, P., Pariante, C., Lazurenko, V., Zelaya, F., Howard, M., Williams, S. (2014). Circadian and Homeostatic Modulation of Functional Connectivity and Regional Cerebral Blood Flow in Humans under Normal Entrained Conditions. Journal of Cerebral Blood Flow \& Metabolism 34(9), 1493-1499. doi:10.1038/jcbfm.2014.109

Hohenfeld, C., Werner, C., Reetz, K. (2018). Resting-state connectivity in neurodegenerative disorders: Is there potential for an imaging biomarker? NeuroImage: Clinical 18, 849-870. doi:10.1016/j.nicl.2018.03.013

Horvath, S. (2013). DNA methylation age of human tissues and cell types. Genome Biology 14(10), 3156. doi:10.1186/gb2013-14-10-r115

Horvath S, Gurven M, Levine ME, Trumble BC, Kaplan H, Allayee H, Ritz BR, Chen B, Lu AT, Rickabaugh TM, Jamieson BD, Sun D, Li S, Chen W, Quintana-Murci L, Fagny M, Kobor MS, Tsao PS, Reiner AP, Edlefsen KL, Absher D, Assimes TL. (2016). An epigenetic clock analysis of race/ethnicity, sex, and coronary heart disease. Genome Biol. 17:171.

Horvath S, Zhang Y, Langfelder P, Kahn RS, Boks MPM, van Eijk K, van den Berg LH, Ophoff RA. (2012). Aging effects on DNA methylation modules in human brain and blood tissue. Genome Biol. 13:R97-18. 
Hu, L., Bentler, P. (1999). Cutoff criteria for fit indexes in covariance structure analysis: Conventional criteria versus new alternatives. Structural Equation Modeling: A Multidisciplinary Journal 6(1), 1-55. doi:10.1080/10705519909540118

Hulshoff Pol, H.E., Baal, G., Schnack, H., Brans, R., Schot, A., Brouwer, R., van Haren, N., Lepage, C., Collins, D., Evans, A., Boomsma, D., Nolen, W., Kahn, R. (2012). Overlapping and Segregating Structural Brain Abnormalities in Twins With Schizophrenia or Bipolar Disorder. Archives of General Psychiatry 69(4), 349. doi:10.1001/archgenpsychiatry.2011.1615

Hulshoff Pol, H.E., Brans, R., van Haren, N., Schnack, H., Langen, M., Baaré, W., Oel, C., Kahn, R. (2004). Gray and white matter volume abnormalities in monozygotic and same-gender dizygotic twins discordant for schizophrenia. Biological Psychiatry 55(2), 126-130. doi:10.1016/s0006-3223(03)00728-5

Hulshoff Pol, H.E, Kahn, R. (2008). What Happens After the First Episode? A Review of Progressive Brain Changes in Chronically Ill Patients With Schizophrenia. Schizophrenia Bulletin 34(2), 354-366. doi:10.1093/schbul/sbm168

Hulshoff Pol, H.E., Schnack, H., Posthuma, D., Mandl, R., Baaré, W., Oel, C., van Haren, N., Collins, D., Evans, A., Amunts, K., Bürgel, U., Zilles, K., Geus, E., Boomsma, D., Kahn, R. (2006). Genetic Contributions to Human Brain Morphology and Intelligence. The Journal of Neuroscience 26(40), 10235-10242. doi:10.1523/jneurosci.1312-06.2006

Hutchison, R., Womelsdorf, T., Allen, E., Bandettini, P., Calhoun, V., Corbetta, M., Penna, S., Duyn, J., Glover, G., Gonzalez-Castillo, J., Handwerker, D., Keilholz, S., Kiviniemi, V., Leopold, D., Pasquale, F., Sporns, O., Walter, M., Chang, C. (2013). Dynamic functional connectivity: Promise, issues, and interpretations. NeuroImage 80, 360-378. doi:10.1016/j.neuroimage.2013.05.079

Huttenlocher PR. (1979). Synaptic density in human frontal cortex - Developmental changes and effects of aging. Brain Research. 163:195-205.

Huttenlocher PR, Dabholkar AS. (1997). Regional differences in synaptogenesis in human cerebral cortex. J Comp Neurol. 387:167-178.

Hwang, K., Hallquist, M.N., Luna, B., (2013). The development of hub architecture in the human functional brain network. Cereb. Cortex 23, 2380-2393. doi:10.1093/cercor/bhs227

Hyvarinen, A. (1999). Fast and robust fixed-point algorithms for independent component analysis. IEEE Transactions on Neural Networks 10(3), 626-634. doi:10.1109/72.761722

Ingalhalikar, M., Smith, A., Parker, D., Satterthwaite, T.D., Elliott, M.A., Ruparel, K., Hakonarson, H., Gur, R.E., Gur, R.C., Verma, R., (2014). Sex differences in the structural connectome of the human brain. Proc. Natl. Acad. Sci. U.S.A. 111, 823828. doi:10.1073/pnas.1316909110

Ismaylova, E., Sante, J., Gouin, J., Pomares, F., Vitaro, F., Tremblay, R., Booij, L. (2018). Associations Between Daily Mood States and Brain Gray Matter Volume, Resting-State Functional Connectivity and Task-Based Activity in Healthy Adults. Frontiers in Human Neuroscience 12, 168. doi:10.3389/fnhum.2018.00168

Jacobus J, Squeglia LM, Meruelo AD, Castro N, Brumback T, Giedd JN, Tapert SF. (2015). Cortical thickness in adolescent marijuana and alcohol users: A three-year prospective study from adolescence to young adulthood. Dev Cogn Neurosci. 16:101-109.

Jaenisch, R., Bird, A. (2003). Epigenetic regulation of gene expression: how the genome integrates intrinsic and environmental signals. Nature Genetics 33(Suppl 3), 245-254. doi:10.1038/ng1089

Jaffe AE, Gao Y, Deep-Soboslay A, Tao R, Hyde TM, Weinberger DR, Kleinman JE. (2015). Mapping DNA methylation across development, genotype and schizophrenia in the human frontal cortex. Nat Neurosci. 19:40-47.

Jaffe AE, Shin J, Collado-Torres L, Leek JT, Tao R, Li C, Gao Y, Jia Y, Maher BJ, Hyde TM, Kleinman JE, Weinberger DR. (2015). Developmental regulation of human cortex transcription and its clinical relevance at single base resolution. Nature Neuroscience 18(1), 154-161. doi:10.1038/nn.3898

Jalbrzikowski, M., Larsen, B., Hallquist, M.N., Foran, W., Calabro, F., Luna, B., (2017). Development of White Matter Microstructure and Intrinsic Functional Connectivity Between the Amygdala and Ventromedial Prefrontal Cortex: Associations With Anxiety and Depression. Biological Psychiatry 82(7), 511-521. doi:10.1016/j.biopsych.2017.01.008 
Jansen, A.G., Mous, S.E., White, T.J.H., Posthuma, D., Polderman, T.J.C., (2015). What Twin Studies Tell Us About the Heritability of Brain Development, Morphology, and Function: A Review. Neuropsychology Review 25(1), 27-46. doi:10.1007/s11065-015-9278-9

Jeon S, Lepage C, Lewis L, Khalili-Mahani N, Bermudez P, Vincent R, Zijdenbos AP, Omidyeganeh M, Adalat R, Evans AC. (2017). Reproducibility of Cortical Thickness Measurement: CIVET (v2.1) vs. Freesurfer (v6.0-beta \& v5.3). On Human Brain Mapping Symposium.

Jeste DV, Wolkowitz OM, Palmer BW. (2011). Divergent trajectories of physical, cognitive, and psychosocial aging in schizophrenia. Schizophr Bull. 37:451-455.

Jia, T., Chu, C., Liu, Y., van Dongen, J., Papastergios, E., Armstrong, N., Bastin, M., Carrillo-Roa, T., den Braber, A., Harris, M., Jansen, R., Liu, J., Luciano, M., Ori, A., Santiañez, R., Ruggeri, B., Sarkisyan, D., Shin, J., Sungeun, K., Gutiérrez, D., Ent, D., Ames, D., Artiges, E., Bakalkin, G., Banaschewski, T., Bokde, A., Brodaty, H., Bromberg, U., Brouwer, R., Büchel, C., Quinlan, E., Cahn, W., Zubicaray, G., Ehrlich, S., Ekström, T., Flor, H., Fröhner, J., Frouin, V., Garavan, H., Gowland, P., Heinz, A., Hoare, J., Ittermann, B., Jahanshad, N., Jiang, J., Kwok, J., Martin, N., Martinot, J., Mather, K., McMahon, K., McRae, A., Nees, F., Orfanos, D., Paus, T., Poustka, L., Sämann, P., Schofield, P., Smolka, M., Stein, D., Strike, L., Teeuw, J., Thalamuthu, A., Trollor, J., Walter, H., Wardlaw, J., Wen, W., Whelan, R., Apostolova, L., Binder, E., Boomsma, D., Calhoun, V., Crespo-Facorro, B., Deary, I., Hulshoff Pol, H.E., Ophoff, R., Pausova, Z., Sachdev, P., Saykin, A., Wright, M., Thompson, P., Schumann, G., Desrivières, S. (2019). Epigenome-wide meta-analysis of blood DNA methylation and its association with subcortical volumes: findings from the ENIGMA Epigenetics Working Group. Molecular Psychiatry doi:10.1038/s41380-019-0605-z

Johansen-Berg, H., Behrens, T. (2013). Diffusion MRI, 2nd edition. Academic Press.

Johnson, M. (2001). Functional brain development in humans. Nature Reviews Neuroscience 2(7), $475-483$. doi:10.1038/35081509

Johnson, M., Grossmann, T., Kadosh, K. (2009). Mapping Functional Brain Development: Building a Social Brain Through Interactive Specialization. Developmental Psychology 45(1), 151-159. doi:10.1037/a0014548

Jonsson BA, Bjornsdottir G, Thorgeirsson TE, Ellingsen LM, Bragi Walters G, Gudbjartsson DF, Stefansson H, Stefansson K, Ulfarsson MO. (2019). Deep learning based brain age prediction uncovers associated sequence variants. bioRxiv. 475:S2.

Jung, R.E., Haier, R.J., (2007). The Parieto-Frontal Integration Theory (P-FIT) of intelligence: converging neuroimaging evidence. Behav Brain Sci 30, 135-54- discussion 154-87. doi:10.1017/S0140525X07001185

Jylhävä, J., Pedersen, N.L., Hägg, S. (2017). Biological Age Predictors. EBioMedicine 21, 29-36. doi:10.1016/j.ebiom.2017.03.046

Kabacik S, Horvath S, Cohen H, Raj K. (2018). Epigenetic ageing is distinct from senescence-mediated ageing and is not prevented by telomerase expression. Aging. 10:2800-2815.

Kang HJ, Kawasawa YI, Cheng F, Zhu Y, Xu X, Li M, Sousa AMM, Pletikos M, Meyer KA, Sedmak G, Guennel T, Shin Y, Johnson MB, Krsnik Z, Mayer S, Fertuzinhos S, Umlauf S, Lisgo SN, Vortmeyer A, Weinberger DR, Mane S, Hyde TM, Huttner A, Reimers M, Kleinman JE, Sestan N. (2011). Spatio-temporal transcriptome of the human brain. Nature 478(7370), 483-489. doi:10.1038/nature10523

Karama S, Ducharme S, Corley J, Chouinard-Decorte F, Starr JM, Wardlaw JM, Bastin ME, Deary IJ. (2015). Cigarette smoking and thinning of the brain's cortex. Molecular Psychiatry. 20:778-785.

Kaufmann T, van der Meer D, Doan NT, Schwarz E, Lund MJ, Agartz I, Alnæs D, Barch DM, Baur-Streubel R, Bertolino A, Bettella F, Beyer MK, Bøen E, Borgwardt S, Brandt CL, Buitelaar J, Celius EG, Cervenka S, Conzelmann A, CórdovaPalomera A, Dale AM, de Quervain DJF, Carlo PD, Djurovic S, Dørum ES, Eisenacher S, Elvsåshagen T, Espeseth T, Fatouros-Bergman H, Flyckt L, Franke B, Frei O, Haatveit B, Håberg AK, Harbo HF, Hartman CA, Heslenfeld D, Hoekstra PJ, Høgestøl EA, Jernigan TL, Jonassen R, Jönsson EG, Kirsch P, Kłoszewska I, Kolskår KK, Landrø NI, Hellard SL, Lesch K-P, Lovestone S, Lundervold A, Lundervold AJ, Maglanoc LA, Malt UF, Mecocci P, Melle I, MeyerLindenberg A, Moberget T, Norbom LB, Nordvik JE, Nyberg L, Oosterlaan J, Papalino M, Papassotiropoulos A, Pauli P, Pergola G, Persson K, Richard G, Rokicki J, Sanders A-M, Selbæk G, Shadrin AA, Smeland OB, Soininen H, Sowa P, 
Steen VM, Tsolaki M, Ulrichsen KM, Vellas B, Wang L, Westman E, Ziegler GC, Zink M, Andreassen OA, Westlye LT. (2019). Common brain disorders are associated with heritable patterns of apparent aging of the brain. Nature Neuroscience 22(10), 1617-1623. doi:10.1038/s41593-019-0471-7

Kelly, A.M.C., Di Martino, A., Uddin, L.Q., Shehzad, Z.E., Gee, D.G., Reiss, P.T., Margulies, D.S., Castellanos, F.X., Milham, M.P., (2009). Development of Anterior Cingulate Functional Connectivity from Late Childhood to Early Adulthood. Cerebral Cortex 19(3), 640-657. doi:10.1093/cercor/bhn117

Kelly, S., Jahanshad, N., Zalesky, A., Kochunov, P., Agartz, I., Alloza, C., Andreassen, O., Arango, C., Banaj, N., Bouix, S., Bousman, C., Brouwer, R., Bruggemann, J., Bustillo, J., Cahn, W., Calhoun, V., Cannon, D., Carr, V., Catts, S., Chen, J., Chen, J., Chen, X., Chiapponi, C., Cho, K., Ciullo, V., Corvin, A., Crespo-Facorro, B., Cropley, V., Rossi, P., Diaz-Caneja, C., Dickie, E., Ehrlich, S., Fan, F., Faskowitz, J., Fatouros-Bergman, H., Flyckt, L., Ford, J., Fouche, J., Fukunaga, M., Gill, M., Glahn, D., Gollub, R., Goudzwaard, E., Guo, H., Gur, R., Gur, R., Gurholt, T., Hashimoto, R., Hatton, S., Henskens, F., Hibar, D., Hickie, I., Hong, L., Horacek, J., Howells, F., Hulshoff Pol, H.E, Hyde, C., Isaev, D., Jablensky, A., Jansen, P., Janssen, J., Jönsson, E., Jung, L., Kahn, R., Kikinis, Z., Liu, K., Klauser, P., Knöchel, C., Kubicki, M., Lagopoulvan Os, J., Langen, C., Lawrie, S., Lenroot, R., Lim, K., Lopez-Jaramillo, C., Lyall, A., Magnotta, V., Mandl, R., Mathalon, D., McCarley, R., McCarthy-Jones, S., McDonald, C., McEwen, S., McIntosh, A., Melicher, T., Mesholam-Gately, R., Michie, P., Mowry, B., Mueller, B., Newell, D., O'Donnell, P., Oertel-Knöchel, V., Oestreich, L., Paciga, S., Pantelis, C., Pasternak, O., Pearlson, G., Pellicano, G., Pereira, A., Zapata, J., Piras, F., Potkin, S., Preda, A., Rasser, P., Roalf, D., Roiz, R., Roos, A., Rotenberg, D., Satterthwaite, T., Savadjiev, P., Schall, U., Scott, R., Seal, M., Seidman, L., Weickert, C., Whelan, C., Shenton, M., Kwon, J., Spalletta, G., Spaniel, F., Sprooten, E., Stäblein, M., Stein, D., Sundram, S., Tan, Y., Tan, S., Tang, S., Temmingh, H., Westlye, L., Tønnesen, S., Tordesillas-Gutierrez, D., Doan, N., Vaidya, J., van Haren, N., Vargas, C., Vecchio, D., Velakoulis, D., Voineskos, A., Voyvodic, J., Wang, Z., Wan, P., Wei, D., Weickert, T., Whalley, H., White, T., Whitford, T., Wojcik, J., Xiang, H., Xie, Z., Yamamori, H., Yang, F., Yao, N., Zhang, G., Zhao, J., van Erp, T., Turner, J., Thompson, P., Donohoe, G. (2017). Widespread white matter microstructural differences in schizophrenia across 4322 individuals: results from the ENIGMA Schizophrenia DTI Working Group. Molecular Psychiatry 23(5), 1261-1269. doi:10.1038/mp.2017.170

Kennedy, D., Makris, N., Herbert, M., Takahashi, T., Caviness, V. (2002). Basic principles of MRI and morphometry studies of human brain development. Developmental Science 5(3), 268-278. doi:10.1111/1467-7687.00366

Keunen, K., Counsell, S.J., Benders, M.J.N.L., (2017). The emergence of functional architecture during early brain development. NeuroImage 160, 2-14. doi:10.1016/j.neuroimage.2017.01.047

Khoury LE, Gorrie-Stone T, Smart M, Hughes A, Bao Y, Andrayas A, Burrage J, Hannon E, Kumari M, Mill J, Schalkwyk LC. (2019). Properties of the epigenetic clock and age acceleration. bioRxiv.

Kim DR, Bale TL, Epperson CN. (2015). Prenatal Programming of Mental Illness: Current Understanding of Relationship and Mechanisms. Current Psychiatry Reports 17(2), 5. doi:10.1007/s11920-014-0546-9

Kim JS, Singh V, Lee JK, Lerch JP, Ad-Dab'bagh Y, MacDonald D, Lee JM, Kim SI, Evans AC. (2005). Automated 3-D extraction and evaluation of the inner and outer cortical surfaces using a Laplacian map and partial volume effect classification. NeuroImage 27(1), 210-221. doi:10.1016/j.neuroimage.2005.03.036

Kirkpatrick, B., Messias, E., Harvey, P.D., Fernandez-Egea, E., Bowie, C.R. (2008). Is Schizophrenia a Syndrome of Accelerated Aging? Schizophrenia Bulletin 34(6), 1024-1032. doi:10.1093/schbul/sbm140

Koch CM, Wagner W. (2011). Epigenetic-aging-signature to determine age in different tissues. Aging. 3:1018-1027.

Kochunov, P., Jahanshad, N., Sprooten, E., Nichols, T., Mandl, R., Almasy, L., Booth, T., Brouwer, R., Curran, J., Zubicaray, G., Dimitrova, R., Duggirala, R., Fox, P., Hong, L., Landman, B., Lemaitre, H., Lopez, L., Martin, N., McMahon, K., Mitchell, B., Olvera, R., Peterson, C., Starr, J., Sussmann, J., Toga, A., Wardlaw, J., Wright, M., Wright, S., Bastin, M., McIntosh, A., Boomsma, D., Kahn, R., den Braber, A., Geus, E., Deary, I., Hulshoff Pol, H.E., Williamson, D., Blangero, J., Ent, D., Thompson, P., Glahn, D. (2014). Multi-site study of additive genetic effects on fractional anisotropy of cerebral white matter: Comparing meta and megaanalytical approaches for data pooling. NeuroImage 95, 136-150. doi:10.1016/j.neuroimage.2014.03.033 
Koenis, M. (2017). Adolescent brain development: a longitudinal twin study into structural brain development and its relation to hormone levels and intelligence. University Utrecht, Netherlands

Koenis, M., Brouwer, R., Baal, G., van Soelen, I., Peper, J., van Leeuwen, M., Waal, H., Boomsma, D., Hulshoff Pol, H.E. (2013). Longitudinal Study of Hormonal and Physical Development in Young Twins. The Journal of Clinical Endocrinology \& Metabolism 98(3), E518-E527. doi:10.1210/jc.2012-3361

Koenis, M.M.G., Brouwer, R.M., Swagerman, S.C., van Soelen, I.L.C., Boomsma, D.I., Hulshoff Pol, H.E., (2018). Association between structural brain network efficiency and intelligence increases during adolescence. Human Brain Mapping 39(2), 822-836. doi:10.1002/hbm.23885

Koenis, M.M.G., Brouwer, R.M., van den Heuvel, M.P., Mandl, R.C.W., van Soelen, I.L.C., Kahn, R.S., Boomsma, D.I., Hulshoff Pol, H.E., (2015). Development of the brain's structural network efficiency in early adolescence: A longitudinal DTI twin study. Human Brain Mapping 36(12), 4938-4953. doi:10.1002/hbm.22988

Kofink, D., Boks, M., Timmers, H., Kas, M. (2013). Epigenetic dynamics in psychiatric disorders: Environmental programming of neurodevelopmental processes. Neuroscience \& Biobehavioral Reviews 37(5), 831-845. doi:10.1016/j.neubiorev.2013.03.020

Kolenic M, Franke K, Hlinka J, Matejka M, Capkova J, Pausova Z, Uher R, Alda M, Spaniel F, Hajek T. (2018). Obesity, dyslipidemia and brain age in first-episode psychosis. Journal of Psychiatric Research. 99:151-158.

Kong, R., Li, J., Orban, C., Sabuncu, M., Liu, H., Schaefer, A., Sun, N., Zuo, X., Holmes, A., Eickhoff, S., Yeo, B. (2018). Spatial Topography of Individual-Specific Cortical Networks Predicts Human Cognition, Personality, and Emotion. Cerebral Cortex 29(6), 2533-2551. doi:10.1093/cercor/bhy123

Koolschijn, P., Crone, E. (2013). Sex differences and structural brain maturation from childhood to early adulthood. Developmental Cognitive Neuroscience 5, 106-118. doi:10.1016/j.dcn.2013.02.003

Koolschijn, P., Peper, J., Crone, E. (2014). The Influence of Sex Steroids on Structural Brain Maturation in Adolescence. PLoS ONE 9(1), e83929. doi:10.1371/journal.pone.0083929

Korgaonkar, M.S., Ram, K., Williams, L.M., Gatt, J.M., Grieve, S.M., (2014). Establishing the resting state default mode network derived from functional magnetic resonance imaging tasks as an endophenotype: A twins study. Human Brain Mapping 35(8), 3893-3902. doi:10.1002/hbm.22446

Koutsouleris, N., Davatzikos, C., Borgwardt, S., Gaser, C., Bottlender, R., Frodl, T., Falkai, P., Riecher-Rössler, A., Möller, H.J., Reiser, M., Pantelis, C., Meisenzahl, E. (2014). Accelerated Brain Aging in Schizophrenia and Beyond: A Neuroanatomical Marker of Psychiatric Disorders. Schizophrenia Bulletin 40(5), 1140-1153. doi:10.1093/schbul/sbt142

Kowalec K, Hannon E, Mansell G, Burrage J, Ori APS, Ophoff RA, Mill J, Sullivan PF. (2019). Methylation age acceleration does not predict mortality in schizophrenia. Translational Psychiatry. 9:157.

Kramer AF, Bherer L, Colcombe SJ, Dong W, Greenough WT. (2004). Environmental influences on cognitive and brain plasticity during aging. J Gerontol A Biol Sci Med Sci. 59:940-957.

Krishnamurthy, V., Krishnamurthy, L., Schwam, D., Ealey, A., Shin, J., Greenberg, D., Morris, R. (2018). Retrospective Correction of Physiological Noise: Impact on Sensitivity, Specificity, and Reproducibility of Resting-State Functional Connectivity in a Reading Network Model. Brain Connectivity 8(2), 94-105. doi:10.1089/brain.2017.0513

Kruschwitz, J., Waller, L., Daedelow, L., Walter, H., Veer, I. (2018). General, crystallized and fluid intelligence are not associated with functional global network efficiency: A replication study with the human connectome project 1200 data set. NeuroImage 171, 323-331. doi:10.1016/j.neuroimage.2018.01.018

Kumar, S., Subramanian, S. (2002). Mutation rates in mammalian genomes. Proceedings of the National Academy of Sciences 99(2), 803-808. doi:10.1073/pnas.022629899

Kuzawa, C., Chugani, H., Grossman, L., Lipovich, L., Muzik, O., Hof, P., Wildman, D., Sherwood, C., Leonard, W., Lange, N. (2014). Metabolic costs and evolutionary implications of human brain development. Proceedings of the National Academy of Sciences 111(36), 13010-13015. doi:10.1073/pnas.1323099111 
Langeslag, S.J.E., Schmidt, M., Ghassabian, A., Jaddoe, V.W.V., Hofman, A., van der Lugt, A., Verhulst, F.C., Tiemeier, H., White, T.J.H., (2013). Functional connectivity between parietal and frontal brain regions and intelligence in young children: the Generation R study. Hum Brain Mapp 34, 3299-3307. doi:10.1002/hbm.22143

Laumann, T., Gordon, E., Adeyemo, B., Snyder, A., Joo, S., Chen, M., Gilmore, A., McDermott, K., Nelson, S., Dosenbach, N., Schlaggar, B., Mumford, J., Poldrack, R., Petersen, S. (2015). Functional System and Areal Organization of a Highly Sampled Individual Human Brain. Neuron 87(3), 657-670. doi:10.1016/j.neuron.2015.06.037

Laursen TM, Nordentoft M, Mortensen PB. (2014). Excess Early Mortality in Schizophrenia. Annual Review of Clinical Psychology 10(1), 425-448. doi:10.1146/annurev-clinpsy-032813-153657

Laursen TM, Trabjerg BB, Mors O, Børglum AD, Hougaard DM, Mattheisen M, Meier SM, Byrne EM, Mortensen PB, Munk-Olsen T, Agerbo E. (2017). Association of the polygenic risk score for schizophrenia with mortality and suicidal behavior - A Danish population-based study. Schizophrenia Research. 184:122-127.

Le Bihan, D., Mangin, J., Poupon, C., Clark, C., Pappata, S., Molko, N., Chabriat, H. (2001). Diffusion tensor imaging: Concepts and applications. Journal of Magnetic Resonance Imaging 13(4), 534-546. doi:10.1002/jmri.1076

Le TT, Kuplicki RT, McKinney BA., Yeh HW, Thompson WK, Paulus MP. (2018). A Nonlinear Simulation Framework Supports Adjusting for Age When Analyzing BrainAGE. Frontiers in Aging Neuroscience, 10(October), 1-11.

Lenroot, R., Giedd, J. (2006). Brain development in children and adolescents: Insights from anatomical magnetic resonance imaging. Neuroscience \& Biobehavioral Reviews 30(6), 718-729. doi:10.1016/j.neubiorev.2006.06.001

Lenroot, R., Gogtay, N., Greenstein, D., Wells, E., Wallace, G., Clasen, L., Blumenthal, J., Lerch, J., Zijdenbos, A., Evans, A., Thompson, P., Giedd, J. (2007). Sexual dimorphism of brain developmental trajectories during childhood and adolescence. NeuroImage 36(4), 1065-1073. doi:10.1016/j.neuroimage.2007.03.053

Lenroot, R.K., Giedd, J.N., (2006). Brain development in children and adolescents: Insights from anatomical magnetic resonance imaging. Neuroscience and Biobehavioral Reviews 30, 718-729. doi:10.1016/j.neubiorev.2006.06.001

Lenroot RK, Giedd JN. (2008). The changing impact of genes and environment on brain development during childhood and adolescence: initial findings from a neuroimaging study of pediatric twins. Dev Psychopathol. 20:1161-1175.

Lenroot RK, Giedd JN. (2010). Sex differences in the adolescent brain. Brain and Cognition 72(1), 46-55. doi:10.1016/j.bandc.2009.10.008

Lenroot RK, Schmitt JE, Ordaz SJ, Wallace GL, Neale MC, Lerch JP, Kendler KS, Evans AC, Giedd JN. (2009). Differences in genetic and environmental influences on the human cerebral cortex associated with development during childhood and adolescence. Human Brain Mapping 30(1), 163-174. doi:10.1002/hbm.20494

Lerch JP, Pruessner J, Zijdenbos AP, Collins DL, Teipel SJ, Hampel H, Evans AC. (2008). Automated cortical thickness measurements from MRI can accurately separate Alzheimer's patients from normal elderly controls. Neurobiol Aging. 29:23-30.

Lessov-Schlaggar, C., Hardin, J., DeCarli, C., Krasnow, R., Reed, T., Wolf, P., Swan, G., Carmelli, D. (2012). Longitudinal genetic analysis of brain volumes in normal elderly male twins. Neurobiology of Aging 33(4), 636-644. doi:10.1016/j.neurobiolaging.2010.06.002

Levine, M., Lu, A., Quach, A., Chen, B., Assimes, T., Bandinelli, S., Hou, L., Baccarelli, A., Stewart, J., Li, Y., Whitsel, E., Wilson, J., Reiner, A., Aviv, A., Lohman, K., Liu, Y., Ferrucci, L., Horvath, S. (2018). An epigenetic biomarker of aging for lifespan and healthspan. Aging, 10(4), 573-591. doi:10.18632/aging.101414

Lewis L, Lepage C, Khalili-Mahani N, Omidyeganeh M, Jeon S, Bermudez P, Zijdenbos AP, Vincent R, Adalat R, Evans AC. (2017). Robustness and reliability of cortical surface reconstruction in CIVET and FreeSurfer. On Human Brain Mapping Symposium.

Li S, Wong EM, Joo JHE, Jung CH, Chung J, Apicella C, Stone J, Dite GS, Giles GG, Southey MC, Hopper JL. (2015). Genetic and Environmental Causes of Variation in the Difference Between Biological Age Based on DNA Methylation and Chronological Age for Middle-Aged Women. Twin Res Hum Genet. 18:720-726.

Li Z, He Y, Ma X, Chen X. (2018). Epigenetic age analysis of brain in major depressive disorder. Psychiatry Res. 269:621624. 
Liang SG, Greenwood TA. (2015). The impact of clinical heterogeneity in schizophrenia on genomic analyses. Schizophrenia Research. 161:490-495.

Liem, F., Mérillat, S., Bezzola, L., Hirsiger, S., Philipp, M., Madhyastha, T., Jäncke, L. (2015). Reliability and statistical power analysis of cortical and subcortical FreeSurfer metrics in a large sample of healthy elderly. NeuroImage 108, 95-109. doi:10.1016/j.neuroimage.2014.12.035

Ligthart, L., Beijsterveldt, C., Kevenaar, S., Zeeuw, E., Bergen, E., Bruins, S., Pool, R., Helmer, Q., Dongen, J., Hottenga, J., Ent, D., Dolan, C., Davies, G., Ehli, E., Bartels, M., Willemsen, G., Geus, E., Boomsma, D. (2019). The Netherlands Twin Register: Longitudinal Research Based on Twin and Twin-Family Designs. Twin Research and Human Genetics 22(6), 623-636. doi:10.1017/thg.2019.93

Lin D, Chen J, Ehrlich S, Bustillo JR, Perrone-Bizzozero N, Walton E, Clark VP, Wang Y-P, Sui J, Du Y, Ho BC, Schulz CS, Calhoun VD, Liu J. (2018). Cross-Tissue Exploration of Genetic and Epigenetic Effects on Brain Gray Matter in Schizophrenia. Schizophr Bull. 44:443-452.

Lindquist, M. (2020). Neuroimaging results altered by varying analysis pipelines. Nature 582(7810), 36-37. doi:10.1038/d41586-020-01282-z

Liu, T. (2016). Noise contributions to the fMRI signal: An overview. NeuroImage 143, $141-151$. doi:10.1016/j.neuroimage.2016.09.008

Logothetis, N., Pauls, J., Augath, M., Trinath, T., Oeltermann, A. (2001). Neurophysiological investigation of the basis of the fMRI signal. Nature 412(6843), 150-157. doi:10.1038/35084005

Logothetis, N., Wandell, B. (2004). Interpreting the BOLD Signal. Annual Review of Physiology 66(1), 735-769. doi:10.1146/annurev.physiol.66.082602.092845

Long, X.-Y., Benischek, A., Dewey, D., Lebel, C., (2017). Age-related functional brain changes in young children. NeuroImage 155, 322-330. doi:10.1016/j.neuroimage.2017.04.059

Lowe D, Horvath S, Raj K. (2016). Epigenetic clock analyses of cellular senescence and ageing. Oncotarget. 7:8524-8531.

López-Vicente M, Tiemeier H, Wildeboer A, Muetzel RL, Verhulst FC, Jaddoe VWV, Sunyer J, White TJH. (2017). Cortical Structures Associated With Sports Participation in Children: A Population-Based Study. Dev Neuropsychol. 42:58-69.

Lu AT, Xue L, Salfati EL, Chen BH, Ferrucci L, Levy D, Joehanes R, Murabito JM, Kiel DP, Tsai P-C, Yet I, Bell JT, Mangino M, Tanaka T, McRae AF, Marioni RE, Visscher PM, Wray NR, Deary IJ, Levine ME, Quach A, Assimes T, Tsao PS, Absher D, Stewart JD, Li Y, Reiner AP, Hou L, Baccarelli AA, Whitsel EA, Aviv A, Cardona A, Day FR, Wareham NJ, Perry JRB, Ong KK, Raj K, Lunetta KL, Horvath S. (2018). GWAS of epigenetic aging rates in blood reveals a critical role for TERT. Nature Communications. 9:387.

Lu, F., Zhou, J., Zhang, J., Xiang, Y., Zhang, J., Liu, Q., Wang, X., Yuan, Z. (2015). Functional Connectivity Estimated from Resting-State fMRI Reveals Selective Alterations in Male Adolescents with Pure Conduct Disorder. PLOS ONE 10(12), e0145668. doi:10.1371/journal.pone.0145668

Luna, B., Sweeney, J. (2004). The Emergence of Collaborative Brain Function: fMRI Studies of the Development of Response Inhibition. Annals of the New York Academy of Sciences 1021(1), 296-309. doi:10.1196/annals.1308.035

Lyttelton O, Boucher M, Robbins S, Evans AC. (2007). An unbiased iterative group registration template for cortical surface analysis. NeuroImage. 34:1535-1544.

Mackay, T., Stone, E., Ayroles, J. (2009). The genetics of quantitative traits: challenges and prospects. Nature Reviews Genetics 10(8), 565-577. doi:10.1038/nrg2612

Madan, C., Kensinger, E. (2017). Test-retest reliability of brain morphology estimates. Brain Informatics 4(2), $107-121$. doi:10.1007/s40708-016-0060-4

Maechler M, Rousseeuw PJ, Struyf A, Hubert M, Hornik K. (2016). cluster: Cluster Analysis Basics and Extensions. https://cran.r-project.org/web/packages/cluster

Majewski, J., Schwartzentruber, J., Lalonde, E., Montpetit, A., Jabado, N. (2011). What can exome sequencing do for you? Journal of Medical Genetics 48(9), 580. doi:10.1136/jmedgenet-2011-100223 
Mak, L.E., Minuzzi, L., MacQueen, G., Hall, G., Kennedy, S.H., Milev, R., (2017). The Default Mode Network in Healthy Individuals: A Systematic Review and Meta-Analysis. Brain Connect 7, 25-33. doi:10.1089/brain.2016.0438

Mandl, R., Schnack, H., Luigjes, J., van den Heuvel, M., Cahn, W., Kahn, R., Hulshoff Pol, H.E. (2008). Tract-based Analysis of Magnetization Transfer Ratio and Diffusion Tensor Imaging of the Frontal and Frontotemporal Connections in Schizophrenia. Schizophrenia Bulletin 36(4), 778-787. doi:10.1093/schbul/sbn161

Manolio, T., Collins, F., Cox, N., Goldstein, D., Hindorff, L., Hunter, D., McCarthy, M., Ramos, E., Cardon, L., Chakravarti, A., Cho, J., Guttmacher, A., Kong, A., Kruglyak, L., Mardis, E., Rotimi, C., Slatkin, M., Valle, D., Whittemore, A., Boehnke, M., Clark, A., Eichler, E., Gibson, G., Haines, J., Mackay, T., McCarroll, S., Visscher, P. (2009). Finding the missing heritability of complex diseases. Nature 461(7265), 747-753. doi:10.1038/nature08494

Marcus, D.S., Harms, M.P., Snyder, A.Z., Jenkinson, M., Wilson, J.A., Glasser, M.F., Barch, D.M., Archie, K.A., Burgess, G.C., Ramaratnam, M., Hodge, M., Horton, W., Herrick, R., Olsen, T., McKay, M., House, M., Hileman, M., Reid, E., Harwell, J., Coalson, T., Schindler, J., Elam, J.S., Curtiss, S.W., Van Essen, D.C., WU-Minn HCP Consortium, (2013). Human Connectome Project informatics: quality control, database services, and data visualization. NeuroImage 80, 202219. doi:10.1016/j.neuroimage.2013.05.077

Marek, S., Hwang, K., Foran, W., Hallquist, M.N., Luna, B., (2015). The Contribution of Network Organization and Integration to the Development of Cognitive Control. PLoS Biology 13, e1002328. doi:10.1371/journal.pbio.1002328

Marioni RE, Shah S, McRae AF, Chen BH, Colicino E, Harris SE, Gibson J, Henders AK, Redmond P, Cox SR, Pattie A, Corley J, Murphy L, Martin NG, Montgomery GW, Feinberg AP, Fallin MD, Multhaup ML, Jaffe AE, Joehanes R, Schwartz J, Just AC, Lunetta KL, Murabito JM, Starr JM, Horvath S, Baccarelli AA, Levy D, Visscher PM, Wray NR, Deary IJ. (2015). DNA methylation age of blood predicts all-cause mortality in later life. Genome Biol. 16:25-12.

Marioni RE, Suderman M, Chen BH, Horvath S, Bandinelli S, Morris T, Beck S, Ferrucci L, Pedersen NL, Relton CL, Deary IJ, Hägg S. (2019). Tracking the epigenetic clock across the human life course: A meta-analysis of longitudinal cohort data. J Gerontol A Biol Sci Med Sci. 74:57-61.

Marroun El H, Tiemeier H, Franken IHA, Jaddoe VWV, van der Lugt A, Verhulst FC, Lahey BB, White TJH. (2016). Prenatal Cannabis and Tobacco Exposure in Relation to Brain Morphology: A Prospective Neuroimaging Study in Young Children. Biol Psychiatry. 79:971-979.

Marrus, N., Eggebrecht, A., Todorov, A., Elison, J., Wolff, J., Cole, L., Gao, W., Pandey, J., Shen, M., Swanson, M., Emerson, R., Klohr, C., Adams, C., Estes, A., Zwaigenbaum, L., Botteron, K., McKinstry, R., Constantino, J., Evans, A., Hazlett, H., Dager, S., Paterson, S., Schultz, R., Styner, M., Gerig, G., Network, T., Schlaggar, B., Piven, J., Pruett, J. (2017). Walking, Gross Motor Development, and Brain Functional Connectivity in Infants and Toddlers. Cerebral Cortex 28(2), $750-763$. doi:10.1093/cercor/bhx313

Martin, N.G., (2010). Imaging genomics. Current Opinion in Neurology 23, 368-373. doi:10.1097/WCO.0b013e32833b764c

Mattfeld, A., Gabrieli, J., Biederman, J., Spencer, T., Brown, A., Kotte, A., Kagan, E., Whitfield-Gabrieli, S. (2014). Brain differences between persistent and remitted attention deficit hyperactivity disorder. Brain 137(9), 2423-2428. doi:10.1093/brain/awu137

McDade TW, Ryan CP, Jones MJ, Hoke MK, Borja J, Miller GE, Kuzawa CW, Kobor MS. (2019). Genome-wide analysis of DNA methylation in relation to socioeconomic status during development and early adulthood. Am J Phys Anthropol. 169:3-11.

McKinney BC, Lin H, Ding Y, Lewis DA, Sweet RA. (2017). DNA methylation evidence against the accelerated aging hypothesis of schizophrenia. npj Schizophrenia 3(1), 13. doi:10.1038/s41537-017-0017-5

McKinney BC, Lin H, Ding Y, Lewis DA, Sweet RA. (2018). DNA methylation age is not accelerated in brain or blood of subjects with schizophrenia. Schizophrenia Research 196, 39-44. doi:10.1016/j.schres.2017.09.025

Meda, S.A., Ruaño, G., Windemuth, A., O'Neil, K., Berwise, C., Dunn, S.M., Boccaccio, L.E., Narayanan, B., Kocherla, M., Sprooten, E., Keshavan, M.S., Tamminga, C.A., Sweeney, J.A., Clementz, B.A., Calhoun, V.D., Pearlson, G.D., (2014). Multivariate analysis reveals genetic associations of the resting default mode network in psychotic bipolar disorder and schizophrenia. Proceedings of the National Academy of Sciences 111(19), E2066-E2075. doi:10.1073/pnas.1313093111 
Mejia, A., Nebel, M., Barber, A., Choe, A., Pekar, J., Caffo, B., Lindquist, M. (2018). Improved estimation of subject-level functional connectivity using full and partial correlation with empirical Bayes shrinkage. NeuroImage 172, 478-491. doi:10.1016/j.neuroimage.2018.01.029

Menon, V. (2011). Large-scale brain networks and psychopathology: a unifying triple network model. Trends in Cognitive Sciences 15(10), 483-506. doi:10.1016/j.tics.2011.08.003

Menon, V., (2013). Developmental pathways to functional brain networks: emerging principles. Trends in Cognitive Sciences 17, 627-640. doi:10.1016/j.tics.2013.09.015

Mill J, Tang T, Kaminsky Z, Khare T, Yazdanpanah S, Bouchard L, Jia P, Assadzadeh A, Flanagan J, Schumacher A, Wang S-C, Petronis A. (2008). Epigenomic Profiling Reveals DNA-Methylation Changes Associated with Major Psychosis. Am J Hum Genet. 82:696-711.

Miller DJ, Duka T, Stimpson CD, Schapiro SJ, Baze WB, McArthur MJ, Fobbs AJ, Sousa AMM, Sestan N, Wildman DE, Lipovich L, Kuzawa CW, Hof PR, Sherwood CC. (2012). Prolonged myelination in human neocortical evolution. Proceedings of the National Academy of Sciences 109(41), 16480-16485. doi:10.1073/pnas.1117943109

Miller, M. (1995). Coefficient alpha: A basic introduction from the perspectives of classical test theory and structural equation modeling. Structural Equation Modeling: A Multidisciplinary Journal 2(3), 255-273. doi:10.1080/10705519509540013

Mills, K., Goddings, A., Herting, M., Meuwese, R., Blakemore, S., Crone, E., Dahl, R., Güroğlu, B., Raznahan, A., Sowell, E., Tamnes, C. (2016). Structural brain development between childhood and adulthood: Convergence across four longitudinal samples. NeuroImage 141, 273-281. doi:10.1016/j.neuroimage.2016.07.044

Mills, K., Lalonde, F., Clasen, L., Giedd, J., Blakemore, S. (2014). Developmental changes in the structure of the social brain in late childhood and adolescence. Social Cognitive and Affective Neuroscience 9(1), 123-131. doi:10.1093/scan/nss113

Mills KL, Goddings A-L, Herting MM, Meuwese R, Blakemore S-J, Crone EA, Dahl RE, Güroğlu B, Raznahan A, Sowell ER, Tamnes CK. (2016). Structural brain development between childhood and adulthood: Convergence across four longitudinal samples. NeuroImage. 141:273-281.

Mills, K.L., Tamnes, C.K., (2014). Methods and considerations for longitudinal structural brain imaging analysis across development. Dev Cogn Neurosci 9, 172-190. doi:10.1016/j.dcn.2014.04.004

Molenaar, D., Middeldorp, C., Willemsen, G., Ligthart, L., Nivard, M., Boomsma, D. (2016). Evidence for Gender-Dependent Genotype by Environment Interaction in Adult Depression. Behavior Genetics 46(1), 59-71. doi:10.1007/s10519-0159752-4

Moodie, C.A., Wisner, K.M., MacDonald, A.W., (2014). Characteristics of canonical intrinsic connectivity networks across tasks and monozygotic twin pairs. Hum Brain Mapp 35, 5532-5549. doi:10.1002/hbm.22568

Mori, S., Zhang, J. (2006). Principles of Diffusion Tensor Imaging and Its Applications to Basic Neuroscience Research. Neuron 51(5), 527-539. doi:10.1016/j.neuron.2006.08.012

Muchinsky, P. (1996). The Correction for Attenuation. Educational and Psychological Measurement 56(1), 63-75. doi:10.1177/0013164496056001004

Mueller, S., Wang, D., Fox, M., Pan, R., Lu, J., Li, K., Sun, W., Buckner, R., Liu, H. (2015). Reliability correction for functional connectivity: Theory and implementation. Human Brain Mapping 36(11), 4664-4680. doi:10.1002/hbm.22947

Murphy, K., Birn, R., Bandettini, P. (2013). Resting-state fMRI confounds and cleanup. NeuroImage 80, 349-359. doi:10.1016/j.neuroimage.2013.04.001

Murray, R., Lewis, S. (1987). Is schizophrenia a neurodevelopmental disorder?British Medical Journal (Clinical research ed.) 295(6600), 681. doi:10.1136/bmj.295.6600.681

Muschelli, J., Nebel, M.B., Caffo, B.S., Barber, A.D., Pekar, J.J., Mostofsky, S.H., 2014. Reduction of motion-related artifacts in resting state fMRI using aCompCor. NeuroImage 96, 22-35. doi:10.1016/j.neuroimage.2014.03.028

Musci, R., Schlomer, G. (2018). The Implications of Genetics for Prevention and Intervention Programming. Prevention Science 19(1), 1-5. doi:10.1007/s11121-017-0837-6

Mwangi B, Hasan KM, Soares JC. (2013). Prediction of individual subject's age across the human lifespan using diffusion tensor imaging: a machine learning approach. Neuroimage, 75:58-67. 
Naumova OY, Lee M, Rychkov SY, Vlasova NV, Grigorenko EL. (2013). Gene expression in the human brain: the current state of the study of specificity and spatiotemporal dynamics. Child Dev. 84:76-88.

Neale, M., Kendler, K. (1995). Models of comorbidity for multifactorial disorders. American journal of human genetics 57(4), 935-53. https://www.ncbi.nlm.nih.gov/pubmed/7573055

Neale, M.C., Cardon, L.R., (1992). Methodology for Genetic Studies of Twins and Families. Springer Netherlands, Dordrecht. doi:10.1007/978-94-015-8018-2

Neale, M.C., Hunter, M.D., Pritikin, J.N., Zahery, M., Brick, T.R., Kirkpatrick, R.M., Estabrook, R., Bates, T.C., Maes, H.H., Boker, S.M., (2016). OpenMx 2.0: Extended Structural Equation and Statistical Modeling. Psychometrika 81(2), 535-549. doi:10.1007/s11336-014-9435-8

Nelson PG, Promislow DEL, Masel J. (2020). Biomarkers for aging identified in cross-sectional studies tend to be noncausative. J Gerontol A Biol Sci Med Sci. 75(3), 466-472. https://doi.org/10.1093/gerona/glz174

Nenadić, I., Dietzek, M., Langbein, K., Sauer, H., Gaser, C. (2017). BrainAGE score indicates accelerated brain aging in schizophrenia, but not bipolar disorder. Psychiatry Research: Neuroimaging 266, 86-89. doi:10.1016/j.pscychresns.2017.05.006

Nguyen TT, Eyler LT, Jeste DV. (2018). Systemic biomarkers of accelerated aging in schizophrenia: A critical review and future directions. Schizophr Bull. 44:398-408.

Noble, S., Scheinost, D., Constable, R. (2019). A decade of test-retest reliability of functional connectivity: A systematic review and meta-analysis. NeuroImage 203, 116157. doi:10.1016/j.neuroimage.2019.116157

Noble, S., Spann, M., Tokoglu, F., Shen, X., Constable, R., Scheinost, D. (2017). Influences on the Test-Retest Reliability of Functional Connectivity MRI and its Relationship with Behavioral Utility. Cerebral Cortex 27(11), 5415-5429. doi:10.1093/cercor/bhx230

Nugent, A., Luckenbaugh, D., Wood, S., Bogers, W., Zarate, C., Drevets, W. (2013). Automated subcortical segmentation using FIRST: Test-retest reliability, interscanner reliability, and comparison to manual segmentation. Human Brain Mapping 34(9), 2313-2329. doi:10.1002/hbm.22068

Okazaki S, Otsuka I, Numata S, Horai T, Mouri K, Boku S, Ohmori T, Sora I, Hishimoto A. (2019). Epigenetic clock analysis of blood samples from Japanese schizophrenia. patients. npj Schizophrenia. 5:4.

Olabi B, Ellison-Wright I, McIntosh AM, Wood SJ, Bullmore E, Lawrie SM. (2011). Are There Progressive Brain Changes in Schizophrenia? A Meta-Analysis of Structural Magnetic Resonance Imaging Studies. Biological Psychiatry 70(1), 88-96. doi:10.1016/j.biopsych.2011.01.032

Oldfield RC. (1971). The assessment and analysis of handedness: The Edinburgh inventory. Neuropsychologia. 9:97-113.

Ori APS, Olde Loohuis LM, Guintivano J, Hannon E, Dempster E, St Clair D, Bass NJ, McQuillin A, Mill J, Sullivan PF, Kahn RS, Horvath S, Ophoff RA. (2019). Schizophrenia is characterized by age- and sex-specific effects on epigenetic aging. bioRxiv doi:10.1101/727859

Ottman, R. (1996). Gene-Environment Interaction: Definitions and Study Design. Preventive Medicine 25(6), 764-770. doi:10.1006/pmed.1996.0117

Ovenden ES, McGregor NW, Emsley RA, Warnich L. (2018). DNA methylation and antipsychotic treatment mechanisms in schizophrenia: Progress and future directions. Prog Neuropsychopharmacol Biol Psychiatry. 81:38-49.

Palaniyappan L, Das T, Dempster K. (2017). The neurobiology of transition to psychosis: Clearing the cache. JPN. 42:294299.

Panizzon, M., Hauger, R., Eaves, L., Chen, C., Dale, A., Eyler, L., Fischl, B., Fennema-Notestine, C., Franz, C., Grant, M., Jacobson, K., Jak, A., Lyons, M., Mendoza, S., Neale, M., Prom-Wormley, E., Seidman, L., Tsuang, M., Xian, H., Kremen, W. (2012). Genetic influences on hippocampal volume differ as a function of testosterone level in middle-aged men. NeuroImage 59(2), 1123-1131. doi:10.1016/j.neuroimage.2011.09.044

Panizzon MS, Fennema-Notestine C, Eyler LT, Jernigan TL, Prom-Wormley EC, Neale MC, Jacobson K, Lyons MJ, Grant MD, Franz CE, Xian H, Tsuang MT, Fischl B, Seidman LJ, Dale AM, Kremen WS. (2009). Distinct Genetic Influences on Cortical Surface Area and Cortical Thickness. Cerebral Cortex 19(11), 2728-2735. doi:10.1093/cercor/bhp026 
Papanastasiou E, Gaughran F, Smith S. (2011). Schizophrenia as segmental progeria. J R Soc Med. 104:475-484.

Park, J., Gail, M., Weinberg, C., Carroll, R., Chung, C., Wang, Z., Chanock, S., Fraumeni, J., Chatterjee, N. (2011). Distribution of allele frequencies and effect sizes and their interrelationships for common genetic susceptibility variants. Proceedings of the National Academy of Sciences 108(44), 18026-18031. doi:10.1073/pnas.1114759108

Parkes, L., Ben Fulcher, Yücel, M., Fornito, A., (2018). An evaluation of the efficacy, reliability, and sensitivity of motion correction strategies for resting-state functional MRI. NeuroImage 171, 415-436. doi:10.1016/j.neuroimage.2017.12.073

Pashayan N, Reisel D, Widschwendter M. (2016). Integration of genetic and epigenetic markers for risk stratification: Opportunities and challenges. Per Med. 13:93-95.

Patenaude, B., Smith, S., Kennedy, D., Jenkinson, M. (2011). A Bayesian model of shape and appearance for subcortical brain segmentation. NeuroImage 56(3), 907-922. doi:10.1016/j.neuroimage.2011.02.046

Paus, T. (2005). Mapping brain maturation and cognitive development during adolescence. Trends in Cognitive Sciences 9(2), 60-68. doi:10.1016/j.tics.2004.12.008

Paus, T. (2010). Growth of white matter in the adolescent brain: Myelin or axon? Brain and Cognition 72(1), 26-35. doi:10.1016/j.bandc.2009.06.002

Paus T, Keshavan M, Giedd JN. (2008). Why do many psychiatric disorders emerge during adolescence? Nature Reviews Neuroscience 9(12), 947-957. doi:10.1038/nrn2513

Peng Q, Schork AJ, Bartsch H, Lo M-T, Panizzon MS, Pediatric Imaging, Neurocognition and Genetics Study, Alzheimer's Disease Neuroimaging Initiative, Westlye LT, Kremen WS, Jernigan TL, Le Hellard S, Steen VM, Espeseth T, Huentelman M, Håberg AK, Agartz I, Djurovic S, Andreassen OA, Dale AM, Schork NJ, Chen C-H. (2016). Conservation of Distinct Genetically-Mediated Human Cortical Pattern. PLOS Genet. 12:1-18.

Peper, J.S. (2008). The early pubertal brain: work in progress. University Utrecht, Netherlands

Peper, J., Brouwer, R., van Leeuwen, M., Schnack, H., Boomsma, D., Kahn, R., Hulshoff Pol, H.E. (2010). HPG-axis hormones during puberty: A study on the association with hypothalamic and pituitary volumes. Psychoneuroendocrinology 35(1), 133-140. doi:10.1016/j.psyneuen.2009.05.025

Peper, J., Hulshoff Pol, H.E., Crone, E., Honk, J. (2011). Sex steroids and brain structure in pubertal boys and girls: a minireview of neuroimaging studies. Neuroscience 191, 28-37. doi:10.1016/j.neuroscience.2011.02.014

Peper, J., van den Heuvel, M., Mandl, R., Hulshoff Pol, H.E., Honk, J. (2011). Sex steroids and connectivity in the human brain: A review of neuroimaging studies. Psychoneuroendocrinology 36(8), 1101-1113. doi:10.1016/j.psyneuen.2011.05.004

Peper JS, Brouwer RM, Boomsma DI, Kahn RS, Hulshoff Pol HE. (2007). Genetic influences on human brain structure: $A$ review of brain imaging studies in twins. Human Brain Mapping 28(6), 464-473. doi:10.1002/hbm.20398

Peper JS, Brouwer RM, Schnack HG, van Baal GCM, van Leeuwen M, van den Berg SM, Delemarre-Van de Waal HA, Janke AL, Collins DL, Evans AC, Boomsma DI, Kahn RS, Hulshoff Pol HE. (2008). Cerebral white matter in early puberty is associated with luteinizing hormone concentrations Psychoneuroendocrinology 33(7), 909-915. doi:10.1016/j.psyneuen.2008.03.017

Peper, J.S., Schnack, H.G., Brouwer, R.M., van Baal, G.C.M., Pjetri, E., Székely, E., van Leeuwen, M., van den Berg, S.M., Collins, D.L., Evans, A.C., Boomsma, D.I., Kahn, R.S., Hulshoff Pol, H.E., (2009). Heritability of regional and global brain structure at the onset of puberty: A magnetic resonance imaging study in 9-year-old twin pairs. Hum Brain Mapp 30, 2184-2196. doi:10.1002/hbm.20660

Perkins, D., Loohuis, L., Barbee, J., Ford, J., Jeffries, C., Addington, J., Bearden, C., Cadenhead, K., Cannon, T., Cornblatt, B., Mathalon, D., McGlashan, T., Seidman, L., Tsuang, M., Walker, E., Woods, S. (2019). Polygenic Risk Score Contribution to Psychosis Prediction in a Target Population of Persons at Clinical High Risk. The American journal of psychiatry 177(2), 155-163. doi:10.1176/appi.ajp.2019.18060721

Petanjek Z, Judaš M, Šimić G, Rašin MR, Uylings HBM, Rakic P, Kostović I. (2011). Extraordinary neoteny of synaptic spines in the human prefrontal cortex. Proceedings of the National Academy of Sciences 108(32), 13281-13286. doi:10.1073/pnas.1105108108 
Peters, S., Peper, J.S., van Duijvenvoorde, A.C.K., Braams, B.R., Crone, E.A., (2017). Amygdala-orbitofrontal connectivity predicts alcohol use two years later: a longitudinal neuroimaging study on alcohol use in adolescence. Developmental Science 20(4), e12448. doi:10.1111/desc.12448

Pfefferbaum, A., Sullivan, E., Carmelli, D. (2004). Morphological changes in aging brain structures are differentially affected by time-linked environmental influences despite strong genetic stability. Neurobiology of Aging 25(2), 175-183. doi:10.1016/s0197-4580(03)00045-9

Pinheiro J, Bates D, DebRoy S, Sarkar D, R Core Team. (2017). nlme: Linear and Nonlinear Mixed Effects Models. https://cran.r-project.org/web/packages/nlme/

Pletikos M, Sousa AMM, Sedmak G, Meyer KA, Zhu Y, Cheng F, Li M, Kawasawa YI, Sestan N. (2014). Temporal Specification and Bilaterality of Human Neocortical Topographic Gene Expression. Neuron. 81:321-332.

Plitt, M., Barnes, K., Wallace, G., Kenworthy, L., Martin, A. (2015). Resting-state functional connectivity predicts longitudinal change in autistic traits and adaptive functioning in autism. Proceedings of the National Academy of Sciences 112(48), E6699-E6706. doi:10.1073/pnas.1510098112

Plomin, R., Fulker, D., Corley, R., DeFries, J. (1997). Nature, Nurture, and Cognitive Development from 1 to 16 Years: A Parent-Offspring Adoption Study. Psychological Science 8(6), 442-447. doi:10.1111/j.1467-9280.1997.tb00458.x

Poldrack, R., Baker, C., Durnez, J., Gorgolewski, K., Matthews, P., Munafò, M., Nichols, T., Poline, J., Vul, E., Yarkoni, T. (2017). Scanning the horizon: towards transparent and reproducible neuroimaging research. Nature Reviews Neuroscience 18(2), 115-126. doi:10.1038/nrn.2016.167

Posthuma, D., Boomsma, D.I., (2000). A note on the statistical power in extended twin designs. Behav Genet 30, $147-158$. doi:10.1023/A:1001959306025

Posthuma, D., de Geus, E.J.C., Neale, M.C., Hulshoff Pol, H.E., Baaré, W.F.C., Kahn, R.S., Boomsma, D.I., (2000). Multivariate genetic analysis of brain structure in an extended twin design. Behav Genet 30, 311-319. doi:10.1023/A:1026501501434

Power, J., Schlaggar, B., Petersen, S. (2015). Recent progress and outstanding issues in motion correction in resting state fMRI. NeuroImage 105, 536-551. doi:10.1016/j.neuroimage.2014.10.044

Power, J.D., Barnes, K.A., Snyder, A.Z., Schlaggar, B.L., Petersen, S.E., (2012). Spurious but systematic correlations in functional connectivity MRI networks arise from subject motion. NeuroImage 59(3), 2142-2154. doi:10.1016/j.neuroimage.2011.10.018

Power, J.D., Cohen, A.L., Nelson, S.M., Wig, G.S., Barnes, K.A., Church, J.A., Vogel, A.C., Laumann, T.O., Miezin, F.M., Schlaggar, B.L., Petersen, S.E., (2011). Functional network organization of the human brain. Neuron 72, 665-678. doi:10.1016/j.neuron.2011.09.006

Price AL, Weale ME, Patterson N, Myers SR, Need AC, Shianna KV, Ge D, Rotter JI, Torres E, Taylor KDD, Goldstein DB, Reich D. (2008). Long-Range LD Can Confound Genome Scans in Admixed Populations. Am J Hum Genet. 83:132-135.

Pulver AE, Mulle J, Nestadt G, Swartz KL, Blouin JL, Dombroski B, Liang KY, Housman DE, Kazazian HH, Antonarakis SE, Lasseter VK, Wolyniec PS, Thornquist MH, McGrath JA. (2000). Genetic heterogeneity in schizophrenia: Stratification of genome scan data using co-segregating related phenotypes. Mol Psychiatry. 5:650-653.

Purcell S, Neale B, Todd-Brown K, Thomas L, Ferreira MAR, Bender D, Maller J, Sklar P, de Bakker PIW, Daly MJ, Sham PC. (2007). PLINK: A tool set for whole-genome association and population-based linkage analyses. Am J Hum Genet. 81:559-575.

R Core Team. (2015). R: A Language and Environment for Statistical Computing version 3.2.2. https://www.r-project.org R Core Team, (2017). R: A Language and Environment for Statistical Computing version 3.4.2. https://www.r-project.org R Core Team. (2018). The R Project for Statistical Computing. https://www.r-project.org

Raichle, M., MacLeod, A., Snyder, A., Powers, W., Gusnard, D., Shulman, G. (2001). A default mode of brain function. Proceedings of the National Academy of Sciences 98(2), 676-682. doi:10.1073/pnas.98.2.676

Raichle, M.E., (2015). The brain's default mode network. Annual Review of Neuroscience 38, 433-447. doi:10.1146/annurevneuro-071013-014030 
Ranlund S, Rosa MJ, de Jong S, Cole JH, Kyriakopoulos M, Fu CHY, Mehta MA, Dima D. (2018). Associations between polygenic risk scores for four psychiatric illnesses and brain structure using multivariate pattern recognition. Neuroimage Clin. 20:1026-1036.

Rapoport, J., Giedd, J., Gogtay, N. (2012). Neurodevelopmental model of schizophrenia: update 2012. Molecular Psychiatry 17(12), 1228-1238. doi:10.1038/mp.2012.23

Rapoport JL, Gogtay N. (2008). Brain neuroplasticity in healthy, hyperactive and psychotic children: insights from neuroimaging. Neuropsychopharmacology. 33:181-197.

Raznahan A, Lerch JP, Lee N, Greenstein D, Wallace GL, Stockman M, Clasen LS, Shaw PW, Giedd JN. (2011). Patterns of coordinated anatomical change in human cortical development: a longitudinal neuroimaging study of maturational coupling. Neuron. 72:873-884.

Raznahan A, Shaw PW, Lalonde F, Stockman M, Wallace GL, Greenstein D, Clasen LS, Gogtay N, Giedd JN. (2011). How does your cortex grow? J Neurosci. 31:7174-7177.

Redolfi A, Manset D, Barkhof F, Wahlund L-O, Glatard T, Mangin J-F, Frisoni GB, neuGRID Consortium for the Alzheimer's Disease Neuroimaging Initiative. (2015). Head-to-head comparison of two popular cortical thickness extraction algorithms: a cross-sectional and longitudinal study. PLoS ONE. 10:e0117692.

Reuter, M., Schmansky, N.J., Rosas, H.D., Fischl, B., (2012). Within-subject template estimation for unbiased longitudinal image analysis. NeuroImage 61, 1402-1418. doi:10.1016/j.neuroimage.2012.02.084

Richiardi, J., Altmann, A., Milazzo, A.-C., Chang, C., Chakravarty, M.M., Banaschewski, T., Barker, G.J., Bokde, A.L.W., Bromberg, U., Büchel, C., Conrod, P.J., Fauth-Bühler, M., Flor, H., Frouin, V., Gallinat, J., Garavan, H., Gowland, P., Heinz, A., Lemaitre, H., Mann, K.F., Martinot, J.-L., Nees, F., Paus, T., Pausova, Z., Rietschel, M., Robbins, T.W., Smolka, M.N., Spanagel, R., Ströhle, A., Schumann, G., Hawrylycz, M., Poline, J.-B., Greicius, M.D., IMAGEN Consortium, (2015). Correlated gene expression supports synchronous activity in brain networks. Science 348, 1241-1244. doi:10.1126/science.1255905

Richmond, S., Johnson, K.A., Seal, M.L., Allen, N.B., Whittle, S., (2016). Development of brain networks and relevance of environmental and genetic factors: A systematic review. Neuroscience and Biobehavioral Reviews 71, 215-239. doi:10.1016/j.neubiorev.2016.08.024

Rijsdijk, F., van Haren, N., Picchioni, M., McDonald, C., Toulopoulou, T., Hulshoff Pol, H.E., Kahn, R., Murray, R., Sham, P. (2005). Brain MRI abnormalities in schizophrenia: same genes or same environment? Psychological Medicine 35(10), 1399-1409. doi:10.1017/s0033291705005167

Rimol LM, Panizzon MS, Fennema-Notestine C, Eyler LT, Fischl B, Franz CE, Hagler DJ, Lyons MJ, Neale MC, Pacheco J, Perry ME, Schmitt JE, Grant MD, Seidman LJ, Thermenos HW, Tsuang MT, Eisen SA, Kremen WS, Dale AM. (2010). Cortical thickness is influenced by regionally specific genetic factors. Biol Psychiatry. 67:493-499.

Ripke, S., Neale, B., Corvin, A., Walters, J., Farh, K., Holmans, P., Lee, P., Bulik-Sullivan, B., Collier, D., Huang, H., Pers, T., Agartz, I., Agerbo, E., Albus, M., Alexander, M., Amin, F., Bacanu, S., Begemann, M., Jr, R., Bene, J., Bergen, S., Bevilacqua, E., Bigdeli, T., Black, D., Bruggeman, R., Buccola, N., Buckner, R., Byerley, W., Cahn, W., Cai, G., Campion, D., Cantor, R., Carr, V., Carrera, N., Catts, S., Chambert, K., Chan, R., Chen, R., Chen, E., Cheng, W., Cheung, E., Chong, S., Cloninger, C., Cohen, D., Cohen, N., Cormican, P., Craddock, N., Crowley, J., Curtis, D., Davidson, M., Davis, K., Degenhardt, F., Favero, J., Demontis, D., Dikeos, D., Dinan, T., Djurovic, S., Donohoe, G., Drapeau, E., Duan, J., Dudbridge, F., Durmishi, N., Eichhammer, P., Eriksson, J., Escott-Price, V., Essioux, L., Fanous, A., Farrell, M., Frank, J., Franke, L., Freedman, R., Freimer, N., Friedl, M., Friedman, J., Fromer, M., Genovese, G., Georgieva, L., Giegling, I., Giusti-Rodríguez, P., Godard, S., Goldstein, J., Golimbet, V., Gopal, S., Gratten, J., Haan, L., Hammer, C., Hamshere, M., Hansen, M., Hansen, T., Haroutunian, V., Hartmann, A., Henskens, F., Herms, S., Hirschhorn, J., Hoffmann, P., Hofman, A., Hollegaard, M., Hougaard, D., Ikeda, M., Joa, I., Julià, A., Kahn, R., Kalaydjieva, L., Karachanak-Yankova, S., Karjalainen, J., Kavanagh, D., Keller, M., Kennedy, J., Khrunin, A., Kim, Y., Klovins, J., Knowles, J., Konte, B., Kucinskas, V., Kucinskiene, Z., Kuzelova-Ptackova, H., Kähler, A., Laurent, C., Keong, J., Lee, S., Legge, S., Lerer, B., Li, M., Li, T., Liang, K., Lieberman, J., Limborska, S., Loughland, C., Lubinski, J., Lönnqvist, J., Jr, M., Magnusson, P., Maher, B., Maier, 
W., Mallet, J., Marsal, S., Mattheisen, M., Mattingsdal, M., McCarley, R., McDonald, C., McIntosh, A., Meier, S., Meijer, C., Melegh, B., Melle, I., Mesholam-Gately, R., Metspalu, A., Michie, P., Milani, L., Milanova, V., Mokrab, Y., Morris, D., Mors, O., Murphy, K., Murray, R., Myin-Germeys, I., Müller-Myhsok, B., Nelis, M., Nenadic, I., Nertney, D., Nestadt, G., Nicodemus, K., Nikitina-Zake, L., Nisenbaum, L., Nordin, A., O’Callaghan, E., O’Dushlaine, C., O’Neill, F., Oh, S., Olincy, A., Olsen, L., van Os, J., Pantelis, C., Papadimitriou, G., Papiol, S., Parkhomenko, E., Pato, M., Paunio, T., PejovicMilovancevic, M., Perkins, D., Pietiläinen, O., Pimm, J., Pocklington, A., Powell, J., Price, A., Pulver, A., Purcell, S., Quested, D., Rasmussen, H., Reichenberg, A., Reimers, M., Richards, A., Roffman, J., Roussos, P., Ruderfer, D., Salomaa, V., Sanders, A., Schall, U., Schubert, C., Schulze, T., Schwab, S., Scolnick, E., Scott, R., Seidman, L., Shi, J., Sigurdsson, E., Silagadze, T., Silverman, J., Sim, K., Slominsky, P., Smoller, J., So, H., Spencer, C., Stahl, E., Stefansson, H., Steinberg, S., Stogmann, E., Straub, R., Strengman, E., Strohmaier, J., Stroup, T., Subramaniam, M., Suvisaari, J., Svrakic, D., Szatkiewicz, J., Söderman, E., Thirumalai, S., Toncheva, D., Tosato, S., Veijola, J., Waddington, J., Walsh, D., Wang, D., Wang, Q., Webb, B., Weiser, M., Wildenauer, D., Williams, N., Williams, S., Witt, S., Wolen, A., Wong, E., Wormley, B., Xi, H., Zai, C., Zheng, X., Zimprich, F., Wray, N., Stefansson, K., Visscher, P., Wellcome Trust Case-Control Consortium, Adolfsson, R., Andreassen, O., Blackwood, D., Bramon, E., Buxbaum, J., Børglum, A., Cichon, S., Darvasi, A., Domenici, E., Ehrenreich, H., Esko, T., Gejman, P., Gill, M., Gurling, H., Hultman, C., Iwata, N., Jablensky, A., Jönsson, E., Kendler, K., Kirov, G., Knight, J., Lencz, T., Levinson, D., Li, Q., Liu, J., Malhotra, A., McCarroll, S., McQuillin, A., Moran, J., Mortensen, P., Mowry, B., Nöthen, M., Ophoff, R., Owen, M., Palotie, A., Pato, C., Petryshen, T., Posthuma, D., Rietschel, M., Riley, B., Rujescu, D., Sham, P., Sklar, P., Clair, D., Weinberger, D., Wendland, J., Werge, T., Daly, M., Sullivan, P., O’Donovan, M. (2014). Biological insights from 108 schizophrenia-associated genetic loci. Nature 511(7510), 421-427. doi:10.1038/nature13595

Robinson, E., Jbabdi, S., Glasser, M., Andersson, J., Burgess, G., Harms, M., Smith, S., Essen, D., Jenkinson, M. (2014). MSM: A new flexible framework for Multimodal Surface Matching. NeuroImage 100, 414-426. doi:10.1016/j.neuroimage.2014.05.069

Roiz-Santiañez R, Suarez-Pinilla P, Crespo-Facorro B. (2015). Brain Structural Effects of Antipsychotic Treatment in Schizophrenia: A Systematic Review. Curr Neuropharmacol. 13:422-434. doi:10.2174/1570159X13666150429002536

Rosazza, C., Minati, L. (2011). Resting-state brain networks: literature review and clinical applications. Neurological Sciences 32(5), 773-785. doi:10.1007/s10072-011-0636-y

Rosenberg, M., Finn, E., Scheinost, D., Papademetris, X., Shen, X., Constable, R., Chun, M. (2016). A neuromarker of sustained attention from whole-brain functional connectivity. Nature Neuroscience 19(1), 165-171. doi:10.1038/nn.4179

Roth RB, Hevezi P, Lee J, Willhite D, Lechner SM, Foster AC, Zlotnik A. (2006). Gene expression analyses reveal molecular relationships among 20 regions of the human CNS. Neurogenetics. 7:67-80.

Rousseeuw PJ. (1987). Silhouettes: A graphical aid to the interpretation and validation of cluster analysis. J Comput Appl Math. 20:53-65.

Ruigrok, A.N.V., Salimi-Khorshidi, G., Lai, M.-C., Baron-Cohen, S., Lombardo, M.V., Tait, R.J., Suckling, J., (2014). A metaanalysis of sex differences in human brain structure. Neuroscience \& Biobehavioral Reviews 39, 34-50. doi:10.1016/j.neubiorev.2013.12.004

Sabunciyan S. (2019). Gene Expression Profiles Associated with Brain Aging are Altered in Schizophrenia. Sci Rep. 9:5896.

Sacher, J., Neumann, J., Okon-Singer, H., Gotowiec, S., Villringer, A., (2013). Sexual dimorphism in the human brain: evidence from neuroimaging. Magnetic Resonance Imaging 31, 366-375. doi:10.1016/j.mri.2012.06.007

Sala-Llonch, R., Bartrés-Faz, D., Junqué, C. (2015). Reorganization of brain networks in aging: a review of functional connectivity studies. Frontiers in Psychology 6, 663. doi:10.3389/fpsyg.2015.00663

Salimi-Khorshidi, G., Douaud, G., Beckmann, C., Glasser, M., Griffanti, L., Smith, S. (2014). Automatic denoising of functional MRI data: Combining independent component analysis and hierarchical fusion of classifiers. NeuroImage 90 , 449-468. doi:10.1016/j.neuroimage.2013.11.046

Santarnecchi, E., Emmendorfer, A., Tadayon, S., Rossi, S., Rossi, A., Pascual-Leone, A., (2017). Network connectivity correlates of variability in fluid intelligence performance. Intelligence 65, 35-47. doi:10.1016/j.intell.2017.10.002 
Satizabal, C., Adams, H., Hibar, D., White, C., Knol, M., Stein, J., Scholz, M., Sargurupremraj, M., Jahanshad, N., Roshchupkin, G., Smith, A., Bis, J., Jian, X., Luciano, M., Hofer, E., Teumer, A., Lee, S., Yang, J., Yanek, L., Lee, T., Li, S., $\mathrm{Hu}$, Y., Koh, J., Eicher, J., Desrivières, S., Arias-Vasquez, A., Chauhan, G., Athanasiu, L., Rentería, M., Kim, S., Hoehn, D., Armstrong, N., Chen, Q., Holmes, A., den Braber, A., Kloszewska, I., Andersson, M., Espeseth, T., Grimm, O., Abramovic, L., Alhusaini, S., Milaneschi, Y., Papmeyer, M., Axelsson, T., Ehrlich, S., Roiz-Santiañez, R., Kraemer, B., Håberg, A., Jones, H., Pike, G., Stein, D., Stevens, A., Bralten, J., Vernooij, M., Harris, T., Filippi, I., Witte, A., Guadalupe, T., Wittfeld, K., Mosley, T., Becker, J., Doan, N., Hagenaars, S., Saba, Y., Cuellar-Partida, G., Amin, N., Hilal, S., Nho, K., Mirza-Schreiber, N., Arfanakis, K., Becker, D., Ames, D., Goldman, A., Lee, P., Boomsma, D., Lovestone, S., Giddaluru, S., Hellard, S., Mattheisen, M., Bohlken, M., Kasperaviciute, D., Schmaal, L., Lawrie, S., Agartz, I., Walton, E., Tordesillas-Gutierrez, D., Davies, G., Shin, J., Ipser, J., Vinke, L., Hoogman, M., Jia, T., Burkhardt, R., Klein, M., Crivello, F., Janowitz, D., Carmichael, O., Haukvik, U., Aribisala, B., Schmidt, H., Strike, L., Cheng, C., Risacher, S., Pütz, B., Fleischman, D., Assareh, A., Mattay, V., Buckner, R., Mecocci, P., Dale, A., Cichon, S., Boks, M., Matarin, M., Penninx, B., Calhoun, V., Chakravarty, M., Marquand, A., Macare, C., Masouleh, S., Oosterlaan, J., Amouyel, P., Hegenscheid, K., Rotter, J., Schork, A., Liewald, D., Zubicaray, G., Wong, T., Shen, L., Sämann, P., Brodaty, H., Roffman, J., Geus, E., Tsolaki, M., Erk, S., Eijk, K., Cavalleri, G., Wee, N., McIntosh, A., Gollub, R., Bulayeva, K., Bernard, M., Richards, J., Himali, J., Loeffler, M., Rommelse, N., Hoffmann, W., Westlye, L., Hernández, M., Hansell, N., van Erp, T., Wolf, C., Kwok, J., Vellas, B., Heinz, A., Loohuis, L., Delanty, N., Ho, B., Ching, C., Shumskaya, E., Singh, B., Hofman, A., Meer, D., Homuth, G., Psaty, B., Bastin, M., Montgomery, G., Foroud, T., Reppermund, S., Hottenga, J., Simmons, A., MeyerLindenberg, A., Cahn, W., Whelan, C., Donkelaar, M., Yang, Q., Hosten, N., Green, R., Thalamuthu, A., Mohnke, S., Hulshoff Pol, H.E., Lin, H., Jack, C., Schofield, P., Mühleisen, T., Maillard, P., Potkin, S., Wen, W., Fletcher, E., Toga, A., Gruber, O., Huentelman, M., Smith, G., Launer, L., Nyberg, L., Jönsson, E., Crespo-Facorro, B., Koen, N., Greve, D., Uitterlinden, A., Weinberger, D., Steen, V., Fedko, I., Groenewold, N., Niessen, W., Toro, R., Tzourio, C., Longstreth, W., Ikram, M., Smoller, J., Tol, M., Sussmann, J., Paus, T., Lemaître, H., Schroeter, M., Mazoyer, B., Andreassen, O., Holsboer, F., Depondt, C., Veltman, D., Turner, J., Pausova, Z., Schumann, G., Rooij, D., Djurovic, S., Deary, I., McMahon, K., Müller-Myhsok, B., Brouwer, R., Soininen, H., Pandolfo, M., Wassink, T., Cheung, J., Wolfers, T., Martinot, J., Zwiers, M., Nauck, M., Melle, I., Martin, N., Kanai, R., Westman, E., Kahn, R., Sisodiya, S., White, T., Saremi, A., Bokhoven, H., Brunner, H., Völzke, H., Wright, M., Ent, D., Nöthen, M., Ophoff, R., Buitelaar, J., Fernández, G., Sachdev, P., Rietschel, M., van Haren, N., Fisher, S., Beiser, A., Francks, C., Saykin, A., Mather, K., Romanczuk-Seiferth, N., Hartman, C., DeStefano, A., Heslenfeld, D., Weiner, M., Walter, H., Hoekstra, P., Nyquist, P., Franke, B., Bennett, D., Grabe, H., Johnson, A., Chen, C., Duijn, C., Lopez, O., Fornage, M., Wardlaw, J., Schmidt, R., DeCarli, C., Jager, P., Villringer, A., Debette, S., Gudnason, V., Medland, S., Shulman, J., Thompson, P., Seshadri, S., Ikram, M. (2019). Genetic architecture of subcortical brain structures in 38,851 individuals. Nature Genetics 51(11), 1624-1636. doi:10.1038/s41588019-0511-y

Sato, J.R., Salum, G.A., Gadelha, A., Picon, F.A., Pan, P.M., Vieira, G., Zugman, A., Hoexter, M.Q., Anés, M., Moura, L.M., Gomes Del'Aquilla, M.A., Amaro, E., Jr, McGuire, P., Crossley, N.A., Lacerda, A.L.T., Rohde, L.A., Miguel, E.C., Bressan, R.A., Jackowski, A.P., (2014). Age effects on the default mode and control networks in typically developing children. 58, 89-95. doi:10.1016/j.jpsychires.2014.07.004

Sato, J.R., Salum, G.A., Gadelha, A., Vieira, G., Zugman, A., Picon, F.A., Pan, P.M., Hoexter, M.Q., Anés, M., Moura, L.M., Del'aquilla, M.A.G., Crossley, N.A., Amaro, E., Jr, McGuire, P., Lacerda, A.L.T., Rohde, L.A., Miguel, E.C., Jackowski, A.P., Bressan, R.A., (2015). Decreased centrality of subcortical regions during the transition to adolescence: a functional connectivity study. NeuroImage 104, 44-51. doi:10.1016/j.neuroimage.2014.09.063

Satterthwaite, T., Wolf, D., Loughead, J., Ruparel, K., Elliott, M., Hakonarson, H., Gur, R., Gur, R. (2012). Impact of inscanner head motion on multiple measures of functional connectivity: Relevance for studies of neurodevelopment in youth. NeuroImage 60(1), 623-632. doi:10.1016/j.neuroimage.2011.12.063

Satterthwaite, T.D., Wolf, D.H., Ruparel, K., Erus, G., Elliott, M.A., Eickhoff, S.B., Gennatas, E.D., Jackson, C., Prabhakaran, K., Smith, A., Hakonarson, H., Verma, R., Davatzikos, C., Gur, R.E., Gur, R.C., (2013). Heterogeneous impact of motion 
on fundamental patterns of developmental changes in functional connectivity during youth. NeuroImage 83, 45-57. doi:10.1016/j.neuroimage.2013.06.045

Sawyer, S., Azzopardi, P., Wickremarathne, D., Patton, G. (2018). The age of adolescence. The Lancet Child \& Adolescent Health 2(3), 223-228. doi:10.1016/s2352-4642(18)30022-1

Schmitt, J., Neale, M., Fassassi, B., Perez, J., Lenroot, R., Wells, E., Giedd, J. (2014). The dynamic role of genetics on cortical patterning during childhood and adolescence. Proceedings of the National Academy of Sciences 111(18), 6774-6779. doi:10.1073/pnas.1311630111

Schmitt, J., Raznahan, A., Clasen, L., Wallace, G., Pritikin, J., Lee, N., Giedd, J., Neale, M. (2019). The Dynamic Associations Between Cortical Thickness and General Intelligence are Genetically Mediated. Cerebral Cortex 29(11), 4743-4752. doi:10.1093/cercor/bhz007

Schmitt JE, Giedd JN, Raznahan A, Neale MC. (2017). The Genetic Contributions to Maturational Coupling in the Human Cerebrum: A Longitudinal Pediatric Twin Imaging Study. Cereb Cortex. 1-8.

Schmitt JE, Lenroot RK, Wallace GL, Ordaz SJ, Taylor KN, Kabani NJ, Greenstein DK, Lerch JP, Kendler KS, Neale MC, Giedd JN. (2008). Identification of genetically mediated cortical networks: a multivariate study of pediatric twins and siblings. Cereb Cortex. 18:1737-1747.

Schmitt JE, Neale MC, Fassassi B, Perez J, Lenroot RK, Wells EM, Giedd JN. (2014). The dynamic role of genetics on cortical patterning during childhood and adolescence. Proc Natl Acad Sci USA. 111:6774-6779.

Schnack, H. (2019). Assessing reproducibility in association studies. eLife 8, e46757. doi:10.7554/eLife.46757

Schnack HG, van Haren NEM, Brouwer RM, Evans AC, Durston S, Boomsma DI, Kahn RS, Hulshoff Pol HE. (2015). Changes in Thickness and Surface Area of the Human Cortex and Their Relationship with Intelligence. Cerebral Cortex 25(6), 1608-1617. doi:10.1093/cercor/bht357

Schnack HG, van Haren NEM, Nieuwenhuis M, Hulshoff Pol HE, Cahn W, Kahn RS. (2016). Accelerated Brain Aging in Schizophrenia: A Longitudinal Pattern Recognition Study. American Journal of Psychiatry 173(6), 607-616. doi:10.1176/appi.ajp.2015.15070922

Seeley, W.W., Menon, V., Schatzberg, A.F., Keller, J., Glover, G.H., Kenna, H., Reiss, A.L., Greicius, M.D., (2007). Dissociable Intrinsic Connectivity Networks for Salience Processing and Executive Control. J. Neurosci. 27, $2349-2356$. doi:10.1523/JNEUROSCI.5587-06.2007

Seitzman, B., Gratton, C., Laumann, T., Gordon, E., Adeyemo, B., Dworetsky, A., Kraus, B., Gilmore, A., Berg, J., Ortega, M., Nguyen, A., Greene, D., McDermott, K., Nelson, S., Lessov-Schlaggar, C., Schlaggar, B., Dosenbach, N., Petersen, S. (2019). Trait-like variants in human functional brain networks. Proceedings of the National Academy of Sciences 116(45), 22851-22861. doi:10.1073/pnas.1902932116

Shah, L., Cramer, J., Ferguson, M., Birn, R., Anderson, J. (2016). Reliability and reproducibility of individual differences in functional connectivity acquired during task and resting state. Brain and Behavior 6(5)doi:10.1002/brb3.456

Shah, S., McRae, A., Marioni, R., Harris, S., Gibson, J., Henders, A., Redmond, P., Cox, S., Pattie, A., Corley, J., Murphy, L., Martin, N., Montgomery, G., Starr, J., Wray, N., Deary, I., Visscher, P. (2014). Genetic and environmental exposures constrain epigenetic drift over the human life course. Genome Research 24(11), 1725-1733. doi:10.1101/gr.176933.114

Shahab S, Mulsant BH, Levesque ML, Calarco N, Nazeri A, Wheeler AL, Foussias G, Rajji TK, Voineskos AN. (2019). Brain structure, cognition, and brain age in schizophrenia, bipolar disorder, and healthy controls. Neuropsychopharmacology. 44:898-906.

Shaw, P., Greenstein, D., Lerch, J., Clasen, L., Lenroot, R., Gogtay, N., Evans, A., Rapoport, J., Giedd, J. (2006). Intellectual ability and cortical development in children and adolescents. Nature 440(7084), 676-679. doi:10.1038/nature04513

Shaw PW, Eckstrand K, Sharp W, Blumenthal JD, Lerch JP, Greenstein DK, Clasen LS, Evans AC, Giedd JN, Rapoport JL. (2007). Attention-deficit/hyperactivity disorder is characterized by a delay in cortical maturation. Proceedings of the National Academy of Sciences 104(49), 19649-19654. doi:10.1073/pnas.0707741104

Shaw PW, Gogtay N, Rapoport JL. (201). Childhood psychiatric disorders as anomalies in neurodevelopmental trajectories. Human Brain Mapping 31(6), 917-925. doi:10.1002/hbm.21028 
Shearer, C. (2020). A resting state functional connectivity analysis of human intelligence: Broad theoretical and practical implications for multiple intelligences theory. Psychology \& Neuroscience 13(2), 127-148. doi:10.1037/pne0000200

Shen, X., Cox, S., Adams, M., Howard, D., Lawrie, S., Ritchie, S., Bastin, M., Deary, I., McIntosh, A., Whalley, H. (2018). Resting-State Connectivity and Its Association With Cognitive Performance, Educational Attainment, and Household Income in the UK Biobank. Biological Psychiatry: Cognitive Neuroscience and Neuroimaging 3(10), 878-886. doi:10.1016/j.bpsc.2018.06.007

Sherman, L.E., Rudie, J.D., Pfeifer, J.H., Masten, C.L., McNealy, K., Dapretto, M., (2014). Development of the default mode and central executive networks across early adolescence: a longitudinal study. Dev Cogn Neurosci 10, 148-159. doi:10.1016/j.dcn.2014.08.002

Shivakumar V, Kalmady SV, Venkatasubramanian G, Ravi V, Gangadhar BN. (2014). Do schizophrenia patients age early? Asian J Psychiatr. 10:3-9.

Shou, H., Eloyan, A., Nebel, M., Mejia, A., Pekar, J., Mostofsky, S., Caffo, B., Lindquist, M., Crainiceanu, C. (2014). Shrinkage prediction of seed-voxel brain connectivity using resting state fMRI. NeuroImage 102, 938-944. doi:10.1016/j.neuroimage.2014.05.043

Shrout, P., Fleiss, J. (1979). Intraclass correlations: Uses in assessing rater reliability. Psychological Bulletin 86(2), 420-428. doi:10.1037/0033-2909.86.2.420

Sideridis, G., Simos, P., Papanicolaou, A., Fletcher, J. (2014). Using Structural Equation Modeling to Assess Functional Connectivity in the Brain. Educational and Psychological Measurement 74(5), 733-758. doi:10.1177/0013164414525397

Siegel, J., Mitra, A., Laumann, T., Seitzman, B., Raichle, M., Corbetta, M., Snyder, A. (2017). Data Quality Influences Observed Links Between Functional Connectivity and Behavior. Cerebral Cortex 27(9), 4492-4502. doi:10.1093/cercor/bhw253

Silbereis JC, Pochareddy S, Zhu Y, Li M, Sestan N. (2016). The Cellular and Molecular Landscapes of the Developing Human Central Nervous System. Neuron. 89:248-268.

Sinclair, B., Hansell, N.K., Blokland, G.A.M., Martin, N.G., Thompson, P.M., Breakspear, M., de Zubicaray, G.I., Wright, M.J., McMahon, K.L., (2015). Heritability of the network architecture of intrinsic brain functional connectivity. NeuroImage 121, 243-252. doi:10.1016/j.neuroimage.2015.07.048

Sled JG, Zijdenbos AP, Evans AC. (1998). A nonparametric method for automatic correction of intensity nonuniformity in MRI data. IEEE T Med Imaging. 17:87-97.

Smeland, O., Bahrami, S., Frei, O., Shadrin, A., O’Connell, K., Savage, J., Watanabe, K., Krull, F., Bettella, F., Steen, N., Ueland, T., Posthuma, D., Djurovic, S., Dale, A., Andreassen, O. (2019). Genome-wide analysis reveals extensive genetic overlap between schizophrenia, bipolar disorder, and intelligence. Molecular Psychiatry 25(4), $844-853$. doi:10.1038/s41380-018-0332-x

Smieskova, R., Fusar-Poli, P., Allen, P., Bendfeldt, K., Stieglitz, R., Drewe, J., Radue, E., McGuire, P., Riecher-Rössler, A., Borgwardt, S. (2010). Neuroimaging predictors of transition to psychosis-A systematic review and meta-analysis. Neuroscience \& Biobehavioral Reviews 34(8), 1207-1222. doi:10.1016/j.neubiorev.2010.01.016

Smit, D., Wright, M., Meyers, J., Martin, N., Ho, Y., Malone, S., Zhang, J., Burwell, S., Chorlian, D., Geus, E., Denys, D., Hansell, N., Hottenga, J., McGue, M., Beijsterveldt, C., Jahanshad, N., Thompson, P., Whelan, C., Medland, S., Porjesz, B., Lacono, W., Boomsma, D. (2018). Genome-wide association analysis links multiple psychiatric liability genes to oscillatory brain activity. Human Brain Mapping 39(11), 4183-4195. doi:10.1002/hbm.24238

Smith, A., Kilaru, V., Klengel, T., Mercer, K., Bradley, B., Conneely, K., Ressler, K., Binder, E. (2015). DNA extracted from saliva for methylation studies of psychiatric traits: Evidence tissue specificity and relatedness to brain. American Journal of Medical Genetics Part B: Neuropsychiatric Genetics 168(1), 36-44. doi:10.1002/ajmg.b.32278

Smith, C. (1970). Heritability of liability and concordance in monozygous twins. Annals of Human Genetics 34(1), 85-91. doi:10.1111/j.1469-1809.1970.tb00223.x

Smith, S., Beckmann, C., Andersson, J., Auerbach, E., Bijsterbosch, J., Douaud, G., Duff, E., Feinberg, D., Griffanti, L., Harms, M., Kelly, M., Laumann, T., Miller, K., Moeller, S., Petersen, S., Power, J., Salimi-Khorshidi, G., Snyder, A., Vu, 
A., Woolrich, M., Xu, J., Yacoub, E., Uğurbil, K., Essen, D., Glasser, M., WU-Minn HCP Consortium. (2013). Restingstate fMRI in the Human Connectome Project. NeuroImage 80, 144-168. doi:10.1016/j.neuroimage.2013.05.039

Smith, S., Fox, P., Miller, K., Glahn, D., Fox, P., Mackay, C., Filippini, N., Watkins, K., Toro, R., Laird, A., Beckmann, C. (2009). Correspondence of the brain's functional architecture during activation and rest. Proceedings of the National Academy of Sciences 106(31), 13040-13045. doi:10.1073/pnas.0905267106

Smith, S., Nichols, T., Vidaurre, D., Winkler, A., Behrens, T., Glasser, M., Ugurbil, K., Barch, D., Essen, D., Miller, K. (2015). A positive-negative mode of population covariation links brain connectivity, demographics and behavior. Nature Neuroscience 18(11), 1565-1567. doi:10.1038/nn.4125

Smith, S.M., Brady, J.M., (1997). SUSAN - A new approach to low level image processing. International Journal of Computer Vision 23, 45-78. doi:10.1023/A:1007963824710

Smith, S.M., Hyvärinen, A., Varoquaux, G., Miller, K.L., Beckmann, C.F., (2014). Group-PCA for very large fMRI datasets. NeuroImage 101, 738-749. doi:10.1016/j.neuroimage.2014.07.051

Soares, J., Magalhães, R., Moreira, P., Sousa, A., Ganz, E., Sampaio, A., Alves, V., Marques, P., Sousa, N. (2016). A Hitchhiker's Guide to Functional Magnetic Resonance Imaging. Frontiers in Neuroscience $10,515$. doi:10.3389/fnins.2016.00515

Solé-Padullés, C., Castro-Fornieles, J., la Serna, de, E., Calvo, R., Baeza, I., Moya, J., Lázaro, L., Rosa, M., Bargalló, N., Sugranyes, G., (2016). Intrinsic connectivity networks from childhood to late adolescence: Effects of age and sex. Dev Cogn Neurosci 17, 35-44. doi:10.1016/j.dcn.2015.11.004

Somel M, Guo S, Fu N, Yan Z, Hu HY, Xu Y, Yuan Y, Ning Z, Hu Y, Menzel C, Hu H, Lachmann M, Zeng R, Chen W, Khaitovich P. (2010). MicroRNA, mRNA, and protein expression link development and aging in human and macaque brain. Genome Res. 20:1207-1218.

Song, M., Zhou, Y., Li, J., Liu, Y., Tian, L.-X., Yu, C., Jiang, T.-Z., (2008). Brain spontaneous functional connectivity and intelligence. NeuroImage 41, 1168-1176. doi:10.1016/j.neuroimage.2008.02.036

Soreq L, Rose J, Soreq E, Hardy J, Trabzuni D, Cookson MR, Smith C, Ryten M, Patani R, Ule J. (2017). Major Shifts in Glial Regional Identity Are a Transcriptional Hallmark of Human Brain Aging. Cell Rep. 18:557-570.

Sowell ER, Peterson BS, Kan E, Woods RP, Yoshii J, Bansal R, Xu D, Zhu H, Thompson PM, Toga AW. (2007). Sex differences in cortical thickness mapped in 176 healthy individuals between 7 and 87 years of age. Cereb Cortex. 17:15501560 .

Sowell, E.R., Trauner, D.A., Gamst, A., Jernigan, T.L., (2002). Development of cortical and subcortical brain structures in childhood and adolescence: a structural MRI study. Developmental Medicine and Child Neurology 44, 4-16. doi:10.1017/S0012162201001591

Spear, L. (2000). The adolescent brain and age-related behavioral manifestations. Neuroscience \& Biobehavioral Reviews 24(4), 417-463. doi:10.1016/s0149-7634(00)00014-2

Spear, L. (2011). Rewards, aversions and affect in adolescence: Emerging convergences across laboratory animal and human data. Developmental Cognitive Neuroscience 1(4), 390-403. doi:10.1016/j.dcn.2011.08.001

Spearman, C. (1904). The Proof and Measurement of Association between Two Things. The American Journal of Psychology 15(1), 72. doi:10.2307/1412159

Speed, D., Hemani, G., Johnson, M., Balding, D. (2012). Improved Heritability Estimation from Genome-wide SNPs. The American Journal of Human Genetics 91(6), 1011-1021. doi:10.1016/j.ajhg.2012.10.010

Spencer, C., Su, Z., Donnelly, P., Marchini, J. (2009). Designing Genome-Wide Association Studies: Sample Size, Power, Imputation, and the Choice of Genotyping Chip. PLoS Genetics 5(5), e1000477. doi:10.1371/journal.pgen.1000477

Spreng, R., Stevens, W., Chamberlain, J., Gilmore, A., Schacter, D. (2010). Default network activity, coupled with the frontoparietal control network, supports goal-directed cognition. Neurolmage 53(1), 303-317. doi:10.1016/j.neuroimage.2010.06.016

Sripada, C., Rutherford, S., Angstadt, M., Thompson, W., Luciana, M., Weigard, A., Hyde, L., Heitzeg, M. (2019). Prediction of neurocognition in youth from resting state fMRI. Molecular Psychiatry doi:10.1038/s41380-019-0481-6 
Steinberg, L. (2005). Cognitive and affective development in adolescence. Trends in Cognitive Sciences 9(2), 69-74. doi:10.1016/j.tics.2004.12.005

Steinberg, L., Morris, A. (2001). Adolescent Development. Annual Review of Psychology 52(1), 83-110. doi:10.1146/annurev.psych.52.1.83

Stevens, F.L., Hurley, R.A., Taber, K.H., (2011). Anterior cingulate cortex: unique role in cognition and emotion. J Neuropsychiatry Clin Neurosci 23, 121-125. doi:10.1176/jnp.23.2.jnp121

Stevens, J.S., Hamann, S., (2012). Sex differences in brain activation to emotional stimuli: A meta-analysis of neuroimaging studies. Neuropsychologia 50, 1578-1593. doi:10.1016/j.neuropsychologia.2012.03.011

Stevens, M.C., (2016). The contributions of resting state and task-based functional connectivity studies to our understanding of adolescent brain network maturation. Neuroscience \& Biobehavioral Reviews 70, 13-32. doi:10.1016/j.neubiorev.2016.07.027

Stiles, J., Jernigan, T. (2010). The Basics of Brain Development. Neuropsychology Review 20(4), $327-348$. doi:10.1007/s11065-010-9148-4

Stilo, S., Murray, R. (2019). Non-Genetic Factors in Schizophrenia. Current Psychiatry Reports 21(10), 100. doi:10.1007/s11920-019-1091-3

Storsve AB, Fjell AM, Tamnes CK, Westlye LT, Overbye K, Aasland HW, Walhovd KB. (2014). Differential longitudinal changes in cortical thickness, surface area and volume across the adult life span: regions of accelerating and decelerating change. J Neurosci. 34:8488-8498.

Streiner, D. (2003). Starting at the Beginning: An Introduction to Coefficient Alpha and Internal Consistency. Journal of Personality Assessment 80(1), 99-103. doi:10.1207/s15327752jpa8001_18

Strike LT, Couvy-Duchesne B, Hansell NK, Cuellar-Partida G, Medland SE, Wright MJ. (2015). Genetics and Brain Morphology. Neuropsychology Review 25(1), 63-96. doi:10.1007/s11065-015-9281-1

Strikwerda-Brown, C., Davey, C.G., Whittle, S., Allen, N.B., Byrne, M.L., Schwartz, O.S., Simmons, J.G., Dwyer, D., Harrison, B.J., (2015). Mapping the relationship between subgenual cingulate cortex functional connectivity and depressive symptoms across adolescence. Social Cognitive and Affective Neuroscience 10(7), 961-968. doi:10.1093/scan/nsu143

Sudre, G., Choudhuri, S., Székely, E., Bonner, T., Goduni, E., Sharp, W., Shaw, P.W., (2017). Estimating the Heritability of Structural and Functional Brain Connectivity in Families Affected by Attention-Deficit/Hyperactivity Disorder. JAMA Psychiatry 74(1), 76. doi:10.1001/jamapsychiatry.2016.3072

Sullivan, P., Agrawal, A., Bulik, C., Andreassen, O., Børglum, A., Breen, G., Cichon, S., Edenberg, H., Faraone, S., Gelernter, J., Mathews, C., Nievergelt, C., Smoller, J., O’Donovan, M. (2018). Psychiatric Genomics: An Update and an Agenda. American Journal of Psychiatry 175(1), 15-27. doi:10.1176/appi.ajp.2017.17030283

Sullivan, P., Kendler, K., Neale, M. (2003). Schizophrenia as a Complex Trait. Archives of General Psychiatry 60(12), 1187. doi:10.1001/archpsyc.60.12.1187

Supekar, K.S., Musen, M., Menon, V., (2009). Development of Large-Scale Functional Brain Networks in Children. PLoS Biology 7(7), e1000157. doi:10.1371/journal.pbio.1000157

Swagerman, S. (2016). Cognitive performance across the lifespan and domains. Vrije University Amsterdam, Netherlands Swagerman, S.C, Brouwer, R.M., Geus, E., Hulshoff Pol, H.E., Boomsma, D.I. (2014). Development and heritability of subcortical brain volumes at ages 9 and 12. Genes, Brain and Behavior 13(8), 733-742. doi:10.1111/gbb.12182

Swathy B, Banerjee M. 2017. Understanding epigenetics of schizophrenia in the backdrop of its antipsychotic drug therapy. Epigenomics. 9:721-736.

Sylvester, C.M., Whalen, D.J., Belden, A.C., Sanchez, S.L., Luby, J.L., Barch, D.M., (2018). Shyness and Trajectories of Functional Network Connectivity Over Early Adolescence. Child Development 89(3), 734-745. doi:10.1111/cdev.13005

Symms, M., Jäger, H., Schmierer, K., Yousry, T. (2004). A review of structural magnetic resonance neuroimaging. Journal of Neurology, Neurosurgery \& Psychiatry 75(9), 1235-1244. doi:10.1136/jnnp.2003.032714 
Talens, R., Boomsma, D., Tobi, E., Kremer, D., Jukema, J., Willemsen, G., Putter, H., Slagboom, P., Heijmans, B. (2010). Variation, patterns, and temporal stability of DNA methylation: considerations for epigenetic epidemiology. The FASEB Journal 24(9), 3135-3144. doi:10.1096/fj.09-150490

Tang B, Chang W-L, Lanigan CM, Dean B, Sutcliffe JG, Thomas EA. (2009). Normal human aging and early-stage schizophrenia share common molecular profiles. Aging Cell. 8:339-342.

Teeuw, J., Brouwer, R.M., Guimarães, J.P.O.F.T., Brandner, P., Koenis, M.M.G., Swagerman, S.C., Verwoert, M., Boomsma, D.I., Hulshoff Pol, H.E. (2019). Genetic and environmental influences on functional connectivity within and between canonical cortical resting-state networks throughout adolescent development in boys and girls. NeuroImage 202, 116073. doi:10.1016/j.neuroimage.2019.116073

Teeuw, J., Brouwer, R.M., Koenis, M.M.G., Swagerman, S.C., Boomsma, D.I., Hulshoff Pol, H.E., (2018). Genetic Influences on the Development of Cerebral Cortical Thickness During Childhood and Adolescence in a Dutch Longitudinal Twin Sample: The BrainSCALE Study. Cereb. Cortex 18, 1707. doi:10.1093/cercor/bhy005

Telzer, E.H., McCormick, E.M., Peters, S., Cosme, D., Pfeifer, J.H., van Duijvenvoorde, A.C.K., (2018). Methodological considerations for developmental longitudinal fMRI research. Developmental Cognitive Neuroscience 33, 149-160. doi:10.1016/j.dcn.2018.02.004

Termenon, M., Jaillard, A., Delon-Martin, C., Achard, S. (2016). Reliability of graph analysis of resting state fMRI using testretest dataset from the Human Connectome Project. NeuroImage 142, 172-187. doi:10.1016/j.neuroimage.2016.05.062

The Brainstorm Consortium, Anttila, V., Bulik-Sullivan, B., Finucane, H., Walters, R., Bras, J., Duncan, L., Escott-Price, V., Falcone, G., Gormley, P., Malik, R., Patsopoulos, N., Ripke, S., Wei, Z., Yu, D., Lee, P., Turley, P., Grenier-Boley, B., Chouraki, V., Kamatani, Y., Berr, C., Letenneur, L., Hannequin, D., Amouyel, P., Boland, A., Deleuze, J., Duron, E., Vardarajan, B., Reitz, C., Goate, A., Huentelman, M., Kamboh, M., Larson, E., Rogaeva, E., George-Hyslop, P., Hakonarson, H., Kukull, W., Farrer, L., Barnes, L., Beach, T., Demirci, F., Head, E., Hulette, C., Jicha, G., Kauwe, J., Kaye, J., Leverenz, J., Levey, A., Lieberman, A., Pankratz, V., Poon, W., Quinn, J., Saykin, A., Schneider, L., Smith, A., Sonnen, J., Stern, R., Deerlin, V., Eldik, L., Harold, D., Russo, G., Rubinsztein, D., Bayer, A., Tsolaki, M., Proitsi, P., Fox, N., Hampel, H., Owen, M., Mead, S., Passmore, P., Morgan, K., Nöthen, M., Schott, J., Rossor, M., Lupton, M., Hoffmann, P., Kornhuber, J., Lawlor, B., McQuillin, A., Al-Chalabi, A., Bis, J., Ruiz, A., Boada, M., Seshadri, S., Beiser, A., Rice, K., Lee, S., Jager, P., Geschwind, D., Riemenschneider, M., Riedel-Heller, S., Rotter, J., Ransmayr, G., Hyman, B., Cruchaga, C., Alegret, M., Winsvold, B., Palta, P., Farh, K., Cuenca-Leon, E., Furlotte, N., Kurth, T., Ligthart, L., Terwindt, G., Freilinger, T., Ran, C., Gordon, S., Borck, G., Adams, H., Lehtimäki, T., Wedenoja, J., Buring, J., Schürks, M., Hrafnsdottir, M., Hottenga, J., Penninx, B., Artto, V., Kaunisto, M., Vepsäläinen, S., Martin, N., Montgomery, G., Kurki, M., Hämäläinen, E., Huang, H., Huang, J., Sandor, C., Webber, C., Muller-Myhsok, B., Schreiber, S., Salomaa, V., Loehrer, E., Göbel, H., Macaya, A., Pozo-Rosich, P., Hansen, T., Werge, T., Kaprio, J., Metspalu, A., Kubisch, C., Ferrari, M., Belin, A., Maagdenberg, A., Zwart, J., Boomsma, D., Eriksson, N., Olesen, J., Chasman, D., Nyholt, D., Anney, R., Avbersek, A., Baum, L., Berkovic, S., Bradfield, J., Buono, R., Catarino, C., Cossette, P., Jonghe, P., Depondt, C., Dlugos, D., Ferraro, T., French, J., Hjalgrim, H., Jamnadas-Khoda, J., Kälviäinen, R., Kunz, W., Lerche, H., Leu, C., Lindhout, D., Lo, W., Lowenstein, D., McCormack, M., Møller, R., Molloy, A., Ng, P., Oliver, K., Privitera, M., Radtke, R., Ruppert, A., Sander, T., Schachter, S., Schankin, C., Scheffer, I., Schoch, S., Sisodiya, S., Smith, P., Sperling, M., Striano, P., Surges, R., Thomas, G., Visscher, F., Whelan, C., Zara, F., Heinzen, E., Marson, A., Becker, F., Stroink, H., Zimprich, F., Gasser, T., Gibbs, R., Heutink, P., Martinez, M., Morris, H., Sharma, M., Ryten, M., Mok, K., Pulit, S., Bevan, S., Holliday, E., Attia, J., Battey, T., Boncoraglio, G., Thijs, V., Chen, W., Mitchell, B., Rothwell, P., Sharma, P., Sudlow, C., Vicente, A., Markus, H., Kourkoulis, C., Pera, J., Raffeld, M., Silliman, S., Perica, V., Thornton, L., Huckins, L., Rayner, N., Lewis, C., Gratacos, M., Rybakowski, F., Keski-Rahkonen, A., Raevuori, A., Hudson, J., Reichborn-Kjennerud, T., Monteleone, P., Karwautz, A., Mannik, K., Baker, J., O’Toole, J., Trace, S., Davis, O., Helder, S., Ehrlich, S., Herpertz-Dahlmann, B., Danner, U., Elburg, A., Clementi, M., Forzan, M., Docampo, E., Lissowska, J., Hauser, J., Tortorella, A., Maj, M., Gonidakis, F., Tziouvas, K., Papezova, H., Yilmaz, Z., Wagner, G., Cohen-Woods, S., Herms, S., Julià, A., Rabionet, R., Dick, D., Ripatti, S., Andreassen, O., Espeseth, T., Lundervold, A., Steen, V., Pinto, D., Scherer, S., Aschauer, H., Schosser, 
A., Alfredsson, L., Padyukov, L., Halmi, K., Mitchell, J., Strober, M., Bergen, A., Kaye, W., Szatkiewicz, J., Cormand, B., Ramos-Quiroga, J., Sánchez-Mora, C., Ribasés, M., Casas, M., Hervas, A., Arranz, M., Haavik, J., Zayats, T., Johansson, S., Williams, N., Elia, J., Dempfle, A., Rothenberger, A., Kuntsi, J., Oades, R., Banaschewski, T., Franke, B., Buitelaar, J., Vasquez, A., Doyle, A., Reif, A., Lesch, K., Freitag, C., Rivero, O., Palmason, H., Romanos, M., Langley, K., Rietschel, M., Witt, S., Dalsgaard, S., Børglum, A., Waldman, I., Wilmot, B., Molly, N., Bau, C., Crosbie, J., Schachar, R., Loo, S., McGough, J., Grevet, E., Medland, S., Robinson, E., Weiss, L., Bacchelli, E., Bailey, A., Bal, V., Battaglia, A., Betancur, C., Bolton, P., Cantor, R., Celestino-Soper, P., Dawson, G., Rubeis, S., Duque, F., Green, A., Klauck, S., Leboyer, M., Levitt, P., Maestrini, E., Mane, S., De-Luca, D., Parr, J., Regan, R., Reichenberg, A., Sandin, S., Vorstman, J., Wassink, T., Wijsman, E., Cook, E., Santangelo, S., Delorme, R., Rogé, B., Magalhaes, T., Arking, D., Schulze, T., Thompson, R., Strohmaier, J., Matthews, K., Melle, I., Morris, D., Blackwood, D., McIntosh, A., Bergen, S., Schalling, M., Jamain, S., Maaser, A., Fischer, S., Reinbold, C., Fullerton, J., Grigoroiu-Serbanescu, M., Guzman-Parra, J., Mayoral, F., Schofield, P., Cichon, S., Mühleisen, T., Degenhardt, F., Schumacher, J., Bauer, M., Mitchell, P., Gershon, E., Rice, J., Potash, J., Zandi, P., Craddock, N., Ferrier, I., Alda, M., Rouleau, G., Turecki, G., Ophoff, R., Pato, C., Anjorin, A., Stahl, E., Leber, M., Czerski, P., Edenberg, H., Cruceanu, C., Jones, I., Posthuma, D., Andlauer, T., Forstner, A., Streit, F., Baune, B., Air, T., Sinnamon, G., Wray, N., MacIntyre, D., Porteous, D., Homuth, G., Rivera, M., Grove, J., Middeldorp, C., Hickie, I., Pergadia, M., Mehta, D., Smit, J., Jansen, R., Geus, E., Dunn, E., Li, Q., Nauck, M., Schoevers, R., Beekman, A., Knowles, J., Viktorin, A., Arnold, P., Barr, C., Bedoya-Berrio, G., Bienvenu, O., Brentani, H., Burton, C., Camarena, B., Cappi, C., Cath, D., Cavallini, M., Cusi, D., Darrow, S., Denys, D., Derks, E., Dietrich, A., Fernandez, T., Figee, M., Freimer, N., Gerber, G., Grados, M., Greenberg, E., Hanna, G., Hartmann, A., Hirschtritt, M., Hoekstra, P., Huang, A., Huyser, C., Illmann, C., Jenike, M., Kuperman, S., Leventhal, B., Lochner, C., Lyon, G., Macciardi, F., Madruga-Garrido, M., Malaty, I., Maras, A., McGrath, L., Miguel, E., Mir, P., Nestadt, G., Nicolini, H., Okun, M., Pakstis, A., Paschou, P., Piacentini, J., Pittenger, C., Plessen, K., Ramensky, V., Ramos, E., Reus, V., Richter, M., Riddle, M., Robertson, M., Roessner, V., Rosário, M., Samuels, J., Sandor, P., Stein, D., Tsetsos, F., Nieuwerburgh, F., Weatherall, S., Wendland, J., Wolanczyk, T., Worbe, Y., Zai, G., Goes, F., McLaughlin, N., Nestadt, P., Grabe, H., Depienne, C., Konkashbaev, A., Lanzagorta, N., Valencia-Duarte, A., Bramon, E., Buccola, N., Cahn, W., Cairns, M., Chong, S., Cohen, D., Crespo-Facorro, B., Crowley, J., Davidson, M., DeLisi, L., Dinan, T., Donohoe, G., Drapeau, E., Duan, J., Haan, L., Hougaard, D., Karachanak-Yankova, S., Khrunin, A., Klovins, J., Kučinskas, V., Keong, J., Limborska, S., Loughland, C., Lönnqvist, J., Maher, B., Mattheisen, M., McDonald, C., Murphy, K., Murray, R., Nenadic, I., van Os, J., Pantelis, C., Pato, M., Petryshen, T., Quested, D., Roussos, P., Sanders, A., Schall, U., Schwab, S., Sim, K., So, H., Stögmann, E., Subramaniam, M., Toncheva, D., Waddington, J., Walters, J., Weiser, M., Cheng, W., Cloninger, R., Curtis, D., Gejman, P., Henskens, F., Mattingsdal, M., Oh, S., Scott, R., Webb, B., Breen, G., Churchhouse, C., Bulik, C., Daly, M., Dichgans, M., Faraone, S., Guerreiro, R., Holmans, P., Kendler, K., Koeleman, B., Mathews, C., Price, A., Scharf, J., Sklar, P., Williams, J., Wood, N., Cotsapas, C., Palotie, A., Smoller, J., Sullivan, P., Rosand, J., Corvin, A., Neale, B. (2018). Analysis of shared heritability in common disorders of the brain. Science 360(6395), eaap8757. doi:10.1126/science.aap8757

Thomason, M.E., Dennis, E.L., Joshi, A.A., Joshi, S.H., Dinov, I.D., Chang, C., Henry, M.L., Johnson, R.F., Thompson, P.M., Toga, A.W., Glover, G.H., Van Horn, J.D., Gotlib, I.H., (2011). Resting-state fMRI can reliably map neural networks in children. NeuroImage 55, 165-175. doi:10.1016/j.neuroimage.2010.11.080

Thomason, M.E., Grove, L.E., Lozon, T.A., Vila, A.M., Ye, Y., Nye, M.J., Manning, J.H., Pappas, A., Hernandez-Andrade, E., Yeo, L., Mody, S., Berman, S., Hassan, S.S., Romero, R., (2015). Age-related increases in long-range connectivity in fetal functional neural connectivity networks in utero. Developmental Cognitive Neuroscience 11, 96-104. doi:10.1016/j.den.2014.09.001

Thompson, P., Jahanshad, N., Ching, C., Salminen, L., Thomopoulos, S., Bright, J., Baune, B., Bertolín, S., Bralten, J., Bruin, W., Bülow, R., Chen, J., Chye, Y., Dannlowski, U., Kovel, C., Donohoe, G., Eyler, L., Faraone, S., Favre, P., Filippi, C., Frodl, T., Garijo, D., Gil, Y., Grabe, H., Grasby, K., Hajek, T., Han, L., Hatton, S., Hilbert, K., Ho, T., Holleran, L., Homuth, G., Hosten, N., Houenou, J., Ivanov, I., Jia, T., Kelly, S., Klein, M., Kwon, J., Laansma, M., Leerssen, J., Lueken, U., Nunes, A., Neill, J., Opel, N., Piras, F., Piras, F., Postema, M., Pozzi, E., Shatokhina, N., Soriano-Mas, C., Spalletta, G., Sun, D., 
Teumer, A., Tilot, A., Tozzi, L., Merwe, C., Someren, E., Wingen, G., Völzke, H., Walton, E., Wang, L., Winkler, A., Wittfeld, K., Wright, M., Yun, J., Zhang, G., Zhang-James, Y., Adhikari, B., Agartz, I., Aghajani, M., Aleman, A., Althoff, R., Altmann, A., Andreassen, O., Baron, D., Bartnik-Olson, B., Bas-Hoogendam, J., Baskin-Sommers, A., Bearden, C., Berner, L., Boedhoe, P., Brouwer, R., Buitelaar, J., Caeyenberghs, K., Cecil, C., Cohen, R., Cole, J., Conrod, P., Brito, S., de Zwarte, S., Dennis, E., Desrivieres, S., Dima, D., Ehrlich, S., Esopenko, C., Fairchild, G., Fisher, S., Fouche, J., Francks, C., Frangou, S., Franke, B., Garavan, H., Glahn, D., Groenewold, N., Gurholt, T., Gutman, B., Hahn, T., Harding, I., Hernaus, D., Hibar, D., Hillary, F., Hoogman, M., Hulshoff Pol, H.E., Jalbrzikowski, M., Karkashadze, G., Klapwijk, E., Knickmeyer, R., Kochunov, P., Koerte, I., Kong, X., Liew, S., Lin, A., Logue, M., Luders, E., Macciardi, F., Mackey, S., Mayer, A., McDonald, C., McMahon, A., Medland, S., Modinos, G., Morey, R., Mueller, S., Mukherjee, P., NamazovaBaranova, L., Nir, T., Olsen, A., Paschou, P., Pine, D., Pizzagalli, F., Rentería, M., Rohrer, J., Sämann, P., Schmaal, L., Schumann, G., Shiroishi, M., Sisodiya, S., Smit, D., Sønderby, I., Stein, D., Stein, J., Tahmasian, M., Tate, D., Turner, J., Heuvel, O., Wee, N., Werf, Y., van Erp, T., van Haren, N., Rooij, D., Velzen, L., Veer, I., Veltman, D., Villalon-Reina, J., Walter, H., Whelan, C., Wilde, E., Zarei, M., Zelman, V. (2020). ENIGMA and global neuroscience: A decade of large-scale studies of the brain in health and disease across more than 40 countries. Translational Psychiatry 10(1), 100. doi:10.1038/s41398-020-0705-1

Thompson PM, Cannon TD, Narr KL, van Erp TGM, Poutanen VP, Huttunen M, Lönnqvist J, Standertskjöld-Nordenstam CG, Kaprio J, Khaledy M, Dail R, Zoumalan CI, Toga AW. (2001). Genetic influences on brain structure. Nat Neurosci. 4:1253-1258.

Thompson, P.M., Ge, T., Glahn, D.C., Jahanshad, N., Nichols, T.E., (2013). Genetics of the connectome. NeuroImage 80, 475488. doi:10.1016/j.neuroimage.2013.05.013

Tomasi, D., Wang, G., Volkow, N. (2013). Energetic cost of brain functional connectivity. Proceedings of the National Academy of Sciences 110(33), 13642-13647. doi:10.1073/pnas.1303346110

Toschi, N., Riccelli, R., Indovina, I., Terracciano, A., Passamonti, L. (2018). Functional Connectome of the Five-Factor Model of Personality. Personality Neuroscience 1, e2. doi:10.1017/pen.2017.2

Turk, E., van den Heuvel, M., Benders, M., Heus, R., Franx, A., Manning, J., Hect, J., Hernandez-Andrade, E., Hassan, S., Romero, R., Kahn, R., Thomason, M., van den Heuvel, M. (2019). Functional Connectome of the Fetal Brain. The Journal of Neuroscience 39(49), 9716-9724. doi:10.1523/jneurosci.2891-18.2019

Tzourio-Mazoyer NEA, Landeau B, Papathanassiou D, Crivello F, Etard O, Delcroix N, Mazoyer B, Joliot M. (2002). Automated anatomical labeling of activations in SPM using a macroscopic anatomical parcellation of the MNI MRI singlesubject brain. NeuroImage. 15:273-289.

Uddin, L.Q., (2014). Salience processing and insular cortical function and dysfunction. Nat Rev Neurosci 16, 55-61. doi:10.1038/nrn3857

Uddin, L.Q., Nomi, J.S., Hébert-Seropian, B., Ghaziri, J., Boucher, O., (2017). Structure and Function of the Human Insula. J Clin Neurophysiol 34, 300-306. doi:10.1097/WNP.0000000000000377

Uddin, L.Q., Supekar, K.S., Ryali, S., Menon, V., (2011). Dynamic reconfiguration of structural and functional connectivity across core neurocognitive brain networks with development. J Neurosci 31, 18578-18589. doi:10.1523/JNEUROSCI.446511.2011

Uğurbil, K., Xu, J., Auerbach, E., Moeller, S., Vu, A., Duarte-Carvajalino, J., Lenglet, C., Wu, X., Schmitter, S., Moortele, P., Strupp, J., Sapiro, G., Martino, F., Wang, D., Harel, N., Garwood, M., Chen, L., Feinberg, D., Smith, S., Miller, K., Sotiropoulos, S., Jbabdi, S., Andersson, J., Behrens, T., Glasser, M., Essen, D., Yacoub, E., WU-Minn HCP Consortium. (2013). Pushing spatial and temporal resolution for functional and diffusion MRI in the Human Connectome Project. NeuroImage 80, 80-104. doi:10.1016/j.neuroimage.2013.05.012

Vaidya, C., Gordon, E. (2013). Phenotypic Variability in Resting-State Functional Connectivity: Current Status. Brain Connectivity 3(2), 99-120. doi:10.1089/brain.2012.0110

Vakhtin, A.A., Ryman, S.G., Flores, R.A., Jung, R.E., (2014). Functional brain networks contributing to the Parieto-Frontal Integration Theory of Intelligence. NeuroImage 103, 349-354. doi:10.1016/j.neuroimage.2014.09.055 
Valiathan R, Ashman M, Asthana D. (2016). Effects of Ageing on the Immune System: Infants to Elderly. Scand J Immunol. 83:255-266.

Valizadeh SA, Hänggi J, Mérillat S, Jäncke L. (2017). Age prediction on the basis of brain anatomical measures. Hum Brain Mapp. 38:997-1008.

van Baal, G.C.M., de Geus, E.J.C., Boomsma, D.I., (1998). Longitudinal study of genetic influences on ERP-P3 during childhood. Dev Neuropsychol 14, 19-45. doi:10.1080/87565649809540699

van Beijsterveldt, C.E.M., Groen-Blokhuis, M., Hottenga, J.-J., Franić, S., Hudziak, J.J., Lamb, D., Huppertz, C., de Zeeuw, E., Nivard, M.G., Schutte, N., Swagerman, S.C., Glasner, T., van Fulpen, M., Brouwer, C., Stroet, T., Nowotny, D., Ehli, E.A., Davies, G.E., Scheet, P., Orlebeke, J.F., Kan, K.-J., Smit, D.J.A., Dolan, C.V., Middeldorp, C.M., de Geus, E.J.C., Bartels, M., Boomsma, D.I., (2013). The Young Netherlands Twin Register (YNTR): longitudinal twin and family studies in over 70,000 children. Twin Res Hum Genet 16, 252-267. doi:10.1017/thg.2012.118

van Beijsterveldt, C.E.M., van Baal, G.C.M., Molenaar, P.C., Boomsma, D.I., de Geus, E.J.C., (2001). Stability of Genetic and Environmental Influences on P300 Amplitude: A Longitudinal Study in Adolescent Twins. Behavior Genetics 31(6), 533543. doi:10.1023/a:1013389226795

van den Heuvel, M.P., Hulshoff Pol, H.E., (2010). Exploring the brain network: A review on resting-state fMRI functional connectivity. European Neuropsychopharmacology 20(8), 519-534. doi:10.1016/j.euroneuro.2010.03.008

van den Heuvel, M.P., van Soelen, I.L.C., Stam, C.J., Kahn, R.S., Boomsma, D.I., Hulshoff Pol, H.E., (2013). Genetic control of functional brain network efficiency in children. European Neuropsychopharmacology 23, $19-23$. doi:10.1016/j.euroneuro.2012.06.007

van Dijk, K., Sabuncu, M., Buckner, R. (2012). The influence of head motion on intrinsic functional connectivity MRI. NeuroImage 59(1), 431-438. doi:10.1016/j.neuroimage.2011.07.044

van Dongen, J., Nivard, M., Willemsen, G., Hottenga, J., Helmer, Q., Dolan, C., Ehli, E., Davies, G., Iterson, M., Breeze, C., Beck, S., Hoen, P., Pool, R., Greevenbroek, M., Stehouwer, C., Kallen, C., Schalkwijk, C., Wijmenga, C., Zhernakova, S., Tigchelaar, E., Beekman, M., Deelen, J., Heemst, D., Veldink, J., Berg, L., Duijn, C., Hofman, B., Uitterlinden, A., Jhamai, P., Verbiest, M., Verkerk, M., Breggen, R., Rooij, J., Lakenberg, N., Mei, H., Bot, J., Zhernakova, D., Hof, P., Deelen, P., Nooren, I., Moed, M., Vermaat, M., Luijk, R., Bonder, M., Dijk, F., Galen, M., Arindrarto, W., Kielbasa, S., Swertz, M., Zwet, E., Isaacs, A., Franke, L., Suchiman, H., Jansen, R., Meurs, J., Heijmans, B., Slagboom, P., Boomsma, D. (2016). Genetic and environmental influences interact with age and sex in shaping the human methylome. Nature Communications 7(1), 11115. doi:10.1038/ncomms11115

van Duijvenvoorde, A., Peters, S., Braams, B., Crone, E. (2016). What motivates adolescents? Neural responses to rewards and their influence on adolescents' risk taking, learning, and cognitive control. Neuroscience \& Biobehavioral Reviews 70, 135-147. doi:10.1016/j.neubiorev.2016.06.037

van Duijvenvoorde, A., Westhoff, B., Vos, F., Wierenga, L., Crone, E. (2019). A three-wave longitudinal study of subcorticalcortical resting-state connectivity in adolescence: Testing age- and puberty-related changes. Human brain mapping 40(13), 3769-3783. doi:10.1002/hbm.24630

van Erp, T., Walton, E., Hibar, D., Schmaal, L., Jiang, W., Glahn, D., Pearlson, G., Yao, N., Fukunaga, M., Hashimoto, R., Okada, N., Yamamori, H., Bustillo, J., Clark, V., Agartz, I., Mueller, B., Cahn, W., de Zwarte, S., Hulshoff Pol, H.E., Kahn, R., Ophoff, R., van Haren, N., Andreassen, O., Dale, A., Doan, N., Gurholt, T., Hartberg, C., Haukvik, U., Jørgensen, K., Lagerberg, T., Melle, I., Westlye, L., Gruber, O., Kraemer, B., Richter, A., Zilles, D., Calhoun, V., Crespo-Facorro, B., Roiz-Santiañez, R., Tordesillas-Gutiérrez, D., Loughland, C., Carr, V., Catts, S., Cropley, V., Fullerton, J., Green, M., Henskens, F., Jablensky, A., Lenroot, R., Mowry, B., Michie, P., Pantelis, C., Quidé, Y., Schall, U., Scott, R., Cairns, M., Seal, M., Tooney, P., Rasser, P., Cooper, G., Weickert, C., Weickert, T., Morris, D., Hong, E., Kochunov, P., Beard, L., Gur, R., Gur, R., Satterthwaite, T., Wolf, D., Belger, A., Brown, G., Ford, J., Macciardi, F., Mathalon, D., O’Leary, D., Potkin, S., Preda, A., Voyvodic, J., Lim, K., McEwen, S., Yang, F., Tan, Y., Tan, S., Wang, Z., Fan, F., Chen, J., Xiang, H., Tang, S., Guo, H., Wan, P., Wei, D., Bockholt, H., Ehrlich, S., Wolthusen, R., King, M., Shoemaker, J., Sponheim, S., Haan, L., Koenders, L., Machielsen, M., Amelsvoort, T., Veltman, D., Assogna, F., Banaj, N., Rossi, P., Iorio, M., Piras, 
F., Spalletta, G., McKenna, P., Pomarol-Clotet, E., Salvador, R., Corvin, A., Donohoe, G., Kelly, S., Whelan, C., Dickie, E., Rotenberg, D., Voineskos, A., Ciufolini, S., Radua, J., Dazzan, P., Murray, R., Marques, T., Simmons, A., Borgwardt, S., Egloff, L., Harrisberger, F., Riecher-Rössler, A., Smieskova, R., Alpert, K., Wang, L., Jönsson, E., Koops, S., Sommer, I., Bertolino, A., Bonvino, A., Giorgio, A., Neilson, E., Mayer, A., Stephen, J., Kwon, J., Yun, J., Cannon, D., McDonald, C., Lebedeva, I., Tomyshev, A., Akhadov, T., Kaleda, V., Fatouros-Bergman, H., Flyckt, L., Project, K., Farde, L., Flyckt, L., Engberg, G., Erhardt, S., Fatouros-Bergman, H., Cervenka, S., Schwieler, L., Piehl, F., Agartz, I., Collste, K., Victorsson, P., Malmqvist, A., Hedberg, M., Orhan, F., Busatto, G., Rosa, P., Serpa, M., Zanetti, M., Hoschl, C., Skoch, A., Spaniel, F., Tomecek, D., Hagenaars, S., McIntosh, A., Whalley, H., Lawrie, S., Knöchel, C., Oertel-Knöchel, V., Stäblein, M., Howells, F., Stein, D., Temmingh, H., Uhlmann, A., Lopez-Jaramillo, C., Dima, D., McMahon, A., Faskowitz, J., Gutman, B., Jahanshad, N., Thompson, P., Turner, J. (2018). Cortical Brain Abnormalities in 4474 Individuals With Schizophrenia and 5098 Control Subjects via the Enhancing Neuro Imaging Genetics Through Meta Analysis (ENIGMA) Consortium. Biological Psychiatry 84(9), 644-654. doi:10.1016/j.biopsych.2018.04.023

van Essen, D., Smith, S., Barch, D., Behrens, T., Yacoub, E., Ugurbil, K., WU-Minn HCP Consortium (2013). The WU-Minn

Human Connectome Project: An overview. NeuroImage 80, 62-79. doi:10.1016/j.neuroimage.2013.05.041

van Gelderen, P., van Gelderen, P., Duyn, J.H., Duyn, J.H., Ramsey, N.F., Ramsey, N.F., Liu, G., Liu, G., Moonen, C.T.W., Moonen, C.T.W., (2012). The PRESTO technique for fMRI. NeuroImage 62, 676-681. doi:10.1016/j.neuroimage.2012.01.017

van Gestel H, Franke K, Petite J, Slaney C, Garnham J, Helmick C, Johnson K, Uher R, Alda M, Hajek T. (2019). Brain age in bipolar disorders: Effects of lithium treatment. Australian \& New Zealand Journal of Psychiatry, 53(12), 1179-88.

van Haren, N., Cahn, W., Hulshoff Pol, H.E, Kahn, R. (2008). Schizophrenia as a progressive brain disease. European Psychiatry 23(4), 245-254. doi:10.1016/j.eurpsy.2007.10.013

van Haren, N., Rijsdijk, F., Schnack, H., Picchioni, M., Toulopoulou, T., Weisbrod, M., Sauer, H., van Erp, T., Cannon, T., Huttunen, M., Boomsma, D., Hulshoff Pol, H.E., Murray, R., Kahn, R. (2012). The Genetic and Environmental Determinants of the Association Between Brain Abnormalities and Schizophrenia: The Schizophrenia Twins and Relatives Consortium. Biological Psychiatry 71(10), 915-921. doi:10.1016/j.biopsych.2012.01.010

van Haren, N., Schnack, H., Koevoets, M., Cahn, W., Hulshoff Pol, H.E., Kahn, R. (2016). Trajectories of subcortical volume change in schizophrenia: A 5-year follow-up. Schizophrenia Research 173(3), 140-145. doi:10.1016/j.schres.2015.09.027 van Haren NEM, Hulshoff Pol HE, Schnack HG, Cahn W, Brans R, Carati I, Rais M, Kahn RS. (2008). Progressive Brain

Volume Loss in Schizophrenia Over the Course of the Illness: Evidence of Maturational Abnormalities in Early Adulthood. Biological Psychiatry 63(1), 106-113. doi:10.1016/j.biopsych.2007.01.004

van Haren NEM, Hulshoff Pol HE, Schnack HG, Cahn W, Mandl RCW, Collins DL, Evans AC, Kahn RS. (2007). Focal

Gray Matter Changes in Schizophrenia across the Course of the Illness: A 5-Year Follow-Up Study. Neuropsychopharmacology 32(10), 2057-2066. doi:10.1038/sj.npp.1301347

van Leeuwen, M. (2008). A study of cognition in pre-adolescent twins. Vrije University Amsterdam, Netherlands van Leeuwen, M., Berg, S., Boomsma, D. (2008). A twin-family study of general IQ. Learning and Individual Differences 18(1), 76-88. doi:10.1016/j.lindif.2007.04.006

van Leeuwen, M., Berg, S., Hoekstra, R., Boomsma, D. (2007). Endophenotypes for intelligence in children and adolescents. Intelligence 35(4), 369-380. doi:10.1016/j.intell.2006.09.008

van Leeuwen, M., Peper, J., Berg, S., Brouwer, R., Hulshoff Pol, H.E., Kahn, R., Boomsma, D. (2009). A genetic analysis of brain volumes and IQ in children. Intelligence 37(2), 181-191. doi:10.1016/j.intell.2008.10.005

van Nierop, M., Janssens, M., Investigators, G., Bruggeman, R., Cahn, W., Haan, L., Kahn, R., Meijer, C., Myin-Germeys,

I., van Os, J., Wiersma, D. (2013). Evidence That Transition from Health to Psychotic Disorder Can Be Traced to SemiUbiquitous Environmental Effects Operating against Background Genetic Risk. PLoS ONE 8(11), e76690. doi:10.1371/journal.pone.0076690

van Os, J., Kapur, S. (2009). Schizophrenia. The Lancet 374(9690), 635-645. doi:10.1016/s0140-6736(09)60995-8 
van Os, J., Rutten, B., Poulton, R. (2008). Gene-Environment Interactions in Schizophrenia: Review of Epidemiological Findings and Future Directions. Schizophrenia Bulletin 34(6), 1066-1082. doi:10.1093/schbul/sbn117

van Soelen, I. (2011). Genetics of structural brain development and cognition in childhood and early adolescence. Utrecht University, Netherlands

van Soelen, I., Berg, S., Dekker, P., van Leeuwen, M., Peper, J., Hulshoff Pol, H.E., Boomsma, D. (2009). Individual differences in dynamic measures of verbal learning abilities in young twin pairs and their older siblings. Learning and Individual Differences 19(4), 440-444. doi:10.1016/j.lindif.2009.03.005

van Soelen, I., Brouwer, R., Peper, J., Beijsterveldt, T., van Leeuwen, M., Vries, L., Kahn, R., Hulshoff Pol, H.E., Boomsma,

D. (2010). Effects of Gestational Age and Birth Weight on Brain Volumes in Healthy 9 Year-Old Children. The Journal of Pediatrics 156(6), 896-901. doi:10.1016/j.jpeds.2009.12.052

van Soelen, I., Brouwer, R., van Leeuwen, M., Kahn, R., Hulshoff Pol, H.E., Boomsma, D. (2011). Heritability of Verbal and Performance Intelligence in a Pediatric Longitudinal Sample. Twin Research and Human Genetics 14(2), 119-128. doi:10.1375/twin.14.2.119

van Soelen ILC, Brouwer RM, Peper JS, van Leeuwen M, Koenis MMG, van Beijsterveldt CEM, Swagerman SC, Kahn RS, Hulshoff Pol HE, Boomsma DI. (2012). Brain SCALE: Brain Structure and Cognition: an Adolescent Longitudinal Twin Study into the Genetic Etiology of Individual Differences. Twin Research and Human Genetics 15(3), 453-467. doi:10.1017/thg.2012.4

van Soelen, I.L.C., Brouwer, R.M., van Baal, G.C.M., Schnack, H.G., Peper, J.S., Chen, L., Kahn, R.S., Boomsma, D.I., Hulshoff Pol, H.E., 2013. Heritability of volumetric brain changes and height in children entering puberty. Hum Brain Mapp 34, 713-725. doi:10.1002/hbm.21468

van Soelen ILC, Brouwer RM, van Baal GCM, Schnack HG, Peper JS, Chen L, Kahn RS, Boomsma DI, Hulshoff Pol HE. (2013). Heritability of volumetric brain changes and height in children entering puberty. Human Brain Mapping 34(3), 713-725. doi:10.1002/hbm.21468

van Soelen, I.L.C., Brouwer, R.M., van Baal, G.C.M., Schnack, H.G., Peper, J.S., Collins, D.L., Evans, A.C., Kahn, R.S., Boomsma, D.I., Hulshoff Pol, H.E., (2012). Genetic influences on thinning of the cerebral cortex during development. NeuroImage 59(4), 3871-3880. doi:10.1016/j.neuroimage.2011.11.044

Vanderwal, T., Kelly, C., Eilbott, J., Mayes, L., Castellanos, F. (2015). Inscapes: A movie paradigm to improve compliance in functional magnetic resonance imaging. NeuroImage 122, 222-232. doi:10.1016/j.neuroimage.2015.07.069

Varikuti, D., Hoffstaedter, F., Genon, S., Schwender, H., Reid, A., Eickhoff, S. (2017). Resting-state test-retest reliability of a priori defined canonical networks over different preprocessing steps. Brain Structure and Function 222(3), 1447-1468. doi:10.1007/s00429-016-1286-x

Viana J, Hannon E, Dempster E, Pidsley R, Macdonald R, Knox O, Spiers H, Troakes C, Al-Saraj S, Turecki G, Schalkwyk LC, Mill J. (2017). Schizophrenia-associated methylomic variation: molecular signatures of disease and polygenic risk burden across multiple brain regions. Human Molecular Genetics 26(1), 210-225. doi:10.1093/hmg/ddw373

Voelcker-Rehage C, Niemann C. (2013). Structural and functional brain changes related to different types of physical activity across the life span. Neuroscience and Biobehavioral Reviews. 37:2268-2295.

Voisey, J., Lawford, B., Morris, C., Wockner, L., Noble, E., Young, R., Mehta, D. (2017). Epigenetic analysis confirms no accelerated brain aging in schizophrenia. npj Schizophrenia 3(1), 26. doi:10.1038/s41537-017-0026-4

Vul, E., Harris, C., Winkielman, P., Pashler, H. (2009). Puzzlingly High Correlations in fMRI Studies of Emotion, Personality, and Social Cognition. Perspectives on psychological science: a journal of the Association for Psychological Science 4(3), 274-90. doi:10.1111/j.1745-6924.2009.01125.x

Waheed, S.H., Mirbagheri, S., Agarwal, S., Kamali, A., Yahyavi-Firouz-Abadi, N., Chaudhry, A., DiGianvittorio, M., Gujar, S.K., Pillai, J.J., Sair, H.I., (2016). Reporting of Resting-State Functional Magnetic Resonance Imaging Preprocessing Methodologies. Brain Connectivity 6(9), 663-668. doi:10.1089/brain.2016.0446

Walhovd KB, Fjell AM, Giedd JN, Dale AM, Brown TT. (2017). Through Thick and Thin: a Need to Reconcile Contradictory Results on Trajectories in Human Cortical Development. Cerebral Cortex 27(2), 1472-1481. doi:10.1093/cercor/bhv301 
Walton E, Hass J, Liu J, Roffman JL, Bernardoni F, Roessner V, Kirsch M, Schackert G, Calhoun V, Ehrlich S. (2016). Correspondence of DNA methylation between blood and brain tissue and its application to schizophrenia research. Schizophr Bull. 42:406-414.

Wang, L. (2010). Disattenuation of Correlations Due to Fallible Measurement. Newborn and Infant Nursing Reviews 10(1), 60-65. doi:10.1053/j.nainr.2009.12.013

Watanabe, K., Taskesen, E., Bochoven, A., Posthuma, D. (2017). Functional mapping and annotation of genetic associations with FUMA. Nature Communications 8(1), 1826. doi:10.1038/s41467-017-01261-5

Watts, M., Pocock, R., Claudianos, C. (2018). Brain Energy and Oxygen Metabolism: Emerging Role in Normal Function and Disease. Frontiers in Molecular Neuroscience 11, 216. doi:10.3389/fnmol.2018.00216

Wechsler, D., (1991). The Wechsler Intelligence Scale for Children administration and scoring manual, 3rd edition. The Psychological Corporation, San Antonio, TX

Wei T, Simko V. (2016). corrplot: Visualization of a Correlation Matrix, version 0.77. https://cran.rproject.org/web/packages/corrplot/

Wei, T., Simko, V. (2017). corrplot: Visualization of a Correlation Matrix, version 0.84. https://cran.rproject.org/web/packages/corrplot/

Weidner CI, Lin Q, Koch CM, Eisele L, Beier F, Ziegler P, Bauerschlag DO, Jöckel K-H, Erbel R, Mühleisen TW, Zenke M, Brümmendorf TH, Wagner W. (2014). Aging of blood can be tracked by DNA methylation changes at just three CpG sites. Genome Biol. 15:R24.

Weinberger, D. (1987). Implications of Normal Brain Development for the Pathogenesis of Schizophrenia. Archives of General Psychiatry 44(7), 660. doi:10.1001/archpsyc.1987.01800190080012

Wen W, Thalamuthu A, Mather KA, Zhu W, Jiang J, de Micheaux PL, Wright MJ, Ames D, Sachdev PS. (2016). Distinct Genetic Influences on Cortical and Subcortical Brain Structures. Sci Rep. 6:1-11.

Wendelken, C., Ferrer, E., Ghetti, S., Bailey, S.K., Cutting, L., Bunge, S.A., (2017). Frontoparietal Structural Connectivity in Childhood Predicts Development of Functional Connectivity and Reasoning Ability: A Large-Scale Longitudinal Investigation. Journal of Neuroscience 37(35), 8549-8558. doi:10.1523/jneurosci.3726-16.2017

Wendelken, C., Ferrer, E., Whitaker, K.J., Bunge, S.A., (2016). Fronto-Parietal Network Reconfiguration Supports the Development of Reasoning Ability. Cereb. Cortex 26, 2178-2190. doi:10.1093/cercor/bhv050

Wheelock, M., Hect, J., Hernandez-Andrade, E., Hassan, S., Romero, R., Eggebrecht, A., Thomason, M. (2019). Sex differences in functional connectivity during fetal brain development. Developmental Cognitive Neuroscience 36, 100632. doi:10.1016/j.dcn.2019.100632

Whitaker KJ, Vértes PE, Romero-Garcia R, Váša F, Moutoussis M, Prabhu G, Weiskopf N, Callaghan MF, Wagstyl K, Rittman T, Tait R, Ooi C, Suckling J, Inkster B, Fonagy P, Dolan RJ, Jones PB, Goodyer IM, NSPN Consortium, Bullmore ET. (2016). Adolescence is associated with genomically patterned consolidation of the hubs of the human brain connectome. Proc Natl Acad Sci USA. 113:9105-9110.

Whiteford, H., Degenhardt, L., Rehm, J., Baxter, A., Ferrari, A., Erskine, H., Charlson, F., Norman, R., Flaxman, A., Johns, N., Burstein, R., Murray, C., Vos, T. (2013). Global burden of disease attributable to mental and substance use disorders: findings from the Global Burden of Disease Study 2010. The Lancet 382(9904), 1575-1586. doi:10.1016/s01406736(13)61611-6

Whitfield-Gabrieli, S., Ford, J. (2012). Default Mode Network Activity and Connectivity in Psychopathology. Annual Review of Clinical Psychology 8(1), 49-76. doi:10.1146/annurev-clinpsy-032511-143049

Whitfield-Gabrieli, S., Nieto-Castanon, A., (2012). CONN: a functional connectivity toolbox for correlated and anticorrelated brain networks. Brain Connectivity 2, 125-141. doi:10.1089/brain.2012.0073

Whitfield-Gabrieli, S., Wendelken, C., Nieto-Castañón, A., Bailey, S., Anteraper, S., Lee, Y., Chai, X., Hirshfeld-Becker, D., Biederman, J., Cutting, L., Bunge, S. (2020). Association of Intrinsic Brain Architecture With Changes in Attentional and Mood Symptoms During Development. JAMA Psychiatry 77(4), 378-386. doi:10.1001/jamapsychiatry.2019.4208 
Wierenga, L., Langen, M., Oranje, B., Durston, S. (2014). Unique developmental trajectories of cortical thickness and surface area. NeuroImage 87, 120-126. doi:10.1016/j.neuroimage.2013.11.010

Wierenga, L., Sexton, J., Laake, P., Giedd, J., Tamnes, C. (2017). A Key Characteristic of Sex Differences in the Developing Brain: Greater Variability in Brain Structure of Boys than Girls. Cerebral Cortex 28(8), 2741-2751. doi:10.1093/cercor/bhx154

Wig, G.S., (2017). Segregated Systems of Human Brain Networks. Trends in Cognitive Sciences 21(12), 981-996. doi:10.1016/j.tics.2017.09.006

Wolf, E., Harrington, K., Clark, S., Miller, M. (2013). Sample Size Requirements for Structural Equation Models. Educational and Psychological Measurement 73(6), 913-934. doi:10.1177/0013164413495237

Wolf EJ, Maniates H, Nugent N, Maihofer AX, Armstrong D, Ratanatharathorn A, Ashley-Koch AE, Garrett M, Kimbrel NA, Lori A, VA Mid-Atlantic MIRECC Workgroup, Aiello AE, Baker DG, Beckham JC, Boks MP, Galea S, Geuze E, Hauser MA, Kessler RC, Koenen KC, Miller MW, Ressler KJ, Risbrough V, Rutten BPF, Stein MB, Ursano RJ, Vermetten E, Vinkers CH, Uddin M, Smith AK, Nievergelt CM, Logue MW. (2018). Traumatic stress and accelerated DNA methylation age: A meta-analysis. Psychoneuroendocrinology. 92:123-134.

Worsley, K., Liao, C., Aston, J., Petre, V., Duncan, G., Morales, F., Evans, A. (2002). A General Statistical Analysis for fMRI Data. NeuroImage 15(1), 1-15. doi:10.1006/nimg.2001.0933

Wray, N., Goddard, M., Visscher, P. (2007). Prediction of individual genetic risk to disease from genome-wide association studies. Genome Research 17(10), 1520-1528. doi:10.1101/gr.6665407

Wu, K., Taki, Y., Sato, K., Hashizume, H., Sassa, Y., Takeuchi, H., Thyreau, B., He, Y., Evans, A.C., Li, X., Kawashima, R., Fukuda, H., (2013). Topological organization of functional brain networks in healthy children: differences in relation to age, sex, and intelligence. PLoS ONE 8, e55347. doi:10.1371/journal.pone.0055347

$\mathrm{Wu}, \mathrm{W} .$, Lien, S., Chang, J., Yang, S. (2014). Caffeine alters resting-state functional connectivity measured by blood oxygenation level-dependent MRI. NMR in Biomedicine 27(4), 444-452. doi:10.1002/nbm.3080

Xiao, Y., Friederici, A.D., Margulies, D.S., Brauer, J., (2016). Longitudinal changes in resting-state fMRI from age 5 to age 6 years covary with language development. NeuroImage 128, 116-124. doi:10.1016/j.neuroimage.2015.12.008

Xu, J., Yin, X., Ge, H., Han, Y., Pang, Z., Liu, B., Liu, S., Friston, K.J., (2016). Heritability of the Effective Connectivity in the Resting-State Default Mode Network. Cereb. Cortex. doi:10.1093/cercor/bhw332

Xu, Z., Niu, L., Li, L., Taylor, J. (2016). ENmix: a novel background correction method for Illumina HumanMethylation450 BeadChip. Nucleic Acids Research 44(3), e20-e20.

Yan, C.-G., Cheung, B., Kelly, A.M.C., Colcombe, S., Craddock, R.C., Di Martino, A., Li, Q., Castellanos, F.X., Milham, M.P., (2013). A comprehensive assessment of regional variation in the impact of head micromovements on functional connectomics. NeuroImage 76, 183-201. doi:10.1016/j.neuroimage.2013.03.004

Yang, Z., Zuo, X.-N., McMahon, K.L., Craddock, R.C., Kelly, A.M.C., de Zubicaray, G.I., Hickie, I.B., Bandettini, P.A., Castellanos, F.X., Milham, M.P., Wright, M.J., (2016). Genetic and Environmental Contributions to Functional Connectivity Architecture of the Human Brain. Cereb. Cortex 26, 2341-2352. doi:10.1093/cercor/bhw027

Yeo, B.T.T., Krienen, F.M., Sepulcre, J., Sabuncu, M.R., Lashkari, D., Hollinshead, M., Roffman, J.L., Smoller, J.W., Zöllei, L., Polimeni, J.R., Fischl, B., Liu, H., Buckner, R.L., (2011). The organization of the human cerebral cortex estimated by intrinsic functional connectivity. Journal of Neurophysiology 106, 1125-1165. doi:10.1152/jn.00338.2011

Yoo, K., Rosenberg, M., Noble, S., Scheinost, D., Constable, R., Chun, M. (2019). Multivariate approaches improve the reliability and validity of functional connectivity and prediction of individual behaviors. NeuroImage 197, $212-223$. doi:10.1016/j.neuroimage.2019.04.060

Zahn-Waxler, C., Shirtcliff, E., Marceau, K. (2008). Disorders of Childhood and Adolescence: Gender and Psychopathology. Clinical Psychology 4(1), 275-303. doi:10.1146/annurev.clinpsy.3.022806.091358

Zatorre RJ, Fields RD, Johansen-Berg H. (2012). Plasticity in gray and white: neuroimaging changes in brain structure during learning. Nat Hum Behav. 15:528-536. 
Zhang, D., Raichle, M. (2010). Disease and the brain's dark energy. Nature Reviews Neurology 6(1), 15-28. doi:10.1038/nrneurol.2009.198

Zhang, H., Yin, W., Lin, W., Shen, D., (2017). Early Brain Functional Segregation and Integration Predict Later Cognitive Performance. Connectomics in NeuroImaging, Lecture Notes in Computer Science. Springer International Publishing, Cham, pp. 116-124. doi:10.1007/978-3-319-67159-8_14

Zhang Q, Vallerga CL, Walker RM, Lin T, Henders AK, Montgomery GW, He J, Fan D, Fowdar J, Kennedy M, Pitcher T, Pearson J, Halliday G, Kwok JB, Hickie I, Lewis S, Anderson T, Silburn PA, Mellick GD, Harris SE, Redmond P, Murray AD, Porteous DJ, Haley CS, Evans KL, McIntosh AM, Yang J, Gratten J, Marioni RE, Wray NR, Deary IJ, McRae AF, Visscher PM. (2018). Improved prediction of chronological age from DNA methylation limits it as a biomarker of ageing. bioRxiv. 80:245.

Zhou, D., Lebel, C., Treit, S., Evans, A., Beaulieu, C. (2015). Accelerated longitudinal cortical thinning in adolescence. NeuroImage, 104, 138-145. doi:10.1016/j.neuroimage.2014.10.005

Zhou, Y., Chen, J., Luo, Y., Zheng, D., Rao, L.-L., Li, X., Zhang, J., Li, S., Friston, K.J., Zuo, X.-N., (2016). Genetic overlap between in-scanner head motion and the default network connectivity. bioRxiv doi:10.1101/087023

Zhou, Y., Friston, K.J., Zeidman, P., Chen, J., Li, S., Razi, A., (2018). The Hierarchical Organization of the Default, Dorsal Attention and Salience Networks in Adolescents and Young Adults. Cereb. Cortex 28, 726-737. doi:10.1093/cercor/bhx307

Zielinski BA, Prigge MBD, Nielsen JA, Froehlich AL, Abildskov TJ, Anderson JS, Fletcher PT, Zygmunt KM, Travers BG, Lange N, Alexander AL, Bigler ED, Lainhart JE. (2014). Longitudinal changes in cortical thickness in autism and typical development Brain 137(6), 1799-1812. doi:10.1093/brain/awu083

Zondervan, K., Cardon, L. (2007). Designing candidate gene and genome-wide case-control association studies. Nature Protocols 2(10), 2492-2501. doi:10.1038/nprot.2007.366

Zuo, X.-N., Ehmke, R., Mennes, M., Imperati, D., Castellanos, F.X., Sporns, O., Milham, M.P., (2011). Network Centrality in the Human Functional Connectome. Cereb. Cortex 22, 1862-1875. doi:10.1093/cercor/bhr269

Zuo, X.-N., Kelly, A.M.C., Di Martino, A., Mennes, M., Margulies, D.S., Bangaru, S., Grzadzinski, R., Evans, A.C., Zang, Y.F., Castellanos, F.X., Milham, M.P., (2010). Growing together and growing apart: regional and sex differences in the lifespan developmental trajectories of functional homotopy. J. Neurosci. 30, 15034-15043. doi:10.1523/JNEUROSCI.2612-10.2010 



\section{NEDERLANDSE}

\section{SAMENVATTING}

\section{GENETISCHE INVLOEDEN OP DE}

ONTWIKKELING VAN DE STRUCTUUR EN

FUNCTIE VAN DE HERSENEN 


\section{ALGEMENE INTRODUCTIE}

De adolescentie is de periode die de overgang representeert tussen kind en volwassene. Het is een periode in het leven waarin grote veranderingen plaatsvinden in gedrag en in lichamelijke kenmerken. Onder andere de toename in kritisch denken, de ontwikkeling van zelfidentiteit en moreel kompas, en binding met leeftijdsgenoten zijn belangrijke ontwikkelingen die tijdens de adolescentie plaatsvinden om tot een onafhankelijk persoon op te groeien. In deze periode kunnen ook gedragsproblemen voorkomen, zoals het onnodig nemen van risico's, impulsiviteit, delinquentie en het gebruik van alcohol of drugs. Daarnaast is de adolescentie een periode waarin sommige psychiatrische stoornissen, zoals schizofrenie, vaak voor het eerst voordoen. Parallel aan deze veranderingen in het gedrag blijven de hersenen zich tot ver in de jongvolwassenheid ontwikkelen. De ontwikkeling is niet bij iedereen hetzelfde, er is sprake van heterogeniteit in de ontwikkeling. De heterogeniteit in de vele aspecten die betrokken zijn bij de ontwikkeling van kinderen en adolescenten leidt tot aanzienlijke verschillen hoe het met hen vergaat als volwassene. Dit roept de vraag op 'waarom sommige kinderen beter gedijen dan anderen'; en welke rol de hersenen, genen, en omgevingsfactoren daarbij hebben. In het veld van de 'imaging genetics' worden methoden uit de neuroimaging en genetica gecombineerd om de relaties tussen gedrag, de hersenen, genen, en omgevingsfactoren te ontcijferen. In voorgaand onderzoek is aangetoond dat structurele en functionele eigenschappen van de hersenen sterk erfelijk bepaald kunnen zijn. Daarnaast is gebleken dat ook de mate van veranderingen in de hersenen erfelijk bepaald zijn. De uitdrukking van de genen die betrokken zijn bij de ontwikkeling van de hersenen worden sterk gereguleerd door onder andere omgeving, voornamelijk tijdens de vroege levensjaren. Maar ook op latere leeftijd kunnen omgevingsfactoren invloed hebben op de expressie van genen, bijvoorbeeld door regulatie via epigenetische modificaties. Er wordt verondersteld dat dit dynamische samenspel van genetische en omgevingsinvloeden op de hersenen verklaart waarom bijvoorbeeld eeneiige tweelingen met dezelfde genetische achtergrond discordant kunnen zijn voor zeer erfelijke aandoeningen zoals schizofrenie. Dit samenspel kan mogelijk ook antwoord geven op de vraag 'waarom sommige kinderen beter gedijen dan anderen'6.

\section{SAMENVATTING}

In dit proefschrift worden de invloeden van genen en omgeving op de ontwikkeling van de hersenen beschreven. Hier wordt specifiek gekeken naar de genetische invloeden op de dikte van de hersenschors en de connectiviteit van functionele netwerken tijdens de ontwikkelingen van de hersenen in de adolescentie (hoofdstuk $2 \& 4$ ). Dit onderzoek is

\footnotetext{
${ }^{6}$ Consortium on Individual Development; https://individualdevelopment.nl
} 
gedaan in een longitudinaal cohort van adolescente tweelingen en een oudere broer of zus die deelnamen toen de tweelingen 9, 12, en 17 jaar oud waren. Voor het onderzoek naar connectiviteit van functionele netwerken wordt gebruik gemaakt van een meetmodel waarvan de bruikbaarheid empirisch is beoordeeld in de openbare 'Young Adult' dataset van het 'Human Connectome Project' (hoofdstuk 3). Daarnaast wordt de genetische en epigenetisch bijdrage aan versnelde veroudering van de hersenen beschreven in een cohort van patiënten met schizofrenie (hoofdstuk 5).

\section{Ontwikkeling van de hersenschors tijdens de adolescentie}

In hoofdstuk 2 worden de genetische invloeden op de dikte van de hersenschors tijdens de kindertijd en adolescentie beschreven. Eerder onderzoek naar de ontwikkeling van de hersenschors in de eerste twee metingen van het BrainSCALE cohort heeft aangetoond dat de mate van veranderingen in de dikte van de hersenschors beïnvloed wordt door genen, en waaruit is gebleken dat tijdens de eerste jaren van de adolescentie nieuwe genen een rol gaan spelen. Dit onderzoek is nu uitgebreid met de derde meting van het BrainSCALE cohort dat is afgenomen toen de tweelingen 17 jaar oud waren. Deze derde meting volgt op een periode van versnelde ontwikkeling van de hersenschors zoals gebruikelijk wordt waargenomen tijdens de adolescentie. Uit dit vervolgonderzoek blijkt dat hoofdzakelijk dezelfde genetische factor de dikte van de hersenschors gedurende de kindertijd en adolescentie bepaald. De verschillende gebieden van de hersenschors worden daarnaast deels beïnvloed door genetische factoren die specifiek zijn voor die gebieden. De verdunning van de hersenschors tijdens adolescentie is voornamelijk toe te schrijven aan fluctuerende invloeden van de gemeenschappelijke genen. Daarnaast zijn er aanwijzingen gevonden voor lokaal-specifieke constante en nieuwe genetische factoren. Dit onderzoek heeft de dynamiek van genetische invloeden op de hersenschors over tijd en locatie laten zien voor de ontwikkelende hersenen tijdens de adolescentie, wat gepaard gaat met een golf aan invloeden van een nieuwe genetische factor.

\section{Betrouwbaar meten van functionele connectiviteit van het brein}

In hoofdstuk 3 wordt het gebruik van een meetmodel voor het betrouwbaarder meten van functionele connectiviteit van de hersenen beschreven. Vrijwel geen eigenschap kan volledig betrouwbaar gemeten worden zonder bijkomstigheid van willekeurige meetruis. Deze meetruis zorgt ervoor dat de sterkte van de verbanden tussen eigenschappen en de erfelijkheid van eigenschappen onderschat worden. Met behulp van een meetmodel kan de betrouwbare component van een eigenschap, dat bepaald wordt door wat gemeenschappelijk is tussen parallelle of herhaalde metingen, bijvoorbeeld de twee helften van een functionele MRI-scan sessie die afgenomen is in rusttoestand, afgezonderd worden van de metingspecifieke variatie. Vervolgens kan de "ware" sterkte van de verbanden tussen bijvoorbeeld 
functionele connectiviteit van de hersenen en gedragsmaten bepaald worden, of kan de erfelijkheid van de betrouwbare component van functionele connectiviteit bepaald worden, alsof de eigenschap feilloos gemeten is. In dit onderzoek is de toegevoegde waarde van het gebruik van een meetmodel voor het betrouwbaarder meten van functionele connectiviteit van de hersenen empirisch beoordeeld in de openbare 'Young Adult' dataset van het 'Human Connectome Project' voor verschillende lengtes van de MRI-scans en aantal participanten in het onderzoek. Daaruit blijkt dat het betrouwbare component van functionele connectiviteit van de hersenen aanzienlijk meer consistent is bij herhaalde metingen die over twee dagen verspreid zijn dan wanneer er geen gebruik gemaakt wordt van een meetmodel. Dit model schat een tot bijna twee keer hogere "ware" sterkte van de associatie tussen de betrouwbare component van functionele connectiviteit en gedragsmaten in, en geeft een hogere schatting voor de erfelijkheid van functionele connectiviteit. Dit onderzoek heeft laten zien dat gedrags- en genetische studies baat hebben bij het gebruik van een meetmodel dat in staat is om de betrouwbare component van een eigenschap te identificeren in het onderzoek naar de functionele connectiviteit van de hersenen.

\section{Ontwikkeling van functionele connectiviteit tijdens de adolescentie}

In hoofdstuk 4 wordt de ontwikkeling van functionele connectiviteit van de grootschalige netwerken in de hersenen tijdens de adolescentie voor de kinderen van het BrainSCALE cohort beschreven. Andere - voornamelijk cross-sectionele - onderzoeken rapporteren inconsistente bevindingen met betrekking tot de ontwikkeling van functionele connectiviteit van de hersenen in kinderen. Dit kan voorkomen bij cross-sectionele studies wanneer veranderingen tussen proefpersonen op verschillende leeftijden groter zijn dan de veranderingen bij longitudinale ontwikkeling van de individuele proefpersonen. Voorgaande genetische studies rapporteren voornamelijk kleine genetisch invloeden op functionele connectiviteit van de hersenen. Dit kan onder andere verklaard worden door grote intra-individuele verschillen die mogelijk toe te wijden zijn aan de relatief onbetrouwbare metingen van functionele connectiviteit in de hersenen. In mijn longitudinale onderzoek wordt het meetmodel, dat in hoofdstuk 3 beschreven wordt, toegepast op de functionele MRI-scans afgenomen in rusttoestand om de invloeden van genen en omgeving op de stabiele en betrouwbare component van functionele connectiviteit te bepalen, en de verschillen te bepalen tussen kindertijd en adolescentie. Uit dit onderzoek blijkt dat de functionele connectiviteit tussen de netwerken afneemt met de leeftijd, terwijl de functionele connectiviteit binnen de netwerken over het algemeen juist toeneemt met de leeftijd. Deze veranderingen in functionele connectiviteit vinden plaatsen ongeacht het cognitief presteren van de kinderen. Daarnaast bepaalt het geslacht van kinderen en jongeren de sterkte van de functionele connectiviteit in bepaalde netwerken; bij meisjes is de functionele connectiviteit in het 'default mode' netwerk - dat actief is tijdens rust en 
onder andere betrokken is bij zelfreflectie - sterker dan bij jongens, en een tegengesteld effect in het 'salience' netwerk - dat onder andere betrokken is bij aandacht en bottom-up stimuli verwerking. De individuele verschillen in de sterkte van functionele connectiviteit van de hersenen kan deels verklaard worden door genetische invloeden. Er zijn geen aanwijzingen gevonden dat deze genetische invloeden veranderen tijdens de ontwikkeling van de functionele netwerken in de adolescentie. Naast genen spelen voor sommige netwerken de invloeden vanuit een gemeenschappelijke omgeving een belangrijke rol bij het verklaren van individuele variatie in de sterke van functionele connectiviteit. Dit onderzoek heeft aangetoond dat ook tijdens de adolescentie er subtiele maar wijdverspreide veranderingen in het functioneren van netwerken van de hersenen plaatsvinden die in vervolgonderzoek mogelijk in verband gebracht kunnen worden met veranderingen in gedrag tijdens de adolescentie. Daarbij spelen genen en gemeenschappelijke omgevingsfactoren een belangrijke rol in het bepalen van de mate van functionele connectiviteit in de hersenen.

\section{Versnelde veroudering in patiënten met schizofrenie}

In hoofdstuk 5 wordt het verband tussen versnelde veroudering in de hersenen en versnelde veroudering zoals gemeten via epi-genetica in het bloed bij patiënten met schizofrenie onderzocht in verhouding tot het genetisch risico op schizofrenie. De versnelde veroudering wordt berekend door het verschil tussen de voorspelde leeftijd van de patiënt aan de hand van een MRI-scan of DNA-monster en de chronologische leeftijd van de patiënt. In twee voorgaande studies is onafhankelijk van elkaar aangetoond dat de hersenen van patiënten met schizofrenie versnelde veroudering laat zien met name in de beginperiode waarin de aandoening zich voor het eerst voordoet, en dat ook de voorspelde biologische leeftijd gemeten via epi-genetische modificaties voorloopt op de chronologische leeftijd. Uit het huidig onderzoek is gebleken dat de versnelde veroudering van de hersenen niet eenduidig samenhangt met de versnelde veroudering gemeten door epi-genetica, ondanks dat beide verouderingsprocessen deels verklaard worden door het genetisch risico op schizofrenie. Nadat de diagnose status van de proefpersonen in acht genomen is en een correctie voor meerdere vergelijkingen is toegepast, waren deze effecten echter niet langer significant. Deze resultaten kunnen mogelijk wijzen op (versnelde) veroudering in de verschillende weefsels die verschillende biologische processen weerspiegelen, ondanks dat beide processen afzonderlijk van elkaar in verband zijn gebracht met genetisch risico op schizofrenie.

\section{SLOTOPMERKING}

In hoofdstuk 6 worden de belangrijkste bevindingen van de onderzoeken uit de hoofdstukken 2 tot en met 5 opgesomd en gevolgd door een algemene discussie van de 
bevindingen. Ik concludeer dat het bestuderen van de gezonde ontwikkeling van de hersenen bij kinderen en adolescenten belangrijk is omdat het een basis vormt voor wat als afwijkende ontwikkeling moet worden beschouwd bij neuro-psychiatrische aandoeningen zoals bijvoorbeeld schizofrenie. Daarnaast helpt deze kennis mee om te begrijpen welke structuren of functies van de hersenen verantwoordelijk zijn voor verandering in het gedrag van adolescenten. Door tweelingen bij de studie te betrekken kan bepaald worden in welke mate individuele variatie in structuur en functie van de hersenen en gedrag van adolescenten door genetische en omgevingsfactoren beïnvloed worden. Samen kunnen deze onderzoeken informeren waarom sommige kinderen beter gedijen dan anderen, en kunnen deze onderzoeken helpen bij de ontwikkeling van diagnostische hulpmiddelen en interventies om kinderen die het moeilijk hebben weer op de juiste weg te helpen.

Vervolgonderzoek van adolescentie naar volwassenheid in dit cohort is niet alleen nodig om de kennis over de ontwikkeling en het gedrag van de hersenen van adolescenten uit te breiden, maar ook om de bestaande onderzoeksresultaten te valideren en repliceren. Grootschalige cohorten die de basis vormen voor populatieonderzoeken kunnen daarbij helpen doordat zij prospectief onderzoek mogelijk maken. De statistische kracht van deze grootschalige studies kan helpen om de associaties tussen de hersenen en gedrag tijdens adolescentie met kleinere effectgroottes betrouwbaarder te detecteren. Gecombineerd met een longitudinale onderzoeksopzet en het betrekken van tweelingen in het onderzoek kunnen deze studies de resultaten van dit proefschrift valideren, en zich vervolgens richten op het uitbreiden van de resultaten door gen-omgevingsinteracties of causale verbanden te onderzoeken. 

DANKWOORD 
Na vier jaar hard zwoegen - oké, inmiddels misschien wat langer dan vier jaar - ligt er dan eindelijk zo'n boekje waar je op mag promoveren. Ook al staat alleen mijn naam op de voorkant, veel personen hebben een bijdrage geleverd aan dit proefschrift waarvoor ik hen graag zou willen bedanken.

Allereerst zou ik graag de tweelingen, hun ouder broers en zussen, en hun ouders willen bedanken voor deelname aan de BrainSCALE studie. Ontzettend veel respect dat de meeste van jullie een tweede of zelfs derde keer zijn op komen dagen, zonder jullie was deze studie nooit van de grond gekomen.

Daarnaast zou ik graag de promotoren en copromotor prof. dr. Hilleke Hulshoff Pol, prof. dr. Dorret Boomsma, en dr. Rachel Brouwer in het algemeen willen bedanken voor het beschikbaar stellen van het promotietraject en hun ondersteuning tijdens het werk. Het was mij een waar genoegen om onder jullie leiding onderzoek te mogen doen. Ik heb er veel van geleerd, niet alleen over ontwikkeling van de hersenen, genetica, en statistiek, maar ook wat "onderzoek doen" inhoudt en hoe onderzoek benut kan worden voor maatschappelijk belang.

Beste Hilleke, jouw energie en vrolijkheid werkt aanstekelijk. Ook als ik zelf wat somber gesteld was over de tegenvallende resultaten wist jij er altijd een positieve twist aan te geven. Het heeft mij geleerd om ook de kleine overwinningen te zegenvieren, want elke stap vooruit is er weer één. Dat optimisme heeft zeker bijgedragen aan het afronden van dit boekwerk. Ik zou jou graag willen bedanken voor het vertrouwen en de hulp bij het doorzetten om dit mogelijk te maken, en bij het zetten van de volgende stap in de onderzoekwereld.

Beste Dorret, de colleges tijdens de cursus Complex Trait Genetics waren ontzettend interessant en hebben veel geholpen bij mijn werk met genetica. Je was altijd bereid om kritisch de pagina's lange teksten te beoordelen, ook als je op het punt stond om op vakantie te gaan. Ik keek altijd erg uit naar het commentaar, want de grote schat aan kennis die jij bezit over alles genetica en tweelingen is van onschatbare waarde. Jij zorgde ervoor dat alles juist en duidelijk opgeschreven stond zodat reviewers er nauwelijks nog een speld tussen kregen. Voor die hulp ben ik jou zeer dankbaar.

Beste Rachel, bedankt dat je altijd beschikbaar was om vragen te beantwoorden of knopen door te hakken. Jouw bereidbaarheid om anderen te helpen met jouw uitgebreide kennis over statistiek is zeer bewonderenswaardig. Daar heb ik dan ook ontzettend veel hulp aan gehad, zeker bij de complexe tweelingmodellen. Ik heb veel plezier gehad aan onze wekelijkse bijeenkomsten waar we konden brainstormen over allerlei mogelijke analyses en bespreken hoe we de resultaten het best op konden schrijven. Ik zal die momenten heel erg gaan missen. 
Tevens zou ik graag de leescommissie, bestaande uit prof. dr. Floor Scheepers, prof. dr. Sarah Durston, prof. dr. Nick Ramsey, prof. dr. Rick Dijkhuizen, en prof. dr. Barbara Franken, willen bedanken voor hun tijd om dit boekje door te lezen, te beoordelen, en zitting te nemen tijdens de promotieplechtigheden.

Mijn dank gaat uit naar de PhD-studenten die mij voorgingen in de BrainSCALE studie: dr. Inge van Soelen, dr. Jiska Peper, dr. Suzanne Swagerman, en dr. Marinka Koenis; bedankt voor jullie inzet bij het verzamelen van data en het opbouwen van een sterke basis aan onderzoeksresultaten om op voort te mogen bouwen. Beste Marinka, het was erg fijn om in het begin iemand te hebben die mij op weg kon helpen met artikelen, software, toegang tot de data, en hulp bij het regelen van allerlei zaken rondom de $\mathrm{PhD}$.

Mijn werkplek was prettig; dank aan alle kantoorgenoten: Elise, Jasper, Pascal, Judith, Bart, Martijn, Jessica, Sonja, Herm, Merel, en Elizabeth; om hulp bij te kunnen zoeken wanneer ik vast kwam te zit, om mijn frustraties te kunnen uiten als het even niet meer lukt of tegen zat, om kennis of interessante feitjes mee te kunnen delen, of gewoon even lekker afgeleid te raken van het harde werk. Het was gezellig om met jullie een kantoor te delen, een gezelligheid die de laatste tijd sterk gemist wordt.

Graag zou ik prof. dr. Roel Ophoff willen bedanken voor het beschikbaar stellen van een stageplek in UCLA; ik kijk nog altijd met veel plezier terug naar mijn tijd in California. Daarnaast ook dank aan dr. Anil Ori en dr. Hugo Schnack voor hun kennis en ondersteuning bij het afronden van het manuscript over epigenetica en brain aging dat is voortgekomen uit deze stage.

Ook bedankt aan de bachelor- en masterstudenten Philip, João, Maxime, Zyneb en Karis. Het was erg leuk om jullie te mogen begeleiden en een fijne afleiding van het dagelijkse ritme. Bedankt voor jullie inzet, waarvan delen ook ten goede zijn gekomen aan de hoofdstukken in dit boekwerk.

Op de achtergrond valt er veel te regelen om een promotie mogelijk te maken. Mijn dank gaat uit naar Jeanette en Janneke voor hun hulp bij het grotendeels over te nemen en in goede banen te leiden van alle administratieve zaken. Daarnaast Thijs en Yumas voor het onderhouden en ondersteunen van het lokale computer infrastructuur, waar dierbaar gebruik van is gemaakt; en het HPC-team voor het onderhouden en de ondersteuning van het centrale rekencluster, zonder die rekenkracht had dit werk er vele jaren langer over gedaan. En BCRM - of tegenwoordig CEN - voor het aanbieden van cursussen en het organiseren van de maandelijkse X-talks en jaarlijkse bijeenkomsten. Bedankt aan iedereen die niet bij naam genoemd is, maar op zijn of haar eigen manier een steentje heeft bijgedragen aan het afronden van mijn promotietraject. 
Ook buiten werk om zijn er personen die ik graag zou willen bedanken voor hun ondersteuning bij het afronden van dit boekje.

Beste Bart, wie had verwacht dat we ooit bij elkaar op hetzelfde kantoor terecht zouden komen? Natuurlijk hadden we tijdens onze studie wilde plannen om onze interesses te combineren tot één project, maar het was uiteindelijk toch een verassing toen bleek dat wij allebei een promotietraject begonnen op dezelfde afdeling! Wij kennen elkaar al zo lang dat $\mathrm{ik}$ niet weet waar ik zou moeten beginnen, laat staan dat er ruimte is om in detail gaan; maar weet dat ik onze vriendschap altijd zeer gewaardeerd heb en blij ben dat wij nog steeds zo'n hechte band hebben. Het is immers niet zonder reden dat wij elkaar als 'broer' beschouwen. Bedankt voor al jouw hulp en ondersteuning in persoonlijke sfeer en op werk; ook in de moeilijke tijden. Ik hoop dat wij nog veel tijd samen mogen doorbrengen, en dat onze dochters net zo'n goede band met elkaar mogen ontwikkelen dat zij zich als 'zusjes' zullen beschouwen.

Beste Marnix en Age, ik denk nog altijd terug aan de tijd die wij samen hebben doorgebracht tijdens onze studie in Enschede. Dat was een heerlijke tijd, waarin wij geregeld met elkaar optrokken om op de Calslaan te barbecueën, films te kijken of te gamen, te sporten bij de Stretchers of deel te nemen aan hardloopwedstrijden, en op vakantie te gaan naar Schotland en Luxemburg. Ook na onze studie heb ik veel plezier aan de contacten die wij onderhouden om samen te wandelen of te gamen. Zo veel leuke herinneringen dat ik trots ben om jullie hier als vrienden te noemen en te kunnen bedanken voor die leuke tijd. $\mathrm{Nu}$ de drukke tijd van het afronden van mijn promotie er bijna op zit kijk ik er naar uit om de gameavonden weer op te pakken.

Beste Inge en Ronald, het voelt wat cliché om de ouders te bedanken, maar met een oprecht hart zou ik jullie willen bedanken voor praktisch alles. Als ouders hebben jullie een belangrijke rol gespeeld bij wie ik ben geworden, zo doende ook mijn academische carrière. Bedankt voor jullie ondersteunende rol en het aanbieden van mogelijkheden om mijn eigen interesses te volgen, de unieke kans om aan tweetalig onderwijs deel te nemen, en de gelegenheid om naar de universiteit te kunnen gaan. De beste eer die ik jullie kan geven is het rolmodel van de ouders die jullie zijn over te nemen bij het opvoeden van mijn eigen kinderen.

Lieve Roos, de afgelopen jaren is een bijzondere tijd geweest waarin veel is gebeurd naast de promotie; wij zijn gaan samenwonen, getrouwd, en hebben onze dochter Vera mogen verwelkomen. Met name het laatste jaar is ook voor jou best pittig geweest, terwijl een groot deel van mijn tijd opging aan werk of afronding van mijn promotie moest jij voor Vera zorgen. Ze is een lekker levendige en eigenwijze dame, en de stilte en rust in huis zal niet snel terugkeren voordat zij oud genoeg is om haar eigen paden te bewandelen. Maar met deze laatste zin kan ik beloven weer meer tijd over te houden om samen met jullie door te brengen. 
To Rosalie, my heloved wife,

"If I could gather all the stars and hold them in my hand, the colours I would then possess would not be half as grand as those which I have seen when I look deep into your eyes or come across when I have kissed your lips, I realize.

If I could ponder all the truths that men have sought since time began they would not teach me more than if I were to touch your hand.

For truth, to know one whom we have touched appears as simple lies and nothing could be truer than your touch, I realize.

If I were given lasting life as only God can do, I'd shun it all and turn away if I could live with you.

For even God has never known the immeasureable size of the love that is within your heart, I realize." 



\section{LIST OF PUBLICATIONS}




\section{PublicATIONS}

Teeuw, J., Ori, A.P.S., Brouwer, R.M., de Zwarte, S.M.C., Schnack, H.G., Hulshoff Pol, H.E., Ophoff, R.A. (under review). Accelerated aging in the brain, epigenetic aging in blood, and polygenic risk for schizophrenia. Available as preprint on MedRxiv; doi:10.1101/2020.08.31.20185066

Teeuw, J., Boomsma, D.I., Hulshoff Pol, H.E., Brouwer, R.M. (in revision). Reliability modelling of functional connectivity in resting-state functional MRI.

Buimer, E.E.L., Pas, P., Brouwer, R.M., Froeling, M., Hoogduin, H., Leemans, A., Luijten, P., van Nierop, B.J., Raemaekers, M., Schnack, H.H., Teeuw, J., Vink, M., Visser, F., Hulshoff Pol, H.E., Mandl, R.C.W. (2020). The YOUth cohort study: MRI protocol and test-retest reliability in adults. Developmental Cognitive Neuroscience 45, 100816. doi:10.1016/j.dcn.2020.100816

Brouwer, R.M., Klein, M., Grasby, K.L., Schnack, H.G., Jahanshad, N., Teeuw, J., Thomopoulos, S.I., Sprooten, E., Franz, C.E., Gogtay, N., Kremen, W.S., Panizzon, M.S., Olde Loohuis, L.M., Whelan, C.D., Aghajani, M., Alloza, C., Alnæs, D., Artiges, E., AyesaArriola, R., Barker, G.J., Blok, E., Bøen, E., Breukelaar, I.A., Bright, J.K., Buimer, E.E.L., Bülow, R., Cannon, D.M., Ciufolini, S., Crossley, N.A., Damatac, C.G., Dazzan, P., de Mol, C.L., de Zwarte, S.M.C., Desrivières, S., Díaz-Caneja, C.M., Doan, N.T., Dohm, K., Fröhner, J.H., Goltermann, J., Grigis, A., Grotegerd, D., Han, L.K.M., Hartman, C.A., Heany, S.J., Heindel, W., Heslenfeld, D.J., Hohmann, S., Ittermann, B., Jansen, P.R., Janssen, J., Jia, T., Jiang, J., Jockwitz, C., Karali, T., Keeser, D., Koevoets, M.G.J.C., Lenroot, R.K., Malchow, B., Mandl, R.C.W., Medel, V., Meinert, S., Morgan, C.A., Mühleisen, T.W., Nabulsi, L., Opel, N., Ortiz-García de la Foz, V., Overs, B.J., Paillère Martinot, M-L, Quinlan, E.B., Redlich, R., Marques, T.R., Repple, J., Roberts, G., Roshchupkin, G.V., Setiaman, N., Shumskaya, E., Stein, F., Sudre, G., Takahashi, S., Thalamuthu, A., Tordesillas-Gutiérrez, D., van der Lugt, A., van Haren, N.E.M., Wen, W., Westeneng, H-J., Wittfeld, K., Zugman, A., Armstrong, N.J., Bralten, J., Dalvie, S., Di Forti, M., Ding, L., Donohoe, G., Forstner, A.J., Gonzalez-Peñas, J., Guimarães, J.P.O.F.T., Homuth, G., Hottenga, J-J., Knol, M.J., Kwok, J.B.J., Le Hellard, S., Mather, K.A., Milaneschi, Y., Morris, D.W., Nöthen, M.M., Papiol, S., Rietschel, M., Santoro, M.L., Steen, V.M., Stein, J.L., Streit, F., Tankard, R.M., Teumer, A., van 't Ent, D., van der Meer, D., Eijk, K.R., Vassos, E., Vázquez-Bourgon, J., Witt, S.H., Alzheimer's Disease Neuroimaging Initiative, Adams, H.H.H., Agartz, I., Ames, D., Amunts, K., Andreassen, O.A., Arango, C., Banaschewski, T., Baune, B.T., Belangero, S.I., Bokde, A.L.W., Boomsma, D.I., Bressan, R.A., Brodaty, H., Buitelaar, J.K., Cahn, W., Caspers, S., Cichon, 
S., Crespo Facorro, B., Dannlowski, U., Elvsåshagen, T., Espeseth, T., Falkai, P., Fisher, S.E., Flor, H., Fullerton, J.M., Garavan, H., Gowland, P.A., Grabe, H.J., Hahn, T., Heinz, A., Hillegers, M., Hoare, J., Hoekstra, P.J., Ikram, M.A., Jackowski, A.P., Jansen, A., Jönsson, E.G., Kahn, R.S., Kircher, T., Korgaonkar, M.S., Krug, A., Lemaitre, H., Malt, U.F., Martinot, J-L., McDonald, C., Mitchell, P.B., Muetzel, R.L., Murray, R.M., Nees, F., Nenadic, I., Oosterlaan, J., Ophoff, R.A., Pan, P.M., Penninx, B.W.J.H., Poustka, L., Sachdev, P.S., Salum, G.A., Schofield, P.R., Schumann, G., Shaw, P., Sim, K., Smolka, M.N., Stein, D.J., Trollor, J., van den Berg, L.H., Veldink, J.H., Walter, H., Westlye, L.T., Whelan, R., White, T., Wright, M.J., Medland, S.E., Franke, B., Thompson, P.M., Hulshoff Pol, H.E. (2020). Dynamics of Brain Structure and its Genetic Architecture over the Lifespan. bioRxiv. doi:10.1101/2020.04.24.031138

Jia, T., Chu, C., Liu, Y., van Dongen, J., Papastergios, E., Armstrong, N.J., Bastin, M.E., Carrillo-Roa, T., den Braber, A., Harris, M., Jansen, R., Liu, J., Luciano, M., Ori, A.P.S., Roiz Santiañez, R., Ruggeri, B., Sarkisyan, D., Shin, J., Sungeun, K., Tordesillas Gutiérrez, D., van ‘t Ent, D., Ames, D., Artiges, E., Bakalkin, G., Banaschewski, T., Bokde, A.L.W., Brodaty, H., Bromberg, U., Brouwer, R.M., Büchel, C., Quinlan, E.B., Cahn, W., de Zubicaray, G.I., Ehrlich, S., Ekström, T.J., Flor, H., Fröhner, J.H., Frouin, V., Garavan, H., Gowland, P., Heinz, A., Hoare, J., Ittermann, B., Jahanshad, N., Jiang, J., Kwok, J.B., Martin, N.G., Martinot, J-L., Mather, K.A., McMahon, K.L., McRae, A.F., Nees, F., Papadopoulos Orfanos, D., Paus, T., Poustka, L., Sämann, P.G., Schofield, P.R., Smolka, M.N., Stein, D.J, Strike, L.T., Teeuw, J., Thalamuthu, A., Trollor, J., Walter, H., Wardlaw, J.M., Wen, W., Whelan, R., Apostolova, L.G., Binder, E.B., Boomsma, D.I., Calhoun, V., Crespo-Facorro, B., Deary, I.J., Hulshoff Pol, H., Ophoff, R.A., Pausova, Z., Sachdev, P.S., Saykin, A., Wright, M.J., Thompson, P.M., Schumann, G., Desrivières, S. (2019). Epigenome-wide meta-analysis of blood DNA methylation and its association with subcortical volumes: findings from the ENIGMA Epigenetics Working Group. Molecular Psychiatry. doi:10.1038/s41380-019-0605-Z

Teeuw, J., Brouwer, R.M., Guimarães, J.P.O.F.T., Brandner, P., Koenis, M.M.G., Swagerman, S.C., Verwoert, M., Boomsma, D.I., Hulshoff Pol, H.E. (2019). Genetic and environmental influences on functional connectivity within and between canonical cortical resting-state networks throughout adolescent development in boys and girls. NeuroImage 202, 116073. doi:10.1016/j.neuroimage.2019.116073

Teeuw, J., Brouwer, R.M., Koenis, M.M.G., Swagerman, S.C., Boomsma, D.I., Hulshoff Pol, H.E. (2018). Genetic Influences on the Development of Cerebral Cortical Thickness During Childhood and Adolescence in a Dutch Longitudinal Twin Sample: The Brainscale Study. Cerebral Cortex 29(3), 978-993. doi:10.1093/cercor/bhy005 
Brouwer, R.M., Teeuw, J., Koenis, M.M.G., Swagerman, S.C., Boomsma, D.I., Hulshoff Pol, H.E. (2018). Fluctuating and emerging genetic influences on cortical development during adolescence. Biological Psychiatry 83(9):S259.

doi:10.1016/j.biopsych.2018.02.669

Koolschijn, P.C.M.P., Caan, M.W.A., Teeuw, J., Olabarriaga, S.D., Geurts, H.M. (2017). Age-related differences in autism: The case of white matter microstructure. Human Brain Mapping 38(1), 82-96. doi:10.1002/hbm.23345

Teeuw, J., Caan, M.W.A., Olabarriaga, S.D. (2015). Robust automated white matter pathway reconstruction for large studies. MICCA 2015: Medical Image Computing and Computer-Assisted Intervention. Lecture Notes in Computer Science 9349, 101108. doi:10.1007/978-3-319-24553-9 13

Mikhaylova, M., Cloin, B.M.C., Finan, K., van den Berg, R., Teeuw, J., Kijanka, M.M., Sokolowski, M., Katrukha, E.A., Maidorn, M., Opazo, F., Moutel, S., Vantard, M., Perez, F., van Bergen en Henegouwen, P.M.P., Hoogenraad, C.C., Ewers, H., Kapitein, L.C. (2015). Resolving bundled microtubules using anti-tubulin nanobodies. Nature Communications 6(1), 7933. doi:10.1038/ncomms8933

\section{ABSTRACTS AND CONFERENCE PROCEEDINGS}

Teeuw, J., Boomsma, D.I., Hulshoff Pol, H.E., Brouwer, R.M. (2020). Reliability modelling of resting-state functional connectivity. On Human Brain Mapping (OHBM) conference.

Teeuw, J., Brouwer, R.M., Guimarães, J.P.O.F.T., Brandner, P., Koenis, M.M.G., Swagerman, S.C., Verwoert, M., Boomsma, D.I., Hulshoff Pol, H.E. (2019). Genetic influences on cortical functional connectivity during longitudinal adolescent development. On Human Brain Mapping (OHBM) conference.

Teeuw, J., Brouwer, R.M., Koenis, M.M.G., Swagerman, S.C., Boomsma, D.I., Hulshoff Pol, H.E. (2017). Genetic influences on development of cortical thickness from a longitudinal adolescent twin sample. On Human Brain Mapping (OHBM) conference.

Teeuw, J., Brouwer, R.M., Koenis, M.M.G., Swagerman, S.C., Boomsma, D.I., Hulshoff Pol, H.E. (2016). Genetic factors of cortical development and intelligence in a longitudinal Dutch twin study. On Human Brain Mapping (OHBM) conference.

Teeuw, J., Brouwer, R.M., Koenis, M.M.G., Swagerman, S.C., Boomsma, D.I., Hulshoff Pol, H.E. (2015). Trajectories of cortical thickness and their relation to cognitive abilities in the BrainSCALE project: preliminary results. Onderzoekschool 
Neurowetenschappen Amsterdam Rotterdam and Brain Center Rudolf Magnus (ONWAR-BCRM) conference.

Caan, M.W.A., Teeuw, J., Shahand, S., Jaghoori, M.M., Huguet, J., van Altena, A., Olabarriaga, S.D. (2015). A neuroscience gateway for handling and processing population imaging studies. Proceedings of the 1st Miccai 2015 Workshop on Management and Processing of images for Population Imaging (MICCAI MAPPING 2015).

\section{SOFTWARE CONTRIBUTION}

Repository of OpenMx models available on https://github.com/jalmar/openmx$\underline{\text { models }}$

\section{STUDENT SUPERVISION}

Karis Colyer Patel, MSc Neuroscience and Cognition, University Utrecht, Netherlands (2020). Shared genetic and environmental influences on altered functional connectivity and schizophrenia liability.

$>$ Zyneb Al-Hassaan, BSc Psychobiology, University of Amsterdam, Netherlands (2020). Altered white matter integrity in the corticospinal tract and the superior longitudinal fasciculus among typically developing children and adolescents with disruptive behavioral problems.

Maxime Verwoert, MSc Neuroscience and Cognition, University Utrecht, Netherlands (2018). Development of the functional brain: a systematic review of longitudinal resting-state $\mathrm{fMRI}$ studies in childhood and adolescence.

> João Guimarães, MSc Biomedical and Biophysical Engineering, Lisbon University, Portugal (2017). BOLD signal properties in brain functioning throughout adolescence: $a$ longitudinal resting-state fMRI study. 



\section{CURRICULUM VITAE}




\section{JALMAR TEEUW}

Born on December $21^{\text {st }} 1987$ in Schiedam, the Netherlands. He finished his bilingual secondary education at the Atrium in Amersfoort in 2006, and continued his academic career at the University of Twente, Enschede, the Netherlands where he finished a dual bachelor's degree in Computer Science (2011) and Telematics (2012). During his study he became interested in computer simulations and artificial intelligence. With the human brain and cognition as epitome of what

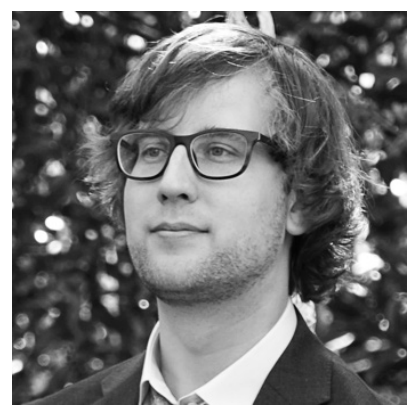
artificial intelligence could become, he picked up extra courses from various schools, including psychology, biotechnology, neuroscience, and educational sciences, to try to decipher the complexities of the human brain and its function. His fascination for how humans and animals learned to solve complex tasks eventually led him to pursue a career in neuroscience. He started his master's degree in Biomedical Image Science at the Utrecht University, Utrecht, the Netherlands in 2011, where he continued his tradition of picking up extra courses on child psychology and artificial intelligence. His master's research project was designing software to trace individual microtubule bundles in super-resolution microscopy images of neurons during an internship in the laboratory of prof. dr. Lukas Kapitein at the Cell Biology department of Utrecht University. He graduated from his master's degree in 2014 with a thesis on different techniques to trace white matter fiber bundles in the brain. He worked as a research assistant at the eBioscience research group of the Amsterdam Medical Center, Amsterdam, the Netherlands to assist researchers in processing MRI datasets on high performance compute clusters through the locally developed eScience Gateway when he applied for a $\mathrm{PhD}$ position on Imaging Genetics at the University Medical Center Utrecht, Utrecht, the Netherlands. Incredibly excited when he was accepted for the position, he began working on the longitudinal adolescent twin cohort BrainSCALE under the supervision of prof. dr. Hilleke Hulshoff Pol, prof. dr. Dorret Boomsma, and dr. Rachel Brouwer. His work, including a brief internship at the laboratory of prof. dr. Roel Ophoff at the Human Genetics department of the University of California Los Angeles, Los Angeles, United States of America, culminated in the thesis presented here. Currently, he is continuing his academic career as a postdoctoral researcher under supervision of prof. dr. Hilleke Hulshoff Pol where he works on studying the neurobiology of anti-social behavior in children.

To read more recent updates on his research, visit his website: https://www.jalmarteeuw.nl/ 


Understanding typical brain development in children and adolescents is important because it provides a baseline for what is to be considered aberrant development. This knowledge can also be used to determine what parts of the brain is responsible for cognitive and behavioral development in adolescence and can inform on psychiatric disorders that have their onset in adolescence, such as schizophrenia. With a longitudinal twin study design, the genetic and environmental influences on brain and behavior, as well as their developmental changes, can be disentangled.

In this thesis, I show that i) the thickness of the cerebral cortex and its rate of development during adolescence is largely under genetic control, with a new genetic factor marking the transition during adolescence; ii) functional connectivity in the adolescent brain is in part also determined by genes, along with several indications of influences from common environment, that remain largely stable over time. This suggests that the development of the structure of the brain is considerably influenced by genes, while the functioning of the brain is more flexible to adapt to the environment. Patients with schizophrenia experience accelerated aging. I.looked at accelerated aging based on the structure of the brain and epigenetic modifications, and found iii) no clear evidence that these two aging processes are related despite that both are partially dètermined by genes implicated in schizophrenia. This suggests the possibility for two distinct aging processes affecting accelerated aging in patients with schizophrenia.

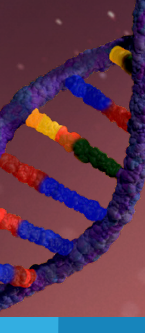

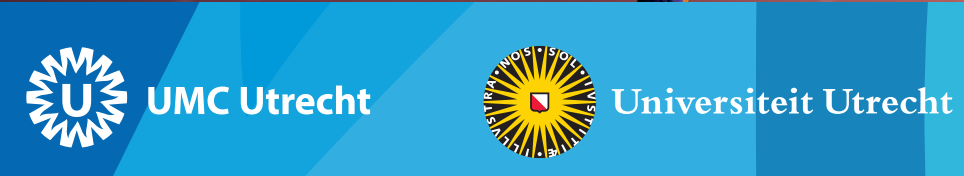

ISBN 978-90-393-7347-7
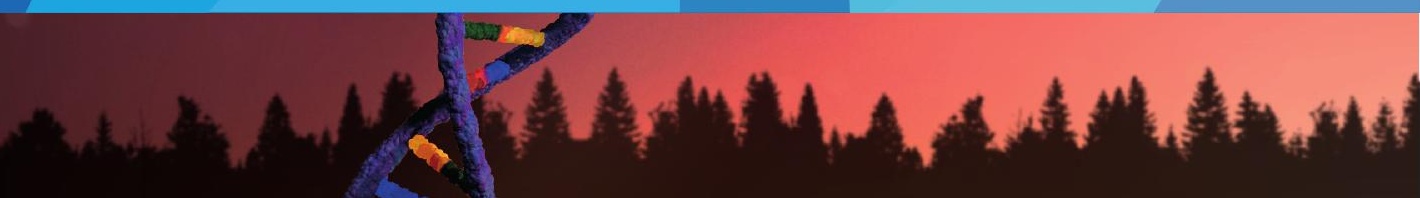